\title{
Monatsschrift
}

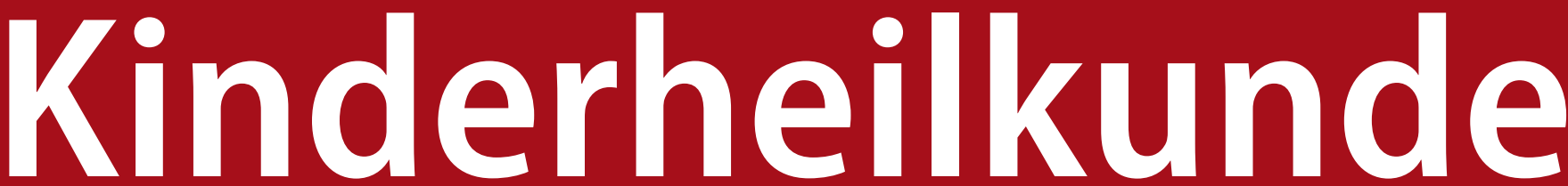

Organ der Deutschen Gesellschaft für Kinder- und Jugendmedizin

Organ der Österreichischen Gesellschaft für Kinder- und Jugendheilkunde

Indexed in Science Citation Index Expanded

\section{Abstracts der 39. Jahrestagung}

der Gesellschaft für Neonatologie und Pädiatrische Intensivmedizin (GNPI)

6.-8. Juni 2013

Freiburg, Konzerthaus
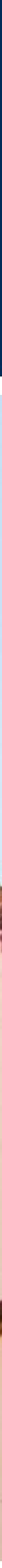

www.MonatsschriftKinderheilkunde.de

Springer Medizin 
Monatsschr Kinderheilkd 2013 · 161:1-93

DOI 10.1007/ s00112-013-2932-5

c) Springer-Verlag Berlin Heidelberg 2013

\section{Abstracts der 39. Jahrestagung der Gesellschaft für Neonatologie und Pädiatrische Intensivmedizin (GNPI)}

\author{
6. bis 8 . Juni 2013 , Freiburg
}

Wissenschaftliche Leitung:

Prof. Dr. Roland Hentschel, Universitätsklinikum

Freiburg, Zentrum für Kinder- und Jugendmedizin

Funktionsbereich Neonatologie / Intensivmedizin

\section{Workshops}

\section{Techniken der genetischen Diagnostik}

\section{WS-6}

Techniken der genetischen Diagnostik

\section{E. Lausch', P. Villavicencio Lorini}

'Universitätsklinikum Freiburg, Zentrum für Kinder- und Jugendmedizin, Freiburg, Deutschland, ${ }^{2}$ Universitaetsklinikum Freiburg, Zentrum für Kinder- und Jugendmedizin, Freiburg, Deutschland

In diesem Workshop werden die Grundlagen der genetischen Diagnostik vermittelt. Die Indikationen, Verfahrensweisen und Aussagemöglichkeiten der zur Verfügung stehenden genetischen Techniken werden demonstriert und an ausgewählten Beispielen diskutiert. Dabei soll die Relevanz eines genetischen Befundes im Hinblick auf daraus abzuleitende Betreuungskonzepte für die Patienten und ihrer Angehörigen beleuchtet werden. Weiterhin werden neue Analyseverfahren des Genoms mit ihren diagnostischen Aussagemöglichkeiten und -grenzen aufgezeigt. Die Teilnehmer erhalten zudem eine Übersicht über den nach Gendiagnostik-Gesetz geforderten Umgang mit genetischen $\mathrm{Be}-$ funden. Es werden Grundkenntnisse über den Aufbau der DNA und das Prinzip der genetischen Informationsspeicherung und Vererbung vorausgesetzt.

\section{Messung von Gewebeoxygenierung und Mikrozirkulation}

\section{WS-14.1}

\section{Was messen wir mit welcher Methode?}

\section{O. Genzel-Boroviczény}

'Klinikum der Universität München Innenstadt, Perinatologie, München, Deutschland

Hintergrund. Bei Neu- und Frühgeborenen wird die Makrozirkulation engmaschig überwacht, wobei die Untergrenze eines normalen Blutdruckes umstritten ist. Letztendlich ist die Gewebsperfusion der entscheidende Parameter, den man aber nicht objektiv direkt messen kann, sondern nur indirekt, wie z. B. durch die Urinausscheidung. Die Messung der Mikrozirkulation steht seit mehr als 20 Jahren im Fokus der Forschung. Die verschiedenen Methoden der Mikrozirkulationsmessungen untersuchen zum Teil sehr unterschiedliche Gefäßbette. Die Ergebnisse können daher nicht direkt verglichen werden und die Fragestellungen müssen der jeweiligen Methode entsprechen. Die diversen Methoden messen von zum Teil Werte aus venösem und arteriellem Blut, mitteln größere und kleine Gefäße oder die Perfusion ganzer Gewebsteile. In diesem Referat werden die diversen Methoden kritisch gegenüber gestellt.

Lernziel. Die unterschiedlichen Methoden kennen und wissen, welche Methode welche Gefäßbette misst.

\section{WS-14.4}

Funktionelle Darstellung der Mikrozirkulation mittels Sidestream Darkfield Imaging

\section{A. Puchwein-Schwepcke', C. Nußbaum², O. Genzel-Boroviczény ${ }^{3}$}

${ }^{1}$ Kinderklinik und Kinderpoliklinik im Dr. von Haunerschen Kinderspital, München, Deutschland, ${ }^{2}$ Walter-Brendel-Zentrum für Experimentelle Medizin, München, Deutschland, ${ }^{3}$ Klinikum der Universität München Innenstadt, Perinatologie, München, Deutschland

In den letzten Jahren konzentrierte sich das Monitoring bei schwerkranken Patienten meist auf makrozirkulatorische Parameter. So lag das Augenmerk auf Werten wie dem mittleren arteriellen Blutdruck, Herzindex, Sauerstoffverbrauch- und -angebot. Neuere Forschungsergebnisse zeigen jedoch, dass insbesondere mikrozirkulatorische Parameter für die Verschlechterung von Zell- und Organfunktionen verantwortlich sind und eine intakte Mikrozirkulation für die Gewebeversorgung und somit für die Organfunktion essentiell ist. Sowohl bei akuten Krankheitsbildern, wie Sepsis und Schock, als auch bei chronischen, wie Diabetes mellitus und peripherer Verschlusskrankheit, finden sich kennzeichnende Veränderungen der Mikrozirkulation. Somit konnte insbesondere bei dem Krankheitsbild der Sepsis die pathogenetische Rolle der mikrovaskulären Dysfunktion nachgewiesen werden. Es wurde gezeigt, dass bei Sepsis die funktionelle Kapillardichte signifikant abfällt, der Fluss in den Kapillaren nicht mehr gewährleistet ist, und eine vermehrte Heterogenität in der Perfusion von verschiedenen Geweben festzustellen ist. Eindrücklich ist auch, dass 
in manchen Fällen eine stark eingeschränkte Mikrozirkulation nachgewiesen werden konnte, obwohl die Labor- und Sauerstoffparameter augenscheinlich normal waren.

Die Sidestream-Darkfield(SDF)-Imaging-Technik ermöglicht die nichtinvasive Untersuchung der Mikrozirkulation bei Neu- und Frühgeborenen. Die SDF-Imaging-Technik beruht auf Leuchtdioden, welche grünes Licht mit einer Wellenlänge von $530 \mathrm{~nm}$ aussenden. Es durchdringt die Haut und trifft auf das oberflächliche Kapillarnetz. Zur Bilddarstellung kommt es, weil Licht dieser Wellenlänge von dem Hämoglobin der Erythrozyten absorbiert aber von anderen Strukturen reflektiert wird. Reflektiertes Licht wird durch das Mikroskop wieder aufgefangen. Eine Linse mit 5-facher Vergrößerung projiziert das Licht auf eine Videokamera. Das durch die Erythrozyten absorbierte Licht ist auf der Videosequenz schwarz, jenes durch die Reflektion hingegen als weiß dargestellt. Somit werden nur durchblutete Gefäße des Kapillarnetzes abgebildet - ein funktionelles Bild entsteht.

Die horizontale Anordnung des Gefäßnetzes und die dünne Hautschicht ermöglichen bei Neugeborenen und Frühgeborenen die Verwendung der SDF-Imaging-Technik an vielen Stellen der Hautoberfläche - im Gegensatz zu Erwachsenen, wo diese überwiegend sublingual verwendet werden muss. Somit bietet SDF-Imaging eine nichtinvasive Darstellung der Mikrozirkulation. Als Parameter werden die funktionelle Gefäßdichte und die Durchmesserverteilung der Gefäße untersucht, sowie die Unterteilung der Qualität des Flusses in kontinuierlich, hyperdynam, sluggish oder keinen Fluss.

Unter der Verwendung dieser Technik konnten wir einerseits den Einfluss von Infektionen bei Neugeborenen auf die Mikrozirkulation, die Auswirkungen eines persistierenden Ductus arteriosus, sowie Veränderungen unter der Gabe von Erythrozytenkonzentraten nachweisen. SDF-Imaging stellt somit eine vielversprechende Methode zum Monitoring der Mikrozirkulation bei Neu- und Frühgeborenen dar.

\section{WS-14.5}

\section{Beurteilung der mikrovaskulären Glykokalyx bei Kindern mittels Sidestream Darkfield Imaging}

\section{Nussbaum', O. Genzel-Boroviczény²}

'Walter-Brendel-Zentrum für Experimentelle Medizin, München, Deutschland, ${ }^{2}$ Klinikum der Universität München Innenstadt, Perinatologie, München, Deutschland

Hintergrund. Die endotheliale Glykokalyx hat in den letzten Jahren zunehmend an wissenschaftlichem und klinischem Interesse gewonnen aufgrund ihrer entscheidenden Bedeutung für die vaskuläre Funktion und Homöostase. Diese komplexe Struktur bestehend aus einem Netzwerk von Proteoglykanen, Glykoproteinen, Glykosaminoglykanen und assoziierten Plasmaproteinen kleidet das gesamte Gefäßsystem aus und kann eine Dicke von mehreren Mikrometern erreichen. Als Grenzschicht zwischen dem Blutstrom und dem Endothel nimmt sie eine zentrale Stellung bei verschiedenen (patho-)physiologischen Prozessen im Gefäßbett ein. So reguliert sie die Permeabilität der Gefäßwand, beeinflusst die rheologischen Eigenschaften der Mikrozirkulation, fungiert als Sensor für Schubspannung und übt antiadhäsive und vaskuloprotektive Effekte aus. Eine Schädigung oder ein vermehrter Abbau („shedding“) der Glykokalyx, wie sie beispielsweise im Rahmen von Sepsis, Ischämie/Reperfusion oder Hyperglykämie beobachtet werden können, beeinträchtigen die dargestellten Funktionen und scheinen den Ausgangspunkt für pathologische Gefäßveränderungen darzustellen.

Methoden. Um die physiologischen Eigenschaften der Glykokalyx genauer zu verstehen und eine mögliche Beteiligung bei der Entstehung vaskulärer Erkrankungen aufzudecken, ist es notwendig Veränderungen der Glykokalyx in vivo messbar zu machen. Mit der Entwicklung des Sidestream Darkfield (SDF) Imaging besteht eine nichtinvasive Möglichkeit zur Darstellung der Mikrozirkulation bei Kindern ver- schiedener Altersstufen, die unter anderem auch eine indirekte Quantifizierung der mikrovaskulären Glykokalyxdicke ermöglicht.

Ergebnisse. Unter Verwendung dieser Technologie konnten wir zeigen, dass es bei Kindern mit Diabetes mellitus Typ I im Vergleich zu gesunden Kontrollen bereits in einem frühen Krankheitsstadium zu einem signifikanten Verlust der Glykokalyx in sublingualen Kapillaren kommt, welcher mit der Höhe der Blutzuckerspiegel korreliert.

Schlussfolgerung. SDF-Imaging stellt somit eine vielversprechende Methode zum Monitoring der Mikrozirkulation und zur Beurteilung der mikrovaskulären Glykokalyx bei Kindern dar.

\section{Vorträge}

\section{Pflege}

\section{PV-02}

\section{Hypothermie nach Reanimation}

\section{T. Lauterbach}

"Universitäts - Kinderklinik, Pädiatrische Intensivstation „Keller", Freiburg, Deutschland

Hintergrund. Die therapeutische Hypothermie ist eine weit verbreitete und praktizierte Begleittherapie, nach erfolgreicher Reanimation. Sie ist eine Maßnahme, um das neurologische Outcome von Patienten positiv zu beeinflussen.

Fragestellung. Was sind die Beweggründe, wo liegen die Risiken und wie sieht die Durchführung dieser Maßnahme im Detail aus? Was besagen die aktuellen Empfehlungen und wie sieht die Zukunft aus?

Methoden. Für die Pädiatrie gibt es kaum konkrete eigene Studien oder Daten zum Thema therapeutische Hypothermie. Handlungsrichtlinien sind denen der Erwachsenentherapie angelehnt. Das Ausmaß des Hirnschadens nach einer Reanimation wird von mehreren Faktoren beeinflusst. Dabei spielen der Grad der Hypoxie, der Reperfusionsschaden und die Apoptose (programmierter Zelltod) eine Rolle. Während der ischämische Hirnschaden nicht mehr behoben werden kann, so kann doch der weitere Verlauf durch eine gezielte Hypothermie positiv beeinflusst werden. Der Zeitpunkt der Induktion, die Art der Kühlung, deren Überwachung und die Kontrolle der Körpertemperatur sind zu beachtende Faktoren. Das Beurteilen der Blutgasanalysen, die Steuerung der Beatmung und die engmaschige Kontrolle des Patienten sind Aufgabenfelder des pflegerischen und ärztlichen Personals.

Ergebnisse. Der positive Effekt von Hypothermie wird immer häufiger auch bei anderen Krankheitsbildern genutzt. Darüber hinaus wird die therapeutische Hypothermie in Kombination mit weiteren Maßnahmen zur Neuroprotektion verwendet. Auch der Grad der notwendigen Sedierungstiefe ist ein Bereich, der erforscht wird.

Schlussfolgerung. Eine milde Hypothermie ist sinnvoll zur Verbesserung des neurologischen Outcomes der Patienten. Die Therapie bedarf vielseitigen Fachwissens und einer guten Überwachung seitens des Intensivteams.

\section{PV-10}

Fütterstörungen und Sondenabhängigkeit nach Früh- und Risikogeburt

\section{Jotzo', S. Becker', B. Lettgen ${ }^{2}$}

'Pädiatrisches Dysphagiezentrum der Darmstädter Kinderkliniken Prinzessin Margaret, Darmstadt, Deutschland, ${ }^{2}$ Darmstädter Kinderkliniken Prinzessin Margaret, Darmstadt, Deutschland

Kinder nach Früh-/Risikogeburt, mit angeborenen Fehlbildungen, Stoffwechsel- oder neurologischen Störungen haben ein erhöhtes Risiko für Fütterstörungen mit oder ohne Sondenabhängigkeit nach Wegfall der medizinischen Indikation. Meist verläuft der orale Nahrungsauf- 
bau bei intensivmedizinisch behandelten Früh-/Risikogeborenen problemlos. Doch treten immer öfter während oder nach dem stationären Aufenthalt massive Fütterprobleme bei Kindern auf, zum Teil bleiben sie ohne medizinische Indikation dauerhaft von der Nahrungssonde abhängig. Insbesondere bei Sondenabhängigkeit nach Entlassung des Kindes aus der Klinik ist die emotionale Belastung der Familien mit betroffenen Kindern hoch, auch die Alltagsgestaltung ist bei einer starken Symptomausprägung ist stressreich und frustrierend.

Der Beitrag geht auf Definition und diagnostische Kriterien der Fütterstörung ein. Erläutert wird, wie sich die Fütterstörung ausbildet und verfestigt, welche Symptome bei einer Fütterstörung auftreten und welche Auswirkungen Fütterstörungen auf Kinder und auf ihre Familien haben.

Dargestellt werden Präventionsansätze in der Neonatologie und den sich zeitlich anschließenden Professionen sowie ein Ansatz interdisziplinärer Diagnostik und Therapie für Kinder mit Fütterstörungen bzw. Sondenabhängigkeit, der auch auf die Belastungen der Eltern eingeht.

\section{PV-11}

\section{Entwicklungssignale Frühgeborener und deren Bedeutung für die frühe Eltern-Kind-Interaktion}

\section{P. Hinner', M. Sommerfeld ${ }^{2}$}

${ }^{1}$ Klinik und Poliklinik für Kinder- und Jugendmedizin, Universitätsklinikum Carl Gustav Carus Dresden, Dresden, Deutschland, ${ }^{2}$ Universitätsklinikum Carl Gustav Carus Kinderklinik, Neonatologie/Päd. Intensivmedizin, Dresden, Deutschland

Die Verbesserung des Entwicklungsoutcomes hinsichtlich neurologischer als auch sozioemotionaler Aspekte rückt in den letzten Jahren bei der Behandlung zu früh geborener Kinder vermehrt in den Fokus der neonatologischen Forschung. Das Interesse gilt unter anderem Angeboten aus dem Kontext der psychosozialen Betreuung der Familien, welche die elterliche Feinfühligkeit und die Eltern-Kind-Interaktion thematisieren. Grundlage für diese Bestrebungen bilden insbesondere Unterschiede in Qualität und Quantität der Entwicklungssignale Frühgeborener gegenüber reifgeborenen Säuglingen. Im Zusammenhang mit der bereits per se belastenden Situation Frühgeburt, wird den Eltern sowohl die Wahrnehmung und Interpretation als auch das angemessene Reagieren auf die Feinzeichen des frühgeborenen Kindes erheblich erschwert.

Der Vortrag bietet basierend auf Videomitschnitten Einblicke in die Klassifizierung von Entwicklungssignalen zu früh geborener Kinder. Weiterführend wird auf die damit assoziierten Herausforderungen für das Etablieren der frühen Eltern-Kind-Interaktion eingegangen. Anhand von Praxisbeispielen aus dem FamilieNetz Dresden wird erläutert, wie Eltern für diese Feinzeichen der Kinder sensibilisiert, im Aufbau einer entwicklungsfördernden Interaktion mit ihrem Kind unterstützt und in ihrem elterlichen Kompetenzerleben gestärkt werden können. Gleichwohl werden dabei Möglichkeiten aufgezeigt, Kompetenzen aus den Professionen Pflege und psychosozialer Beratung für eine adäquate Betreuung und Anleitung der Eltern zusammen zu bringen.

Die Verbesserung des kurz- wie langfristigen neurologischen als auch sozioemotionalen Entwicklungsoutcomes rückt in den zurückliegenden Jahren bei der Behandlung zu früh geborener Kinder vermehrt in den Fokus neonatologischer Forschung. Eine besondere Beachtung finden dabei die unterschiedlichen Angebote psychosozialer Betreuung der Familien, in deren Mittelpunkt die elterliche Feinfühligkeit und die Eltern-Kind-Interaktion stehen. Ein Grund hierfür liegt in der eingeschränkten Qualität und Quantität der Entwicklungssignale Frühgeborener gegenüber reifgeborenen Säuglingen. Im Zusammenhang mit der bereits per se belastenden Situation der Frühgeburt wird den Eltern sowohl die Wahrnehmung und Interpretation als auch das angemessene Reagieren auf die Feinzeichen des frühgeborenen Kindes erheblich erschwert.
Der Vortrag bietet basierend auf Videomitschnitten Einblicke in die Klassifizierung von Entwicklungssignalen zu früh geborener Kinder. Weiterführend wird auf die damit assoziierten Herausforderungen für das Etablieren der frühen Eltern-Kind-Interaktion eingegangen. Praxisbeispiele aus dem FamilieNetz Dresden machen deutlich, wie Eltern für diese Feinzeichen der Kinder sensibilisiert, im Aufbau einer entwicklungsfördernden Interaktion mit ihrem Kind unterstützt und in ihrem elterlichen Kompetenzerleben gestärkt werden können. Hierbei ergeben sich neue Möglichkeiten des Zusammenwirkens der Bereiche der Kinderkrankenpflege und der psychosozialen Beratung, um die Betreuung und Anleitung der Eltern sicherzustellen und zu optimieren.

\section{PV-12}

Babyschalenanpassung für Früh- und Neugeborene als Bestandteil der Entlassungsroutine

\section{Diekerhoff}

'Kinderklinik Datteln - Neonatologie, Datteln

Jeden Tag werden in Deutschland Frühgeborene nach Hause entlassen, die in herkömmlichen Rückhaltesystemen der Gruppe o+ nur mit zusätzlichen Hilfsmitteln zu sichern sind. Oft wird dieses Problem nicht erkannt, oder Eltern und Pflegepersonal sind überfordert und greifen zu inadäquaten Maßnahmen. Dieser Umstand führt dazu, dass Frühgeborene im Straßenverkehr sehr häufig nur unzureichend gesichert sind und während der Autofahrt in eine Position rutschen, die ihnen die Atmung erschwert (• Abb. 1). Ausgehend von umfangreichen Studien zu diesem Thema ist es deshalb in den USA Pflicht, dass Neonatologien, die Frühgeborene behandeln, sogenannte „car seat fitter" beschäftigen, deren Aufgabe es ist, die Eltern fachkundig zu beraten, damit das Frühgeborene sicher im Auto transportiert werden kann.

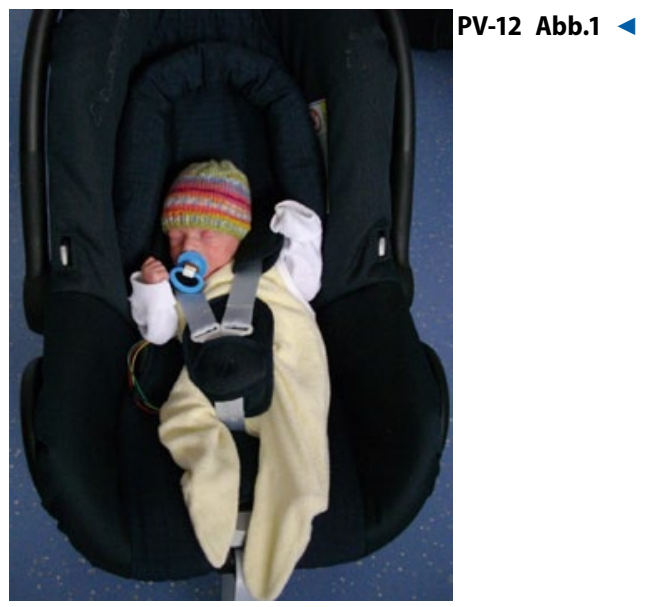

Gemeinsam mit europäischen Verkehrssicherheitsexperten und Herstellern von Babyschalen haben Mitarbeiter der Vestischen Kinder- und Jugendklinik die Empfehlungen der amerikanischen Gesellschaft für Pädiatrie europäischen Sicherheitsstandards angepasst und Informationsmaterial für Eltern entwickelt. Seit 2009 führen wir Babyschalenanpassung in unserer Abteilung durch. Für dieses Engagement wurde die Vestische Kinder- und Jugendklinik Universität Witten/Herdecke 2010 in die Liste „Beispielhaftes Projekt Landesinitiative Gesundes Land NRW“ aufgenommen und im Jahr 2012 mit dem „Roten Ritter" geehrt. Damit Eltern fachkundige Hilfe bekommen, haben wir in 2012 mit Unterstützung der Hersteller und in Kooperation mit Verkehrssicherheitsexperten einen Flyer zu diesem Thema entwickelt und bieten ab Sommer 2013 Schulungen zu diesem Thema an.

Die Probleme bei der Nutzung unterschiedlicher Babyschalen werden dargestellt und die korrekte Anpassung, sowie das Schulungskonzept im Vortrag erläutert und diskutiert. 


\section{PV-13}

\section{Umsetzung von Hygienemaßnahmen bei MRSA-Ausbrüchen}

\section{N. Frömming-Doll \\ 'Universitätsspital beider Basel, Basel, Schweiz}

MRSA-Ausbrüche können jederzeit und überall passieren und stellen hohe Anforderungen an Patienten und Personal. Massnahmen müssen strikt und unverzüglich umgesetzt und eingehalten werden, um einen Ausbruch schnell einzudämmen. Hygienisches Fachpersonal muss vorhanden sein um vorzubeugen, zu schulen und um rechtzeitig einzugreifen.

Eine wirksame Prävention der Übertragung von potentiell pathogenen (empfindlichen und resistenten) Erregern kann nur erreicht werden, wenn jeder Patient als besiedelt betrachtet wird und wenn im Umgang mit jedem Patienten die Standard-Hygienemaßnahmen beachtet werden. In erster Linie ist hier eine hinreichende und effektive Händehygiene, d. h. in der Regel Händedesinfektion nach den fünf Indikationen der WHO zu nennen. Der sinnvolle Gebrauch von Einmal-Handschuhen, d. h. Wechsel der Handschuhe nach Beendigung der Tätigkeit, für die man sie benötigt hat. Aber auch das Tragen von Schutzkleidung bei entsprechenden Tätigkeiten (Schutzkittel/Schürze und Mund-Nasen- und Augen-Schutz) sowie die regelmäßige Flächendesinfektionen von Handkontaktflächen, Materialien und Geräten sind ein wichtiger Bestandteil der Transmissionsprävention. Wesentlich weniger wichtig ist die Arbeitskleidung, die aber natürlich sauber sein soll. Wenn diese Standard-Hygienemaßnahmen nicht ausreichend beachtet werden, helfen weder Einzelzimmer noch Screening-Untersuchungen. Das Problem der Übertragung multiresistenter Erreger, wie MRSA, kann zu einem wesentlichen Teil nur durch Verbesserung der hygienischen Grundversorgung aller Patienten gelöst werden, nicht durch ausgetüftelte „Isolierungsmaßnahmen“.

In der Kinderheilkunde sollte außerdem immer die ganze Familie mit einbezogen werden. Hier ist es wichtig „Familienprävention“ zu betreiben und die Eltern über Maßnahmen wie sie ihr Kind und sich selbst schützen können aufzuklären.

Ein Personalscreening bedarf gründlicher Überlegungen im Vorfeld. Es muss gut geplant sein und die möglichen Konsequenzen müssen den Mitarbeitern transparent gemacht werden. Ein mit MRSA besiedelter Mitarbeiter, besonders wenn er direkten Kontakt zum Patienten hat muss eine strapaziöse Prozedur über sich ergehen lassen. Wenn die Dekolonisierung versagt, kann das verheerende berufliche Auswirkungen haben.

\section{PV-14}

Binasale CPAP-Beatmung - eine effektive Therapiealternative zur invasiven Beatmung in der Neonatologie, aber auch eine pflegerische Herausforderung

\section{B. Schröder ${ }^{1}$}

'Universitätsklinikum Schleswig Holstein, Kinderklinik, Lübeck, Deutschland

Hintergrund. Seit vielen Jahren hat sich die nichtinvasive Therapie des Atemnotsyndroms Frühgeborener mit der binasalen CPAP Beatmung in Deutschland etabliert und es gibt eine Vielzahl an Applikationssystemen auf dem Markt. Keine andere Beatmungsform fällt so sehr in den pflegerischen Bereich, von Seiten der Ärzte wird die Therapie verordnet, aber die Durchführung obliegt allein der verantwortlichen Pflegekraft. Die Versorgung dieser Kinder ist oftmals eine große pflegerische Herausforderung. Die Haut ist dünn und extrem empfindlich, Maske oder Prongs müssen im Gesicht fixiert werden, die Gefahr von Hautirritationen bis hin zu Druckstellen und sogar Nekrosen im Nasenbereich ist groß. Um einen möglichst guten therapeutischen Effekt zu erzielen muss der PEEP aufrechterhalten werden. Wissenschaftliche Publikationen zu den pflegerischen Aspekten sind jedoch kaum zu finden. Das war für mich ausschlaggebend, meine Bachelor-Thesis diesem Thema zu widmen.

Fragestellung. Haben Pflegekräfte in anderen Kliniken ähnliche Probleme? Verwenden alle binasale Systeme oder kommt der mononasale CPAP über einen gekürzten Tubus noch zum Einsatz? Wechseln die Anwender zwischen Maske und Prongs, um eine Druckpunktverlagerung zu erzielen? Was ist der optimale Personalschlüssel für eine adäquate Versorgung dieser Patienten?

Material und Methoden. Die oben genannten pflegerisch relevanten Probleme wurden anhand einer deskriptiven, explorativen Studie wissenschaftlich hinterfragt. 41, im Netzwerk der GNN integrierte, Kliniken wurden kontaktiert und jeweils drei Fragebögen an jede neonatologische Intensivstation versandt. Die Bögen wurden per SPSS ausgewertet und die Ergebnisse in Diagrammen dargestellt.

Ergebnisse. Insgesamt $49 \%$ verwenden nur noch ein binasale CPAPSystem. Die größte pflegerische Herausforderung gaben die Befragten an, sei die Vermeidung von Druckstellen $(63,7 \%)$, gefolgt von der Aufrechterhaltung des PEEPs (45\%). 17,7\% gaben an „häufig“ Probleme mit Hautirritationen zu haben, 55,8\% haben "gelegentlich “ angekreuzt und $26,5 \%$ gaben an ,selten“ Hautprobleme zu haben. Wichtigstes Kriterium zur Vermeidung von Druckstellen ist die richtige Größe von Maske und Prongs (90,4\%), gefolgt von einem routinierten Handling/Schulung des Personals $(84,8 \%)$ sowie dem Wechsel von Maske und Prongs $(68,8 \%)$. Zwei bis maximal drei Kinder können von einer Pflegekraft versorgt werden, sind die Patienten aber sehr instabil kann auch eine 1:1 Betreuung notwendig sein.

Schlussfolgerung. Der hohe Rücklauf von über $85 \%$ verdeutlicht die Relevanz dieses Themas im pflegerischen Bereich. Aufgrund der Umfrage konnten die Hypothesen abgeleitet werden, dass sowohl die richtige Wahl der Größe von Maske und Prongs und deren Wechsel sowie die adäquate Schulung des Personals Hautdefekte vermeiden oder zumindest minimieren können.

\section{PV-18}

Achtsamer Umgang mit Sterben und Tod auf pädiatrischen Intensivstationen

\section{J. Schirrmeister', R. Abel', D. Vlajnic', M. Sticker', J. Breuer}

'Universitätsklinikum Bonn, Zentrum Kinderheilkunde, Bonn, Deutschland, ${ }^{2}$ Universitätsklinik Zentrum f. Kinderheilkunde, Bonn, Deutschland

In vielen Bereichen der Medizin ist durch Fortschritte der Palliativbetreuung häufig ein Sterben zu Hause möglich. Dennoch gehört Sterben und Tod weiterhin zum klinischen Alltag auf pädiatrischen und neonatologischen Intensivstationen. Bei kritisch herzkranken Kindern - oder extrem Frühgeborenen - wird, um Heilung zu ermöglichen, maximale Intensivtherapie eingesetzt, z. B. Katecholamintherapie, Oszillationsbeatmung, extrakorporale Membranoxygenierung. Misslingt dies, sind die Kinder nicht entlassbar und versterben auf der Intensivstation, oft mit einem „Abschalten“ der Maschinen. Für Kind, Familie und Behandlungsteam ist solch ein Sterbeprozess sehr belastend. Es führt bei den Angehörigen oft zu anhaltenden Depressionen, bei den Behandlern häufig zu Schuldgefühlen und Burn-out-Symptomatik. Im Pflegebereich oft zu Stationswechsel oder Kündigung.

Mit meinem Vortrag möchte ich dazu beitragen, ein Qualitätsbewusstsein für diesen Aspekt klinischer Behandlung zu entwickeln. Sterben auf pädiatrischen Intensivstationen kann und soll human und würdevoll geschehen. Dadurch wird der Trauerprozess der Familie eingeleitet. Dies und weitere psychohygienische Maßnahmen erhalten eine gute Arbeitsatmosphäre und die Gesundheit beim Team.

Am Beispiel unserer Intensivstation stelle ich dar, wie wir eine Sterbekultur etabliert haben. Dies begann mit Engagement und Weiterbildung einzelner Teammitglieder in Palliativmedizin inkl. Selbsterfahrung und Persönlichkeitsentwicklung. Daraus entwickelten sich Verbesserungen der Klinikstrukturen und -abläufe. Diese beinhalten auch räumliche Verbesserungen, (Verabschiedungsraum/Gedenkbaum). 
Gestaltung und Umgang mit dieser Problematik wird wenig gelehrt. Im letzen Jahr haben meine Frau Renate Abel und ich Fortbildungsseminare für Behandlungsteams päd. Intensivstationen entwickelt, um die Ausbildung in diesem Bereich zu verbessern. Eine größere Arbeitszufriedenheit und positive Rückmeldung der Eltern und Teammitgliederbestätigen uns in unserer Arbeit.

Schlussfolgerung. Ebenso wie andere Aspekte pädiatrischer Intensivmedizin, benötigt gute klinische Sterbebegleitung ein geregelte Strukturen und Abläufe, die geübt werden müssen.

1. Kappstein I (1999) Erläuterungen zu den RKI-Empfehlungen bei Nachweis von MRSA

\section{Das gesunde Neugeborene}

\section{V-04}

\section{Jaundice in the newborn}

\section{Bratlid \\ 'NTNU-Trondheim Norwegian University of Science and Technology, Department of Laboratory Medicine Children's and Womens Health, Trondheim, Norwegen}

Neonatal hyperbilirubinemia affects nearly all newborns. Most hyperbilirubinemic infants will be visibly jaundiced during the second day of life when serum bilirubin (TSB) reaches $70-130 \mu \mathrm{mol} / \mathrm{L}$. Twenty per cent of all newborns will have TSB measured and 5-10\% all newborns will be treated with phototherapy.

This physiologic hyperbilirubinemia is mainly caused by increased production and reduced excretion of bilirubin. It can, however, be calculated that with no bilirubin excretion, TSB levels would by the 3 rd postnatal day have reached $>1000 \mu \mathrm{mol} / \mathrm{L}$. Since average TSB in normal newborns then is around $200 \mu \mathrm{mol} / \mathrm{L}$, even significantly jaundiced newborns excrete most of their bilirubin load.

Assessment of jaundice typically begins with the mother or caregivers recognizing the yellow color of the skin which develops in a cranio-caudal extension. Physiologic jaundice usually peaks at day 3 to 4 . Visible jaundice $<24$ hours of life should always be considered "pathologic". Transcutaneous bilirubinometry $(\mathrm{TcB})$ correlates reasonably well with TSB, but should not be used alone as basis for treatment.

The distinction between physiologic and pathologic hyperbilirubinemia is not clear. It can be helpful to compare the situation with measuring the height of a mountain topped by an ice cap. It is difficult to tell which height is related to the mountain (physiology) and which is the ice cap (pathology). Early onset jaundice with TSB $100-200 \mu \mathrm{mol} / \mathrm{L}$ is usually caused by hemolytic diseases. Hyperbilirubinemia during days 1-10 in the range of $200-400 \mu \mathrm{mol} / \mathrm{L}$ can in addition be caused by inborn errors of metabolism, endocrine disorders or breast milk jaundice. In infants who remain jaundiced after 10 days cholestasis should be considered.

Most infants can be treated with phototherapy, also immune hemolytic diseases. In such cases intravenous immunoglobulins can also be used, exchange transfusion is rarely indicated. All treatment indications are strictly empirical and based on TSB in combination with gestational age, birth weight, postnatal age, rate of TSB increase, and presence of risk factors. Treatment with tin-mesoporphyrin to reduce bilirubin production by inhibition of heme-oxygenase has only been used in small trials and has not yet been approved.

The mechanisms of bilirubin-induced neurologic dysfunction (BIND) remain elusive. Some term or near-term infants appear to tolerate TSB levels of $5-600 \mu \mathrm{mol} / \mathrm{L}$ without harm, while others may suffer permanent damage at much lower levels. Recent studies show that TSB-levels as high as $400-450 \mu \mathrm{mol} / \mathrm{L}$ are not harmful as long as the infant does not show signs of acute bilirubin encephalopathy. Treatment indications should, however, be much lower than this.

All infants should be assessed for jaundice at discharge, particularly when discharged before 24-48 hours of life. A TSB should be added when a jaundiced infant returns for the metabolic screen. Parents of infants with risk factors (large cephalhematoma, phototherapy of previous sibling, late preterm infants, Asian ethnicity) should be told to return if infant becomes more jaundiced or feeds poorly.

Since bilirubin has significant effects on cellular functions, hyperbilirubinemia and/or phototherapy has been linked to later development of other conditions. Based on research findings it seems difficult to conclude on any causative relationships between physiologic hyperbilirubinemia and later development of conditions like cow's milk intolerance, diabetes, asthma, melanocytic nevi and autism spectrum disorders.

\section{Sauerstoff-Therapie und Monitoring}

\section{V-07 \\ Messung der zerebralen und peripheren Sauerstoffsättigung - welche Erkenntnisse bringt sie für die tägliche Praxis?}

\section{B. Urlesberger \\ 'Universitätsklinik für Kinder- und Jugendheilkunde, Abteilung für Neo- natologie, Graz, Österreich}

Hintergrund. Die Messung der regionalen Sauerstoffsättigung ist eine Domäne der Nahinfrarotspektroskopie (NIRS). Sie kann grundsätzlich in jedem Gewebskompartiment des menschlichen Körpers durchgeführt werden. Aufgrund der entwicklungsbedingten Besonderheiten ist der Schädel von Früh- und Neugeborenen für die NIRS-Anwendung besonders gut geeignet. Da das entwicklungsneurologische Ergebnis weiterhin der relevanteste Parameter der Ergebnisqualität neonatologischer Therapie ist, hat sich die NIRS-Forschung in den letzten 20 Jahren besonders intensiv mit zerebralen Messungen beschäftigt.

Methoden. Wie Eingangs erwähnt, ist natürlich jedes Gewebskompartiment der NIRS-Technologie zugänglich, jedoch sicherlich mit unterschiedlich guter Messqualität. Ausgehend von der Messtechnik ist Luft das größte Messhindernis, aber auch größere Gefäße mit hohem Flussraten sind problematisch, da viele Photonen im Blutfluss mitgerissen werden. Wichtig für die Messqualität ist auch eine Konstanz in der Gewebszusammensetzung während der Messung, sodass Veränderungen NIRS-Parameter wirklich zuverlässig auf Änderungen der Oxygenierung oder Perfusion zurück zuführen sind. Somit eignen sich solide Gewebskompartimente gut, ganz schlecht eigenen sich Organe mit Hohlräumen, wie z. B. der Darm.

Ergebnisse. Trotz vielversprechender Studienergebnisse hat sich die NIRS Technologie jedoch über die Jahre nicht zu einem Routine-Monitoring im klinischen Sinn entwickelt. Das größte Hindernis hierbei liegt in der Messgenauigkeit. Diese ist trotz Verbesserungen noch immer nicht groß genug, um beim Individuum im klinischen Einsatz als Entscheidungsbasis dienen zu können. Somit bleibt vor allem der Trend als Verlaufsparameter im Fokus klinischen Arbeitens. Bei der Beurteilung von Veränderungen im Trend bedarf es eines geänderten klinischen Zugangs mit Detailwissen um die möglichen Einflussgrößen. Dies erschwert die Interpretation von Veränderungen der regionalen Sättigung.

Schlussfolgerung. Derzeit gibt es einige Konzepte zur Anwendung von NIRS als Monitoring-Tool, es handelt sich hierbei vor allem um prospektive wissenschaftliche Beobachtungsstudien. Außerhalb von Studienprotokollen erscheint ein klinischer Einsatz derzeit problematisch. Im Sinne der Evidenz Based Medicine stellt sich grundsätzlich die Frage, welchen beweisbaren Vorteil bringt der Einsatz von NIRS im Bereich der Neonatologie. Jetzt ist die letzte Möglichkeit sich dieser Herausforderung zu stellen. In einigen Jahren wird es zu spät sein, und die Gefahr eines unkritischen klinischen Einsatzes dieser Technologie kann dann Realität werden. 


\section{Anämie - Hämotherapie - Transfusionsmedizin}

\section{V-13}

\section{Pathophysiologie der neonatalen Anämie}

\section{A. Pollak \\ 'Universitätsklinik für Kinder- und Jugendheilkunde Wien, Abteilung für Allgemeinpädiatrie und Neonatologie, Wien, Österreich}

Der Begriff neonatale Anämie beschreibt das - schon seit über einem Jahrhundert bekannte - Phänomen des Absinkens des Hämoglobins von 14,6-22,5 g/dl post partum auf 10.0-12.0 g/dl in der 8.-10. Lebenswoche. Bei Frühgeborenen erfolgt dieser Abfall wesentlich schneller und auf tiefere, meist auch von kardiorespiratorischen Symptomen begleitete Werte um 7.0-8.0 g/dl. Die Frühgeborenenanämie (FA) stellt besonders für extrem unreife Frühgeborene (unter der 28. SSW) ein klinisch relevantes Problem dar, zumal die meisten dieser Patienten in der Phase ihrer initialen intensivmedizinischen Versorgung nach wie vor zumindest eine Bluttransfusion erhalten. Für die FA wird eine Vielzahl "physiologischer" wie pathologischer und iatrogener Faktoren ursächlich diskutiert. Inadäquate Erythropoietin-Produktion (King et al., J Pediatr 1996) und -Sensitivität (niedriger Schwellenwert des O2-Sensors: Dallman; J Pediatr 1984), rasches postnatales Wachstum bei unzureichender Zufuhr von Protein, Vitaminen und Kalorien (Haiden et al., Pediatrics 2006) sowie im Besonderen Eisen (Pollak et al., Pediatrics 2001) und eine verminderte Erythrozyten-Überlebenszeit (Widness et al., PAS Denver 2011) sind ätiologisch bedeutsam.

Andererseits spielen trotz aller Bemühungen wie restriktive Blutabnahmeprotokolle, Inline-Blutgasanalyse-Katheter (Widness et al., Pediatrics 2005) etc. iatrogene Blutverluste nach wie vor eine entscheidende Rolle, weiters Blutungen, Infektionen, Sepsis, nekrotisierende Enterokolitis etc.

Bis zur Entwicklung weiterer „Nanotechnologien“ in der Labormedizin ist es wichtig, den pathophysiologischen Hintergrund der FA zu verstehen, um entsprechende präventive und therapeutische Maßnahmen zu ergreifen.

\section{Perinatologie}

\section{V-17 \\ Perinatale Programmierung - der Effekt von diabetischer Feto- pathie und IUGR \\ G. Desoye \\ 'Universitätsklinik für Frauenheilkunde und Geburtshilfe, Medizinische Universität, Graz, Österreich}

Experimentelle und epidemiologische Daten zeigen, dass intrauterine Vorgänge langfristige Auswirkungen auf die Gesundheit der Neugeborenen und Kinder haben, die bis ins Erwachsenenalter reichen. Auf dieser Basis wurde das Konzept der „Perinatalen Programmierung" entwickelt (DOHAD - Developmental Origins of Health and Adult Disease). Da intrauterine Vorgänge schwer zu messen bzw. erfassen sind, wurde das Geburtsgewicht als Annäherung genommen. Es soll den Effekt des intrauterinen Milieus auf den heranwachsenden Feten abbilden. Auf dieser Basis wurde gezeigt, dass ein Zusammenhang besteht zwischen Geburtsgewicht und den Risken für hypertensive Erkrankungen, Diabetes mellitus, und Adipositas später im Leben. Dieser Zusammenhang zwischen Geburtsgewicht und späterem Krankheitsrisiko ist in den meisten Fällen U-förmig, d. h. ein zu niedriges oder zu hohes Geburtsgewicht ist mit einem höheren Risiko assoziiert.

Schwangerschaftsprobleme, die zu erhöhtem Geburtsgewicht führen, sind Gestationsdiabetes und Adipositas der Mutter („diabesity“ - „diabetes and obesity"). Das Charakteristikum der Neugeborenen solcher Schwangerschaften, ist ein disproportional erhöhter Fettgehalt, un- abhängig vom Geburtsgewicht. Nach einem weit gehend akzeptierten Konzept, ist eine erhöhte fetale Insulinkonzentration verantwortlich für diese exzessive Fettakkumulation im Feten. Fetaler Hyperinsulinismus am Anfang der Schwangerschaft erhöht das Risiko des Feten für ein erhöhtes Geburtsgewicht. Fetaler Hyperinsulinismus im dritten Schwangerschaftstrimester ist assoziiert mit einem erhöhten Risiko für reduzierte Insulinsensitivität und Entwicklung von Adipositas im Kindesalter. Mechanistisch wird derzeit spekuliert, dass der fetale Hyperinsulinismus zu einer Veränderung des Epigenoms im Hypothalamus führt, was das Hungergefühl der Neugeborenen und der Kinder und damit auch ihre Essgewohnheiten beeinflussen könnte.

Das erhöhte Krankheitsrisiko bei kleinen Kindern bzw. Kinder mit geringem Geburtsgewicht wird mechanistisch nicht wirklich verstanden. Eine gängige Arbeitshypothese („Match-Mismatch-Hypothese“) postuliert, dass eine Abweichung der Wachstumstrajektorie, die intrauterin festgelegt wird, nach der Geburt mit dem erhöhten Risiko verbunden ist. Auch hier werden Veränderungen des fetalen Epigenoms durch das intrauterine Milieu postuliert.

Epigenetische Veränderungen sind grundsätzlich reversibel. Geeignete Interventionen bzw. Verbesserungen des Lebensstils könnten auf dieser Basis das Krankheitsrisiko reduzieren - ein Themenfeld das derzeit wegen der weltweit explodierenden und epidemische Ausmaße annehmenden metabolischen Erkrankungen intensiv erforscht wird.

\section{Immunologie - Inflammation}

\section{V-21}

Probiotika im Frühchendarm: etwas Licht im Dunkeln

\section{U. Grundmann'}

'Uniklinik Freiburg, Freiburg, Deutschland

Der Einsatz von Probiotika zur Prophylaxe der nekrotisierenden Enterokolitis (NEC) des Frühgeborenen ist in vielen Kliniken Routine geworden, aber die Studienlage ist weiterhin limitiert. Das Verständnis der pathophysiologischen Mechanismen der NEC dagegen wächst stetig, ebenso die Erkenntnis über den Einfluss probiotischer Mikroorganismen auf einzelne Aspekte dieser hochkomplexen Vorgänge. Im Rahmen des Vortrags soll hierüber ein Überblick gegeben werden. Prädisponierend für die Entwicklung einer NEC sind die Unreife des Gastrointestinaltrakts beim Frühgeborenen, kombiniert mit einem hochreaktiven angeborenen Immunsystem der intestinalen Mukosa, eine mikrobielle Fehlbesiedelung des Darms, sowie untergeordnet auch Schwankungen des kapillären Blutflusses und eine genetische Prädisposition. Bifidobakterien und Laktobazillen stehen aktuell im Zentrum der Forschungsbemühungen, da sie den Großteil der natürlichen gastrointestinalen Flora Muttermilch-ernährter Säuglinge ausmachen und ihr Einsatz zur Prävention der NEC am besten dokumentiert ist. Obgleich viele Effekte nicht nur Spezies-spezifisch sind, sondern sogar zwischen den einzelnen Stämmen variieren, ist insbesondere für diese beiden Spezies klar, dass sie an verschiedenen pathophysiologischen Teilaspekten zugleich angreifen. Dies geht häufig einher mit einer Korrektur von Imbalancen, die die Immaturität des Gastrointestinaltraktes des Frühgeborenen ausmachen.

So stärken Probiotika die noch unterentwickelte epitheliale Barrierefunktion durch Stabilisierung der "tight junctions“, durch Stimulation der Mucin-Sekretion sowie durch Apoptoseverhinderung. Gastrointestinale Motilität und Neurotransmission werden stimuliert und so die Passagezeit für potentielle Toxine oder Pathogene verkürzt. Verbesserungen des intestinalen Blutflusses durch Probiotika sind vermutlich das Ergebnis der Produktion vasoaktiver Amine. Probiotika wirken regulierend auf die mikrobielle Kolonisation, indem sie sowohl indirekte als auch direkte antimikrobielle Effekte gegenüber potentiellen Pathogenen vermitteln. Eine Hauptrolle spielen sie wahrscheinlich insbesondere bei der Begrenzung exzessiver Immunantworten auf Stimuli von Pathogenen, die durch eine Überexpression von TLR-4 im imma- 
turen Darm ausgelöst werden. Eine TLR-4-Aktivierung führt via $\mathrm{Zy}$ tokin-Sekretion zur Rekrutierung von Entzündungszellen, außerdem zu Apoptoseinduktion und Schwächung von mukosalen Reparaturmechanismen. Die resultierende mukosale Schädigung ermöglicht die Translokation pathogener Bakterien, was den für die Endstrecke der NEC typischen Teufelskreis aus Inflammation und Gewebeschädigung zur Folge hat. Probiotika können dabei offenbar direkt hemmend in die Signalkaskade nach TLR-4-Aktivierung eingreifen. Darüber hinaus können Bakterien über die Aktivierung weiterer Oberflächenrezeptoren, wie TLR-9 und NOD-2, durch einen Cross-Talk mit TLR-4-Rezeptoren deren Herabregulation bewirken. So könnte insgesamt eine verringerte Sensitivität gegenüber der fortschreitenden Kolonisation des unreifen Darms im Sinne einer erwünschten Toleranzinduktion erreicht werden.

Die bessere Kenntnis der molekularbiologischen Vorgänge und der sich ergebenden Wechselwirkungen auch im immaturen Darm des Frühgeborenen könnten dazu beitragen, alltagstaugliche Protokolle für den zielgerichteten Einsatz probiotischer Keime zu entwickeln und deren Sicherheit in der klinischen Routine zu erhöhen.

\section{V-22}

Veränderungen fetaler Organe und des Immunsystems nach Chorioamnionitis

\section{B. Kramer}

'Universitätsklinik Maastricht, Pädiatrie, Maastricht, Niederlande

Die fötale Entwicklung kann von diversen endogenen und exogenen Faktoren beeinflusst werden. In den letzten Jahren konnte mithilfe von klinischen Studien und verschiedenen Tiermodellen Erkenntnisse über die Wirkung von histologischer Chorioamnionitis auf die Lungenreifung und -entwicklung, den Darm, die Haut sowie auf das sich entwickelnde Immunsystem im Thymus und Blut gewonnen werden. Dabei zeigte sich, dass Chorioamnionitis die Entstehung von Alveolarmarkophagen induziert und eine Verletzung des Lungengewebes auslöst. Durch eine Entzündungsreaktion und sich daran anschließenden Umbauprozessen wird die Lungenstruktur simplifiziert. Als eines der wesentlichen Zytokine, das in die Vermittlung dieser Prozesse involviert ist, konnte Interleukin-1 (IL-1) identifiziert werden, verbunden mit einer Modulation von Wachstumsfaktoren wie TGF- $\beta$, CTGF, FGF-10 oder BMP-4, die alle eine wesentliche Rolle in der Lungenentwicklung besitzen.

In verschiedenen Chorioamnionitismodellen wurde der Effekt von Chorioamnionitis auf den fötalen Darm untersucht. Dabei konnte gezeigt werden, dass die Kolonisation mit Ureaplasma urealyticum zur Entzündung des Darms mit folgender Atrophie der Darmzotten führte. Die Bildung von "tight junctions" an der Darmoberfläche wurde ebenfalls durch die Chorioamnionitis verhindert. Durch die systemische Gabe von Interleukin-1-Rezeptorantagonisten (Anakinra) konnte die fötale Darmentzündung und die Darmschädigung verhindert werden. Die weitere Entwicklung der Lunge und des Darms nach dieser intrauterinen Schädigung können ebenfalls indirekt durch die Wirkung der Chorioamnionitis auf die Effizienz des fötalen Immunsystems beeinflusst werden. Chorioamnionitis kann eine Toleranz gegenüber Endotoxin (LPS-Toleranz, Immunparalyse) induzieren, die wohl weiteren Schaden des Feten verhindern kann, aber auch das Risiko für postnatale Infektionen erhöht. Die postnatale Reparaturreaktion scheint daher in hohem Maße von pränatalen Faktoren abhängig zu sein. Daher erscheint die Hypothese von sekundären Verletzungen sehr plausibel, wobei histologische Chorioamnionitis eine Prädisposition für eine postnatal beeinträchtigte Entwicklung ist. Durch ein Prädiktionsmodell aus klinischen Daten ist es möglich geworden, das Risiko für das Vorliegen einer histologischen Chorioamnionitis direkt nach der Geburt zu bestimmen. Damit werden prospektive klinische Interventionsstudien in Abhängigkeit von histologischer Chorioamnionitis möglich.

\section{Neurologisches "Outcome" nach Asphyxie und Frühgeburt}

\section{V-27 Teilleistungsstörungen ehemaliger Frühgeborener - 5-Jahres-Out- comedaten aus Tirol}

\section{U. Kiechl-Köhlendorfer ${ }^{1}$}

'Medizinische Universität Innsbruck, Universitätsklinik für Pädiatrie IV, Neonatologie, Neuropädiatrie, Stoffwechselerkrankungen, Innsbruck, Österreich

Hintergrund. Frühgeborene zeigen ein erhöhtes Risiko für die Entwicklung von spezifischen Lernstörungen und Schulproblemen. Das Ziel dieses Vortrages ist es, eine Übersicht über vorliegende Studien zum Thema Ehemalige Frühgeborene und Teilleistungsstörungen im Vorschulalter zu geben und die Ergebnisse der Kinder, die an der Neonatologie der Universitätsklinik Innsbruck betreut wurden, zu präsentieren. Methodik. Im Zeitraum von Jänner 2003 bis August 2006 wurden alle Frühgeborenen mit einem Gestationsalter von weniger als 32 Schwangerschaftswochen, die an der Neonatologie Innsbruck behandelt wurden, inkludiert $(n=303) .223$ dieser Kinder wurden zur Nachsorge eingeladen, davon konnten 161 ehemalige Frühgeborene (Follow-up Rate $72,2 \%)$ im Alter von 5 Jahren standardisiert nachkontrolliert werden (kognitives Leistungsprofil: WPPSI-III; schulische Vorläuferfertigkeiten: TEDI-MATH, BISC; exekutive Funktionen: BRIEF; Verhalten: SDQ).

Ergebnisse. Bei etwa einem Drittel der untersuchten Kinder befindet sich das intellektuelle Leistungsniveau im grenzwertigen Bereich der Altersnorm (Hauptproblembereich: Verarbeitungsgeschwindigkeit), $20 \%$ aller Kinder $(n=27)$ zeigen verzögerte numerische Fähigkeiten. Insgesamt haben zwei Drittel der ehemaligen Frühgeborenen einen Bedarf an spezifischen Fördermaßnahmen im Vorschulalter.

Schlussfolgerung. Sowohl die Ergebnisse der Literatur als auch unsere Resultate bestätigen die Notwendigkeit der frühen Erfassung von schulischen Vorläuferfertigkeiten. Nur durch eine standardisierte Frühgeborenennachsorge, die bis ins Vorschulalter reicht, ist es möglich, der Entwicklung von Teilleistungsproblemen entgegenzuwirken.

\section{Pränatale Pathologie - angeborene Fehlbildungen}

\section{V-37}

\section{Angeborene Fehlbildungen des Gehirns}

\section{R. Korinthenberg}

'Universitätsklinikum Freiburg, Zentrum für Kinderheilkunde und Jugendmedizin Klinik II, Neuropädiatrie und Muskelerkrankungen, Freiburg, Deutschland

In der Ära der Magnetresonanztomographie werden Hirnfehlbildungen häufiger diagnostiziert als zuvor: Ihre Inzidenz wird mit 3,32/10oo und die Prävalenz bis zum 14. Lebensjahr mit 2,21/100o angegeben; in einer Population von Kindern mit Cerebralparese lag die Inzidenz bei 88/100o. Die Klassifikation der Hirnfehlbildungen kann nach anatomischen und embryologischen Kriterien erfolgen. Die Tatsache, dass häufig mehrere Hirnabschnitte gleichzeitig betroffen sind, die Weiterentwicklung der MRT-Technik und neuroradiologischen Erfahrung, und - last but not least - eine Flut molekulargenetischer Befunde machen aber eine definitive Klassifikation schwierig. Dies führte in den vergangenen Jahren zu Weiterentwicklungen in immer kürzeren Zeitabständen. Neben genetischen Defekten und primären Fehlanlagen können Hirnfehlbildungen ursächlich auch extrinsische Faktoren zugrunde liegen, wie frühe intrauterine vaskuläre Komplikationen und Infektionen, mütterlicher Diabetes, Strahlenbelastung und verschiedene Toxine. $\mathrm{Zu}$ beachten ist auch, dass bestimmte Hirnfehlbildungen im Rahmen 
von metabolischen Syndromen anzutreffen sind, z. B. pontocerebelläre Hypoplasie bei CDG1A, Lissencephalien bei O-Glykosylierungsstörungen, Pachygyrie/Polymikrogyrie bei peroxisomalen Erkrankungen. Enzephalocelen treten bei o,8 bis 5/10.00o Lebendgeborenen auf, sie sind meist occipital, seltener frontal und sehr selten basal (Nasengänge, Pharynx) lokalisiert. Syndromatisch treten sie bei mehreren Syndromen auf: Meckel-Gruber S., Dandy-Walker S., Walker-Warburg S., Joubert S, Knobloch S, Robert's S. Anders als die Meningomyelocele sind sie nicht durch Folsäure zu verhindern.

Holoprosencephalien, davon zwei Drittel höchsten Schweregrades (alobare HPE), treten bei 1/10.0oo Lebendgeburten auf. Sie beruhen auf einer mangelnden Separierung von Hemispären-, Zwischen- und Mittelhirnstrukturen, wobei bei den leichteren Formen die posterioren Strukturen weitergehend separiert sind als die frontalen. Die Ursache ist teratogen oder genetisch. In 30-50\% lässt sich eine Chromosomenanomalie oder eine syndromatische Kombination finden (Smith-LemlyOpitz S., Pallister-Hall S.), für die isolierten HPE wurden Mutationen in mindestens neun Genen gefunden, von denen vier $\left(\mathrm{SHH}, \mathrm{ZIC} 2, \mathrm{SIX}_{3}\right.$, TGIF) für etwa $25 \%$ der Fälle verantwortlich sind.

Die Corpus-callosum-Agenesie beruht auf einer mangelnden Entwicklung der interhemispheralen Kommissuren und ist die häufigste Hirnfehlbildung (1:300o Lebendgeborene). Sie ist häufig mit anderen Fehlbildungen assoziiert und ihre Ätiologie und Pathogenese ist komplex. Chromosomenanomalien, Mikrodeletionen/Duplikationen und monogenetische Erkrankungen mit bekanntem oder bislang unbekanntem Gen machen einen nennenswerten Prozentsatz der Fälle aus. Cerebelläre Hypoplasien manifestieren sich meist mit globaler psychomotorischer Retardierung und nicht selten mit Epilepsie. Anders als Kleinhirnatrophien verlaufen sie nicht progredient. Neben infektiös-exogenen Ursachen sind Mikrodeletionen, mehrere monogene Erkrankungen (VLDLR, DKC1, OPHN1, PTF1A) und das CDG Syndrom Typ $1 \mathrm{~A}$ als Ursache nachgewiesen worden. Die pontocerebellären Hypoplasien umfassen mehrere genetische Entitäten, mit $(\mathrm{PCH})$ und ohne ( $\left.\mathrm{PCH}_{2} \mathrm{ff}\right)$ spinale Muskelatrophie und mit generell schlechter Prognose. Das Joubert-Syndrom und Joubert-ähnliche Erkrankungen mit Ataxie, Atemantriebsstörungen, mentaler Retardierung und dem charakteristischen „Molar-Zahn-Zeichen“ im MRT bilden die häufigsten genetischen angeborenen Kleinhirnerkrankungen. Mit steigender Überlebensdauer werden Nieren- und Leberbeteiligung bei diesen auf einen Ziliendefekt zurückzuführenden Erkrankungen bedeutsam.

Das Dandy-Walker-Syndrom wird heute als primäre Entwicklungsstörung des Kleinhirns bei lokaler Dysfunktion von Transkriptionsfaktoren und nicht mehr als Folge eines Verschlusses der Foramina des 4. Ventrikels verstanden. Andere Kleinhirnhypoplasien, retrocerebelläre Zysten und eine Megacisterna magna sollten als solche diagnostiziert und nicht mehr als Dandy-Walker-Variante bezeichnet werden.

Die Mikrozephalie ist ein bezüglich Ätiologie, Anatomie und Prognose extrem variables Symptom. Die genetischen Formen lassen sich nach Cortexdicke, Erweiterung der extracerebralen Räume, infratentorieller Beteiligung, lissenzephaler Cortexstruktur und extracerebralen Symptomen einteilen. Die Syndromdiagnostik, aber auch die Auswahl von Kandidatengenen für die genetische Diagnostik folgt diesen Prinzipien. Störungen der radialen oder tangentialen Wanderung der Neurone führen zu den verschiedenen Erscheinungsbildern der Migrationsstörungen. Die Lissenzephalien mit fehlender oder verminderter Gyrierung und verdicktem Cortex lassen sich nach dem Anterior-posterior-Gradienten der kortikalen Veränderungen und Begleitsymptomen verschiedenen Genen zuordnen (LIS1, CDX, TUBA1A, ARX, RELN). Eine pflastersteinartige Oberflächenstruktur („,cobblestone lissencephaly“), meist mit Kleinhirnfehlbildungen weist auf eine O-Glykolsylierungsstörung als Ursache hin (Walker-Warburg Syndrom). Periventrikuläre und andere noduläre Heterotypien treten häufig sporadisch auf, sind aber auch als $\mathrm{x}$-dominant (Filamin A) und autosomal-rezessiv (ARF$\mathrm{GEF}_{2}$ ) vererbt bekannt. Polymikrogyrien entstehen nach Abschluss der Migration in der Organisationsphase der Cortexentwicklung. Sie können auf exogene Noxen zurückgeführt werden (vaskulär, CMV), sind aber auch typisch für peroxisomale Stoffwechselleiden (Zellweger
S.) und können syndromatisch oder nichtsyndromatisch auf monogene Mutationen zurückzuführen sein. Die Schizenzephalien werden in erster Linie als Folge früher Insulte aufgefasst.

Fokale kortikale Dysplasien (FCD) werden nach dem radiologischen und histologischen Zellbild klassifiziert. Sie sind häufig das Substrat therapieresistenter fokaler Epilepsien im Kindesalter und deshalb in der Epilepsiechirurgie besonders relevant. Mit Ausnahme der tuberösen Sklerose, deren charakteristische Tubera Typ IIb FCDs entsprechen, sind keine genetischen Ursachen bekannt. Die Hemimegalencephalie, die häufig mit kutanen Auffälligkeiten des Schädels vergesellschaftet ist, kann als Maximalform der FCD angesehen werden.

1. Swaiman KF, Ashwal S, Ferriero DM, Schor NF (2012) Swaimans's Pediatric Neurology, 5th edtn. Elsevier Saunders

2. Barkovich AJ, Raybaud C (2012). Pediatric Neuroimaging, 5th edition, Wolters Kluwer, Lippincott Williams \& Wilkins

\section{Freie Vorträge}

\section{Ernährung - Wachstum - Gedeihen}

\section{FV-01}

\section{Cholinmangel bei Frühgeborenen - postnatale Plasmakonzentra- tionen als Indikator der Unterversorgung}

\section{W. Bernhard', M. Raith', V. Koch', R. Kunze', C. Maas', C. Poets', A. Franz'} 'Eberhard-Karls-Universität, Universitätsklinik für Kinderheilkunde und Jugendmedizin, Neonatologie, Tübingen, Deutschland

Hintergrund. Cholin ist ein essentieller Nährstoff und notwendig zur Synthese von Phosphatidylcholin (Ptd'Cholin) und Sphingomyelin als Hauptbestandteile von Zellmembranen und lipidhaltigen Sekreten (Surfactant, Galle) sowie des Neurotransmitters Acetylcholin. Als Komponente der „very low density lipoproteins“ (VLDL) der Leber sichert Ptd'Cholin zudem die Versorgung des ZNS und anderer Organe mit essentiellen Fettsäuren wie Dokosahexaensäure. Cholinmangel beeinträchtigt im Tierversuch und beim Menschen die kognitive Entwicklung. Der Cholinbedarf ist wachstumsabhängig und invers proportional zum Körpergewicht. In utero erfolgt eine kontinuierliche diaplazentare Anreicherung im Fötus. Die Cholin-Aufnahme in periphere Organe ist proportional zur Plasmakonzentration, die bei reifen Neugeborenen etwa 3-mal höher ist (ca. $35 \mu \mathrm{mol} / \mathrm{L}$ ) als bei Schwangeren. Die Plasmakonzentrationen bei Feten und Frühgeborenen sind unbekannt. Methoden. Nabelschnur-EDTA-Plasmen von Frühgeborenen $(n=146$; Gestationsalter bei Geburt: 24-36 Wochen) und reifen Neugeborenen $(\mathrm{n}=28 ; 37-41$ Wochen) sowie Rest-Plasmen klinisch indizierter Blutentnahmen $(n=163)$ von 55 Frühgeborenen $[27,9(23,4-35,0)$ Wochen] wurden mit Chloroform/Methanol extrahiert und die Cholinkonzentration mittels Tandemmassenspektrometrie (LC-MS/MS) gemessen. Alle Ergebnisse sind als Median (25.-75. Perz.) angegeben.

Ergebnisse. Die mittlere Cholinkonzentration im Nabelschnurplasma betrug $41,4(31,8-51,2) \mu \mathrm{mol} / \mathrm{L}$ und korrelierte invers $(\mathrm{r}=-0,3452$, p<0,0001) mit dem Gestationsalter: 24-27 Wochen: 47,1 (32,0-60,o) $\mu \mathrm{mol} / \mathrm{L} ; 37-42$ Wochen: $34,9(29,35-41,3) \mu \mathrm{mol} / \mathrm{L}$. In postnatalen Plasmaproben Frühgeborener sank der Cholinspiegel unabhängig vom Gestationsalter bei Geburt innerhalb von $48 \mathrm{~h}$ von $42,5(26,6-52,0 ; n=13)$ auf ein konstantes Niveau von $20,3(15,2-26,0) \mu \mathrm{mol} / \mathrm{L}(\mathrm{d} 2-\mathrm{d} 84 ; \mathrm{n}=15 \mathrm{O}$; $\mathrm{p}<0,001$ )

Schlussfolgerung. Die Cholinkonzentration im fetalen Plasma ist während des schnellen Wachstums im frühen 3. Trimenon höher als am Ende der Schwangerschaft. Postnatal sinkt die Cholinkonzentration innerhalb von $48 \mathrm{~h}$ auf die Hälfte der intrauterinen Konzentration ab. Dies könnte Ausdruck einer ungenügenden Cholinversorgung Frühgeborener sein und eine weitere Erklärung für ihre beeinträchtigte kognitive Entwicklung bieten. 


\section{FV-02}

Vergleich zwischen 3-Kammerbeutel-Konzept vs. individuelle parenterale Ernährung - Auswirkungen auf die Nährstoffzufuhr von Frühgeborenen

\section{A. Kreissl', A. Repa', C. Binder', A. Berger', N. Haiden'}

${ }^{1}$ Universitätsklinik für Kinder- und Jugendheilkunde, Medizinische Universität Wien, Abteilung für Neonatologie, pädiatrische Intensivmedizin und Neuropädiatrie, Wien, Österreich

Hintergrund. Frühgeborene benötigen in den ersten Lebenstagen häufig parenterale Ernährung (PN) um Ihren Energie und Nährstoffbedarf suffizient zu decken. Dafür wurde ein neues „ready to use“ Produkt mit einem 3-Kammerbeutel-Konzept (Numeta ${ }^{\star}$, Baxter) entwickelt, das auf den Empfehlungen der European Society for Pediatric Gastroenterology, Hepatology and Nutrition (ESPGHAN) und der European Society for Clinical Nutrition and Metabolism (ESPEN) für Frühgeborene basiert.

Fragestellung. Das Ziel dieser Studie ist es Nährstoffzufuhr und Kosten individuell rezeptierter parenteraler Ernährungslösungen mit denen von den neuen Fertigmischbeuteln zu vergleichen.

Material und Methoden. Mittels des elektronischen Rezepturprogramms Cato $\mathrm{Pan}^{\circ}$ (Cato software solutions) wurden in dieser Studie die Vorschreibungen für parenterale Ernährungslösungen von Frühgeborenen ( $\mathrm{n}=50 ;<37$. Gestationswoche) verglichen. Jede Infusionslösung wurde einmal individuell und einmal mittels Mischbeutel rezeptiert. Bei Spiegelung der Ernährungslösungen wurden die Parameter Gewicht, venöser Zugang, Gesamtvolumen und Menge an enteraler $\mathrm{Zu}$ fuhr exakt gleich belassen - die Steuerung der Nährstoffe erfolgte wie bei den Fertigmischbeuteln vorgesehen über die Proteinzufuhr. Die Ergebnisse der Nährstoffanalysen von Fertigbeutel vs. individuellen PN wurden mit den aktuellen ESPGHAN-Empfehlungen verglichen. Weiters wurden die Kosten für beide Rezepturwege verglichen.

Ergebnisse. Bis Juni 2013 werden 400 parenterale Ernährungsrezepturen gespiegelt und ausgewertet. Bis dato wurden $n=40$ Infusionen analysiert und gespiegelt. Die Proteinzufuhr bei Anwendung der Fertigbeutel würde mit $3.04 \mathrm{~g} / \mathrm{kg} / \mathrm{d}$ (vs. $3.53 \mathrm{~g} / \mathrm{kg} / \mathrm{d}$ individuelle PN) unter dem empfohlenen Zielbereich von ca. $3.6 \mathrm{~g} / \mathrm{kg} / \mathrm{d}$ Protein liegen. Der Fettanteil des 3-Kammerbeutel-Konzeptes wäre um $0.92 \mathrm{~g} / \mathrm{kg} / \mathrm{d}$ höher als die Empfehlungen (Fertigbeutel: $4.42 \mathrm{~g} / \mathrm{kg} / \mathrm{d}$ vs. individuell: $4.05 \mathrm{~g} /$ $\mathrm{kg} / \mathrm{d}$ ). Bei der Verwendung von Numeta-Beutel würde den Patienten eine höherprozentige Glucose (11\%) im Vergleich zur individuellen PN (9.5\%) verabreicht werden.

Diskussion. Erste Analysen zeigten, dass individuelle Rezeptur von PN den Bedarf von Frühgeborenen optimal abdeckt. Bei Verwendung der neuen Fertigmischbeutel liegt man bei der Glucose und Fettzufuhr eher über den Empfehlungen, bei der Proteinzufuhr unter den Empfehlungen, was im klinischen Einsatz kritisch zu betrachten ist.

\section{Immunologie - Inflammation II}

\section{FV-03 \\ Verteilung von zirkulierenden T-Zell-Subpopulationen im periphe- ren Blut während der Ontogenese}

\section{Zemlin', S. Markert', S. Kerzel', J. Wagner', R. Maier', T. Rogosch' 'Universitätsklinikum Gießen und Marburg GmbH, Klinik für Kinder- und Jugendmedizin Marburg, Marburg, Deutschland}

Hintergrund. Das Neugeborene erwirbt postnatal durch Kontakt mit Umweltantigenen (Nahrung, Mikroben) ein immunologisches „Gedächtnis“. Andererseits setzen sich ontogenetische Reifungsprozesse fort. In den letzten Jahren sind die T-Zell-Subpopulationen teilweise neu definiert worden, wobei noch unklar ist, inwieweit sich die Verteilung der T-Zell-Subpopulationen während der Ontogenese verändert.
Fragestellung. Das Ziel unserer Studie war es, die Verteilung zirkulierender T-Zell-Subpopulationen zwischen Nabelschnurblut und peripherem Blut von gesunden Erwachsenen zu vergleichen.

Material und Methoden. Aus Nabelschnurblut von 10 gesunden Reifgeborenen und aus peripherem Blut von 10 gesunden Erwachsenen wurden die mononukleären Zellen mittels Ficoll-Gradient aufgereinigt, mit fluoreszierenden Antikörpern gefärbt und im Durchflusszytometer analysiert. Folgende Zellpopulationen wurden untersucht: T-Helfer-1-Zellen (Th1, CD $3+\mathrm{CD}_{4}+\mathrm{CCR} 6+\mathrm{CXCR}_{3}+$ ), T-Helfer-2-Zellen (Th2, $\left.\mathrm{CD}_{3}+\mathrm{CD}_{4}+\mathrm{CRTH}_{2}+\mathrm{CCR} 4+\right)$, regulatorische T-Zellen (Treg) $\left(\mathrm{CD}_{3}+\mathrm{CD}_{4}+\mathrm{CD}_{25}+\mathrm{CD} 127-\right)$, Th17-Zellen (CD3+CD4+CD161+CCR6+), zytotoxische T-Zellen (CD3+CD8+), natürliche Killer-T-Zellen $\left(\mathrm{CD}_{3}+\mathrm{CD} 56+\right)$.

Ergebnisse. Während im peripheren Blut Erwachsener T-Helfer (Th) Zellen $63,6 \pm 3,3 \%$ aller $\mathrm{CD}_{3}+$ T-Zellen ausmachten, war ihre Häufigkeit im Nabelschnurblut mit $72,9 \pm 2,1 \%$ höher ( $\mathrm{p}<0,05)$, wobei sich der Quotient aus Th1-/Th2-Zellen nicht unterschied. Auch der Anteil von

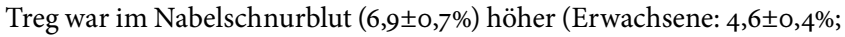
$\mathrm{p}<0,01$ ), während Th17-Zellen ( $0,5 \pm 0,1 \%$ vs. $13,0 \pm 1,1 \%, p<0,001)$, zytotoxische T-Zellen $(23,7 \pm 1,6 \%$ vs. $30,6 \pm 2,9 \%$, p $<0,05)$ und natürliche KillerT-Zellen (o, $5 \pm 0,1 \%$ vs. $6,2 \pm 1,8 \%, \mathrm{p}<0,001)$ im Nabelschnurblut seltener vorkamen.

Diskussion. Funktionell befindet sich das Immunsystem des Neugeborenen am Übergang von Toleranz (intrauterin) zu Abwehr (extrauterin). Dazu passen unsere Beobachtungen, dass zytotoxische T-Zellen, Th17-Zellen und natürliche Killer-T-Zellen, also Zellen der Pathogenabwehr, im Nabelschnurblut im Vergleich zu Erwachsenen seltener vorkommen, wohingegen regulatorische T-Zellen, die eine wichtige Rolle in der Toleranzentwicklung spielen, und auch T-Helfer Zellen, häufiger sind. Allerdings können aus diesen Beobachtungen noch keine endgültigen Rückschlüsse auf funktionelle Auswirkungen, wie z. B. die Infektionsanfälligkeit bei Neugeborenen, gezogen werden.

Schlussfolgerung. Die T-Zell-Subpopulationen unterliegen während der Ontogenese erheblichen quantitativen Veränderungen. Dies reflektiert eine Überlagerung aus ontogenetischen Reifungsprozessen und der Etablierung eines adaptiven Immungedächtnisses und muss bei der Beurteilung von Spezialdiagnostik, z. B. im Rahmen der Suche nach einem angeborenen Immundefekt, berücksichtigt werden.

\section{FV-04 \\ Myeloide Suppressorzellen hemmen die T-Zell-Antwort während der Schwangerschaft}

H. Kugel', N. Köstlin', B. Spring', A. Marmé2, N. Rieber', C. Poets', C. Gille

'Eberhard-Karls-Universität Universitätsklinik für Kinderheilkunde und Jugendmedizin, Neonatologie, Tübingen, Deutschland, ${ }^{2}$ Frauenarztpraxis, Tübingen, Deutschland, ${ }^{3}$ Eberhard-Karls-Universität Universitätsklinik für Kinderheilkunde und Jugendmedizin, Infektiologie, Tübingen, Deutschland

Hintergrund. Während der Schwangerschaft muss das mütterliche Immunsystem die Balance halten zwischen immunologischer Toleranz gegenüber dem semiallogenen Feten und gleichzeitiger Fähigkeit zur Infektabwehr. Störungen dieses Gleichgewichts können zu Aborten oder Frühgeburtlichkeit führen. Myeloide Suppressorzellen (MDSC) sind Vorläuferzellen, die bei pathologischen Zuständen wie Tumorerkrankung und Infektion stark vermehrt sind und T-Zell-Antworten hemmen. Ob sie auch eine Bedeutung für die physiologische Immunregulation in der Schwangerschaft haben ist unklar.

Hypothese. MDSC zirkulieren vermehrt im Blut Schwangerer und hemmen die T-Zell-Aktivierung.

Methoden. Mononukleäre Zellen aus peripherem Blut nicht schwangerer Erwachsener (PBMC) und gesunder Schwangerer (S-PBMC) wurden isoliert und der Anteil granulozytärer (CD66b+/CD33+/CD14-/ HLA-DR-) und monozytärer (CD14+/HLA-DR-/low) MDSC sowie die Expression der Enzyme Arginase I und iNOS durchflusszytome- 
trisch bestimmt. Zur Anreicherung der granulozytären MDSC wurden CD66b-positive Zellen mittels "magnetic activated cell sorting" isoliert Isolierte MDSC aus PBMC und S-PBMC wurden zu CFSE-markierten PBMC bzw. S-PBMC gegeben und die $\mathrm{CD}_{4}$ - und CD8-T-Zell-Proliferation nach $96 \mathrm{~h}$ Stimulation mit anti- $\mathrm{CD}_{3}\left(\mathrm{OKT}_{3}\right)$ und Interleukin-2 (IL-2) durchflusszytometrisch gemessen. Als Vergleich dienten PBMC ohne Zugabe von MDSC.

Ergebnisse. Der Anteil an granulozytären MDSC war in S-PBMC mehr als zehnfach höher (median 2,545\%, Range: $0,32-9,41 \%, n=10$ ) als in PBMC (median o, $2 \%$, Range: $0,1-0,6, n=13 ; \mathrm{p}<0,0001$ ). Keine Unterschiede gab es dagegen beim Anteil an monozytären MDSC (median 1,3\%, Range: $0,4-4,4 ; n=7$ für S-PBMC und median 1,8\%, Range: $0,7-5,5 ; n=10$; $\mathrm{p}=0,6237$ für PBMC). Im Scatter-Plot lag die Population der granulozytären MDSC in typischer Position über der Lymphozytenpopulation. Die granulozytären MDSC aus S-PBMC exprimierten die Effektorenzyme Arginase I (MFI 28,18 $\pm 12,13 ; n=16)$ und iNOS (MFI 84,92 $\pm 66,35$; $\mathrm{n}=16$ ). Die $\mathrm{OKT}_{3}$ - und IL-2-induzierte $\mathrm{CD}_{4}$-T-Zell-Proliferation betrug

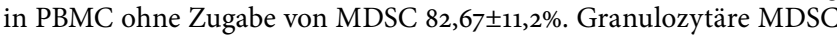
aus S-PBMC hemmten CD4-T-Zellproliferation konzentrationsabhängig auf $62,0 \pm 2,7 \%$ bzw. $38,0 \pm 21,7 \%$. Die CD8-T-Zellproliferation wurde ebenfalls konzentrationsabhängig von $89,0 \pm 7,81 \%$ auf $64,67 \pm 10,1 \%$ bzw. $42,3 \pm 24,4 \%$ gehemmt.

Schlussfolgerung. Granulozytäre MDSC sind im peripheren Blut Schwangerer stark erhöht und exprimieren die Effektorenzyme Arginase I und iNOS. In vitro hemmen sie die CD4- sowie die CD8-T-ZellProliferation. Die erhöhte Anzahl an funktionell aktiven MDSC im Blut Schwangerer könnte eine wichtige Rolle für die maternofetale Toleranz spielen und sich zur gezielten Therapie immunologischer Schwangerschaftskomplikationen eignen.

\section{FV-05}

Der postpartal steigende Sauerstoffpartialdruck hemmt myeloide Suppressorzellen (MDSC) und reguliert die Immunadaptation des Neugeborenen

\section{H. Spieles', N. Köstlin', B. Spring', N. Rieber', C. Poets', C. Gille}

'Eberhard-Karls-Universität Universitätsklinik für Kinderheilkunde und Jugendmedizin, Neonatologie, Tübingen, Deutschland, ${ }^{2}$ Eberhard-KarlsUniversität Universitätsklinik für Kinderheilkunde und Jugendmedizin, Infektiologie, Tübingen, Deutschland

Hintergrund. Neugeborene sind anfälliger für Infektionen als Erwachsene. Ein Unterschied zwischen fetalem und adultem Organismus ist der intrauterin physiologisch niedrige Sauerstoffpartialdruck ( $\left.\mathrm{pO}_{2}\right)$. Myeloide Suppressorzellen (MDSC) sind Vorläuferzellen, die im Nabelschnurblut vermehrt sind und Immunzellen v. a. durch Expression des Effektorenzyms Arginase I hemmen. Niedriger $\mathrm{pO}_{2}$ führt zur Akkumulation von MDSC.

Fragestellung. $\mathrm{Ob}$ der intrauterin niedrige $\mathrm{pO}_{2}$ zur Akkumulation von MDSC und der postnatal steigende $\mathrm{pO}_{2}$ zur Inaktivierung von MDSC und Immunadaptation des Neugeborenen führt, ist unklar.

Methoden. Nabelschnurblut wurde in luftleere, heparinisierte Spritzen entnommen, der $\mathrm{pO}_{2}$ ermittelt und Mononukleäre Zellen (CBMC) in einer Hypoxiewerkbank ( $\mathrm{pO}_{2} 10-35 \mathrm{mmHg}$ ) isoliert. Gleichzeitig wurden mononukleäre Zellen Erwachsener (PBMC) bei Raumluft ( $\mathrm{pO}_{2}$ $165 \mathrm{mmHg})$ aufgereinigt. Der Anteil granulozytärer $\left(\mathrm{CD} 66 \mathrm{~b}+/ \mathrm{CD}_{33}+/\right.$ CD14-/HLA-DR-) MDSC wurde durchflusszytometrisch bestimmt. Nach 48 h wurde die Arginase-I-Expression durchflusszytometrisch bestimmt. Zur Anreicherung der MDSC wurden CD66b+ Zellen mittels "magnetic activated cell sorting" isoliert. MDSC aus PBMC und CBMC wurden zu CFSE-markierten PBMC bzw. CBMC gegeben. Nach 96 h Stimulation mit anti- $\mathrm{CD}_{3}\left(\mathrm{OKT}_{3}\right)$ und Interleukin-2 (IL-2) und Kultur unter hypoxischen bzw. normoxischen Bedingungen wurde die T-Zellproliferation gemessen.

Ergebnisse. Der Anteil an MDSC war in CBMC (Median: 4,5\%, Range: 2,8-11,8) dreifach höher als in PBMC (Median: 1,5\%, Range: 0,6-3,2, je $\mathrm{n}=10 ; \mathrm{p}=0,01$ ). MDSC aus CBMC exprimierten nach Kultur in Hypoxie deutlich mehr Arginase I (Median: 60\%, Range: 18-85) als nach Kultur in Normoxie (Median: $26 \%$, Range: $5-66, n=7 ; p=0,02$ ), während sich bei MDSC aus PBMC keine Unterschiede in der Arginase-I-Expression zwischen hypoxisch (Median: 47\%, Range: 11-87) und normoxisch (Median: $43 \%$, Range: $32-95$ ) kultivierten Zellen zeigten ( $n=5 ; p=1,0)$. MDSC aus CBMC hemmten die T-Zellproliferation in Hypoxie stärker als in Normoxie (Median: 23\%, Range: 7-69 vs. Median: 70,5\%, Range: $23-96$, $\mathrm{n}=8)$. Dagegen hatte Hypoxie keinen Einfluss auf MDSC aus PBMC (Median: $39 \%$, Range: $23-74$ vs. Median: $57 \%$, Range: $37-91, n=8 ; p=0,2$ ). Schlussfolgerung. Steigender $\mathrm{SpO}_{2}$ führt zu einer verminderten Expression des Effektorenzyms Arginase I in fetalen MDSC und reduziert deren Kapazität T-Zellen zu hemmen. Der physiologisch niedrige $\mathrm{SpO}_{2}$ des Feten könnte eine Ursache für die Immunsuppression in utero sein und der postpartale Wechsel in Normoxie als Signal für die neonatale Immunadaptation dienen.

\section{FV-06}

Konzentration von Melatonin, Superoxiddismutase, GlutathionPeroxidase und 8-Hydroxydesoxyguanosin im Nabelschnurblut des Neugeborenen

\section{Katzer', A. Mueller', L. Welzing', H. Reutter', W. Merz'², J. Reinsberg', P. Bartmann', S. Bagci'}

'Zentrum für Kinderheilkunde, Universitätsklinikum Bonn, Abt. Neonatologie, Bonn, Deutschland, ${ }^{2}$ Zentrum für Geburtshilfe und Frauenheilkunde, Universitätsklinikum Bonn, Bonn, Deutschland, ${ }^{3}$ Zentrum für Geburtshilfe und Frauenheilkunde, Universitätsklinikum Bonn, Abt. Gynäkologische Endokrinologie und Reproduktionsmedizin, Bonn, Deutschland

Einleitung. Melatonin (MT) ist ein lipophiles Neurohormon, welches in der Epiphyse produziert und in einem zirkadianen Rhythmus in das Blut abgegeben wird. Neben der Fähigkeit als direktes Antioxidans vor oxidativem Stress zu schützen, konnte in mehreren Tiermodellen gezeigt werden, dass MT auch antioxidative Enzyme wie die Superoxiddismutase (SOD) und die Glutathion-Peroxidase (GSH-Px) stimuliert. In der vorliegenden Studie wurde untersucht, ob die MT-Konzentration einen Einfluss auf die Konzentration von SOD, GSH-Px und 8-Hydroxydesoxyguanosin (8-OHdG, ein Biomarker für oxidativen Stress), im arteriellen (NA) und venösen (NV) Nabelschnurblut von Neugeborenen hat und ob diese Parameter vom Geburtsmodus abhängig sind.

Methodik. Das Patientenkollektiv wurde bezüglich des Geburtsmodus [Spontanpartus (SP, $\mathrm{n}=45$ ), Sectio (CS, $\mathrm{n}=90)$ ] und der Geburtszeit in vier Gruppen eingeteilt. Das Tagesintervall $(n=60)$ wurde definiert von 9:0o Uhr bis 21:00 Uhr, das Nachtintervall $(\mathrm{n}=75)$ von 21:00 $\mathrm{Uhr}$ bis 9:00 Uhr.

Ergebnisse. Das mediane Gestationsalter (Interquartilsabstand) der SPGruppe war statistisch signifikant höher als der CS-Gruppe [39,1 SSW $(38,1-40,4)$ vs. 37,9 SSW $(37,3-38,4), \mathrm{p}<0,001]$. Die MT-Konzentration zeigte in beiden Gruppen sowohl in der NA als auch in der NV einen zirkadianen Rhythmus mit hohen Konzentrationen in den Nacht- und niedrigen Konzentrationen in den Tagproben $(\mathrm{p}<0,001)$. In beiden Gruppen waren die SOD-, GSH-Px3- und 8-OHdG-Konzentrationen unabhängig von der Geburtszeit $(\mathrm{p}>0,05)$ und von der MT-Konzentration $(p>0,05)$. Im Vergleich zur CS-Gruppe fanden wir in der SP-Gruppe sowohl in der NA als auch in der NV höhere MT-, SOD- und GSH$\mathrm{Px}_{3}$ - und niedrigere 8-OHdG-Konzentrationen $(\mathrm{p}<0,05)$. In beiden Gruppen zeigten sich in NA und NV mit zunehmendem Gestationsalter signifikant höhere GSH-Px3 Konzentrationen $(\mathrm{p}<\mathrm{o}, 01)$. MT, SOD und 8-OHdG-Konzentrationen waren unabhängig vom Gestationsalter ( $>0,05)$.

Schlussfolgerung. Die in der SP-Gruppe höheren Melatonin, SOD3- und GSH-Px3-Konzentrationen lassen auf eine höhere antioxidative Kapazität und somit einen besseren Schutz gegenüber oxidativem Stress des spontan geborenen Kindes schließen. Dies spiegelt sich auch in der bei Spontangeburten erniedrigten Konzentration des 8-OHdG wider. Die 
erhöhte Enzymsynthese könnte ein protektiver Mechanismus für den unter Spontanpartus bevorstehenden Anstieg des oxidativen Stresses darstellen.

\section{FV-07}

Toll-like Rezeptor-Agonisten als potenzielle Impfstoffadjuvantien beim Neu- und Frühgeborenen - In-vitro-Studie neonataler dendritischer Zellen

\section{S. Schüller', L. Wisgrill', K. Sadeghi', A. Spittler², H. Helmer', P. Husslein ${ }^{3,}$ A. Berger', A. Pollak', E. Förster-Waldl' \\ 'Medizinische Universität Wien, Universitätsklinik für Kinder- und Jugend- heilkunde, Wien, Österreich, ${ }^{2}$ Medizinische Universität Wien, Universi- tätsklinik für Chirurgie \& Core Facility Flow Cytometry, Wien, Österreich, ${ }^{3}$ Medizinische Universität Wien, Universitätsklinik für Frauenheilkunde, Wien, Österreich}

Hintergrund. Neu- und insbesondere Frühgeborene zeigen im Bereich des angeborenen und erworbenen Immunsystems eine funktionelle Unreife, welche neben einer erhöhten Infektanfälligkeit auch eine unzureichende Immunantwort nach Impfungen verursachen kann. Frühe und vor allem effiziente Impfstrategien könnten das Risiko für Infektionserkrankungen bei Früh- und Reifgeborenen senken. Ein wesentlicher Schritt zur Optimierung der Impfantwort besteht in der Zugabe von Adjuvantien. Das herkömmliche Impfadjuvans Aluminiumhydroxid (Alum) stellt nicht für alle Antigene und Impfpopulationen eine optimale Immunstimulation dar. Toll-like Rezeptor(TLR)-Agonisten wie R-848(TLR7/8) und CpG B(TLR9), die direkt das angeborene Immunsystem stimulieren, sind neue potenzielle Adjuvantien.

Fragestellung. Die vorliegende In-vitro-Studie untersucht den Effekt von Impfstoffadjuvantien des klinischen und präklinischen Bereiches auf plasmazytoide dendritische Zellen (pDC) und myeloide dendritische Zellen (mDCs) des Nabelschnurblutes. Ziel dieser Studie ist die immunmodulatorische Wirkung von Impfstoffadjuvantien beim Neuund Frühgeborenen detailliert zu definieren.

Material und Methoden. Aus dem Nabelschnurblut von Reifgeborenen $(n=9)$ und Frühgeborenen $(n=10)$, sowie aus dem peripheren Blut von gesunden Erwachsene $(n=10)$ - als immunkompetente Vergleichsgruppe - wurden mononukleäre Zellen isoliert und anschließend über 8 Stunden mit Alum, CpG ODN20o6 (TLR9), R-848 (TLR7/8) oder LPS stimuliert. Nach der Inkubation wurden Reife- und Aktivierungsmarker von DCs mittels 9-fach-Färbung durchflusszytometrisch ermittelt. Ergebnisse. Durch R-848 und CpG ODN2006 kommt es zu einer signifikant gesteigerten Expression von $\mathrm{CD}_{40}$, CD80, CD86, CCR7 und HLA-DR auf pDCs von Früh- und Reifgeborenen. Weiters führt die TLR- spezifische Stimulation zur gesteigerten CD40-, CD86- und CCR7-Expression auf neonatalen mDCs. Im Vergleich zu R-848 und CpG ODN2006, hat Alum keinen signifikanten Einfluss auf Aktivierungs- und Reifemarkern der neonatalen DCs.

Diskussion. Die TLR-spezifischen Agonisten R-848 und CpG ODN20o6 führen zur Aktivierung und Reifung von neonatalen DCs in vitro Diese Daten sprechen für die potentielle immunstimulatorische Wirkung moderner TLR-Agonisten auch für die Altersgruppe der Neuund Frühgeborenen. Die Entwicklung moderner Impfstoffadjuvantien könnte zukünftig auch für diese sensible Altersgruppe zusätzliche Effizienz in der Infektionsprophylaxe bewirken.

\section{Perinatale neurologische Schädigung und „Outcome“}

\author{
FV-08 \\ CEACAM1 und sein Einfluss auf die Myelinisierung
}

S. Prager', B. Singer ${ }^{2}$, I. Bendix', G. Schlager ${ }^{3}$, F. Bertling', B. Ceylan', M. Keller ${ }^{3}$, S. Ergün ${ }^{4}$, U. Felderhoff-Müser ${ }^{1}$

${ }^{1}$ Klinik für Kinder und Jugendmedizin der Universität, Klinik für Kinderheilkunde , Essen, Deutschland, ${ }^{2}$ Institut für Anatomie Universitätsklinikum Essen, Essen, Deutschland, ${ }^{3}$ Kinderklinik Dritter Orden, Passau, Deutschland, ${ }^{4}$ Institut für Anatomie und Zellbiologie II, Universität Würzburg, Würzburg, Deutschland

Hintergrund. In Deutschland werden jährlich rund 700.0oo Kinder geboren, der Anteil der Frühgeborenen beträgt 7-10\%. Obwohl die Mortalität auch bei sehr kleinen Frühgeborenen rückläufig ist, bleibt die Morbidität weiterhin hoch. Insbesondere perinatale Hirnschäden mit anschließenden lebenslangen neurologischen Defiziten stellen eine Belastung für die Kinder und ihre Familien dar. Ursächlich für die Hirnschädigung sind bei einem Großteil der Frühgeborenen nicht akute Ereignisse wie intrakranielle Blutungen, sondern die aufgrund der extrauterinen Hirnreifung auftretende längerfristige Exposition gegenüber einer Vielzahl diffuser Noxen wie Inflammation, Hyperoxie, Hypoxie/Ischämie und Medikamente. Als Schädigungsmuster tritt hauptsächlich eine diffuse Schädigung der weißen Substanz in Kombination mit einer neuronalen Verknüpfungsstörung auf. Die zugrunde liegenden Mechanismen sind nur unzureichend verstanden, daher ist zur Entwicklung neuer Therapieansätze ein besseres Verständnis der molekularen Grundlagen notwendig. CEACAM1, ein Mitglied der Familie der „carcinoembryonic antigen-related cell adhesion molecules“ (CEACAMs), ist hier aufgrund seiner komplexen Funktion in der Regulierung von Zellproliferation und -differenzierung sowie seiner Beteiligung an inflammatorischen Prozessen ein geeignetes Zielprotein.

Fragestellung. Welche Rolle spielt CEACAM1 während der Myelinisierung sowie bei der Hirnschädigung durch Frühgeburtlichkeit?

Material und Methoden. Ontogenetische CEACAM1-Expressionsanalysen wurden an unbehandelten Wistar-Ratten von Lebenstag $1\left(\mathrm{P}_{1}\right)$ bis P28 durchgeführt. Die Modulation der CEACAM1-Expression im Rahmen eines Modells der Frühgeborenen-Hirnschädigung (WistarRatten, Hyperoxie an $\mathrm{P}_{3}$, Inflammation an $\mathrm{P}_{5}$ ) wurde an P6 und $\mathrm{P}_{11}$ analysiert. In-vitro-Untersuchungen erfolgten an primären Oligodendrozyten-Kulturen. Immunhistochemische Färbungen, Protein-/RNAIsolierung, Western-Blot- sowie PCR- und qRT-PCR-Analysen wurden nach Standardprotokollen durchgeführt.

Ergebnisse. CEACAM1 wird in Oligodendrozyten des sich entwickelnden Gehirns exprimiert, das immunhistochemische Expressionsmuster zeigt eine große zeitlich-räumliche Ähnlichkeit zu Expressionsmustern von Myelinisierungsmarkern. Zudem ist in einem In-vivo-Modell der Frühgeborenen-Hirnschädigung durch Inflammation und Hyperoxie die CEACAM1-Expression auf RNA-Ebene moduliert. In vitro wird durch Stimulation primärer Oligodendrozyten mit CEACAMı die Myelinisierungsrate dieser Zellen erhöht.

Schlussfolgerung. CEACAM1 ist aufgrund seines ontogenetischen Expressionsprofils sowie der in vitro nachgewiesenen Fähigkeit zur Beeinflussung der Myelinisierung ein potentielles Zielprotein für therapeutische Ansätze zur Behandlung der Frühgeborenen-Hirnschädigung. 


\section{FV-09}

Antenatale Kortikosteroide verringern die durch intraamniotische Lipopolysaccharidgabe ausgelöste Entzündungsreaktion der subkortikalen weißen Substanz im Schaffeten

E. Kuypers', R. Jellema', D. Ophelders', M. Hütten' ${ }^{2}$, T. Orlikowsky ${ }^{3}$, M. Nikiforou', T. Wolfs', M. Kemp 4 , I. Nitsos ${ }^{5}$, J. Pillow ${ }^{4}$, G. Polglase ${ }^{5}$, J. Newnham ${ }^{4}$, A. Jobe ${ }^{6}$, S. Kallapur 6 , B. Kramer

${ }^{1}$ Maastricht University - Faculty of Health, Medicine and Life Science, Pediatrics Neonatology, Maastricht, Niederlande, ${ }^{2}$ Universitätsklinik für Kinder- und Jugendmedizin und Pediatrics Neonatology, Maastricht University - Faculty of Health, Medicine and Life Sciene, Maastricht, Sektion für Neonatologie, Aachen/Maastricht, Deutschland, ${ }^{3}$ Universitätsklinik für Kinder- und Jugendmedizin, Sektion für Neonatologie, Aachen, Deutschland, 'School of Women's and Infant's Health, The University of Western Australia, Subiaco, Perth, Australien, ${ }^{5} 5$ chool of Women's and Infant's Health, The University of Western Australia and The Ritchie Centre, Monash Institute of Medical Research, Australia, Subiaco, Perth, Australien, ${ }^{6} \mathrm{Cincin}-$ nati Children's Hospital Medical Center and School of Women's and Infant's Health, The University of Western Austrialia, Subiaco, Perth, Division of Pulmonary Biology, Cincinnati/Perth, USA

Hintergrund. Chorioamnionitis ist mit Frühgeburtlichkeit assoziiert und kann eine systemische fetale Entzündungsreaktion (FIRS) induzieren. Diese steht in enger Verbindung zu pathologischen Veränderungen der weißen Substanz (,white matter disease“, WMD), die u. a. zu Cerebralparese (CP) und neurokognitiven Einschränkungen prädisponieren. Bei drohender Frühgeburtlichkeit werden der Mutter antenatal Glukokortikoide verabreicht. Die kombinierte Wirkung von antenatalen Glukokortikoiden und Chorioamnionitis auf die zerebrale Entzündungsreaktion ist dabei unklar.

Fragestellung. Unsere Hypothese ist, dass die fetale zerebrale Entzündungsantwort in der subkortikalen weißen Substanz durch maternale antenatale Kortikosteroidgabe moduliert wird.

Material und Methoden. Trächtigen Schafen wurden 7 und/oder 14 Tage vor der Entbindung eine intraamniotische Lipopolysaccharid(LPS)-Injektion und/oder intramuskulär Betamethason verabreicht (bzw. Kochsalzlösung als Kontrolle). Die Feten wurden mit einem Gestationsalter von $120 \mathrm{~d}$ (Termin $150 \mathrm{~d}$ ) per Kaiserschnitt geboren. Immunhistochemische Untersuchungen der subkortikalen weißen Substanz umfassten Marker für Mikrogliaproliferation („,ionized calcium-binding adapter molecule“, IBA1), der Astrozytenpopulation („glial fibrillary acidic protein“, GFAP) und der Myelinisierung („myelin basic protein“, MBP).

Ergebnisse. Nach intraamniotischer LPS-Gabe ist in der subkortikalen weißen Substanz immunhistochemisch eine erhöhte Reaktivität für IBA1 und GFAP nachweisbar. Maternale Glukokortikoide vor LPS-Exposition verhinderten den Anstieg von IBA1, während die Glukokortikoide nach LPS-Exposition mit niedrigerer GFAP Reaktivität einhergingen. MBP ist, unabhängig von Glukokortikoiden, $14 \mathrm{~d}$ nach LPS vermindert.

Schlussfolgerung. Intrauterine LPS-Exposition bewirkt eine fetale Entzündungsreaktion in der subkortikalen weißen Substanz, die durch Glukokortikoide moduliert wird. Glukokortikoide allein zeigten keine negativen Effekte.
FV-10

Tumor necrosis factor-inducible gene 6 protein: eine neue neuroprotektive Substanz gegen inflammatorische Schädigungen während der Hirnentwicklung

F. Bertling', S. Prager ${ }^{2}$, H. Wisniweski', R. Herrmann ${ }^{4}$, K. Drommelschmidt ${ }^{5}$, U. Felderhoff-Müser ${ }^{6}$, M. Keller', I. Bendix ${ }^{8}$

${ }^{1}$ Universitätsklinik Essen, Kinderheilkunde I, Essen, Deutschland, ${ }^{2}$ Klinik für Kinder und Jugendmedizin der Universität, Klinik für Kinderheilkunde, Essen, Deutschland, ${ }^{3} \mathrm{NYU}$ School of Medicine, Dept. of Microbiology, New York, Deutschland, ${ }^{4}$ Klinik für Kinder und Jugendmedizin der Universität, Neonatologie, Essen, Deutschland, ${ }^{5}$ Klinik für Kinder und Jugendmedizin der Universität, Klinik für Kinderheilkunde, Essen, Deutschland ${ }^{6}$ Universitätsklinikum Essen, Direktorin der Klinik für Kinderheilkunde I, Zentrum für Kinder- und Jugendmedizin, Essen, Deutschland, ${ }^{7}$ Universitätsklinikum Essen, Essen, Deutschland, ${ }^{8} \mathrm{Klinik}$ für Kinder und Jugendmedizin der Universität, Klinik für Kinderheilkunde I, Essen, Deutschland

Hintergrund. Inflammation spielt eine entscheidende Rolle bei der Entstehung von Gehirnschäden während der Entwicklung. „Tumor necrosis factor-inducible gene 6 protein" (TSG-6) ist ein endogenes Protein mit antiinflammatorischen Eigenschaften bei zahlreichen inflammatorischen Krankheitsprozessen. Bislang ist nichts bekannt über eine physiologische Funktion des TSG-6 während der Gehirnentwicklung oder im Rahmen einer neonatalen Neuroinflammation. Ebenfalls unbekannt ist das therapeutische Potenzial von TSG-6.

Methoden. PCR und Western Blotting wurden nach Standardprotokollen durchgeführt. Expressionsmuster von Serumcytokinen wurden mit Hilfe eines Multiplex-Elisas (Luminex 200) analysiert. Hemisphären von unbehandelten Wistar Ratten (p1-p15) wurden auf Änderungen der TSG-6-Expression während der Gehirnentwicklung untersucht. LPS behandelte Ratten (o,25 mg/kg LPS i.p. an p3) wurden auf Änderungen der TSG-6-Expression während einer Inflammation des entwickelnden Gehirn untersucht. Um das therapeutische Potenzial von TSG-6 zu analysieren, wurden neugeborene Wistarratten an p3 mit LPS behandelt und mit vier Dosen TSG-6 à 2,25 mg/kg im Abstand von jeweils $12 \mathrm{~h}$ therapiert. Die erste Applikation fand 3 h vor LPS-Gabe statt.

Ergebnisse. Analysen der entwicklungsabhängigen Expression von TSG-6 im Gehirn zeigten eine lineare Steigerung auf Genebene von p1 zu p15. Unterschiedliche TSG-6-Expressionen konnten zwischen Hemisphären, Cortex, Thalamus und Striatum auf Genebene an p6 nachgewiesen werden. Auf Proteinebene zeigten sich Expressionsunterschiede zwischen Thalamus und den anderen Gebieten. Nach LPS-induzierter Neuroinflammation (o-24 h) zeigte sich eine signifikant erhöhte Expression von TSG-6 auf Genebene. Cleaved Caspase-3, ein Apoptosemarker, zeigte eine signifikante Herabregulation von ca. 30\% unter zusätzlicher TSG-6-Therapie im Vergleich zur alleinigen LPS Applikation $(\mathrm{n}=12-15 ; \mathrm{p}=0,037)$. Das Expressionsmuster von Serumcytokinen zeigte unter LPS eine massiv erhöhte Expression o-8 h nach LPS Gabe. Eine signifikante Verringerung der Expression unter LPS im Vergleich zur Kontrolle konnte nach 48 h (p5) detektiert werden. Die zusätzliche Gabe von TSG-6 verstärkte die Verringerung der Cytokinexpression, mit Ausnahme von IL-18 (p>0,05), jedoch nur tendenziell.

Schlussfolgerung. Die TSG-6-Expression im Gehirn ist entwicklungsabhängig reguliert und gesteigert während einer Inflammation des neonatalen Gehirns. Die reduzierte Expression von aktivierter cleaved Caspase-3 demonstriert das neuroprotektive Potential einer TSG-6Therapie zur Behandlung einer inflammatorischen Schädigung des sich entwickelnden Gehirns. 


\section{FV-11}

\section{Akute Mikroglia-Aktivierung durch Hyperoxie im unreifen Hirn geht mit langfristig gestörter Integrität der weißen Substanz einher und wird durch Minozyklin blockiert}

\section{T. Schmitz' , G. Krabbe'2, S. Endesfelder', H. Kettenmann², C. Bührer' ${ }^{1}$ Charité - Universitätsmedizin Berlin, Klinik für Neonatologie, Berlin, Deutschland, ${ }^{2}$ Max Delbrück Centrum für Molekulare Medizin, Zelluläre Neurowissenschaften, Berlin, Deutschland}

Hintergrund. Trotz deutlich verbesserter Überlebensrate von sehr unreifen Frühgeborenen durch moderne medizinische Behandlungen leiden viele der Frühgeborenen an neurologischen Defiziten. Ursache ist oft eine Schädigung der weißen Hirnsubstanz mit Hypomyelinisierung. Perinatale Infektion, Hypokapnie und Hyperoxie sind Faktoren, die zu Schäden der weißen Substanz bei Frühgeborenen führen können.

Methode. Zur Untersuchung der akuten und langfristigen Auswirkungen von Sauerstoff-Toxizitäten auf die weiße Substanz haben wir neugeborene Ratten im Alter von 6 Tagen 80\% Sauerstoff über 24 h ( $\mathrm{P}_{6}$ bis $\left.\mathrm{P}_{7}\right)$ exponiert. Nach Erholung in Raumluft wurden im Alter von P11, $\mathrm{P}_{30}$ und P6o Myelinisierung und Diffusionseigenschaften der weißen Substanz mit Western Blot und Diffusion Tensor Imaging (DTI) im MRT analysiert. Eine Aktivierung von Mikroglia nach Hyperoxie wurde durch Zytokinmessungen mit ELISA und durch immunhistologische Beurteilung der Morphologie im Alter $\mathrm{P}_{7}$ unmittelbar nach Hyperoxie bestimmt. Schließlich wurde getestet, ob eine Behandlung mit Minozyklin die Hyperoxie-Effekte verhindert oder mindert.

Ergebnisse. Nach $24 \mathrm{~h}$ Hyperoxie wiesen Mikroglia in der weißen Substanz von $\mathrm{P}_{7}$-Ratten eine aktivierte, amöboide Morphologie auf, die Expression einiger proinflammatorischer Zytokine und Mediatoren war erhöht. Die Myelin-Synthese, gemessen anhand der Expression von Myelin-basischem Protein (MBP), war nach Hyperoxie im Alter P11 signifikant reduziert, bei $\mathrm{P}_{3} 0$ aber spontan kompensiert. Im Alter $\mathrm{P}_{30}$ und P6o war die fraktionale Anisotropie (FA) in den DTI-Messungen des Corpus callosum bei Tieren nach Hyperoxie signifikant erniedrigt In Tieren mit Minozyklin-Behandlung waren diese Hyperoxie-induzierten Veränderungen nicht zu finden.

Schlussfolgerung. Hyperoxie im unreifen Hirn führt in unseren Studien zu rascher Aktivierung von Mikroglia und zu nachhaltiger Schädigung der Integrität der weißen Substanz. Beides wird durch Behandlung mit Minozyklin verhindert.

\section{FV-12}

Therapeutische Hypothermie nach schwerer Hypoxie-Ischämie: unwirksam und potenziell gefährlich im neonatalen Rattenmodel

\section{H. Sabir', E. Scull-Brown', X. Liu', M. Thoresen' \\ 'University of Bristol, School of Clinical Sciences, Neonatal Neuroscience, St Michael's Hospital, Bristol, United Kingdom}

Hintergrund. Die therapeutische Hypothermie (HT) sollte nach perinataler Asphyxie innerhalb der ersten 6 Lebensstunden begonnen werden. Es gibt klinische Hinweise darauf, dass HT vor allem für die moderate Enzephalopathie wirksam ist. Es gibt jedoch keine Hinweise darauf, ob ein verzögerter Start von HT nach moderater bzw. schwerer Enzephalopathie effektiv oder gar schädlich ist.

Material und Methoden. 107 sieben Tage alte Ratten $\left(\mathrm{P}_{7}\right)$ wurden in einem moderaten unilateralen Hypoxie-Ischämie-Model (HIE) untersucht $\left(8 \%\right.$ Sauerstoff für 90 min bei $\left.36^{\circ} \mathrm{C}\right)$. Die Ratten wurden anschließend randomisiert, um 5 h Normothermie (NT, Trec $=37^{\circ} \mathrm{C}$ ) oder HT $\left(\operatorname{Trec}=32^{\circ} \mathrm{C}\right) \mathrm{zu}$ erhalten. Die Therapie wurde entweder direkt im Anschluss an die Ischämie-Hypoxie oder mit 3 h, 6 h oder 12 h Verzögerung gestartet. Weitere $105 \mathrm{P} 7$-Ratten wurden in einem schweren unilateralen Ischämie-Hypoxie Model ( $8 \%$ Sauerstoff für 150 min bei $\left.37^{\circ} \mathrm{C}\right)$ untersucht und anschließend nach dem gleichen Design randomisiert und behandelt. Nach einer Woche wurden die Rattengehirne mittels „brain area loss" (BAL) analysiert.
Ergebnisse. In der moderaten NT-Gruppe zeigte sich ein mittlerer BAL von $40,5 \%$. Mittels direkter HT wurde dieser signifikant auf $24,8 \%$ reduziert $(\mathrm{p}<0,05)$. Ein verzögerter HT Beginn resultierte in einem linearen Anstieg des BAL von 1,788\%/Stunde, bis zu einer Verzögerung von $6 \mathrm{~h}$ (lineare Regressionsanalyse; $\mathrm{p}=\mathrm{O}, \mathrm{026}$ ). Ein $12 \mathrm{~h}$ verzögerter Therapiebeginn zeigte einen gleichen BAL, wie in der NT Gruppe (41,1\%). Nach schwerer HIE zeigte sich in der NT Gruppe ein mittlerer BAL von 59,3\%. Die direkte, als auch die $3 \mathrm{~h}$ und $6 \mathrm{~h}$ verzögerte HT resultierte in einem ähnlichen BAL. Nach 12 h verzögerter HT zeigte sich ein signifikant erhöhter BAL (69,5\%; $\mathrm{p}=0,032)$.

Diskussion und Schlussfolgerung. Die direkte und bis zu $6 \mathrm{~h}$ verzögerte HT ist neuroprotektiv nach moderater HIE im neonatalen Rattengehirn. Diese Neuroprotektion nimmt linear mit zunehmender Verzögerung ab. Nach schwerer HIE ist weder die direkte noch die bis zu $6 \mathrm{~h}$ verzögerte HT neuroprotektiv. Ein um 12 h verzögerter Therapiebeginn erhöht den Gehirnschaden nach schwerer HIE.

\section{FV-13}

Neugeborene drogenabhängiger Mütter - Management und neonatales Outcome

\section{Hüseman', S. Muecke', M. Nagel', C. Bührer', W. Henrich' ' J. Siedentopf ${ }^{2}$}

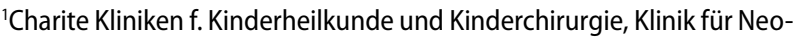
natologie, Berlin, Deutschland, ${ }^{2}$ Charité Campus Virchow-Klinikum, Klinik für Geburtsmedizin, Berlin, Deutschland

Hintergrund. Neugeborene mit chronischer intrauteriner Opiatexposition auf Grund maternaler Substanzabhängigkeit sind durch fetale Retardierung, Frühgeburtlichkeit, durch eine komplexe Entzugssymptomatik (neonatales Abstinenzsyndrom, NAS) sowie durch Entlassung in unsichere familiäre Verhältnisse bedroht. Die Heterogenität und eingeschränkte Erreichbarkeit dieser Patientengruppe erschweren die Datenerhebung. Buprenorphin als mütterliches Substitut und Morphinlösung zur neonatalen Entzugstherapie werden durch existierende Studien favorisiert.

Fragestellung. Wir berichten über die regionalisierte geburtsübergreifende Betreuung in unserem Perinatalzentrum in einem 12-JahresZeitraum. Die Analyse umfasst anthropometrische Daten, neonatale Komorbiditäten, den Verlauf des NAS sowie Entlassungsmodalitäten in Abhängigkeit vom maternalen Konsummuster und der kindlichen medikamentösen Therapie.

Methoden. Eingeschlossen wurden 378 Mutter-Kind-Paare aus den Geburtsjahrgängen 2000-2011, bei denen entweder ein mütterlicher Opioid-Konsum bekannt war oder aus kindlicher Klinik und Mekoniumtoxikologie die Diagnose NAS (ICD-10 P96.1) gestellt wurde. Maternale Daten wurden prospektiv, neonatale Verlaufsdaten retrospektiv erhoben. Zur statistischen Analyse wurden SPSS, Microsoft Excel und EpiInfo7 verwendet.

Ergebnisse. Insgesamt 94\% der Mütter wurden zum Zeitpunkt der Geburt kontrolliert substituiert. Das Geburtsgewicht lag bei $32 \%$ der Kinder $\leq 10$. Perzentile, bei $14 \% \leq \mathrm{P}_{3} . \mathbf{2 0} \%$ der Kinder waren Frühgeborene, $6 \%$ waren HIV-exponiert. Ein medikamentös therapiepflichtiges NAS trat nach Buprenorphin-Substitution der Mutter mit 48\% signifikant seltener auf als nach Methadon-Substitution ( $71 \% ; \mathrm{p}<0,01)$, die mediane Behandlungsdauer betrug 6 bzw. 10 Tage $(\mathrm{p}<0,05)$. Bei der medikamentösen Therapie der Kinder zeigte Phenobarbital eine auf 9 Tage verkürzte mediane Behandlungsdauer gegenüber 19 Tagen unter Morphin $(\mathrm{p}<\mathrm{0}, 01)$. Im Lauf der 12 Jahre nahmen sowohl die Dauer der Behandlung als auch die maternale Substitutionsdosis zu. $72 \%$ der Kinder wurden in den mütterlichen Haushalt entlassen, während der letzten 3 Jahre wurde eine vorübergehende Betreuung in Mutter-Kind-Einrichtungen in $16 \%$ der Fälle organisiert.

Diskussion. Die Betreuung von opiatkonsumierenden Schwangeren und ihren Neugeborenen erfordert geburts- und insititutionsübergreifende interdisziplinäre Strukturen. Die Substitutionsbehandlung mit Buprenorphin scheint Vorteile für die Neugeborenen zu haben, lässt sich aber 
aus mütterlicher Sicht häufig nicht durchführen. Die in unserer Gruppe beobachtete längere Therapiedauer bei Einsatz von Morphin steht im Widerspruch zu international publizierten Daten. Zur Prävention einer Kindeswohlgefährdung erscheint eine professionalisierte kinderschutzorientierte psychosoziale Begleitung der Mutter-Kind-Paare von großer Wichtigkeit.

\section{Medizinethik - schwierige klinische Entscheidungen}

\section{FV-14 \\ Nur zu deinem Besten - Reanimation zweier Jugendlicher mit Selbsttötungsabsicht in klinisch-ethischer Sicht}

\section{Schoberer', S. Trepels-Kottek', E. Rose', K. Heimann', V. Umlauf', D. Groß $\beta^{2}$, T. Orlikowsky' \\ 'Universitätsklinik für Kinder- und Jugendmedizin, Sektion für Neonato- logie, Aachen, Deutschland, ${ }^{2}$ Institut für Geschichte, Theorie und Ethik der Medizin, Universitätsklinikum Aachen, Aachen, Deutschland}

Einleitung. Die Durchführung einer kardiopulmonalen Reanimation (CPR) ist nach gängigen Leitlinien beim präklinischen Auffinden lebloser Patienten ohne sichere Zeichen des Todes geboten. Sofern die CPR nicht im Rahmen einer Patientenverfügung ausdrücklich abgelehnt wird, gilt sie als Maßnahme im besten Interesse des Patienten. Anhand zweier Kasuistiken skizzieren wir klinisch-ethische Dilemmata, die bei retrospektiver Betrachtung das eigene Vorgehen in Frage stellen, obwohl es kunstgerecht war.

Kasuistiken. Zwei männliche Jugendliche im Alter von 15 und 17 Jahren wurden jeweils durch enge Familienangehörige nach suizidal herbeigeführter Strangulation aufgefunden, sofort aus der Strangulation befreit und zunächst durch die Laien, nach Eintreffen der Rettungsdienste professionell reanimiert. Eine Rückkehr der spontanen Zirkulation (ROSC) trat nach 20 bzw. 12 Minuten ein. Beide postpubertären $\mathrm{Pa}$ tienten wurden gemäß DGN-Leitlinie „Hypoxische Enzephalopathie (AWMF-Register-Nr. 030/119) gekühlt. Ein Patient verstarb nach $7 \mathrm{Ta}-$ gen nach Hirntodfeststellung, der andere Patient überlebte mit schwerer Stammganglienschädigung, Occipitalhirnschädigung mit partieller Hirnstammareflexie in einem Zustand maximal eingeschränkter Kommunikations- und Handlungsfähigkeit.

Diskussion. Anhand der dargestellten Kasuistiken ist zu diskutieren, ob eine CPR bei offensichtlicher Selbsttötungsabsicht gegen die Autonomie des Patienten verstößt und ob die Stellvertreterentscheidung zur Reanimation tatsächlich im besten Interesse der Patienten erfolgte. Aus klinisch-ethischer Sicht sind hierbei vier Problembereiche zu identifizieren:

1. So ist der ärztliche Entscheidungskorridor in der beschriebenen Akutsituation ausgesprochen eng; er erschwert in vielen Fällen eine sorgfältige Güterabwägung.

2. Auch wenn die Suizidhandlung prima vista als Willensäußerung zu werten ist, kann nicht vorausgesetzt werden, dass sie in einem mental kompetenten Zustand getroffen wurde.

3. Zudem erlaubt die Auffindesituation nicht in jedem Fall einen sicheren Ausschluss von Fremdverschulden.

4. Schließlich ist auch die Evidenzlage schwierig: Empirische Daten zum Überleben der präklinischen Reanimation bei Kindern sind rar. Aufgrund von von Daten des Cardiac Arrest Registry to Enhance Survival (CARES) der Centers for Disease Control and Prevention (CDC) ist anzunehmen, dass bei fehlender Bezeugung des Eintritts des Kreislaufstillstandes durch Dritte nur rund 10\% der primär erfolgreich reanimierten Patienten langfristig überleben. Besonders kritisch ist, dass für rund die Hälfte der Langzeitüberlebenden von einem schwer bis schwerst eingeschränkten neurologischen Status (Cerebral Performance Category 3 und 4) auszugehen ist. Ein zusätzliches Problem ergibt sich aktuell für die Gruppe hypothermiebehandelter Patienten mit hypoxischer Enzephalopathie daraus, dass herkömmliche Frühindikatoren für die Prognoseabschätzung ihre Validität verloren haben.

\section{Anämie - Hämotherapie - Transfusionsmedizin}

\section{FV-15}

Hepcidinkonzentration im Nabelschnurblut von Früh- und Reifgeborenen korrelieren mit Gestationsalter und Eisenstatus

\section{Lorenz', J. Herbst', A. Peter', G. Olbina ${ }^{3}$, M. Westerman ${ }^{3}$, C. Poets', A. Franz}

'Eberhard-Karls-Universität Universitätsklinik für Kinderheilkunde und Jugendmedizin, Neonatologie, Tübingen, Deutschland, ${ }^{2}$ Eberhard-KarlsUniversität Universitätsklinik für Innere Medizin, Labormedizin, Tübingen, Deutschland, ${ }^{3}$ Intrinsic LifeSciences, La Jolla, USA

Hintergrund. Das 2001 entdeckte Peptidhormon Hepcidin spielt eine wichtige Rolle in der Bereitstellung von Eisen aus Enterozyten, Makrophagen, Hepatozyten und auch Syncytiotrophoblasten. Durch Bindung an den Eisentransporter Ferroportin wird die Abgabe von Eisen aus der Zelle in das Plasma verhindert. Dieser 2012 erstmalig bei Frühgeborenen (FG) nachgewiesene Parameter könnte langfristig eine nichtinvasive Überwachung einer individualisierten Eisensupplementierung ermöglichen.

Fragestellung. Ziel war, potenzielle Einflussfaktoren auf die fetale Serum-Hepcidin-Konzentration $(\mathrm{Hep}(\mathrm{S}))$ wie Gestationsalter, Eisenstatus, Entbindungsmodus, perinatale Infektion und perinatale Azidose $\mathrm{zu}$ untersuchen und gestationsalterspezifische Referenzbereiche für die Hep(S) zu etablieren.

Material und Methoden. In die Studie wurden 162 Neugeborene (Gestationsalter: 24.-42. SSW) eingeschlossen. Aus Nabelschnurblut erfolgte die Hepcidinbestimmung mittels Bioassay-validiertem ELISA, außerdem wurden Ferritin und IL- 6 bestimmt. Die nicht normalverteilten Daten werden als Median (Interquartilabstand) dargestellt und mittels Spearman's Rangkorrelationskoeffizient sowie Wilcoxon-Mann-Whitney-Test ausgewertet.

Ergebnisse. Hep(S) betrug $75(39,125) \mathrm{ng} / \mathrm{ml}$. Hepcidin konnte auch bei extrem unreifen FG im Nabelschnurblut nachgewiesen werden. Mit steigendem Gestationsalter nahm $\operatorname{Hep}(\mathrm{S}) \mathrm{zu}(\mathrm{r}=0,51 ; \mathrm{p}<0,0001)$. Bei $\mathrm{FG}<30$ SSW betrug Hep(S) $30 \mathrm{ng} / \mathrm{ml}[(17,60), \mathrm{n}=19]$, bei FG zwischen $30-$ $36 \mathrm{SSW} 54 \mathrm{ng} / \mathrm{ml}[(34,78), \mathrm{n}=56]$ und bei Reifgeborenen $(\mathrm{RG})>36 \mathrm{SSW}$ $107 \mathrm{ng} / \mathrm{ml}[(72,153), \mathrm{n}=87]$. Hep(S) von RG mit einem Nabelarterien-pH $(\mathrm{NapH})<7,15$ war höher $[130 \mathrm{ng} / \mathrm{ml}(116,198)]$ als von RG mit einem $\mathrm{NapH}>7,15[97 \mathrm{ng} / \mathrm{ml}(68,145) ; \mathrm{p}=0,02]$. Spontan entbundene RG hatten höhere Hep(S) $[121 \mathrm{ng} / \mathrm{ml}(87,153)]$ im Vergleich zu RG nach primärer Sektio [ $95 \mathrm{ng} / \mathrm{ml}(47,123)$; p=0,06]. Die höchste Hep(S) (437 ng/ml) wurde im Rahmen einer neonatalen Infektion mit einem hohen IL-6-Wert (1760 ng/L) beobachtet. Es konnte eine Korrelation zwischen Hep(S) und Ferritin nachgewiesen werden $(\mathrm{r}=0,67 ; \mathrm{p}<0,0001)$. In der Subgruppe der Kinder ohne Infektion, Azidose oder versuchter vaginaler Entbindung $(n=48)$ zeigte sich eine deutlich bessere Korrelation zwischen $\operatorname{Hep}(\mathrm{S})$ und Ferritin $(\mathrm{r}=0,86 ; \mathrm{p}<0,0001)$, sowie zwischen $\operatorname{Hep}(\mathrm{S})$ und dem Gestationsalter ( $r=0,70 ; p<0,0001)$.

Schlussfolgerung. Dies ist die erste Beobachtungsstudie über Hepcidin im Nabelschnurblut von FG. Es konnte gezeigt werden, dass auch sehr unreife FG bereits intrauterin Hepcidin produzieren und dass Hep $(\mathrm{S})$ mit steigendem Gestationsalter zunimmt. Hep(S) korreliert stark mit Ferritin und ist damit zur Evaluation des Eisenstatus geeignet, kann allerdings von verschiedenen Faktoren wie z. B. einer perinatalen Inflammationsreaktion beeinflusst werden. 


\section{Monitoring der Vitalfunktionen und der Oxygenierung}

\section{FV-16}

\section{Postpartale Sauerstoffsättigung reifer Neugeborener}

\section{J. Aicher', M. Burgmann'2, U. Hasbargen ${ }^{2}$, A. Schulze', A. Flemmer'}

'LMU-München, Neonatologie der Kinderklinik am Perinatalzentrum Großhadern, München, Deutschland, ${ }^{2}$ Klinikum Großhadern, Perinatalzentrum Großhadern, München, Deutschland

Hintergrund und Zielsetzung. Die ersten Lebensstunden nach der Geburt werden reife Neugeborene in der Regel bei der Mutter belassen und durch die versorgende Hebamme überwacht. In dieser Zeit der postnatalen Anpassung besteht aber möglicherweise ein erhöhtes Risiko für Atemregulationsstörungen. Ziel dieser Pilotstudie war, Abfälle der Sauerstoffsättigung und der Herzfrequenz reifer Neugeborener in dieser Phase zu quantifizieren. Des Weiteren wurden Risikofaktoren untersucht, die einen Einfluss auf das Auftreten von Sauerstoffsättigungs- bzw. Herzfrequenzabfällen haben können.

Methodik. 100 reife, gesunde Neugeborene $(37+0 / 7$ bis $42+0 / 7$ SSW) wurden unmittelbar nach der Geburt mittels Pulsoxymetrie $(0,5 \mathrm{~Hz})$ untersucht. Eine Sättigung unter $85 \%$ und Herzfrequenz unter $80 / \mathrm{min}$ bei guter Signalqualität wurden als pathologisch gewertet. Als klinisch relevant wurden Episoden mit Entsättigung oder Bradykardie gewertet, die eine Intervention benötigten. Mittels logistischer Regression wurde der Einfluss von Risikofaktoren für das Auftreten von Desaturierungen im Kreißsaal untersucht.

Ergebnisse. Bei 26/10o Kindern traten Desaturierungen, bei o/10o Bradykardien auf. Bei Kindern nach primärer Sectio $(n=49)$ fanden sich tendenziell häufiger Desaturierungen ( $37 \%$ vs. $14 \%, p=0,18$ im unkorrigierten Modell bzw. 0,23 im korrigierten Modell). Diese wurden dabei signifikant früher entbunden $(\mathrm{p}<0,01)$. Des Weiteren traten bei $50 \%$ der Kinder mit mütterlichem Gestationsdiabetes oder später diagnostizierten Neugeboreneninfektion Desaturierungen auf. Kinder mit Desaturierungen hatten im Mittel einen signifikant niedrigeren APGAR bei 5 Minuten $(\mathrm{p}=0,03)$.

Schlussfolgerungen. Diese Daten zeigen, dass bei gesunden reifen Neugeborenen Desaturierungen in den ersten Lebensstunden auftreten. Potenzielle Risikofaktoren scheinen eine primäre Schnittentbindung, GDM oder ein niedriger APGAR bei 5 Minuten zu sein. Ob eine kontinuierliche Überwachung aller Neugeborenen zu einer zusätzlichen Sicherheit im Kreißsaal führt und damit den zusätzlichen Aufwand rechtfertigt, muss an einer größeren Studienpopulation untersucht werden.

\section{FV-17}

Definition von Perzentilen für die zerebrale regionale Gewebssättigung bei Neugeborenen in den ersten 15 Minuten nach Geburt

G. Pichler', C. Binder', A. Avian², M. Pocivalnik', E. Beckenbach', G. Schmoelzer ${ }^{1}$, B. Urlesberger

'Universitäts Klinik für Kinder- und Jugendheilkunde Graz, Abteilung für Neonatologie, Graz, Österreich, ${ }^{2}$ Institut für Informatik, Statistik und Dokumentation, Med. Universität Graz, Graz, Österreich

Hintergrund. Die mittels Nahinfrarot Sektroskopie (NIRS) durchgeführte Monitorisierung der zerebralen regionalen Gewebssättigung findet zunehmend Anwendung bei Neugeborenen in der Adaptationsphase.

Fragestellung. Ziel der Studie war es Perzentilen der zerebralen regionalen Gewebssättigung ( $\mathrm{crSO}_{2}$ ) während der ersten 15 Minuten nach Geburt bei Neugeborenen mit unauffälliger Adaptation zu erstellen.

Material und Methodik. In dieser prospektiven Beobachtungsstudie wurden reife Neugeborene und Frühgeborene inkludiert. crSO2 wurde mit NIRS (INVOS 5100) während der ersten 15 Minuten nach Geburt gemessen. Die NIRS-Sensoren wurden links frontal am Kopf fixiert.
Die Neugeborenen wurden exkludiert, wenn sie eine Unterstützung benötigten.

Ergebnisse. Insgesamt wurden 381 Neugeborene in diese Studie inkludiert: 82 reife Neugeborene nach vaginaler Spontangeburt, 272 reife Neugeborene und 27 Frühgeborene nach Kaiserschnittentbindung. In der Gesamtgruppe betrug 2, 5, 10 und 15 Minuten nach Geburt $\mathrm{crSO}_{2}$ $41 \%(23-64), 68 \%(45-85), 79 \%$ (65-9o) bzw. 77\% (63-89) [Median (10.90. Perzentile)]. Bei den reifen Neugeborenen nach Spontangeburt betrug 2, 5, 10 und 15 Minuten nach Geburt crSO2 41\% (25-60), 72\% (5486), $78 \%$ (64-88) bzw. 75\% (59-87). Bei den reifen Neugeborenen nach Kaiserschnittentbindung betrug 2, 5, 10 und 15 Minuten nach Geburt $\mathrm{crSO}_{2} 41 \%$ (22-64), 67\% (42-85), 80\% (66-90) bzw. 78\% (65-89). Bei den Frühgeborenen nach Kaiserschnittentbindung betrug 2, 5, 10 und $15 \mathrm{Mi}$ nuten nach Geburt crSO2 44\% (25-67), 70\% (52-85), 83\% (66-94) bzw. $80 \%(63-92)$.

Diskussion. In der vorliegenden Studie konnten erstmals Perzentilen für die zerebrale regionale Gewebssättigung bei Neugeborenen in der Adaptationsphase definiert werden.

\section{FV-18}

\section{Zusammenhang zwischen BNP und zerebraler Gewebssättigung} bei Neugeborenen

\section{Binder', B. Urlesberger', B. Schwaberger', E. Ziehenberger', G. Pichler'}

'Univ. Klinikum für Kinder- und Jugendheilkunde, Medizinische Universität Graz, Klinische Abteilung für Neonatologie, Graz, Österreich

Hintergrund. Die regionale zerebrale Gewebssättigung (zrSO2) wird von der Sauerstoffanlieferung zum Gehirn und somit auch vom zerebralen Blutfluss beeinflusst. Der zerebrale Blutfluss hängt einerseits von der Herzauswurfsleistung und andererseits vom Gefäßwiderstand ab. Das B (brain) type natriuretic peptide (BNP) ist ein Hormon welches von der Herzmuskelzelle synthetisiert und bei zu starker Dehnung des Ventrikels in das Blut abgegeben wird. BNP kann somit als Marker für die Herzfüllung/-leistung und Herzinsuffizienz herangezogen werden. Fragestellung. Gibt es einen Zusammenhang zwischen dem BNP und der $\mathrm{zrSO}_{2}$ bei Neugeborenen während des ersten Lebenstages?

Methoden. Bei dieser prospektiven Beobachtungsstudie wurden zerebrale Nahinfrarotspektroskopie (NIRS) Messungen, zur Bestimmung der $\mathrm{zrSO}_{2}$, an der rechten Stirnseite von Früh/Reifgeborenen durchgeführt. Die Messdauer betrug 24 Stunden mit Beginn innerhalb der ersten 6 Lebensstunden. Zusätzlich wurden die Neugeborenen mit einem Pulsoxymeter überwacht. Am Ende der NIRS-Messung wurde eine Blutprobe zur Analyse des BNP abgenommen. Die zerebrale „fractional tissue oxygen extraction" (zFTOE) wurde für jede Stunde berechnet. Es wurden Mittelwerte der $\mathrm{zrSO}_{2}$ und der zFTOE über die gesamte Messdauer ( $\mathrm{rrS}_{2} \_24 \mathrm{~h}, \mathrm{zFTOE} \_24 \mathrm{~h}$ ) und für die Stunde vor der Blutabnahme (zrSO2_b, zFTOE_b) berechnet. $\mathrm{zrSO}_{2} \_24 \mathrm{~h} / \mathrm{zFTOE} \_24 \mathrm{~h}$ und $\mathrm{zrSO}_{2} \_\mathrm{b} / \mathrm{zFTOE} \_\mathrm{b}$ wurden mit den BNP Werten korreliert.

Ergebnisse. 54 Neugeborene ( $35 \pm 3$ Wochen, $2337 \pm 801 \mathrm{~g}$ ) wurden in die Studie inkludiert. Die NIRS-Messungen begannen $4 \pm 4$ Stunden nach der Geburt und die Blutabnahmen zur Bestimmung des BNP erfolgten $25 \pm 9$ Stunden nach der Geburt. Das BNP betrug im Mittel $5121 \pm 3718$ pg/ $\mathrm{ml}$. Die mittlere $\mathrm{zrSO}_{2} \_24 \mathrm{~h}$ war $75 \pm 9 \%$, die $\mathrm{zrSO}_{2} \_\mathrm{b} 76 \pm 9 \%$, die zFTOE_24 h 0,24 $\pm 0,11$ und die zFTOE_b o,19 $\pm 0,08$. BNP korrelierte negative mit der $\mathrm{zrSO}_{2} \_\mathrm{b}(\mathrm{r}=-0,554 ; \mathrm{p} \leq \mathrm{O}, \mathrm{Ooo})$ und der $\mathrm{zrSO}_{2} \_24 \mathrm{~h}$ $(\mathrm{r}=-0,516 ; \mathrm{p} \leq \mathrm{o}, 000)$ und positive mit $\mathrm{zFTOE} \_\mathrm{b}(\mathrm{r}=0,610 ; \mathrm{p} \leq \mathrm{o}, 000)$ und zFTOE_24 h (o,291; p=0,049).

Schlussfolgerung. Mit dieser Studie konnte gezeigt werden, dass es einen Zusammenhang zwischen dem BNP, einem Marker der Herzfüllung/-leistung, und der $\mathrm{zrSO}_{2}$ bzw. der zFTOE am ersten Lebenstag bei Neugeborenen gibt. 


\section{FV-19}

\section{Auswirkungen von Sättigungsabfällen und Bradykardien auf die} zerebrale Oxygenierung von Frühgeborenen

\section{Schmid', R. Hopfner', S. Lenhof', H. Hummler', H. Fuchs' \\ ${ }^{1}$ Klinik für Kinder- und Jugendmedizin, Universitätsklinikum Ulm, Sektion Neonatologie und Pädiatrische Intensivmedizin, Ulm, Deutschland}

Hintergrund. Sättigungsabfälle und Bradykardien treten im Rahmen des Apnoe- und Bradykardiesyndroms bei fast allen sehr unreifen Frühgeborenen auf und sind mit Störungen der psychomotorischen Entwicklung assoziiert. Ereignisse welcher Art (Sättigungsabfälle, Bradykardien oder kombinierte Ereignisse), Häufigkeit und Intensität mit welchen Mitteln zu behandeln sind, ist derzeit unklar. Die Nahinfrarotspektroskopie könnte zur Erforschung der Auswirkungen dieser Ereignisse auf die zerebrale Sauerstoff- und Blutversorgung und mögliche Kompensationsmechanismen des Gehirns beitragen. Bisher liegen hierzu keine vergleichenden Studien vor.

Zielsetzung. Ziel dieser Arbeit ist es, den Einfluss von Sättigungsabfällen und Bradykardien auf die zerebrale Gewebeoxygenierung ( $\left.\mathrm{StO}_{2}\right)$ und auf Änderungen des zerebrales Gesamthämoglobin (HbT), der Sauerstoffextraktion (FTOE) und der Hämoglobin-Differenz (HbD) zu untersuchen.

Methoden. Bei 16 Frühgeborenen mit ausgeprägten Sättigungsabfällen und Bradykardien wurden alle 2 Sekunden arterielle Sauerstoffsättigung (SpO2), EKG Herzfrequenz (HF) und mittels absoluter Oxymetrie (FORE-SIGHT, Casmed) zerebrale $\mathrm{StO}_{2}, \mathrm{HbT}$, FTOE und $\mathrm{HbD}$ simultan aufgezeichnet. Ereignisse wurden einer von fünf Kategorien zugeordnet: (1) nur Bradykardie ( $\mathrm{HF}<8 \mathrm{o} / \mathrm{min}),(2)$ nur Sättigungsabfall (SpO2<75\%), (3) Sättigungsabfall und Bradykardie gleichzeitig, (4) Bradykardie mit nachfolgendem Sättigungsabfall, (5) Sättigungsabfall mit nachfolgender Bradykardie. Für jedes der Ereignisse wurde ein zerebraler Desaturierungsscore berechnet, der die Fläche unter dem $\mathrm{StO}_{2}$ Ausgangswert vor Beginn des Ereignisses widerspiegelt.

Ergebnisse. In $\bullet$ Tab. 1 sind die Hauptergebnisse (Median und Quartilen) wiedergegeben.

Schlussfolgerung. Bradykardien ohne Sättigungsabfälle hatten die geringste Auswirkung auf die zerebrale Oxygenierung, die stärkste Auswirkung hatten Sättigungsabfälle mit nachfolgender Bradykardie. Kombinierte Ereignisse wirkten sich stärker aus als Bradykardien oder Sättigungsabfälle alleine. Die zerebralen Desaturierungen waren weniger ausgeprägt als die arteriellen Sättigungsabfälle.

\section{FV-20}

Video Apgar Trial: Was man beim Filmen von Erstversorgungen im Kreißsaal beachten sollte

\section{H. Küster' ${ }^{1}$ M. Rüdiger ${ }^{2}$}

${ }^{1}$ Universitäts- Kinderklinik, Neonatologie, Göttingen, Deutschland, ${ }^{2}$ Univ. Klinikum Carl Gustav Carus, Klinik für Kinder- und Jugendmedizin, Neonatologie, Dresden, Deutschland

Hintergrund. Bei Erstversorgungen ist häufig der zeitliche und inhaltliche Ablauf der durchgeführten Maßnahmen im Nachhinein nicht exakt erinnerlich. Dies erschwert die Diskussion um potentielle Verbesserungsmöglichkeiten. Videoaufnahmen von Erstversorgungen sind ein schon seit Jahren verwendetes Werkzeug, um dieses Problem zu umgehen. Sowohl Aufnahme wie Interpretation dieser Videos erfolgen jedoch lokal sehr unterschiedlich, was ein Vergleich zwischen Kliniken unmöglich macht.

Fragestellungen. Wie können Videoaufnahme der Erstversorgung im Kreißsaal standardisiert und damit vergleichbar gemacht werden? Welche Voraussetzungen sind zu schaffen und welche Probleme zu beachten?

Material und Methode. Zwischen Kliniken mit Erfahrung bei Videoaufnahmen von Erstversorgungen wurde ein detailliertes Studienprotokoll mit Standardisierung der Aufnahmetechnik sowie der rechtlichen Rahmenbedingungen für Videoaufnahmen erarbeitet.

Ergebnisse. Vorab sollte mit der Verwaltung geklärt werden, welche Regelungen bei einer derartigen Qualitätssicherungsmaßnahme jenseits des Aufnahmevertrags getroffen werden müssen: Involvierung von Ethikkommission, Betriebsrat/Personalrat, Pflegedienstleitung, Datenschutzbeauftragte. Wichtig sind die frühzeitige Einbeziehung des Personals und die Betonung, dass die Aufnahmen ohne Schuldzuweisung analysiert werden. Die Akzeptanz wird erleichtert, wenn das Hervorheben der positiven Aspekte gezielt forciert wird und in der ersten Zeit bei der Nachbesprechung nur direkt Beteiligte anwesend sind. Wird der Kreis der Zuschauer sukzessive erweitert, ist je nach lokaler Rechtslage und Umfang der Verwendung der Videos eine Einwilligung der Erziehungsberechtigten notwendig. Die Aufnahme kann mit einer preisgünstigen Web-Kamera erfolgen, der Ton sollte abgeschaltet sein. Kameraposition und Lichteinfall müssen zu einem spiegelfreien Bildausschnitt von Kind und Monitoring sowie weiteren Messwerten (Thermometer) führen. Köpfe und Hände dürfen nicht die Sicht versperren, Gesichter nicht zu erkennen sein. Die Videos sollten auf einem separaten, passwortgesicherten PC ohne Internetzugang gespeichert werden, eine Anonymisierung von Dateiname und Aufnahmezeit ist wichtig. Auf diese Weise wird sichergestellt, dass im unwahrscheinlichen Fall einer Beschlagnahmung von Videos, z. B. durch die Staatsanwaltschaft, diese nicht nachträglich identifiziert werden können.

Schlussfolgerung und Ausblick. Die Standardisierung der Aufnahmetechnik erlaubt den klinikübergreifenden Vergleich des Vorgehens im Kreißsaal anhand einfach durchzuführender Videoaufnahmen. Im Rahmen des Video Apgar Trial soll mit dieser Technik 1. das Spektrum der Vorgehensweisen bei der Erstversorgung in Europa und deren potentielle Auswirkungen auf die Kurzzeitmorbidität und 2. der Effekt von Aufnahmen und deren interner Besprechung auf die Versorgungsqualität erfasst werden.

FV-19 Tab. 1 Hauptergebnisse (Median und Quartilen)

\begin{tabular}{|c|c|c|c|c|c|c|c|}
\hline $\begin{array}{l}\text { Ereigni- } \\
\text { styp }\end{array}$ & $\begin{array}{l}\text { Anzahl } \\
\text { (n) }\end{array}$ & $\begin{array}{l}\text { Zerebrale StO2: } \\
\text { Abfall vom StO2- } \\
\text { Ausgangswert }\end{array}$ & $\begin{array}{l}\text { Zerebrale St02: } \\
\text { Fläche unter dem } \\
\text { St02-Ausgang- } \\
\text { swert }\end{array}$ & $\begin{array}{l}\text { Zerebrale St02: } \\
\text { Fläche unter 55\% }\end{array}$ & $\begin{array}{l}\text { Sp02: Abfall vom } \\
\text { Sp02-Ausgang- } \\
\text { swert }\end{array}$ & Ereignisdauer (s) & $\begin{array}{l}\text { HbD: Abfall vom } \\
\text { HbD-Ausgang- } \\
\text { swert }\end{array}$ \\
\hline 1 & 20 & $8,0(4,6-11,8)$ & 98 (61-209) & $0(0-0)$ & $9,0(5,3-10,8)$ & $63(47-80)$ & $2,6(1,7-4,1)$ \\
\hline 2 & 393 & $10,5(6,5-16,5)$ & $523(177-1078)$ & $0(0-10)$ & $20,0(15,0-26,0)$ & 176 (102-298) & $5,3(3,1-7,7)$ \\
\hline 3 & 122 & $14,0(9,5-19,5)$ & $539(276-1132)$ & $0(0-41)$ & $24,0(18,5-29,0)$ & $176(104-286)$ & $5,7(3,7-8,0)$ \\
\hline 4 & 99 & $16,5(10,5-22,0)$ & 739 (407-1202) & $0(0-168)$ & $23,0(17,0-29,0)$ & $192(126-294)$ & $5,8(4,2-9,7)$ \\
\hline 5 & 122 & $17,3(11,4-25,3)$ & $1172(602-2571)$ & $0(0-353)$ & $30,0(20,0-40,3)$ & $274(170-538)$ & $7,6(5,0-12,3)$ \\
\hline
\end{tabular}


FV-21

Plötzliche Todesfälle und schwere lebensbedrohliche Ereignisse bei (Fast-)Reifgeborenen am 1. Lebenstag - Ergebnisse einer deutschlandweiten Erhebung

\author{
A. Poets ${ }^{1}$ \\ ${ }^{1}$ Kinderklinik, UKT, Abteilung Neonatologie, Tübingen, Deutschland
}

Hintergrund. Anlass war das Auftreten zweier solcher Fälle in unserer Klinik. Bei der Literaturrecherche fanden wir Fallberichte aus verschiedenen Ländern, jedoch keine landesweite Erhebung.

Fragestellung. Wie häufig treten solche Fälle in Deutschland auf? Was sind Risikofaktoren hierfür?

Material und Methoden. Deutschlandweite ESPED-Erhebung in den Jahren 2009 und 2010. Gemeldet werden sollten Reifgeborene (und FastReifgeborene in 2010), welche sich initial gut adaptiert hatten (10-Minuten-APGAR mindestens 8) und innerhalb der ersten 24 Lebensstunden plötzlich verstarben („sudden infant death“, SID) oder ein schweres lebensbedrohliches Ereignis („,apparent life threatening event“, ALTE) erlitten, welches einer Maskenbebeutelung, Intubation und/oder Herzdruckmassage bedurfte. Die Auswertung erfolgte anhand von Fragebögen zur Schwangerschaft, Geburt und Auffindesituation der Kinder. Für jeden Fall mit unklarer Ursache sollten 3 Kontrollen rekrutiert werden, für die in derselben Klinik entsprechende Fragebögen ausgefüllt werden sollten.

Ergebnisse. Von 85 Meldungen erfüllten 34 die Einschlusskriterien, was einer Inzidenz von 2,6/100.00o Lebendgeburten entspricht. Zwei dieser Kinder waren Fast-Reifgeborene und in drei Fällen wurde eine Ursache für das Ereignis identifiziert. Von den 31 Fällen mit unklarer Ursache traten 19 in den ersten zwei Stunden nach der Geburt auf. Neun Neugeborene erlitten ein SID, 22 ein ALTE. Für die 31 Fälle rekrutierten wir 93 Kontrollen. Im Fall-Kontroll-Vergleich konnten Erstgebärende [OddsRatio (OR) 6,22; 95\% Konfidenzintervall (95\% CI) 2,11-18,32] und eine mögliche Erstickungsposition des Kindes (OR 6,45; 95\% CI 1,22-34,10) als statistisch signifikante Risikofaktoren identifiziert werden.

Schlussfolgerung. Es ist wichtig, auch Reifgeborene in den ersten Stunden nach der Geburt engmaschig zu überwachen, v. a. bei Erstgebärenden. Eine Position, in der Ersticken vorstellbar ist, sollte durch entsprechende Aufklärung der Eltern vermieden werden.

1. Poets A, Steinfeldt R, Poets CF (2011) Sudden deaths and severe apparent life-threatening events in term infants within 24 hours of birth. Pediatrics 127:e869-e873

2. Poets A, Urschitz MS, Steinfeldt R, Poets CF (2012) Risk factors for early sudden deaths and severe apparent life-threatening events. Arch Dis Child Fetal Neonatal 97:F395-F397

\section{Ernährung - Wachstum - Gedeihen II}

\section{FV-22}

\section{Plasmakonzentrationen und molekulare Zusammensetzung von Phosphatidylcholin bei Frühgeborenen als Indikator der Unterver- sorgung mit essenziellen polyunsaturierten Fettsäuren}

W. Bernhard', M. Raith', R. Kunze', V. Koch', C. Maas', C. Poets', A. Franz ${ }^{1}$ 'Eberhard-Karls-Universität, Klinik für Kinderheilkunde und Jugendmedizin, Neonatologie, Tübingen, Deutschland

Hintergrund. Die polyunsaturierten Fettsäuren (PUFA) Docosahexaensäure (DHA) und Arachidonsäure (ARA) werden durch die Plazenta aktiv in den Fötus transportiert, während Linolsäure (LA) überwiegend zurückgehalten wird. DHA und ARA sind essentiell für die Entwicklung des Zentralnervensystems und als Präkursoren inflammationsregulierender Eicosanoide und Resolvine. Der PUFA-Transport im Plasma geschieht mit hohem Turnover im Wesentlichen als Phosphatidylcholin (PtdCho). Ziel war, die Versorgung Frühgeborener mit PUFA anhand der PtdCho-Konzentrationen in Nabelschnur- und postnatalen Blutproben zu untersuchen.

Methoden. Mütterliches Blutserum zum Geburtszeitpunkt ( $\mathrm{n}=121)$ und Nabelschnur-EDTA-Plasmen von Früh- und Reifgeborenen [n=144, Gestationsalter (GA): 24-42 Wochen] sowie Rest-Plasmen klinisch indizierter Blutentnahmen ( $\mathrm{n}=173$ von 55 Frühgeborenen [GA: 27,9 (23,435,o) Wochen] wurden mit Chloroform/Methanol extrahiert und die Konzentrationen und Zusammensetzung der individuellen PtdChoKomponenten mittels Tandemmassenspektrometrie (LC-MS/MS) gemessen. Alle Ergebnisse sind als Median (25.-75. Perz.) angegeben.

Ergebnisse. Die Plasmakonzentrationen von PtdCho der Feten [1,1 (o,9$1,3) \mathrm{mmol} / \mathrm{l}]$ waren niedriger als die Schwangerer $[3,3(2,8-3,8) \mathrm{mmol} / \mathrm{l}$; $\mathrm{p}<0,0001]$. Nach der (Früh-)Geburt stieg das Gesamt-PtdCho zunächst auf 1,6 (1,5-1,8) $\mathrm{mmol} / \mathrm{L}(\mathrm{d} \mathrm{o-2})$ und schließlich auf 2,7 (2,2-2,9) $\mathrm{mmol} / \mathrm{L}$ (d 14-28). Dieser Anstieg war durch eine Zunahme an LA- und Ölsäure(OA)-haltigen PtdCho-Komponenten bedingt, so dass der Anteil an PUFA-PtdCho vom Nabelschnur-Plasma mit 50 (46-53)\% und von frühen postnatalen Proben mit 50 (45-53)\% (d o-2) innerhalb einer Woche auf 31 (30-33)\% (d 7-14; p<0,001) abnahm. Im Nabelschnur-Plasma stieg DHA-PtdCho von 7,9 (7,0-8,6)\% (GA: 24-27 Wochen) auf 14,1 (12,415,8)\% (GA: 37-42 Wochen). In postnatalen Plasmaproben von für das korrigierte GA gematchten Frühgeborenen war DHA-PtdCho um ca. $50 \%$ erniedrigt und betrug $4,5(4,2-5,1) \%$ bzw. 6,6 $(6,2-7,0) \%(\mathrm{p}<0,001)$. AA-PtdCho war bei Frühgeborenen ebenfalls von 35 (33-38)\% im NSPlasma auf 25 (22-28)\% postnatal erniedrigt $(\mathrm{p}<0,001)$. Hingegen stieg LA-PtdCho von 27 (25-29)\% im NS-Plasma auf 40,3 (35,1-45,1)\% postnatal und OA-PtdCho von $20(18-22) \%$ auf $28(26-32) \%$.

Schlussfolgerung. Konzentration und molekulare Zusammensetzung von Plasma-PtdCho als Carrier zur Versorgung mit essentiellen Fettsäuren unterschieden sich bei den Frühgeborenen stark von intrauterinen Werten. Während die Absolutzunahme an Plasma-PtdCho Ausdruck der postnatal verstärkten Lipoproteinsekretion der Leber ist, ist die relative Abnahme polyunsaturierter zugunsten LA-haltiger Lipide Ausdruck einer metabolischen Abweichung von der für das Entwicklungsstadium physiologischen Norm. Die möglicherweise zugrundeliegende Lipidfehlernährung könnte zur beeinträchtigten kognitiven Entwicklung von Frühgeborenen und zur fehlerhaften Regulation von Inflammationsprozessen, z. B. im Rahmen einer bronchopulmonalen Dysplasie, beitragen. 


\section{FV-23}

Use of fatty acid profiles to investigate potential differences in absorption of commercially available lipid emulsions vs. breast milk: a preliminary study

\section{N. Fink', G. Fusch', N. Rochow', C. Fusch' \\ 'McMaster University, Department of Pediatrics, Hamilton, ON, Kanada}

Background. Growth and development of the preterm infant is influenced by energy intake and nutrient composition. Lipid emulsions (LE) are the main source for energy and essential fatty acids during the critical phase of postnatal adaption of preterm infants. Adverse side effects in infants fed LEs vs. breast milk (BM) suggest that these products may not be metabolically optimal for neonates. This could be linked to the physical properties and the biochemical composition of dietary lipids as the fatty acid profiles of commercial LEs are not similar to breast milk. In North America, access to various LEs is limited but it is possible that other LEs or combinations of various LEs may exhibit a more balanced fatty acid profile for preterm infants.

Aim. To determine how the fatty acid profile of BM obtained from mothers of preterm infants compares to that of LEs used in North America (mainly Intralipid) and internationally (ClinOleic, Lipidem and Omegaven).

Method. Extraction and esterification of lipids from $2 \mathrm{~mL}$ samples of $\mathrm{BM}(\mathrm{n}=14)$ and LE products (Intralipid, ClinOleic, Lipidem, Omegaven) and subsequent analysis of the fatty acid composition using a previously published method and gas chromatography/mass spectrometry. Left over BM samples were obtained from mothers of infants admitted to McMaster Children's Hospital NICU and pooled for each mother.

Preliminary results. Preliminary results indicate that preterm BM samples exhibit a markedly different fatty acid profile compared to LEs (- Fig. 1). Different profiles of fatty acid chain length categories were observed between samples; Lipidem has a high proportion of mediumchain fatty acids compared to $\mathrm{BM}$, and Omegaven has a high proportion of polyunsaturated long-chain fatty acids compared to BM and other LEs.

Clinical significance. LEs do not display a comparable fatty acid profile to that of BM, yet they are intended to replace the lipid portion of $\mathrm{BM}$ for infants. Future comparison of fatty acid profiles in nutrition sources to resulting serum profiles may reveal differences in lipid digestion and absorption that could influence whether the fatty acids are utilized for either $\beta$-oxidation or lipogenesis.

\section{FV-24}

Target fortification of breast milk for preterm infants - What is the ideal measurement frequency?

\section{N. Rochow', G. Fusch', B. Zapanta', A. Ali', C. Fusch}

'McMaster University, Department of Pediatrics, Hamilton, ON, Kanada

Background. Target fortification (TFO) of breast milk (BM) with fat (F), protein $(\mathrm{P})$ and carbohydrates $(\mathrm{CHO})$ standardizes the macronutrient composition. Recently, we have shown that preterm infants receiving TFO demonstrate a linear relationship between growth and milk intake over the whole range of feeding intake $(140-155 \mathrm{~mL} / \mathrm{kg} / \mathrm{d} ; \mathrm{R} 2$ of 0.68 ), while in infants with only routine fortification, growth rates seem to be independent of milk intake. However, TFO adds considerable amount of work load to the NICU team: the total time required to measure BM composition is approximately 5 to $7 \mathrm{~min}$ per sample. The time required for calculation of TFO, documentation, and printing of the prescription is 5-10 min per sample. We acknowledge that daily BM analysis may not be feasible in all locations, particularly in units with limited resources. Objective. To analyze the variation of macronutrients in BM when TFO is done at different frequencies.

Method. Single-center, prospective study of 10 stable, fully enteral BM fed VLBW infants receiving TFO. Calories, F, P and CHO were measured on a daily basis for at least 21 days. For TFO to native BM, a routine fortifier (F: 0.36, P: 1 and CHO: $1.8 \mathrm{~g} / 100 \mathrm{~mL}$ ) and single additives of F, P and $\mathrm{CHO}$ was added to achieve a target concentration of BM (F: 4.4, P: 3, CHO: $8.8 \mathrm{~g} / 100 \mathrm{~mL}$ ). The variation in calories and macronutrients in TFO was modelled for when the BM would be target fortified based on measurements daily, every weekday, Mo-We-Fr, Mo-Th, and Mo only. The resulting variation of TFO BM was further compared to native BM. Results. $\mathrm{n}=\mathbf{2 9 5}$ native BM samples were used for model calculations. This data was analyzed and showed an average composition of kcal: 65 \pm 8 , F: $4.1 \pm 0.9$, P: $1.2 \pm 0.2$, CHO: $5.8 \pm 0.2 \mathrm{~g} / 100 \mathrm{~mL}$. When BM was tar-

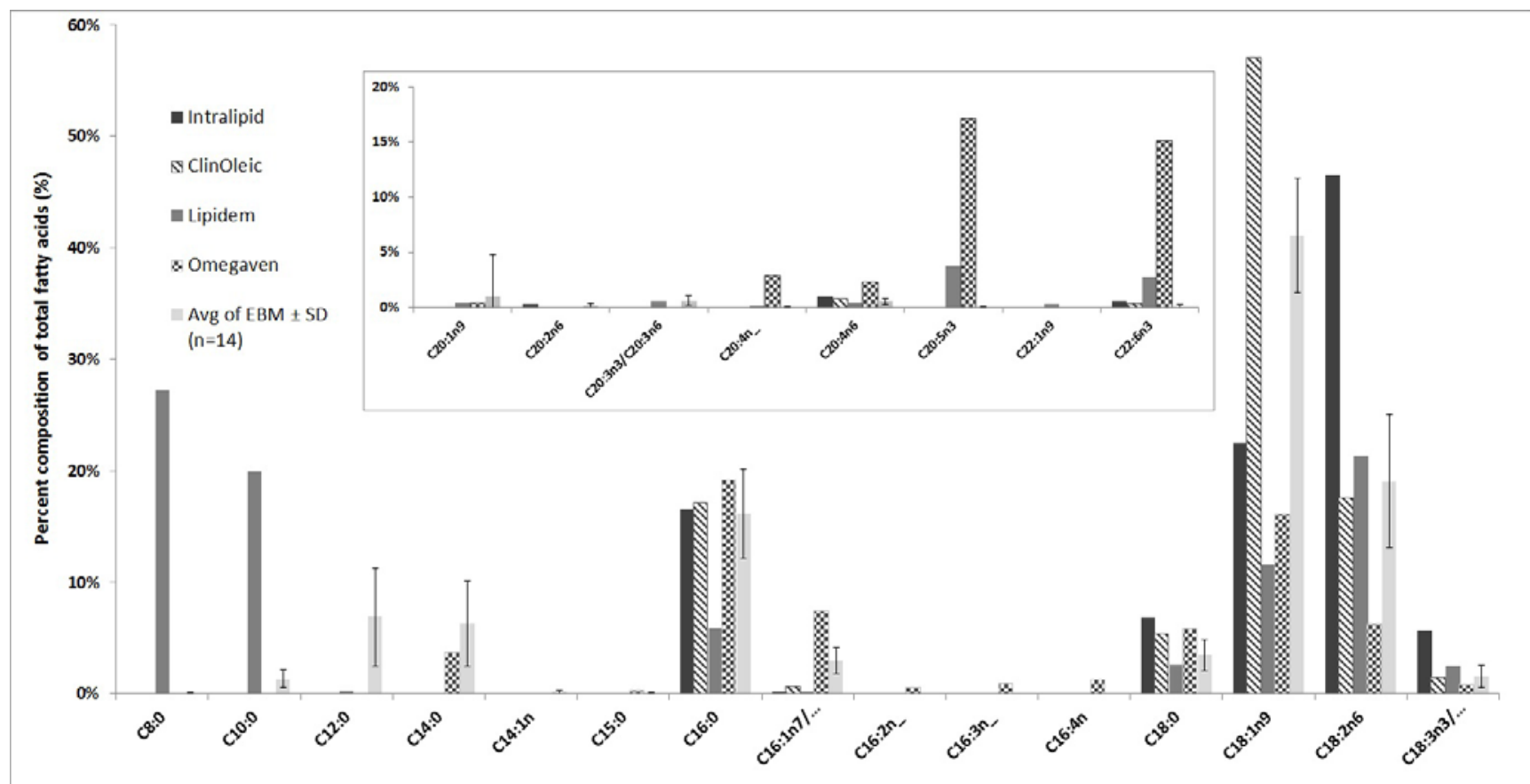

FV-23 Fig. $1 \Delta$ Percent composition of fatty acids 
get fortified using different schedules, the variation of macronutrients increased as the frequency of milk analysis decreased, while the average intake remained stable.

Conclusions. The mean macronutrient intake was stable and independent of which approach was used. The positive deviation of mean calories and $\mathrm{F}$ from target concentration in a considerable amount of native $B M$ is due to fat-rich BM. As TFO adds a considerable workload to routine practice, a balance between workload and outcome needs to be found. We observed an increase in variation of macronutrient intake across all of our proposed measurement frequencies. This may affect growth and development; and needs to be studied in future RCTs.

\section{FV-25}

Preliminary report: body composition of preterm infants using air displacement plethysmography during the first weeks of life

\section{J. Chin', N. Rochow', G. Fusch', P. Murthy', D. Pogorzelski', D. Radke C. Fusch}

${ }^{1}$ McMaster University, Division of Neonatology, Department of Pediatrics, Hamilton, Ontario, Kanada

Background. Inadequate nutrition during the postnatal period may be associated with deficits in growth, development and adverse outcomes in later life. Tailoring nutrition to promote optimal growth requires growth patterns and more ideally body composition (BC) be monitored. This data could guide nutritional strategies to promote optimal outcomes, however longitudinal BC reference data of preterm infants is currently lacking.

Objective. Establish longitudinal reference data of preterm infant $\mathrm{BC}$ and growth using air displacement plethysmography and anthropometric measurements at bedside.

Design and methods. Ongoing, single-centre, longitudinal, observational study of physically stable preterm infants. Inclusion criteria: 24-36 weeks gestational age (GA), infants without IV lines and stable off respiratory support for $7 \mathrm{~min}$, written and informed consent. Exclusion criteria: chromosomal or congenital abnormalities, hydrops fetalis. BC assessed by PEA POD daily for the first 21 days of life, then twice per week. Weight, length, head circumference collected once per week. Infants assessed from study inclusion to hospital discharge.

FV-24 Tab. 1 Modelling of TFO when fortifier was adjusted on different schedules: deviation from the target concentration of macronutrients (achieved macronutrient content by TFO - target concentration of TFO).

\begin{tabular}{|c|c|c|c|c|c|c|c|}
\hline & & $\begin{array}{l}\text { Every } \\
\text { day }\end{array}$ & $\begin{array}{l}\text { Week- } \\
\text { days }\end{array}$ & $\begin{array}{l}\text { Mo- } \\
\text { We-Fr }\end{array}$ & Mo-Th & Mo & $\begin{array}{l}\text { No } \\
\text { TFO }\end{array}$ \\
\hline \multirow{4}{*}{$\begin{array}{l}\text { KCAL } \\
(\mathrm{kcal} / 100 \mathrm{ml})\end{array}$} & Mean & 3 & 3 & 3 & 3 & 3 & -8 \\
\hline & SD & 5 & 6 & 6 & 7 & 7 & 8 \\
\hline & IQR & 4 & 5 & 6 & 7 & 7 & 10 \\
\hline & Range & 27 & 34 & 39 & 41 & 41 & 46 \\
\hline \multirow{4}{*}{$\begin{array}{l}\text { FAT } \\
(\mathrm{g} / 100 \mathrm{ml})\end{array}$} & Mean & 0.3 & 0.4 & 0.3 & 0.3 & 0.4 & 0.0 \\
\hline & SD & 0.6 & 0.6 & 0.7 & 0.8 & 0.7 & 0.9 \\
\hline & IQR & 0.5 & 0.6 & 0.6 & 0.7 & 0.8 & 1.1 \\
\hline & Range & 3.0 & 3.8 & 4.3 & 4.5 & 4.5 & 5.1 \\
\hline \multirow{4}{*}{$\begin{array}{l}\text { PROTEIN } \\
(\mathrm{g} / 100 \mathrm{ml})\end{array}$} & Mean & 0.0 & 0.0 & 0.0 & 0.0 & 0.0 & -0.8 \\
\hline & SD & 0.0 & 0.1 & 0.1 & 0.1 & 0.1 & 0.2 \\
\hline & IQR & 0.0 & 0.0 & 0.0 & 0.1 & 0.1 & 0.2 \\
\hline & Range & 0.0 & 0.8 & 1.1 & 1.3 & 1.0 & 1.0 \\
\hline \multirow{4}{*}{$\begin{array}{l}\text { CHO } \\
(\mathrm{g} / 100 \mathrm{ml})\end{array}$} & Mean & 0.0 & 0.0 & 0.0 & 0.0 & 0.0 & -1.2 \\
\hline & SD & 0.0 & 0.1 & 0.1 & 0.1 & 0.1 & 0.2 \\
\hline & IQR & 0.0 & 0.0 & 0.0 & 0.1 & 0.1 & 0.2 \\
\hline & Range & 0.0 & 0.8 & 0.8 & 0.9 & 0.8 & 1.0 \\
\hline
\end{tabular}

Results. A total 161 measurements of 55 preterm infants with a mean GA at birth $32 \pm 2$ wks and mean birth weight $1773 \pm 500 \mathrm{~g}$ were performed. At test, mean post menstrual age was 33 4/7 \pm 1 wks (range: $304 / 7-38$ 3/7 wks) and mean percent fat mass (FM) was $8.8 \%$. Fat free mass and FM accretion occurred at different rates leading to an overall\% FM increase after sufficient caloric intakes were established. Preliminary data suggests that FM is not predicted by GA at birth. No infant experienced an adverse event relating to a measurement.

Discussion. Successfully established the infrastructure needed to routinely measure preterm infant $\mathrm{BC}$ at bedside indicating PEA PODs feasibility for clinical use. To our knowledge, this is the first longitudinal, observational data of preterm infants measured so soon after birth using PEA POD.

\section{FV-26}

A multi-center study to develop new longitudinal postnatal growth references for preterm infants

\section{P. Raja', N. Rochow', S. Göttler', A. Olbrich', J. Cheng' ${ }^{4}$, S. Seigel'5, D. Camp-} bell' ${ }^{6}$, M. Heckmann ${ }^{3}$, J. Poeschl', L. Thabane ${ }^{4}$, C. Fusch

'McMaster University, Department of Pediatrics, Hamilton, ON, Kanada, ${ }^{2}$ Klinikum d.Ruprecht-Karl- Universität, Kinderklinik, Heidelberg, Deutschland, ${ }^{3}$ Ernst-Moritz-Arndt Universität, Kinderklinik, Greifswald, Deutschland, ${ }^{4}$ McMaster University, Department of Clinical Epidemiology \& Biostatistics, Hamilton, Kanada, ${ }^{5}$ St. Joseph's Healthcare, Department of Pediatrics, Hamilton, Ontario, Kanada, ${ }^{6}$ St Michael's Hospital, Department of Paediatrics, Toronto, Kanada, ${ }^{7}$ Universitätsklinikum Heidelberg, Klinik für Kinder- und Jugendmedizin, Heidelberg, Deutschland

Background. Postnatal growth of preterm infants is monitored using birth weight percentiles (BW\%iles) based on cross-sectional estimates of intrauterine growth. Due to postnatal contraction of extracellular water space, infants adjust to a percentile below that at birth. However, it is unknown to which percentile a preterm infant should ideally adjust to after postnatal adaptation.

Objective. Develop postnatal growth references for preterm infants, by 1) Characterizing the growth of low-risk preterm infants with minimal postnatal support; 2) Using this data to develop a model that uses gestational age (GA) and BW to predict the hypothesized ideal trajectory to adjust to after postnatal adaptation.

Methods. Inclusion criteria: admitted between 2008-2011 to participating hospitals, 30-36 completed weeks GA. Exclusion criteria: maternal diabetes or substance use, unavailable growth data until day of life (DoL) 14, did not attain full enteral feeds by DoLio, CPAP $>3$ days, proven sepsis, or major congenital malformations. GA and either \% of 5 oth BW\%ile or $\mathrm{z}$-scores infants (30-35 completed weeks GA) adjust to by DoL 1, 7, 14, 21 were used to develop predictive model.

Results. 391 infants were eligible of 4319 that met inclusion criteria, however infants born at 36 completed weeks GA $(n=34)$ were excluded from the analysis. On average, infants had a maximum weight loss of $5.5 \%$ by DoL 5 and regained their BW by DoL 11. Postnatal growth during DoL 7-14 remained constant for preterm infants stratified by GA at birth. At birth, infants were on average $95 \pm 17.7 \%$ of 5 oth BW\%ile, but adjusted to $83 \pm 13.1 \%$ by DoL 14 . On average, the $\mathrm{z}$-score difference from birth was $-0.74 \pm 0.820$ at DoL 14 . Preliminary linear regression models $(\mathrm{n}=304)$ accurately predicted weight at DoL 7 and 14 (as \% of 5oth BW\%ile: $\mathrm{r} 2=0.93,0.75$; or $\mathrm{z}$-score: $\mathrm{r} 2=0.92,0.88$ ) using GA and BW (expressed as \% of BW\%ile or Z-score; 1 Fig. 1).

Conclusions. These results provide preliminary postnatal growth references for preterm infants that take into account postnatal adaptation. 


\section{FV-27}

Wachstum von Frühgeborenen mit einem Geburtsgewicht von $<1500 \mathrm{~g}$ unter proteinreicher Ernährung

\section{A. Loui', C. Bührer' \\ ${ }^{1}$ Charité - Universitätsmedizin, Klinik für Neonatologie, Berlin, Deutschland}

Ziel. Untersucht wurde die Auswirkung einer neuen Ernährungsstrategie mit einer höheren Menge an parenteralen Aminosäuren und enteralem Protein auf das Wachstum in den ersten 5 Lebenswochen bei mit Muttermilch ernährten Frühgeborenen mit einem Geburtsgewicht von unter $1500 \mathrm{~g}$.

Design. Gewicht, Körperlänge und Kopfumfang der Frühgeborenen nach Einführung der Strategie der "proteinreicheren Ernährung“ (2010, 43 Frühgeborene) wurden verglichen mit den Daten der kürzlich beschriebenen Kohorte geboren im Jahre 2000 (45 Frühgeborene).

Ergebnisse. Die klinischen Basisdaten und die Häufigkeiten der typischen Frühgeborenenerkrankungen waren in den beiden Kohorten vergleichbar. Im Jahr 2010 war die Dauer der parenteralen Ernährung kürzer als im Jahr 2000 ( $\mathrm{p}<\mathrm{0}, 001)$, während die Gesamtaufnahme von Fetten, Aminosäuren und Protein höher war $(\mathrm{p}<0,001)$. Im Jahr 2010 wurde Muttermilch zusätzlich zum Fortifier mit einem Proteinpulver mit einer Menge von 0,5-2,3 g/kg/d angereichert. In 2010 war das Wachstum schneller als in 2000: mediane Gewichtszunahme [Quartile] von Lebenstag 8-35: 17,6 [14,9-20,5] g/kg/d vs. 14,1 [11,6-11,o] g/kg/d, $\mathrm{p}<0,001$; Zunahme des Kopfumfanges von Lebenstag 1-35: 0,70 [0,50$0,80]$ vs. $0,56[0,40-0,70] \mathrm{p}<0,01$.

Schlussfolgerung. Die Ernährung mit einer höheren Menge an parenteralen Aminosäuren und enteralem Protein erhöht die Gewichtszunahme und das Kopfumfangswachstum bei Frühgeborenen mit einem Geburtsgewicht von unter $1500 \mathrm{~g}$ in den ersten 5 Lebenswochen signifikant. Diese Daten unterstützen die kürzlich veröffentlichten Empfehlungen der ESPGHAN zur Ernährung dieser Frühgeborenen.

\section{Versorgungsqualität - Nosokomiale Infektionen}

\section{FV-28}

Nach dem BSG-Urteil zur Mindestmenge: Gibt es einen neuen Zentralisierungsanlauf?

\section{B. v. Wolff'}

'lindenpartners Rechtsanwälte, Berlin, Deutschland

Das Bundessozialgericht (BSG) hat kurz vor Weihnachten die Entscheidung des Landessozialgerichts Berlin-Brandenburg im Ergebnis bestätigt, wonach die Anhebung der Mindestmenge für die Versorgung von Frühgeborenen mit einem Geburtsgewicht von unter $1250 \mathrm{~g}$ von 14 auf 30 rechtswidrig und damit nichtig ist. Die Urteilsgründe des BSG liegen noch nicht vor.

Der Vortrag bzw. das Poster wird - auch unter Rückgriff auf das bereits vollständig begründete Knie-TEP-Urteil des BSG - versuchen, die wesentlichen Anforderungen für eine nochmalige Erhöhung der Mindestmenge herauszuarbeiten. Das BSG hat ausdrücklich festgehalten, dass die Versorgungssteuerung durch Mindestmengen auf Ausnahmelagen beschränkt bleiben müsse. Entscheidend ist für das BSG gerade auch mit Blick auf die Berufsfreiheit der Klinikträger, ob ohne Mengensteuerung eine anders (d. h. insbesondere durch verhaltens- und qualifikationsbezogene Anforderungen) nicht aufzufangende Qualitätseinbuße zu besorgen ist. Das BSG hat dem Gemeinsamen Bundesausschuss (G-BA) u. a. auch aufgegeben, Ausnahmetatbestände zu erwägen, um die Rigidität des mit der Mindestmenge verknüpften Leistungserbringungsverbots abzumildern. Hierbei ist insbesondere an eine Korridorlösung zu denken, bei der ein Bereich unterhalb der festgesetzten Mindestmenge zu bestimmen; Kliniken, die sich in diesem Korridor bewegten und vom G-BA im Einzelnen festzulegende Qualitätsanforderungen erfüllten, könnten dann weiterhin an der Versorgung teilnehmen.

Die Entscheidungen des BSG werden der Einführung von Mindestmengen Grenzen setzen. Ausgeschlossen erscheinen sie aber bei sorgfältiger Sachverhaltsaufklärung und ausgewogener Normsetzung durch den G-BA nicht.

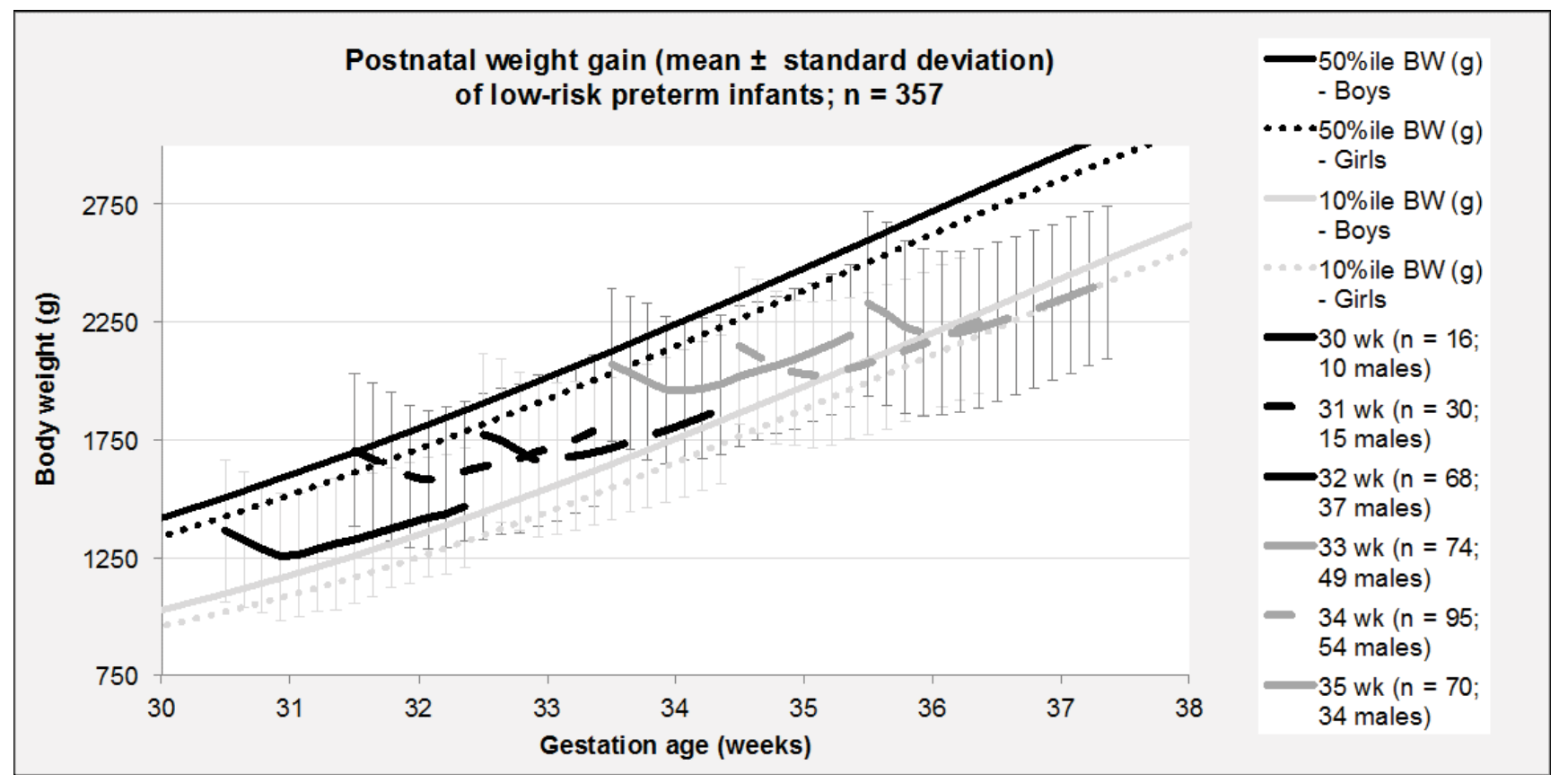

FV-26 Fig.1 $\Delta$ 


\section{Kreislauftherapie - Kardiologie II}

\section{FV-29}

\section{Arterielle Hypotension bei sehr kleinen Frühgeborenen am ersten} Lebenstag

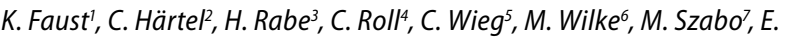 \\ Herting $^{8}$, W. Göpel ${ }^{9}$
}

'Universitätsklinikum Schleswig Holstein, Kinderklinik, Lübeck, Deutschland, ${ }^{2}$ Universitätsklinikum Schleswig Holstein/Campus Lübeck, Klinik für Kinder- und Jugendmedizin, Lübeck, Deutschland, ${ }^{3}$ Brighton \& Sussex University Hospitals, Neonatology, Brighton, United Kingdom, ${ }^{4}$ Vestische Kinder- und Jugendklinik Datteln, Neonatologie, Datteln, Deutschland, ${ }^{5}$ Klinikum Aschaffenburg, Neonatologie und pädiatrische Intensivmedizin, Aschaffenburg, Deutschland, ${ }^{6}$ Vivantes Klinikum Neukölln, Kinder- und Jugendmedizin - Perinatalzentrum, Berlin, Deutschland, ${ }^{7}$ Semmelweis Universität, Budapest, Ungarn, ${ }^{8}$ Universitätsklinikum Schleswig-Holstein Campus Lübeck, Direktor der Klinik für Kinder- und Jugendmedizin, Lübeck, Deutschland, 'ソniversitätsklinikum Schleswig-Holstein, Klinik für Kinder- und Jugendmedizin, Lübeck, Deutschland

Hintergrund. Bisher gibt es keine einheitlichen Richtlinien zur Definition und möglichen Therapie der arteriellen Hypotension bei sehr kleinen Frühgeborenen (VLBW, „very low birth weight") mit einem Geburtsgewicht von unter $1500 \mathrm{~g}$.

Fragestellung. Diese Untersuchung hatte das Ziel, an einer großen Gruppe von VLBW-Frühgeborenen zum einen Blutdruckwerte am ersten Lebenstag zu beschreiben, zum anderen Korrelationen zu assoziierten Komplikationen zu identifizieren.

Methoden. Für die hier dargestellte Analyse des GNN wurden 3332 VLBW-Frühgeborene mit einem Gestationsalter von unter 32 Schwangerschaftswochen (SSW) eingeschlossen. Zusammen mit verschiedenen antenatalen und postnatalen Daten wurde der jeweils niedrigste mittlere arterielle Blutdruck (nMAD) am ersten Lebenstag dokumentiert. Arterielle Hypotension wurde definiert als nMAD kleiner als der Median der gestationsaltergleichen Gruppe.

Ergebnisse. Der nMAD war erwartungsgemäß sowohl vom Gestationsalter als auch vom Geburtsgewicht abhängig. Bei Kindern mit einem Gestationsalter $\leq 28$ vollendeten SSW lag der Median des gemessenen nMAD 1 bis $2 \mathrm{mmHg}$ unter dem Gestationsalter (in vollen Wochen). In einer multivariaten Analyse zeigte sich für die Kinder, die am ersten Lebenstag eine arterielle Hypotension hatten, auch nach Einbeziehung von Kovariaten wie Gestationsalter, Early-onset-Sepsis und Katecholamintherapie am ersten Lebenstag sowohl eine erhöhte Mortalität (OR 2,0, 95\% CI 1,25-3,15) als auch ein erhöhtes Risiko für die Entwicklung einer intraventrikulären Hämorrhagie (IVH; OR: 1,4, 95\% CI 1,1-1,7). Das Risiko für die Entwicklung einer periventrikulären Leukomalazie war nicht erhöht.

Schlussfolgerung. Die arterielle Hypotension sehr kleiner Frühgeborener am ersten Lebenstag ist in einer aktuellen Kohortenstudie mit einer erhöhten Sterblichkeit und der Entwicklung einer IVH assoziiert.

\section{FV-30}

Reproduzierbarkeit echokardiographischer Parameter zur Abschätzung der hämodynamischen Relevanz eines persistierenden Ductus arteriosus Botalli (PDA)

C. Schwarz', W. Baden², A. Preusche', C. Poets' , A. Franz'

'Eberhard-Karls-Universität Universitätsklinik für Kinderheilkunde und Jugendmedizin, Neonatologie, Tübingen, Deutschland, ${ }^{2}$ Eberhard-KarlsUniversität Universitätsklinik für Kinderheilkunde und Jugendmedizin, Kinderkardiologie, Pulmologie, Intensivmedizin, Tübingen, Deutschland

Hintergrund. Die Entscheidung zum PDA-Verschluss bei Frühgeborenen (FG) wird aufgrund der echokardiographischen Einschätzung des
Links-Rechts-Shunts (LRS) getroffen. Hinreichende Daten über die Reproduzierbarkeit der Messergebnisse fehlen, sind aber für eine Bewertung der Methode unabdingbar.

Fragestellung. Ermittlung der Wiederholbarkeit echokardiographischer Parameter zur Abschätzung des LRS über den PDA.

Material und Methoden. Bei FG mit klinischem Verdacht auf PDA wurden prospektiv von jeweils 2 (von 3 ) Untersuchern innerhalb von 30 min folgende Parameter gemessen: LA/Ao-Ratio (linkes Atrium/ Aortenwurzel), LVPEP/LVET (linksventrikuläre Präejektionsperiode/ LV-Ejektionszeit), VTI_Ao/VTI_Pa (Volumen-Zeit-Integral Aorta/ VTI Pulmonalarterie), Resistance Index (RI) im Truncus coeliacus (TC) und einer Arteria cerebri anterior (ACA), Durchmesser des PDA an der engsten Stelle/pulmonalem Ende, sowie maximale systolische und minimale diastolische Flussgeschwindigkeit. Die statistische Auswertung umfasste die Berechnung des Repeatability coefficient (RepC) und des Repeatability Index (RepI) nach Bland \& Altman sowie eine Confidence-Step-Analyse (CSA). Der RepC $(=2 \times$ Standardabweichung der Differenzen) repräsentiert den geringsten Unterschied zwischen 2 Messwerten, der (bei 5\% Fehlerwahrscheinlichkeit) nicht mehr allein durch die Schwankung wiederholter Messungen erklärbar ist. Der RepI (RepC/arithmetisches Mittel aller Messwerte) setzt den RepC in Bezug zur Größe der Messwerte. Ein hoher CSA-Wert (CSA: Differenz zwischen kleinstem und größtem Messwert/RepC), signalisiert eine hohe Sensitivität, um in wiederholten Messungen tatsächliche Unterschiede feststellen zu können. "Ausreißer" wurden mittels Outlier-Test nach Dean \& Dixen identifiziert und von der Auswertung ausgeschlossen.

Ergebnisse. 14 wiederholte Messungen wurden ausgewertet (medianes Gestationsalter der FG: 29 SSW, Interquartilenabstand 26-31 SSW; Geburtsgewicht 1240 (808-1550) g, postnatales Alter 9,3 (4,9-18,0) Tage und Gewicht 1522 (860-2434) g bei Untersuchung).Ein PDA mit LRS wurde farbdopplersonographisch in 8/14 Messungen festgestellt. PDADurchmesser sowie maximale systolische und minimale diastolische Flussgeschwindigkeit konnte jedoch nur selten dargestellt werden. In abfallender Reihenfolge zeichneten sich die Parameter hinsichtlich ihrer Reproduzierbarkeit wie folgt aus: RI_TC $(\operatorname{RepI}=12 \%$; $\mathrm{RepC}=0,09$; CSA=6,o) > RI_ACA $(16 \% ; 0,12 ; 2,1)>$ TI_Ao/VTI_Pa $(21 \% ; 0,22$; $5,9)>$ LA/Ao-Ratio $(30 \% ; 0,41 ; 2,6)>\operatorname{LVPEP} / \operatorname{LVET}(33,5 \%$; 0,10; 2,2)

Schlussfolgerung. Die Wiederholbarkeit der echokardiographischen Messwerte war gering. Am zuverlässigsten können die RIs in ACA und TC sowie das Verhältnis des VTI_Ao/VTI_Pa beurteilt werden. Der RepI für die LA/Ao ratio \& LVPEP/LVET ist so groß, dass man diese Parameter nicht für eine Therapieentscheidung in Betracht ziehen sollte.

Mit Unterstützung der Else-Kröner-Fresenius Stiftung.

\section{FV-31}

Tissue-Doppler basierte-Strain- und Strain-rate-Messung bei Frühgeborenen unter $1500 \mathrm{~g}$ Geburtsgewicht während der ersten 28 Lebenstage

\section{Czernik', S. Helfer², C. Bührer', L. Schmitz²}

${ }^{1}$ Charité - Universitätsmedizin Berlin, Klinik für Neonatologie, Berlin, Deutschland, ${ }^{2}$ Charité - Universitätsmedizin Berlin, Klinik für Pädiatrie m.S. Kardiologie, Berlin, Deutschland

Hintergrund. Frühgeborene (FG) können aufgrund eines persistierenden Duktus arteriosus (PDA) oder einer bronchopulmonalen Dysplasie (BPD) einer erhöhten kardialen Belastung ausgesetzt sein. Die Einschätzung der Herzfunktion mittels der neuen Tissue-Dopplerechokardiographie wurde bei FG bisher nicht beschrieben.

Fragestellung. Wir untersuchten die Entwicklung der Herzfunktion von FG mit Hilfe der Tissue-Doppler-basierten Peak-systolic-Strain(PSS) und Strain-Rate (PSSR) in den ersten 28 Lebenstagen in Abhängigkeit vom klinischen Verlauf der Kinder.

Material und Methoden. Wir bestimmten PSS und PSSR in der rechten und linken Herzwand sowie im Septum an den Lebenstagen (LT) 1, 7, 14 
und 28 von 121 FG unter 1500 g Geburtsgewicht. Körpergewicht, Herzfrequenz sowie das Vorliegen eines hämodynamisch signifikanten (hs) PDA und die Entwicklung einer BPD wurden dokumentiert.

Ergebnisse. PSS und PSSR des rechten Ventrikels nahmen während der ersten 28 Lebenstage signifikant zu. FG, die eine BPD entwickelten, hatten an LT 14 und 28 einen signifikant niedrigeren PSS im rechten Ventrikel, während ein hsPDA an LT 14 zu einem signifikant niedrigeren PSS im linken Ventrikel führte.

Diskussion. Eine beginnende BPD und ein hsPDA wirken sich auf die PSS aus. Da sich ein hsPDA und eine beginnende BPD oft bei den gleichen Patienten finden, ist ihr Einfluss auf PSS und PSSR schwer auseinanderzuhalten.

Schlussfolgerung. PSS scheint bei FG zur Erfassung eines erhöhten Afterloads (erniedrigter PSS im rechten Ventrikel bei Kindern mit BPD) und eines erhöhten Preloads (erniedrigter PSS im linken Ventrikel be Kindern mit PDA) herangezogen werden zu können. Fragen zum klinischen Einsatz bedürfen weiterer Untersuchungen.

\section{FV-32}

Reproduzierbarkeit und Optimierung der Analyseparameter der Tissue-Doppler-basierten Strain- und Strain-rate-Messung bei Frühgeborenen unter $1500 \mathrm{~g}$ Geburtsgewicht

\section{S. Helfer', L. Schmitz', C. Bührer², C. Czernik ${ }^{3}$}

${ }^{1}$ Charite Kliniken f. Kinderheilkunde und Kinderchirurgie, Berlin, Deutschland, ${ }^{2}$ Charité - Universitätsmedizin Berlin, Klinik für Neonatologie, Berlin, Deutschland, ${ }^{3}$ Charite Campus Virchow-Klinikum, Berlin, Klinik für Neonatologie, Berlin, Deutschland

Hintergrund. Tissue-Doppler-basierte Strain- (S) und Strain-Rate (SR) sind echokardiographische Parameter, die die Verformung des Herzmuskels wiedergeben. Während ihre Bestimmung in der Versorgung von Erwachsenen und Kindern einen größer werdenden Stellenwert einnimmt, ist wenig darüber bekannt, welchen Einfluss Messfaktoren wie die Größe der Region-of-interest (ROI) oder der Strain-length (SL) auf die Reproduzierbarkeit der Messungen bei Frühgeborenen mit einem Geburtsgewicht von unter $1500 \mathrm{~g}$ haben.

Fragestellung. Welchen Einfluss haben ROI-Größe und SL auf die Durchführbarkeit und Reproduzierbarkeit der S- und SR-Bestimmung bei Frühgeborenen mit einem Geburtsgewicht unter 1500 g?

Material und Methoden. Wir bestimmten die Beat-to-beat-Variation (BBV) für die S und SR-Kurven bei unterschiedlichen Kombinationen von ROI-Breite (RW), ROI-Länge (RL) und SL anhand von je 20 qualitativ hochwertigen Tissue-Doppler-Aufnahmen in drei Herzwänden. Wir untersuchten den Einfluss von RW, RL und SL auf die BBV und bestimmten Inter- und Intra-Operator-Variabilität.

Ergebnisse. Die geringste BBV fanden wir für eine RW von $2 \mathrm{~mm}$. Eine Erhöhung der SL (auch auf Kosten der RL) führte zu einem Absinken der BBV. Mehrere Kombinationen führten zu niedriger BBV ohne sich untereinander signifikant darin zu unterscheiden. Die Variationskoeffizienten für die Intra- und Inter-Operator-Variabilität lag zwischen 15,1\% und $65,2 \%$. Beide waren für systolische Werte niedriger als für diastolische Werte.

Diskussion. Wir zeigten die technische Durchführbarkeit und Reproduzierbarkeit der S- und SR-Bestimmung bei einer kleinen, ausgewählten Gruppe unserer Grundpopulation. Die Reproduzierbarkeit ist vergleichbar mit ähnlichen Studien. Die Durchführbarkeit und klinische Aussagekraft in einer größeren, unselektierten Population bleibt noch zu bestimmen.

Schlussfolgerung. S- und SR-Messungen mittels Tissue-Doppler sind bei Frühgeborenen mit einem Geburtsgewicht unter $1500 \mathrm{~g}$ technisch möglich und in Grenzen reproduzierbar. ROI-Größe und SL haben einen signifikanten Einfluss auf diese Werte. Das RW-Optimum liegt bei ca. $2 \mathrm{~mm}$, die SL sollte groß und die RL klein gehalten werden.

\section{Neue Studien}

\section{FV-33}

Permissive Hyperkapnie bei extrem untergewichtigen Frühgeborenen

U. Thome', O. Genzel-Boroviczény'2, B. Bohnhorst ${ }^{3}$, O. Rohde', H. Fuchs's, S. Avenarius ${ }^{6}$, H. Topf ${ }^{7}$, A. Zimmermann ${ }^{8}$, D. Faas ${ }^{9}$, K. Timme $^{10}$, B. Kleinlein ${ }^{11}, \mathrm{H}$. Buxmann ${ }^{12}$, W. Schenk ${ }^{13}$, H. Segerer ${ }^{14}$, N. Teig ${ }^{15}$, R. Hentschel ${ }^{16}$, W. Lindner ${ }^{17}$, G. Hansen ${ }^{18}$, E. Cloppenburg ${ }^{19}$, G. Jorch ${ }^{20}$, M. Schroth ${ }^{21}$, M. Heckmann ${ }^{22}$, R. Rossi ${ }^{23}$, J. Peters ${ }^{11}$, R. Schlößer ${ }^{24}$, J. Seidenberg ${ }^{25}$, W. Rascher ${ }^{26}$, P. Neuhaus ${ }^{27}$, M. Zernickel28, G. Alzen ${ }^{29}$, J. Dreyhaupt ${ }^{30}$, R. Muche ${ }^{31}$, H. Hummler ${ }^{32}$

'Universitätsklinikum Leipzig, Klinik und Poliklinik für Kinder und Jugendliche der Universität/Leiter der Abteilung für Neonatologie, Leipzig, Deutschland, ${ }^{2}$ Klinikum der Universität München Innenstadt, Perinatologie, München, Deutschland, ${ }^{3}$ Medizinische Hochschule Hannover, Abteilung für Pädiatrische Pneumologie und Neonatologie, Hannover, Deutschland, ${ }^{4}$ Elisabeth-Kinderkrankenhaus, Kinder- und Jugendmedizin, Oldenburg, Deutschland, ${ }^{5}$ Klinik für Kinder und Jugendmedizin, Universitätsklinikum Ulm, Neonatologie und päd. Intensivmedizin, Ulm, Deutschland, ${ }^{6}$ Universitätsklinik für Allgemeine Pädiatrie und Neonatologie, Magdeburg, Deutschland, ${ }^{7}$ Universitäts-Kinderklinik, Neonatologie, Erlangen, Deutschland, ${ }^{8}$ Kinderklinik der TU Krankenhaus München - Schwabing, Klinik und Poliklinik für Kinder- und Jugendmedizin, München, Deutschland, ${ }^{9}$ Zentrum für Kinderheilkunde der Justus-v.-Liebig-Universität, Allgemeinpädiatrie und Neonatologie, Giessen, Deutschland, ${ }^{10}$ Vivantes-Klinikum Neukölln, Kinder- und Jugendmedizin, Berlin, Deutschland, "Kinderklinik Dritter Orden, Klinik für Kinder- und Jugendmedizin, München, Deutschland, ${ }^{12}$ Klinikum der J.W. Goethe Universität, Klinik für Kinder- und Jugendmedizin, Neonatologie, Frankfurt am Main, Deutschland, ${ }^{13}$ Klinikum Augsburg, II. Klink für Kinder und Jugendliche, Augsburg, Deutschland, ${ }^{14}$ Klinik St. Hedwig, Krankenhaus Barmherzige Brüder Regensburg, Abteilung für Neonatologie, Regensburg, Deutschland, ${ }^{15} \mathrm{Klinik}$ für Kinder- und Jugendmedizin St. Josefs-Hospital, Abteilung für Neonatologie, Bochum, Deutschland, ${ }^{16}$ Universitätsklinikum Freiburg, Zentrum für Kinderheilkunde/Jugendmedizin, Neonatologie/Intensivmedizin, Freiburg, Deutschland, ${ }^{17}$ Universitätsklinikum Ulm, Klinik für Kinder- und Jugendmedizin, Ulm, Deutschland, ${ }^{18}$ Medizinische Hochschule Hannover, Zentrum für Kinderheilkunde und Jugendmedizin, Hannover, Deutschland, ${ }^{19}$ Elisabeth Kinderkrankenhaus, Klinikum Oldenburg, Neonatologische Intensivstation, Oldenburg, Deutschland, ${ }^{20}$ Universitätskinderklinik, Klinikdirektor, Magdeburg, Deutschland, ${ }^{21}$ Universitätsklinikum Erlangen, Kinder- und Jugendklinik, Erlangen, Deutschland, ${ }^{22} \mathrm{Klinik}$ und Poliklinik für Kinder und Jugendmedizin, Abt. Neonatologie u. Päd. Intensivmedizin, Greifswald, Deutschland, ${ }^{23}$ Vivantes Klinikum Neukölln, Klinik für Kinder - und Jugendmedizin, Berlin, Deutschland, ${ }^{24}$ Klinikum der J.W.Goethe-Universität, Zentrum der Kinderheilkunde, Abteilung für Neonatologie, Frankfurt am Main, Deutschland, ${ }^{25} \mathrm{Klinikum}$ Oldenburg gGmbH, Klinik für Kinder- und Jugendmedizin, Oldenburg, Deutschland, ${ }^{26}$ Kinder- und Jugendklinik, Universitätsklinikum Erlangen, Erlangen, Deutschland, ${ }^{27}$ Universitätskinderklinik, Zentrum für klinische Studien Leipzig - KKS, Leipzig, Deutschland, ${ }^{28}$ Universitätsklinikum Ulm, Kinder- und Jugendmedizin, Ulm, Deutschland, ${ }^{29}$ Medizinische Fakultät JLU Gießen, Kinderradiologie, Gießen, Deutschland, ${ }^{30}$ Universität Ulm, Institut für Epidemiologie und Medizinische Biometrie, Ulm, Deutschland, ${ }^{31}$ Universitäts-Ulm, Ulm, Deutschland, ${ }^{32} \mathrm{Klinik}$ für Kinder- und Jugendmedizin, Universitätsklinikum Ulm, Sektion Neonatologie und Pädiatrische Intensivmedizin, Ulm, Deutschland

Hintergrund. Die mechanische Beatmung ist für extrem kleine Frühgeborene (ELBW) oftmals lebensrettend, führt jedoch zu Lungenschäden und ist in erheblichem Umfang an der Pathogenese der bronchopulmonalen Dysplasie (BPD) beteiligt. Tierversuche und Sekundäranalysen voran gegangener Studien legen nahe, dass die Beatmungseinstellungen gesenkt und die Lunge geschont werden können, wenn man hö- 
here $\mathrm{CO}_{2}$-Partialdrücke ( $\left.\mathrm{PCO}_{2}\right)$ im kindlichen Blut toleriert. In einer randomisierten Multizenterstudie wurde die Hypothese getestet, dass ein höherer $\mathrm{PCO}_{2}$-Zielbereich die Rate überlebender Kinder ohne BPD erhöht.

Methoden. Frühgeborene mit einem Gewicht von 400-100o g, die innerhalb von 24 Stunden nach Geburt intubiert und beatmet waren, wurden randomisiert zwei verschiedenen $\mathrm{PCO}_{2}$-Zielbereichen zugeteilt, die für die ersten 14 Tage verbindlich waren. In beiden Gruppen stieg der Zielbereich mit dem postnatalen Alter an. Er betrug für die Zeiträume Tag 1-3, Tag 4-6, Tag 7-14 in der Hyperkapniegruppe 55-65, 6o-70, 65-75, in der Normokapniegruppe 40-50, 45-55, 50-6o (alle Angaben in $\mathrm{mmHg}$ ). Das primäre Zielkriterium war BPD, definiert als Beatmungs-, CPAP- oder $\mathrm{FiO}_{2}$-Bedarf $>0,21$ im postmenstruellen Alter von 36 Wochen oder Tod vor Erreichen dieses Alters. Bei FiO2 zwischen 0,21 und 0,3 wurde ein standardisierter Sauerstoffreduktionstest durchgeführt. Die Auswertung der zu vorherbestimmten Zeitpunkten durchgeführten Schädelsonographien erfolgte zentral.

Ergebnisse. Die Rekrutierungsrate blieb hinter den Erwartungen zurück, insbesondere da ein zunehmender Anteil von Kindern der Zielgruppe nicht intubiert und beatmet wurde und damit die Einschlusskriterien nicht erfüllte. Die Rekrutierung wurde aufgrund einer Zwischenanalyse nach Einschluss von 362 Patienten gestoppt. Die Ergebnisse der Zwischenanalyse, basierend auf 312 Patienten, waren wie folgt (Hyperkapnie/Normokapnie): Anzahl 156/156, Geburtsgewicht $714 \pm 159 / 710 \pm 155$, BPD oder Tod $57(38 \%) / 51(35 \%)$, IVH1-4 $53(34 \%) / 55$ (36\%), IVH3-4 21 (14\%)/15 (10\%). Kinder der Hyperkapniegruppe unterschritten den $\mathrm{PCO}_{2}$-Zielbereich doppelt so häufig ( $73 \pm 19$-mal pro $\mathrm{Pa}$ -

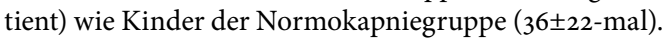

Schlussfolgerung. In dieser bisher größten Studie zur permissiven Hyperkapnie bei Frühgeborenen führte der höhere $\mathrm{PCO}_{2}$-Zielbereich nicht $\mathrm{zu}$ einer höheren Überlebensrate ohne BPD und beeinflusste auch nicht die Rate schwerer Hirnblutungen. Viele Patienten der Hyperkapniegruppe glichen offenbar die Reduktion der Beatmung durch vermehrte Eigenatmung aus und blieben unter dem vorgesehenen PCO2-Zielbereich. Die neurologische Nachuntersuchung der Studienteilnehmer wird derzeit durchgeführt. Vorläufige Endergebnisse aller 362 rekrutierten Patienten werden bis zum Kongress zur Verfügung stehen und präsentiert.

\section{FV-34}

Eine endotracheale Surfactantgabe unter CPAP-unterstützter Spontanatmung optimiert das Outcome extrem unreifer Frühgeborener - Ergebnisse der NINSAPP-Studie (Non Intubated Surfactant Application)

\section{A. Kribs', C. Roll', W. Göpel', C. Wieg ${ }^{4}$, P. Groneck', R. Laux 6 , N. Teig ${ }^{7}$,} T. Höhn ${ }^{8}$, R. Burghard ${ }^{9}$, L. Welzing ${ }^{10}$, M. Vochem ${ }^{11}$, M. Hoppenz ${ }^{12}$, C. Bührer ${ }^{13}$ 'Universitätskinderklinik Köln, Neonatologie und Pädiatrische Intensivmedizin, Köln, Deutschland, ${ }^{2}$ Vestische Kinder- und Jugendklinik Datteln, Neonatologie, Datteln, Deutschland, ${ }^{3}$ Universitätsklinikum SchleswigHolstein, Klinik für Kinder- und Jugendmedizin, Lübeck, Deutschland, ${ }^{4}$ Klinikum Aschaffenburg, Neonatologie und pädiatrische Intensivmedizin, Aschaffenburg, Deutschland, ${ }^{5}$ Klinikum Leverkusen, Kinderklinik, Leverkusen, Deutschland, ${ }^{6}$ AK Barmbek, Neonatologie, Hamburg, Deutschland, ${ }^{7}$ Klinik für Kinder- und Jugendmedizin St. Josefs-Hospital, Abteilung für Neonatologie, Bochum, Deutschland, ${ }^{8}$ Med. Einricht. d. Universität Kinderklinik, Düsseldorf, Deutschland, ${ }^{9}$ DRK Kinderklinik Siegen, Siegen, Deutschland, ${ }^{10}$ Zentrum für Kinderheilkunde, Universitätsklinikum Bonn, Abt. Neonatologie, Bonn, Deutschland, "Olgahospital, Neonatologie, Stuttgart, Deutschland, ${ }^{12}$ Städt. Krankenanstalten Kinderkrankenhaus, Neonatologie und pädiatrische Intensivmedizin, Köln, Deutschland, ${ }^{13}$ Charité - Universitätsmedizin Berlin, Klinik für Neonatologie, Berlin, Deutschland

Hintergrund. Für die Surfactantapplikation über eine dünne endotracheale Sonde unter CPAP unterstützter Spontanatmung als Methode zur Therapie des Atemnotsyndroms Frühgeborener (FG) fehlten lan-

ge Daten aus prospektiven, randomisierten, kontrollierten Studien (PRCT). Die AMV- Studie (Lancet 2011; 5:1627-34) zeigte die Sicherheit der Methode sowie die Wirksamkeit im Hinblick auf Beatmungsvermeidung für FG mit einem Gestationsalter (GA) von 26 o/7 bis 28 6/7 Wochen. Die NINSAPP-Studie untersucht FG mit einem GA von 23 o/7 bis 26 6/7 Wochen

Fragestellung. Verbessert die Surfactantapplikation über eine dünne endotracheale Sonde unter CPAP-unterstützter Spontanatmung das Outcome extrem kleiner FG?

Patienten und Methode. Zwischen April 2009 und Juni 2012 wurde eine multizentrische PRCT in 13 deutschen Zentren durchgeführt. FG mit einem GA von 23 o/7 bis 26 6/7 Wochen, die bei der Erstversorgung zur Spontanatmung kamen und im Alter zwischen 10 und $120 \mathrm{~min}$. klinisch eindeutige Zeichen eines Surfactantmangels zeigten (Silverman-Score $\geq_{5}, \mathrm{FiO}_{2} \geq 0,3$ für $\mathrm{SO}_{2}>83 \%$ ), wurden nach Einholung des elterlichen Einverständnisses randomisiert. FG im Kontrollarm wurden intubiert und beatmet und erhielten Surfactant über den Endotrachealtubus, Patienten im Interventionsarm erhielten unter CPAP unterstützter Spontanatmung Surfactant über eine dünne endotracheale Sonde. Primäres Zielkriterium war das „Überleben ohne BPD“, sekundäre Zielkriterien waren verschiedene neonatale Morbiditäten sowie das ,Überleben ohne $\mathrm{BPD}, \mathrm{IVH}>\mathrm{II}^{\circ}$, PVL, OP-bedürftige NEC oder FIP und therapiebedürftige ROP“. Die Auswertung erfolgte auf Basis der Intention-to-treat-Population.

Ergebnisse. 211 Frühgeborene wurden in die Studie eingeschlossen, davon 104 in die Kontrollgruppe und 107 in die Interventionsgruppe. Die neonatalen Basisparameter unterschieden sich nicht in beiden Gruppen. Alle neonatalen Morbiditäten mit Ausnahme der operationsbedürftigen NEC od. FIP waren häufiger in der Kontroll- als in der Interventionsgruppe, wobei dies nur für die IVH signifikant wurde (• Tab. 1). Hinsichtlich des primären Zielkriteriums „Überleben ohne BPD“ fand sich ein nicht signifikanter Unterschied zugunsten der Interventionsgruppe (67vs.59\%; p=0,2). Das sekundäre Zielkriterium „Überleben ohne BPD, IVH $>\mathrm{II}^{\circ}$, PVL, OP-bedürftige NEC od. FIP und therapiebedürftige ROP“ war in der Interventionsgruppe signifikant häufiger als in der Kontrollgruppe (50 vs. $36 \%$; $p=0,023$, NNT 6,7, 95\% CI 3,5-71,4). Schlussfolgerung. Die Surfactantapplikation unter CPAP unterstützter Spontanatmung ist bei extrem kleinen FG möglich und erhöht die Wahrscheinlichkeit eines Überlebens ohne eine der neonatalen Morbiditäten, die zu einer Behinderung prädisponieren.

\begin{tabular}{|llll|}
\hline FV-34 Tab. 1 & $\begin{array}{l}\text { Kontrollgruppe } \\
(\mathbf{n}=104)\end{array}$ & $\begin{array}{l}\text { Interventions- } \\
\text { gruppe }(\mathbf{n}=107)\end{array}$ & p-Wert \\
\hline Tod & $12(12)$ & $10(9)$ & 0,59 \\
\hline BPD & $31(30)$ & $25(23)$ & 0,34 \\
\hline IVH (alle) & $41(39)$ & $26(24)$ & 0,017 \\
\hline IVH>II & $23(22)$ & $11(10)$ & 0,019 \\
\hline Cystische PVL & $11(11)$ & $4(4)$ & 0,054 \\
\hline ROP alle & $68(65)$ & $66(62)$ & 0,5 \\
\hline $\begin{array}{l}\text { ROP mit Thera- } \\
\text { pie }\end{array}$ & $7(7)$ & $2(2)$ & 0,08 \\
\hline $\begin{array}{l}\text { NEC od. FIP mit } \\
\text { OP }\end{array}$ & $17(16)$ & $19(18)$ & 0,74 \\
\hline Angaben in Klammern sind Prozentangaben. & \\
\hline
\end{tabular}




\section{FV-35}

Surfactant-Gabe an spontan atmende Frühgeborene - 2-JahresFollow-up-Daten der AMV-Studie und epidemiologische Daten aus dem Deutschen Frühgeborenen Netzwerk

W. Göpel', A. Kribs', T. Höhn', R. Laux', C. Wieg', J. Siegel', S. Avenarius', A. von der Wense ${ }^{8}$, M. Vochem ${ }^{9}$, P. Groneck ${ }^{10}$, U. Weller ${ }^{11}$, J. Möller ${ }^{12}$, C. Härtel ${ }^{13}$, B. Roth ${ }^{14}$, E. Herting ${ }^{15}$

'Universitätsklinikum Schleswig-Holstein, Klinik für Kinder- und Jugendmedizin, Lübeck, Deutschland, ${ }^{2}$ Universitätskinderklinik Köln, Neonatologie und Pädiatrische Intensivmedizin, Köln, Deutschland, ${ }^{3}$ Med. Einricht. d. Universität Kinderklinik, Düsseldorf, Deutschland, ${ }^{4}$ AK Barmbek, Neonatologie, Hamburg, Deutschland, ${ }^{5}$ Klinikum Aschaffenburg, Neonatologie und pädiatrische Intensivmedizin, Aschaffenburg, Deutschland, ${ }^{6}$ Kinderkrankenhaus auf der Bult, Neonatologie, Pädiatrie I, Hannover, Deutschland, ${ }^{7}$ Universitätsklinik für Allgemeine Pädiatrie und Neonatologie, Magdeburg, Deutschland, ${ }^{8}$ Altonaer Kinderkrankenhaus, Neonatologie und pädiatrische Intensivmedizin, Hamburg, Deutschland, ${ }^{9}$ Olgahospital, Neonatologie, Stuttgart, Deutschland, ${ }^{10} \mathrm{Klinikum}$ Leverkusen, Kinderklinik, Leverkusen, Deutschland, "Krankenanstalten Gilead, Kinderklinik, Bielefeld (Bethel), Deutschland, ${ }^{12}$ Klinikum Saarbrücken, Klinik für Kinderund Jugendmedizin, Saarbrücken, Deutschland, ${ }^{13}$ Universitätsklinikum Schleswig Holstein/Campus Lübeck, Klinik für Kinder- und Jugendmedizin, Lübeck, Deutschland, ${ }^{14}$ Universitäts-Kinderklinik Köln, Neonatologie und pädiatrische Intensivmedizin, Köln, Deutschland, ${ }^{15}$ Universitätsklinikum Schleswig-Holstein Campus Lübeck, Direktor der Klinik für Kinder- und Jugendmedizin, Lübeck, Deutschland

Hintergrund. Die randomisierte „Avoiding Mechanical Ventilation“ (AMV)-Studie zeigte eine kürzere Beatmungszeit und eine niedrigere Beatmungsrate am 2. und 3. Lebenstag bei Kindern, denen unter Spontanatmung Surfactant verabreicht worden war.

Fragestellung. Wir berichten über die 2-Jahres-Follow-up-Daten der AMV-Studie und epidemiologische Daten aus dem Deutschen Frühgeborenen Netzwerk.

Material und Methode. Die 2-Jahres-Follow-up-Daten der an der AMVStudie teilnehmenden Frühgeborenen wurden mittels standardisierten Fragebögen von den jeweiligen sozialpädiatrischen Zentren bzw. den betreuenden Kinderärzten erhoben. In der GNN-Datenbank wurden alle Kinder mit einem Gestationsalter unter 32 Schwangerschaftswochen, bei denen Informationen zur Art der Surfactantapplikation vorlagen ausgewertet. Kinder die an der AMV- oder NINSAPP-Studie teilgenommen hatten wurden aus der GNN-Auswertung ausgeschlossen. Ergebnisse. Von 179 (86\%) der 208 lebend entlassenen Frühgeborenen der AMV-Studie lagen Outcome-Daten vor (mittleres Untersuchungsalter 27,4 $\pm 3,4$ Monate). Es fanden sich keine signifikanten Unterschiede zwischen Interventions- und Kontrollgruppe in Bezug auf Körpermaße, Bayley MDI, Freies Laufen, Hör- und Sehstörungen. In der Kontrollgruppe wurde in den letzten 12 Monaten vor der Erhebung häufiger die Diagnose einer obstruktiven Bronchitis gestellt, aber auch diese Differenz war statistisch nicht signifikant ( $49 \%$ vs. $34 \%$ in der Interventionsgruppe; $\mathrm{p}=0,056$; Fisher's exact Test). Die GNN-Daten für das Jahr 2012 sind noch nicht vollständig gemonitort. Eine vorläufige Auswertung zeigte einen zunehmenden Einsatz der Surfactantgabe ohne Intubation (34\% in 2009 ansteigend auf $40 \%$ im Jahr 2012). Insgesamt wurden mehr als 1000 Frühgeborene unter 32 Schwangerschaftswochen mit Surfactant unter Spontanatmung behandelt. Diese Gruppe zeigte im Vergleich zur den konventionell, bzw. nicht mit Surfactant behandelten Frühgeborenen eine deutlich niedrigere BPD-Rate (11,1\% vs. $17,7 \%)$. Weitere Subgruppenanalysen und Matched-pair-Vergleiche sind geplant.

Diskussion. Die Gabe von Surfactant an spontan atmende Frühgeborene mit Atemnotsyndrom und einem Gestationsalter zwischen 26+o und 28+6 Schwangerschaftswochen reduziert die Beatmungsrate und die Beatmungsdauer und hat keine negativen Auswirkungen auf den Outcome mit 2 Jahren.

\section{Neurologisches „Outcome“ nach Asphyxie und Frühgeburt}

\section{FV-36}

Entwicklungsprognose extrem unreifer Frühgeborener S. Herber-Jonat', S. Streiftau' ${ }^{2}$, E. Knauss ${ }^{3}$, F. Voigt ${ }^{3}$, H. Hummler', H. Bode ${ }^{2}$,
A. Schulze

'Perinatalzentrum Großhardern, Neonatologie, München, Deutschland, ${ }^{2}$ Klinik für Kinder- und Jugendmedizin, Universitätsklinikum UIm, Sozialpädiatrisches Zentrum, Ulm, Deutschland, ${ }^{3}$ Kinderzentrum München, München, Deutschland, ${ }^{4}$ Klinik für Kinder- und Jugendmedizin, Universitätsklinikum Ulm, Sektion Neonatologie und Pädiatrische Intensivmedizin, Ulm, Deutschland

Hintergrund. Im Rahmen intensiver perinataler Betreuung und dem Einsatz modernster medizinischer Techniken gelingt es heute immer häufiger, dass extrem unreifen Frühgeborenen an der Grenze der Lebensfähigkeit [<25 vollendete Schwangerschaftswochen (SSW)] überleben. Es besteht jedoch eine erhebliche Unsicherheit darüber, ob und ggf. wie sich die langfristige Entwicklungsprognose von Frühgeborenen des Gestationsalter 22+o-23+6 im Vergleich zu Frühgeborenen mit 24+o-6 unterscheidet. Nullhypothese: Zwischen Frühgeborenen der Gestationsaltersgruppen $22+0-23+6$ vs. 24+0-6 SSW der Jahrgänge 19992003 besteht hinsichtlich ihrer Langzeitprognose kein Unterschied.

Methodik. Im Rahmen einer prospektiven Kohortenstudie an Frühgeborenen <25+o SSW der Geburtsjahrgänge 1999-2003, die in den Perinatalzentren München Großhadern und der Universität Ulm geboren und nach Hause entlassen worden sind eine standardisierte entwicklungsneurologische Nachuntersuchung der Kinder im Alter zwischen 7 bis 9 Jahren (pädiatrisch-neurologische Untersuchung, HAWIK IV, LOS KF 18, Frostigs Entwicklungstest-2 und GMFCS) durchgeführt. Es erfolgte eine Kategorisierung anhand festgelegter Kriterien als mild, moderat oder schwer beeinträchtigt bzw. als unauffällig. Eine Subgruppe der Kinder wurde zusätzlich mittels kranialer MRT untersucht. Neben einem Vergleich der beiden Gestationsaltersgruppen ( $\chi^{2}$-Test, ANOVA) wurde eine logistische Regressionsanalyse zur Identifikation möglicher Assoziationen zwischen Langzeitentwicklung und Gestationsalter, Geburtsgewicht, antenataler Steroidprophylaxe und neonataler Morbidität durchgeführt.

Ergebnisse. 79/105 (75\%) ehemalige Frühgeborene $<25$ Wochen der Jahrgänge 1999-2003 wurden im Alter zwischen 7 und 9 Jahren (im Mittel 8,7 Jahre) nachuntersucht. 6o Kinder (75,9\%) zeigten keine oder eine milde Entwicklungsbeeinträchtigung. Hinsichtlich der Entwicklungsprognose bestand kein Unterschied zwischen den beiden Gestationsaltersgruppen [22-23 SSW ( $n=18): 77,8 \% ; 24$ SSW ( $n=61): 75,4 \%]$. Eine intrazerebrale Blutung $>\mathrm{II}^{\circ}$ und/oder periventrikuläre Leukomalazie und eine Frühgeborenenretinopathie $>\mathrm{II}^{\circ}$ waren signifikante Risikofaktoren für die Entwicklung einer moderaten oder schweren Behinderung. Weder das Gestationsalter, noch das Geburtsgewicht waren mit der Langzeitprognose assoziiert. In der Volumetrie der einzelnen Hirnregionen zeigten die untersuchten Frühgeborenen im Vergleich $\mathrm{zu}$ reifgeborenen Kindern einen geringeren Anteil grauer und weißer Substanz, Hippocampus, Kleinhirn und Hirnstamm, während das Ventrikelsystem einen größeren Raum einnahm ( $\mathrm{p}<\mathrm{o}, \mathrm{oo1})$.

Schlussfolgerung. In unserer Studienkohorte hatten ehemalige Frühgeborene mit 22-23 vollendeten Schwangerschaftswochen eine ähnliche Entwicklungsprognose wie Kinder der 24. Schwangerschaftswoche. Unsere Ergebnisse stützen die Hypothese, dass das Gestationsalter allein eine geringe Aussagekraft in Bezug auf die Langzeitentwicklung von Frühgeborenen $<25$ SSWhat. 


\section{Kreislauftherapie - Kardiologie}

\section{FV-37}

Auswirkungen von nasalem "continuous positive airway pressure" auf die Herzfunktion von Frühgeborenen mit fortgeschrittenem Atemnotsyndrom

\section{F. Beker', C. Wong', P. Davis', S. Hooper', S. Rogerson ${ }^{2}$}

'Southernhealth, Clayton, Victoria, Monash Newborn, North Melbourne, Australien, ${ }^{2}$ Royal Women's Hospital, Neonatal Intensive and Special Care Units, Parkville, Victoria, Australien, ${ }^{3}$ Department of Physiology, Monash Institute for Medical Resarch, Monash University, Clayton, Victoria, Australien

Einführung. Studien an beatmeten Frühgeborenen sowie an Lämmern haben gezeigt, dass ein ansteigender positiver end- expiratorischer Druck (PEEP) das Schlagvolumen der rechten Herzkammer reduziert. Hohe Beatmungsdrücke führen möglicherweise zum Anstieg des Widerstandes in den Lungengefäße und schränken den venösen Rückfluss zum Herzen ein. Die Lungen von Frühgeborenen mit fortgeschrittenem Atemnotsyndrome haben eine reduzierte Compliance und der Atemwegsdruck wird daher möglicherweise nicht oder nur in reduziertem Maße auf die Thoraxhöhle und damit auf die torakalen Blutgefäße übertragen. Wir haben die Auswirkungen von drei verschiedenen CPAP-Drücken auf das Herzschlagvolumen untersucht.

Methoden. Wir haben Frühgeborene mit einem korrigiertem Alter von 28-34 Wochen, die mindestens zwei Wochen alt waren und nCPAP-Drücke von $5-7 \mathrm{cmH}_{2} \mathrm{O}$ und $25-40 \%$ Sauerstoff brauchten, untersucht. Die Eltern hatten zuvor der Untersuchung schriftlich zugestimmt. Frühgeborene mit Ductus arteriosus, Atriumseptumdefekt oder anderen Herzfehlbildungen wurden von der Studie ausgeschlossen. Es wurden jeweils drei CPAP Drücke $\left(4,6\right.$, und $\left.8 \mathrm{cmH}_{2} \mathrm{O}\right)$ für jeweils 15 Minuten in randomisierter Reihenfolge appliziert. Mit einem Vivid-I-Ultraschallgerät wurde nach jeder Änderung des nCPAP-Druckes das Schlagvolumen des rechten und linken Herzens, der Fluss in der linken Lungenarterie sowie der Vena cava superior gemessen. Alle Messungen wurden von einem Untersucher durchgeführt, der die aktuellen CPAP-Drücke nicht kannte. Ergebnisse. Dreiundzwanzig Frühgeborene nahmen an der Studie teil. Es wurden keine signifikanten Unterschiede der erhobenen Daten zwischen den drei nCPAP-Drücke festgestellt ( $\bullet$ Tab. 1).

Diskussion. Es ist nicht klar, ob nCPAP die Herzfunktion beeinflusst. Zwei vorangegangene Studien an Neugeborenen mit und ohne nasales CPAP haben kein übereinstimmendes Ergebnis gefunden. Die hier gezeigten Ergebnisse sind der zweite Teil unserer Studie, die Auswirkungen von nasalem CPAP auf die Herzfunktion von Frühgeborenen mit 1) schnell überwundenem Atemnotsyndrom und 2) fortgeschrittenem Atemnotsyndrom untersucht.

Schlussfolgerung. Wir stellen fest, dass bei unseren Untersuchungen ansteigende nCPAP-Drücke zwischen 4 und $8 \mathrm{cmH}_{2} \mathrm{O}$ keinen messbaren Einfluss auf die Herzfunktion von stabilen Kindern mit fortgeschrittenem Atemnotsyndrom haben.

\section{Infektionen}

\section{FV-38}

Evaluation des Oberflächenantigens CD64 auf neutrophilen Granulozyten als diagnostischer Marker nosokomialer bakterieller Infektionen bei Frühgeborenen

\section{F. Kipfmüller', J. Schneider', J. Prusseit', P. Bartmann', B. Zur², A. Müller \\ 'Universitätsklinik Zentrum f. Kinderheilkunde, Neonatologie, Bonn, Deutschland, ${ }^{2}$ Institut für Klinische Chemie und Klinische Pharmakologie, Bonn, Deutschland}

Hintergrund. Bakterielle Infektionen sind insbesondere bei Frühgeborenen mit einem Geburtsgewicht $<1500$ g (VLBW) mit einer erhöhten
Mortalität und Morbidität verbunden. Der frühzeitigen Diagnosestellung und Therapie kommt eine große Bedeutung zu. Neben den länger etablierten Parametern wie CrP und IL6, konnte in mehreren Studien gezeigt werden, dass das auf neutrophilen Granulozyen exprimierte Oberflächenantigen CD64 im Rahmen bakterieller Infektionen ansteigt und eine gute diagnostische Aussagekraft besitzt. Alle zu CD64 bisher durchgeführten Studien an Früh- und Neugeborenen beschränken sich auf die diagnostische Aussagekraft bei bestehendem klinischem Infektionsverdacht.

Fragestellung. Kommt es im Rahmen einer bakteriellen Infektion bei VLBW zu einer CD64-Expremierung bevor klinische Symptome auftreten und andere Infektionsparameter ansteigen?

Material und Methoden. Einschlusskriterien zur Studienteilnahme waren ein Geburtsgewicht $<1500 \mathrm{~g}$ und eine schriftliche Einwilligung der Eltern. Bei teilnehmenden Kindern wurde prospektiv im Rahmen von Routineblutabnahmen zwischen dem 4. und 28. Lebenstag eine CD64Bestimmung durchgeführt. Ein Infektionsausschluss mittels $\mathrm{CrP}$ und IL6 erfolgte nach Einschätzung des behandelnden Arztes. Infektionsereignisse wurden gemäß den NEO-KiSS-Kriterien definiert. Als Infektionsperiode wurde der Zeitraum zwei Tage vor bis 4 Tage nach Diagnosestellung festgelegt. Als Cut-off-Werte für IL6, CrP und CD64-Index wurde folgende Grenzwerte definiert: $\geq 25 \mathrm{pg} / \mathrm{ml}, \geq 10 \mathrm{mg} / \mathrm{l} \mathrm{bzw} . \geq 1,5$.

Ergebnisse. Es wurden 50 Patienten in die Studie aufgenommen. Bei 14 Patienten musste die CD64-Bestimmung vor dem 28. Lebenstag beendet werden (2-mal Wunsch der Eltern, 12-mal Verlegung). Eine CD64Messung erfolgte an 380/1046 Tagen. Während des Studienzeitraums kam es zu 24 Infektionsepisoden (8 mit positiver Blutkultur, 11 klinische Sepsis, 3 NEC, 1 Pneumonie). Am Tag der Diagnosestellung fanden sich erhöhte Werte für IL6, CrP und CD64-Index in 83,3\%, 33,3\% und 50\% der Fälle. Bei 33,3\% der untersuchten Kinder konnte ein erhöhter CD64Index am Tag vor Diagnosestellung gemessen werden. Im Vergleich fand sich am Tag vor Infektionsbeginn bei ein erhöhtes CrP bzw. IL6 bei $12,5 \%$ bzw. o\% der Patienten. Insgesamt hatten $70,8 \%$ der Patienten während der Infektionsperiode einen erhöhten CD64-Index und 66,6\% ein erhöhtes CrP.

Diskussion. CD64 wird im Rahmen von bakteriellen Infektionen auf neutophilen Granulozyten bei VLBW exprimiert. Teilweise findet sich bereits $24 \mathrm{~h}$ vor Diagnosestellung ein erhöhter CD64-Index. Bezüglich der diagnostischen Aussagekraft erscheint CD64 bei Frühgeborenen im Vergleich CrP und IL6 nicht überlegen. Größere prospektive Studien mit konsistenterer Bestimmung sind notwendig um das vorliegende Ergebnis zu bestätigen.

\begin{tabular}{|c|c|c|c|c|}
\hline & $4 \mathrm{cmH} 2 \mathrm{O}$ & $6 \mathrm{cmH} 2 \mathrm{O}$ & $8 \mathrm{cmH} 20$ & p-Wert \\
\hline $\begin{array}{l}\text { Fluss in der } \\
\text { Lungenarterie } \\
\text { (ml/kg/min) }\end{array}$ & $399 \pm 123$ & $404 \pm 103$ & $401 \pm 91$ & $>0,05$ \\
\hline $\begin{array}{l}\text { Fluss in der } \\
\text { Aorta (ml } / \mathrm{kg} / \\
\mathrm{min})\end{array}$ & $397 \pm 129$ & $398 \pm 151$ & $389 \pm 128$ & $>0,05$ \\
\hline $\begin{array}{l}\text { Fluss in der } \\
\text { Vena cava (ml/ } \\
\mathrm{kg} / \mathrm{min})\end{array}$ & $154 \pm 49$ & $142 \pm 41$ & $157 \pm 46$ & $>0,05$ \\
\hline $\begin{array}{l}\text { Fluss in der } \\
\text { linken Lungen- } \\
\text { arterie (ml/kg/ } \\
\mathrm{min} \text { ) }\end{array}$ & $138 \pm 64$ & $145 \pm 69$ & $132 \pm 59$ & $>0,05$ \\
\hline
\end{tabular}




\section{Pränatale Pathologie - angeborene Fehlbildungen}

\section{FV-39}

Fetale, intratracheale Instillation eines Aktivators der löslichen Guanylatzyclase als Therapieoption der pulmonalen Hypertension im Modell der kongenitalen Zwerchfellhernie

\section{S. Herber-Jonat', A. Vuckovic ${ }^{2}$, J. Jani ${ }^{3}$, A. Flemmer ${ }^{1}$}

'LMU-München, Neonatologie der Kinderklinik am Perinatalzentrum Großhadern, München, Deutschland, ${ }^{2}$ Laboratory of Physiology and Physiopathology, Université Libre de Bruxelles, Brussels, Belgien, ${ }^{3}$ University Hospital Brugmann, Department of Obstetrics and Gynecology, Brussels, Deutschland

Hintergrund. Die postnatale pulmonale Vasodilatiation beim Neugeborenen wird unter Anderem durch die Aktivierung der sGC vermittelt und ist bei fetaler Lungenhypoplasie gestört. Bei Neugeborenen mit kongenitaler Zwerchfellhernie ( $\mathrm{CDH})$ weisen die Arterien eine Mediahypertrophie mit bis weit in die Peripherie reichender Muskulatur auf. BAY 41-2272 (BAY) ist ein neuer Aktivator der sCG und könnte so einer Gefäßhypoplasie bei CDH entgegenwirken.

Fragestellung. Welchen Einfluss hat die fetale, intratracheale Instillation von BAY auf die postnatale Hämodynamik, vaskuläre Morphologie und Expression vasodilatativer Mediatoren bei Kaninchen mit fetaler Lungenhypoplasie?

Methoden. Am Tag 23/31 erhielten jeweils 2 Feten pro Muttertier eine Inzision des linksseitigen Zwerchfells im Rahmen einer EXIT-Prozedur. Am Tag 28/31 erfolgte eine zweite Operation zur intratrachealen BAY- (CDH-BAY; $\mathrm{n}=9$ ) bzw. $\mathrm{NaCl}$ o,9\% Instillation (CDH-Saline; $\mathrm{n}=9$ ). Nach Entbindung am Termin wurden die operierten Feten sowie nichtoperierte Kontrollen beatmet, der Thorax eröffnet und der rechtsventrikuläre Blutdruck invasiv gemessen. Als Marker einer kardialen Schädigung wurde die „heart to body weight ratio“ (HBWR) bestimmt und das NT-pro-BNP im Serum gemessen. Im Anschluss wurden mittels qRT-PCR cGMP, eNOS und VEGFA im Lungengewebe bestimmt.

Ergebnisse. Der mittlere rechtsventrikuläre Druck war in den CDHBAY Tieren im Vergleich zu den $\mathrm{CDH}$-Saline Tieren signifikant vermindert $(\mathrm{p}=0,006)$. Die HBWR der $\mathrm{CDH}-\mathrm{Saline}$ Tiere als auch der mittlere Serumspiegel von NT-pro-BNP waren im Vergleich zu den gesunden Kontrolltieren erhöht ( $\mathrm{p}<\mathrm{0}, \mathrm{o1})$. In Bezug auf beide Parameter unterschieden die CDH-BAY Tiere nicht von den gesunden Kontrollen. Es zeigte sich eine signifikanter Anstieg der Expression von eNOS in der CDH-BAY Gruppe im Vergleich zu CDH-Saline Tieren $(p=0,004)$. Die Expression von VEGFA war in der Therapiegruppe im Vergleich zur Kontrollgruppe erniedrigt $(\mathrm{p}=$ ?).

Schlussfolgerung. Die einmalige fetale Instillation eines Aktivators der löslichen Guanylatzyklase bei Lungenypoplasie durch CDH führt zu einer Reduktion des rechtsventrikulären Drucks und NT-pro-BNP Konzentration. Dieser funktionale Effekt ist mit einer verstärkten eNOS Expression assoziiert. Damit ist die fetale Instillation von Aktivatoren der sGC möglicherweise eine vielversprechende Therapieoption der Lungenhypoplasie induzierten pulmonalen Hypertonie.

\section{FV-40}

Regulation und Expression von NOS3 in arteriellen Nabelschnurendothelien von Feten mit Plazentainsuffizienz

\section{A. Jenke', S. Wirth' ${ }^{2}$ J. Postberg}

${ }^{1}$ Klinikum Wuppertal GmbH Zentrum f. Kinder- u. Jugendmedizin, Wuppertal-Barmen, Deutschland, ${ }^{2}$ Helios Klinikum Wuppertal, Zentrum für Kinderund Jugendmedizin, Wuppertal, Deutschland

Background. To contribute to our limited knowledge about placental insufficiency and the later risk for hypertension and other disorders, identification of intra uterine adaptive molecular mechanisms by the fetus might be helpful.

Methods. Epidemiological parameters, Doppler examinations as well as DNA/RNA samples purified from endothelial umbilical artery cells of newborns were collected during a 6 months period. Expression of $\mathrm{NOS}_{3}$ was quantified using $\mathrm{qPCR}$. As putative adaptive epigenomic signatures involved in gene regulation $5 \mathrm{meC} / 5 \mathrm{hmeC}$ levels as well as histone posttranslational modification patterns of the $\mathrm{NOS}_{3}$ promoter were analyzed using (h)MeDIP-qPCR and ChIP-qPCR, respectively. Moreover genotyping of a putative miRNA locus was performed.

Results. 42 patients were included in this study (gestational age range: $23+6$ to $41+7$, median $31+3$ ). eNOS copy numbers were significantly higher in infants with impaired perfusion in the uterine and mediocerebral artery (mean 15.7 vs. 228.6). The NOS 3 promoter region was hypomethylated and hyperhydroxymethlated at the transcription start site in all patients but Histone $\mathrm{H}_{3} \mathrm{~K} 9$ and $\mathrm{H}_{2}$. $\mathrm{AZ}$ acetylation directly correlated with the NOS 3 mRNA copy number. Treatment of human umbilical venous cells in vitro with either the histone acetyl transferase inhibitor C646 or the histone deacetylase inhibitor trichostatin A also significantly altered the eNOS expression profile. No association between intronic 27-nt miRNA genotype status and eNOS expression was observed. Conclusion. Endothelial expression of $\mathrm{NOS}_{3}$ in umbilical arteries is an adaptive responsive mechanism in placental insufficiency. Adaptive regulation of $\mathrm{NOS}_{3}$ expression apparently depends on differential posttranslational modifications of histone $\mathrm{N}$-termini, such as $\mathrm{H}_{3}$ ac.

\section{Freie Themen}

\section{FV-41}

Establishment and validation of a method to detect circulating endothelial cells in preterm infants

S. Lee', B. Brar', N. Rochow', K. Kalaniti', S. el Helou', G. Fusch', D. Snider', C. Fusch ${ }^{7}$

'McMaster University, Department of Pediatrics, Hamilton, ON, Kanada, ${ }^{2}$ McMaster University, Department of Pathology and Molecular Medicine, Hamilton, Kanada

Background. Preterm infants undergo medical treatments which may compromise vascular integrity. Since circulating endothelial cells (CEC) have been shown to be a marker of vascular injury in adolescents and adults, levels of CEC could be used to assess vascular health in preterm infants. Published methods to enumerate CECs require blood volume too large $(>4 \mathrm{~mL})$ for sampling in preterm infants.

Objective. To establish and validate a precise and accurate method to enumerate CECs using $450 \mu \mathrm{L}$ of blood that is feasible for use in neonatal medicine.

Method. Adult peripheral blood and cord blood from term placentas were used to establish a peripheral blood mononuclear cell isolation method to enumerate CECs by flow cytometry (identified as CD146+ CD 45- CD14- CD133- Syto16+ CD 31+ cells). The identity of these cells was validated by morphological assessment under $20,000 \times$ magnification (TEM). Reproducibility was evaluated by processing 5 aliquots of the same sample in parallel (aliquot size of $450 \mu \mathrm{L}$ cord blood, $\mathrm{n}=3 ; 5 \mathrm{~mL}$ adult blood, $n=5$ ). Accuracy was evaluated by processing duplicates of 
$450 \mu \mathrm{L}$ and $5 \mathrm{~mL}$ of the same cord blood sample in parallel $(\mathrm{n}=2)$. To compare the influence of cell fixation, stained cells were either unfixed or fixed with $1 \%$ formaldehyde prior to analysis at o d, $1 \mathrm{~d}$ and $2 \mathrm{~d}$ post sample preparation $(n=10)$. Temporal decline in levels of CEC was evaluated by storing adult peripheral blood in $10 \%$ acid citrate dextrose and EDTA at $4^{\circ} \mathrm{C}$ until sample preparation and analysis at o h, $12 \mathrm{~h}$ and $24 \mathrm{~h}$ post sampling $(\mathrm{n}=6)$.

Results. Average CV between 5 aliquots of $450 \mu \mathrm{L}$ cord blood samples was $22 \%$ and $5 \mathrm{~mL}$ of adult peripheral blood samples was $19 \%$. Both the forward and side scatter properties of fixed cells decreased in comparison to unfixed cells; the relative fluorescence intensities of the fluorochromes remained unchanged. There was no difference in the levels of CEC between fixed and unfixed samples over the course of analysis. Significant decline in the levels of CEC was observed in samples prepared and analyzed at 24 hours but not 12 hours post sampling.

Conclusions. We have established a method to enumerate CECs in $450 \mu \mathrm{L}$ of whole blood that is precise, accurate and feasible for use in the preterm population. Our results suggest that studies can be carried out at sites remote to a flow cytometer and in clinical settings, where babies are born at unpredictable hours and a technician is unavailable to immediately process the samples.

\section{FV-42}

\section{DMBT1, ein in der Lunge von Früh- und Neugeborenen expri- miertes Protein, zeigt in vitro und in vivo pro-angiogenetische Eigenschaften}

\section{H. Müller', J. Hu², R. Popp'2, J. Mollenhauer ${ }^{3}$, J. Pöschl', I. Fleming ${ }^{2}$}

${ }^{1}$ Abteilung Neonatologie, Zentrum für Kinder- und Jugendmedizin, Universitäts-Klinikum Heidelberg, Heidelberg, Deutschland, ${ }^{2}$ Institute for Vascular Signalling, Goethe Universität Frankfurt/Main, Frankfurt/Main, Deutschland, ${ }^{~}$ Lundbeckfonden Center of Excellence NanoCAN and Molecular Oncology, University of Southern Denmark, Odense C, Dänemark, ${ }^{4}$ Abteilung Neonatologie, Zentrum für Kinder- und Jugendmedizin, Universitäts-Klinikum Heidelberg, Heidelberg, Deutschland

Hintergrund. Das Protein DMBT1 („deleted in malignant brain tumors $1^{\prime \prime)}$ hat Funktionen bei der angeborenen Immunabwehr, bei der Zellpolarität und bei der epithelialen Differenzierung. Es wird in der Lunge von Früh- und Neugeboren und auch im adulten Lungengewebe exprimiert. Bei einer Inflammation kommt es zu einer erhöhten DMBT1-Expression.

Fragestellung. Das Ziel der Studie war, die Funktionen von DMBT1 bei Endothelzellen zu analysieren.

Material und Methoden. Es wurden HUVECs (humane Nabelschnurvenen-Endothelzellen) und Lungenendothelzellen aus Wildtyp- und DMBT1-/--Mäusen kultiviert. Die DMBT1-Detektion erfolgte mittels Immunfluoreszenz und Western Blot. Die Bindung von DMBT1 an Interaktionspartner wurde mit Immunopräzipitation und Elisa-Bindungsversuchen nachgewiesen. Die angiogenetischen Eigenschaften von DMBT1 wurden in vitro sowohl mit dem Endothelial-tube-formation-Assay als auch mit dem Aortenring-Assay und in vivo mit dem Hinterbeinischämie-Versuch bei Wildtyp- und DMBT1-/--Mäusen untersucht.

Ergebnisse. Endothelzellen sezernieren DMBT1 in die extrazelluläre Matrix und erhöhen damit die Adhärenz von Endothelzellen. DMBT interagiert mit Galectin-3. Humanes rekombinantes DMBT1 bindet an VEGF, EGF und Dll4. DMBT1-/--Endothelzellen zeigen eine verminderte Proliferation, Migration und weniger tubes beim endothelial tube formation-Assay im Vergleich zu Wildtyp-Endothelzellen. Das VEGFinduzierte Endothelzellen-Sprouting beim Aortenring-Assay als auch das Aussprossen der Gefäße in vivo beim Hinterbeinischämie-Versuch war bei der DMBT1-/--Maus vermindert. In den DMBT1-/--Endothelzellen konnte eine erniedrigte Expression von Dllı, Dll4, Hesı, Hey1 (Notch-Signalweg) und von EphB4, aber eine erhöhte Expression von EphrinB2 beobachtet werden.
Schlussfolgerung. Die Ergebnisse zeigen, dass DMBT1 ein wichtiges Protein in der extrazellulären Matrix von Endothelzellen ist und dabei Funktionen bei der Angiogenese und Gefäßreparatur ausübt. Diese Eigenschaften von DMBT1 könnten bei der Pathogenese verschiedener Lungenerkrankungen wie z. B. der bronchopulmonalen Dysplasie von Bedeutung sein, was durch weitere Studien untermauert werden sollte.

\section{FV-43}

\section{Copeptin als Plasmamarker perinataler Stressreaktionen}

\section{S. Wellmann', T. Burkhardt' ${ }^{2}$, C. Bührer ${ }^{3}$, H. Bucher', V. Cannizzaro ${ }^{5}$} 'Universitäts-Kinderspital beider Basel (UKBB), Basel, Schweiz, ${ }^{2}$ UniversitätsSpital Zürich, Klinik für Geburtshilfe, Zürich, Schweiz, ${ }^{3}$ Charité - Universitätsmedizin Berlin, Klinik für Neonatologie, Berlin, Deutschland, ${ }^{4}$ UniversitätsSpital Zürich, Klinik für Neonatologie, Zürich, Schweiz, ${ }^{5}$ Kinderspital Zürich, Universitäts-Kinderkliniken, Intensivmedizin, Zürich, Schweiz

Hintergrund. Copeptin ist ein stabiles Spaltprodukt der Synthese von Arginin Vasopressin (AVP) und wird in äquimolarer Menge zu AVP sezerniert. Im Gegensatz zu AVP ist Copeptin zuverlässig im Plasma messbar und hat sich als idealer Surrogatmarker von AVP in der Erwachsenenmedizin etabliert. Im Gegensatz dazu ist Copeptin in der Neonatologie und pädiatrischen Intensivmedizin kaum untersucht. In eigenen prospektiven Querschnittsuntersuchungen wurden Plasmaproben von mehr als 400 früh- und termingeborenen Kindern bei Geburt und in der ersten Lebenswoche untersucht. Wir konnten zeigen, dass eine vaginale Geburt für das Neugeborene unabhängig vom Gestationsalter mit äußerst hohen Copeptinspiegeln verbunden ist. Erfolgte die Geburt per elektivem Kaiserschnitt waren die Copeptinwerte im Nabelschnurblut niedrig mit Ausnahme von Kindern mit signifikanter intrauteriner Wachstumsverzögerung. Hier waren die Copeptinspiegel auch ohne Geburtsstress erhöht, was ein Beleg für die Hypothese der fetalen Programmierung ist. Wichtige Copeptin Prädiktoren in der ersten Lebenswoche sind postnatale Dehydratation und das Ausmaß respiratorischer Unterstützung.

Fragestellung. Wie beeinflussen verschiedene neonatologisch-intensivmedizinische Parameter die Stressreaktion gemessen als Copeptin.

Material und Methoden. Copeptinbestimmung im Plasma von infantilen Wistar-Ratten $\left(\mathrm{P}_{14}\right)$ in einem Ventilationsmodell mit 10 Tieren je Versuchsgruppe.

Ergebnisse. Niedrige Messwerte mit durchschnittlich $22 \mathrm{pmol} / \mathrm{L}$ zeigten sich in ungestressten nichtventilierten und protektiv beatmeten Tieren. Respiratorische Alkalose bewirkte einen signifikanten 5-fachen Anstieg der Copeptinwerte. 10-fach erhöhte Copeptinwerte ergaben sich in den Gruppen Hypoxie, Beatmung mit hohem PEEP, akuter Blutverlust und psycho-emotionaler Stress ( $\mathrm{p}<0,05$ für alle Gruppen). Hingegen kam es zu keinem Anstieg der Copeptinwerte bei respiratorischer Azidose, Hyperoxie, niedrigem PEEP und Hypothermie.

Schlussfolgerung. Diese Beobachtungen geben Einblick in die Regulation der AVP-Sekretion unter intensivmedizinischen Bedingungen. Es kann geschlussfolgert werden, dass kritisch kranke Neugeborene, insbesondere Frühgeborene mit langer Beatmungszeit einem hohen Maß an kumulativem Stress ausgesetzt sind, dessen Auswirkung auf die weitere Entwicklung der Kinder kaum untersucht ist. 


\section{FV-44}

Glukokortikoidrezeptor-Genpolymorphismen und neonatales Outcome VLBW-Frühgeborener: vorläufige Ergebnisse einer deutschen multizentrischen Studie

\section{Poralla', F. Schreiner' ${ }^{2}$, C. Härtel', M. Heckmann ${ }^{4}$, A. Heep ${ }^{5}$, P. Bartmann ${ }^{5}$, E. Herting ${ }^{3}$, W. Göpel ${ }^{6}$ \\ 'Universitätsklinik Zentrum f. Kinderheilkunde, Neonatologie, für das Deutsche Frühgeborenen Netzwerk (German Neonatal Network, GNN), Bonn, Deutschland, ${ }^{2}$ Universitätsklinik Zentrum f. Kinderheilkunde, Pädiatrische Endokrinologie, Bonn, Deutschland, ${ }^{3}$ Universitätsklinikum Schleswig Holstein/Campus Lübeck, Klinik für Kinder- und Jugendmedizin, Lübeck, Deutschland, ${ }^{4}$ Klinik und Poliklinik für Kinder und Jugendmedizin, Abt. Neonatologie u. Päd. Intensivmedizin, Greifswald, Deutschland, ${ }^{5}$ Uni- versitätsklinik Zentrum f. Kinderheilkunde, Neonatologie, Bonn, Deutsch- land, ${ }^{6}$ Universitätsklinikum Schleswig Holstein/Campus Lübeck, Klinik für Kinder- und Jugendmedizin, für das Deutsche Frühgeborenen Netzwerk (German Neonatal Network, GNN), Lübeck, Deutschland}

Hintergrund. Durch die Betamethason-Prophylaxe zur Induktion der fetalen Lungenreife werden neben dem Risiko für das Auftreten eines ANS u. a. auch Mortalität und Inzidenz von NEC, Sepsis und IVH vermindert. Die Bedeutung genetisch bedingter individueller Unterschiede in der Glukokortikoidrezeptor (GR) -Sensitivität für das neonatale Outcome ist dagegen bislang kaum untersucht worden.

Studiendesign. In einem 2211 VLBW-Frühgeborene umfassenden Kollektiv des Deutschen Frühgeborenen Netzwerks (German Neonatal Network, GNN) untersuchten wir den Einfluss der GR- Genpolymorphismen BclI, $\mathrm{N}_{3} 6_{3} \mathrm{~S}$ und $\mathrm{R}_{23} \mathrm{~K}$ auf die neonatale Morbidität.

Ergebnisse. Perinatale Basisparameter wie Gestationsalter, Geburtsgewicht und Geburtsmodus unterschieden sich nicht zwischen den GR-Genotyp-Gruppen. Sowohl BclI als auch R23 K waren mit einem erhöhten Risiko für eine Blutkultur-positive Sepsis assoziiert (BclI homozygot: $\mathrm{OR} 1,85 ; \mathrm{p}=0,002 ; \mathrm{R} 23 \mathrm{~K}$-Träger: $\mathrm{OR} 2,08 ; \mathrm{p}=0,016$; adjustiert für Gestationsalter). BclI-Träger entwickelten außerdem signifikant häufiger eine BPD (OR 1,41 pro Allel [95\% CI 1,14-1,75], $\mathrm{p}<0$,o1 adjustiert für Gestationsalter und mechanische Beatmung). Ein vergleichbares relatives Risiko war in der Subgruppe der Kinder mit Betamethason-Prophylaxe nachweisbar $(\mathrm{OR} 1,44 ; \mathrm{p}<0,01)$, nicht aber in der kleineren Untergruppe ohne Betamethason-Prophylaxe ( $\mathrm{n}=185$, OR 1,03, n.s.). Träger der $\mathrm{N}_{3} 63 \mathrm{~S}$-Variante zeigten niedrigere mittlere arterielle Blutdruckwerte am ersten Lebenstag (Gesamtkohorte: beta $=-0,050 ; \mathrm{p}=0,010$; reine $\mathrm{N}_{363}$ S-Träger, d. h. BclI-/R23K-Wildtyp: beta $\left.-0,055 ; \mathrm{p}=0,074\right)$ und benötigten signifikant häufiger eine Katecholamintherapie $(\mathrm{p}<0,01)$. Keine der analysierten GR-Varianten zeigte dagegen einen signifikanten Einfluss auf die IVH-, NEC- oder ROP-Inzidenz.

Schlussfolgerung. Die Studiendaten unterstützen die Hypothese, dass endogene, durch GR-Genpolymorphismen bedingte Unterschiede der GR-Sensitivität die neonatale Morbidität VLBW-Frühgeborener mitbeeinflussen. Zur Bestätigung der beobachteten Assoziationen wird aktuell eine Replikationskohorte untersucht.

\section{FV-45}

Populationsbasierte Studie zum Outcome Neugeborener in Abhängigkeit vom Geburtsmodus (SNiP-Studie)

\section{A. Lange', R. Thyrian' ${ }^{2}$, K. Zindel', W. Hoffmann ${ }^{2}$, M. Heckmann ${ }^{7}$ ${ }^{1}$ Klinik und Poliklinik für Kinder und Jugendmedizin, Abt. Neonatologie u. Päd. Intensivmedizin, Greifswald, Deutschland, ${ }^{2}$ Community Medicine, Greifswald, Deutschland}

Hintergrund. Das Ziel der Studie ist es, den Zusammenhang vom Geburtsmodus zum neonatalem Schwangerschaftsoutcome zu beschreiben.

Probanden und Methode. Im Rahmen des Survey of Neonates in Pommerania (SNiP-Studie) untersuchten wir im Zeitraum von $03 / 2003$ bis
11/2008 n=5394 Mütter und ihre Kinder hinsichtlich des Outcomes in Bezug zum Geburtsmodus. Es wurden $n=3989$ Kinder spontan geboren und $n=1387$ per Sectio. 15 vs. 63 Kinder $<32$ SSW, 40 vs. 67 von $32-34$ SSW, 358 vs. 278 von $35-37$ SSW und 3576 vs. $979>37$ SSW wurden spontan vs. Sectio entbunden. Ein pathologischer Ultraschall präpartal führte signifikant häufiger in den höheren Schwangerschaftswochen zur Sectio (<32 SSW: $p<0,126$; 32-34 SSW: $p<0,086$; 35-37 SSW: $<<0,001$; $>37$ SSW: $<<0,001)$. Bei der Häufigkeit angeborener Fehlbildungen, der Altersverteilung der Mütter sowie der Gewichtsklassen der Kinder zeigten sich keine Unterschiede in der Verteilung beider Gruppen. Es gab keinen signifikanten Unterschied bzgl. des Auftretens eines Amnioninfektionssyndroms ( $<32$ SSW: $\mathrm{p}<0,459 ; 32-24$ SSW: $\mathrm{p}<0,484 ; 35-37$ SSW: $\mathrm{p}<0,725)$, in der Durchführung einer Amniocentese ( $<32$ SSW: $p<0,363$; 32-34 SSW: $p<0,432 ; 35-37$ SSW: $p<0,085)$ und mütterlicher Grunderkrankungen in den unteren Schwangerschaftswochen ( $<32$ SSW; p>0,658; 32-34 SSW: $\mathrm{p}<0,501 ; 35-37$ SSW: $\mathrm{p}<0,041 ;>37$ SSW: $\mathrm{p}<0,002)$. Ergebnisse. Die Prävalenz per Sectio entbunden zu werden betrug $25,7 \%$. Als Ursachen einer Sectio fanden sich die pathologische Geburtslage, ein pathologischer Ultraschallbefund bei der Mutter und die Mehrlingsschwangerschaft. Als fetale Risikoparameter konnten die Frühgeburtlichkeit und ein Gewicht des Kindes $>3500$ g aufgezeigt werden. Als negatives Outcome zeigt sich bei den Neugeborenen, die per Sectio entbunden wurden, häufiger ein 5 Minuten APGAR-Wert $<5$ ( $p<0,007)$, sie wurden häufiger primär erstversorgt [spontangeboren 217/3931 $(5,5 \%)$ vs. Sectio $162 / 1252(12 \%)>35$ SSW; p <0,001] sowie stationär aufgenommen [SG 566/3925 (14\%) vs. 317/1255 (25\% ) >35 SSW; p<0,002]. Das Fruchtwasser wurde bei den spontan geborenen Kindern $>37$ SSW häufiger als auffällig beurteilt als bei denen, die per Sectio (558/3467 vs.142/1387) entbunden wurden ( $\mathrm{p}<0,005)$. Der arterielle $\mathrm{NspH}$ war bei den reifgeborenen Kinder die spontan entbunden wurden, signifikant häufiger azidotisch (ph-Wert<7,25); (<32 SSW. p<0,914, 32-34 SSW: $\mathrm{p}<0,797,35-37$ SSW: $\mathrm{p}<0,007,>37$ SSW: $\mathrm{p}<0,001)$.

Schlussfolgerung. Jedes 4 . Neugeborene einer Mutter die per Kaiserschnitt entbunden wird, wurde stationär aufgenommen und erhielt eine erweiterte Neugeborenen Erstversorgung obwohl die Fruchtwasserqualität und der 5-min-Apgar bei den spontangeborenen Kindern schlechter waren. Die Daten unterstützen die hochaktuelle Diskussion über die Sicherheit und Indikationsstellung der Sectio-Entbindung.

\section{FV-46}

Deutschlandweite Umfrage zur Entlassungssituation Frühgeborener: erste Ergebnisse

\section{Peter', B. Boberski', S. Pirr', B. Bohnhorst ${ }^{1}$}

'Kinderklinik der Med. Hochschule, Abteilung Pädiatrische Pneumologie, Neonatologie und Allergologie, Hannover, Deutschland

Hintergrund. Zur Entlassungssituation Frühgeborener (FG) liegen in Deutschland aktuell keine Daten vor.

Methode. Wir entwarfen einen vierseitigen Fragebogen zur Entlassungssituation FG, dessen Schwerpunkte auf der Beurteilung und Therapie kardiorespiratorischer Ereignisse und auf dem Vorgehen bezüglich einer Entlassung mit Heimsauerstoff lagen. Dieser Fragebogen wurde an 293 Kinderkliniken deutschlandweit versendet.

Ergebnisse. 247 der 293 Kinderkliniken (84\%) beantworteten den Fragebogen. 61 Kliniken gaben an, keine FG mit einem Geburtsgewicht (GG) <1500 g zu versorgen, 186 Kliniken versorgten FG mit einem GG von $<1500 \mathrm{~g}$. Von diesen Kliniken gaben 70 Kliniken an, pro Jahr durchschnittlich weniger als 14 FG mit einem GG $<1250$ g zu versorgen (Perinatalzentren Level 2), 116 Kliniken versorgten durchschnittlich pro Jahr mehr als 14 solche FG (Perinatalzentren Level 1). Die Mehrzahl der Kliniken definierte ein kardiorespiratorisches Ereignis als Sauerstoffsättigungsabfall auf $<80 \%$ oder einen Herzfrequenzabfall auf unter $80 /$ Minute. Ein Apnoescore wurde nur von wenigen Kliniken ( 15 Kliniken, davon 11 Level 1, 4 Level 2) verwendet. Coffein wurde von der überwiegenden Anzahl der Kliniken (157 Kliniken, 96 Level 1, 59 Level 2) in einer Loading-Dosis von $10 \mathrm{mg} / \mathrm{kg}$ und einer Erhaltungsdosis von 2,5- 
$5 \mathrm{mg} / \mathrm{kg} /$ tag eingesetzt. Ein Drittel der Kliniken (67 Kliniken, 37 Level 1, 30 Level 2) gab an, Coffeinspiegel zu bestimmen, wobei dies in der Regel nur nach Indikation erfolgte. Nur vier Kliniken gaben an, regelmäßig einmal wöchentlich Coffeinspiegel zu bestimmen. Eine Hochdosiscoffeintherapie führten nur 48 Kliniken durch (37 Level 1, 11 Level 2), Level 1 Kliniken doppelt so häufig wie Level 2 Kliniken (32\% vs. 16\%). Doxapram wurde von gut der Hälfte der Kliniken (98 Kliniken, davon 68 Level 1, 30 Level 2) zumindest dann, wenn Coffein ausgereizt war, eingesetzt. Eine kumulative Doxapramdosis berechneten nur 13 der Kliniken, die Doxapram verordneten (9 Level 1, 4 Level 2). 151 Kliniken entließen FG mit Heimsauerstoff, hiervon 9o Kliniken durchschnittlich ein Kind pro Jahr, 61 Kliniken mehr als ein Kind pro Jahr. Die Verordnung von Heimsauerstoff erfolgte durchschnittlich bei Sauerstoffsättigungswerten unterhalb von $90 \%$, wobei der angegebene Bereich, unterhalb dessen Heimsauerstoff verordnet wurde, zwischen 80 und $94 \%$ variierte. Kliniken, die mehr als $50 \mathrm{FG}<1500 \mathrm{~g}$ GG pro Jahr betreuten, wählten signifikant häufiger höhere Sauerstoffsättigungsgrenzwerte als Kliniken die weniger als $50 \mathrm{FG}<1500 \mathrm{~g}$ pro Jahr betreuten.

Schlussfolgerung. Bezüglich der Definition und Therapie kardiorespiratorischer Ereignisse, für die Leitlinien vorliegen, werden diese Leitlinien ganz überwiegend umgesetzt. Bezüglich der Heimsauerstofftherapie, für die in Deutschland keine Leitlinien vorliegen, bestehen erhebliche Unterschiede im Vorgehen. Für diesen Bereich sollten dringend Leitlinien entwickelt werden, um das Vorgehen zu vereinheitlichen.

\section{FV-47 \\ Auswirkungen von Hygienemaßnahmen auf die Keimbelastung in abgepumpter Muttermilch}

\section{B. Pimpel', C. Binder', R. Durina', A. Berger', N. Haiden'}

'Universitätsklinik für Kinder- und Jugendheilkunde, Abteilung für Neonatologie, pädiatrische Intensivmedizin und Neuropädiatrie, Wien, Österreich

Hintergrund. Muttermilch (MM) ist der „Goldstandard“ in der Ernährung frühgeborener Kinder. Allerdings kann die Verfütterung nativer MM bei mangelnder Pumphygiene eine potentielle Infektionsquelle für Frühgeborene darstellen. Die Literatur liefert keine klare Hilfestellung, wie hygienisches Abpumpen von MM für Frühgeborene zu erfolgen hat. Aufgrund eines ESBL-coli-Sepsisfalles bei Zwillingen an der Medizinischen Universität Wien wurden gemeinsam mit der Krankenhaushygiene zwei standardisierte Hygienemaßnahmenpakete festgelegt und deren Wirksamkeit miteinander verglichen. Ziel der vorliegenden Studie war es, zu evaluieren, unter welchem Paket weniger pathogene Keime in der abgepumpten MM nachweisbar sind.

Fragestellung. Treten unter verstärkten (A) oder vereinfachten (B) Hygienemaßnahmen vermehrt pathogene Keime in der abgepumpten MM auf und welche?

Methode. MM von Müttern frühgeborener Kinder wurde ab dem 7 . Lebenstag des Kindes wöchentlich mikrobiologisch gescreent und anschließend pasteurisiert. Zwei Pakete von Hygienemaßnahmen wurden verglichen. Maßnahme A beinhaltete ein verstärktes Hygienemanagement (Desinfektion der Brust, verstärkte Handhygiene) und Maßnahme B ein stark vereinfachtes Management mit einmaliger Händedesinfektion. Unter beiden Hygieneregimen wurde vermehrt Personal für Laktationsschulungen bereitgestellt, den Müttern Lehrvideos gezeigt und laufend Pumpnachschulungen durchgeführt. Entsprechend den gesetzlichen Vorgaben werden in $\mathrm{MM}$ bis $\mathrm{zu} \max .10^{\wedge} 5 \mathrm{cfu} / \mathrm{ml}$ grampositive Keime toleriert. Bei einer Keimzahl von $>10^{\wedge} 6 \mathrm{cfu} / \mathrm{ml}$ grampositiven Keimen und bei jeglicher Anzahl an gramnegativen Keimen, ist die MM nicht zum unpasteurisierten Verzehr für Frühgeborene geeignet.

Ergebnisse. In die Studie wurden 316 laktierende Mütter frühgeborener Kinder der Neonatologischen Intensivstationen (NICU) der Abteilung für Kinder- und Jugendheilkunde, Medizinische Universität Wien, aufgenommen. Bei Maßnahme A konnten bei 685 MM-Proben von insgesamt 148 Müttern 90 pathogene Proben $(13,10 \%)$ festgestellt werden. Bei Maßnahme B waren bei 788 MM-Proben von insgesamt 168 Müttern
100 Proben $(12,70 \%)$ kontaminiert. Es wurden ausschließlich Kontaminationen mit gramnegativen Keimen nachgewiesen. Pathologisch hohe Konzentrationen mit grampositiven Keimen $>10^{\wedge} 6 \mathrm{cfu} / \mathrm{ml}$ konnten nicht gefunden werden. Die Variationsbreite an Keimen war bei den strengeren Maßnahmen signifikant höher ( 35 Keimarten) als bei den vereinfachten Maßnahmen (25 Keimarten, p<0,05).

Diskussion. Trotz Aufstockung des Personals und Intensivierung der Pumpschulungen ist es uns nicht gelungen, die Anzahl an kontaminierter MM zu senken. Das Keimspektrum ist bei strengeren Maßnahmen signifikant breiter als bei vereinfachten Maßnahmen, was auf einen höheren Selektionsdruck unter dem intensiven Regime schließen lässt. Aufgrund der nach wie vor unzufriedenstellend hohen Keimzahl in abgepumpter MM wird das Pasteurisieren der MM bis auf weiteres beibehalten.

\section{FV-48}

Auswertbarkeit von Schädelsonographien Frühgeborener anhand von Videosequenzen

\section{S. Hübner', F. Hermann', J. Pons-Kühnemann², N. Görner', U. Thome ${ }^{3,}$ G. Alzen ${ }^{1}$}

'Medizinische Fakultät der Justus-Liebig-Universität Gießen, Kinderradiologie, Gießen, Deutschland, '2Institut für Medizinische Informatik JLU Gießen, Arbeitsgruppe Medizinische Statistik, Gießen, Deutschland, ${ }^{3}$ Universitätsklinikum Leipzig, Klinik und Poliklinik für Kinder und Jugendliche der Universität/Leiter der Abteilung für Neonatologie, Leipzig, Deutschland

Hintergrund. Von 2008 bis 2012 wurde die deutschlandweite randomisierte kontrollierte prospektive multizentrische PHELBI-Studie („permissive hypercapnia in extremely low birth weight infants“) durchgeführt, bei der ELBW-Infants (400-999 g, Gestationsalter von der 23. bis 28. vollendeter SSW) unterschiedlich beatmet wurden. Im Rahmen dieser Studie wurden anonymisierte videoaufgezeichnete Schädelsonographien zentral nachbefundet. Dies erfolgte vollständig geblindet ohne Kenntnis jeglicher klinischer Daten.

Fragestellung. Neben der Beurteilung der durch die Sonografien festgestellten Pathologien war Hauptziel dieser Studie die Beurteilung der jeweiligen Gerätequalität und die untersucherabhängige Aufzeichnungsqualität der beteiligten Zentren. Hierzu wurde deren Einfluss auf die Auswertbarkeit der eingesandten Videosequenzen analysiert. Es wurde untersucht, ob ein unabhängiger Beurteiler anhand standardisierter Videoaufzeichnungen qualifizierte Diagnosen mit ausreichender Sicherheit erkennen kann.

Material und Methoden. Die von 16 unterschiedlichen Studienzentren eingesandten Schädelsonographien wurden gemäß des Studienprotokolls auf Blutungen (IVH), periventrikuläre Echogenitätsabweichungen, Hydrocephalus internus und Fehlbildungen von einem erfahrenen Kinderradiologen untersucht. Darüber hinaus wurden auch die Bildqualität und die sie beeinflussenden Parameter beurteilt. Die eingesandten Videosequenzen waren am 1. Lebenstag, am 12.-14. LT und in der 36 . Woche post menstruationem ( $\mathrm{pm}$ ) entsprechenden Lebenswoche erstellt worden.

Ergebnisse. Von insgesamt 1053 eingesandten Ultraschalluntersuchungen waren 903 Untersuchungen auswertbar. Von diesen wurden 336 Untersuchungen am 1. Lebenstag, 300 am 12.-14. Lebenstag und 267 in der 36 . Woche pm erstellt. Es wurden 156 Hirnblutungen (IVH) unterschiedlichen Ausmaßes diagnostiziert von denen 114 einen posthämorrhagischen Hydrocephalus entwickelten. Bei 20 Kindern wurde eine Erweiterung der äußeren Liquorräume diagnostiziert, die als Folge einer hypoxischen Hirnvolumenminderung angesehen werden können. Aufgrund der Untersuchungsintervalle waren Blutungen vereinzelt nur anhand ihrer Folgen erkennbar. 52\% der eingesandten Videoaufzeichnungen waren gut bis sehr gut in ihrer Bildqualität. $2 \%$ waren diagnostisch unbrauchbar. Die Ursachen für nicht optimale, aber auswertbare Bildqualität waren in $61 \%$ gerätebedingt und in $39 \%$ auf mangelnde Sorgfalt des Untersuchers zurückzuführen. 
Schlussfolgerung. Die Durchführung der Studie erforderte einen hohen organisatorischen und logistischen Aufwand. Es konnte die Machbarkeit der Multicenterstudie bestätigt werden. Die gewonnen Erfahrungen können in zukünftige Projekte einfließen. Wesentliche Einschränkungen ergaben sich vorwiegend durch unterschiedliche Gerätequalitäten der beteiligten Zentren und in weniger gravierendem Umfang in der mangelnden Erfahrung der beteiligten Untersucher.

\section{Zelluläre und molekulare \\ Regulationsvorgänge in der Lunge}

\section{FV-49}

Residente mesenchymale Stamm- oder Stromalzellen der unreifen humanen Lunge (hFLMSCs) nehmen nach Sauerstoffexposition einen potenziell schädlichen Phänotyp an

\section{Möbius', S. McConaghy'2, R. Oh/s's, F. Eaton', S. Zhong ${ }^{4}$, S. Koss ${ }^{5}$, M. Rüdiger ${ }^{5}$, B. Thébaud}

'Department of Pediatrics, Women and Children's Health Research Institute, University of Alberta, Edmonton, Kanada und Bereich Neonatologie und Pädiatrische Intensivmedizin, Universitätskinderklinikum und Hochschulmedizin "Carl Gustav Carus", Dresden, Deutschland, ${ }^{2}$ University of New Mexico, Children's Hospital of New Mexico, Department of Pediatrics/Division of Neonatology, Albuquerque, USA, ${ }^{3}$ University of Alberta, Department of Pediatrics, Women \& Children's Health Research Institute, Edmonton Alberta, Kanada, ${ }^{4}$ Ottawa Hospital Research Institute, University of Ottawa, Children's Hospital of Eastern Ontario, Sprott Centre for Stem Cell Research, Ottawa, Ontario, Kanada, ${ }^{5}$ Universitätskinderklinikum und Hochschulmedizin "Carl Gustav Carus", Bereich Neonatologie und Pädiatrische Intensivmedizin, Dresden, Deutschland, ${ }^{6}$ Department of Pediatrics, Women and Children's Health Research Institute, University of Alberta, Edmonton, Kanada und Children's Hospital of Eastern Ontario, Sprott Centre for Stem Cell Research, Ottawa Hospital Research Institute, University of Ottawa, Ottawa, Kanada

Hintergrund. Die fetale Lunge wächst und reift in strenger Hypoxie. Durch eine zu frühe Geburt des Kindes ist das Organ jedoch - selbst unter Raumluft - unphysiologisch hohen Sauerstoffpartialdrücken ausgesetzt. Hieraus entwickelt sich häufig die bronchopulmonale Dysplasie (BPD), eine durch den histomorphologischen Arrest der Lungenentwicklung charakterisierte Erkrankung. Alle derzeitigen Therapien sind supportiv, aber regenerative Therapieansätze mit exogenen MSCs aus Knochenmark und Nabelschnur zeigen in Tierexperimenten eine deutliche Verbesserung der Alveolarisierung. Die Rolle der endogenen, lungenresidenten MSCs in der BPD ist dennoch unklar.
Methoden. hFLMSCs wurden aus humanen Lungengewebe $(n=4$, Gestationsalter $15+6$ bis $17+4$ Wochen) per enzymatischem Verdau und anschließender Dichtegradientenzentrifugation isoliert. Rückschlüsse auf Identität des Feten und Abortgrund waren zu keinem Zeitpunkt möglich. Nabelschnüre von per Kaiserschnitt termingeborenen, gesunden Kindern wurden für die Isolation von humanen Umbilical Cord MSCs (hUCMSCs) herangezogen. Beide Zelltypen wurden charakterisiert und 7 Tage entweder 5\%, 21\% oder $60 \% \mathrm{O}_{2}$ ausgesetzt. Colony Formation Efficiency (CF-E) und Proliferation sowie die Expression und Sekretion von inflammation- und fibroseassoziierten Proteinen wurden verglichen. Alle Experimente wurden von den Ethikkommissionen der University of Alberta, Edmonton, Canada und der University of New Mexico, Albuquerque, USA genehmigt.

Ergebnisse. Normoxie und Hyperoxie reduzieren die hohe CF-E der hFLMSCs signifikant und führen zu massiver Proliferation (Abb. 1). Erhöhter aSMA-Gehalt und vermehrte ACTA2 Transkription weisen auf eine sauerstoffinduzierte Dedifferenzierung der MSCs in Myofibroblasten hin. Die Elastin und Collagen-1-Produktion wird durch Sauerstoff deutlich eingeschränkt; zudem wird vermehrt TGF $\beta 1$ und IL-6 sezerniert. hUCMSCs stellen unter den gleichen hyperoxischen Kulturbedingungen ihre Proliferation ein und sezernieren weniger TGF $\beta_{1}$ als hFLMSCs.

Schlussfolgerungen. Die residenten, spezialisierten MSCs der humanen fetalen Lunge dedifferenzieren unter Sauerstoffeinfluss und entwickeln einen profibrotischen, hoch proliferativen Phenotyp der für die Pathogenese der BPD entscheidend sein könnte. hUCMSCs zeichnen sich im Vergleich dazu durch eine deutlich geringere Sensitivität gegenüber oxidativen Schäden aus. Weitere funktionelle Charakterisierungen beider Zelltypen sind für ein besseres Verständnis der Erkrankung und neuer, regenerativer Therapieansätze erforderlich.

\section{FV-50 \\ Assoziation zwischen frühem Influx CD14+CD16+ Monozyten und der Entwicklung einer schweren BPD bei Frühgeborenen <29 SSW}

\section{H. Ehrhardt', N. Hofmann², J. Buchner' ${ }^{2}$, T. Reicherzer ${ }^{2}$, A. Schulze ${ }^{2,}$ A. Hilgendorff ${ }^{2}$}

'Zentrum für Kinderheilkunde und Jugendmedizin, Abteilung Allgemeine Pädiatrie und Neonatologie, Gießen, Deutschland, ${ }^{2}$ Neonatologie der Kinderklinik am Perinatalzentrum Großhadern, LMU München, München, Deutschland

Hintergrund. Die bronchopulmonale Dysplasie (BPD) wird wesentlich durch die lebensrettenden, aber lungenschädigenden Therapien der mechanischen Beatmung und der Sauerstofftherapie verursacht. Die pulmonale Inflammationsreaktion spielt in der Pathogenese der BPD eine zentrale Rolle und ist charakterisiert durch eine proinflammatorische
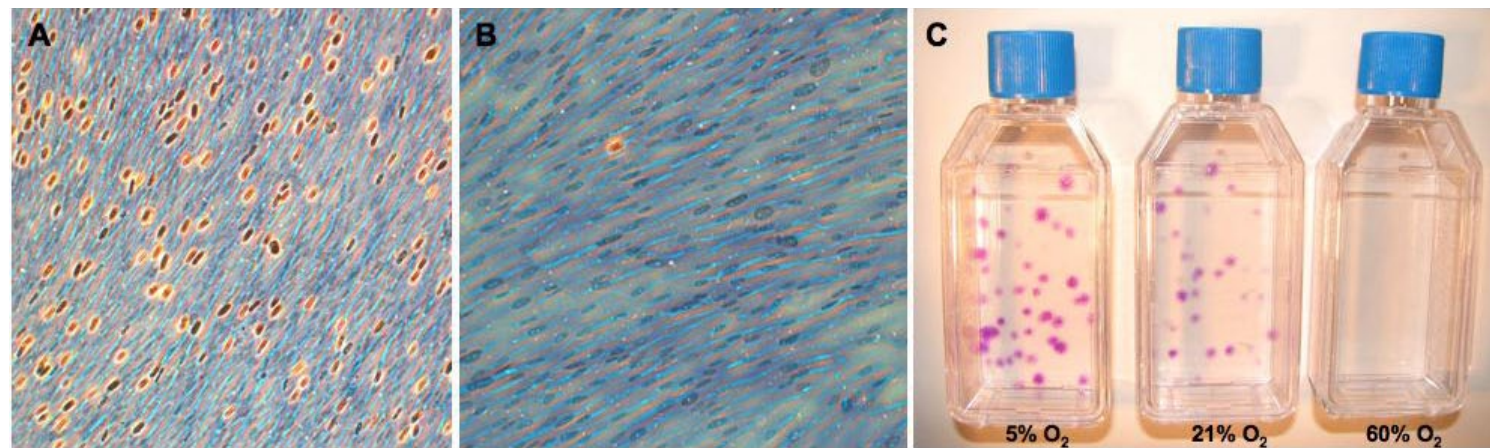

FV-49 Abb.1

Abbildung 1. Proliferation und Colony Forming - Efficiency von MSCs in $60 \% \mathrm{O}_{2}$

Immunozytochemie von BrdU-gepulsten hFLMSCs (A) und hUCMSCs (B), gefärbt mit DAB und CytoBlue nach 7 Tagen in $60 \% \mathrm{O}_{2}$ - $20 \times 10$. Repräsentative Resultate von Colony Forming-Unit Assays für hFLMSCs (C). 50 einzelne Zellen wurden per $25 \mathrm{~cm}^{2}$ Flasche gesät und 9 Tage unter den angegebenen Sauerstoffkonzentrationen inkubiert. Keine Kolonien konnten in der $60 \% \mathrm{O}_{2}$ Gruppe beobachtet werden, ein deutliches Zeichen von Dedifferentiation und Verlust des Stammzellcharakters. Färbung mit Methanol und Kristallviolett. 
Reaktion mit Einstrom von Monozyten und neutrophilen Granulozyten.

Fragestellung. Der spezifische Beitrag der Monozyten zur Pathogenese der BPD ist bisher weder in Tiermodellen noch in Frühgeborenenkohortenstudien ermittelt worden. Lässt sich eine Assoziation zwischen spezifischen Charakteristika der zellulären pulmonalen Inflammationsreaktion und dem Schweregrad der BPD herstellen?

Material und Methoden. Die Zellfraktion aus dem Trachealsekrete von $\mathrm{n}=54$ Frühgeborenen $<29$ Schwangerschaftswochen, die für mindestens 7 Tage invasiv beatmet wurden, wurde in der Multicolordurchflusszytometrie an einem 9-Kanal-FACS-Gerät longitudinal analysiert. Die Kinder wurden in eine Kohorte mit milder BPD $(n=20)$ und eine mit moderater/schwerer BPD $(n=34)$ aufgeteilt.

Ergebnisse. Am 5. Lebenstag bestand kein Unterschied in der Gesamtzahl der Entzündungszellen im Trachealsekret zwischen den beiden Gruppen (200 vs. 220/U sIgA; p>0,05). Der Anteil der Monozyten im Trachealspirat in der Gruppe mit moderater/schwerer BPD war jedoch signifikant erhöht (o,38 vs. o,17; $\mathrm{p}=0,026$ ). Die Auftrennung in die einzelnen Monozytensubpopulationen ergab, dass die CD14+CD16+ Monozyten Fraktion in dieser Gruppe ebenfalls signifikant erhöht war ( 0,684 vs. 0,$544 ; \mathrm{p}=0,01$ ). In einer Subgruppe von $\mathrm{n}=32$ Kindern bestand zusätzlich ein Trend zu einer höheren Expression der Chemokinrezeptoren $\mathrm{CXCR} 1$ (o,o8 vs. 3,11; $\mathrm{p}=0,13$ ) und $\mathrm{CXCR}_{3}$ (o,02 vs. 2,52; $\mathrm{p}=0,09$ ). $\mathrm{Zu}$ späteren Zeitpunkten der Analyse waren die Unterschiede nicht mehr nachweisbar ( $\mathrm{p}>0,05)$.

Diskussion und Schlussfolgerung. Unsere Daten zeigen eine Assoziation zwischen spezifischen Charakteristika der Inflammationsreaktion und der Entwicklung einer schweren BPD. Wir postulieren, dass frühe Veränderungen der pulmonalen Inflammationsreaktion bei Frühgeborenen wesentlichen Einfluss nehmen auf die Entwicklung der BPD. Wir planen, die klinischen Daten durch molekulare Studien an Monozytendepletierten Mäusen im Modell der Langzeitbeatmung und Sauerstofftherapie zu untermauern.

\section{FV-51}

Bronchopulmonary dysplasia (BPD) in a double-hit mouse model induced by intrauterine hypoxia and postnatal hyperoxia: are human cord blood mononuclear cells (UCB-MNCs) a therapeutic option?

\section{E. Tutdibi', D. Monz', C. Mildau', J. Shen', M. Kasoha', M. Laschke 3 , T.} Roolfs ${ }^{4}$, A. Schmiedl ${ }^{4}$, C. Meier ${ }^{5}$, T. Tschernig ${ }^{5}$, L. Gortner

'Unversitätskliniken für Kinder und Jugendmedizin, Klinik für Neonatologie und Allg. Pädiatrie, Homburg, Deutschland, ${ }^{2}$ Third Military Medical University, Daping Hospital, Department of Pediatrics, Chongqing, China ${ }^{3}$ Universität des Saarlandes, Institut für Klinisch-Experimentelle Chirurgie, Homburg, Deutschland, ${ }^{4}$ Institut für Funktionelle und Angewandte Anatomie, Medizinische Hochschule Hannover, Hannover, Deutschland, ${ }^{5}$ Universität des Saarlandes, Institut für Anatomie und Zellbiologie, Homburg, Deutschland

Background. BPD remains a major threat of very preterm neonates especially with intrauterine growth restriction and treatment options still are limited. Stem cell-based regenerative therapy offers an innovative option in the prevention and treatment of BPD but is controversial in neonatology.

Aim. As an adequate experimental model of BPD in small animals for evaluation of novel therapies is still lacking, the goal of the present study was to develop a perinatal double-hit model of BPD in neonatal mice, which is close to clinical features in humans. It was aimed to test whether allogenic transplantation of human UCB-MNCs improves experimental BPD in mice.

Methods. In the experimental setting date-mated pregnant mice (C57BL/6, Charles River, Sulzfeld, Germany) were randomly assigned to a control group (normoxia) or to a double-hit group: prenatal hypoxia $\left(\mathrm{FiO}_{2}\right.$ 0.1) from gestational day E14 until spontaneous delivery and postnatal hyperoxia $\left(\mathrm{FiO}_{2}\right.$ 0.75) from postnatal day $\mathrm{P}_{1}$ to $\mathrm{P}_{14}$. At day $\mathrm{P}_{7}$ double-hit pups were randomly allocated to receive intraperitoneally a single dose of UCB-MNCs with median cell numbers of $2 \times 105$. At day $\mathrm{P}_{14}$ pups were sacrificed and lung tissues were immediately excised for further histological and molecular analyses.

Results. In double-hit exposed neonatal mice the development of body, lung and brain weight was retarded and pups showed a less morphological lung maturity by day $\mathrm{P}_{14}$ compared with normoxic controls. Histomormphometric analysis revealed reduced alveolarization and increased septal thickness without pronounced inflammatory lesions. A down-regulation of transcriptional markers of lung maturation Sftpb, Sftpc and Mtor genes was observed in double-hit animals compared with controls. Transplantation of human UCB-MNCs did not lead to adverse events; mortality was in the same range as for untreated animals. Furthermore there was tendency to increased body weight, lung and brain weight in the treated animals in comparison to the controls. Pulmonary architecture was slightly improved in treated animals and showed increased Mtor gene expression.

Conclusion. We established a new experimental model of BPD mimicking closely the pathogenesis in human preterm neonates using preand postnatal hits as a basis for research on restoration of normal lung growth. Our study results demonstrate that UCB-MNC therapy of BPD in a double-hit rodent model was efficient by reducing the severity of the disorder without adverse effects. It thus might be considered as a translational option, given further studies will improve efficacy without loss in safety.

\section{FV-52}

Fgf-10-Expression schützt vor Hyperoxie-induzierter Lungenschädigung in einem Mausmodell der bronchopulmonalen Dysplasie

\section{Chao', C. Tiozzo', D. Al Alam², K. Zimmer', S. Bellusci ${ }^{3}$}

'Zentrum für Kinderheilkunde der Justus-v.-Liebig-Universität, Neonatologie, Gießen, Deutschland, ${ }^{2}$ Saban Research Institute of Children's Hospital Los Angeles, Los Angeles, USA, ${ }^{3}$ Excellence Cluster Cardio-Pulmonary System (ECCPS), Gießen, Deutschland

Hintergrund. Die bronchopulmonale Dysplasie (BPD) ist eine chronische Lungenerkrankung der Frühgeborenen. Charakteristika der BPD sind Arrest des Lungenwachstums, eine verminderte Alveolarisierung und dysmorphe Blutgefäße. Hauptfaktoren, die zur Entstehung der BPD beitragen, sind $u$. a. Verletzung der unreifen Lunge durch die mechanische Ventilation, Sauerstofftoxizität und Entzündungsprozesse. Die spezifischen molekularen Mechanismen, die zur BPD führen sind bis heute noch nicht vollständig bekannt. Der Fibroblasten-Wachstumsfaktor Fgf-10 spielt eine wesentliche Rolle in der Alveolarisierung. Bei Patienten mit BPD konnte immunhistochemisch eine verminderte Fgf-10-Konzentration nachgewiesen werden. Im Fgf-1o hypomorphen Mausmodell führte die Verminderung der Fgf-10-Expression zur gestörten Alveolarisierung. Weiterhin zeigen neueste Daten im Tiermodell, dass Fgf-10 an der Regeneration des respiratorischen Epithels auch in der adulten Lunge zentral beteiligt ist.

Fragestellung. Spielt Fgf-10 während der Alveolarisierung im transgenen Mausmodell der BPD eine Rolle?

Material und Methoden. Es wurden konditional transgene Mauslinien [SPC-rtTA;tet(o)Fgf-10, Rosa26-rtTA;tet(o)Fgf-10] verwendet, die durch Induktion von oral verabreichtem Doxycyclin Fgf-10 exprimieren. Durch eine Hyperoxie-Behandlung (85\% Sauerstoff) für 8 Tage wurde ein BPD-ähnlicher Phänotyp der Lunge erzeugt. Die Induktion der

\begin{tabular}{|c|c|c|c|}
\hline Parameter & Normoxie WT & Hyperoxie WT & $\begin{array}{l}\text { Hyperoxie } \\
\text { Transgen }\end{array}$ \\
\hline $\mathrm{MLI}(\mu \mathrm{m})$ & 34 & 87 & 75 \\
\hline RAC & 5,0 & 2,4 & 3,5 \\
\hline
\end{tabular}

MLI "mean linear intercept", RAC "radial alveolar count", WT Wildtyp. 
Fgf-10-Expression wurde während der Hyperoxie-Behandlung durchgeführt. Die Auswertung erfolgte mit Hilfe der Histologie und Lungenmorphometrie.

Ergebnisse. Im Vergleich zur Normoxie-Gruppe zeigte die HyperoxieGruppe eine deutliche Rarefizierung der Alveolen ( $\bullet$ Tab. 1). Jedoch konnte durch eine Expression von Fgf-10 während der Hyperoxie-Behandlung die Schädigung der Alveolen signifikant $(\mathrm{p}<0,05)$ vermindert werden. Die Quantifizierung ist in $\bullet$ Tab. 1 dargestellt. Es ist zu erwähnen, dass in der Gruppe der Fgf-1o Expression eine Hyperzellularität der Septen und eine erhöhte Erythrozytenanzahl intraalveolar zu verzeichnen waren. Dies ist eventuell auf eine gestörte Vaskularisierung zurückzuführen.

Schlussfolgerung. Fgf-10 zeigte einen schützenden Effekt in Bezug auf die Hyperoxie-induzierte Schädigung der Lunge im Mausmodell der BPD. Allerdings sollten potentielle Nebenwirkungen auf die Gefäßentwicklung berücksichtigt werden. Hierfür werden noch differenzierte Untersuchungen durchgeführt.

\section{FV-53 \\ Antenatal glucocorticoids counteract LPS-induced changes in TGF- $\beta$ signalling pathway in fetal lung}

S. Kunzmann', J. Collins², E. Kuypers², J. Newnham², S. Kallapur², A. Jobe², C. Speer', B. Kramer ${ }^{2}$

${ }^{1}$ Universitäts-Kinderklinik, Neonatologie, Würzburg, Deutschland, ${ }^{2}$ Maastricht University- Faculty of Health, Medicine and Life Science, Pediatrics Neonatology, Maastricht, Niederlande

Background. Inflammation and antenatal glucocorticoids affect lung development and may contribute to the development of bronchopulmonary dysplasia (BPD). Transforming growth factor- $\beta$ (TGF- $\beta$ ), its activated Smad signalling pathway and its downstream mediators, like connective tissue growth factor (CTGF) and Caveolin-1 (Cav-1), are implicated in the etiology of BPD. The effects of the combined exposures on inflammation and antenatal glucocorticoids on these members of TGF- $\beta$ family are unknown. Therefore we asked whether glucocorticoids altered intra-amniotic lipopolysaccharide (LPS) effects on TGF- $\beta$ expression, its signaling molecule pSmad2, and CTGF and Cav-1 expression.

Methods. Ovine singleton fetuses were randomized to receive either an intra-amniotic injection of LPS and/or maternal betamethasone (BTM) intra-muscularly 7 and/or 14 days prior to delivery at 120 days GA (term $=150$ days GA). Saline was used for controls. Protein levels of TGF- $\beta 1$ and 2 were measured by ELISA. Smad 2 phosphorylation was assessed by immuno-histochemistry. CTGF and Cav-1 mRNA and protein levels were determined by RT-PCR and Western blot.

Results. Free TGF- $\beta_{1}$ and -2 and total TGF- $\beta_{1}$ levels were unchanged after LPS and/or BTM exposure, although total TGF- $\beta_{2}$ increased in animals exposed to BTM 7 days before LPS. Smad 2 phosphorylation increased 7 days after LPS exposure. Similarly, CTGF mRNA and protein levels increased 7 days after LPS exposure as Cav-1 mRNA and protein levels decreased. BTM exposure prior to LPS prevented Smad2 phosphorylation, CTGF induction and Cav-1 down-regulation.

Conclusion. This study demonstrated that the intra-uterine inflammation induced TGF- $\beta$ signaling can be inhibited by antenatal glucocorticoids in fetal lungs, which may influence the development of BPD.

\section{FV-54}

Abca3 haploinsufficiency results in an altered BAL phospholipid ratio predisposing to hyperoxic lung injury in a murine model

R. Mittal', S. Herber-Jonat ${ }^{2}$, G. Liebisch', M. Hammel', A. Bischof', G. Schmitz ${ }^{6}$, A. Holzinger ${ }^{7}$, A. Flemmer ${ }^{8}$

${ }^{1}$ Klinikum der Universität München; Dr. von Haunersches Kinderspital, Neonatologie, München, Deutschland, ${ }^{2}$ Klinikum Großhadern, Neonatologische Abteilung, München, Deutschland, ${ }^{3} U$ niversitätsklinikum Regensburg, Regensburg, Deutschland, ${ }^{4}$ Klinikum der Universität München, München, Deutschland, ${ }^{5}$ Klinikum der Universität München, Neonatologie, München, Deutschland, ${ }^{6}$ Klinikum Universität Regensburg, Institut für Klinische Chemie und Laboratoriumsmedizin, Regensburg, Deutschland, ${ }^{7}$ Kinderklinik im Dr. von Haunerschen Kinderspital, LMU-München, Neonatologie, München, Deutschland, ${ }^{8}$ LMU-München, Neonatologie der Kinderklinik am Perinatalzentrum Großhadern, München, Deutschland

Background. Biallelic $\mathrm{ABCA}_{3}$ deficiency leads to fatal lung failure at birth or chronic interstitial lung disease. Heterozygous $\mathrm{ABCA}_{3}$ mutations, estimated to be present in $2-4 \%$ of general population, have been found to be associated with respiratory distress syndrome (RDS) in neonates, who are often treated with oxygen. In addition, preterm neonates with heterozygous $\mathrm{ABCA}_{3}$ mutations and RDS are observed to have an increased incidence of pneumothorax and chronic lung disease. We investigated in an adult murine model whether Abcaz haploinsufficiency might be a predisposing factor for lung injury induced by hyperoxia. Methods. Abca3 haploinsufficient (Abca3+/-) and wild-type (WT) mice were prospectively randomized to 72 hours of hyperoxia or left unchallenged in air.

Results. As compared to WT, unchallenged Abca3+/- mice had significantly decreased lung phosphatidylcholine (PC) and phosphatidylglycerol (PG) levels $(\mathrm{p}<0.02)$, and decreased lung compliance $(\mathrm{p}<0.05)$. The ratio of PC to PG in bronchoalveolar lavage fluid (BALF) - relevant for surfactant dysfunction - was significantly elevated in the Abca3+/- mice in room air $(\mathrm{p}=0.03)$. Hyperoxia for 72 hours resulted in increased total lung capacity (TLC; $\mathrm{p}=0.01$ ), increased compliance $(\mathrm{p}<0.05)$ and emphysematous changes only in the lungs of $\mathrm{Abca3}^{+/-}$mice. The ratio of BALF PC to PG was significantly elevated by oxygen exposure in both $\mathrm{WT}$ and $\mathrm{Abca} 3+/-$ mice with the greatest increase in Abca3+/- mice. Conclusions. In a murine model, Abcaz haploinsufficiency results in an altered biochemical and lung mechanical phenotype as well as a greater lung injury induced by hyperoxia. The inability to maintain a normal PC to PG ratio appears to play a key role.

\section{FV-55}

Physiologische Interaktion von Coffein und Glucocorticoiden bei der Expression von Surfactant Protein $B$ in vitro und in vivo

\section{Fehrholz', B. Kramer', C. Speer', S. Kunzmann}

${ }^{1}$ Universitäts-Kinderklinik, Neonatologie, Würzburg, Deutschland, ${ }^{2}$ Maastricht University Medical Center, Department of Pediatrics, Maastricht, Niederlande

Hintergrund. Die Gabe von Glucocorticoiden und Coffein dient als wichtige therapeutische Intervention innerhalb der Neonatologie. Kürzlich haben wir eine synergistische Induktion von Surfactant Protein B (SP-B) durch eine Kombination dieser beiden Substanzen beschrieben (Fehrholz et al., PLOS One, 2012). Hierbei wurde Coffein in hohen, nichtphysiologischen Dosen eingesetzt. Das Ziel dieser Studie war es, mögliche Effekte von Coffein auf die Glucocorticoid-vermittelte Expression von SP-B auch in physiologischen Dosen in vitro zu untersuchen und zu ermitteln, ob ein vergleichbarer Effekt auch in vivo besteht. Methoden. Hierzu wurden SP-B mRNA und reifes SP-B mittels Realtime-PCR und Western Blot in $\mathrm{H}_{441}$ Zellen, welche zuvor mit $250 \mu \mathrm{M}$ Coffein und/oder $1 \mu \mathrm{M}$ Dexamethason (DEX) behandelt wurden, bestimmt. In vivo wurde SP-B in bronchoalveolärer Lavage (BAL) aus mit 
$20 \mathrm{mg} / \mathrm{kg}$ Coffein und/oder $0,5 \mathrm{mg} / \mathrm{kg}$ DEX behandelten, frühgeborenen Schafen untersucht. Zusätzlich wurden mRNA-Level der Proteasen Pepsinogen C (PGC), Cathepsin H und Napsin A bestimmt, welche an der Reifung von SP-B beteiligt sind.

Ergebnisse. Die Anwesenheit von Coffein bei DEX-vorbehandelten Zellen führte zu einer signifikanten Induktion der SP-B mRNA $(p=0,496)$ und der SP-B-Menge über einen Zeitraum von 6 Tagen. Schon nach einer 48-stündigen Vorbehandlung mit DEX wurden durch zusätzliches Coffein signifikant höhere SP-B mRNA und SP-B Level im Vergleich mit nur DEX-behandelten Zellen erreicht. Höhere Mengen SP-B wurden auch in BAL aus Coffein-behandelten, frühgeborenen Schafen nach maternaler Vorbehandlung mit DEX gemessen. Eine zusätzliche Behandlung von $\mathrm{H}_{441}$ Zellen mit Coffein bewirkte außerdem eine Erhöhung der Expression von PGC, welche wiederum zu einem Anstieg von reifem SP-B führen könnte.

Schlussfolgerung. In dieser Studie konnten wir zeigen, dass eine kontinuierliche Anwesenheit von physiologischen Mengen an Coffein in vitro als auch in vivo zu einer Verstärkung der Glucocorticoid-vermittelten SP-B Expression führt, welche durch eine Erhöhung der SP-B mRNA-Menge und eine verstärkte Reifung von pro-SP-B durch PGC vermittelt sein könnte.

\section{Beatmung - Lunge}

\section{FV-56}

Welche Vorhersagekraft für den Bedarf an Atemwegsinterventionen hat Surfactant aus oronasalen Absaugsekreten der neonatalen Erstversorgung?

\section{G. Stichtenoth', R. Lange', G. Walter', E. Herting ${ }^{1}$ \\ 'Universitätsklinikum Schleswig Holstein, Campus Lübeck, Klinik für Kin- der- und Jugendmedizin, Lübeck, Deutschland}

Hintergrund. Die Prädiktion der Lungenreife, insbesondere von kleinen, unreifen Frühgeborenen war bereits in der Frühphase der Surfactantforschung von Interesse. Hierzu wurden verschiedene Tests, wie das Lecithin/Sphingomyelin-Verhältnis im Fruchtwasser bestimmt. Jedoch wurden solche Tests nicht langfristig etabliert. Der Captive Bubble Surfactometer (CBS) ermöglicht die Messung der Oberflächenaktivität aus kleinsten Probenmengen.

Fragestellung. Hat die Oberflächenaktivität von Surfactant präpariert aus oronasalen Absaugsekret von Früh- und Neugeborenen einen prädiktiven Wert für den postnatalen klinischen Verlauf?

Methoden. Nach einem positiven ethischen Votum des klinischen Ethik-Komittees der Universität zu Lübeck wurden von 159 Neonaten, die aufgrund einer postnatalen Depression aufgenommen wurden und deren obere Luftwege abgesaugt wurden, oro-nasale Absaugsekrete von $>300 \mu \mathrm{L}$ gewonnen. Diese wurden ultrazentrifugiert und das Pellet relativ zum Original-Sekretvolumen in $\mathrm{NaCl} 0,9 \%$ resuspendiert. Aus 1-5 $\mu \mathrm{L}$ Probevolumen wurde die Oberflächenaktivität im CBS bestimmt. Die gewonnenen biophysikalischen Daten wurden mit den perinatalen Daten (Gestationsalter, Gewicht, Geschlecht), dem Bedarf an Atemwegsinterventionen und Surfactanttherapie verglichen und statistisch analysiert.

Ergebnisse. Die Oberflächenaktivität der präparierten Sekrete korreliert mit dem Gestationsalter, Geburtsgewicht und dem Beatmungsbedarf. Eine ROC-Analyse zeigte eine Sensitivität von o,77 und eine Spezifität von 0,72 für den Bedarf an Atemwegsinterventionen bei einem „Cutoff" von $44 \mathrm{mN} / \mathrm{m}$.

Diskussion. Aus kleinsten Mengen oronasaler Absaugsekrete kann oberflächenaktives Surfactant so präpariert werden, dass die Unterschiede in der Oberflächenaktivität mit den klinischen Beobachtungsdaten korrelieren. Derzeit unklar sind die Konzentration und die $\mathrm{Zu}$ sammensetzung des darin befindlichen Surfactants.
Schlussfolgerung. Weitere Untersuchungen sind nötig, um den Nutzen dieser Methode zu überprüfen.

\section{FV-57}

Changes of supportive respiratory care in extremely low birth weight infants: a representative survey in German-speaking countries 1997 and 2011

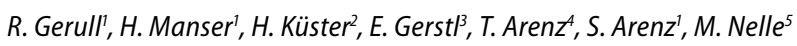
'Inselspital Bern, Neonatologie, Bern, Schweiz, ${ }^{2}$ Universitäts- Kinderklinik, Neonatologie, Göttingen, Deutschland, ${ }^{3}$ Kinderklinik Dritter Orden Passau, Pädiatrie, Passau, Deutschland, ${ }^{5}$ Medizinische Kinderklinik/Inselspital, Abteilungsleiter Neonatologie, Bern, Schweiz, ${ }^{4}$ Inselspital Bern, Abteilung für Neonatologie, Bern, Schweiz

Introduction. In the last 20 years an increasing number of studies have been published which substantially changed the existing evidence in numerous areas of neonatal care. However, little is known about changes of treatment strategies. Therefore, we conducted a survey in German speaking countries in order to assess to which extent the treatment intentions of extremely low birth weight (ELBW) infants have changed between 1997 and 2011.

Methods. A questionnaire regarding the routine treatment of newborns between $750 \mathrm{~g}$ and $1000 \mathrm{~g}$ was developed in 1996. This questionnaire was sent to all German neonatal intensive care units (NICUs) assumed to care for VLBW infants in 1997. Based on the first questionnaire, a web based follow-up survey was sent to the head of neonatal departments of all Swiss $(n=9)$, German $(n=171)$ and Austrian $(n=15)$ neonatal units caring for extremely low birth weight ELBW infants in 2011. Intensive care units were categorized into university and non university hospitals. Small, medium and large NICUs were arbitrarily defined as units with annual admissions of $=12,13-30$ and $>30$ ELBW infants, respectively. Results. In 1997, a completed questionnaire was available for analysis from 126 NICUs. In 2011, 195 neonatal departments were approached and 129 answered (66.2\%). Comparison between countries indicated that most therapies are applied at similar frequencies. Similarly, treatment strategies did not differ between University hospitals and non-university hospitals, as well as between small, medium and large volume NICUs. Mechanically ventilated ELBW infants were treated less frequently with sedatives in 2011 compared to 1997 ( $p<0.0001$ ); the frequency of analgesia did not change. Routine administration of muscle relaxants was low in both surveys but even lower in $2011(\mathrm{p}=0.008)$. The therapy of apnea of prematurity showed an increased use of caffeine $(\mathrm{p}<0.0001)$ and decreased administration of theophylline and doxapram (both $\mathrm{p}<0.0001$ ). Treatment of BPD with corticosteroids was lower in 2011 ( $\mathrm{p}<0.0001)$. Furthermore, the drug of choice changed significantly with a decreased use of dexamethasone $(\mathrm{p}<0.0001)$ and increased use of hydrocortisone $(\mathrm{p}<0.0001)$ in 2011 compared to 1997. Inhalation and mucolytics were reduced in 2011 compared to 1997 ( $\mathrm{p}=0.0002$ and $\mathrm{p}<0.0001$, respectively). There was a trend towards increased use of diuretics $(p=0.056)$ in 2011. Conclusion. Treatment strategies in ELBW infants, regarding administration of muscle relaxants, caffeine, theophylline, doxypram, corticosteroids, inhalations and mucolytics changed significantly between 1997 and 2011 in Germany. No substantial differences exist between German, Austrian and Swiss neonatal centers as well as between centers of different size or level of care in 2011. 


\section{FV-58}

Duration of mechanical ventilation and neurodevelopmental outcome in extremely preterm infants - preliminary data from the Swiss Neonatal Network

\section{Schlapbach', G. Natalucci' ${ }^{2}$, E. Proietti ${ }^{3}$, M. Adams 4 , S. Grunt ${ }^{5}$, C. Borrado- ri $^{6}$, B. Latal ${ }^{7}$, M. Bickle ${ }^{8}$, H. Bucher ${ }^{4}$ \\ ${ }^{1}$ Medizinische Universitäts-Kinderklinik, Abteilung für Neonatologie, Bern, Schweiz, ${ }^{2}$ Kinderspital Zürich, Entwicklungspädiatrie, Zürich, Schweiz, ${ }^{3}$ Medizinische Universitäts-Kinderklinik, Pneumologie, Bern, Schweiz, ${ }^{4}$ Universitätsspital, Klinik für Neonatologie, Zürich, Schweiz, ${ }^{5}$ Medizinische Universitäts-Kinderklinik, Neuropädiatrie, Bern, Schweiz, ${ }^{6}$ Department of Pediatrics, University Hospial Geneva, Geneva, Schweiz, ${ }^{7}$ Universitäts- Kinderklinike, Entwicklungspädiatrie, Zürich, Schweiz, ${ }^{8}$ Centre Hospitalier Universitaire Vaudois, Division de Néonatologie, Lausanne, Schweiz}

Background and aims. Invasive ventilation (IV) of extremely premature infants harbours potentially harmful effects, such as decreased cerebral blood flow, ventilator-induced lung injury, and increased risk of nosocomial infections. We assessed the impact of the duration of IV on outcome at two years of age in a recent national cohort of extremely premature infants.

Methods. This study is based on the national registry of preterm infants born in Switzerland between 240/7 and 276/7 weeks during 2000-2008 Neurodevelopmental disability (ND) was assessed using Bayley Scales of Infant Development-II and the Gross Motor Function Classification System at 18 to 24 months corrected for prematurity. Adverse outcome (death or severe ND), and unfavourable outcome (death or any ND) were analysed using multivariable logistic regression with adjustment for perinatal factors known to affect outcome.

Results. Of 856 infants surviving to 36 weeks postmenstrual age, followup information was available in $81 \%$. $74 \%$ of infants required IV (median duration $4 \mathrm{~d}$ ), and $21 \%$ developed bronchopulmonary dysplasia (BPD). 440 (63\%) showed normal development, 246 (37\%) unfavourable outcome, and $90(13 \%)$ adverse outcome. IV duration was one of the strongest predictors of BPD $(\mathrm{p}<0.001)$. The duration of IV was significantly associated with adverse outcome (per day of IV: multivariate OR 1.03; 95\% CI 1.01-1.05, $\mathrm{p}=0.005$ ) and with unfavourable outcome (OR 1.02, 95\% CI 1.00-1.04, $\mathrm{p}=0.03$ ).

Conclusions. Prolonged duration of IV increased the risk for poor outcome of extremely premature infants independently of other neonatal morbidities. Whether IV duration is primarily a marker of disease severity, or whether it may directly contribute to poor outcome merits further investigation.

\section{FV-59}

\section{Wie verlässlich ist die Lungenschwimmprobe bei Neonaten?}

\section{A. Müller', A. Große Ostendorf', M. Rothschild², S. Banaschak ${ }^{2}$ \\ 'Universitätsklinik Bonn, Abteilung für Paidopathologie, Bonn, Deutsch- land, ${ }^{2}$ Klinikum der Universität zu Köln, Rechtsmedizin, Köln, Deutschland}

Einleitung. In Deutschland ist - im Gegensatz zu anderen Ländern - die Durchführung der Lungenschwimmprobe zur juristisch belastbaren Beantwortung der Frage, ob ein Neugeborenes tot oder lebend geboren wurde, verbindlich. Zur Beantwortung der Frage, ob dieser Test bei dem heute gegenüber früher veränderten Geburtenkollektiv noch korrekte Aussagen liefert, wurden Lungen von Neugeborenen systematisch der Lungenschwimmprobe unterzogen.

Material und Methode. Lungen von 209 induzierten Aborten und Totgeburten $(n=195)$ sowie Neonaten $(n=4)$ und Säuglingen $(n=10)$, die zwischen 2 Tagen und 1o Monaten post partum verstorben waren, wurden mit der Lungenschwimmprobe untersucht.

Ergebnisse. Der Test war negativ für die Lungen aller Totgeborenen. Jedoch fiel der Test bei den 14 Lebendgeborenen in 10 Fällen positive aus. Diskussion. Die Lungenschwimmprobe war in all den Fällen korrekt negativ, in denen die Kinder definitive nicht geatmet hatten bzw. tot geboren wurden. Sie war falsch negativ in 4 von 14 Fällen, in denen Kinder definitive geatmet hatten. Daher beträgt die Sicherheit der Lungenschwimmprobe bei Kindern, die (vielleicht) geatmet haben, nur $71 \%$.

\section{Intensiv- und Notfallmedizin}

\section{FV-60}

Einfluss von Maskentyp und Maskenpostition auf die Effektivität der Beutel-Maskenbeatmung an einer Neugeborenen-Reanimationspuppe

\section{P. Deindl', G. Schmölzer', M. O'Reilly², A. Berger', A. Pollak', J. Schwindt'}

'Universitätskinderklinik AKH Wien, Klinik für Neonatologie, Wien, Deutschland, 'Department of Neonatology, Royal Alexandra Hospital, Edmonton, Canada, Edmonton, Canada

Einleitung. Anatomisch geformte Luftpolster-Beatmungsmasken können falsch auf dem Gesicht eines Neugeborenen aufgesetzt werden. Das Luftpolster der Maske kann mit unterschiedlichen Mengen an Luft befüllt werden. Sowohl falsche Orientierung als auch unterschiedliche Füllung des Luftpolsters können ein luftdichtes Abschließen der Maske verhindern und damit eine effektive Beatmung beeinträchtigen.

Ziel. Messung der Effektivität der Beutel-Masken-Beatmung in Abhängigkeit vom verwendeten Maskentyp sowie von der Maskenpositionierung.

Material und Methoden. 20 MitarbeiterInnen einer Neugeborenen-Intensivstation beatmeten eine modifizierte leckfreie Reanimationspuppe. Während der Beatmung mit einem Beutel und einer anatomisch geformten Intersurgical Neugeborenen Luftpolster-Maske (IS) und einer Laerdal Neugeborenen-Rundmaske (Größe o/1) wurden jeweils Beatmungsparameter (in- und expiratorisches Tidalvolumen, Druck und Fluss) aufgezeichnet. Drei verschiedene Maskenpositionierungen wurden mit der IS getestet: Korrekte Position, 90 und 180 Grad rotiert, bezogen auf die Mittellinie des Gesichtes. Die IS-Masken in korrekter Position, jedoch mit unterschiedlicher Füllung des Luftpolsters: Leer, $10 \mathrm{~mL}, 20 \mathrm{~mL}$, $30 \mathrm{~mL}$ Füllung (• Abb. 1, • Abb. 2, $\bullet$ Abb. $\mathbf{3}$ )

Ergebnisse. Das Maskenleck war bei Fehlpositionierung in 90 bzw. 180 Grad Rotation ähnlich. Ein leeres Luftpolster führte jedoch zu einer signifikanten Zunahme des Maskenlecks auf 50 (22)\% im Vergleich zu einem adäquat gefüllten Luftpolster [28 (16\%)].

Schlussfolgerung. Anatomisch geformte Luftpolstermasken weisen ähnliche Maskenlecks wie runde Beatmungsmasken auf. Das Maskenleck steigt durch einen leeren Luftpolsterrand erheblich an und kann daher möglicherweise zu einer ineffektiven Beutel-Maskenbeatmung führen. Eine falsche Positionierung einer anatomisch geformten Luftpostermaske beeinflusst das Maskenleck nicht.

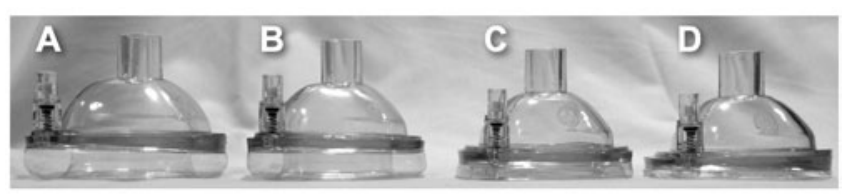

FV-60 Abb. $1 \Delta$ Anatomisch geformte Neugeborenen-Beatmungsmaske mit Luftpolsterrand und Ventil (Intersurgical, Wokingham, UK). Rand gefüllt mit $30 \mathrm{~mL}$ (A), $20 \mathrm{~mL}$ (B), $10 \mathrm{~mL}$ (C) Luft und leer (D) 


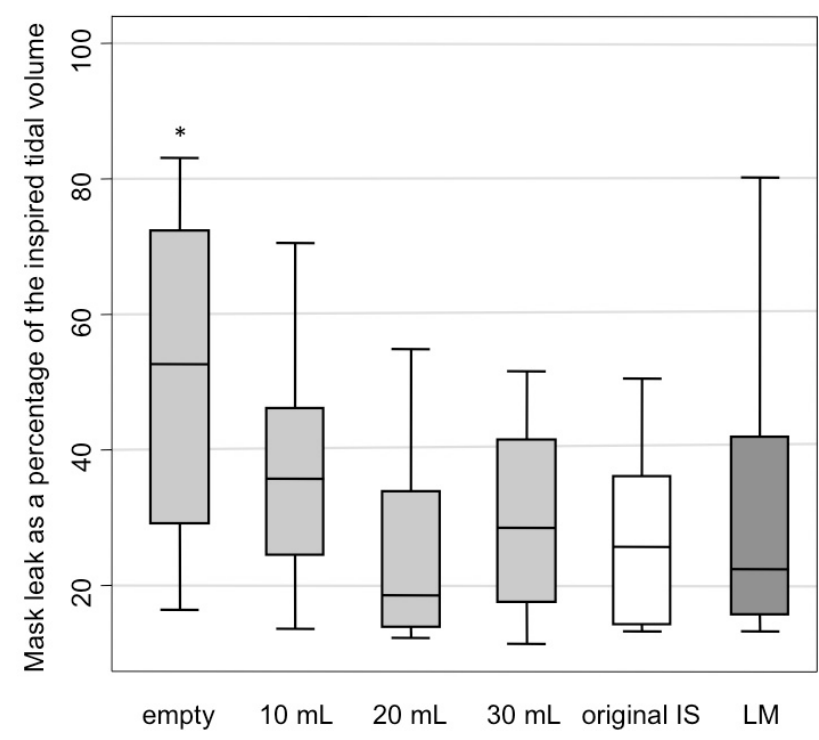

FV-60 Abb. 2ム Masken-Leck bei unterschiedlich gefülltem Luftpolsterrand einer anatomisch geformten Intersurgical Neugeborenen Beatmungsmaske (IS) in korrekter Position verglichen mit einer original Intersurgical Neugeborenen Beatmungsmaske und einer Laerdal Rundmaske (LM)

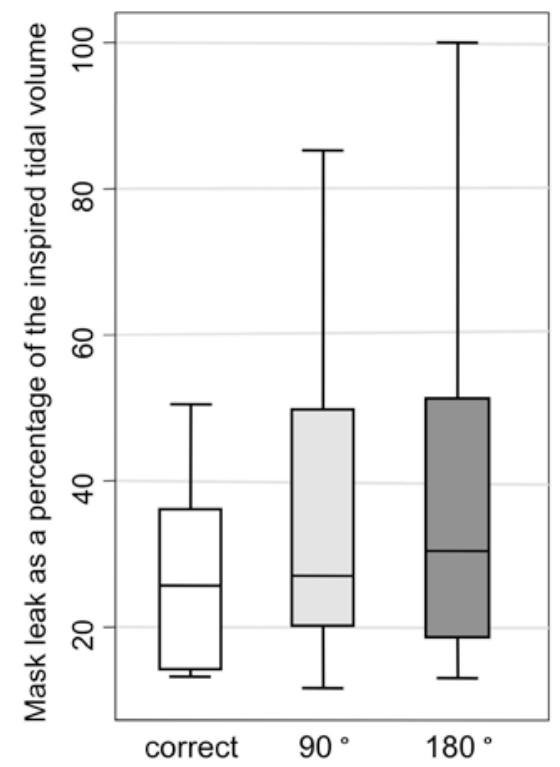

FV-60 Abb.3 $\Delta$ Einfluss unterschiedlicher Maskenpositionen (bezogen auf die Mittellinie des Gesichtes) auf das Masken-Leck bei Verwendung einer anatomisch geformten Intersurgical Neugeborenen-Beatmungsmaske

\section{FV-61}

\section{Anwendung permissiver Hyperkapnie bei immunsupprimierten Kindern mit schwerem Lungenversagen}

\section{H. Fuchs', N. Rossmann', W. Lindner', H. Hummler', M. Schmid'}

${ }^{1}$ Klinik für Kinder und Jugendmedizin, Universitätsklinikum Ulm, Neonatologie und päd. Intensivmedizin, Ulm, Deutschland

Hintergrund. Lungenschonende Beatmung bei schwerem Lungenversagen beinhaltet die Verwendung niedrigen Tidalvolumens, hoher Beatmungsfrequenz und der Begrenzung des inspiratorischen Spitzen- drucks. Es ist unklar, ob eine daraus resultierende Hyperkapnie in Kauf genommen werden darf.

Fragestellung. Ziel dieser Studie war es, Outcome und klinischen Verlauf von immunsupprimierten Kindern mit Lungenversagen zu charakterisieren, bei denen mit Ziel der lungenschonenden Beatmung auf der Intensivstation der Universitätskinderklinik in Ulm eine moderate bis extreme Hyperkapnie akzeptiert wurde.

Methoden. In dieser retrospektiven Kohortenstudie wurden Outcome und klinischer Verlauf von Kindern mit primärem Immundefekt, onkologischer Erkrankung oder Stammzelltransplantation und $\geq 5$ Tage beatmungsbedürftigem Atemversagen analysiert, bei denen ein $\mathrm{PCO}_{2}$ Zielbereich über $60 \mathrm{mmHg}$ bis max. $140 \mathrm{mmHg}$ sukzessive akzeptiert wurde, um das Tidalvolumen zu limitieren. Die Mortalität und Beatmungsdauer aus der Zeit vor und nach Einführung der Beatmungsstrategie mit permissiver Hyperkapnie (1.1996-10.2003 vs. 11.2003-12.2010) wurden verglichen. Median (1.; 3. Quartile) sind angegeben

Ergebnisse. Seit 11.2003 erfüllten 32 Kinder [Alter $3,8(0,6 ; 7,3)$ Jahre] die Einschlusskriterien und wurden unter Inkaufnahme permissiver Hyperkapnie für $20(14 ; 36)$ Tage beatmet. Die maximal erreichten $\mathrm{PCO}_{2}$-Werte erreichten $112(90 ; 134) \mathrm{mmHg}$. Aufgrund einer raschen metabolischen Kompensation kam es in der Regel nicht zur Azidose. Die Überlebensrate der hyperkapnisch beatmeten Kohorte betrug 12/32 (37,5\%). Auch langandauernde extreme Hyperkapnie konnte ohne neurologische Einschränkungen überlebt werden. Die Überlebensrate der Kohorte $\geq 5$ Tage beatmeter Kindern vor Einführung der permissiven Hyperkapnie als Beatmungsstrategie betrug 8/25 (31\%) vs. 18/41 (44\%) nach Einführen dieser Strategie (nicht signifikanter Unterschied). Diskussion und Schlussfolgerung. Lungenschonende Beatmung unter Inkaufnahme moderater bis extremer Hyperkapnie ist durchführbar und war in unserem Kollektiv mit einer nicht signifikanten Reduktion der Mortalität assoziiert. Größere Studien sind notwendig, um den Einfluss dieser Beatmungsstrategie auf den Patientenoutcome zu klären.

\section{FV-62} Regionale Zitrat-Antikoagulation mit der Prismaflex und dem
Prismaflex ${ }^{\circledast}$ HF20-Set

\section{S. Rödl', G. Wendelin', I. Marschitz' ', S. Ring', C. Mache ${ }^{3}$, G. Zobel'}

'Universitätsklinik für Kinder und Jugendheilkunde, Pädiatrische Intensivstation, Graz, Österreich, ${ }^{2}$ Universitätsklinik für Kinder- und Jugendheilkunde, Neonatologie, Salzburg, Österreich, ${ }^{3} U$ niversitätsklinik für Kinder und Jugendheilkunde, Medizinische Universität Graz, Nephrologie, Graz, Österreich

Hintergrund. Die regionale Zitrat-Antikoagulation (RCA) wurde bereits vor über 10 Jahren bei extrakorporalen Therapien bei Erwachsenen erfolgreich eingesetzt. Die Anpassung der Flussverhältnisse an pädiatrische PatientInnen wurde in Einzelfällen mit Hilfe von externen Infusionspumpen und engmaschigen Laborkontrollen erfolgreich durchgeführt. Die Prismaflex ${ }^{\oplus}$-Maschine (PF) unterstützt bei allen Filtersets $\mathrm{ab}$ der Softwareversion 5.0 die Option einer automatische Zitrat- und Kalziumdosierung.

Zielsetzung. Vorstellung einer optimalen Kombination an Infusionslösungen für die RCA in HDF Filtern mit kleiner Oberfläche (Prismaflex ${ }^{\ominus}-\mathrm{HF}_{20} \mathrm{O}$-Set) für pädiatrische Patienten.

Patienten und Methoden. Von 9/2010 bis 1/2013 wurden an unserer pädiatrischen Intensivstation für Patienten bis 30 kg Körpergewicht $(\mathrm{n}=12)$ die Nierenersatztherapien bei akutem und chronischem Nierenversagen (NV) mit der PF (SW 5.0, 6.10) und dem HF-20-Set durchgeführt. Die RCA erfolgte mit Zitratlösungen unterschiedlicher Konzentrationen. Als Zitratlösungen wurde Prismocitrat 18/o, Zitrat Fresenius 4\% und eine Natrium isotone Lösung mit $47,5 \mathrm{mmol} / \mathrm{l}$ Zitrat verwendet. Als kalziumfreie Dialysatlösungen wurden verwendet: PrismoCal, CICA $\mathrm{K}_{2}$, CICA K4 (Fresenius) und PrismoCalB22 (Gambro). Als Postdilutionssubstitution wurde in allen Fällen Phoxilium (Gambro) verwendet. Die Kalziumsubstitution wurde mit 0.2 molarer $\mathrm{CaCl}_{2}$-Lösung 
durchgeführt. Die Steuerung der Flussgeschwindigkeit sowohl der Zitrat- als auch der Kalziumlösung erfolgte automatisiert durch die PF in Abhängigkeit von der eingestellten Zitratdosis pro Liter Blut (mmol/l) und dem vorgewählten Kalzium Ersatz in Prozent vom berechneten Verlust.

Ergebnisse. Insgesamt wurden 7 Patienten mit akutem NV 1200 h kontinuierlich und 5 Patienten mit chronischem NV über insgesamt 800 mal ambulant behandelt. Die Kombination von Prismocitrat 18/o als Zitratlösung und PrismoCal als Dialysatlösung hatte, bedingt durch die niedrige Zitratkonzentration, einen für den HF-2o-Filter zu hohen transmembranösen Fluss, und musste nach wenigen Stunden beendet werden. Die Kombination von Citrat Fresenius $4 \%$ und $\mathrm{CiCaK}_{2} / 4$ ließ sich über einen weiten Flussbereich des HF-20-Sets einsetzen, hatte aber zur Bedingung, dass die hohe über die Zitratlösung zugeführte $\mathrm{Na}$-Konzentration durch einen entsprechend hohen Dialysatfluss kompensiert wird. Dies war bei einem Verhältnis für Butfluss:Dialysatfluss von 3:1 gegeben. Die Natrium isotone Zitratlösung mit 47,5 mmol/l Zitrat in Kombination mit PrismoCalB22 als Dialysatlösung hat in allen Lösungen eine isotone Natrium-Konzentration und ist mit Flussraten über den ganzen Bereich des HF-2O-Sets kompatibel.

Schlussfolgerung. Die RCA mit dem Prismaflex ${ }^{\circ}-\mathrm{HF}-20-S e t$, ohne Einschränkungen auf mögliche Flussraten und ohne zusätzliche Natriumbelastung, konnte am besten mit der Kombination einer Natrium isotonen Zitratlösung mit $47,5 \mathrm{mmol} / \mathrm{l}$ Zitrat und PrismoCalB22 als Dialysatlösung umgesetzt werden.

\section{FV-63 \\ Erfahrungen mit einem klinikinternen Notfallzubringer bei ex- ternen geburtshilflichen Notfällen - ein erheblicher Zeitgewinn}

\section{F. Eifinger', B. Roth', F. Alshut' ${ }^{2}$, J. Dötsch}

'Universitäts-Kinderklinik, Neonatologie und pädiatrische Intensivmedizin, Köln, Deutschland, ${ }^{2}$ Universitäts-Klinik, Werksfeuerwehr, Köln, Deutschland

Hintergrund. Die organisatorische Verantwortung für die Erstversorgung von Neugeborenen liegt laut AWMF-Leitlinie bei den Geburtshelfern. Bei Geburtskliniken ohne pädiatrischen Anwesenheitsdienst erfolgt die Versorgung bis zum Eintreffen des „Neugeborenen-Notarztes“ i. d. R. durch die Geburtshilfe und Anästhesie. Die Universitäts-Kinderklinik Köln verfügte bis zum 1.10.2012 über kein vor Ort stationiertes Einsatzfahrzeug. Das hinzugerufene Neugeborenen-Notarztteam alarmiert den Rettungsdienst und wird, gemeinsam mit dem Transportinkubator, mittels Rettungswagen (RTW) zu der Zielklinik gefahren.

Fragestellung. Gibt es einen Zeitgewinn, wenn das Notfallteam unabhängig von dem Transportinkubator in die Geburtsklinik transportiert wird (Rendezvous-System) und wird das Outcome dieser Patienten maßgeblich verbessert?

Material und Methoden. Seit 1.10.2012 hält die Werkfeuerwehr der Uniklinik Köln ein Notarzt-Einsatzfahrzeug (NEF) vor. Im Einsatzfall befördert das NEF das Neugeborenen-Notfallteam mit Sonderrechten zur Geburtsklinik. Mittels eines gesonderten Transportes (RTW) wird sekundär der Inkubator zur Geburtsklinik gebracht. Verglichen wurden zwei Patientengruppen externer Geburtskliniken, zu denen das pädiatrische Notfallteam innerhalb der ersten drei Lebensstunden (Umkreis: $2 \mathrm{~km}$ bis $30 \mathrm{~km}$ ) hinzugerufen wurde. Beide Gruppen bestehen aus Notfallpatienten mit mindestens einem der folgenden Kriterien: a) Reanimation, b) schwere Asphyxie oder anhaltende ( $>5$ min) Hypoxie c) schweres Atemnotsyndrom. Gruppe A $(n=39)$ besteht aus Patienten (1.1.2010-30.9.2012), die mittels RTW-transportiertem Team versorgt wurden. Gruppe B $(n=15)$ besteht aus Patienten, die primär durch das im NEF transportierte Notfallteam (seit 1.10.2012, d. h. ohne Transportinkubator) versorgt wurden.

Ergebnisse. Die Gruppen unterscheiden sich hinsichtlich APGAR-Werten, Schwangerschaftdauer, Nabelarterien-pH, Geburtsgewicht, -länge und Kopfumfang nicht. Die Gesamtzeit, d. h. von der Alarmierung bis zum ersten Kindeskontakt beträgt in Gruppe A: $27,1 \pm 1,1$ min (Mittelwert \pm S.E.M) und ist damit signifikant länger $(\mathrm{p}<\mathrm{o}, \mathrm{oo1})$ als in Gruppe
B mit 14.0 $\pm 2,0$ min. Der Zeitgewinn wird v. a. durch die unmittelbare Beförderung des Notfallteams gewonnen. Einen Einfluss auf die Beat-

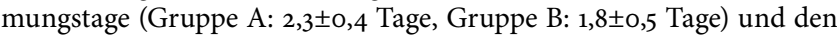
ersten, im Verlauf gemessenen, $\mathrm{pH}$-Wert zeigt sich nicht.

Schlussfolgerung. Durch das Hinzuziehen des Kinder-Notfallteams mittels Notfallzubringer (Rendezvous-System) wird ein deutlicher Zeitgewinn gegenüber dem Inkubator-gekoppelten Systems erzielt. Das System erweist sich als äußerst flexibel. Der Zeitgewinn von ca. $14 \mathrm{Mi}-$ nuten kann v. a. in Notfallsituation, wenn eine spezielle neonatologische Erstversorgung essentiell ist, von großem Nutzen sein. Es zeigt sich, dass v. a. weiter entfernte Geburtskliniken $(>4 \mathrm{~km})$ von diesem Zubringer profitieren.

\section{Poster}

\section{Pflege}

\section{Pfl.PO-1}

Beeinflussung der Eltern/Mutter-Kind-Interaktion durch den elterlichen Kompetenzerwerb in der Pflege und Versorgung des frühgeborenen Kindes

\section{A. Steinhardt' ${ }^{1}$ J. Reichert ${ }^{1}$, M. Rüdiger ${ }^{1}$, R. Rossi ${ }^{2}$}

'Uniklinikum, Neonatologie und pädiatrische Intensivmedizin, Dresden, Deutschland, ${ }^{2}$ Vivantes Klinikum Neukölln, Perinatalzentrum, Berlin, Deutschland

Hintergrund. Neben der intensivmedizinischen Versorgung Frühgeborener ist eine individualisierte Pflege durch die Eltern für die Kindesentwicklung sehr wichtig. Das Beachten entwicklungsfördernder Aspekte durch die Eltern sowie theoretische und praktische Kenntnisse der Eltern hinsichtlich des kindlichen Verhaltens, der Feinzeichen und des feinfühligen Reagierens erlangen eine besondere Bedeutung. Frühe Interventionen sollen zur Stabilisierung der emotionalen Situation der Eltern und zur Förderung der Eltern-Kind-Bindung und damit zur Verbesserung der Kindesentwicklung beitragen. Eltern frühgeborener Kinder erhalten am Uniklinikum Dresden und am Vivantes-Klinikum Berlin-Neukölln unterschiedliche Angebote, um weitgehende Selbstständigkeit in der Pflege und Versorgung zu erlangen. Die videogestützte Auswertung einer Versorgungssituation liefert die Grundlage für ein Feedback an die Eltern, um ihr Kompetenzgefühl zu stärken und erlaubt zudem die Beurteilung elterlicher Kompetenzen. Anhand von Verhaltensbeobachtungen werden Rückschlüsse auf die Qualität der Eltern-Kind Interaktion und dem Fürsorge- und Bindungssystems gezogen.

Fragestellung. Ist die Qualität der Eltern/Mutter-Kind Interaktion abhängig vom Strukturgrad theoretischer Wissensvermittlung und von den Rahmenbedingungen praktischer Elternanleitung?

Methodik. Der Strukturgrad theoretischer Wissensvermittlung und die Rahmenbedingungen praktischer Elternanleitung wurden erfasst. Zur Beurteilung der Qualität der Eltern/Mutter-Kind Interaktion wurden Videoaufnahmen einer definierten Versorgungssituation mittels Experten-Fremdrating analysiert. Einschlusskriterien: Eltern von $\mathrm{FG}<1500$ g. Ausschlusskriterien: infauste Prognose (FG), fehlende Deutschkenntnisse oder Psychopathologie der Kindeseltern.

Ergebnisse. Die einjährige Studie läuft bis 08/13 und soll 50 Patienten einschließen. In der bisherigen Analyse zeigt sich, dass sich strukturelle und inhaltliche Angebote zum Kompetenzerwerb an den Kliniken unterscheiden, die personellen Einschätzungen zur Umsetzung einer elternorientierten Pflege hingegen kaum. Eine Voranalyse ausgewählter Videosequenzen ergab Hinweise auf Unterschiede in den Eltern-KindInteraktionen. Rückschlüsse auf die Bedingungen des Kompetenzerwerbes werden aus der noch ausstehenden Videoanalyse erwartetet. 
Diskussion. Die Ergebnisse dienen der weiteren formalen und inhaltlichen Qualifizierung der Wissensvermittlung und praktischen Anleitung von Eltern in der Pflege und Versorgung von Frühgeborenen sowie der Adaptation an elterliche Bedürfnisse, um die Bedingungen für den Beziehungsaufbau zu ihrem Kind optimal zu unterstützen.

\section{Pfl.PO-2}

\section{Einschätzung der Analgosedierungstiefe in der pädiatrischen Intensivpflege}

\section{T. Lauterbach ${ }^{7}$}

'Universitäts - Kinderklinik, Pädiatrische Intensivstation „Keller", Freiburg, Deutschland

Die Einschätzung der Analgosedierungstiefe in der pädiatrischen Intensivpflege spielt eine sehr wichtige Rolle. Während bei Neonaten und Säuglingen der Berner-Schmerzscore und die KUSS-Skala zum Einsatz kommen, gibt es für beatmete Kinder kaum Assessment-Instrumente. Die Comfort-Scale wurde speziell für die Erfassung von Stress bei Kindern auf der Pädiatrischen Intensivstation entwickelt. Acht Parameter werden beurteilt: Wachheit/Aufmerksamkeit, Agitation, Atmung, Körperhaltung, Mimik, mittlerer arterieller Blutdruck, Herzfrequenz und Muskeltonus. Dieser Scale ist für die englische Sprache validiert. Die Comfort-Behavioral-Scale oder Comfort-B-Scale wurde daraus entwickelt. Dieses Assessment-Instrument arbeitet ohne die Parameter mittlere arterieller Blutdruck und Herzfrequenz. Bei nicht beatmeten Kindern wird der Punkt Atmung durch Weinen ersetzt. Die Comfort$\mathrm{B}$-Scale bietet eine verlässliche Alternative zur Comfort-Scale. Beide Skalen bieten gute Möglichkeiten die Analgosedierungstiefe pädiatrischer Patienten besser einschätzen und Medikamente entsprechen titrieren zu können.

\section{Pfl.PO-3}

\section{Intranasale Medikamentenapplikation}

\section{T. Lauterbach ${ }^{7}$}

'Universitäts - Kinderklinik, Pädiatrische Intensivstation „Keller", Freiburg, Deutschland

Die Nasenhöhle ist leicht zugänglich, die Schleimhaut bietet eine große Oberfläche und ist sehr gut durchblutet. Die Gabe von Medikamenten, mittels Applikation in vernebelter Form, ist eine vielversprechende $\mathrm{Me}$ thode. Das Einsatzgebiet ist weit gestreut. Neben Sedierung und Analgesie während Kurznarkosen, steht die Therapie des epileptischen Anfalls im Mittelpunkt. Die Anwendung ist einfach, kostengünstig und in der Regel schmerzfrei. Es ist unter Umständen kein i.v. Zugang vonnöten. Zudem wird der Magen-Darmtrakt umgangen. Die Auswahl an Medikamenten die verabreicht werden können ist allerdings begrenzt. $\mathrm{Zu}$ hohe Medikamentenkonzentrationen und zu große Mengen stellen eine Kontraindikation dar. Mögliche Anwendungsgebiete sind in der Therapie chronischer Schmerzen, Migräne, Verabreichung von Impfstoffen und Insulin zu finden. Diese finden sich zum Teil noch in der klinischen Erforschung.

\section{Pfl.PO-4}

What a beautiful skin - Sequel: ein Forschungsanwendungsprojekt

\section{Burgermeister', H. Nicolai', U. Emmenegger ${ }^{3}$, B. Dinten-Schmid ${ }^{4}$,} L. Stoffel ${ }^{5}$

'Universitätskinderklinik Bern Inselspital, Neonatologische Intensivstation, Bern, Schweiz, ${ }^{2}$ Universitätsspital Zürich/Klinik für Neonatologie, Departement Frauenheilkunde, Zürich, Schweiz, ${ }^{3}$ Universitäts Kinderspital beider Basler (UKBB), Pädiatrische Intensivpflegestation Neonatologie, Basel, Schweiz, ${ }^{5}$ Medizinische Universitätskinderklinik, Inselspital Bern, Neonatologie, Bern, Schweiz, ${ }^{4}$ Frauenklinik, Neonatologie, Zürich, Schweiz

Einleitung und Fragestellung. Fundiertes Wissen, Verständnis über die Prinzipien der Hautpflege bei den frühgeborenen Kindern $<32$. Schwangerschaftswochen (SSW) und eine einheitliche Vorgehensweise bezüglich Hautschutz/Hautpflege während der neonatalen Intensivpflege kann die Risiken und Kosten dieser Population senken. Eine Literaturreview zeigte, dass durch die Anwendung eines Assessments eine systematische Hautbeurteilung gewährleistest wird. Dies führt schneller zu präventiven Massnahmen. Die Anwendung von Sonnenblumenöl zum Hautschutz bewirkt eine signifikante Reduktion der Infektionsrate. Prinzipiell wird die Anwendung von semipermeablen Pflastern und Hydrokolloid zur präventiven und therapeutischen Anwendung empfohlen, insbesondere den Pflastergebrauch auf ein Minimum zu beschränken. Um den transepidermalen Wasserverlust zu reduzieren, sollen befeuchtete Doppelwandinkubatoren eingesetzt werden. Die Keimbesiedlung der frühgeborenen Haut nimmt nicht zu, wenn das Reinigungsintervall auf vier Tage ausgedehnt wird. Wie werden diese evidenzbasierten Erkenntnisse nachhaltig in die Praxis implementiert? Methode. Für die Projektumsetzung diente die partizipative Aktionsforschung.

Implementierungsprozess und Ergebnisse. Schritt 1: Die Studienresultate wurden mit einer Peergruppe im Hinblick auf die Umsetzung der Resultate in die klinische Praxis diskutiert. Schritt 2: Entwicklung der evidenzbasierten Leitlinie Hautschutz/Hautpflege <32. SSW. Schritt 3: Wissensvermittlung mittels online Schulung. Schritt 4: Einführung der Leitlinie und Praxiscoaching. Schritt 5: Wissensüberprüfung bezüglich Praxistransfer. Schritt 6: Evaluation der Leitlinie und Update der Literatur. Die dreijährige Praxiserfahrung zeigt, dass die eingeführten Maßnahmen in der Pflege umgesetzt werden. Die Haut wird systematisch beurteilt, die Haut ist ein Thema bei der täglichen Visite. Das Feuchtigkeitsmanagement sowie das reduzierte Bade-Waschintervall wurden sehr gut akzeptiert. Seit der Einführung der Sonnenblumenöl-Prophylaxe und dem bewussten Einsatz von Hautschutzverbänden und Pflasterprodukten werden deutlich weniger Hautschädigungen dokumentiert.

Diskussion und Schlussfolgerung. Die Pflegepraxis hat erkannt, dass die strukturierte Einschätzung der Frühgeborenenhaut mit dem Hautscore wichtig ist. Jedoch wurde von der Arbeitsgruppe bemängelt, dass das Assessment weder die Hautunreife noch die Hautdefekte ausreichend misst. Deshalb wurde im Januar 2012 ein validiertes Einschätzungsinstrument mit Zusatzkriterien implementiert.

\section{Pfl.PO-5}

Wie können Schädelverformungen unter langandauernder NasenCPAP-Therapie bei Frühgeborenen durch pflegerische Maßnahmen verhindert werden?

\section{S. Wiederkehr, Lörrach}

Hintergrund. Um die Beatmungsdauer der Frühgeborenen so kurz wie möglich zu halten oder eine Beatmung ganz zu vermeiden, setzen wir auf unserer Station so früh wie möglich den Nasen-CPAP („continous positive airway pressure") ein. Über längere Zeit habe ich beobachtet, dass es unter mehrwöchiger Nasen-CPAP-Therapie auf Grund der notwendigen Fixierung mittels Mützen zu deutlichen Schädelverformungen kommt (in der Fachliteratur als Dolichocephalus beschrieben). 
Daraus ergibt sich für Ärzte und Pflegekräfte z. B. Schwierigkeiten bei der korrekten Fontanellen- und Kopfumfangbeurteilung, Eltern sehen es oft als kosmetisches Problem.

Fragestellung. Wie können Schädelverformungen unter langandauernder Nasen-CPAP-Therapie bei Frühgeborenen durch pflegerische Maßnahmen verhindert werden?

Methoden. Über das Internet, Hersteller und ehemalige Kollegen habe ich Experten gefunden. Direkt auf unserer Station habe ich Daten von Kindern unter CPAP-Therapie gesammelt. Zum Schluss hatte ich Kontakt zu einem Physiotherapeuten zum Thema Bauchlage unter CPAPTherapie.

Ergebnisse. Beim Anbringen des CPAP, achten wir auf unserer Station darauf, dass wir bei jeder Versorgung zwischen Prongs und Maske, sowie den beiden Befestigungsmöglichkeiten (Mütze und Klettverschlusssystem) wechseln. Unsere Mützen wählen wir nicht zu klein Dies habe ich herausgefunden, als ich Mützengröße, Körpergewicht und Kopfumfang entsprechend Herstellerangaben verglichen habe. Trotzdem treten Schädelverformungen weiterhin auf. Ich hatte zu mehreren Fachpflegekräften sowie Ärzten verschiedener Kliniken Kontakt. Alle halten die richtige Mützengröße für essentiell! Die Anwenden greifen nicht genau auf die Größenangaben der Hersteller zurück, sondern wählen in der Regel eine Mützennummer größer. Aber Vorsicht, zu große Mützen rutschen, dann ist mehr Kraft zur Fixierung notwendig und so nimmt die Deformierung zu. Einige Kliniken setzen auf eine kurze CPAP-Therapie, indem schnellstmöglich die High-Flow-Brille zum Einsatz kommt. Als wichtig wird auch erachtet, die Mützen am Ende zuzubinden und weiche und elastische Mützen zu nutzen. Auch der Rachen-CPAP wird angesprochen. Dabei muss allerdings bedacht werden, dass dieser bei unruhigen Kindern oft zu Verletzungen im Nasen-Rachen-Raum führt. Die Lagerung der Schläuche hinter dem Kopf kann den Druck auf diesen verringern. Dazu werden die Schläuche oberhalb des Kopfes auf einer Unterlage gelagert oder man benutzt an der Inkubatorrückseite die Öffnung in der Klappe. In Bauchlage sind Kinder stabiler und zufriedener, weniger Zug ist zur CPAP-Fixierung notwendig. Die Kombination aus CPAP-Therapie und Bauchlage führt aber zu stärkeren Verformungen. Der Physiotherapeut meint, dass sich der Schädel später in Rückenlage wieder rundet. Eine Herstellerfirma weiß um das Problem und suchen nach Verbesserungen. Sie vertrauen aber grundsätzlich auf Pflegekräfte, die systematisch die Mützen überprüfen, um rechtzeitig eine Größere zu wählen. Auf Nachfrage habe ich zudem erfahren, dass die korrekte Mützengröße über die Messung des Hutumfangs ermittelt wird.

Schlussfolgerung. Die Schädelverformung ist bei allen angefragten Kliniken ein Problem. Alle haben auch Erfahrungswerte zur Verminderung der Verformung gesammelt, jedoch liegen keine nachgewiesen wirksamen Maßnahmen vor. Es gibt mehrere Expertenmeinungen, die vor allem auf die perfekte Passform der Mütze sowie einer CPAP-Therapie-Verkürzung setzen.

\section{Pfl.PO-6 \\ Saug- und Schluckreflexförderung bei langzeitbeatmeten Früh- geborenen}

\section{K. Felberg, Lörrach}

Sehr unreife Frühgeborene haben ein erhöhtes Risiko eine Saug- und/ oder Schluckstörung zu entwickeln. Diese kann schwerwiegend sein und eine langfristige, intensive Therapie nach sich ziehen. Grundlagen zur Beurteilung, wann und wie ein Training gestaltet werden kann, sind: neurologische Reife, beteiligte Strukturen (Nerven, Muskeln, Gehirn) und die Physiologie des Saug- und Schluckaktes.

Es gibt ein einziges evidenzbasiertes Konzept für Frühgeborene: „nonnutritive sucking“. Es hat viele positive Auswirkungen, u. a. auf die Nahrungsverträglichkeit, die Koordination der beiden Reflexe sowie die Stärkung der Muskulatur, aber auch auf die Psyche des Frühgeborenen und sogar auf die spätere Sprachentwicklung. Ein Review von Pinelli und Symington bestätigte den positiven Effekt von „non-nutritive sucking" auf FG im Zusammenhang mit der Dauer des Krankenhausaufenthalts. Ein zweites Review beschäftigte sich mit der schnelleren Entwöhnung von der Sonde durch dieses Konzept.

Wenn mit einem frühzeitigen Training begonnen wird und einige Aspekte beim oralen Absaugen beachtet werden, haben diese kleinen FG gute Chancen ein normales Saug- und Schluckverhalten zu erlernen.

\section{Pfl.P0-7}

\section{Atemunterstützende Lagerungen bei extremen Frühgeborenen}

\section{Engler, Lörrach}

Hintergrund. Viele Frühgeborene fallen mit Apnoen und periodischer Atmung auf, welche Sättigungsabfälle zur Folge haben. Aufgrund von Erfahrungen werden diese Kinder häufig in Oberkörperhoch- oder Bauchlagerung gelagert. In dieser Facharbeit soll geklärt werden, ob diese Erfahrungen auch evidenzbasiert sind und ob noch andere Möglichkeiten zur Atemunterstützenden Lagerung verfügbar sind.

Methoden. Hierfür wurde Fachliteratur aus dem deutschsprachigen Raum gesichtet, 10 Intensivstationen Level 1 befragt, eine Fachkinderkrankenschwester und MH Infant-Handling Grundkurstrainerin interviewt sowie die 1. Fachtagung für CPAP und NIV in Neonatologie und Pädiatrie besucht.

Ergebnisse. Die Studie der Eberhard-Karls Universität für Kinderheilkunde und Jugendmedizin Tübingen ergab keinen Vorteil einer Oberkörperhochlagerung. Die Umfrage und Fachgespräche wiesen auf positive Effekte hin.

Schlussfolgerung. Recherchen haben ergeben, dass das MH Kinaesthetik-Infant-Handling-Konzept und eine 3-Stufen-Lagerung generell bei der Pflege dieser Kinder berücksichtigt werden sollte, da sie sich positiv auf die respiratorische Situation auswirkt.

\section{Freie Themen}

\section{PO-01}

\section{Zwei Fälle einer ausgeprägten neonatalen kardialen Hypertrophie}

\section{Katrin', S. Armbrust ${ }^{2}$}

'Dietrich-Bonhoeffer-Klinikum Neubrandenburg, Klinik für Kinder- und Jugendmedizin, Neubrandenburg, Deutschland, ${ }^{2}$ Dietrich-Bonhoeffer-Klinikum, Klinik für Kinder- und Jugendmedizin, Neubrandenburg, Deutschland

Hintergrund. Kongenitale Myokardhypertrophien treten zumeist bei mütterlichem Diabetes mellitus auf. Andere, seltene Ursachen sind Stoffwechselerkrankungen, sowie auch z. B. aortale Vitien. Wir beschreiben zwei außergewöhnliche Verläufe einer neonatalen myokardialen Hypertrophie, deren Ätiologie, Behandlung und Outcome.

Fallbericht 1. 23-jährige G1/Po, Epilepsie - anfallsfrei seit 6 Jahren, Triple-X-Syndrom, insulinpflichtiger Gestationsdiabetes (ab 31. SSW behandelt), Maximale Werte über $20 \mathrm{mmol} / \mathrm{l}, \mathrm{HbA1c}$ präpartal: 7,0\% Reifes NG, keine ausreichende Sättigung trotz CPAP und O2-Gabe, rasche Entwicklung einer Herzinsuffizienz mit Tachypnoe, Trinkschwäche, Schwitzen unter Belastung, Ödeme. Ausgeprägte generalisierte LVHypertrophie mit kaum sichtbarem Cavum. Ausschluss von Stoffwechselerkrankungen. Beginn einer Propranolol-Therapie, hierunter Besserung der klinischen Symptomatik. Milde Hypoglykämien im Verlauf, sonst stabil. In den Folgemonaten kontinuierliche Besserung aller myokardialen Parameter.

Fallbericht 2. 25-jährige G1/Po, Polyhydramnion 21. SSW, FG $29+4$ SSW mit persistierender fetaler Zirkulation, IVH $\mathrm{II}^{\circ}$ links und $\mathrm{III}^{\circ}$ rechts mit Shuntableitung, Krampfanfälle, kurze Extremitäten, Hypertelorismus, nach oben ausgezogener Kopf. Ab LT 35 zunehmende Tachyarrhythmie, dann myokardiale Hypertrophie mit Vorwärtsversagen, LT 42: Massive LV-Hypertrophie, intrakardiales Pumpversagen, nachfolgend Propanololtherapie. Klinisch hiernach besser, aber weiter zunehmende RV-Hypertrophie. Bei V. a. Costello-Syndrom Untersuchung des HRAS 
Exon 2: hier heterozygote Missense-Mutation und Nachweis desselben. Propranololtherapie, Exitus im 4. Lebensmonat.

Diskussion. Die Ätiologie der diabetischen Fetopathie führt über die maternale Hyperglykämie zur kindlichen Hyperglykämie und nachfolgend zum Wachstum von Bindegewebe, Fett und Muskel durch Insulin als Wachstumsfaktor. Die myokardiale Hypertrophie stellt sich zumeist als Septumhypertrophie dar, eine therapeutische Notwendigkeit ergibt sich bei guter Prognose und Rückbildung innerhalb von 2 bis 12 Monaten nicht. Die Notwendigkeit von Beta-Blockern ist in der Literatur nicht beschrieben. Das Costello-Syndrom ist eine seltene genetische Erkrankung im HRAS-Gen, die häufig mit kardiovaskulären Malformationen und Rhythmusstörung einhergeht. Es kommt zu einer peri- und intrazellulären Ansammlung von Chondroitin 6-sulfate-enthaltenden Glycosaminoglycanen und zur Akkumulation von Chondroitin 6-sulfate in Cardiomyocyten. Die Fibroblasten produzieren normales Tropoelastin aber keine elastischen Fasern wg. sek. Mangel an $67-k D$ elastin-binding protein. Der Einsatz von $\beta$-Blockern stabilisiert den Krankheitsverlauf, ändert aber nichts an der infausten Prognose. Schlussfolgerung. Bei ausgeprägter myokardialer Hypertrophie kann auch bei der diabetischen Fetopathie der Einsatz von Beta-Blockern notwendig sein. Das Costello-Syndrom ist differentialdiagnostisch zu erwägen bei Befundprogredienz und zunehmender Rhythmusstörung.

\section{PO-02}

\section{Zwei kritische angeborene Herzerkrankungen bei einem Säugling}

\section{F. Riede', R. Gebauer', F. Löffelbein', M. Kostelka², I. Dähnert ${ }^{1}$}

'Herzzentrum, Universität Leipzig, Kinderkardiologie, Leipzig, Deutschland, ${ }^{2}$ Herzzentrum, Universität Leipzig, Kinderherzchirurgie, Leipzig, Deutschland

Einleitung. Neugeborene und Säuglinge mit kritischen angeborenen Herzerkrankungen fallen in der Regel nur durch eine unspezifische Symptomatik auf und haben das Risiko, rasch einen akut lebensbedrohlichen Zustand zu entwickeln. Auch wenn ein typisches Leitsymptom vorliegt, ist eine vollständige kardiologische Diagnostik unerlässlich.

Kasuistik. Bei einem bislang gesunden männlichen Säugling fielen bei der $\mathrm{U}_{3}$ eine Tachydyspnoe und ein Herzgeräusch auf. Rückblickend war auch eine Trinkschwäche zu erfragen. Wegen abgeschwächter Pulse an den unteren Extremitäten erfolgte die sofortige Einweisung in unsere Klinik unter dem Verdacht einer kritischen Aortenisthmusstenose (ISTA). Bei Aufnahme sahen wir einen 5 Wochen alten Säugling in reduziertem Allgemeinzustand. Die ISTA konnte echokardiographisch bestätigt werden. Zusätzlich waren ein kräftiger Links-Rechts-Shunt über einen Vorhofseptum-sekundum-Defekt (ASD) und eine Mitralinsuffizienz $\mathrm{II}^{\circ}$ nachweisbar. Ein trotz morphologischer Enge dopplersonographisch nur gering ausgeprägter Gradient $(19 \mathrm{mmHg})$ wurde zunächst durch die bereits eingeschränkte linksventrikuläre Funktion erklärt; die Indikation zur dringlichen Operation mit ISTA-Resektion wurde gestellt. Aufgrund des ebenfalls gering ausgeprägten klinischen Blutdruckgradienten zwischen oberer und unterer Extremität erfolgte eine erneute Echokardiographie mit der Fragestellung weiterer Anomalien. Dabei fielen echoreiche Papillarmuskeln der Mitralklappe auf. Die linke Koronararterie (LCA) konnte zwar mit diastolisch antegradem Fluss dargestellt werden, eine sichere Zuordnung ihres Abgangs zur Aortenwurzel gelang jedoch nicht. Unter dem Verdacht auf einen zusätzlich zur ISTA vorliegenden Fehlabgang der LCA aus der Pulmonalarterie (ALCAPA) wurde die Indikation zur Herzkatheteruntersuchung gestellt. Dabei zeigte sich eine seltene Form der ALCAPA mit Abgang der LCA aus der rechten Pulmonalarterie. Am Folgetag der Aufnahme konnte die Korrekturoperation mit ISTA-Resektion, Re-Implantation der LCA und ASD-Verschluss durchgeführt werden. Postoperativ war für 3 Tage eine Unterstützung mit einem linksventrikulären Assistsystem nötig. 6 Wochen postoperativ ist der Säugling in stabilem Allgemeinzustand. Die kardiale Funktion ist unter medikamentöser Therapie deutlich gebessert; die Nahrung wird noch teilsondiert.
Diskussion. Die kritische ISTA als Ursache einer raschen Verschlechterung in den ersten Lebenswochen ist gut bekannt. Das zusätzliche Vorliegen einer ALCAPA ist eine Rarität. In der Literatur liegen nur einzelne Fallberichte vor, bei denen die Diagnose erst postoperativ gestellt wurde. In unserem Fall ermöglichte die präoperative Diagnose eine Anpassung des therapeutischen Vorgehens und eine Reduktion der perioperativen Morbidität.

\section{PO-03}

Anfangs nicht erkannte Aortenisthmusstenose bei SFM-Syndrom (Kasuistik) - Gefahr des Übersehens angeborener Herzfehler bei seltenem gravierendem Hautbefund

\section{R. Eyermann}

'Dr. Eyermann, Kinder- und Jugendmedizin, Kinderkardiologie, Sportmedizin, München, Deutschland

Hintergrund. 1957 beschrieben Schimmelpenning und 1962 Feuerstein und Mims den neuroektodermalen Anomaliekomplex unklarer Ätiopathogenese mit facialem Naevus sebaceus linearis, multiplen Naevi, geistiger Entwicklungsverzögerung und Krampfanfällen. In wenigen Kasuistiken noch Abnormitäten an Augen (Nystagmus, Kornealvaskularisierung, Choroideakolobom, Pupillenektopie, subkonjunktivales Lipodermoid und Amaurose) und Gehirn (Hydrocephalus internus) beschrieben. Kardiale Erkrankungen bei Reizleitungsstörungen für möglich gehalten. Bislang berichteten wenige Autoren, zuerst Marden et al. (1966), über Coinzidenz mit Vitium (CoA und Aortenhypoplasie). Kasuistik. Anamnese: Mädchen, 4 Mo., wegen Kardiomegalie (Rö-Thorax) zur Diagnostik in Kinder-Herzzentrum verlegt. SSV und Geburt unauffällig. Seit Geburt ausgedehnter Talgdrüsennaevus im Gesicht und Hals. Beginn Hautdiagnostik mit 3 Mo. in Uni-Hautklinik. Aufnahmebefund: $5120 \mathrm{~g}$ schwerer, weiblicher Säugling, mit exzessivem, v. a. im Gesichts- u. Halsbereich ausgeprägtem Naevus sebaceus und adäquaten Schleimhautveränderungen im Mundbereich (forme fruste; - Abb. 1, - Abb. 2). Pigmentnaevi an beiden Beinen. Oberlidkolobom links u. von temporal auf die Cornea übergreifende Conjunctiva mit Pupillenektopie (- Abb. 3). Strabismus. Keine Dyspnoe, Zyanose oder Ödeme nachweisbar. Cor: rhythmische HA. 2/6 Systolikum p.m. 3L2 und dorsal paravertebral li. u. re.. Pulmo: ausk. o. B.. Hepar $2 \mathrm{~cm}$ unter RIBO palpabel. Femoralispulse beidseits abgeschwächt. RR-Messung: re. Arm 140 mmHg, re. Bein 120 mmHg. Sonstiger interner Status kli-

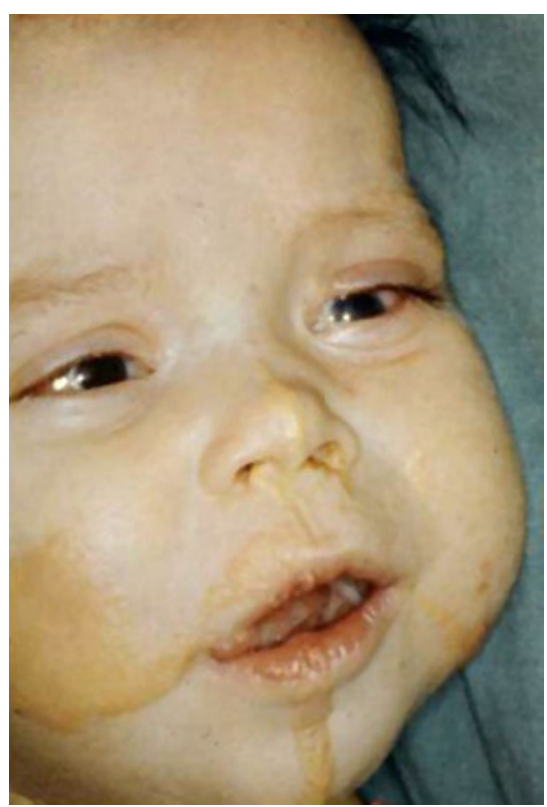




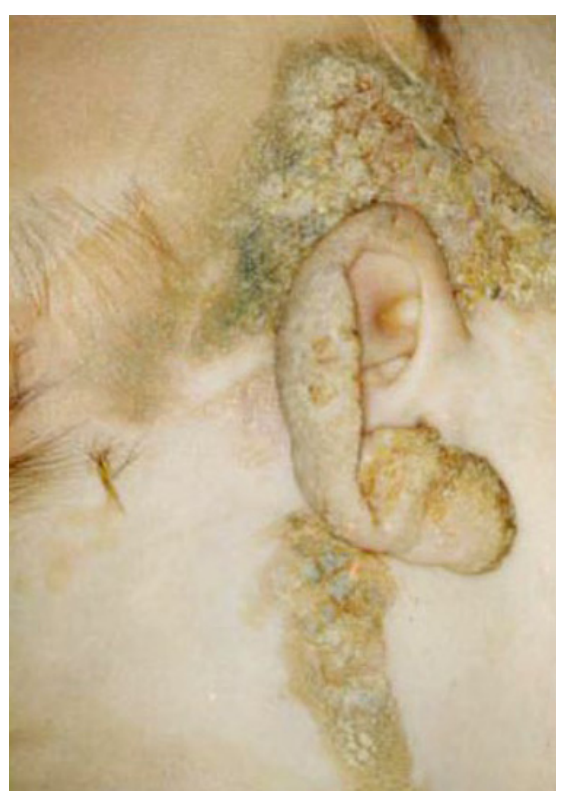

P0-03 Abb. 24

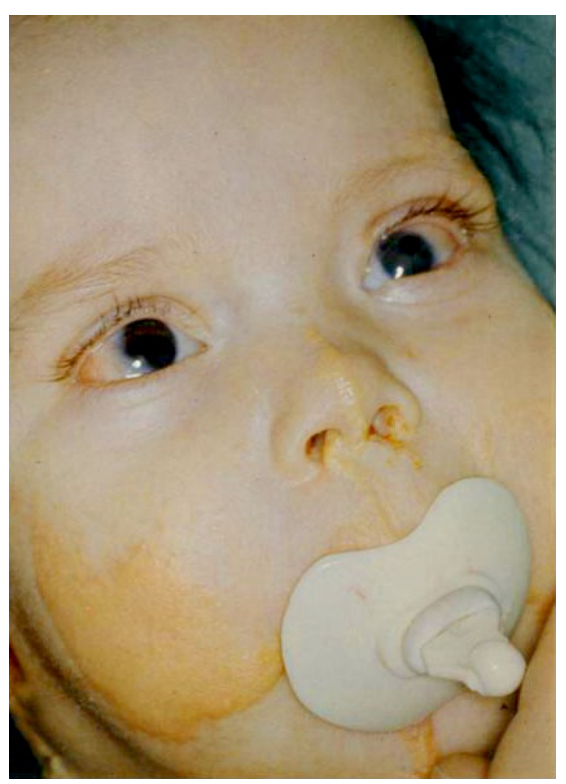

PO-03 Abb. 3

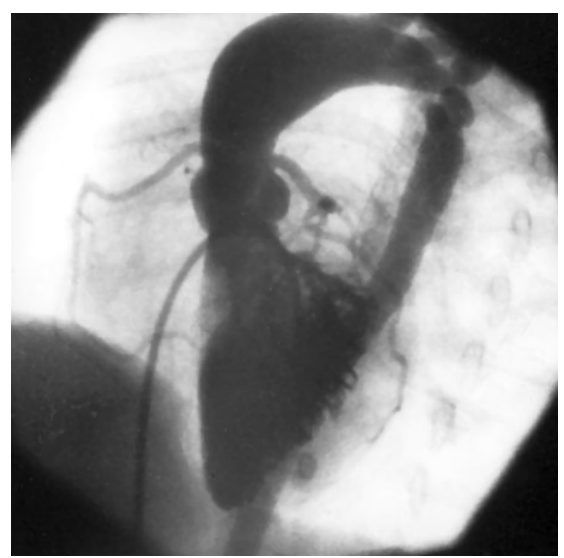

PO-03 Abb. 44 nisch unauffällig. Wesentliche weitere Befunde: Rö-Thorax: Linksherzverbreiterung, Gefäßfüllung normal. EKG: SR. Ausgeprägte LV. Echo: Linker Ventrikel mit ausgeprägter konzentrischer Hypertrophie und Einengung des Cavums. Herzkatheteruntersuchung (Druckwerte in Torr): re. Vorhof MD O-1, re. Ventrikel 25/-/3, A. pulmonalis 20/12 MD 15, li. Vorhof MD 4, li. Ventrikel 175/-/4, Ergebnis: CoA mit Involvierung Abgang li. A. subclavia in den Stenosebereich (• Abb. 4). Umschriebener, schleifenförmiger Aortenverlauf im Isthmusbereich. Mäßige Erweiterung Ao. ascendens. Signifikante Hypertrophie li. Ventrikel ohne Hinweis auf kinetische Störungen. EEG: Alters- und vigilanzentsprechende Allgemeinfunktion; auftretende Seitendifferenzen noch physiologisch; keine gesteigerte Anfallsbereitschaft. Sonographie Schädel: Leichte Erweiterung der lateralen und des 3. Ventrikels. Cerebrales CT: Linksbetonte, mäßige Erweiterung der Seitenventrikel.

Schlussfolgerung. Gravierende Hautbefunde sollten nicht von subtiler Erhebung des Gesamtstatus eines Neugeborenen ablenken. Das SFMSyndrom ist eine Blickdiagnose bei typischen Hautveränderungen $u$. bei Nachweis pathologischer Befunde an Auge und Gehirn zu sichern. An mögliche Coinzidenz mit einem angeborenen Herz- u. Gefäßfehler (CoA oder Aortenhypoplasie, auch Stenose aortaler Äste, z. B. Nierengefäße - persönliche Mitteilungen) sollte gedacht werden. Synonym: Haut-Augen-Hirn-Herz-Syndrom, berechtigt.

\section{PO-04}

\section{Neonatale eosinophile Pustulose bei einem Frühgeborenen}

\section{A. Stein', A. Körber², U. Hillen², U. Felderhoff-Müser ${ }^{3}$}

${ }^{1} K$ linik für Kinder und Jugendmedizin der Universität, Klinik für Kinderheilkunde I, Essen, Deutschland, ${ }^{2}$ Klinik für Dermatologie der Universität, Essen, Deutschland, ${ }^{3}$ Klinik für Kinder und Jugendmedizin der Universität, Essen, Deutschland

Hintergrund. Die Differenzialdiagnosen von vesikopustulösen Hauterkrankungen des Neugeboren sind vielfältig und umfassen neben primär infektiösen Ursachen auch einige seltene Erkrankungen wie die eosinophile Pustulose.

Fallbericht. Wir berichten über ein männliches Frühgeborenes, das bei Amnioninfektionssyndrom mit einem Gestationsalter von 25+1 SSW geboren wurde (Geburtsgewicht $690 \mathrm{~g}$, APGAR 3/7/7). Auffällig war eine Rötung des gesamten Integuments mit Nachweis von Candida albicans. Bei blandem Hautzustand wurde die seit Geburt durchgeführte systemische antibiotische und antimykotische Therapie am 8. Lebenstag (LT) und die topische Therapie mit Nystatin am 2o. LT beendet. Im Alter von 4 Wochen traten, teils gruppierte, vesikulo-pustulöse Effloreszenzen auf gerötetem Hof mit gelblicher Kruste im Gesicht auf. Im Abstrich sowie in der Blutkultur wurde Staph. aureus nachgewiesen. Bei systemischen Infektionszeichen (CrP max. 1,6 mg/dl) wurde für $14 \mathrm{~d}$ mit Flucloxacillin i.v. behandelt. Im weiteren Verlauf traten rezidivierend Effloreszenzen im Gesicht und vereinzelt an Stamm und Extremitäten auf. Bei erneuten systemischen Entzündungszeichen (CrP $\max 2,1 \mathrm{mg} / \mathrm{dl}$ ) und einmaligem Nachweis von Raoultella planticola im Abstrich wurde für 3 Wochen mit Meropenem behandelt. Unter dieser Therapie, sowie unter Lokaltherapie mit Retapamulin kam es jeweils nur transient zu einer Besserung. Weitere Abstriche blieben steril. Herpes oder Varizella konnte nicht nachgewiesen werden. Auffällig war eine Eosinophilie im peripheren Blutbild von max. $47 \%$ sowie von etwa $50 \%$ im Bläschenausstrich. Das Ergebnis der dermatohistopathologischen Untersuchung bestätigte die Verdachtsdiagnose der eosinophilen Pustulose.

Diskussion. Die eosinophile Pustulose, auch Ofuji-Syndrom genannt, ist eine seltene Hauterkrankung mit intraepidermal gelegenen eosinophilen Pusteln bei Säuglingen und Erwachsenen. Dies ist der erste Fallbericht eines Frühgeborenen. Typischerweise treten über Monate bis Jahre wiederkehrende vesikulopustulöse Effloreszenzen mit stark gerötetem Hof v. a. im Gesicht auf. Die histopathologisch nachgewiesenen eosinophilen Infiltrate sind jedoch nicht pathognomonisch, so dass in unse- 
rem Fall auch ein Zusammenhang mit der initialen Candidosis und der späteren Staphylodermie sowie immunologischen Faktoren diskutiert werden muss.

\section{PO-05}

\section{Ungewöhnliche Ursache eines Atemnotsyndroms bei einem Neu-} geborenen

\section{Z. el Hafid', M. Vochem², P. Neuberger ${ }^{3}$}

${ }^{1}$ Klinikum Stuttgart, Olgahospital, Stuttgart, Deutschland, ${ }^{2}$ Olgahospital, Neonatologie, Stuttgart, Deutschland, ${ }^{3}$ Olgahospital Stuttgart, Päd. Zentrum, Neonatologie, Leinfelden-Echterdingen, Deutschland

Hintergrund. Das Surfactantmangelsyndrom ist eine häufige Diagnose auf jeder neonatologischen Intensivstation. Neben der Unreife der Lunge spielen beim akuten Atemnotsyndrom des Neugeborenen in seltenen Fällen auch andere Faktoren eine Rolle. Wir stellen den Fall eines reifen Neugeborenen mit Hypothyreose und Atemnotsyndrom, bei dem wir einen Zusammenhang zwischen den beiden Entitäten vermuten.

Kasuistik. Geburt per Re-Sectio in der 37+6 SSW, Nabelarterien-pH 7,35, Apgar 10/10/10, Gewicht 3045 g, postnatal sekundäre respiratorische Verschlechterung, unter CPAP zunehmender Sauerstoffbedarf bis 60\%, daher Intubation, maschinelle Beatmung, Surfactantgabe. Extubation nach $18 \mathrm{~h}$ bei $\mathrm{FiO}_{2}$ 0,21; danach wieder respiratorische Verschlechterung am CPAP mit FiO2 0,45, noch weitere 2 Surfactantgaben ohne Effekt, Röntgen-Thorax: RDS; CPAP bis zum 8. Lebenstag. Im Neugeborenenscreening TSH $330 \mathrm{mIE} / \mathrm{l}, \mathrm{fT}_{4} \mathrm{o}, 3 \mathrm{ng} / \mathrm{dl}$. Therapie mit L-Thyroxin ab dem 3. Lebenstag, darunter rasche Normalisierung von TSH und $\mathrm{fT}_{4}$. Schilddrüsensonographie: rechter Lappen o. B., links nur kleine, fragliche Schilddrüsenstrukturen erkennbar. Entlassung am 26. Lebenstag. Im Verlauf immer wieder Krankengymnastik zur Unterstützung der Entwicklung; rezidivierende pulmonale Infekte. Erreichen der Meilensteine der Entwicklung sehr spät, aber noch zeitgerecht.

Diskussion. Nach Durchsicht der Literatur bei ungewöhnlichem Verlauf eines ANS bei einem reifen Neugeborenen fanden wir mehrere Veröffentlichungen, die einen Zusammenhang zwischen konnataler Hypothyreose und Atemnotsyndrom beschreiben. Meist auf der Grundlage eines Gendefekts, der neben rezidivierenden Infektionen der Lunge auch eine Entwicklungsverzögerung begünstigen kann.

Schlussfolgerung. Bei Atemnotsyndrom und konnataler Hypothyreose ist auch an seltene Ursachen zu denken. Eine Konsequenz aus der Diagnosestellung wäre in unserem Fall ein besonderes Augenmerk auf die Entwicklung zu legen und das Kind frühzeitig und adäquat zu fördern, besonders da bereits durch die Hypothyreose die Gefahr einer gestörten Entwicklung gegeben ist. Außerdem sollte frühzeitig rezidivierenden pulmonalen Infekten entgegengewirkt und diese frühzeitig behandelt werden.

\section{PO-06}

\section{Diaphragmatische Hernie nach Lebertransplantation}

\section{J. Kalk', T. Diehl', M. Koch'2, R. Ganschow', G. Hillebrand', D. Singer'}

'Universitätsklinikum Hamburg-Eppendorf, Neonatologie und pädiatrische Intensivmedizin, Hamburg, Deutschland, 'Universitätsklinikum Hamburg-Eppendorf, Hepatobiliäre Chirurgie und Transplantationschirurgie, Hamburg, Deutschland, ${ }^{3}$ Zentrum für Frauen-, Kinder- und Jugendmedizin, Päd. Gastroenterologie/Hepatologie, Hamburg, Deutschland

Hintergrund. Die Lebertransplantation (LTX) ist heute eine etablierte Therapie der schweren Lebererkrankungen im Kindesalter mit einer 1-Jahres-Überlebensrate von 90\%. Dabei werden häufig die linken Leberanteile eines geteilten Organs (Split-Organ) verwendet. $\mathrm{Zu}$ den häufigsten Komplikationen gehören Blutungen, Gallengangsstenosen, Gallelecks, Pfortader- und Leberarterienthrombosen.

Fallbericht. Wir stellen eine 7-jährige Patientin nach Split-LTX vor, die 3 Monate nach Transplantation mit unstillbarem Erbrechen ohne
Fieber auffiel. Als Ursache war zunächst bei noch vorhandenen Darmgeräuschen eine "Gastroenteritis" vermutet worden. Zudem bestand radiologisch ein pulmonales „Infiltrat“ mit einem auch sonographisch nachweisbaren „gekammerten Erguss“. Bei zunehmender Ausprägung der abdominellen Beschwerden mit Rückfluss von stuhlverdächtigem Darminhalt über die Magensonde wurde ein Thorax-/Abdomen-CT durchgeführt. So wurde die Diagnose einer erworbenen linksseitigen diaphragmatischen Hernie mit Enterothorax gestellt. Nach operativer Versorgung mit direkter Naht des Zwerchfells war der weitere Verlauf problemlos.

Ergebnisse. Als Ursachen für die Entstehung solcher diaphragmatischer Hernien werden $u$. a. eine durch Mangelernährung bedingte erhöhte Vulnerabilität des Zwerchfells (1) und latente Verletzungen während der Operation in Kombination mit erhöhtem intraabdominellem Druck durch unverhältnismäßig große Transplantate (2) diskutiert. Hinzu kommt, dass die Split-Organe in der Regel medial in die Bauchhöhle transplantiert werden, so dass eine Lücke zwischen rechter Bauchwand und Transplantat entsteht, in der das Zwerchfell ungeschützt und daher für die Entstehung einer rechtsseitigen $\mathrm{DH}$ prädestiniert ist. Die klinischen Symptome einer nach LTX erworbenen DH differieren nach Alter: Bei Kindern unter 1 Jahr kommt es eher zu respiratorischen, bei Kindern über 1 Jahr eher zu gastrointestinalen Symptomen. Der von uns beschriebene Fall zeigt als Besonderheit eine linksseitige DH. Dies ist untypisch und bisher nicht für diese Komplikation beschrieben. Allerdings finden sich, wie auch bei rechtsseitigen DH, die Faktoren Mangelernährung, Split-Organ und großes Transplantat in Relation zur Körpergröße.

Schlussfolgerung. Bei Kindern nach Lebertransplantation sollte bei akuten gastrointestinalen und/oder respiratorischen Symptomen auch die Differenzialdiagnose einer erworbenen DH bedacht werden.

1. Mc Cabe AJ, Orr JD, Sharik K et al (2005) Right-sided diaphragmatic hernia in infants after liver transplantation. Journal of Pediatr Surg 40:1181

2. Moon S-B, Jung S-M, Kwon C-H et al (2012) Posteromedial diaphragmatic hernia following pediatric liver transplantation, Pediatr Transplantation 16:106

\section{PO-07}

\section{Die frühzeitige Kallusdistraktion als alternative Therapieoption bei} Glossoptose

\section{J. Wilbrand', M. Frey², M. Wilbrand', P. Streckbein', D. Faas'², H. Howaldt', H. Ehrhardt ${ }^{2}$}

'Universitätsklinikum Gießen, Abt. für Mund-, Kiefer- und Gesichtschirurgie, Gießen, Deutschland, ${ }^{2}$ Universitätsklinikum Gießen, Zentrum für Kinderheilkunde der Justus-v.-Liebig-Universität, Gießen, Deutschland

Einführung. Glossoptose ist ein häufiges Phänomen bei Säuglingen mit mandibulärer Hypoplasie und Pierre-Robin-Sequenz. Rezidivierende Atemwegsverlegungen machen das postnatale Management dieser $\mathrm{Pa}$ tienten zu einer intensivmedizinischen Herausforderung. Lip-to-tongue-Adhäsionen (LTA) und permanente Tracheotomien (PT) werden in Einzelfällen notwendig. Diese Eingriffe sind jedoch für die Patienten, deren Eltern und das betreuende medizinische Personal mit hohen Belastungen verknüpft.

Material und Methoden. Ein Neugeborenes mit Franceschetti-Syndrom und konsekutiver Pierre-Robin-Sequenz zeigte postpartal rezidivierende Verlegungen der oberen Atemwege aufgrund der ausgeprägten Retrognathie mit Glossoptose. Trotz aller konservativen Maßnahmen inklusive Rachen-CPAP-Beatmung häuften sich die obstruktiven Ereignisse mit Hypoxie und Bradykardie. In einer interdisziplinären Therapiekonferenz von Neonatologen und Mund-, Kiefer- und Gesichtschirurgen wurde die frühzeitig Kallusdistraktion des Unterkiefers als alternative Behandlungsmethode zur LTA und PT diskutiert. Zusammen mit den Eltern wurde nachfolgend die Entscheidung für eine Distraktionstherapie getroffen. Einem Therapieprotokoll der Uni- 
versität Rom mit $\mathrm{n}=12$ Kindern folgend wurde im 2. Lebensmonat eine bilaterale Osteotomie der Mandibula im Bereich des Kieferwinkels vorgenommen und zwei unidirektionale externe Distraktoren inseriert. Es erfolgte eine rapide Distraktion des Unterkiefers um $2 \mathrm{~mm}$ pro Tag auf eine Gesamtstrecke von $20 \mathrm{~mm}$ in Allgemeinanästhesie.

Ergebnisse. Es wurde eine deutliche Überkorrektur des zuvor hypoplastischen Unterkiefers erreicht. Eine sechswöchige Ausheilungsphase führte zu suffizienter knöcherner Konsolidierung der distrahierten Mandibula. Atemwegsverlegungen wurden nach der Unterkieferdistraktion nicht mehr beobachtet und dem Kind der Saug-Schluck-Akt ermöglicht. Die Eingliederung einer Gaumen-Nasen-Trennplatte zur Abdeckung der U-förmigen Gaumenspalte führte zu einer Verbesserung der peroralen Ernährung des Kindes. Lokale fremdmaterialbedingte oder narkoseassozierte Komplikationen traten nicht auf. Das Behandlungsergebnis des exemplarischen Falls wird mit denen der römischen Kohorte abgeglichen.

Schlussfolgerung. Die präsentierten Fallbeispiele zeigen, dass die frühzeitige Kallusdistraktion des hypoplastischen Unterkiefers bei PierreRobin-Sequenz eine effiziente Methode darstellen kann, um obstruktive Apnoen zu minimieren und die LTA oder PT zu vermeiden. Der chirurgische Eingriff kann mit einem akzeptablen perioperativen Risiko durchgeführt werden. Die ostogene Potenz im frühen Säuglingsalter ist hoch genug, um schnelle Distraktionen durchzuführen und kurze Konsolidierungsphasen einzuhalten.

\section{PO-08}

Positive Erfahrungen mit der Videolaryngoskopie zur endotrachealen Intubation in Neonatologie und Pädiatrie für Ausbildung und Lehre

\section{T. Hoppen', S. Wien'², L. Nilles', V. Schneider', A. Hildebrandt', A. Krötz} I. Weis', T. Nüßlein

'Gemeinschaftsklinikum Koblenz-Mayen, Kemperhof Koblenz, Klinik für Kinder- und Jugendmedizin, Koblenz, Deutschland, ${ }^{2}$ Universitätsklinikum Bonn/Zentrum für Kinderheilkunde, Bonn, Deutschland

Hintergrund. Videoassistierte Laryngoskopietechniken können die Sicht auf den Larynx bei normalem und schwierigem Atemweg verbessern. In unserer Klinik kamen verschiedene indirekte Intubationssysteme zeitlich befristet zur Anwendung. Über unsere Erfahrungen mit dem C-MAC-Videolaryngoskop möchten wir berichten. Patienten: Bei 11 Patienten mit einem Alter zwischen 27+2 Schwangerschaftswochen (Gewicht $995 \mathrm{~g}$ ) und 11 Jahren (Gewicht $20 \mathrm{~kg}$ ) wurde eine Intubation mittels indirekter Laryngoskopie durchgeführt. Es handelte sich dabei um 6 Frühgeborene/Neonaten und 5 ältere Kinder.

Methode. Über einen Zeitraum von 6 Wochen erfolgte bei sämtlichen intubations- und beatmungspflichtigen Patienten, die im Frühdienst (7-15.00 Uhr) auf die interdisziplinäre neonatologisch-pädiatrische Intensivstation der Klinik aufgenommen wurden, die Intubation mittels indirekter Laryngoskopie. Zu Anwendung kam ausschließlich das C-MAC der Firma Karl Storz GmbH \& Co. KG (Tuttlingen) mit dem neuen Spatel MILLER o und Spateln MACINTOSH 2 bis 4. Zusätzlich erfolgte bei 3 Patienten eine videoskopische Larynxinspektion unter Beatmung.

Ergebnisse. Bis auf 1 Patienten gelang jede Intubation auf Anhieb. In 6 Fällen war die Glottis komplett, in 3 Fällen partiell einsehbar. Bei $2 \mathrm{~Pa}$ tienten mit anatomischen Besonderheiten waren auch unter optimaler Einstellung nur die Aryknorpel bzw. die hinteren Anteile der Stimmlippen einsehbar. Bei sämtlichen 5 Frühgeborenen kam der neue MILLERo-Spatel erfolgreich zum Einsatz. Insbesondere beim jüngsten Frühgeborenen dieser Serie war die Anwendung des MILLER-o-Spatels sicher und für alle Beteiligten komfortabel. Sieben Intubationen wurden von Assistenzärzten im 2.-4. Weiterbildungsjahr und 4 Intubationen von Fachärzten erfolgreich durchgeführt.

Schlussfolgerungen. Die indirekte Laryngoskopie stellt eine wesentliche Bereicherung in der Neonatologie und Pädiatrie dar. Dies gilt nicht nur für den schwierigen Atemweg sondern auch unter Weiterbildungs- und Dokumentationsgesichtspunkten für grundsätzlich jede Intubationsnotwendigkeit. Bei geübter Anwendung kann bei schwieriger Anatomie eine auch für Nebenstehende nachvollziehbar ausreichende Visualisierung des Trachea-Eingangs gelingen. Das C-MAC-Videolaryngoskop erlaubt neben der videoassistierten Technik auch eine direkte Laryngoskopie und somit eine kontinuierliche visuelle Kontrolle der Laryngoskop- und Tubusspitze. Während der kurzen Untersuchungsphase dieser Studie kam der MILLER-o-Spatel zur Markteinführung und wurde sogleich bei 5 Frühgeborenen sicher und für alle Beteiligten komfortabel eingesetzt. Die Präsenz eines Videolaryngoskops auf einer neonatologisch-pädiatrischen Intensivstation ist heutzutage insbesondere $\mathrm{zu}$ Weiterbildungszwecken wünschenswert. Inzwischen verfügen wir über das komplette System inklusive sämtlicher Spatel und zusätzlichem Pocket-Monitor. Eine vergleichende Wertung sämtlicher am Markt befindlicher Systeme für Kinder ist zu fordern.

\section{PO-09}

\section{Zilienschlag/-funktion bei reifen, gesunden Neugeborenen}

\section{W. Beckmann', M. Tekaat', H. Omran', E. Rieger-Fackeldey'}

'Westf. Wilhelms-Univ.- Kinderklinik, Allgemeine Pädiatrie, Münster, Deutschland

Hintergrund. Eine effektive Reinigung der Atemwege durch Zilienschlag ist bereits im Neugeborenenalter etabliert. Ziel dieser Untersuchung ist die Schaffung von Referenzwerten des Zilienschlages bei gesunden Neugeborenen in unserem Labor.

Ziel. Evaluation Zilienschlag/-funktion bei reifen, gesunden Neugeborenen.

Material und Methoden. Nasenbürstung bei 30 gesunden reifen Neugeborenen (inborn UKM September 2012 bis Januar 2013) in den ersten fünf Lebenstagen im Rahmen der U2, Einbringung des Materials in RPMI-Medium und anschließende Hochgeschwindigkeits-Videomikroskopie (Olympus IMT-2 Phasen-Kontrast Mikroskop mit Redlake ES-310-Turbo Monochrom Hochgeschwindigkeits-Videokamera) Vergrößerung des Objektivs 40X, digitale Sammelrate 125 frames/s, $640 \times 480$ Auflösung) in Raumtemperatur. Die Zilienschlagfrequenz wird mit dem SAVA-System ausgewertet (Sava Software Solutions) nach publizierten Kriterien von O'Callaghan.

Ergebnisse. Bei allen untersuchten Neugeborenen waren zilierte Zellen im Bürstenmaterial nachweisbar, die Zilienqualität wurde bei $20 \mathrm{~Pa}$ tienten als gut bewertet und das Material in die Auswertung einbezogen. Die Zilien Neugeborener erscheinen lang und gebogen, sie schlagen in einer Ebene vor und zurück. Die Zilienschlagfrequenz liegt bei $5,6 \mathrm{~Hz}$ (Median; Range: 2,2-12,4).

Schlussfolgerung. Damit liegt die Zilienschlagfrequenz dieser Gruppe gesunder Neugeborener niedriger als von O'Callaghan 1991 publiziert $(12,7 \mathrm{~Hz} / 0,84)$ [Mean; Stabw], wobei diese Messungen bei $37^{\circ} \mathrm{C}$ und mit einer anderen Methode vorgenommen wurden. Wir werden Messungen bei $37^{\circ} \mathrm{C}$ anschließen.

\section{PO-10}

Beschleunigter mikrozirkulatorischer Fluss als frühes Zeichen neonataler Infektionen

\section{A. Schwepcke', D. Preußer', O. Genzel-Boroviczény}

${ }^{1}$ Klinikum der Universität München Innenstadt, Perinatologie, München, Deutschland

Hintergrund. Infektionen stellen eine häufige Komplikation in der Versorgung reifer Neugeborener dar. Eine frühzeitige Erkennung und Therapie gefährdeter Neugeborener ist erforderlich, um schwere Verläufe zu vermeiden. Da die wiederholten Blutentnahmen Neugeborene belasten, wäre ein nichtinvasives Screening wünschenswert. Mittels nicht- 
invasiver Lichtmikroskopie können Veränderungen der Mikrozirkulation bei Infektionen gemessen werden.

Fragestellung. Können Änderungen der Mikrozirkulation bei Infektionen bei Neugeborenen frühzeitig direkt gemessen werden?

Material und Methoden. Im Zeitraum März bis April 2011 wurden prospektiv alle konsekutiv geborenen reifen Neugeborenen (Gestationsalter $37+0-42+0$ SSW, Geburtsgewicht $>2000$ g) rekrutiert und mit Einverständnis der Eltern untersucht. Die Untersuchung der Mikrozirkulation erfolgte nicht invasiv mittels Sidestream Dark Field Imaging an der Ohrmuschel einmalig zwischen 1.-3. Lebenstag. Es wurden folgende Parameter zur Bewertung der Mikrozirkulation erhoben: die funktionelle Gefäßdichte, Gefäßoberfläche, sowie die Qualität des Flusses. Die Qualität des Flusses wurde mit einem Scoresystem bewertet. So wurde zwischen einem kontinuierlichen (Score $=3$ ), einem hyperdynamen $($ Score $=4)$, einem intermittierenden $($ Score $=2)$, einem zähen (Score $=1$ ) und keinem Fluss $($ Score $=0$ ) unterschieden. Die Auswertung erfolgte anhand eines automatisierten Auswertungsprogramms (AVA von Microvision). Der Untersucher war zum Zeitpunkt der Messung bzgl. der Infektionswerte der Kinder geblindet. Zur Auswertung wurden die untersuchten Kinder hinsichtlich der Infektionsparameter in 2 Gruppen unterteilt: Kinder mit einem $\mathrm{CrP}>0,5 \mathrm{mg} / \mathrm{dl}$ wurden der Infektionsgruppe zugeordnet, der Rest der Kinder bildete die Vergleichsgruppe.

Ergebnisse. Von den insgesamt 110 reifen Neugeborenen mit einem mittleren Gestationsalter von 40 Schwangerschaftswochen war bei $29 \mathrm{Neu}-$ geborenen das $\mathrm{CrP}>0,5 \mathrm{mg} / \mathrm{dl}$ [mittleres $\mathrm{CrP}$ 1,6 mg/dl (95\% CI 1-2,1); Infektionsgruppe (Inf)]. 81 Neugeborene bildeten die Vergleichsgruppe (Kontrolle). In der Inf zeigte sich ein höherer Anteil an hyperdynamen Flüssen als in der Kontrolle, sowohl bei sofortiger manueller als auch bei der automatischen Auswertung [Score MW (95\% CI); Inf 3,2 (3,1-3,3) vs. Kontrolle 3,1 (3-3,1); p<0,0oo1]. Die Gefäßoberfläche war in der Inf signifikant niedriger [Inf $25(24,1-25,9)$ vs. Kontrolle $26[25,5-26,6] \%$; $\mathrm{p}<0,05]$. Die funktionelle Gefäßdichte war nicht unterschiedlich.

Schlussfolgerung. Bei neonatalen Infektionen zeigen sich in der Intravitalmikroskopie bereits bei geringen CrP-Erhöhungen Veränderungen der Mikrozirkulation mit einem höheren Anteil an hyperdynamen Fluss und einer Reduktion der Gefäßoberfläche. Der hyperdyname Fluss ist bereits während der Untersuchung mit dem bloßen Auge erkennbar. Die Einsetzbarkeit als nichtinvasiver Screeningtest in der frühen Infektionsdiagnostik sollte deshalb in größeren Studien evaluiert werden.

\section{PO-11}

Schmerzevaluierung einer intramuskulären, passiven Hepatitis-BImmunisierung beim reifen Neugeborenen

\section{Steiner', K. Klebermaß-Schrehof', T. Werther', J. Straub', A. Pollak', A. Berger}

'Universitätsklinik für Kinder- und Jugendheilkunde, Medizinische Universität Wien, Abt. für Neonatologie, pädiatrische Intensivmedizin und Neuropädiatrie, Wien, Österreich

Hintergrund. Die neonatale Hepatitis-B-Postexpositionsprophylaxe mittels aktiver und passiver Immunisierung hat $\mathrm{zu}$ einer dramatischen Reduktion der perinatalen Hepatitis-B-Transmission geführt. An unserer Abteilung wurde bislang die aktive Impfung intramuskulär gegeben, während die passive Immunisierung in Ermangelung eines entsprechenden Präparates intravenös verabreicht wurde. Im Jahr 2011 erfolgte die Umstellung der passiven Immunisierung von einer intravenösen Verabreichung auf eine intramuskuläre Gabe (Uman$\mathrm{Big}^{\circledR}$ ). Wichtiger Beweggrund für die Umstellung war es, im Sinne einer maximal möglichen Schmerz- und Stressreduktion in dieser sensiblen Lebensphase die Notwendigkeit eines peripher-venösen Zuganges zu vermeiden. Andererseits muss auch für die intramuskuläre Gabe eine möglichst schmerzarme Verabreichung gewährleistet sein.
Fragestellung. Unser Ziel war es, im Zuge der Umstellung auf die intramuskuläre passive Immunisierung deren klinische Tolerabilität bei Neugeborenen anhand des Schmerzverhaltens zu erfassen.

Material und Methoden. Von Juli 2011 bis Dezember 2012 wurden insgesamt 17 reife Neugeborene im Rahmen einer prospektiven Beobachtungsstudie untersucht. Aktive und passive Immunisierung erfolgten rasch nacheinander intramuskulär in den jeweils kontralateralen M. vastus lateralis. Es wurden die Herzfrequenz vor, unmittelbar nach und 10 Minuten nach der Immunisierung erfasst, sowie die Schmerzscores NPASS und NIPS unmittelbar nach und 10 Minuten nach Immunisierung evaluiert. Zudem wurde die Schreidauer nach beiden intramuskulären Injektionen erfasst.

Ergebnisse. Die Herzfrequenz zeigte einen geringen Anstieg unmittelbar nach Immunisierung $(141 \pm 11 / \mathrm{min}$ vs. $160 \pm 15 / \mathrm{min})$, und lag nach 10 Minuten wieder auf Höhe des Ausgangswertes ( $141 \pm 13 / \mathrm{min})$. Die Schreidauer nach Immunisierung betrug im Mittel $16 \pm 15$ Sekunden und beide Schmerzscores zeigten unmittelbar nach Immunisierung niedrige Werte, die nach 10 Minuten noch weiter abgesunken waren (NPASS und NIPS $2 \pm 2$ vs. $0 \pm 0$ ).

Schlussfolgerung. Die aktive und passive intramuskuläre Hepatitis-BImmunisierung ist beim Neugeborenen mit einer minimalen Schmerzund Stressreaktion assoziiert.

\section{PO-12}

\section{Homöopathie in der Neonatologie - Gibt es eine Evidenz?}

\section{Thiel ${ }^{1}$}

'Sana-Klinikum Remscheid, Klinik für Kinder und Jugendliche, Remscheid, Deutschland

Hintergrund. Alternative und komplementäre Methoden sind in der Medizin weltweit sehr beliebt. Wirkung, auch Wirkungsweisen sowie potentielle Nebenwirkungen sind nicht geklärt. Theoretisch sind Neugeborene auch aus wissenschaftlicher Sicht eine sehr interessante $\mathrm{Pa}$ tientengruppe, da der Placebo-Effekt außer durch die Beeinflussung durch die Eltern oder die verabreichende Bezugsperson sehr gering ist. Methodik. Aus diesem Grund führten wir eine systematische Review der Literatur zu diesem Thema durch.

Ergebnisse. Die Medline-Recherche nach den PRISMA-Kriterien lieferte bei der Suche nach „Neonates“, „newborn“, „neonatology“ und „homeopathy“ insgesamt 61 Studien. Davon waren 41 in den letzten 10 Jahren publiziert worden. Insgesamt beschäftigten sich aber nur 4 mit Neonaten i. e. S. Dabei handelte es sich um Fallberichte und Übersichtsarbeiten. Eine Kasuistik berichtet von einem 18 Tage alten Neugeborenen mit schwerer Atemstörung und myoklonischen Anfälle, die sich unter Therapie mit Opium $\mathrm{C}_{3}$ o besserten. Eine weitere Kasuistik berichtete über eine Nebenwirkung und mögliche Erklärungen nach der Anwendung eines homöopathischen Präparates. Zwei Übersichten zur Anwendung komplementärer und alternativer Verfahren im Kindesalter berührte die Homöopathie in der gesuchten Altersgruppe nur marginal. Es fanden sich 7 klinische Studien, von denen keine sich mit Neonaten beschäftigte. Systematische Reviews zum Thema fehlen.

Schlussfolgerung. Für den Einsatz der Homöopathie in der Neonatologie gibt es keine durch Studien abgesicherte Grundlage. Vereinzelten anekdotischen Erfolgsgeschichten stehen auch Berichte über Nebenwirkungen gegenüber. 


\section{PO-13}

Zeitpunkt, Art und Umfang des Überbringens schwieriger Nachrichten an die Eltern von Neugeborenen - am Beispiel des pränatal nicht erkannten Down-Syndroms

\section{N. Lorenz' ' S. Schmidt' ' A. Nordwig' ', M. Kabus' ', G. Heubner ${ }^{1}$ \\ 'Städtisches Krankenhaus, Zentrum für Kinder- und Jugendmedizin, Dresden, Deutschland}

Einleitung. Bereits 1964 wurde in einer wegweisenden Arbeit gezeigt, dass Mütter umso zufriedener mit dem Zeitpunkt der Überbringung der Information über das Vorliegen einer Trisomie 21 bei ihrem Kind sind, je früher ihnen diese Nachricht überbracht wird [1]. Trotz einer seitdem verbesserten Information der breiten Öffentlichkeit und des medizinischen Fachpersonals über das Down-Syndrom bestehen auch heute noch eminenzbasierte Unsicherheiten über den richtigen Umgang mit dieser Nachricht. Das Problem ist aktuell, so wurden 2006-2012 im Perinatalzentrum Dresden-Neustadt 16 Neugeborene mit klinischem Verdacht auf Down-Syndrom aufgenommen, und die Eltern unmittelbar informiert. 13 Neugeborene bedurften aufgrund downsyndromassoziierter neonataler Anpassungsstörungen oder Fehlbildungen einer medizinischen Behandlung, alle Neugeborenen wurden in die Häuslichkeit entlassen.

Methode. Pubmed-Recherche (OLDMEDLINE, MEDLINE, PubmedCentral) mit: [Down syndrome. Trisomy 21. genetic counselling], [communication, newborn, down syndrome], [bad news OR difficult news, newborn]. Google-Recherche: nach Leitlinien zur Kommunikation der Diagnose Trisomie 21.

Ergebnisse. 89 Arbeiten und eine US-amerikanische Leitlinie wurden selektiert. Mehr als die Hälfte der selektierten Arbeiten (46) erschienen erst nach 1999. Alle aktuellen evidenzbasierten Empfehlungen raten, bei Neugeborenen mit Verdacht auf das Vorliegen einer Trisomie 21 die Nachricht an beide Eltern unmittelbar, strukturiert, mit standardisierten Inhalten, gemeinsam durch Geburtshelfer und Kinderarzt zu überbringen. In diesem Erstgespräch sollten neben dem Erklären der klinischen Zeichen des Downsyndroms und dem Hinweis auf mögliche Fehlbildungen vor allem die positiven Aspekte des Lebens von Menschen und mit Menschen mit Down-Syndrom sowie Möglichkeiten der Förderung herausgestellt werden. Den betroffenen Eltern sollte der Kontakt zu lokalen Selbsthilfegruppen empfohlen werden.

Diskussion. Für betroffene Familien regelt im Perinatalzentrum eine interne Handlungsanweisung (SOP) diese Abläufe. Das Verschweigen des Verdachtes auf Down-Syndrom ist heute mehr denn je obsolet.

1. Drillien CM, Wilkinson EM (1964) Mongolism: When should Parents be told?. Brit med J 2:1306-13

\section{Gastroenterologie - Ernährung - Stoffwechsel}

\section{PO-14}

\section{Gastroösophageale Perforationen bei Frühgeborenen - meist eine} iatrogene Komplikation

\section{T. Dresbach', P. Bartmann', A. Müller'}

'Zentrum für Kinderheilkunde am Universitätsklinikum Bonn, Neonatologie, Bonn, Deutschland

Hintergrund. Eine Perforation von Ösophagus oder Magen in der Neonatalperiode ist ein seltenes Ereignis, welches meist iatrogen verursacht ist, und mit einer hohen Mortalität einhergeht. Wir berichten von drei Patienten mit gastroösophagealen Perforationsverletzungen. Patient 1: Traumatische Ösophagusruptur nach Anlage einer nasogastralen Ernährungssonde. Patient 2: Magenruptur durch nasogastrale Ernährungssonde. Patient 3: Magenruptur infolge von Drucknekrose durch nasogastrale Ernährungssonde.

Diskussion. Die meisten Perforationen des Magens und des Ösophagus in der Neonatalperiode sind iatrogen und mit einer Mortalität bis zu
$30 \%$ beschrieben. Ursächlich sind häufig akute traumatische Ereignisse, durch die Anlage von Ernährungssonden oder fehlplazierte Trachealtuben. Aber auch Perforationen, die durch Druckulzera infolge einer Fehllage oder längerer Liegedauer von Ernährungssonden entstehen, wurden beschrieben. Spontane Perforationen sind selten. Das Risiko einer akzidentellen Verletzung durch Ernährungssonden steigt mit der Unreife der Patienten, sowie in Phasen mit CPAP-Atemtherapie. Bei akuter klinischer Verschlechterung eines zuvor stabilen Patienten sollte immer an eine gastroösophageale Verletzung gedacht werden. Zur Minimierung des Verletzungsrisikos sollten weiche Silikonsonden verwendet werden. Bei schwieriger Anlage sollte die Lage des Kindes modifiziert werden und ein erneuter Versuch erfolgen. Eine umgehende Lagekontrolle der Ernährungssonde ist obligat, Untersuchungen zeigen, dass nahezu jede zweite Ernährungssonde bei neonatologischen Patienten einer Lagekorrektur bedarf Bei der Aspiration an der Magensonde ist darauf zu achten, nicht zu viel Sog aufzubauen und somit mögliche ischämische Verletzungen der Magenschleimhaut zu verhindern. Eine Sensibilisierung des Pflegepersonals hinsichtlich dieser Risiken, sowie der Notwendigkeit der Dokumentation über die Liegedauer und Lage sollte regelmäßig erfolgen, und ist von großer Bedeutung.

\section{PO-14a}

Nekrotisierende Enterokolitis ist ein Risikofaktor für nosokomiale Infektionen bei Neugeborenen

\section{A. Edle von Oetinger', S. Gfrörer' ${ }^{2}$, D. Fischer ${ }^{3}$, U. Rolle ${ }^{4}$, R. Schlößer}

'Klinikum der J.W.Goethe-Universität, Zentrum der Kinderheilkunde, Abteilung für Neonatologie, Frankfurt am Main, Deutschland, ${ }^{2}$ Klinikum der J.W.Goethe-Univ. Zentrum der Chirurgie, Kinderchirurgie, Frankfurt, Deutschland, ${ }^{3}$ Klinikum der Johann Wolfgang Goethe-Universität, Zentrum der Kinderheilkunde, Klinik I, Neonatologie, Frankfurt, Deutschland, ${ }^{4}$ Universitätsklinikum der Johann-Wolfgang-Goethe-Universität Frankfurt am Main, Klinik für Kinderchirurgie, Frankfurt, Deutschland

Hintergrund. Nosokomiale Infektionen (NI) sind ein wichtiger Grund für Mortalität und Morbidität von Neugeborenen auf Intensivstationen. Fragestellung. Ist eine voran gegangene Nekrotisierende Enterokolitis (NEC) ein Risikofaktor für nachfolgende nosokomiale Infektionen? Material und Methoden. In einer retrospektiven Analyse wurden alle Patienten des Schwerpunktes Neonatologie in der Universitätsklinik Frankfurt/Main mit der Diagnose NEC aller Grade aus den Jahren 2009 bis 2011 identifiziert. Der Patientengruppe wurde durch Matchedpairs-Analyse (zuerst Gestationsalter, dann Geburtsgewicht) eine Kontrollgruppe gegenübergestellt. Neben der Häufigkeit von NI wurden auch Risikofaktoren (HELLP-Syndrom der Mutter, Nahrungsmenge am 7. und 14. Lebenstag) oder andere Folgen (Ernährung, Wachstum und Cholestase nach NEC) analysiert und mit den Daten der Kontrollgruppe verglichen.

Ergebnisse. In der Patientengruppe und der Kontrollgruppe waren jeweils 59 Patienten, deren Charakteristika (Gestationsalter und Geburtsgewicht) vergleichbar waren. In der Patientengruppe erkrankten 59 an einer NI, in der Kontrollgruppe 51. Die mittlere Anzahl der Sepsis-Episoden nach NEC betrug 2,9, in der Kontrollgruppe 2,2 ( $\mathrm{p}<0,05)$. Es ergab sich eine Risikoerhöhung von 2,3 (CI=1,01-5,35). Ein HELLPSyndrom der Mutter kam in den Anamnesen der NEC-Gruppe 5-mal, in der Kontrollgruppe zweimal vor. Der Nahrungsaufbau erfolgte in der NEC-Gruppe zögerlicher als in der Kontrollgruppe. Eine Cholestase war nach NEC signifikant häufiger ( $\mathrm{OR} 1,5 ; \mathrm{CI}=1,08-2,20)$. Die Kinder mit NEC wurden länger parenteral ernährt als die ohne (im Mittel 34 Tage vs. 16 Tage; $\mathrm{p}<0,001)$. Nach korrigierten 38 SSW waren die Körperlänge und der Kopfumfang bei NEC-Patienten statistisch signifikant geringer als bei den Patienten der Kontrollgruppe ( $<<0,001)$.

Schlussfolgerung. Die vorliegende Untersuchung belegt ein höheres Risiko für nosokomiale Infektionen nach einer statt gehabten NEC. Als Grund dafür käme die längere parenterale Ernährung mit zentralen 
Venenkathetern, aber auch der funktionelle oder anatomische Verlust von immunologisch wichtigen Darmanteilen in Frage.

\section{PO-15}

\section{Colon-transversum-Atresie - eine Fallvorstellung}

\section{Irion', T. Bielefeld', H. Engels', A. Trotter ${ }^{1}$ \\ 'HBH-Kliniken, Klinik für Kinder und Jugendliche, Singen, Deutschland}

Hintergrund. Die Kolon-Atresie stellt eine seltene Form der intestinalen Fehlbildungen dar, die Inzidenz wird auf 1:40.0oo geschätzt. Die Pathogenese der Kolon-Atresie ist ungeklärt.

Befund. Eutrophes reifgeborenes Mädchen, unauffällige Schwangerschaft und Geburt, problemlose postnatale Adaptation bis zur Vorstellung. Im Alter von 30 Stunden Vorstellung mit Subileussymptomatik, rezidivierendem galligem Erbrechen und massiv geblähtem Abdomen, bis zur Vorstellung kein Absetzen von Mekonium. Kein Hinweis auf äußere Fehlbildungen.

Diagnostik. Abdomen-Sonographie: Magen und Darm im rechten Oberbauch massiv erweitert und luft- und flüssigkeitsgefüllt. Deutlich Aszites, Hepatomegalie. Röntgen Abdomen mit Kontrastmittel: Massiv aufgeweitete Darmschlinge, die sich auf den rechten Abdominalraum projiziert mit kaudaler koprostatischer Verschattung. Unauffällige Darstellung des Magens. Keine Luftfüllung im Bereich des Rektums. Labor: Leukozyten $17.400 / \mu \mathrm{l}, \mathrm{Hb} 17,9 \mathrm{~g} / \mathrm{dl}, \mathrm{Hkt} 48 \%$, Thrombozyten $27400 \mathrm{o} / \mu \mathrm{l}$, $\mathrm{CrP}$ o,3 mg/dl, IL-6 76 pg/ml.

Therapie. Bei dringendem Verdacht einer intestinalen Atresie Entscheidung zur Laparatomie. Intraoperativ zeigte sich eine Colon-transversum-Atresie ohne Verbindung zwischen dem Colon ascendens und dem Colon descendens. Aufgrund des intraoperativen Befundes erfolgte nach Ausschluss weiterer Fehlbildungen die Anlage einer primären End-zu-Seit-Anastomose. Postoperativ zunächst Nahrungskarenz für 5 Tage, danach sehr langsamer Beginn des Kostaufbaus mit Glukose und Muttermilch nach dem Minimal-Feeding-Prinzip. Nach 4 Wochen war der orale Nahrungsaufbau abgeschlossen und das Kind konnte nach Hause entlassen werden.

Diskussion und Schlussfolgerung. Die hier dargestellte klinische Präsentation zeigt die typischen Symptome einer distalen intestinalen Atresie mit Subileussymptomatik und rezidivierendem Erbrechen. In diesem Fall erfolgte aufgrund des intraoperativen Befundes mit deutlicher Lumenverkleinerung und guter Tonisierung des proximalen Anteils nach Entfernung von Mekonium eine primäre Anastomosierung. Der langsame Kostaufbau nach dem Minimal-Feeding-Prinzip über 4 Wochen mit Glukose und Muttermilch wurde zur Konditionierung des Kolon mit weiterhin erhaltener Resorptionsfähigkeit und möglichst rascher Normalisierung der Darmmotilität durchgeführt.

\section{PO-16}

\section{Milchpfropf-Syndrom des Colon bei einem ELBW-Frühgeborenen}

\section{B. Albayrak', S. Horsch', R. Tröbs' ${ }^{2}$, C. Roll}

'Vestische Kinder- und Jugendklinik Datteln Universität Witten/Herdecke, Abteilung Neonatologie und Pädiatrische Intensivmedizin, Datteln, Deutschland, ${ }^{2}$ Kinderchirurgische Universitätsklinik der Ruhr Universität Bochum im Marienhospital Herne, Kinderchirurgie, Herne, Deutschland

Hintergrund. Das sogenannte Milchpfropf-Syndrom ist eine sehr seltene gastrointestinale Komplikation des Früh- und Neugeborenen. Ein Konglomerat aus nicht verdauter, eingedickter Milch (Hauptbestandteil sind Fett und Kalzium) und abgeschilferten Darmepithelien - auch als Lactobezoar bezeichnet - führt zu gastrointestinalen Obstruktion und Ileus, in deren Folge es zur Sepsis kommen kann. Ursächlich werden eine hochkalorische Nahrung mit hohem Anteil an Fett, Protein und Kalzium, Dehydratation sowie die Unreife des gastrointestinalen Trakts des Früh- und Neugeborenen per se diskutiert.
Fallvorstellung. Wir präsentieren den Fall eines männlichen Frühgeborenen (Gestationsalter 27+3 Wochen, Geburtsgewicht $790 \mathrm{~g}$ ) mit Milchpfropf-Syndrom. Zunächst war der Verlauf unkompliziert, und der Junge war seit dem 11. Lebenstag voll enteral ernährt. Die Muttermilch wurde mit 5\% FMS und zusätzlich Protein angereichert. In der 4. Lebenswoche traten vermehrt Apnoen und Sättigungsabfälle auf, der Bauch war ausladend. In der linken Flanke und im Unterbauch waren derbe Resistenzen zu tasten. Die Röntgenaufnahme zeigte einen Ileus und eine röntgendichte Masse im Darmlumen mit umgebendem intraluminalem Luftsaum - das typische, „pathognomonische“ radiologische Bild des Milchpfropf-Syndroms. Nach rektalem Anspülen mit Laxantien wurde zwar reichlich fester weißlicher Stuhl abgesetzt, die Resistenzen und die Ileussymptomatik bestanden jedoch fort. Daher wurde bei Verschlechterung des Allgemeinzustandes mit Symptomen einer Sepsis eine Laparotomie durchgeführt. Intraoperativ zeigte sich das gesamte Colon descendens mit dem Milchpfropf obstruiert. Eine Kolostomie wurde angelegt. Der Junge erholte sich und ist im Alter von 7 Monaten klinisch unauffällig.

Diskussion. Ob die Proteinsubstitution zur Optimierung der Eiweißzufuhr, wie sie aktuell von der ESPGHAN für Frühgeborene mit einem Geburtsgewicht $1000 \mathrm{~g}$ empfohlen wird, das Risiko für die Entstehung des Milchpfropf-Syndroms erhöht und dieses somit in Zukunft häufiger auftreten wird, steht zur Diskussion. In unserem Fall ungewöhnlich ist die Lokalisation der Obstruktion im Colon. In den meisten Fällen ist der Magen oder Dünndarm betroffen. Die Kenntnis des typisch radiologischen Befundes des Milchpfropf-Syndroms ermöglicht eine frühzeitige, präoperative Diagnose, die bei einem stabilen Kind einen konservativen Therapieversuch rechtfertigt - auch wenn dieser in unserem Fall nicht erfolgreich war.

\section{PO-17}

Die Bedeutung der Zeichen der Nahrungsintoleranz zur Diagnosestellung einer nekrotisierenden Enterokolitis bei Frühgeborenen

\section{Frost', A. Philos', A. Mueller', H. Reutter', L. Welzing', P. Bartmann ${ }^{1}$ S. Bagci'}

'Zentrum für Kinderheilkunde, Universitätsklinikum Bonn, Abt. Neonatologie, Bonn, Deutschland

Hintergrund und Fragestellung. Isolierte blutige oder gallige Magenreste sowie Blut im Stuhl sind klinische Zeichen der Nahrungsintoleranz oder der nekrotisierenden Enterokolitis (NEK). Das Ziel dieser Studie war es, die Bedeutung der klinischen Zeichen einer Nahrungsintoleranz bei der Diagnosestellung einer NEK zu evaluieren.

Patienten und Methoden. In dieser retrospektiven Fall-Kontroll-Studie (im Verhältnis 1:2) wurden 65 NEK-Patienten ab Stadium 2a nach Bell und 130 Kontrollpatienten aus einem Zeitraum von 11 Jahren (20002010) eingeschlossen. Das Gestationsalter und Geburtsgewicht war in beiden Gruppen ähnlich ( $\mathrm{p}>0,05)$. Der Manifestationszeitpunkt der NEK [Median (Interquartilabstand(IQR)] lag bei den betroffenen Kindern bei 9 (7-14) Tagen. Die Zeichen einer Nahrungsintoleranz, wie Magenrestvolumen, gallige oder blutige Magenreste oder Blut im Stuhl, wurden zwischen beiden Gruppen ab Geburt bis zum Manifestationszeitpunkt der NEK [Tag o (To)] verglichen.

Ergebnisse. In den ersten 72 Lebensstunden fand keine Mekoniumausscheidung bei $15 \%$ der Kinder in der NEK-Gruppe (NEK-G) gegenüber $19 \%$ der Kinder in der Kontrollgruppe (K-G) statt $(\mathrm{p}=0,691)$. Der maximale Magenrest (mMR proKg) war in letzten 6 Tagen vor To in beiden Gruppen ähnlich (NEK-G vs. K-G, p>0,05 für T6, T5, T4, T3, T2, T1; mMR pro Tag war wechselnd zwischen $2,1 \mathrm{ml} / \mathrm{kg} / \mathrm{d}(1,5-4,6)$ und $3,1 \mathrm{ml} / \mathrm{kg} / \mathrm{d}(1,6-4,8)$ in der NEK-G und zwischen $2,1 \mathrm{ml} / \mathrm{kg} / \mathrm{d}(1,2-4,6)$ und $2,9 \mathrm{ml} / \mathrm{kg} / \mathrm{d}(1,5-5,6)$ in der K-G). Im Vergleich mit den letzten 6 Tagen vor To zeigte sich ein signifikanter Anstieg der Magenreste in der NEK-G (mMR am To=5,9 ml/kg/d (3,4-8,3), p<o,oo1 für To vs. T61), während keine signifikante Änderung in der K-G auftrat (mMR am To=2,6 ml/kg/d (1,6-4,o), p>0,05 für To vs. T6-1; mMR in der NEK-G 
vs. mMR in der K-G am To, p<o,oo1). Die beiden Gruppen unterschieden sich nicht hinsichtlich des Auftretens von blutigen (NEK-G vs. K-G, $46 \%$ vs. $48 \%$, $p=0,880$ ) oder galligen MR (NEK-G vs. K-G, $66 \%$ vs. $59 \%$, $p=0,269)$. Sowohl der erste blutige als auch der erste gallige MR trat in beiden Gruppen im Median am 3. LT auf ( $p>0,05)$. Es gab keinen Unterschied zwischen beiden Gruppen hinsichtlich der Anzahl an Tagen mit blutigen oder galligen MR [NEK-G vs. K-G, 2 Tage (1-3) vs. 1 Tage (1-3) und 3 Tage (1-4) vs. 2 Tage (1-3), $p=0,734$ und $p=0,769$ für blutigen und galligen MR]. 46\% der Kinder in der NEK-G hatten Blut (okkult oder makroskopisch) im Stuhl gegenüber $15 \%$ der Kinder in der K-G $(\mathrm{p}<\mathrm{o}, 001)$. Blut im Stuhl trat erstmalig am 6. LT (6-13) in der NEK-G gegenüber am 7. LT (5-12. LT) in der K-G $(\mathrm{p}=0,515)$ auf.

Diskussion. Die großen Überschneidungen zwischen der NEK-G und der K-G zeigen, dass die Zeichen einer Nahrungsintoleranz unsichere klinische Indikatoren für eine NEK sind. Erst in den letzten $24 \mathrm{~h}$ vor Diagnosestellung waren die Magenreste in der NEK-G signifikant erhöht. Die untersuchten gastrointestinalen Symptome geben also keinen früheren Hinweis auf die Entwicklung einer NEK.

\section{PO-18}

Einfluss früh-infantiler Ernährung auf Stoffwechselparameter und Körperzusammensetzung im Grundschulalter bei extrem leichten Frühgeborenen (ELBW)

\section{S. Stutte', A. Peiler', M. Born' ${ }^{3}$, P. Bartmann ${ }^{4}$, B. Gohlke ${ }^{5}$, J. Wölfle ${ }^{5}$}

'Universitätsklinik Zentrum f. Kinderheilkunde, Neonatologie, Bonn, Deutschland, ${ }^{2}$ Universitätsklinik Zentrum f. Kinderheilkunde, Endokrinologie, Bonn, Deutschland, ${ }^{3}$ Radiologische Klinik Universitätsklinikum Bonn, Bonn, Deutschland, ${ }^{4}$ Universitätsklinikum Bonn, Zentrum für Kinderheilkunde, Abt. Neonatologie, Bonn, Deutschland, ${ }^{5}$ Universitätsklinik Zentrum f. Kinderheilkunde, Bonn, Deutschland

Fragestellung. Eine rasche Gewichtsnormalisierung bei Frühgeborenen scheint sich nicht nur positiv auf das unmittelbar postnatale Wachstum auszuwirken sondern im Erwachsenenalter auch mit einem höheren Risiko für die Entwicklung eines metabolischen Syndroms assoziiert zu sein. Gibt es bereits im Grundschulalter messbare negative Auswirkungen der früh-postnatalen Ernährung auf Stoffwechselparameter und Körperzusammensetzung bei extrem leichten Frühgeborenen?

Patienten und Methodik. Das Patientenkollektiv umfasst 39 (17 männlich) gesunde, altersentsprechend entwickelte Kinder (mittleres Alter: 9,5 Jahre), die mit einem Geburtsgewicht $<1000 \mathrm{~g}$ an der Universitätsklinik Bonn geboren wurden. Die Geburtsmaße sowie Angaben über die frühe postnatale Ernährung wurden retrospektiv den stationären Akten entnommen, die Wachstumsparameter im Verlauf im Rahmen ambulanter Vorstellungen erhoben. In einer aktuellen Follow-up-Untersuchung wurden die Körperzusammensetzung aus Knochen-, Fett- und Muskelmasse mittels Dual-Röntgen-Absorptiometrie(DEXA)-Messung bestimmt sowie Nüchternblut abgenommen. Aufholwachstum Gewicht $(A w G)$ bedeutet: Gewichtszunahme >0,5 SDS zwischen Entlassung +6. Lebensmonat Aufholwachstum Länge (AwL) bedeutet: Längenzunahme $>0,5$ SDS zwischen 6.+12. Lebensmonat.

Ergebnisse. Die höchste Gewichtszunahme des gesamten Beobachtungszeitraumes findet statt zwischen Entlassung und 6. Lebensmonat $(+1,21$ SDS). Die Phase der größten Zunahme der Körperlänge wird zwischen dem 6 . und 12. Lebensmonat beobachtet $(+4,28$ SDS) - nach deutlichem Catch-down im ersten Lebenshalbjahr. Unter den Komponenten der früh-infantilen Ernährung korreliert besonders die Höhe der Aminosäuren-Zufuhr mit dem Zeitraum der höchsten Gewichtszunahme. Die Höhe der Aminosäuren-Zufuhr beeinflusst zudem signifikant den Körperfettanteil, nicht die Gesamt-Muskelmasse. Es besteht nicht nur eine Assoziation der Aminosäurenzufuhr zu einer signifikant höheren Gesamt-Fettmasse $(r=0,481 ; p=0,007)$, sondern vor allem $\mathrm{zu}$ einem höheren abdominellen Fettanteil $(r=0,488 ; p=0,006)$ im Grundschulalter. Das Körperlängen-Aufholwachstum hat weder Einfluss auf den späteren Körperfettanteil noch auf die Gesamt-Muskelmasse.
Bezüglich der Stoffwechselparameter im Grundschulalter korreliert die Aminosäuren-Zufuhr von Geburt zur Entlassung positiv mit dem Nüchtern-Insulinwert $(\mathrm{r}=0,581 ; \mathrm{p}=0,003)$ und ist negativ assoziiert mit dem nüchtern HDL-Cholesterin-Wert $(\mathrm{r}=-0,445 ; \mathrm{p}=0,014)$.

Schlussfolgerung. Die unmittelbar postnatale Gewichts- und Längenentwicklung verläuft bei extrem leichten Frühgeborenen stark divergent. Besonders eine hohe Aminosäurenzufuhr von Geburt bis zur Entlassung bewirkt eine überdurchschnittliche Gewichtszunahme im ersten Lebenshalbjahr sowie eine erhöhte abdominelle Fettmasse und auch eine beginnende Insulinresistenz im Grundschulalter. Somit finden sich schon im durchschnittlichen Alter von 9,5 Jahren unter den extrem leichten Frühgeborenen erste Anzeichen eines metabolischen Syndroms.

\section{PO-19}

Vitamin B12- und Folsäurestoffwechsel bei Frühgeborenen $<1500 \mathrm{~g}$ und/oder $<32$ SSW

\section{Witt' ${ }^{1}$ T. Ott' ${ }^{1}$, H. Omran' ${ }^{1}$, T. Marquardt' ${ }^{1}$, E. Rieger-Fackeldey'}

'Westf. Wilhelms-Univ.-Kinderklinik, Klinik für Allgemeine Pädiatrie, Münster, Deutschland

Fragestellung. Sind Frühgeborene (FG) ausreichend mit Vit B12 versorgt und ergeben sich Unterschiede im Vit-B-12-Haushalt zwischen Formula- und Muttermilchernährung?

Methodik. Prospektive longitudinale Studie aller Frühgeborenen und dystrophen Neugeborenen des Universitätsklinikums Münster von Oktober 2010 bis Dezember 2011. Einschlusskriterien: Geburtsgewicht $<1500 \mathrm{~g}$ und/oder Gestationsalter $<32$ Schwangerschaftswochen. Ausschluss von FG mit gastrointestinalen Erkrankungen und Stoffwechselstörungen.

Material. Einmalurinproben wöchentlich ab der zweiten Lebenswoche bis zum Ende des stationären Aufenthaltes. Parameter im Urin: Methylmalonsäure (MMA), Homocystin. Bestimmung von Vit 12 und Homocystein im Blut bei pathologisch hoher MMA-Ausscheidung und/ oder anderer organischer Säuren oder mehrerer aufeinander folgender hoher Werte. Bestimmung der AUC der Chromatogramme der MMAAusscheidung und Berechnung des Quotienten MMA/Standard. Ergebnisse. Von 83 Frühgeborenen konnten 58 in die Studie eingeschlossen werden, davon hatten 29 auffällige MMA-Werte, 45 erhöhte Homocystin-Werte. Auffällige MMA-Werte korrelierten nicht mit auffälligen Homocystin-Werten ( $\mathrm{p}<0,05)$. Dreizehn Kinder erfüllten die Kriterien für eine Blutabnahme, 9 Kinder erhielten diese. Bei allen 9 Kindern fanden sich auffällige Chromatogramme mit einem Quotienten MMA/Standard >0,6. Ausschließliche Muttermilchernährung hatten 7/9 Kindern, 6/9 waren Mehrlinge. Die im Blut bestimmten Werte zeigten zweimal eine als leicht erhöhte (als grenzwertig zu beurteilende) Homocysteinkonzentration bei normalen Werten für Vit B12 und Folsäure, 6-mal einen Normalbefund. Nur ein FG zeigte eine stark erhöhte Homocysteinkonzentration mit auffälligen Vit-B12- und Folsäurewerten. Es erfolgte eine Vit-B12-Substitution s.c., worunter alle pathologischen Werte rückläufig waren.

Schlussfolgerung. Im Gegensatz zu bisher publizierten Daten wies die Hälfte unserer Patienten erhöhte MMA-Werte im Urin auf. Es besteht keine Korrelation der MMA-Ausscheidung mit der Homocystin-Ausscheidung, Homocystein im Blut korreliert nicht mit Homocystin im Urin. Mehrlinge und muttermilchernährte Kinder hatten vermehrt auffällige MMA-Ausscheidungen im Urin. Der Stellenwert der erhöhten MMA-Ausscheidung im Urin muss weiter abgeklärt werden, möglicherweise sind die erhöhten MMA-Werte ein Hinweis auf einen latenten Vit-B12-Mangel. 


\section{PO-20}

Osteopenische Frakturen bei Frühgeborenen $<1500 \mathrm{~g}$ - eine retrospektive Datenanalyse an der Medizinischen Universität Wien (MUW) in den Jahren 2003-2012

\section{Binder', A. Repa', A. Kreissl', A. Berger', N. Haiden'}

${ }^{1}$ Medizinische Universität Wien, Neonatologie, Pädiatrische Intensivmedizin und Neuropädiatrie, Wíen, Österreich

Hintergrund. Die Osteopenie des Frühgeborenen (FG) ist eine häufige Erkrankung die gelegentlich mit schwerwiegenden Komplikationen wie Frakturen der Röhrenknochen, der Rippen oder einer dolichozephalen Verformung des Kopfes sowie Schmelzhypoplasien einhergeht. Die Mineralisation des fetalen Knochens erfolgt zu 80\% im letzten Trimenon der Schwangerschaft weshalb FG $<1500$ g Geburtsgewicht ein erhöhtes Risiko haben eine Osteopenie (39\%) zu entwickeln und manchmal spontane Frakturen (2\%) erleiden. Neben der zu geringen Zufuhr von $\mathrm{Ca} / \mathrm{Ph}$ und Vit. $\mathrm{D}_{3}$ sind Immobilisierung, ein verzögerter enteraler Nahrungsaufbau sowie Nebenwirkungen von Medikamenten (Steroide, Diuretika) weitere Risikofaktoren. Ziel dieser retrospektiven Datenanalyse ist es, die Prävalenz sowie Risikofaktoren für Frakturen bei $\mathrm{FG}<1500 \mathrm{~g}$ zu analysieren.

Material und Methoden. Die Krankengeschichten von allen FG<1500 g Geburtsgewicht, die im Zeitraum von 2003 bis 2012 an der Neonatologie der MUW aufgenommen waren, wurden auf die Diagnose Frakturen gescreent sowie assoziierte neonatale Co- Morbiditäten erhoben. Ergebnisse. Bei 10 von 1698 (o,6\%) FG<150o g fanden sich eine oder mehrere osteopenische Frakturen. Das durchschnittliche Gestationsalter dieser Kinder bei Geburt war $26+3$ SSW $(23+4-33+4)$, das durchschnittliche Geburtsgewicht lag bei $716 \mathrm{~g}$ (422-1400). Die Frakturen traten im Mittel in der korr. SSW $42+3(35+6-52+3)$ auf. Bei $7 / 10$ Fällen waren Rippen, bei 5/10 Fällen lange Röhrenknochen betroffen. Bei 50\% der FG mit osteopenischen Frakturen wurde ein bauchchirurgischer Eingriff durchgeführt, davon musste bei $40 \%$ eine Darmresektion durchgeführt werden. Die Dauer der parenteralen Ernährung betrug im Mittel 123 Tage (57-210). Eine Cholestase konnte bei 90\% der FG nachgewiesen werden (konjugiertes Bilirubin 15,6 mg/dl (o,3-37). Alle FG hatten zumindest einmal eine Infektion mit positiver Blutkultur. Die Dauer der maschinellen Beatmung betrug im untersuchten Kollektiv 32,3 Tage (751). $40 \%$ der FG hatten einen zusätzlichen $\mathrm{O}_{2}$-Bedarf nach SSW $36+0$. $70 \%$ der Kinder erhielten Diuretika sowie bei 30\% der FG fand sich eine Nephrokalzinose. Die für den Knochenstoffwechsel relevanten Laborwerte: AP im Mittelwert 915 IU/L (563-1301). Eine Hypocalcämie konnte in $40 \%$ und eine Hypophosphatämie in $20 \%$ der Fälle nachgewiesen werden. Die nach Fraktur bestimmten $25 \mathrm{OH}$-Cholecalciferol-Spiegel waren bei $90 \%$ der FG $<75 \mathrm{nmol} / \mathrm{l}$ und im Median bei 43,3 $\mathrm{nmol} / \mathrm{l}(34,4-$ $89,8)$.

Diskussion und Schlussfolgerung. In den letzten zehn Jahren fanden sich bei o,6\% unserer VLBW-infants osteopenische Frakturen. Langzeitparenterale Ernährung war hierfür ein starker Risikofaktor Weiters spielten Immobilisation und Steroid/Diuretika-Therapie in Verbindung mit zu niedrigen Vit.-D-Spiegeln eine pathogenetische Rolle. Schlussfolgernd scheinen die Empfehlungen von 40-16o Einheit Vit. D pro kg i.v. bei Langzeitparenteral ernährten Kindern nicht ausreichend zu sein.

\section{PO-21}

Schädelvolumina von extremen und späten Frühgeborenen am Termin

S. Ifflaender', M. Rüdiger ${ }^{1}$, W. Burkhardt ${ }^{1}$

'Universitätsklinikum Carl Gustav Carus Kinderklinik, Abteilung für Neonatologie und Pädiatrische Intensivmedizin, Dresden, Deutschland

Hintergrund. Nicht nur extrem unreife sondern auch „späte“ Frühgeborene (FG) haben sowohl eine höhere Morbidität und Mortalität als reife Neugeborene, als auch ein erhöhtes Risiko für Probleme der neurologischen Entwicklung. Die neurologische Entwicklung von Frühgebore- nen (FG) ist eng mit dem zerebralen Wachstum und damit auch dem Schädelwachstum assoziiert. Der Kopfumfang (KU) wird bislang zur Beschreibung des Kopfwachstums benutzt, allerdings konnten wir zeigen, dass Neonaten mit vergleichbaren KU sehr unterschiedliche Hirnschädelvolumina (HSV) haben. Daher ist zu vermuten, dass die Bestimmung des HSV eine bessere Abschätzung des zerebralen Wachstums erlaubt.

Fragestellung. Unterscheiden sich die Schädelvolumina von extrem unreifen (euFG) bzw. späten (sFG) Frühgeborenen zum Zeitpunkt der Entlassung von den Schädelvolumina Reifgeborener?

Methoden. Auf der Intermediate Care Station eines Level 1 Perinatalzentrums wurden im Rahmen einer Fall-Kontroll-Studie von April 2011 bis Januar 2013127 FG eingeschlossen. Im korrigierten Alter von 37-40 SSW wurden im Rahmen von Routinemessungen zur Entlassung mittels eines 3 D-Laserscanners (STARScanner laser shape digitizer, Vorum Research Corp., Vancouver, BC) die Schädelvolumina erfasst. Zwei Gruppen nach Gestationsalter (GA; <34 SSW, 34-36+6 SSW) wurden unterschieden, 50 Reifgeborene dienten als Kontrollgruppe.

Ergebnisse. 177 Patienten (m 88, w 89; Geburtsgewicht 2000 \pm 860 g, GA $239 \pm 28 \mathrm{~d})$ wurden untersucht. Die HSV von euFG $(\mathrm{n}=68,365 \pm 58 \mathrm{ml}) \mathrm{am}$ Termin unterschieden sich nicht signifikant von denen der $\mathrm{sFG}(\mathrm{n}=59$, $352 \pm 42 \mathrm{ml}, \mathrm{p}=0.16)$ und denen Reifgeborener $(\mathrm{n}=50,377 \pm 56 \mathrm{ml}, \mathrm{p}=0,28)$. Die Zunahme des HSV im Untersuchungszeitraum (37-40 SSW) unterschied sich nicht signifikant zwischen den Gruppen und lag bei 14-26 ml/Woche ( $\mathrm{p}=0,64 ; \mathrm{b}=3,03 \mathrm{ml} / \mathrm{d})$.

Diskussion. Trotz erhöhtem Risiko für neurologische Langzeitschäden zeigen, anders als aus Daten in der Literatur zu erwarten gewesen wäre, sowohl extrem unreife als auch späte Frühgeborene am Termin keine unterschiedlichen Hirnschädelvolumina im Vergleich zu Reifgeborenen. Diese Daten sind mit Vorsicht zu interpretieren, da (I) das HSV keine Rückschlüsse auf das zerebrale Volumen erlaubt, (II) Verlaufsuntersuchungen des HSV und (III) eine Korrelation mit der neurologischen Entwicklung erforderlich sind.

\section{PO-22}

Symptomatische Hypoglykämie bei einem reifen Neugeborenen mit Septum-pellucidum-Agenesie

S. Wiegand ', A. Stahl', K. Gründler', A. Weitkämper ${ }^{3}$, B. Jurklies', , C. Heyer ${ }^{5}$, U. Menken ${ }^{2}$, E. Hamelmann ${ }^{2}$, N. Teig ${ }^{6}$

${ }^{1}$ Klinik für Kinder- und Jugendmedizin St. Josefs-Hospital, Abteilung für Neonatologie und Intensivmedizin, Bochum, Deutschland, ${ }^{2}$ Klinik für Kinder- und Jugendmedizin St. Josefs-Hospital, Bochum, Deutschland, ${ }^{3}$ Klinik für Kinder- und Jugendmedizin St. Josefs-Hospital, Abteilung für Neonatologie und Intensivmedizin, Bochum, Deutschland, ${ }^{4} \mathrm{Helios}-K$ Klinikien Wuppertal, Augenheilkunde, Wupperetal, Deutschland, ${ }^{5}$ Bergmannsheil Bochum, Radiologie und Nuklearmedizin, Bochum, Deutschland, ${ }^{6} \mathrm{Klinik}$ für Kinder- und Jugendmedizin St. Josefs-Hospital, Abteilung für Neonatologie und Intensivmedizin, Bochum, Deutschland,

Hintergrund. Die Agenesie des Septum pellucidum ist eine bereits mittels fetaler Sonografie feststellbare Fehlbildung des Telencephalons. Sie tritt meist sporadisch mit einer Häufigkeit von ca. 1:30.00o auf und ist oft mit anderen ZNS-Fehlbildungen (septo-optische Dysplasie, neuronale Migrationsstörungen, Mittelliniendefekte) assoziiert. Wir berichten über den Verlauf eines Neugeborenen, bei dem die präpartal festgestellte Agenesie des Septum pellucidum zunächst als harmlos eingestuft wurde, dann aber mit erheblichen Komplikationen assoziiert war.

Kasuistik. Das weibliche Neugeborene wurde mit 39 SSW in einer auswärtigen Klinik ambulant entbunden. Die Eltern brachten es am Ende des 1. Lebenstages wegen Trinkschwäche in eine Kinderarztpraxis, dort fielen eine tiefe Zyanose, Bradykardie, Hypopnoe und muskuläre Hypotonie auf. Nach unserem Eintreffen wurde eine Blutglucosekonzentration von $12 \mathrm{mg} / \mathrm{dl}$ (venös) gemessen. Unter intravenöser Glucose-Infusion und Sauerstoffvorlage stabilisierte sich der Zustand. Anamnestisch berichteten die Eltern, dass bei ihrem Kind präpartal eine 
Sepum-pellucidum-Agenesie festgestellt worden sei. Die am 13. Lebenstag durchgeführte kranielle Magentresonanztomographie zeigte neben dieser Auffälligkeit Veränderungen der Nn. Optici und des Chiasma opticum sowie das nahezu vollständige Fehlen der Adenohypophyse, passend zur Diagnose einer septo-optischen Dysplasie. Okzipital fanden sich Veränderungen, die einer posthypoglykämischen Schädigung entsprechen könnten. Endokrinologisch ergaben sich die Befunde einer sekundären NNR-Insuffizienz, eines Wachstumshormonmangels und eines zentralen Diabetes insipidus. Unter der Substitution mit Hydrocortison, Somatropin und Desmopressin konnte eine ausgeglichene Stoffwechsellage erzielt werden. Augenärztlich zeigte sich eine deutliche Hypoplasie der Papille, im weiteren Verlauf eine hochgradige Visusminderung. Eine Mutation im HESX1-Gen konnte ausgeschlossen werden.

Schlussfolgerung. Eine präpartal festgestellte Septum-pellucidum-Agenesie sollte postnatal stets mittels Bildgebung sowie endokrinologischer und augenärztlicher Untersuchungen genauer abgeklärt werden. Bei allen Kindern sind postnatale Blutzucker-Kontrollen erforderlich. Unser Fall illustriert zudem, dass Schnittstellen zwischen Pränataldiagnostik, Geburtshilfe und Neonatologie systematischer verzahnt werden müssen.

\section{PO-23}

Schwere hypoketotische Hypoglykämie und Azidose eines 6 Tage alten Neugeborenen - seltene Erkrankung: $30 \mathrm{H}$-MethylglutarylCoA-Lyase-Mangel

\section{K. Heimann', S. Trepels-Kottek', M. Hütten', M. Schoberer', T. Meissner2, T. Orlikowsky}

'Universitätsklinik für Kinder- und Jugendmedizin, Sektion Neonatologie und Kinderintensivmedizin, Aachen, Deutschland, ${ }^{2}$ Universitätsklinik Düsseldorf, Allgemeine Pädiatrie, Düsseldorf, Deutschland, ${ }^{3}$ Universitätsklinik für Kinder- und Jugendmedizin, Sektion für Neonatologie, Aachen, Deutschland

Kasuistik. Ein eutrophes, 6 Tage altes Neugeborenes nicht konsanguiner Eltern wurde nach unauffälliger Schwangerschaft und Geburt zwei Tage nach seiner Entlassung wegen Trinkschwäche (Stilldauer 1-3 min), Schläfrigkeit, fahlem Hautkolorit und Hypothermie $\left(34,4^{\circ} \mathrm{C}\right.$ rektal) aufgenommen. Es war dehydriert, hatte gegenüber dem Entlassgewicht $15 \%$ abgenommen, war tachypnoeisch mit grauem Hautkolorit und vigilanzgemindert. Sauerstoffsättigung $>90 \%$. Erste BGA: $\mathrm{pH} 6,8$, BE - $27 \mathrm{mmol} / \mathrm{l}, \mathrm{BZ} 17 \mathrm{mg} / \mathrm{dl}$. Das Blutbild war unauffällig, CRP und Interleukin-6 waren negativ, die Echokardiographie war ohne pathologischen Befund. Die Initialtherapie bestand in Volumensubstitution und Glucose- sowie Natriumbicarbonatgabe. Die initiale Ammoniakkonzentration betrug $339 \mu \mathrm{mol} / \mathrm{l}$. Wir führten neben Stopp der Eiweiß zufuhr die Glucoseinfusion fort und ergänzten um Natriumbenzoat bis zum Ausgleich der Azidose. Das Stoffwechselscreening erbrachte die Diagnose eines 3-OH-Methylglutaryl-CoA-Lyase-Mangels, der durch Bestimmung der Acylcarnitine im Plasma und der organischen Säuren im Urin mit typischem Metabolitenprofil bestätigt wurde. Beim 3-Hydroxy-3-Methyl-Gutaryl(HMG)-CoA-Lyase-Mangel ist die Bildung des Ketonkörpers Acetoacetat aus 3-Hydroxy-3-Methylglutaryl-CoA gehemmt. Die Reaktion ist gleichzeitig der letzte Schritt des Abbaus der ketogenen Aminosäure Leucin. Wie bei unserem Kind sind die typischen Symptome eine schwere Hypoglykämie, hypoketotische metabolische Azidose, Hyperammonämie, Erbrechen und Lethargie. Nicht selten versterben betroffene Kinder in einer akuten Reye-Syndrom-artigen Krise. Nach Stabilisierung der Stoffwechsellage erfolgte bei unserem Neugeborenen eine Umstellung auf Muttermilchernährung ohne Eiweißrestriktion. Die Langzeittherapie besteht in einer fettarmen (ca. $25 \%$ des täglichen Kalorienbedarfs) und eiweißreduzierten Diät. Zusätzlich kann Carnitin verabreicht werden.

Schlussfolgerung. Trinkschwäche und Gewichtsabnahme über 10\% des Geburtsgewichtes sollten immer zeitnah kinderärztlich abgeklärt wer- den. Stoffwechselstörungen sind auch bei erfolgtem Stoffwechselscreening eine wichtige Differenzialdiagnose.

\section{PO-24}

Fallvorstellung eines Neugeborenen mit Granulopenie, Thrombozytopenie, Hypotonie und Hypothermie - kombinierte Methylmalonacidämie und Homocystinurie: Cobalamin-C-Defekt

R. Brangenberg', S. Wörnle', G. Wolf', H. Korall', J. Kohlhase', P. Freisinger ${ }^{4}$ 'Klinikum Traunstein, akad. Lehrkrankenhaus der LMU München, Klinik für Kinder- und Jugendmedizin, Traunstein, Deutschland, ${ }^{2}$ Zentrum für Stoffwechseldiagnostik Reutlingen $\mathrm{GmbH}$, Reutlingen, Deutschland, ${ }^{3}$ Praxis für Humangenetik Freiburg, Freiburg, Deutschland, ${ }^{4}$ Kreiskliniken Reutlingen, Klinik für Kinder- und Jugendmedizin, Reutlingen, Deutschland

Klinische Präsentation. Entbindung eines männlichen NG per Sectio bei Beckenendlage, 38+o SSW, Geburtsgewicht 2900 g. Unauffälliges NGStoffwechselscreening. Stationäre Aufnahme am 13. LT bei zunehmender Lethargie, Trinkschwäche, Hypotonie und Hypothermie $\left(33,8^{\circ} \mathrm{C}\right.$ rektal). Blutdruck 58/35 mmHg, Herzfrequenz 100/min. Diagnostik. Thrombozyten 55.00o/ $\mu$ l, Leukozyten $3500 / \mu \mathrm{l}$, Hb 15,3 g/dl, Ret. $3 \%$. CRP o mg/l, Glucose $63 \mathrm{mg} / \mathrm{dl}$, Laktat 1,34 mmol/l, pH 7,4, Anionenlücke 15,0 mmol/l, Ammoniak $65 \mu \mathrm{g} / \mathrm{dl}$. PCR-Untersuchungen aus EDTA-Blut und Urin ohne Hinweis für konnatale Infektion. Immunhämatologisch keine typischen, eine neonatale Alloimmunthrombopenie auslösenden AK bei der Mutter. Deutlich erniedrigtes Methionin i. S. von $4 \mu \mathrm{mol} / 1$ (10-6o). Im Trockenblut erhöhtes totales Homocystein (LC-MS/MS) von $232 \mu \mathrm{mol} / \mathrm{l}(<8)$. In der Quantifizierung der Acylcarnitine erhöhtes $\mathrm{C}_{3}$ von $6,54 \mu \mathrm{mol} / 1(<5)$ und erhöhte $\mathrm{C}_{3} / \mathrm{C}_{16}$ Ratio von $10,3(<4,7)$. Methylmalonsäure im Urin mit $2226 \mathrm{mmol} / \mathrm{mol} \mathrm{Crea}(<20)$ deutlich erhöht. Somit dringender V. a. Cobalamin C/D-Defekt. Molekulargenetisch Mutationen c.[271dupA];[457C> T], p.[R91Kfs* $\left.{ }_{14}\right] ;\left[\mathrm{R}_{153}{ }^{*}\right]$ im MMACHC-Gen. Beide Mutationen werden als krankheitsverursachend bei Cobalamin-C-Defekt beschrieben.

Management. Initial antibiotische Behandlung und Aciclovir-Therapie. Hierunter Zunahme von Hypotonie und Trinkschwäche, intermittierende Sättigungsabfälle. Gabe eines TK bei Thrombozyten von 29.0oo/ $\mu l$. Nach Erhalt der Stoffwechselergebnisse am 16. LT Gabe von Hydroxycobalamin zunächst i.v. $1 \mathrm{mg} / \mathrm{kg} / \mathrm{Tag}$, Betain $100 \mathrm{mg} / \mathrm{kg} / \mathrm{Tag}$ p.o., Folsäure $5 \mathrm{mg} / \mathrm{Tag}$ p.o., L-Carnitin $3 \mathrm{mal} 50 \mathrm{mg} / \mathrm{kg}$ i.v. Adäquat kalorische parenterale Ernährung (10o kcal/kg), zunächst mit Eiweißrestriktion auf $1 \mathrm{~g} / \mathrm{kg} /$ Tag und Steigerung bis $1,5 \mathrm{~g} / \mathrm{kg}$ unter Kontrolle der Stoffwechselparameter. Hierunter rasche klinische Besserung von Vigilanz und Trinkverhalten. Im EEG zunehmende Mischaktivität bei zuvor überwiegenden Aktivitäten im Bereich der Theta- und Delta-Wellen. Unter Therapie rasche Normalisierung von MMA im Urin sowie Homocystein und Methionin im Trockenblut. Im Alter von 4 Wochen Entlassung ohne neurologische Auffälligkeiten. In der Dauertherapie Reduktion von Hydroxycobalamin auf 3-mal $1500 \mu \mathrm{g} /$ Woche i.m. Eine entwicklungsneurologische Nachuntersuchung mit 3 Monaten zeigte eine altersentsprechende motorische und kognitive Entwicklung (Bayley Scales).

Schlussfolgerung. Der Cobalamin-C-Defekt ist die häufigste angeborene Störung des Cobalamin-Stoffwechsels. Ursachen sind Mutationen im MMACHC-Gen, die zu einer verminderten intrazellulären Synthese von Adenosyl-Cobalamin (Coenzym der Methylmalonyl-CoA-Mutase) und Methylcobalamin (Coenzym der Methionin-Synthase) führen. Leitsymptome wie Lethargie, Hypothermie und aregeneratorische Blutbildveränderungen sollten an diesen therapierbaren Defekt denken lassen und zu einer raschen Diagnostik und Therapie führen. 


\section{PO-25}

\section{Neue Mutation beim Zellweger-Syndrom im PEX3-Gen}

\section{E. Block', J. Kohlhase' ${ }^{2}$ H. Kaiser ${ }^{3}$, H. Fahnenstich'}

'Zentrum für Kinder und Jugendmedizin St. Elisabethen-Krankenhaus Lörrach, Neonatologie, Lörrach, Deutschland, ${ }^{2}$ Universität, Freiburg, Deutschland, ${ }^{3}$ Universitätsspital, Basel, Deutschland

Hintergrund. Das Zellweger-Syndrom ist die schwerste Erkrankungsform der peroxisomalen Stoffwechseldefekte. Es wird durch eine Mutation in einem von mindestens 12 verschiedenen PEX-Genen verursacht. Am häufigsten sind die Gene PEX 1, 6, und 26 betroffen. Bisher wurde kein Zusammenhang des klinischen Phänotyps mit der zugrundeliegenden Mutation dokumentiert.

Kasuistik. Wir berichten über eine Familie mit 5 Kindern von denen 4 am Zellweger-Syndrom erkrankt sind. Aufgrund eines außergewöhnlichen Phänotyps mit einem besonders auffallenden breiten Digitus I aller 4 Extremitäten, war bei den ersten beiden betroffenen Kindern ( $\mathrm{Ge}$ mini) die Diagnose Rubinstein-Taybi-Syndrom gestellt worden. Dies ließ sich genetisch nicht bestätigen. Bei Geburt des dritten betroffenen Kindes war die Diagnose weiterhin unklar. Erst der augenärztliche Befund makulärer Pigmentveränderungen war, in Zusammenhang mit den weiteren Symptomen, pathognomonisch für das Zellweger-Syndrom. Daraufhin konnten wir die Diagnose durch Nachweis erhöhter überlangkettiger Fettsäuren bestätigen. Die genetische Untersuchung des am häufigsten betroffenen PEX1-Gen ergab keine Auffälligkeiten. Erst in der erweiterten Diagnostik fand sich die Mutation c.160C $>\mathrm{T}$, p.R54X in Exon 2 des PEX3-Gens. Sie wurde heterozygot bei beiden Eltern nachgewiesen. Bei allen betroffenen Kindern lag sie homozygot vor. Die gefundene Mutation wurde bisher nicht beschrieben. Sie führt zu einem vorzeitigen Stoppkodon und damit zum Funktionsverlust des betroffenen Allels.

Schlussfolgerung. Die typischen Merkmale, die schnell zur Diagnose Zellweger-Syndrom beim Neugeborenen führen sollten sind (1) schwere muskuläre Hypotonie, (2) neonatale Krampfanfälle, (3) Hepatomegalie und aufgrund der gestörten Leberfunktion, erhöhte Leberenzyme und verlängerter Neugeborenenikterus, (4) Nierenzysten, sowie (5) Hör- und Sehstörungen. Manchmal können bisher nicht beschriebene Phänotypen mit zusätzlichen Merkmalen die Diagnose, wie in unserem Fall, erschweren. Sobald ein Hinweis für ein Zellweger-Syndrom besteht, sollte die biochemische Analyse mit Bestimmung der überlangkettigen Fettsäuren im Blut erfolgen. Bei auffälligem Ergebnis folgen dann die Untersuchung der PEX-Gene und die genetische Beratung der betroffenen Familien. Diese könnten bei bekannter Mutation von einer PID profitieren.

\section{PO-26}

\section{Case-Management bei einem Neugeborenen mit ausgeprägtem Smith-Lemli-Opitz-Syndrom}

N. Börner', J. Hennermann², D. Horn', B. Opgen-Rhein ${ }^{4}$, D. Haas' ${ }^{5}$, C. Bart$\mathrm{ram}^{6}$, C. Czernik', C. Bührer', C. Dame ${ }^{1}$

${ }^{1}$ Charité - Universitätsmedizin Berlin, Klinik für Neonatologie, Berlin, Deutschland, ${ }^{2}$ Charité - Universitätsmedizin Berlin, Klinik für Pädiatrie m.S. Endokrinologie, Berlin, Deutschland, ${ }^{3}$ Charité - Universitätsmedizin Berlin, Institut für Medizinische Genetik und Humangenetik, Berlin, Deutschland, ${ }^{4}$ Charité - Universitätsmedizin Berlin, Klinik für Pädiatrie m.S. Kardiologie, Berlin, Deutschland, ${ }^{5}$ Universitätskinderklinik Heidelberg, Abteilung für Stoffwechselerkrankung und Neugeborenen-Screening, Heidelberg, Deutschland, ${ }^{6}$ Universitätsklinik Heidelberg, Institut für Humangenetik, Heidelberg, Deutschland

Hintergrund. Das Smith-Lemli-Opitz-Syndrom (SLOS) ist eine autosomal rezessiv vererbte Erkrankung, die durch eine abnormal niedrige Aktivität der 3 b-Hydroxysteroid- $\Delta 7$-Reduktase (7-Dehydrocholesterol Reduktase, DHCR7) gekennzeichnet ist. Dieser Defekt führt zu einer Akkumulation von 7-Dehydroxycholesterol und seinem Isomer, 8-De- hydroxycholesterol. Bislang sind etwa 150 verschiedene Mutationen im DHCR7-Gen als Ursache des SLOS beschrieben worden, denen eine unterschiedliche Ausprägung des Phänotyps zugeordnet werden kann. Methoden. Wir berichten über ein reifes Neugeborenes (Chromosomensatz in der AC: $46, \mathrm{XY})$ einer gesunden Mutter $\left(\mathrm{G}_{3} / \mathrm{P}_{3}\right)$, bei dem in der pränatalen Sonographie eine fetale Wachstumsretardierung, ein Atrio-Ventrikel-Septum Defekt und dilatierte Darmschlingen, eine ausgeprägte Hypospadie und eine auffällige Facies diagnostiziert wurden. Geburt spontan nach 40+3 SSW, GG $2770 \mathrm{~g}$ (3. P.), KL $45 \mathrm{~cm}(<3$. P.), KU $31 \mathrm{~cm}$ (<3. P.). Multiple Fehlbildungen (Balkenhypoplasie, Mikrogyrierung, mediane Spalte des weichen Gaumens, Hypertelorismus, Epikanthus, kurze Nase, Mikrognathie und kurzer Hals, Syndaktylie der 2. und 3. Zehe, Hexadaktylie bds., Fußfehlstellung, Mikrophthalmie, AVSD, Aortenbogenhypoplasie, fortbestehende Atemstörung (CPAP, $\mathrm{FiO}_{2}$ bis $40 \%$ ), M. Hirschsprung-artige intestinale Transportstörung, ausgeprägte Hypospadie mit Leistenhoden) führten zur Diagnose eines schweren SLOS (Physical Severity Score; Am J Med Genet C 2012;160C:250-262), bestätigt durch das Sterolprofil im Plasma (4. LT): Cholesterol 14,4 mg/dl, 7-Dehydrocholesterol 7,28 mg/dl (Ref.: o-o,11 mg/dl.), 8-Dehydrocholesterol 6,88 mg/dl (Ref.: o-o,11 mg/dl.), Ratio (7+8-DHC)/Cholesterol o,983. Nachweis der zwei krankheitsrelevanten heterozygoten Mutationen p.W151X (c.452G>A) in Exon 6 und p.V326L (c.976G>T) in Exon 9 des DHCR7 Gens; nebenbefundlich mehrere hetero- und homozygote Polymorphismen.

Ergebnisse. Nach Bestätigung der schweren Form des SLOS im Konsens mit den Eltern und dem Team Verzicht auf eine Fortführung der postnatal begonnenen Prostaglandin-Therapie und eine Operation der ISTA. Auf eine probatorische Behandlung mit Cholesterin oder Simvastatin wurde ebenso verzichtet. Palliation im Rooming-in mit Perspektive auf eine spätere (teil-)ambulante Versorgung.

Schlussfolgerung. Das ausgeprägte SLOS erfordert die intensive Kommunikation mit den beteiligten Fachdisziplinen und ein familienorientiertes Konzept der Palliationsbehandlung.

\section{Pädiatrische Intensivmedizin}

\section{PO-27}

Langzeiterfahrungen in der pädiatrischen Nierenersatztherapie mit Prismaflex ${ }^{\circledast}$ Maschine und dem Prismaflex ${ }^{\circledast}$ HF 20 Set

\section{S. Rödl', G. Wendelin', I. Marschitz², S. Ring', C. Mache ${ }^{3}$, G. Zobel'}

'Universitätsklinik für Kinder und Jugendheilkunde, Pädiatrische Intensivstation, Graz, Österreich, ${ }^{2}$ Universitätsklinik für Kinder- und Jugendheilkunde, Neonatologie, Salzburg, Österreich, ${ }^{3}$ Universitätsklinik für Kinder und Jugendheilkunde, Medizinische Universität Graz, Graz, Österreich

Hintergrund. Für die Hämodiafiltration (HDF) von Säuglingen und Kleinkindern steht nur eine stark eingeschränkte Auswahl an geeigneten Geräten und Filtersystemen zur Verfügung. Mit der Prismaflex ${ }^{\circ}$ Maschine (PF) und dem Prismaflex ${ }^{\circ}$ HF 20 Set (HF2O) ist seit 2009 ein System am Markt, das speziell auf den pädiatrischen Bereich ausgelegt wurde.

Zielsetzung. Präsentation unserer Erfahrung mit der PF und dem HF2O nach 4 Jahren Einsatz auf der pädiatrischen Intensivstation.

Patienten und Methoden. 12 Patienten mit akutem Nierenversagen (NV) und 1 Patient mit einer Stoffwechselstörung wurden über insgesamt 93 Tage kontinuierlich mit der PF und dem HF2O an unserer Intensivstation hämodiafiltriert. Sechs Patienten hatten zusätzlich ein Multiorganversagen, zwei Patienten benötigten zusätzlich die ECMO. Im gleichen Zeitraum wurden bei 5 Säuglingen und Kleinkindern mit chronischem Nierenversagen und bestehender Kontraindikation für eine Peritonealdialyse insgesamt 1100 Therapien einer ambulanten intermittierenden HDF mit der PF und dem $\mathrm{HF}_{2} \mathrm{O}$ durchgeführt.

Ergebnisse. Sowohl für die kontinuierliche als auch intermittierende Nierenersatztherapie war die PF in Kombination mit dem HF2o von 
Seiten der wählbaren Einstellungen für unsere kleinen Patienten geeignet. Bei Neugeborenen und Säuglingen mit Multiorganversagen und Hypervolämie war mittels HDF eine Volumsreduktion unter stabilen Blutdruckverhältnissen infolge der Bilanzgenauigkeit des Systems problemlos zu erreichen. Die Software der PF unterstützt sowohl die Antikoagulation mit Heparin als auch eine automatisch an die jeweiligen Flussraten von Blut, Filtratersatz und Dialysat angepasste Zitratantikoagulation mit nachfolgendem Kalziumausgleich. Von Seite der Filterleistung ist der Abfluss mit 2,5 1/h begrenzt. Damit lassen sich im akuten NV Patienten mit einem Gewicht von 3 bis $30 \mathrm{~kg}$ mittels kontinuierlicher HDF behandeln, im chronischen NV Patienten von 3-15 kg mittels intermittierender HDF. Die maximale Clearence für Harnstoff beträgt $25 \pm 0,3 \mathrm{ml} / \mathrm{h}$, für Kreatinin $20 \pm 0,2 \mathrm{ml} / \mathrm{h}$. Die Alarmgrenzen der PF stellen sich nach dem Start automatisch ein. Schnelle Druckänderungen führen zu einem Pumpenstillstand. Falls sich ein negativer Ansaugdruck oder positiver Rückflussdruck innerhalb von 8 Sekunden wieder normalisiert, startet die Maschine von selbst neu. Bilanzverletzungen werden $\mathrm{ab} 20 \mathrm{ml}$ durch einen Alarm angezeigt, im Falle einer Bilanzverletzung von mehr als $10 \mathrm{ml} / \mathrm{kg}$ Körpergewicht wird die Therapie irreversibel beendet.

Schlussfolgerung. Die Anforderungen an ein System zur Hämodiafiltration von Säuglingen und Kleinkindern werden von der PF in Kombination mit dem HF 20 Set in zufriedenstellender Weise erfüllt. Das System lässt sich gleichermaßen zur Behandlung des akuten und chronischen NV einsetzen.

\section{PO-28}

\section{Rasche Entfernung von Myoglobin mit dem HCO 1100-Dialysefilter bei 5 Kindern mit Rhabdomyolyse}

\section{O. Amon', E. Heimberg', I. Gerbig', M. Kumpf', H. Billing', C. Fink' \\ 'Eberhard-Karls-Universität Universitätsklinik für Kinderheilkunde und Jugendmedizin, Tübingen, Deutschland}

Hintergrund. Der High cut off (HCO) 1100 Dialysefilter wurde entwickelt, um freie Leichtketten bei Plasmozytom aus dem Blut zu entfernen und ist in der Lage, Plasmabestandteile bis 45 KiloDalton (KDa) zu beseitigen. Myoglobin (Molekulargewicht 17,8 KDa) kann zu Rhabdomyolyse-assoziiertem Nierenversagen führen. Alle bisher eingesetzten extrakorporalen Verfahren haben Myoglobin nicht in relevantem Umfang beseitigen können.

Fragestellung. Effektivität des HCO-110o-Filters in der Elimination von Myoglobin bei Kindern.

Material und Methoden. Fallbeschreibungen bei 5 konsekutiven Fällen von schwerer Rhabdomyolyse bei Kindern.

Ergebnisse. Seit 2011 wurden 5 Kinder mit Rhabdomyolyse mit dem HCO-110o-Filter behandelt. Es handelte sich um 4 Jungen und 1 Mädchen im Alter von 2-15 Jahren mit einem Körpergewicht von 11-6o kg. Ursachen der Rhabdomyolyse waren Ischämie von Extremitäten $(\mathrm{n}=2)$ und Trauma, Infektion sowie Multiorganversagen (jeweils $\mathrm{n}=1$ ) Der mittlere initiale Myoglobin-Wert im Blut betrug 43.227 ( Range: 1876-102.300) $\mu \mathrm{g} / \mathrm{L}$. Die Patienten wurden mit dem HCO-Filter für 12 Stunden behandelt, gefolgt von konventioneller Hämofiltration/ Hämodialyse (CVVH/CVVHD). Am Ende der 12 Stunden HCO-Filter-Behandlung war der mittlere Myoglobin-Wert auf 12.250 ( Range: 396-42.000) $\mu \mathrm{g} / \mathrm{L}$ gefallen. Bei 2 Kindern wurden 2 weitere HCO-Filter-Behandlungen angeschlossen, bei 1 Patienten wurde 1 zusätzliche HCO-Filter-Behandlung durchgeführt - jeweils gefolgt von 12 Stunden CVVH oder CVVHD. Nach diesen weiteren HCO-Filter-Behandlungen war der Myoglobin-Wert auf 927, 983 bzw.1483 $\mu \mathrm{g} / \mathrm{L}$ gesunken. Während der Perioden konventioneller CVVH/CVVHD kam es nicht zum Absinken des Myglobin-Wertes. 4 Kinder überlebten. Ein Kind verstarb im Multiorganversagen. Bei den Überlebenden konnte die Dialysebehandlung durchschnittlich 6 Tage ( Range: 1-14 Tage) nach der HCO-Filter-Behandlung beendet werden. Wir substituierten während der HCO-Filter-Behandlungen Humanalbumin.
Schlussfolgerung. Nach unserer Erfahrung ist die Behandlung mit dem HCO-110o-Dialysefilter allen anderen bisher bei kindlicher Rhabdomyolyse eingesetzten extrakorporalen Verfahren überlegen und führt zu einer raschen Entfernung von Myoglobin aus dem Blut. Auf die Entfernung weitere Plasmaproteine während der Behandlung muss geachtet werden.

\section{PO-29}

Plasmaseparation als Therapie des akuten Leberversagens bei Paracetamolüberdosierung im Kleinkindalter

\section{R. Vargha', G. Mostafa', M. Hermon', G. Burda ${ }^{4}$, A. Pollak ${ }^{5}$, G. Trittenwein ${ }^{6}$,} J. Golej ${ }^{6}$

'Universitätsklinik für Kinder und Jugendheilkunde Wien, Neonatologie, pädiatrische Intensivmedizin und Neuropädiatrie, Wien, Österreich, ${ }^{2}$ Universitätsklinik für Kinder und Jugendheilkunde Wien, Neonatologie, Pädiatrische Intensivmedizin und Neuropädiatrie, Wien, Österreich, ${ }^{3}$ Universitätsklinik für Kinder und Jugendheilkunde, Neonatologie, Pädiatrische Intensivmedizin und Neuropädiatrie, Wien, Österreich, ${ }^{4} U n i v$. Klinik für Kinder und Jugendheilkunde, Neonatologie, Pädiatrische Intensivmedizin

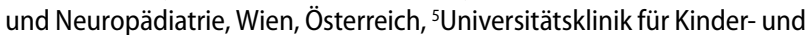
Jugendheilkunde Wien, Abteilung für Allgemeinpädiatrie und Neonatologie, Wien, Österreich, ${ }^{6} U n i v$. Klinik für Kinder- und Jugendheilkunde, Neonatologie, Pädiatrische Intensivmedizin und Neuropädiatrie, Wien, Österreich

Hintergrund. Paracetamol ist ein weit verbreitetes Analgetikum und Antipyretikum mit potentiell hepatotoxischem Nebenwirkungsprofil. Gelingt es im Falle einer Überdosierung nicht, mit N-Acetyl-Cystein die hepatische Funktion wieder zu bessern, kann daraus ein irreversibles Leberversagen entstehen.

Fragestellung. Ist die Plasmaseparation (PS) eine geeignete Therapieform um Paracetamol induziertes Leberversagen bei Kleinkindern zu therapieren?

Material und Methoden. Fallbericht von 2 Kleinkindern, die nach Paracetamol Überdosierung einen toxischen Paracetamolspiegel aufwiesen und ein akutes Leberversagen entwickelten.

Patienten. 1. Patientin: 19 Monate, $11 \mathrm{~kg}$, litt seit 5 Tagen an einem Infekt der oberen Atemwegen, der mit Clarithromycin und Paracetamol $(=100 \mathrm{mg} / \mathrm{kg} / \mathrm{d}$ über $72 \mathrm{~h})$ therapiert wurde. Die Vorstellung in der Ambulanz erfolgte wegen Durchfall und Somnolenz, wobei im Rahmen der Abklärung eine gravierende Leberfunktionsstörung entdeckt wurde. Nach 24 h stationärer Therapie mit FFP-Substitution und NAcetyl-Cystein Gabe, weitere Verschlechterung des neurologischen $\mathrm{Zu}$ stands [hepatische Enzephalopathie (HE) II] sowie der Leberfunktion: GOT>9999 U/l, GPT 8856 U/l, LDH>9999 U/l, Ammoniak $270 \mu \mathrm{mol} / \mathrm{l}$, PTZ 15\%. Deshalb Intubation und Beginn mit PS sowie Abklärung wegen allfälliger LTx. 2. Patientin: 28 Monate, $10 \mathrm{~kg}$, wurde wegen eines Infekts der oberen Atemwege mit Codein und Paracetamol $(=80 \mathrm{mg} /$ $\mathrm{kg} / \mathrm{d})$ seit 7 Tagen therapiert. Die Vorstellung im auswärtigen Krankenhaus erfolgte wegen Somnolenz, wobei sich im Labor eine schwere Leberfunktionsstörung zeigte: Blutglucose $9 \mathrm{mg} / \mathrm{dl}$, GOT $>14.237 \mathrm{U} / \mathrm{l}$, GPT 13.161 U/l, LDH>17.861 U/l, Ammoniak $105 \mu \mathrm{mol} / \mathrm{l}$, Normotest 15\%. Unter laufender N-Acetyl-Cystein Gabe und FFP-Substitution erfolgte der Transfer an unsere Station, wo sich das klinische Bild einer HE III mit nochmals verschlechterten Leberfunktionsparametern fand. Daraufhin Intubation und Beginn mit PS. Evaluation für mögliche LTx. Ergebnis. Beide Kleinkinder zeigten nach ein- (Pat 1), bzw. zweimaliger Durchführung (Pat 2) eine deutlich gebesserte neurologische Funktion. Die pathologischen Leberfunktionsparameter im weiteren Verlauf deutlich rückläufig, eine FFP-Substitution war nicht mehr erforderlich. Die Kinder überlebten defektfrei.

Schlussfolgerung. Bei Paracetamol-induziertem Leberversagen konnte durch Einsatz der PS die Leberfunktion bei zwei Kleinkindern deutlich gebessert und die drohende LTx abgewendet werden. 
PO-30

Plötzlicher Atemstillstand nach Ingestion von Gamma-Butyrolacton bei einer 2-Jährigen

\section{A. Beyersdorff', M. Heckmann ${ }^{7}$ \\ 'Zentrum für Kinder und Jugendmedizin, Universitätsmedizin, Neonatolo- gie und Pädiatrische Intensivmedizin, Greifswald, Deutschland}

Einführung. Bei GBL (Gamma-Butyrolacton) handelt es sich um das Lacton der Gamma-Hydroxybuttersäure (GHB). In höheren Dosen wirkt es sedierend bis narkotisierend über die Metabolisierung zu GHB. GBL wird in der Industrie als Lösungsmittel verwendet. Es wird missbraucht als Partydroge unter dem Namen „Liquid Ecstasy“. Es ist farblos und hat einen leicht seifigen und sauren Geschmack. In geringeren Dosen verursacht es einen dem Alkohol ähnlichen Rausch, dessen Beginn nach 5-20 min nach oraler Aufnahme einsetzt. Die Serumspitze wird nach 15-40 min erreicht, die Wirkdauer beträgt 90-180 min. Bisher liegen nur wenige Berichte über eine GBL-Intoxikation im Kindesalter vor.

Kasuistik. Wir berichten über ein 2-jähriges Mädchen, dass nach Ingestion einer unbekannten Menge (50-100 $\mathrm{ml} \mathrm{mit} \mathrm{anschließendem} \mathrm{Erbre-}$ chen) einer klaren, farblosen Flüssigkeit (als Wasser fehlgedeutet) im Beisein des Vaters eine rasche Eintrübung entwickelte. Das Kind wurde somnolent, intubiert und beatmet eingewiesen. Bei Aufnahme war sie tief komatös, Pupillen bds. mittelweit, keine Lichtreaktion, keine Reaktion auf Schmerzreize, kein Cornealreflex, GCS 3 . Das Drogenscreening war negativ. Die Toxikologie ergab initial GHB i. S. $500 \mu \mathrm{g} / \mathrm{ml}$, Urin $8800 \mu \mathrm{g} / \mathrm{ml}$ (toxische Plasmakonzentration ab $260 \mu \mathrm{g} / \mathrm{ml}$ ), nach $8 \mathrm{~h}$ i. S. $59 \mu \mathrm{g} / \mathrm{ml}$, Urin $2020 \mu \mathrm{g} / \mathrm{ml}$, nach 12 h i. S. $21 \mathrm{ng} / \mathrm{ml}$, im Urin nicht mehr nachweisbar. Das EEG zeigte bei Aufnahme ein Burst suppression Muster. Nach $4 \mathrm{~h}$ setzte eine unregelmäßige Eigenatmung ein, nach $8 \mathrm{~h}$ erfolgte die Extubation. Nach $18 \mathrm{~h}$ war sie neurologisch unauffällig. Das Jugendamt wurde informiert. Das GBL wurde von den Eltern in einer unbeschrifteten Flasche aufbewahrt. Die ursprüngliche Flasche war entgegen der gesetzlichen Vorschriften mit einem Etikett ohne Gefahrenkennzeichnung versehen aber mit Angabe des Inhaltsstoffes in deutscher Sprache, und dem Zweck „Reinigungsmittel“. Keine Angaben wurden zum Erwerb der Flasche gemacht.

Schlussfolgerung. GBL oder die Partydroge „Liquid Ecstasy“ kann eine lebensbedrohliche Ingestion hervorrufen. Die Substanz wird z. B. im Internet gehandelt. In diesem Fall führte die Zusammenarbeit mit den beteiligten Personen zur raschen Identifikation der das Koma verursachenden Substanz.

\section{PO-31}

\section{Untersuchung zu Dexmedetomidin auf einer pädiatrischen Inten- sivstation}

\section{Richter', J. Dinger', M. Rüdiger', S. Brenner ${ }^{1}$}

'Universitätskinderklinik C.G. Carus Dresden, Fachbereich Neonatologie und Pädiatrische Intensivmedizin, Dresden, Deutschland

Einleitung. Dexmedetomidin, ein selektiver Alpha2-AdrenozeptorAgonist, spielt in Amerika und Asien zunehmend eine Rolle zur additiven oder auch monotherapeutischen Analgosedierung schwerstkranker Kinder und Frühgeborener. Aufgrund der häufig auftretenden Sedierungsschwierigkeiten bei langzeitsedierten Kindern nutzen wir zunehmend Dexmedetomidin wegen seiner sedierenden, analgetischen und anxiolytischen Wirkung.

Untersuchung. Wir berichten über erste Erfahrungen bei 20 Patienten (insgesamt 22 Anwendungsepisoden) auf unserer pädiatrischen Intensivstation. Folgende Daten wurden retrospektiv erfasst: Alter und Gewicht der Patienten, Diagnosen, Behandlungsdauer auf ITS, Beatmungsdauer, Extubationszeitpunkt, Dexmedetomidindosis (kumulative Dosis, mittlere und maximale Stundendosis), Therapiedauer, zusätzlicher Bedarf an Analgosedativa.
Ergebnisse. Die Kinder waren zwischen 1,5 und 124 Monate alt, Körpergewicht 3-30 kg. Krankheitsbilder waren akute Infektionen meist mit schwerem ARDS (bei chromosomalen und syndromalen Erkrankungen: Trisomie 18, 21, Angelman-Syndrom, Pierre-Robin-Sequenz), postoperativ komplizierte Verläufe (nach Kraniotomie, Volvulus, parapharyngealem Abszess), oder schwer verlaufende Infektionen mit Sepsis oder Waterhouse-Friderichsen-Syndrom. Die Aufenthaltsdauer auf ITS lag zwischen 8 bis 50 Tagen, die Beatmungsdauer zwischen 65 bis $522 \mathrm{~h}$. Bei vier Anwendungsepisoden wurde die Extubation vor Dexmedetomidingabe durchgeführt, die Therapie zur Entzugstherapie begonnen. Bei den anderen 18 Anwendungsepisoden wurde die Therapie zur Optimierung der Analgosedierung oder zum Weaning begonnen, die Extubation erfolgte zwischen 1 und 430 h nach Beginn mit Dexmedetomidin. Die kumulative Gesamtdosis lag zwischen 36,5 und $14.397 \mu \mathrm{g}$, die mittlere Stundendosis zwischen 0,27 und $1,5 \mu \mathrm{g} / \mathrm{kg} / \mathrm{h}$, die maximale Dosis zwischen 0,94 und $2,5 \mu \mathrm{g} / \mathrm{kg} / \mathrm{h}$, die Therapiedauer zwischen 30 und $456 \mathrm{~h}$. Alle Kinder erhielten während des stationären Aufenthaltes weitere Sedativa (im Mittel 6,7 Präparate: Kombination aus Opioiden wie Sufentanil, Fentanyl, Morphin, Sedativa wie Ketamin, Levomepromazin, Gamma-Hydroxybuttersäure, Midazolam und Clonidin und häufig (95\% der Patienten) Einzelgaben von Propofol.

Schlussfolgerung. Dexmedetomidin scheint eine Erweiterung der therapeutischen Optionen bei schwerstkranken Kindern im Rahmen der Analgosedierung auf der Intensivstation darzustellen. Weitere Anwendungsbeobachtungen und Studien sind notwendig um zu prüfen, ob Dexmedetomidin eine Therapieoption zur sicheren Reduktion einer Mehrfachsedierung darstellt. Darüber hinaus muss geprüft werden, ob Dexmedetomidin auch zur Therapie einer Entzugssymptomatik im Kindes- und Jugendalter geeignet ist.

\section{PO-32}

Effekt eines pflegegesteuerten Analgosedierungsprotokolls für beatmete Kinder auf die Beatmungsdauer und die Aufenthaltsdauer auf der Intensivstation

\section{F. Neunhoeffer', N. Berneck', M. Kumpf', I. Gerbig', E. Heimberg', M. Hof-} beck', C. Fink

'Universitätsklinik für Kinderheilkunde und Jugendmedizin/EberhardKarls-Universität /, Kinderheilkunde II (Kardiologie, Pulmologie, Intensivmedizin), Tübingen, Deutschland, ${ }^{2} \mathrm{Hegau}-\mathrm{Klinikum} \mathrm{GmbH}$, Kinderklinik, Singen, Deutschland

Hintergrund. Pflegegesteuerte („nurse-controlled-sedation“) Analgosedierungsprotokolle (ASP) für beatmete erwachsene Patienten auf Intensivstationen sind hinreichend evaluiert und Standard. Es gibt jedoch nur wenige Daten über den Gebrauch und Nutzen von standardisierten pädiatrischen Analgosedierungsprotokollen (pASP) bei kritisch kranken Kindern auf der Intensivstation.

Ziel. Untersuchung des Effektes eines pflegegesteuerten pASP für beatmete pädiatrische Patienten auf die Beatmungsdauer und die Aufenthaltsdauer auf der Intensivstation.

Patienten und Methode. Retrospektive Studie vor und nach Implementierung eines pASP auf einer interdisziplinären pädiatrischen Intensivstation mit 14 Betten (ca. 8oo Patienten/Jahr) an einem Universitätsklinikum. Eingeschlossen wurden alle Kinder (o-18 Jahre), die für mindestens 24 Stunden intubiert und beatmet waren. Ausschlusskriterien waren Verlegung intubiert und beatmet und Versterben. Vor Implementierung wurde die Analgosedierung nach Protokoll auf ärztliche Anweisung gesteuert, nach Implementierung wurde die Analgosedierung durch das Pflegepersonal anhand eines Protokolls (pASP), basierend auf der Comfort-B-Scale und dem NISS-Score, gesteuert.

Ergebnisse. Die Beobachtungsgruppe bestand aus 298 Patienten, die innerhalb von 12 Monaten vor Implementierung aufgenommen wurden. Die Interventionsgruppe bestand aus 356 Patienten, die innerhalb der ersten 12 Monate nach Implementierung des pASP erhoben wurden. Die mittlere Beatmungsdauer betrug in der Interventionsgruppe 4 
(1-88) vs. $5(1-115)$ Tage $(\mathrm{p}=0,189)$, die mittlere Intensivaufenthaltsdauer betrug 7,5 (1-97) vs. $9(1-120)$ Tage $(p=0,108)$. Es zeigten sich keine signifikanten Unterschiede bzgl. Pneumonie oder ZVK-Infektion.

Schlussfolgerung. In der Interventionsgruppe zeigte sich ein Trend $\mathrm{zu}$ einer Verkürzung von Beatmungsdauer und Aufenthaltsdauer auf der Intensivstation, die Unterschiede erreichten aber keine statistische $\mathrm{Si}$ gnifikanz.

\section{PO-33}

\section{Intensivmedizinische Aspekte des postoperativen Managements} bei kongenitalem Hyperinsulinismus

\section{H. Bahlmann', A. Weidner', W. Barthlen ${ }^{3}$, M. Heckmann}

${ }^{1}$ Klinik und Poliklinik für Kinder und Jugendmedizin, Abt. Neonatologie u. Päd. Intensivmedizin, Greifswald, Deutschland, ${ }^{2}$ Ernst-Moritz-Arndt Universität, Kinderklinik, Greifswald, Deutschland, ${ }^{3}$ Ernst-Moritz-Arndt Universität, Klinik für Kinderchirurgie, Greifswald, Deutschland

Hintergrund. Kongenitaler Hyperinsulinismus (CHI) bezeichnet ein heterogenes Krankheitsbild, bei dem rezidivierend schwere Hypoglykämien auftreten. Zur Stabilisierung des Butzuckers können eine kontinuierliche Nahrungszufuhr und eine medikamentöse Therapie notwendig sein. Eine Genanalyse differenziert zwischen diffuser und fokaler Form. Ein F-L-Dopa-PET-CT sichert die Lokalisation des Fokus. Ein Fokus kann durch gezielte Pankreas-Chirurgie entfernt werden. Durch restriktive Pankreasteilresektion kann bei der diffusen Form die Gefahr gefährlicher Hypoglykämien gebannt werden. Zur postoperativen Intensivtherapie dieser seltenen Erkrankung (Inzidenz in Mitteleuropa 1:40.00o) liegen bisher keine systematischen Daten vor.

Fragestellung. Evaluation postoperativer intensivmedizinischer Aspekte nach Pankreasteilresektion bei CHI.

Patienten und Methode. 25 Patienten (11 Mädchen) im Alter von 7 Wochen bis 10 Jahren (Median 10 Monate) wurden in eine retrospektive Analyse ihres postoperativen Aufenthaltes auf der pädiatrischen Intensivstation (PICU) eingeschlossen. Bei 18 Kindern lag die genetische Prädisposition einer fokalen Form vor, bei 7 Kindern die einer diffusen. Die Pankreasteilresektion wurde bei 12 Patienten laparoskopisch und bei 13 mittels Laparotomie durchgeführt. Postoperativ wurde unter stündlichen Blutzuckerkontrollen ein permissiv hyperglykämisches (3,5-12 mmol/l) Regime angestrebt. Die medikamentöse Therapie (u. a Somatostatin) wurde nicht fortgesetzt. Wundflüssigkeit, Urinproduktion und Konzentration der Plasma-Lipase wurden aufgezeichnet.

Ergebnisse. Die Patienten verbrachten zwischen 2 und 62, im Median 6 Tage (d) auf der PICU. Drei wurden über max. 8 Stunden nachbeatmet, 2 über $3 \mathrm{~d}$ (Kapillary Leak), 1 über $8 \mathrm{~d}$ (Reanimation). Bei 2 Patienten mit ausgeprägtem Kapillary Leak waren Noradrenalin bzw. Dopamin über jeweils $3 \mathrm{~d}$ notwendig. Ein Patient erhielt $\mathrm{u}$. a. Adrenalin über $7 \mathrm{~d}$ während einer Hypothermiebehandlung nach Reanimation nach intraoperativer Asystolie. Die Nierenfunktion stellte sich bei allen Patienten spontan ein. Die Glukoseinfusion direkt postoperativ betrug $0,6-8,3$ im Median $4 \mathrm{mg} / \mathrm{kg} / \mathrm{min}$. Bei Verlegung lag sie im Median bei 3,1 mg/ $\mathrm{kg} / \mathrm{min}$ und variierte von 1,o bis 12,5 bzw. 13,7 (2 diffuse Formen). Bei 3 Patienten wurde erneut eine medikamentöse Therapie initiiert. Die Wunddrainagen förderten zwischen 20 und $360 \mathrm{ml} / \mathrm{d}$ (Median $58,5 \mathrm{ml}$ ) und waren bei 11 Patienten zu vernachlässigen. Die Plasma-Lipase-Werte lagen bei 13 Patienten über dem Cut-off von $6,36 \mu \mathrm{katal} / \mathrm{l}$ und waren schnell rückläufig. Ein standardisiertes Schmerzmanagement mit Metamizol und Piritramid bewährte sich. Der Nahrungsaufbau war bei Verlegung bei allen Patienten unvollständig.

Schlussfolgerung. Der postoperative Verlauf war in 22 Fällen unkompliziert. Ein schneller und anhaltender Rückgang des postoperativen Glukosebedarfs war sowohl nach Resektion des Fokus als auch in der Mehrzahl der Fälle nach sparsamer Pankreasteilresektion zu beobachten

\section{PO-34}

Toxic-Schock-Syndrom: 20 Jahre Erfahrung auf der pädiatrischen Intensivstation

\section{S. Rödl', G. Wendelin', I. Marschitz' , G. Zobel', W. Zenz}

'Universitätsklinik für Kinder und Jugendheilkunde, Pädiatrische Intensivstation, Graz, Österreich, ${ }^{2}$ Universitätsklinik für Kinder- und Jugendheilkunde Salzburg, Neonatologie, Salzburg, Österreich, ${ }^{3}$ Univ. Klinik für Kinder- und Jugendheilkunde Graz, Klin. Abt. für Allgemeinpädiatrie, Graz, Österreich

Hintergrund. Das Toxic-Schock-Syndrom (TSS) ist eine akute systemische Erkrankung charakterisiert durch hohes Fieber, arterielle Hypotonie, Erbrechen, Bauchschmerzen, Durchfall, Muskelschmerzen, nichtfokale neurologische Auffälligkeiten und ein Erythem. Das TSS tritt in Assoziation mit Wunden mit einem anaeroben Milieu auf. Das für das TSS hauptverantwortliche TSS Toxin wird von Staphylococcus aureus gebildet und führt zu einem massiven Verlust von Flüssigkeit aus dem Gefäßsystem in Folge einer erhöhten Freisetzung von Interleukin 1 und Tumornekrosefaktor.

Zielsetzung. Es wird ein Überblick über die innerhalb der letzten 20 Jahre an unserer Pädiatrischen Intensivstation behandelten Patienten mit TSS geboten.

Methoden. Prospektive Studie an der interdisziplinären pädiatrischen Intensivstation eines Universitätsklinikums mit 12 Betten. Die Patientendaten wurden seit 1990 mittels Datenbanksystem gesammelt und bei Bedarf ausgewertet.

Patienten. 12 Patienten mit TSS im Zeitraum von 1990 bis 2010. Die Patienten wurden in Gruppen zu je 3 Jahren zusammenfasst um Veränderungen des Patientenkollektivs im Lauf der Zeit erfassen zu können. Endpunkte waren die Beatmungsdauer, die Aufenthaltsdauer an der Intensivstation und ein Scoring zur Erfassung von physiologischen Parametern. In allen Fällen wurde eine Infektion mit TSS Toxin bildenden Staphylococcus Aureus Stämmen nachgewiesen.

Ergebnisse. Bei unseren 12 Patienten betrug das mittlere Alter 9,5 $\pm 7,9$ Jahre. Als Ausgangspunkt für die Infektion konnte in einem Patienten eine Pneumonie, in 2 Patienten eine kleine Hautwunde nach einer oberflächlichen Brandverletzung und in 9 Patientinnen die Verwendung eines Tampons im Rahmen der Regelblutung identifiziert werden. Die mittlere Aufenthaltsdauer auf der Intensivstation betrug 14 Tage, die mittlere Beatmungsdauer 3 Tage, der PRISM III Score bei Aufnahme betrug im Mittel ${ }_{15}$ Punkte. Alle Patienten überlebten. Mit Ausnahme von einem Patienten mit Defektheilung konnte bei allen Patienten eine Resitutio ad integrum erreicht werden. Die häufigsten Symptome bei Aufnahme waren: Fieber $>39^{\circ} \mathrm{C}$, Hautauschlag mit diffus makulöser Erythrodermie, Kreislaufinsuffizienz mit arterieller Hypotonie und Zentralisation, Erbrechen, Durchfall, Myositis, Hyperämie der Wundregion (Verbrennungspatienten), prärenales Nierenversagen, Somnolenz.

Schlussfolgerung. Das TSS ist eine ernste systemische Komplikation von primär unkompliziert verlaufenden bakteriellen Lokalinfektionen. Für die Prognose erscheint die rechtzeitige Diagnose mit frühem Beginn einer aggressiven Schocktherapie von essentieller Bedeutung.

\section{PO-35}

Intensivmedizinische Behandlung von 60 Kindern mit RotavirusInfektion

\section{S. Rödl', I. Marschitz², G. Wendelin', G. Zobel', W. Zenz ${ }^{3}$}

'Universitätsklinik für Kinder und Jugendheilkunde, Pädiatrische Intensivstation, Graz, Österreich, ${ }^{2}$ Universitätsklinik für Kinder- und Jugendheilkunde, Neonatologie, Salzburg, Österreich, ${ }^{3}$ Univ. Klinik für Kinder- und Jugendheilkunde Graz, Klin. Abt. für Allgemeinpädiatrie, Graz, Österreich

Hintergrund. Rotavirus-Infektionen (RVI) sind eine der Hauptursachen der schweren akuten Gastroenteritis bei Kindern auf der ganzen Welt. Selbst in Ländern mit hohem Hygienestandard und guten medizini- 
schen Behandlungsmöglichkeiten kommen schwere und tödliche Fälle vor.

Zielsetzung. Wir bieten einen Überblick über schwer verlaufende RVI bei später Diagnosestellung.

Patienten und Methoden. Von 1990-2012 analysierten wir alle an unserer pädiatrischen Intensivstation aufgenommenen Kinder mit viraler Gastroenteritis und drohendem Organversagen. Patienten mit schweren Grunderkrankungen wurden von dieser Analyse ausgeschlossen.

Ergebnisse. 373 Patienten mit schwerer Gastroenteritis mussten aufgrund metabolischer Entgleisung und beginnendem Organversagen auf die Intensivstation stationär aufgenommen werden. Von diesen waren 60 RVI-positiv, davon 25 weiblich (41\%) und 35 männlich (59\%). Das mittlere Alter der RVI-positiven Patienten lag bei 16 Monaten [Median 10, (2-190)]. Bei 7 Patienten (11\%) war eine Intubation notwendig. Ein Patient starb trotz aller Bemühungen. Bei 59 Patienten führte eine Kombination von oraler und parenteraler Rehydratation zu einem stabilen Flüssigkeitshaushalt und vollständiger Genesung. Die mittlere Dauer des Aufenthalts auf der Intensivstation lag bei $4 \pm 6$ Tage. Die physiologischen Scoringsysteme APSC, OSF und PRISM III waren bei RVI positiven Patienten (APSC: $5,8 \pm 0,9$; OSF: 0,45 $\pm 0,14$; PRISMIII: $2,8 \pm 0,8$ ) deutlich höher als bei den Rotavirus negativen (APSC: $3,9 \pm 0,3$; OSF:

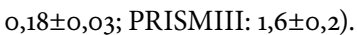

Schlussfolgerung. Obwohl RVI eine häufige Ursache für einen Krankenhausaufenthalt von kleinen Kindern darstellt, sind schwere Komplikationen unter rechtzeitiger Therapie selten. Auch in entwickelten Ländern in unseren Breiten mit modernen therapeutischen Möglichkeiten kann es durch RVI zu einem lebensbedrohlichen Ereignis bei zu später Diagnosestellung kommen.

\section{PO-36 \\ Lange Aufenthaltsdauer auf der Intensivstation durch Pneumokok- ken-Infektionen}

\section{S. Rödl', G. Wendelin', I. Marschitz², G. Zobel', W. Zenz ${ }^{3}$}

'Universitätsklinik für Kinder und Jugendheilkunde, Pädiatrische Intensivstation, Graz, Österreich, ${ }^{2}$ Universitätsklinik für Kinder- und Jugendheilkunde, Neonatologie, Salzburg, Österreich, ${ }^{3}$ Univ. Klinik für Kinder- und Jugendheilkunde Graz, Klin. Abt. für Allgemeinpädiatrie, Graz, Österreich

Hintergrund. Invasive Pneumokokken Infektionen wie Sepsis, Pneumonie und Meningitis stellen lebensbedrohliche Erkrankungen dar. Schwere Dauerfolgen mit bleibender Beeinträchtigung wie totalem Gehörverlust oder motorischen Ausfällen infolge einer ZNS Schädigung sind möglich. Die Übertragung von Pneumokokken erfolgt über den Nasen Rachen Raum via Tröpfcheninfektion oder engen direkten Kontakt.

Zielsetzung. Wir präsentieren unsere Erfahrungen mit Pneumokokken Infektionen verglichen mit anderen bakteriellen Infektionen über einen Zeitraum von 20 Jahren.

Methoden. Prospektive Studie an der interdisziplinären pädiatrischen Intensivstation eines Universitätsklinikums mit 12 Betten. Die Patientendaten wurden seit 1990 mittels Datenbanksystem gesammelt und bei Bedarf ausgewertet. Patienten: 315 Patienten mit schweren bakteriellen Infektionen im Zeitraum von 1990 bis 2010. Die Einteilung der Patienten erfolgte nach dem Zeitpunkt der Behandlung in Gruppen zu je 3 Jahren, nach der Art des Erregers und nach der Lokalisation der Infektion. Endpunkte waren die Beatmungsdauer, die Aufenthaltsdauer an der Intensivstation und die Ergebnisse von verschiedenen Scoring-Systemen zur Erfassung von physiologischen Parametern.

Ergebnisse. Bei 27 von 315 Patienten konnten Pneumokokken mittels Bakterienkultur nachgewiesen werden. Von 103 Patienten mit der klinischen Diagnose einer bakteriellen Meningitis war bei 15 der Pneumokokken Nachweis positiv. Von 139 Patienten mit bakterieller Pneumonie waren 10 Patienten Pneumokokken positiv. Von 73 Patienten mit septischem Schock war bei 2 die Untersuchung auf Pneumokokken positiv. Das physiologische Scoring erbrachte innerhalb der Untergruppen der
Erreger bezüglich der Erkrankungsschwere keine Unterschiede. Bei einem Vergleich der Untergruppen nach Erregern wurde die höchste Beatmungsdauer in der Untergruppe mit Pneumokokken Infektion und septischem Schock gefunden (10 Tage vs. o,6 bis 2,8 Tage). Der größte Unterschied zwischen Patienten mit Pneumokokken positiven und Pneumokokken negativen Infektionen bestand in der längeren Aufenthaltsdauer auf der Intensivstation in der Gruppe mit Pneumokokken positiven Infektionen (Meningitis 16 vs. 10 Tage, Pneumonie 22 vs. 12 Tage, septischer Schock 17 vs. 9 Tage).

Schlussfolgerung. Pneumokokken-Infektionen bedingen verglichen mit anderen bakteriellen Infektionen deutlich längere Aufenthalte auf der Intensivstation aufgrund häufiger Komplikationen.

\section{PO-37}

Cholangitis im Rahmen einer hämorrhagischen Enterokolitis und nachfolgendem hämolytisch-urämischem Syndrom

\section{Stamm ${ }^{1}$, H. Schwalm ${ }^{1}$, P. Lasch ${ }^{7}$}

${ }^{1}$ Klinikum Bremen-Mitte gGmbH Kinderklinik Prof. Hess, Pädiatrische Intensivmedizin, Bremen, Deutschland

Ein 5 Jahre altes Mädchen entwickelte nach einem Türkei-Urlaub eine hämorrhagische Enterokolitis mit hohen Entzündungswerten (Leukozytose max. 33/nl mit massiver Linksverschiebung, CrP max. $142 \mathrm{mg} / \mathrm{l})$. Bei zunehmender Verschlechterung des Allgemeinzustandes, abfallenden Hämoglobin-Werten und Thrombozyten sowie steigenden Kreatinin-Werten erfolgte am vierten Tag die Verlegung auf die Kinderintensivstation bei V. a. hämolytisch-urämisches Syndrom. Bei rasch zunehmender Ödem- und Aszitesbildung mit beidseitigen Pleuraergüssen kam es zur respiratorischen Dekompensation, die ab dem fünften Tag eine maschinelle Beatmung notwendig machte. Im Verlauf bestand eine Olig- bis Anurie mit stark steigenden Retentionswerten, einer hämolytischen Anämie und einer Thrombopenie (Laborwerte bei Aufnahme vs. schlechteste Laborwerte: Hämoglobin 14,7 g/dl vs. 5,7 g/ dl, Thrombozyten 301/nl vs. 17/nl, Kreatinin 0,36 mg/dl vs. 5,03 mg/dl). Es gelang der Nachweis eines enteropathogenen E. coli 026:K6o.

Neun Tage nach Aufnahme entwickelte sich ein ausgeprägter Ikterus mit Anstieg der Cholestaseparameter, der Transaminasen und der Lipase (Bilirubin max. $30,8 \mathrm{mg} / \mathrm{dl}$, Bilirubin direkt $26,2 \mathrm{mg} / \mathrm{dl}$, Alkalische Phosphatase max. 1395 U/l, gGT 4012 U/l, GOT 255 U/l, GPT 547 U/l, Lipase $806 \mathrm{U} / \mathrm{l})$. Eine MRCP am elften Tag bestätigte eine Cholangitis. Wir therapierten die Patientin mit Meropenem, Desoxycholsäure, Phenobarbital und einem 3-tägigen Steroidpuls. Die Symptomatik besserte sich langsam, eine Hypercholesterinämie und gGT-Erhöhung besteht bis zum heutigen Tage.

Systemerkrankungen aus dem rheumatischen Formenkreis, Autoimmunprozesse oder Vaskulitiden konnten wir ausschließen. In der Erwachsenen-Intensivmedizin wird eine Bilirubinerhöhung bei Intensivpatienten wiederholt mit Medikamenten, Toxinen, maschineller Beatmung, Sepsis und total parenteraler Ernährung in Zusammenhang gebracht [1]. Vor einigen Jahren wurde erstmals der Begriff der sekundär sklerosierenden Cholangitis bei kritisch-kranken Patienten (SC-CIP: „sclerosing cholangitis in critically ill patients") geprägt [2]. Hierbei kommt es bei schwerster Erkrankung des Patienten zu einer Cholangitis mit hoher Wahrscheinlichkeit der biliären Zirrhose. Der klinische Verlauf unserer Patientin erinnert an dieses Krankheitsbild. Literatur hierzu fehlt jedoch in der Pädiatrie.

1. Kredel, Brederlau, Roewer, Wunder (2008) Cholestase und Leberinsuffizienz beim Intensivpatienten. Anaesthesist 57:1172-1182. doi 10.1007/s00101-008-1459-y

2. Gelbmann, Ruemmele, Wimmer, Hofstaedter, Goehlmann, Endlicher, Kullmann, Langgartner, Schoelmerich (2007) Ischemic-like cholangiopathy with secondary sclerosing cholangitis in critically ill patients. Am J Gastroenterol 102:1221-1229 


\section{PO-38}

\section{Akuter Thoraxschmerz mit 17 Jahren - nicht immer harmlos!}

\section{A. Hildebrandt ${ }^{1}$, D. Fatehpour', R. Douwa' ${ }^{2}$, W. Müller', T. Nüßlein ${ }^{1,}$} T. Hoppen

${ }^{1}$ Klinik für Kinder- und Jugendmedizin, Gemeinschaftsklinikum KoblenzMayen, Kemperhof Koblenz, Koblenz, Deutschland, ${ }^{2}$ Klinik für Innere Medizin II, Schwerpunkt Kardiologie, Gemeinschaftsklinikum Koblenz-Mayen, Kemperhof Koblenz, Koblenz, Deutschland

Einleitung. Die Liste der Differenzialdiagnosen beim akuten Thoraxschmerz umfasst neben zahlreichen harmlosen Erkrankungen wie Interkostalneuralgie, akute Pankreatitis, Pneumonie/Pleuritits und Ösophagitis selten auch potenziell lebensbedrohliche Ursachen wie akutes Koronar- und Aortensyndrom, Peri-/Myokarditis, Lungenembolie, Spontanpneumothorax und Ösophagusruptur.

Kasuistik. Ein 17 Jahre alter Junge wurde mit starken Schmerzen „hinter dem Herzen" vom Notarzt gesehen. Er habe den adipösen Jungen auf dem Sofa sitzend vorgefunden und ihm Piritramid verbreicht. Das 12-Kanal-EKG sei unauffällig gewesen. Seit 10 Tagen habe er einen viralen Infekt der Atemwege mit Husten jedoch ohne Fieber. Bei Aufnahme klagte der Patient weiterhin über starke retrosternale Schmerzen und Kribbeln in beiden Armen. Im EKG ST-Streckenhebung in I, aVR, aVL, V2-V6, und ST-Senkung in II, III, aVF mit ventrikulären Extrasystolen. Im Verlauf zunehmender R-Verlust in $\mathrm{V}_{2}-\mathrm{V}_{5}$. Laborchemisch waren die herzmuskelassoziierten Enzyme leicht erhöht. Echokardiographisch zeigte sich eine ausreichende Kontraktilität, in der Verlaufskontrolle dann Hypokontraktilität des anterioren Myokards. Primär bestand der Verdacht auf eine Peri-/Myokarditis. Aortendissektion, Lungenembolie, Ösophagusruptur und Pneumothorax wurden ausgeschlossen. Bei zunehmenden Hinweisen auf einen akuten Herzinfarkt erfolgte die zügige Verlegung zur Herzkatheteruntersuchung in die invasive Kardiologie. Hier zeigte sich in der Koronarangiographie ein Verschluss des proximalen Ramus interventrikularis anterior (Typ-C1-Stenose). Unmittelbar erfolgte eine Rekanalisation mittels DE-Stentimplantation (Intengrity Resolute 3,5/15 $\mathrm{mm}$ ) und eine begleitende medikamentöse Therapie mit Clopidogrel und Acetylsalicylsäure. Das NT-proBNP stieg bis auf $1340 \mathrm{pg} / \mathrm{ml}$. Die Verlaufskontrollen dokumentierten eine deutliche körperliche Leistungsminderung mit Belastungsdyspnoe bei hochgradig reduzierter linksventrikulärer Pumpfunktion unter ischämischer Kardiomyopathie mit apikoseptaler Narbe und Wandausdünnung. 15 Monate nach dem Ereignis wurde der Patient mit einem intrakardialen Defibrillator versorgt.

Schlussfolgerung. Ein Herzinfarkt mit 17 Jahren bei einem zuvor herzgesunden Patienten ist ein äußerst seltenes Ereignis. Weder der internistische Notarzt und der Aufnahmearzt der Klinik noch die Erstbehandelnden auf der Kinderintensivstation hatten diese schwerwiegende Diagnose primär vermutet. Ätiologisch ist neben der Beachtung etwaiger Risikofaktoren und nach Ausschluss von Gerinnungsstörung und anatomischer Anomalie bei diesem Patienten auch ein zugrundeliegender Intimaschaden der Koronarien zu diskutieren. Die typischen progredienten klinischen und in kurzer zeitlicher Abfolge wiederholt erfassten und unmittelbar interpretierten laborchemischen, echokardiographischen und gefäßarealkorrelierten elektrokardiographischen Zeichen können bei der Diagnose- und Indikationsstellung zur Koronarangiographie eindeutig richtungsweisend sein.

\section{PO-39}

„Bagatelltrauma?" - Kalottenfraktur, epidurales Hämatom und Hemiparese bei einem Säugling nach Sturz aus geringer Höhe

N. Köhler', A. von der Wense², T. Maiberger', C. Junge ${ }^{3}$, P. Kunkel ${ }^{4}$

${ }^{1}$ Altonaer Kinderkrankenhaus, Neonatologie und Intensivmedizin, Hamburg, Deutschland, ${ }^{2}$ Altonaer Kinderkrankenhaus, Neonatologie und pädiatrische Intensivmedizin, Hamburg, Deutschland, ${ }^{3}$ Altonaer Kinderkrankenhaus, Kinderradiologie, Hamburg, Deutschland, ${ }^{4}$ Altonaer Kinderkrankenhaus, Kinderneurochirurgie, Hamburg, Deutschland

Anamnese. Sechs Monate alter Säugling, der aus dem Bett der Eltern aus ca. $50 \mathrm{~cm}$ Höhe auf Parkettboden gestürzt war, sofort geweint habe und kurz danach einschlief. Keine Hinweise auf eine körperliche Kindesmisshandlung. Vorstellung beim Kinderarzt bei blassem Hautkolorit direkt im Anschluss. Bei Anisokorie, Verlegung der Patientin per Hubschrauber in unsere Klinik.

Diagnostik. Kraniales MRT: notfallmäßig nur transversale T2-gewichtete Sequenz, Darstellung eines ausgedehnten epiduralen Hämatoms links mit massiver Mittellinienverlagerung. Postoperativ im MRT Nachweis von ödematösen Veränderungen links mit Betonung im Stromgebiet der A. cerebri media.

Therapie und Verlauf. Bei Aufnahme komatöses Kind, GCS 6, spontan atmend. Sofortige Intubation, MRT und Kraniektomie, Hämatomausräumung und Anlage einer Hirndrucksonde. Im Neurointensivmonitoring postoperativ isokore Pupillen und wiederholt normale Dopplerflussprofile der intrakraniellen Arterien. Deutliche armbetonte Hemiparese rechts, sowie Schluck-Koordinationsstörung, im Verlauf deutliche Besserung. Nach 13 Tagen Verlegung in eine neurologische Rehabilitationsklinik.

Schlussfolgerung. Bei Schädelhirntraumen im Säuglingsalter nach „Bagatelltrauma“ ist nach der Literatur eine epidurale Blutung sehr selten. Unser Fall zeigt mit zusätzlicher druckbedingter Schädigung der linken Hemisphäre einen besonders schweren Verlauf trotz umgehender Therapie.

\section{Kardiologie - Kreislauf}

\section{PO-40}

Medikamentöser Ductusverschluss mit Paracetamol bei Frühgeborenen

\section{A. Oberthür', K. Mehler', T. Keller', B. DeCarolis', B. Roth' ', A. Kribs'}

'Univ.-Kinderklinik, Neonatologie und Pädiatrische Intensivmedizin, Köln, Deutschland

Hintergrund. Ein medikamentöser Ductusverschluss bei Frühgeborenen erfolgt standardmäßig mit einem Cyclooxygenase(COX)-Hemmer (Ibuprofen oder Indomethazin). Beide Substanzen können potentiell gravierende Nebenwirkungen hervorrufen. Kürzlich wurde über einen alternativen Therapieansatz mit Paracetamol (PCM) berichtet. Effektivität und Verträglichkeit einer PCM-Therapie bei Frühgeborenen sind bislang nur unzureichend untersucht.

Fragestellung. Überprüfung der Effektivität und Verträglichkeit von PCM zur Behandlung eines offenen Ductus arteriosus Botalli bei Frühgeborenen mit einem Gestationsalter $<28$ Wochen und unzureichendem Ansprechen auf oder Kontraindikationen für eine Therapie mit COX-Hemmern.

Material und Methoden. Gemäß dem internen Standard unserer Klinik wurde bei allen Frühgeborenen im Jahr 2012 ein frühtherapeutischer Ductusverschluss mit Indomethazin durchgeführt. Im Falle eines unzureichenden Therapieansprechens, mehrfach wieder eröffnetem Ductus oder bestehenden Kontraindikationen gegenüber einer Therapie mit Indomethazin oder Ibuprofen, erfolgte eine medikamentöse Therapie mit PCM $(15 \mathrm{mg} / \mathrm{kg}$ alle 6 Stunden bis zum Verschluss). Der Therapieerfolg wurde mittels Echokardiographie dokumentiert. 
Ergebnisse und Diskussion. Acht Frühgeborene (Gestationsalter 23 1/7 bis 25 1/7 Wochen, mittleres Geburtsgewicht $547 \mathrm{~g}$ (420-670 g), die mit mindestens einem Zyklus ( $\geq_{3}$ Gaben) eines COX-Hemmers vorbehandelt waren (3-mal 1 Zyklus, 4-mal 2 Zyklen und 1-mal 3 Zyklen) wurden mit PCM behandelt. In 6 dieser 8 Fälle konnte im Mittel nach 7 (4-9) Gaben ein Ductusverschluss erreicht werden (75\%). In einem Fall erfolgte eine notfallmäßige chirurgische Ductusligatur nach nur zwei Gaben PCM aufgrund einer Anurie infolge renaler Minderperfusion. In einem zweiten Fall erfolgte nach insgesamt 8 Gaben PCM aufgrund eines anhaltend hämodynamsich signifikanten Ductus arteriosus ein erneuter Wechsel auf Indomethazin. In zwei Fällen mit initial erfolgreichem Ductusverschluss nach PCM erfolgte ein zweiter PCM-Therapiezyklus aufgrund eines wieder eröffneten Ductus nach 10 bzw. 16 Tagen (Wiedereröffnungsrate 30\%). In beiden Fällen war der Ductusverschluss erfolgreich, so dass die kumulative Effektivität $80 \%$ (8 von 10 Zyklen) beträgt. In keinem Fall traten Therapie-bedingte Nebenwirkungen auf. Epidemiologische Daten belegen eine deutliche Assoziation zwischen einer Therapie mit PCM und asthmatischen Beschwerden im Kindesalter. Derartige Effekte konnten aufgrund der noch kurzen Nachbeobachtungszeit in der vorliegenden Arbeit nicht erfasst werden. Schlussfolgerung. PCM kann eine effektive und verträgliche Therapieoption zum Ductusverschluss bei Frühgeborenen mit fehlendem Ansprechen auf oder bestehender Kontraindikationen gegenüber einer Behandlung mit Cyclooxygenasehemmern darstellen. Langzeiteffekte (erhöhtes Asthmarisiko) sollten im Rahmen kontrollierter Studien erhoben werden.

\section{PO-41}

\section{Einfluss der Thrombozytenwerte auf den Erfolg einer pharmako- logischen Therapie des persistierenden Ductus arteriosus bei VLBW-Frühgeborenen}

\section{H. Sallmon', S. Weber', J. Dirks', T. Schiffer', G. Hansmann ${ }^{3}$, C. Bührer', P. Koehne ${ }^{1}$ \\ 'Klinik für Neonatologie, Charité - Universitätsmedizin Berlin, Berlin, Deutschland, ${ }^{2}$ Pädiatrische Intensivmedizin, Campus Virchow-Klinikum, Charité - Universitätsmedizin Berlin, Berlin, Deutschland, ${ }^{3}$ Pädiatrische Kardiologie und Intensivmedizin, Medizinische Hochschule Hannover, Hannover, Deutschland}

Hintergrund und Fragestellung. Der Einfluss niedriger Thrombozytenwerte auf die Spontanverschlussrate des Ductus arteriosus wird kontrovers diskutiert. Kürzlich konnten wir zeigen, dass eine Thrombozytopenie am ersten Lebenstag nicht mit einer niedrigeren Spontanverschlussrate des Ductus bei VLBW-Frühgeborenen assoziiert ist. Die vorliegende Arbeit untersuchte den Einfluss der Thrombozytenwerte auf den Erfolg einer pharmakologischen Ductustherapie.

Methoden. In dieser retrospektiven Studie wurde untersucht, ob das Vorliegen einer Thrombozytopenie unmittelbar vor einer pharmakologischen Ductustherapie (Indometacin oder Ibuprofen) bei VLBWFrühgeborenen $(n=471)$ mit einer erhöhten Therapieversagerrate assoziiert ist.

Ergebnisse. Von 471 eingeschlossenen VLBW-Frühgeborenen, die eine pharmakologische Ductustherapie erhielten, wurden 167 als Therapieversager eingestuft, da weiterhin ein hämodynamisch relevanter Ductus nach Therapie nachweisbar war. Es konnte keine Assoziation zwischen der Thrombozytenzahl und dem Therapieerfolg der pharmakologischen Ductustherapie gezeigt werden. Hingegen waren ein niedrigeres Geburtsgewicht, ein niedrigeres Gestationsalter und ein höherer CRIB-Score signifikant mit einem Versagen der medikamentösen Ductustherapie assoziiert.

Schlussfolgerungen. Die Höhe der Thrombozytenwerte vor pharmakologischer Therapie scheint nicht mit einer höheren Rate von Therapieversagern assoziiert zu sein. Die klinische Bedeutung der Thrombozytopenie ist daher nicht nur für die Rate therapiebedürftiger PDAs sondern auch für den Therapieerfolg kritisch zu hinterfragen. Zukünf- tige prospektive Studien zur Bedeutung der Thrombozyten für den PDA sollten neben der Thrombozytenzahl auch Parameter der Thrombozytenfunktion berücksichtigen.

\section{PO-42}

Prävalenz eines persistierenden Ductus arteriosus zum Zeitpunkt der Entlassung sehr unreifer Frühgeborener

\section{S. Weber', H. Sallmon'2, J. Pepper' ${ }^{2}$, T. Klippstein' ${ }^{2}$ I. Prühs², K. Weiss ${ }^{3}$ \\ S. Akanbi', C. Bührer', P. Koehne ${ }^{2}$}

'Pädiatrische Intensivmedizin, Campus Virchow-Klinikum, Charité - Universitätsmedizin Berlin, Berlin, Deutschland, ${ }^{2}$ Klinik für Neonatologie, Charité - Universitätsmedizin Berlin, Berlin, Deutschland, ${ }^{3}$ Klinik für Pädiatrie m. S. Kardiologie, Charité - Universitätsmedizin Berlin, Berlin, Deutschland

Einleitung. Bei Frühgeborenen mit einem Geburtsgewicht $<1500 \mathrm{~g}$ (VLBW) beträgt die Prävalenz eines persistierenden Ductus arteriosus (PDA) nach dem dritten Lebenstag bis zu 6o\%. Zur medikamentösen Therapie stehen zwei Cyclooxygenasehemmer, Indometacin und Ibuprofen, zur Verfügung. Bei den Therapieversagern der medikamentösen Ductustherapie erfolgt häufig eine chirurgische Ductusligatur. Einige Kinder werden jedoch bei Nichtvorliegen einer Indikation zum chirurgischen Ductusverschluss mit einem PDA entlassen.

Methodik. In dieser retrospektiven Analyse wurden alle zwischen 1998-2008 in der Klinik für Neonatologie der Charité am Campus Virchow-Klinikum geborenen VLBW-Frühgeborenen, die mit einem nachweisbaren PDA entlassen wurden, eingeschlossen. Es erfolgte die Auswertung der poststationär vornehmlich im Rahmen der Weiterbetreuung im Sozialpädiatrischen Zentrum erhobenen echokardiographischen Befunde.

Ergebnisse. Von 352 VLBW-Frühgeborenen hatten 70 nach der Entlassung aus der stationären Betreuung noch einen PDA. Bei 54 kam es zu einem Spontanverschluss, der überwiegend in den ersten 24 Monaten nach der Entlassung auftrat. Bei fünf Kindern erforderte ein hämodynamisch relevanter PDA den interventionellen Verschluss mittels Coil bzw. Amplatzer Duct Occluder (ADO) (3/2), bei weiteren drei Kindern war echokardiographisch ein PDA ohne hämodynamische Relevanz nachweisbar, zwei Kinder verstarben nach der Entlassung und bei sechs Kindern konnte kein poststationäres Follow-up nachvollzogen werden. Schlussfolgerung. Obwohl sich ein PDA in den meisten Fällen nach der Entlassung spontan verschließt, ist eine engmaschige poststationäre echokardiographische Weiterbetreuung notwendig, um eine drohende kardiale Dekompensation durch einen hämodynamisch relevanten Ductus arteriosus zu vermeiden und diese Patienten frühzeitig einer Katheterintervention zuzuführen.

\section{PO-43}

Ultrasonic cardiac output monitor for cardiac output measurement: is it equal to conventional echocardiography?

\section{P. Meyer Schiffer', C. Däster', M. Adams' ${ }^{1}$ R. Arlettaz Mieth \\ 'Universitätsspital Zürich, Klinik für Neonatologie, Zürich, Schweiz}

Background. Measurement of cardiac output as an additional parameter in assessing the circulatory status of premature and term infants has become more and more useful in guiding therapy of circulatory compromised babies. Echocardiography is the gold standard to do so but requires time and expertise. Ultrasonic cardiac output monitor (USCOM) is a rapidly available method to estimate cardiac output. However, it's accuracy for use in a neonatal intensive care unit (NICU) needs to be determined.

Methods. Cardiac output measurements of left ventricular cardiac output by echocardiography and USCOM were done paired. Measurements were obtained for premature as well as for term born infants $(n=49)$. Measurements were then compared. 


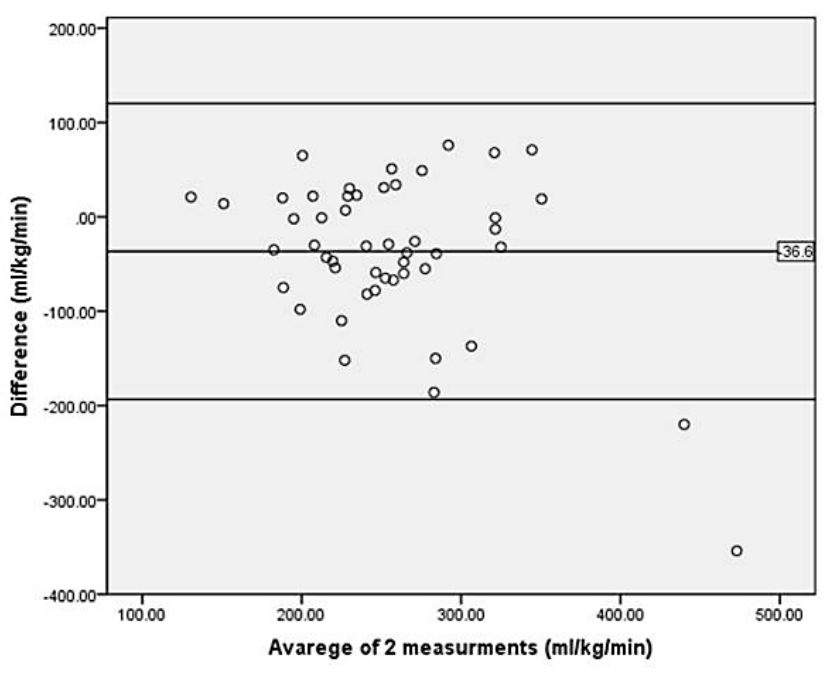

PO-43 Fig. 14

Results. Mean USCOM measurement differed from echocardiography in measuring left ventricular cardiac output by $-36.6 \mathrm{ml} / \mathrm{kg} / \mathrm{min}$ $(\mathrm{p}=0.003)$. The Bland-Altmann plot shows low measuring agreement (- Fig. 1). Variations for single results were up to $265 \mathrm{ml} / \mathrm{kg} / \mathrm{min}$. Conclusions. Agreement between the ultrasonic cardiac output monitor and echocardiography for left ventricular cardiac output measurement is poor. At this point USCOM measurement of left ventricular cardiac output as stand alone assessment tool in a NICU can not be recommended.

\section{PO-44}

\section{Arterio-ventrikuläres Coupling bei Frühgeborenen}

S. Baumgartner', M. Steiner', M. Olischar', M. Wald', G. Fischer ${ }^{3}$, T. Waldhör', T. Binder ${ }^{5}$, U. Salzer-Muhar

${ }^{1}$ Medizinische Universität Wien, Abt. für Neonatologie, pädiatrische Intensivmedizin und Neuropädiatrie, Wien, Österreich, ${ }^{2}$ Landeskrankenhaus Salzburg, Klinische Abteilung für allgemeine Pädiatrie und Intensivmedizin, Salzburg, Österreich, ${ }^{3}$ Medizinisches Informationsmanagement und Bildverarbeitung, Wien, Österreich, ${ }^{4}$ Center of Public Health, Abteilung für Epidemiologie, Wien, Österreich, ${ }^{5}$ Medizinische Universität Wien, Innere Medizin II, Wien, Österreich, 'Medizinische Universität Wien, Kardiologie, Wien, Österreich

Hintergrund. Das arterioventrikuläre Coupling (AVC) ist ein Index, der die Interaktion zwischen dem linken Ventrikel (ELV) und dem arteriellen systemischen Kreislauf (EA) beschreibt. Da das AVC mittels M-Mode Echokardiographie errechnet werden kann, könnte es routinemäßig an der NICU erfasst und zur Beurteilung der Hämodynamik beim kranken Frühgeborenen (FG) herangezogen werden.

Fragestellung. Besteht ein Unterschied in Bezug auf AVC, EA und ELV von FG mit pathologischer Hämodynamik in der transitorischen Phase der postpartalen Kreislaufumstellung i.e. FG mit therapiepflichtiger pulmonaler Hypertension (PH), FG mit hämodynamisch wirksamen Ductus arteriosus (hPDA) und von FG mit adäquater Anpassung der postpartalen Hämodynamik und unkompliziertem/"normalem" Verlauf?

Patienten und Methodik. AVC, EA und ELV wurden bei FG der SSW $23+0-31+6(n=103)$ jeweils in einer Gruppe von FG mit PH $(n=21)$ und hPDA $(\mathrm{n}=19)$ analysiert und mit den Werten von FG mit unkompliziertem Verlauf („normal“: $n=63$ ) verglichen. In die PH-Gruppe wurden FG inkludiert, die aufgrund ihrer klinischen Symptomatik und echokardiographisch nachgewiesener (supra-)systemischer $\mathrm{PH}$ eine
PH-Therapie erhalten hatten. In die hPDA- Gruppe wurden FG mit PDA inkludiert, wenn sie echokardiographisch einen enddiastolischen Fluss in der linken Pulmonalarterie (LPAd) von $>0,2 \mathrm{~m} / \mathrm{s}$ aufwiesen. In die „Normalgruppe“ fielen jene FG, die keinen Katecholaminbedarf hatten, keine $\mathrm{PH}$-Therapie benötigten und echokardiographisch einen LPAd $<0,2 \mathrm{~m} / \mathrm{s}$ und eine Ratio des linken Vorhofes zum Aortendurchmesser von $<1,4$ hatten. Alle Parameter wurden im zeitlichen Verlauf (Kategorie 1: 1.-3. Lebenstag; Kategorie 2: 4.-7. Lebenstag und Kategorie 3: 8.-30. Lebenstag) analysiert.

Ergebnisse. Das AVC war sowohl bei der PH- als auch bei der hPDAGruppe im Vergleich zur Normalgruppe niedriger. Im Vergleich zur Normalgruppe waren EA und ELV in der hPDA-niedriger und in der PH-Gruppe höher.

Schlussfolgerung. Der Verlauf von ELV, EA und AVC der Normalgruppe gibt Einblick in die Interaktion des linken Ventrikels mit dem arteriellen System beim FG mit adäquater postpartaler kardiovaskulärer Adapatation. Das höhere ELV und EA in der PH-Gruppe kann durch die interventrikuläre Dependenz mit konsekutiv verminderter Füllung des linken Ventrikels aber auch durch die in der PH-Therapie verwendeten Katecholamine erklärt werden. Das geringere ELV und EA in der hPDA-Gruppe resultiert aus der gesteigerten Volumsbelastung des linken Ventrikels und dem diastolischen Stealeffekt der Aorta. Das in beiden Gruppen geringere AVC spiegelt die pathologische Interaktion des linken Ventrikels mit dem arteriellen System wider und könnte somit zur Beurteilung der pathologischen Hämodynamik beim Frühgeborenen an der NICU beitragen.

\section{PO-45}

Dopplersonographische Diagnostik der Hypovolämie des Neugeborenen

\section{Langhammer', A. Reich', U. Fleischer', E. Robel-Tillig' 'Städtisches Klinikum St. Georg Kinderklinik, Leipzig, Deutschland}

Problem. Als Symptom vielfältiger neonatologischer Krankheitsbilder ist das Auftreten einer Hypovolämie bei Frühgeborenen oder reifen Neugeborenen zu beobachten. Das klinische Bild ist dabei recht unspezifisch und eine adäquate Therapie bedarf deshalb einer möglichst spezifischen Diagnostik. Dopplersonographische Untersuchungen des Herzens und der peripheren Arterien sind bewährte Untersuchungsmethoden in der Neonatologie. Ziel der vorliegenden Studie sind die Etablierungen validierter Werte zur Diagnostik der Hypovolämie im Rahmen einer neonatologischen Erkrankung.

Fragestellung. Ist es möglich, mit Hilfe der Bewertung der Accelerationszeit, der systolischen Spitzengeschwindigkeit und der Dauer der Systole in der Arteria cerebri anterior, die Diagnose Hypovolämie zu stellen und den Therapieverlauf zu kontrollieren?

Patienten und Methoden. Integriert werden 100 Patienten von denen 50 Neugeborene klinische Symptome der Hypovolämie (Tachykardie, zentral-periphere Temperaturdifferen $\mathrm{z}>\mathbf{2} \mathrm{s}$, verlängerte Rekapillarisierungszeit) boten und 50 Patienten hämodynamisch unauffällig waren. Als leicht zu erhebender peripherer Parameter wurden die Flussgeschwindigkeiten (PI und RI) in der A. cerebri anterior gemessen. Wir bestimmten außerdem die systolische Akzelerationszeit, Systolenlänge und Länge der Diastole in der ACA und verglichen diese mit den echokardiographischen Parametern (rechts- und linksventrikuläre systolische Zeitintervalle, Herzzeitvolumina). Die erhobenen Werte wurden vor und nach Volumengabe bei Patienten mit klinischem Verdacht auf Hypovolämie verglichen. Die Ausgangswerte aller Parameter wurden zur Sicherung der Diagnose Hypovolämie in beiden Gruppen verglichen.

Ergebnisse. Bei Patienten mit den klinischen Symptomen der Hypovolämie waren deutliche verkürzte Ejektionszeiten innerhalb der linksventrikulären Zeitintervalle darstellbar. Cardiac output wurde durch die erhöhte Herzfrequenz konstant gehalten. Es ließ sich eine signifikante Korrelation zur Akzelerationszeit und Länge der Systole in der Arteria cerebri anterior nachweisen. Damit war die Form der Kurve in 
der Arteria cerebri anterior durch eine spitze, schmale Systole gekennzeichnet. Nach Volumengabe kam es einerseits zur Verlängerung der linksventrikulären Ejektionszeit (12 ms) und ebenso zur Verlängerung der Akzelerationszeit $(150 \mathrm{~cm} / \mathrm{s} 2)$ und der Systolenlänge in der A. cerebri ant. (10 ms).

Schlussfolgerung. Es lässt sich damit aufzeigen, dass durch die technisch unkomplizierte Messung der Blutflussparameter in der A. cerebri ant. signifikante Rückschlüsse auf die Volumensituation bei Neugeborenen gezogen werden können.

\section{PO-46}

Verwertbarkeit von 24-h-Messungen der zerebralen und peripheren regionalen Sauerstoffsättigung bei Früh- und Reifgeborenen am ersten Lebenstag

\section{B. Schwaberger', J. Schmitz', C. Binder', B. Urlesberger', G. Pichler ${ }^{7}$ \\ 'Universitäts Klinik für Kinder- und Jugendheilkunde Graz, Abteilung für Neonatologie, Graz, Österreich}

Hintergrund. Nahinfrarotspektroskopie (NIRS) ist eine Methode zur kontinuierlichen, non-invasiven Messung der regionalen Sauerstoffsättigung $\left(\mathrm{rSO}_{2}\right)$ in Geweben wie Gehirn oder Muskel. Fehlmessungen durch Artefakte stellen ein Problem dar und sind ein Grund für die derzeit noch fehlende Anwendung in der klinischen Routine.

Fragestellung. Primäres Ziel dieser retrospektiven Analyse von zerebralen und peripher-muskulären 24-h-NIRS-Messungen am 1. Lebenstag von Früh- und Reifgeborenen war es, die Artefakthäufigkeit und den Einfluss von Artefakten auf die mittlere zerebrale und peripher-muskuläre Gewebesättigung zu erheben.

Material und Methode. Die rSO2 wurde bei 40 Neugeborenen (31 Frühund 9 Reifgeborene) mit dem INVOS Cerebral/Somatic Oximeter (Somanetics Corporation, USA) über jeweils $24 \mathrm{~h}$ mit Messbeginn innerhalb der ersten 6 Lebensstunden aufgezeichnet. Die Optoden wurden jeweils frontoparietal links (zerebral) sowie präduktal am rechten Unterarm (peripher-muskulär) befestigt und alle $6 \mathrm{~h}$ repositioniert, um der Entstehung von Druckstellen entgegenzuwirken. Die rSO2-Werte wurden in Abständen von 5-6 bzw. nach Umstellung der Software von 7-8 Sekunden registriert. Die arterielle Sauerstoffsättigung ( $\left.\mathrm{SpO}_{2}\right)$ wurde mittels Pulsoxymetrie (IntellVue MP5o Monitor; Philips, Niederlande) zu jeder Minute aufgezeichnet. Für die Identifizierung von Artefakten wurden 3 Kriterien (K) definiert und die Fehlmessungen aus dem Datensatz entfernt

- K 1: Nullwerte, die bei der Datenverarbeitung anstelle von fehlenden Messwerten aufscheinen,

- $\mathrm{K}$ 2: sprunghafte Veränderungen der $\mathrm{rSO}_{2}>15 \%$ mit einer neuerlichen sprunghaften Rückkehr aufs Ursprungsniveau innerhalb einer Minute, - $\mathrm{K}_{3}$ : gleiche Größe oder Übersteigen der $\mathrm{rSO}_{2}$ im Vergleich zur korrespondierenden $\mathrm{SpO}_{2}$ in den 1-Minuten-Kontrollen.

Um den Einfluss der Artefakte auf die mittlere $\mathrm{rSO}_{2}$ über $24 \mathrm{~h}$ zu untersuchen, wurden zwei Datensätze definiert und mittels t-Test verglichen - Datensatz 1: Rohdatensatz bearbeitet mit K 1,

- Datensatz 2: Rohdatensatz bearbeitet mit $\mathrm{K}_{1}+\mathrm{K}_{2}+\mathrm{K}_{3}$

Ergebnisse. In den zerebralen bzw. peripher-muskulären Messungen zeigte sich in K 1 eine Artefakthäufigkeit von $7,37 \%$ bzw. 10,83\%, in K 2 von 0,03\% bzw. 0,07\% und in K 3 von 1,50\% bzw. 6,89\%. Beim Vergleich der Datensätze 1 und 2 zeigte sich bei der mittleren $\mathrm{rSO}_{2}$ über $24 \mathrm{~h}$ in den zerebralen $(78,58 \pm 1,25 \%$ bzw. $78,54 \pm 1,24 \%$; $p=0,90)$ und periphermuskulären Messungen (83,74 $\pm 0,89 \%$ bzw. $83,50 \pm 0,87 \%$; $\mathrm{p}=0,35)$ kein signifikanter Unterschied.

Schlussfolgerung. Die Analyse unserer Daten zeigt, dass die mittlere $\mathrm{rSO}_{2}$ in der NIRS-Langzeitmessung über $24 \mathrm{~h}$ durch Artefakte nicht signifikant beeinflusst wurde. Dennoch wurden insgesamt $8,89 \%$ (zerebral) bzw. $17,78 \%$ (peripher-muskulär) der Messwerte als Artefakte identifiziert. Deshalb müssen bei Kurzzeitmessungen und der Anwendung als Routinemonitoring die rSO2-Werte hinsichtlich des möglichen Auftretens von Artefakten kritisch beurteilt werden.

\section{PO-47}

Zerebrale und periphere regionale Sauerstoffsättigung in der Adaptationsphase bei Frühgeborenen mit und ohne Atemunterstützung

\section{B. Schwaberger', G. Pichler', C. Binder', A. Avian², M. Pocivalnik', B. Urles-} berger

'Universitätsklinik für Kinder- und Jugendheilkunde, Abteilung für Neonatologie, Graz, Österreich, ${ }^{2}$ Medizinische Universität Graz, Institut für medizinische Informatik, Statistik und Dokumentation, Graz, Österreich

Hintergrund. Nahinfrarotspektroskopie (NIRS) ermöglicht kontinuierliche non-invasive Messungen der regionalen Sauerstoffsättigung $\left(\mathrm{rSO}_{2}\right)$ in Gewebe wie Gehirn oder Muskel. Durch zeitgleiche Positionierung von Optoden an Stirn, rechten Oberarm und Unterschenkel kann die regionale cerebrale ( $\left.\mathrm{rcSO}_{2}\right)$, prä- ( $\mathrm{rpSO}_{2}$ prä) und postduktale regionale periphere Sauerstoffsättigung (rpSO2post) von Frühgeborenen (FG) gemessen werden, und anhand unterschiedlicher Verlaufskurven die Dynamik beurteilt werden.

Fragestellung. Finden sich bei FG mit milder Atemnotsymptomatik und Notwendigkeit respiratorischer Unterstützung am Reanimationstisch unterschiedlich dynamische Verläufe der Sättigungskurven im Vergleich zu FG mit problemloser Adaptation?

Methodik. Die Studie wurde an der Abteilung für Neonatologie der Medizinischen Universität Graz nach positivem Votum der Ethikkommission durchgeführt. Eingeschlossen wurden FG nach Entbindung per sectionem mit Gestationsalter $=30+0$ und $<37+0$ bei vorliegendem schriftlichen Einverständnis der Eltern. Ausschlusskriterien waren schwerwiegende Malformationen und die Notwendigkeit einer primären Intubation. Die Erstversorgung der FG erfolgte gemäß den ERC Richtlinien 2010. Beatmung (PPV) oder Atemunterstützung (CPAP) wurden mit Neopuff Infant T-Piece-Resucitator (Perivent, Fisher\&Paykel; Neuseeland) appliziert. NIRS Messungen (Invos Cerebral/Somatic Oximeter Monitor, Covidien; USA) wurden durch zusätzliches Personal in den ersten 15 Lebensminuten vorgenommen ohne Routinemaßnahmen zu behindern. Mittels Pulsoxymetrie wurde die prä- (SpO2prä) und postduktale (SpO2post) Sauerstoffsättigung aufgezeichnet (IntelliVue $\mathrm{MP}_{3} \mathrm{O} / \mathrm{X}_{2}$ Monitor, Philips; Niederlande).

Ergebnisse. $\mathrm{SpO}_{2}$ : Beatmete $\mathrm{FG}$ zeigen eine kontinuierlich von $65 \%$ in Lebensminute (LM) 3 auf $90 \%$ in $\mathrm{LM}_{15}$ steigende $\mathrm{SpO}_{2}$ prä und SpO2post, wobei postduktal geringfügig niedrigere Werte gemessen werden. Bei nicht beatmeten FG steigt die $\mathrm{SpO}_{2}$ prä und SpO2post von $75 \%\left(\mathrm{LM}_{3}\right)$ auf 95\% (LM15) ohne Unterschied der prä- und postduktalen Werte. $\mathrm{rSO} 2$ : In der Gruppe der beatmeten FG zeigt sich ein kontinuierlicher Anstieg von 30\% (LM3) auf 75\% (LM15) der rcSO2, von $20 \%\left(\mathrm{LM}_{3}\right)$ auf $70 \%$ (LM15) der rpSO2prä und von $15 \%\left(\mathrm{LM}_{3}\right)$ auf 60\% (LM15) der rpSO2post. Bei den nicht beatmeten FG findet sich in $\mathrm{LM}_{3}$ ein Unterschied zwischen rpSO2prä (40\%) und $\mathrm{rpSO}_{2}$ post (30\%), ab LM1o zeigt sich kein Unterschied mehr, beide erreichen einen Steady state von $70 \%$. Die $\mathrm{rCSO}_{2}$ weist auch in dieser Gruppe im Vergleich die höchsten Werte auf: $50 \%$ in $\mathrm{LM}_{3}$, ab LM10 $80 \%$.

Schlussfolgerung. Trotz Leitlinien-konformer Sauerstoffanwendung zeigte sich ein deutlicher Unterschied der $\mathrm{SpO}_{2}$ zwischen den Gruppen. Auffallend war die unterschiedliche Dynamik im Verhalten der rpSO2prä und rpSO2post. Während diese bei FG mit Atemunterstützung keine Annäherung aufwiesen, zeigten sie in der Kontrollgruppe kongruente Werte ab LM1o. Die rcSO2 wies im Vergleich zur rpSO2prä und rpSO2post in beiden Gruppen die höheren Werte auf. 


\section{PO-48}

\section{Die Wirkung von Magnesium auf die fetalen Herzzeitintervalle -} eine Magnetokardiographiestudie

\section{A. Wacker', J. Brändle', M. Weiss', J. Muenssinger ${ }^{3}$, A. Zimmermann ${ }^{1,}$ H. Abele', R. Goelz', H. Preiss及}

'Universitätsklinik für Kinderheilkunde und Jugendmedizin, Neonatologie, Tübingen, Deutschland, ${ }^{2}$ Universitäts- Frauenklinik, Tübingen, Deutschland, ${ }^{3} \mathrm{fMEG}$ Zentrum, Tübingen, Deutschland

Hintergrund. Magnesiummangel in der Schwangerschaft ist ein häufiges Problem. Daher ist eine entsprechende Supplementation weit verbreitet. Magnesium passiert die Plazentaschranke. Da die fetale Niere das Magnesium jedoch nicht so effizient ausscheiden kann wie die mütterliche Niere, sind Effekte auf die fetalen Herzzeitintervalle möglich, jedoch bislang nicht bekannt.

Studiendesign. 6o Schwangere wurden im Rahmen einer Beobachtungsstudie rekrutiert. 31 gesunde Frauen erhielten im Rahmen der Schwangerschaft orales Magnesium. In Ergänzung zum Ultraschall wurde eine fetale Magnetokardiographie (fMKG) durchgeführt, um die fetalen Herzzeitintervalle zu bestimmen. Diese Bestimmung der Herzzeitintervalle erfolgte nach einer festgelegten Vorgehensweise. Messparameter (P-Welle, PQ-Segment, PR-Interval, QRS Komplex, ST Segment, T-Welle and QTc Interval) wurden erfasst. Diese wurden sodann mit einer gesunden Kontrollgruppe verglichen.

Ergebnisse. Es zeigte sich im Vergleich zur Kontrollgruppe eine signifikante Verbreiterung des QRS-Komplexes $(\mathrm{p}=\mathrm{0}, \mathrm{0O} 4)$ bei Feten, deren Mütter orales Magnesium (240 mg/Tag) einnahmen.

Schlussfolgerung. Feten, die Magnesium ausgesetzt waren, zeigten eine intraventrikuläre Leitungsverzögerung im Vergleich zur Kontrollgruppe. Das neonatale Outcome war bei allen Neugeborenen jedoch nich beeinträchtigt. Obwohl das fMKG derzeit eine präklinische Methode ist, könnte sich diese Methode als ergänzendes pränatales Monitoring eignen.

\section{PO-49 \\ Monotherapie der postoperativen junktional ektopen Tachykardie mit R-Wellen-getriggerter Vorhofstimulation anhand von zwei Fallbeispielen}

\section{F. Egender', A. Entenmann', M. Michel', H. Kramer ${ }^{1}$ \\ 'Univ.-Klinikum Schleswig-Holstein, Campus Kiel, Klinik für Kinderkardio- logie, Kiel, Deutschland}

Einleitung. Nach Operationen bei Kindern mit angeborenen Herzfehlern kommt es in 5-14\% der Fälle zum Auftreten einer junktional ektopen Tachykardie (JET). Der Erregungsursprung liegt im Bereich des Hisschen-Bündels. Die fehlende synchronisierte Vorhofaktion führt zu einer deutlich reduzierten Auswurfleistung des Herzens. Die etablierte Therapie besteht aus der Gabe von Antiarrhythmika und der Kühlung des Patienten. Ein neuer Therapieansatz ist die R-Wellen getriggerte Vorhofstimulation (AVT-Pacing). Durch Vertauschen der externen Schrittmacherkabel werden hierbei die Vorhöfe vor den QRS-Komplexen der JET stimuliert. Nach anfänglicher additiver Verwendung dieser Technik in unserer Klinik beschreiben wir hier die Kasuistiken von zwei Patienten, die mit AVT-Pacing als Monotherapie erfolgreich behandelt werden konnten.

Hintergrund. Im Zeitraum 7/o9 bis 12/11 wurden in unserem Zentrum 780 Patienten am Herzen operiert. Neunundzwanzig Patienten entwickelten eine JET, davon wurden 18 Patienten mit AVT-Pacing behandelt. Bei 2 Patienten wurde AVT-Pacing als Monotherapie eingesetzt (keine Kühlung $<36,5^{\circ} \mathrm{C}$, kein Amiodaron).

Kasuistik I. Alter 8 Monate, AV-Kanal, Beginn der JET 1,5 h postoperativ, Beginn mit AVT-Pacing 1,5 h postoperativ, Frequenzkontrolle ( $\mathrm{HF}<16 \mathrm{o} /$ min) 19,o h nach Pacing-Beginn, Re-Sinusrhythmus 43,5 h nach PacingBeginn, max. Laktat 2,6 mmol/l, Freiheit von Intensivmedizin nach 4 Tagen postoperativ, Entlassung nach 7 Tagen postoperativ.
Kasuistik II. Alter 7 Monate, VSD-Verschluss, Beginn der JET 23,5 h postoperativ, Beginn mit AVT-Pacing 23,5 h postopertiv, Frequenzkontrolle $(\mathrm{HF}<160 / \mathrm{min}) 19,5 \mathrm{~h}$ nach Pacing-Beginn, Re-Sinusrhythmus $21,5 \mathrm{~h}$ nach Pacing-Beginn, max. Laktat $3 \mathrm{mmol} / \mathrm{l}$, Freiheit von Intensivmedizin nach 3 Tagen postopertiv, Entlassung nach 6 Tagen postoperativ. Diskussion. AVT-Pacing kann offenbar auch als Monotherapie mit gutem Erfolg zur Behandlung der postoperativen JET eingesetzt werden. Es erscheint daher sinnvoll, sie als ersten Therapieschritt vor der Kühlung und Antiarrhytmika-Gabe zu verwenden, um die Nebenwirkungen dieser Therapieformen zu vermeiden.

\section{PO-50}

Ektope atriale Tachykardie (EAT) mit intraatrialem Mikroreentry seltene Differenzialdiagnose der supraventrikulären Tachykardie (SVT) bei einem Frühgeborenen

\section{F. Riede', R. Gebauer', J. Hambsch', U. Thome'2, I. Dähnert'}

'Herzzentrum, Universität Leipzig, Kinderkardiologie, Leipzig, Deutschland, ${ }^{2}$ Klinik und Poliklinik für Kinder- und Jugendmedizin der Universität Leipzig, Neonatologie, Leipzig, Deutschland

Einleitung. SVT sind die häufigsten symptomatischen Tachyarrhythmien bei Früh- und Neugeborenen. Sie werden in der Regel über viele Stunden gut toleriert, können aber im Verlauf zu einer ausgeprägten Herzinsuffizienz führen. Meist liegen atrioventrikuläre Reentry-Mechanismen zugrunde, die sich gut erkennen und behandeln lassen. In Einzelfällen können sich der klinische Verlauf und die Therapie allerdings sehr schwierig gestalten.

Kasuistik. Nach Diagnose einer fetalen Tachykardie am Vortag erfolgte die Geburt eines weiblichen hypertrophen Frühgeborenen durch Sectio beim Gestationsalter von $36+4$ Schwangerschaftswochen mit einem Geburtsgewicht von 3400 g. Eine postnatale Anpassungsstörung (Apgar $5 / 6 / 7$ ) besserte sich unter pharyngealer Beatmung nur unzureichend. Im EKG zeigte sich eine SVT mit einer Herzfrequenz um $245 / \mathrm{min}$. Nach Gabe von Adenosin demaskierte sich eine EAT mit 1:1 Überleitung. Unter Behandlung mit Digoxin und Sotalol kam es zu einer kardiorespiratorischen Insuffizienz mit Katecholamin- und Beatmungspflichtigkeit. Eine pulmonale Hypertension wurde mit inhalativem Stickstoffmonoxid behandelt. Nach Verlegung in unsere Einrichtung im Alter von 30 Stunden bestand nach einmaliger elektrischer Kardioversion Sinusrhythmus um 120/min. Im Verlauf kam es zu einer Stabilisierung des Allgemeinzustandes, die eine schnelle Beendigung der Katecholamintherapie, die Extubation am 3. Lebenstag und schließlich eine Entwöhnung von der CPAP-Atemhilfe ermöglichte. Bei ab dem 6. Lebenstag rezidivierenden Tachykardien waren wiederholte elektrische Kardioversionen erforderlich, die erst nach Gabe von Amiodaron erfolgreich waren. Eine Anfallsfreiheit konnte erst unter kombinierter antiarrhythmischer Therapie mit Digoxin, Sotalol und Propafenon erreicht werden. Bei Entlassung am 18. Lebenstag bestand Sinusrhythmus mit zahlreichen isolierten Vorhofextrasystolen. Im Verlauf sind bis zum Alter von 6 Wochen unter Fortführung der Therapie keine erneuten Tachykardien aufgetreten.

Diskussion. Die elektrische Kardioversion ist bei der EAT aufgrund der meist zugrundeliegenden abnormalen Automatie in der Regel nicht erfolgreich bzw. nicht indiziert, führte aber bei unserer Patientin zumindest initial zu einer Konversion in einen normofrequenten Sinusrhythmus. Ursache hierfür dürfte ein intraatrialer Microreentry-Mechanismus sein, der bei der Entstehung der Tachykardie beteiligt war. Die Möglichkeit des Vorliegens dieser ausgesprochen seltenen Form einer EAT rechtfertigt unseres Erachtens den Versuch einer elektrischen Kardioversion insbesondere beim hämodynamisch instabilen Kind. 


\section{PO-51}

\section{Fetale Myokardnekrose nach intrauteriner Ballonvalvuloplastie}

\section{A. Müller', U. Gembruch'2, J. Breuer ${ }^{3}$}

'Universitätsklinik Bonn, Abteilung für Paidopathologie, Bonn, Deutschland, ${ }^{2}$ Universitäts-Frauenklinik Bonn, Klinik für Geburtshilfe und Pränataldiagnostik, Bonn, Deutschland, ${ }^{3}$ Universitätsklinik Zentrum f. Kinderheilkunde, Bonn, Deutschland

Einführung. Der vorzeitige Verschluss des Foramen ovales ist ein Risikofaktor für das postnatale Outcome von Kindern mit kritischer Aortenstenose und hypoplastischem Linksherzsyndrom. Es tritt bei ca. 6\% der betroffenen Feten auf. Eine pränatale linksatrial druckentlastende Sprengung des Foramen ovale gilt angesichts des fortschreitenden pulmonalvenösen Gefäßremodelings als indiziert, insbesondere, wenn eine Aortenklappensprengung nicht möglich ist.

Fallbericht. Wir berichten über einen in der $24+1$ SSW intrauterin verstorbenen Fetus mit hypoplastischem Linksherzsyndrom, bei dem im pränatalen Ultraschall eine kritische Aortenstenose mit intaktem Vorhofseptum ohne linksatrial druckentlastende Venen diagnostiziert worden war. Eine intrauterine Sprengung der Aortenklappe in der $23+1$ SSW, bei der routinemäßig Suprarenin transventrikulär injiziert wurde, blieb erfolglos. Da sich in den darauf folgenden Tagen der $\mathrm{Zu}$ stand des Feten kritisch verschlechterte, wurde eine Woche später eine interventionelle intrauterine Eröffnung des Foramen ovale durchgeführt. Der Fetus verstarb wenige Stunden nach dem Eingriff.

Obduktionsbefund. Bei der Obduktion fand sich ein moderates Hämatoperikard, ein intaktes Vorhofseptum bei einem kleinen linken Ventrikel und eine hochgradig stenotische bikuspide Aortenklappe mit septumnahen linksventrikulären im Epikard nachweisbaren Einstichstellen. In enger räumlicher Assoziation war das Myokard des apikalen Anteils sowohl des linken als auch rechten Ventrikels infolge einer zentral gelegenen, schichtförmigen Nekrose geteilt in eine lumenwärts intakte endokardiale sowie eine nach außen hin intakte epikardiale Myokardschicht. Histologisch fand sich in den an die midmyokardialen Nekrosen angrenzenden Schichten eine Abräumreaktion und Organisation. Diskussion. Es liegt hier der ungewöhnliche Befund einer unphysiologischen und nicht durch eine pathophysiologisch bedingte Ischämie erklärbaren zentralen, schichtförmigen Myokardnekrose vor, die die Herzwand in zwei Schichten teilt. Mangels einer pathophysiologischen Erklärung ist als auslösender Faktor für die Nekrose eine lokale Minderperfusion nach Suprarenin-Injektion im Rahmen des transventrikulären Zugangs bei der versuchten intrauterinen Aortenklappensprengung zu diskutieren. Dafür spricht, dass sich a) die nekrotischen Areale in engem räumlichem Bezug zu den linsventrikulären septumnahen Einstichstellen fanden und b) das Alter der Nekrose mit dem Zeitpunkt der versuchten Klappensprengung deckte.

\section{PO-52 \\ Komplizierte Mitralklappenendokarditis eines immunologisch und herzgesunden Jugendlichen bei Staph. aureus-Sepsis}

\section{E. Böschen', T. Maiberger' ${ }^{2}$ C. Thiel ${ }^{3}$, A. Riso ${ }^{4}$, A. von der Wense ${ }^{5}$}

${ }^{1}$ Altonaer Kinderklinik, Pädiatrische Intensivmedizin, Hamburg, Deutschland, ${ }^{2}$ Altonaer Kinderkrankenhaus, Neonatologie/pädiatrische Intensivmedizin, Hamburg, Deutschland, ${ }^{3}$ Universitäres Herzzentrum Hamburg Eppendorf, Kinderkardiologie, Hamburg, Deutschland, ${ }^{4}$ Universitätsklinik Eppendorf, Klinik für Kinderherzchirurgie, Hamburg, Deutschland, ${ }^{5}$ Altonaer Kinderkrankenhaus, Neonatologie und pädiatrische Intensivmedizin, Hamburg, Deutschland

Anamnese. Ein 13 Jahre alter, bisher gesunder Junge mit leichtgradiger Neurodermitis stellte sich mit akuten Symptomen von hohem Fieber, Durchfall und Kopfschmerzen in einer auswärtigen Klinik vor. Bei zunehmender respiratorischer Insuffizienz und Vigilanzstörung erfolgte die Verlegung in unsere Klinik.
Diagnostik. Kraniales MRT: mehrere hyperintense Herde im Bereich von Kleinhirn und Großhirn. Liquordiagnostik ohne Hinweis auf bakterielle oder virale Meningitis/Encephalitis. Rö-Thorax: beidseits ausgeprägte pneumonische Infiltrate. Labor: ausgeprägte CrP-Erhöhung. Stark erhöhte D-Dimere bei pathologischer INR und PTT-Verlängerung im Sinne einer beginnenden intravasalen Koagulation. Unspezifische Erhöhung der Transaminasen und indirektem Bilirubin. Mikrobiologie: Nachweis von Staph. Aureus in der Blutkultur und Salmonella brandenburg in der Stuhlkultur. Echokardiographie: flottierende Vegetationen im Bereich des anterioren Mitralsegels mit im Verlauf zunehmender Mitralinsuffizienz.

Therapie und Verlauf. Zunächst empirische Therapie mit Cefotaxim und Doxycyclin, nach Erhalt der Blutkultur mit Tobramycin statt Doxycyclin. Darunter rasche Besserung der neurologischen Symptome und der respiratorischen Insuffizienz, die zu Beginn eine NIV-Beatmung erforderte. Normalisierung der Leberwerte und rückläufige Inflammationsparameter. Die aufgrund eines Systolikums diagnostizierte Mitralklappenendokarditis zeigte zunehmende Vegetationen unter antikoagulatorischer Behandlung mit niedermolekularem Heparin und parallel eine zunehmende Klappeninsuffizienz. Deshalb Weiterverlegung zur kinderherzchirurgischen Intervention. Intraoperativ ausgeprägte Klappendestruktion mit Notwendigkeit der Implantation einer Bioprothese. Die kardiale Funktion zeigte sich im Anschluss suffizient. Perioperativ entwickelte sich ein ischämischer Insult mit Hemiparese rechts. Die neurologischen Symptome besserten sich unter Therapie.

Schlussfolgerung. Eine bakterielle Endokarditis bei immunologisch gesunden Kindern ohne Herzfehler ist selten. Eintrittspforte für die Staph.-aureus-Sepsis waren hier möglicherweise die Hautveränderungen bei Neurodermitis. In diesem Fall kam es trotz umgehender antibiotischer Therapie zu einem komplizierten Verlauf mit möglicherweise embolisch bedingten cerebralen Herdbefunden und rascher Klappendestruktion der Mitralklappe.

\section{Lungenfunktion - Beatmung - Pneumologie}

\section{PO-53}

Die mesenchymalen Zellen der fetalen Lunge zeigen Phänotyp und Charakteristika mesenchymaler Stamm- oder Stromazellen (MSCs)

M. Möbius', S. McConaghy², R. Ohls's, F. Eaton 3 , S. Zhong ${ }^{4}$, M. Rüdiger ${ }^{5}$, B. Thébaud ${ }^{6}$

'Department of Pediatrics, Women and Children's Health Research Institute, University of Alberta, Edmonton, Kanada und Bereich Neonatologie und Pädiatrische Intensivmedizin, Universitätskinderklinikum und Hochschulmedizin "Carl Gustav Carus", Dresden, Deutschland, ${ }^{2}$ University of New Mexico, Children's Hospital of New Mexico, Department of Pediatrics/Division of Neonatology, Albuquerque, USA, ${ }^{3}$ University of Alberta, Department of Pediatrics, Women \& Children's Health Research Institute, Edmonton, Alberta, Kanada, ${ }^{4}$ Ottawa Hospital Research Institute, University of Ottawa, Children's Hospital of Eastern Ontario, Sprott Centre for Stem Cell Research, Ottawa, Ontario, Kanada, ${ }^{5}$ Universitätskinderklinikum und Hochschulmedizin "Carl Gustav Carus", Bereich Neonatologie und Pädiatrische Intensivmedizin, Dresden, Deutschland, ${ }^{6}$ Department of Pediatrics, Women and Children's Health Research Institute, University of Alberta, Edmonton, Kanada und Children's Hospital of Eastern Ontario, Sprott Centre for Stem Cell Research, Ottawa Hospital Research Institute, University of Ottawa, Ottawa, Kanada

Hintergrund. Das Mesenchym spielt eine zentrale Rolle für Wachstum und Reifung der humanen Lunge; viele pulmonale Probleme des Frühgeborenen gehen mit einer Pathologie des unreifen Mesenchymes einher. Dennoch ist so gut wie nichts über die genauen Eigenschaften und Funktionen der Zellen des fetalen pulmonalen Mesenchymes bekannt. Methoden. Humane fetale Lungen $(n=5)$ im Gestationsalter von $10+4$ bis $17+4$ Wochen wurden für diese Studie verwendet; Rückschlüsse auf 
Identität und Abortgrund waren zu keinem Zeitpunkt möglich. Nach mechanischer und enzymatischer Dissoziation des Lungengewebes erfolgte eine Aufreinigung des pulmonalen Mesenchymes mittels eines speziellen Dichtegradienten und selektiver Plastikädheränz in 5\% $\mathrm{O}_{2}$. Die so erhaltenen Zellen wurden mittels Multicolor-FACS, Colonyforming Unit Assays und induzierten Differenzierungskulturen charakterisiert und mit zuvor isolierten MSCs der humanen Nabelschnur verglichen. Alle Experimente wurden von den Ethikkommissionen der University of Alberta, Edmonton, Canada und der University of New Mexico, Albuquerque, USA genehmigt.

Ergebnisse. Die überwiegende Anzahl (>98\%) der Zellen des pulmonalen Mesenchymes exprimiert die MSC Oberflächenmarker $\mathrm{CD}_{73}$, CD9o, CD105 und CD146 bei gleichzeitiger Abwesenheit von CD11b, CD19, $\mathrm{CD}_{34}$, CD45, HLA-DR und GD-2. Eine Colony-forming Efficiency von über $70 \%$ weist auf den deutlich ausgeprägten Stammzellcharakter dieser fetalen Zellen hin. Eine rasche Transdifferenzierung in aSMA-positive Myofibroblasten unter TGF $\beta$-Induktion wurde beobachtet. Zudem konnte eine ausgeprägte Elastinproduktion durch die fetalen Lungen-MSCs, nicht jedoch durch die MSCs der Nabelschnur nachgewiesen werden.

Schlussfolgerungen. Wir beschreiben eine neue Methode um die mesenchymalen Zellen der fetalen Lunge hochselektiv zu isolieren. Zudem weisen wir nach, dass das von uns isolierte fetale Lungenmesenchym fast ausschließlich aus hochpotenten und spezialisierten MSCs besteht. Diese Zellen könnten unter anderem durch ihre endogene Elastinproduktion entscheidend zur Lungenentwicklung beitragen. Die weitere funktionelle Charakterisierung dieser MSCs kann das Verständnis für die physiologische und pathologische Lungenentwicklung des Feten entscheidend erweitern und dazu beitragen, neue Therapieansätze für Krankheiten wie die bronchopulmonale Dysplasie (BPD) und interstitielle Lungenfibrosen zu entwickeln.

\section{PO-54}

\section{Lung assist device for term and preterm newborns with severe} respiratory failure: „artificial placenta“ - an update of development

\section{N. Rochow', A. Manan', M. Shelly', G. Fusch', W. Wu' ${ }^{2}$, R. Selvaganapathy ${ }^{2}$ C. Fusch ${ }^{1}$ \\ 'McMaster University, Department of Pediatrics, Hamilton, ON, Kanada, ${ }^{2}$ McMaster University, Department of Mechanical Engineering, Hamilton, ON, Kanada}

Background. Respiratory failure is a major cause of mortality and long term morbidity in preterm infants. Based on the concept of the placenta we previously reported the development of a lung assist device (LAD) which is connected via the umbilical vessel and is pumpless as it is only driven by the heart. Previously we reported that our LAD provides effective gas exchange in a newborn hypoxic piglet model comparable to $10 \%$ of a neonate's oxygen consumption. Subsequent testing with different membrane types demonstrated that a polydimethylsiloxane (PDMS) membrane could provide improved gas exchange in LAD.

Objective. To assess the feasibility of a LAD and the effects of extracorporeal bypass on cardiovascular parameter in a piglet model.

Method. The LAD is an array of parallel stacked PDMS membrane single micro fluidic oxygenators (SOU) which was tested in a newborn piglet model. The piglet was anaesthetized with Phenobarbital and mechanical ventilated (FiO2: 0.21, PIP: 12 mbar, PEEP: 5 mbar, Freq.: 35/ min). Both umbilical arteries and the umbilical vein were cannulated with customized umbilical catheters (artery $5 \mathrm{Fr}$ by $11 \mathrm{~cm}$, vein $6.5 \mathrm{Fr}$ by $7 \mathrm{~cm}$ ). Customized angio-catheters ( $14 \mathrm{G}$ by $2.5 \mathrm{~cm}$ ) were placed into the carotid artery and jugular vein. Heparin was given for systemic anticoagulation. Cardiovascular parameters were studied applying a short cut to create a bypass between: i) umbilical artery and vein, and ii) carotid artery and jugular vein. Test flow rates ranged were i) 1-10 and ii) $1-30 \mathrm{~mL} / \mathrm{kg} / \mathrm{min}$, respectively. The feasibility of the LAD was tested in a hypoxic piglet model. Muscles were relaxed with Rocuronium and hy- poxic conditions were applied by mechanical ventilator ( $\mathrm{FiO}_{2}$ : 0.12, PIP: 6 mbar, PEEP: o mbar, Freq.: 30/min). Gas exchange rate was measured. Results. Four newborn piglets with a weight of 1.5 to $2.3 \mathrm{~kg}$ were studied. Heart rate remained stable by extracorporeal circulation up to $25 \mathrm{~mL} /$ $\mathrm{kg} / \mathrm{min}$ and increased by an average of 15 beats when blood flow was up to $30 \mathrm{~mL} / \mathrm{kg} / \mathrm{min}$ while blood pressure remained stable. Via umbilical vessel flow rate up to $10 \mathrm{~mL} / \mathrm{kg} / \mathrm{min}$ could be achieved. The average gas exchange for oxygen in the PDMS LAD was $1.5 \mu \mathrm{l} / \mathrm{cm} 2 / \mathrm{min}$ and $6 \mu \mathrm{l} /$ $\mathrm{cm} 2 / \mathrm{min}$ for carbon dioxide.

Conclusion. The high gas exchange rates allow lower residence time for the blood. High blood flows through our miniature device provide appropriate gas exchange. The vascular access via umbilical vessels needs to be improved to enhance performance of LAD.

\section{PO-55}

Auswirkungen einer partiellen Flüssigkeitsbeatmung im Tiermodell des LPS-induzierten Lungenschadens

\section{Mense', L. Köthe ${ }^{2}$, M. Ochs², M. Rüdiger', W. Burkhardt ${ }^{1}$}

'Univ. Klinikum Carl Gustav Carus, Klinik für Kinder- und Jugendmedizin, Neonatologie, Dresden, Deutschland, ${ }^{2}$ Medizinische Hochschule Hannover, Institut für Funktionelle und Angewandte Anatomie, Hannover, Deutschland

Hintergrund. In der frühen Phase des akuten Atemnotsyndrom (ARDS) spielt eine pulmonale Inflammation eine wichtige Rolle. Die zur Flüssigkeitsbeatmung beim ARDS eingesetzten Perfluorcarbone zeigen antiinflammatorische Eigenschaften. Welchen Einfluss die frühe Inflammation und die nachfolgende PFC-Applikation auf das endogene Surfactant-System und die Typ-II-Zellen haben ist bisher unklar.

Fragestellung. Welche Effekte an Lungenstruktur und Surfactant-System sind Folge einer intravenösen LPS-Gabe und sind Unterschiede zwischen konventioneller mechanischer Beatmung (CMV) und partieller Flüssigkeitsbeatmung (PLV) zu beobachten?

Material und Methoden. Die frühe, inflammatorische Phase des ARDS wurde mittels LPS Applikation (1 mg/KG) bei männlichen Wistar-Ratten $(\mathrm{n}=10)$ simuliert (Kontrollgruppe $\mathrm{NaCl}$ o, $9 \%, \mathrm{n}=10$ ). Jeweils die Hälfte der Tiere pro Gruppe erhielt PFC intratracheal appliziert, alle Tiere wurden anschließend für $2,5 \mathrm{~h}$ mechanisch beatmet. Nach Fixierung der Lungen wurden Surfactant-Struktur und Lungenparenchym in Licht- und Elektronenmikroskopie stereologisch ausgewertet.

Ergebnisse. Die LPS-Gabe führte zu einer signifikant reduzierten Anzahl von Typ-II-Zellen unter CMV (LPS 209,6 \pm 36 ,o, Kontrolle $258,8 \pm 28,8 \times 10^{\wedge} 6 /$ Lunge). Auswirkungen auf die intraalveoläre Surfactant-Menge oder die Lungenstruktur (Oberfläche des Alveolarepithels, Lungenvolumen) fanden sich nicht. Unter PFC-Einfluss veränderte sich die Anzahl von Typ-II-Zellen nach LPS-Gabe nicht im Vergleich zur Kontrollgruppe unter CMV. Es war in beiden Gruppen (LPS/Kontrolle) eine Zunahme des Lungenvolumens mit Vergrößerung der Alveolarepithel-Oberfläche als Folge der Flüssigkeitsbeatmung zu beobachten. Schlussfolgerungen. Die Durchführung einer partiellen Flüssigkeitsbeatmung scheint protektive Effekte auf die Typ-II-Zellen während einer LPS-induzierten pulmonalen Inflammationsreaktion zu haben und führt zu einer Vergrößerung der Lungenoberfläche. 


\begin{tabular}{|l|l|l|l|c|}
\hline Gruppe & Respirator & T-Piece-System & Beatmungsbeutel & p \\
\hline $\mathrm{SaO}_{2}(\%)$ & $43.5(38.2-54.6)$ & $27.2(23.4-40.9)$ & $25.9(14.8-52.5)$ & 0.120 \\
\hline $\mathrm{paO}_{2}(\mathrm{mmHG})$ & $54.4(46.9-62.7)$ & $41.2(38.5-48.9)$ & $42.2(28.9-59.8)$ & 0.142 \\
\hline $\mathrm{paCO}_{2}(\mathrm{mmHg})$ & $75.5(70.1-83.1)$ & $96.2(82.5-112.9)$ & $103.3(83.5-109.6)$ & 0.131 \\
\hline $\mathrm{pH}$ & $6.9(6.9-7,0)$ & $6.9(6.8-6.9)$ & $6.8(6.8-6.9)$ & 0.257 \\
\hline Laktat $(\mathrm{mmol} / \mathrm{l})$ & $9.5(8.7-10.8)$ & $8.9(7.8-10.9)$ & $9.0(7.1-9.2)$ & 0.477 \\
\hline $\mathrm{BE}(\mathrm{mmol} / \mathrm{l})$ & $-16.4(-18.2--14.3)$ & $-15.8(-17.4--15.5)$ & $-16.2(-18,1--15,0)$ & 0.969 \\
\hline $\mathrm{MAD}\left(\mathrm{mmHg}^{2}\right)$ & $24.1(19.9-25.3)$ & $22.7(20.6-25.1)$ & $21.2(18.2-22.7)$ & 0.198 \\
\hline $\mathrm{Paw}\left(\mathrm{cmH}_{2} \mathrm{O}\right)$ & $8.4(8.2-8.7)$ & $8.2(7.2-8.8)$ & $4.4(3.7-4.8)$ & $<0.001^{*}$ \\
\hline
\end{tabular}

PO-56 Abb. 14

Durchschnittswerte aus den letzten 3 Blutgasanalysen (Min. 16-20), dargestellt als Median mit 1. und 3. Quartile;

Paw = mittlerer Atemwegsdruck, MAD = mittlerer arterieller Blutdruck;

${ }^{*}=p<0.001$ : Respirator vs. Beatmungsbeutel, T-Piece vs. Beatmungsbeutel,

Durchschnittswerte aus gesamter CPR (Min.0-20)

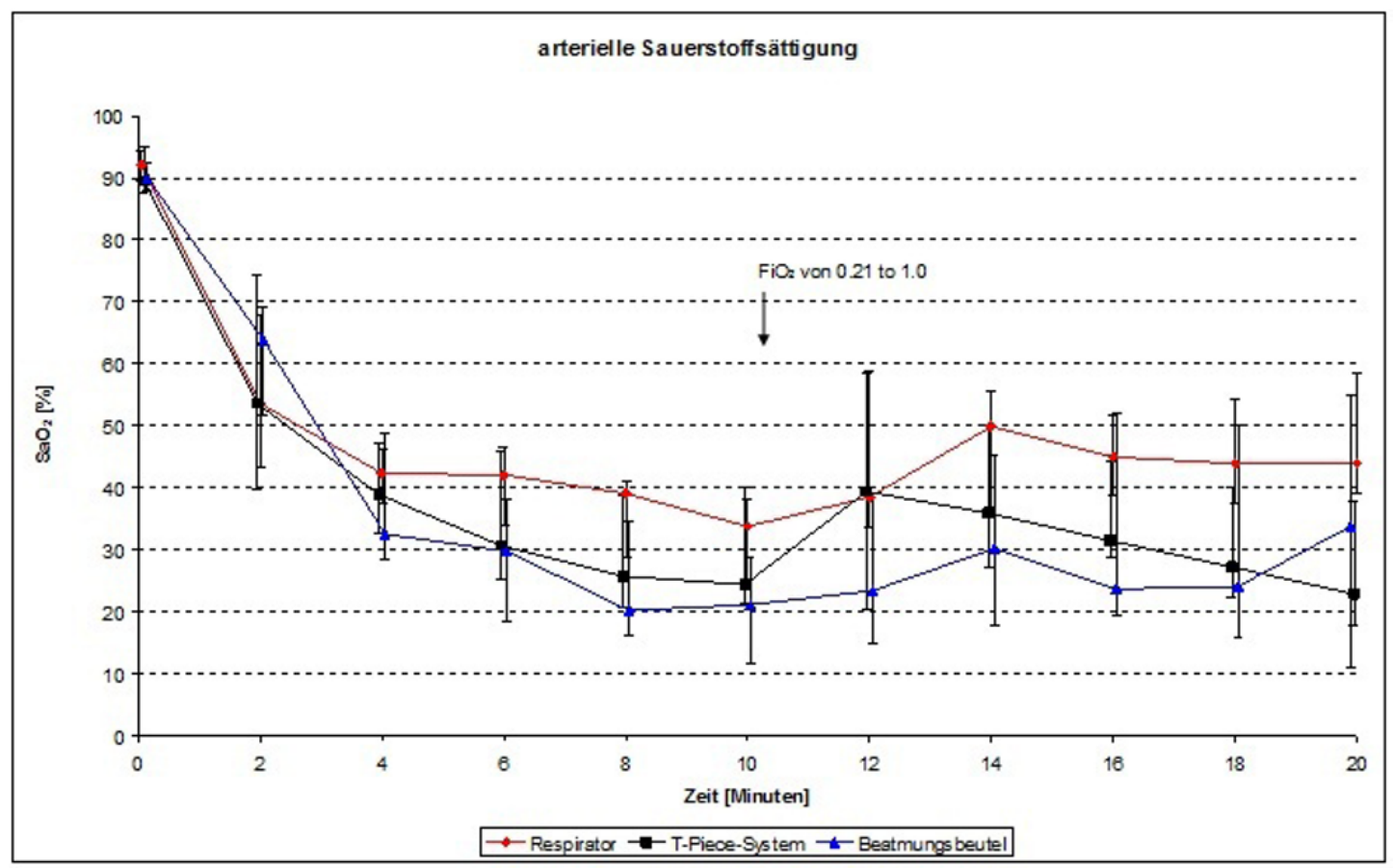

P0-56 Abb. 24

Arterielle Sauerstoffsättigung $\left(\mathrm{SaO}_{2}\right)$, dargestellt als Median mit 1. und 3. Quartile für die 3 Behandlungsgruppen

\section{PO-56}

Respiratorische Unterstützung während der kardiopulmonalen Reanimation bei neugeborenen Ferkeln: Einfluss verschiedener Beatmungsverfahren auf den Gasaustausch und die Hämodynamik

M. Mendler', M. Maurer', L. Huang', M. Hassan', M. Waitz', H. Hummler' ${ }^{1}$ Klinik für Kinder- und Jugendmedizin, Universitätsklinikum Ulm, Sektion Neonatologie und pädiatrische Intensivmedizin, Ulm, Deutschland

Hintergrund. Die aktuellen ILCOR-Richtlinien für die kardiopulmonale Reanimation (CPR) von Neugeborenen im Kreißsaal sehen ein Kompressions-/Ventilationsverhältnis von 3:1 vor und empfehlen gleichzeitig, die Atemhübe zwischen den kardialen Kompressionen zu interponieren. Allerdings gibt es keine Untersuchungen, die der Frage nachgegangen sind, ob diese Interposition der Atemhübe gegenüber nichtsynchroner kontinuierlicher Fortführung der kardialen Kompression mit 120 Kompressionen pro Minute notwendig oder vorteilhaft ist. Die derzeit gültigen Richtlinien empfehlen kein spezifisches Beatmungsverfahren während der CPR. Weiterhin ist unklar, ob die Anwendung von positivem endexpiratorischem Druck (PEEP) während der CPR gegenüber einer Beatmung mit Beatmungsbeutel tatsächlich vorteilhaft ist, zumal sich unter Verwendung eines Beatmungsbeutels in der Regel kein PEEP aufrechterhalten lässt.

Fragestellung. Das Ziel dieser Studie war die Untersuchung der Einflüsse verschiedener Formen der respiratorischen Unterstützung während der CPR auf den Gasaustausch und die Hämodynamik bei neugeborenen Ferkeln im Kreislaufstillstand. Unsere Annahme war hierbei, dass die Verwendung von PEEP und die Interposition der Beatmungshübe zwischen den kardialen Kompressionen den Gasaustausch und die Hä- 


\begin{tabular}{|c|c|c|c|c|}
\hline Gruppe & Respirator & $\begin{array}{l}\text { T-Piece- } \\
\text { System }\end{array}$ & $\begin{array}{l}\text { Beatmungs- } \\
\text { beutel }\end{array}$ & p-Wert \\
\hline $\mathrm{S}_{\mathrm{a}} \mathrm{O}_{2}(\%)$ & $\begin{array}{l}43,5(38,2- \\
54,6)\end{array}$ & $\begin{array}{l}27,2(23,4- \\
40,9)\end{array}$ & $\begin{array}{l}25,9(14,8- \\
52,5)\end{array}$ & 0,120 \\
\hline $\mathrm{p}_{\mathrm{a}} \mathrm{O}_{2}(\mathrm{mmHg})$ & $\begin{array}{l}54,4(46,9- \\
62,7)\end{array}$ & $\begin{array}{l}41,2(38,5- \\
48,9)\end{array}$ & $\begin{array}{l}42,2(28,9- \\
59,8)\end{array}$ & 0,142 \\
\hline $\begin{array}{l}\mathrm{p}_{\mathrm{a}} \mathrm{CO}_{2} \\
(\mathrm{mmHg})\end{array}$ & $\begin{array}{l}75,5(70,1- \\
83,1)\end{array}$ & $\begin{array}{l}96,2(82,5- \\
112,9)\end{array}$ & $\begin{array}{l}103,3(83,5- \\
109,6)\end{array}$ & 0,131 \\
\hline $\mathrm{pH}$ & $6,9(6,9-7,0)$ & $6,9(6,8-6,9)$ & $6,8(6,8-6,9)$ & 0,257 \\
\hline $\begin{array}{l}\text { Laktat } \\
(\mathrm{mmol} / \mathrm{l})\end{array}$ & $9,5(8,7-10,8)$ & $8,9(7,8-10,9)$ & $9,0(7,1-9,2)$ & 0,477 \\
\hline $\mathrm{BE}(\mathrm{mmol} / \mathrm{l})$ & $\begin{array}{l}-16,4 \\
(-18,2- \\
-14,3)\end{array}$ & $\begin{array}{l}-15,8 \\
(-17,4- \\
-15,5)\end{array}$ & $\begin{array}{l}-16,2 \\
(-18,1- \\
-15,0)\end{array}$ & 0,969 \\
\hline $\begin{array}{l}\text { MAD } \\
(\mathrm{mmHg})\end{array}$ & $\begin{array}{l}24,1(19,9- \\
25,3)\end{array}$ & $\begin{array}{l}22,7(20,6- \\
25,1)\end{array}$ & $\begin{array}{l}21,2(18,2- \\
22,7)\end{array}$ & 0,198 \\
\hline Paw $\left(\mathrm{cmH}_{2} \mathrm{O}\right)$ & $8,4(8,2-8,7)$ & $8,2(7,2-8,8)$ & $4,4(3,7-4,8)$ & $<0,001^{*}$ \\
\hline \multicolumn{5}{|c|}{$\begin{array}{l}\text { Durchschnittswerte aus den letzten } 3 \text { Blutgasanalysen (Min. 16-20), dargestellt als } \\
\text { Median mit 1. und 3. Quartile. Paw mittlerer Atemwegsdruck, MAD mittlerer arteri- } \\
\text { eller Blutdruck, }{ }^{*} p<0,001 \text { : Respirator vs. Beatmungsbeutel, T-Piece vs. Beatmungs- } \\
\text { beutel, Durchschnittswerte aus gesamter CPR (Min. 0-20). }\end{array}$} \\
\hline
\end{tabular}

modynamik, verbessert. Als primäres Zielkriterium wurde die arterielle Sauerstoffsättigung definiert.

Material und Methoden. Prospektive, kontrollierte, randomisierte Studie an 30 intubierten neugeborenen Ferkeln in 3 Gruppen:

- Gruppe 1: Neugeborenenbeatmungsgerät mit PIP/PEEP 20/5 cm ${ }_{2} \mathrm{O}$ und einer Frequenz (f) von 30 Beatmungshüben/Minute (Inspirationszeit 0,4 s). Die kardialen Kompressionen (CC) wurden mit einer Frequenz von 120/min verabreicht, ohne auf Synchronisation mit der Beatmung zu achten.

- Gruppe 2: T-piece-System mit einem PIP/PEEP 20/5 cmH2O, $\mathrm{f}=3 \mathrm{O} /$ min, wobei die Beatmungshübe zwischen CC mit einer 3:1 Ratio interponiert wurden.

- Gruppe 3: Beatmungsbeutel mit einem PIP $20 \mathrm{cmH}_{2} \mathrm{O}$ ohne PEEP, $\mathrm{f}=3 \mathrm{o} / \mathrm{min}$, wobei die Beatmungshübe zwischen CC mit einer 3:1 Ratio interponiert wurden.

Ergebnisse. Zwischen den einzelnen Gruppen konnte kein signifikanter Unterschied bezüglich der arteriellen Sauerstoffsättigung festgestellt werden. Allerdings ließ sich ein Trend zu einer höheren Sauerstoffsättigung und zu niedrigeren $\mathrm{paCO}_{2}$-Werten in der Gruppe feststellen, in der das Beatmungsgerät verwendet wurde (- Tab. 1, $\bullet$ Abb. 1, $\bullet$ Abb. 2). Diskussion und Schlussfolgerung. Mit vorliegender Studie konnte unsere Hypothese nicht bestätigt werden. Außerdem konnten wir keinen signifikanten Vorteil für die Synchronisation von Beatmung und Kompression, sowie für die Applikation von PEEP finden. Möglicherweise hat die erhöhte Rate an Kompressionen in der Respiratorgruppe einen vorteilhaften Einfluss. Auf Grund dessen sind weitere Studien notwendig, um einen möglichen Einfluss verschiedener Beatmungsformen auf die Hämodynamik und die Rückkehr des Spontankreislaufs in einem Lebendmodell zu untersuchen.

\section{PO-57}

Ein kurzes Druckplateau reicht aus, um die Lungen von sehr kleinen Frühgeborenen komplett zu füllen

\section{Kirchner', V. Jeitler', A. Pollak', M. Weninger', W. Oczenski', M. Wald ${ }^{3}$}

'Universitätsklinik für Kinder- und Jugendheilkunde Wien, Abteilung für Allgemeinpädiatrie und Neonatologie, Wien, Österreich, ${ }^{2}$ Krankenhaus Hietzing, Anästhesie, Wien, Österreich, ${ }^{3}$ Salzburger Universitätsklinikum, Neonatologie, Salzburg, Österreich

Einleitung. Bei der druckkontrollierten Beatmung von sehr kleinen Frühgeborenen wird üblicherweise ein langes inspiratorisches Druckplateau empfohlen. Jüngste Studien haben allerdings ergeben, dass eine längere Druckanstiegszeit, die bei gleichbleibender Inspirationszeit ein kürzeres inspiratorisches Druckplateau bedingt, das Risiko von beatmungsbedingten Lungenschäden deutlich reduziert. Die vorliegende Arbeit untersucht, ob die Lungen sehr kleiner Frühgeborener auch mit einem kurzen inspiratorischen Druckplateau komplett gefüllt werden können.

Methode. Insgesamt 19 konventionell beatmete Frühgeborene mit einem mittleren Körpergewicht von $909( \pm 194) \mathrm{g}$ wurden prospektiv in die Studie inkludiert. Durch langsame Reduktion des Gasflusses wurde die Druckkurve so stark abgeflacht, dass der inspiratorische Flow über mehr als 90\% der Inspirationszeit bestehen blieb, aber trotzdem noch ausreichte, um ein kurzes inspiratorisches Druckplateau aufzubauen. Diese Einstellung wurde fünf Minuten lang belassen.

Ergebnisse. Der inspiratorische Flow wurde von $6,681 / \mathrm{min}( \pm 1,0)$ auf $3,82 \mathrm{l} / \mathrm{min}( \pm 0,88)$ reduziert $(\mathrm{p}=0,001)$. Die Dauer des inspiratorischen Flows stieg dabei von $60,2 \%( \pm 11,8)$ auf $97,1 \%( \pm 5,2)$ der Inspirationszeit $(\mathrm{p}=0,001)$ an; die Druckanstiegszeit stieg gleichzeitig von $45,2 \%( \pm 7,1)$ auf $84,1 \%( \pm 8,5 ; p=0,001)$ der Inspirationszeit, wodurch sich die Dauer des inspiratorischen Druckplateaus von $54,8 \%$ auf $15,9 \%$ der Inspirationszeit verkürzte. Es bestand eine signifikante direkte Korrelation zwischen dem Gasfluss der Maschine und dem inspiratorischen Spitzendruck $(\mathrm{p}=0,001)$.

Schlussfolgerung. Unsere Studie zeigt, dass ein inspiratorisches Druckplateau von nur $15-20 \%$ der gesamten Inspirationszeit ausreicht, um die Lungen von beatmeten Frühgeborenen komplett zu füllen.

\section{PO-58}

Effektivere Beatmung unter Neurally Adjusted Ventilatory Assist (NAVA)

\section{K. Schuberth', A. Schwepcke', O. Genzel-Boroviczény'}

${ }^{1}$ Klinikum der Universität München, Perinatalzentrum Innenstadt, München, Deutschland

Hintergrund. Maschinelle Beatmung ist ein unumgänglicher Bestandteil der Frühgeborenenversorgung. Hohe Beatmungsdrücke und lange Beatmungszeiten stellen jedoch ein hohes Risiko für BPD und Langzeitschäden dar. Deshalb wird nach immer physiologischeren Beatmungsformen gesucht. Durch NAVA-Beatmung ist es nun dem Patienten selbst möglich, die Beatmung durch die Aktivität des Zwerchfelles zu steuern.

Fragestellung. Treten Veränderungen in Vital- und Beatmungsparametern auf, wenn Frühgeborene von einer konventionellen Beatmungsform auf die NAVA-Beatmung umgestellt werden?

Material und Methoden. Retrospektiv wurden die Akten aller im Zeitraum von 12/2010 bis 04/2012 mit NAVA beatmeten Frühgeborenen (Gestationsalter bei Geburt <32.SSW, Geburtsgewicht <1500 g) zur Auswertung herangezogen. Analysiert wurden die Daten folgender Tage: 7 Tage vor Umstellung, der Tag vor Umstellung, der Umstellungstag, Tag 1-3 unter NAVA-Beatmung, der letzte ganze NAVA-Tag, der Absetzungstag und der Tag danach. Untersucht wurden die Beatmungsparametern vor, unter und nach NAVA. Hierfür wurden für jeden Tag und jedes Kind Tagesmittelwerte errechnet. Ebenfalls wurde ein Mittelwert 
für NAVA-Tag 1-3 (NAVA123) und allen analysierten NAVA-Tagen (NAVAges.) gebildet und mit den Vor- und Nachwerten verglichen.

Ergebnisse. In dem Beobachtungszeitraum wurden 10 Frühgeborenen mit NAVA beatmet [mittlere NAVA-Zeit $9,3(3,5-15,6)$ d]. Die positiven inspiratorischen Spitzendrücke waren unter NAVA-Beatmung signifikant niedriger als unter der konventionellen Beatmung. Die maximal benötigte inspiratorische Sauerstoffkonzentration unter NAVA war niedriger. Die O2-Sättigung zeigte unter NAVA-Beatmung höhere Werte. Ebenfalls waren $\mathrm{SaO}_{2}$-Abfälle unter NAVA-Beatmung seltener (• Tab. 1).

Schlussfolgerung. Unter NAVA-Beatmung zeigten sich signifikant niedrigere Spitzendrücke bei gleichzeitig geringerem Sauerstoffbedarf und besserer arterieller Sauerstoffsättigung im Vergleich zu der konventionellen Beatmung. In wie weit diese zu einer Reduktion von beatmungsassoziierten Gesundheitsschäden führt, müssen weitere Studien mit längerem Follow-up zeigen.

\section{PO-59}

Effects of synchronization during noninvasive intermittent mandatory ventilation in very low birth weight infants (VLBWI) recovering from RDS immediately after extubation

\section{Huang', H. Hummler', M. Mendler ${ }^{3}$, M. Waitz', M. Hassan}

'Universitätsklinikum Ulm, Sektion Neonatologie und Pädiatrische Intensivmedizin Klinik für Kinder- und Jugendmedizin, Ulm, Deutschland, ${ }^{2}$ Klinik für Kinder- und Jugendmedizin, Universitätsklinikum Ulm, Sektion Neonatologie und Pädiatrische Intensivmedizin, Ulm, Deutschland, ${ }^{3}$ Universitätsklinik für Kinder- und Jugendmedizin, Sektion für Neonatologie und Pädiatrische Intensivmedizin, Ulm, Deutschland, ${ }^{4}$ Universitätsklinik für Kinder- und Jugendmedizin, Sektion Neonatologie und Pädiatrische Intensivmedizin, Ulm, Deutschland, ${ }^{5}$ Universitätsklinikum Ulm, Neonatologie und Pädiatrische Intensivmedizin Klinik für Kinder- und Jugendmedizin, Ulm, Deutschland

Background. Non-invasive ventilation i.e. nasal ventilation is increasingly being used in VLBWI for respiratory support. The physiologi- cal effects of synchronization during non-invasive nasal IMV have not been tested in VLBWI immediately after extubation.

Objective. The objective was to study the short-term effects of synchronized nasal IMV (S-NIMV) as compared to non-synchronized nasal IMV (NIMV) on breathing effort as measured by phasic esophageal pressure deflection $(\mathrm{Pe})$, respiratory rate $(\mathrm{RR})$, and gas exchange, cerebral saturation, and intermittent episodes of bradycardia or hypoxemia in VLBWI immediately after extubation recovering from RDS.

Methods. Thirteen VLBWI with RDS were studied using a randomized cross-over design during either S-NIMV or NIMV for 2 hours each immediately after extubation. The characteristics of the 13 infants were: five female, eight male; GA $25.9 \pm 2.3 \mathrm{wk}$; birth weight (BW) 740 (470, 1290) g; and postnatal age $13(2-43) \mathrm{d}$.

Results. All results are shown in Tab. 1. RR and Pe significantly decreased and there was a trend towards a lower $\mathrm{PCO}_{2}$, and less bradycardia and desaturation events, whereas $\mathrm{PO}_{2}$ and cerebral saturation $\left(\mathrm{StO}_{2}\right)$ tended to increase during S-NIMV compared with NIMV.

Conclusion. Synchronization during nasal ventilation immediately after extubation in VLBWI recovering from RDS seems to result in a more efficient support and may help to stabilize gas exchange by decreasing the respiratory effort of infants.

PO-60

Anwendung von Analgetika und Sedativa bei Frühgeborenen $<1500 \mathrm{~g}$ in Deutschland von 2003-2010. Spielen Art der Surfactantgabe und Form der respiratorischen Unterstützung eine Rolle?

K. Mehler', C. Härtel', A. Oberthür' , E. Herting ${ }^{2}$, A. Kribs' ', B. Roth', W. Göpel ${ }^{1}$ Universitätskinderklinik Köln, Neonatologie und Pädiatrische Intensivmedizin, Köln, Deutschland, ${ }^{2}$ Universitätsklinikum Schleswig-Holstein, Klinik für Kinder- und Jugendmedizin, Lübeck, Deutschland

Hintergrund. Analgosedierung findet bei kleinen Frühgeborenen häufig im Kontext der endotrachealen Intubation und der mechanischen Beatmung statt. Strategien zur Beatmungsvermeidung wie die primäre Anwendung von CPAP und die nichtinvasive Surfactantgabe tragen zur

\begin{tabular}{|c|c|c|c|c|c|c|c|c|}
\hline & -1 Tag & Tag 1 & Tag 2 & Tag 3 & NAVA $^{123}$ & 1 vor ex. & $\mathrm{NAVA}_{\text {ges }}$ & Nach \\
\hline PIP (cmH2) & $\begin{array}{l}15,4^{\text {bcdlg }} \\
{[12,9-17,8]}\end{array}$ & $\begin{array}{l}12,1^{\mathrm{b}} \\
{[10,7-13,6]}\end{array}$ & $\begin{array}{l}111,8^{c} \\
{[10,2-13,4]}\end{array}$ & $\begin{array}{l}10,5 \mathrm{~d} \\
{[8,3-12,6]}\end{array}$ & $\begin{array}{l}11,91 \\
{[10,5-13,3]}\end{array}$ & $\begin{array}{l}11,2 \\
{[8,6-13,7]}\end{array}$ & $\begin{array}{l}11,8^{9} \\
{[10,3-13,2]}\end{array}$ & $\begin{array}{l}13,0 \\
{[10,2-15,8]}\end{array}$ \\
\hline $\mathrm{FiO}_{2}(\%)$ & $\begin{array}{l}36,4^{\mathrm{bg}} \\
{[26,9-55,0]}\end{array}$ & $\begin{array}{l}29,9^{\mathrm{b}} \\
{[22,9-36,9]}\end{array}$ & $\begin{array}{l}25,5 \\
{[22,0-36,9]}\end{array}$ & $\begin{array}{l}34,5 \\
{[20,9-29,1]}\end{array}$ & $\begin{array}{l}27,9 \\
{[23,2-32,6]}\end{array}$ & $\begin{array}{l}24,1 \\
{[20,8-27,5]}\end{array}$ & $\begin{array}{l}27,3^{9} \\
{[23,0-31,6]}\end{array}$ & $\begin{array}{l}26,9 \\
{[21,2-32,6]}\end{array}$ \\
\hline $\mathrm{SaO}_{2}(\%)$ & $\begin{array}{l}93,7^{\text {cdl }} \\
{[92,2-95,3]}\end{array}$ & $\begin{array}{l}94,5 \\
{[92,6-96,4]}\end{array}$ & $\begin{array}{l}95,5^{c} \\
{[93,7-97,3]}\end{array}$ & $\begin{array}{l}96,1^{\mathrm{d}} \\
{[94,4-97,9]}\end{array}$ & $\begin{array}{l}94,91 \\
{[93,3-96,5]}\end{array}$ & $\begin{array}{l}95,2 \\
{[92,8-97,5]}\end{array}$ & $\begin{array}{l}95,0 \\
{[93,4-96,5]}\end{array}$ & $\begin{array}{l}94,5 \\
{[93,1-95,8]}\end{array}$ \\
\hline $\mathrm{SaO}_{2}$-Abfälle & $\begin{array}{l}22^{\text {beffg }} \\
{[13-32]}\end{array}$ & $\begin{array}{l}9^{b} \\
{[5-14]}\end{array}$ & $\begin{array}{l}7^{c} \\
{[2-12]}\end{array}$ & $\begin{array}{l}5 \\
{[0-10]}\end{array}$ & $\begin{array}{l}8^{1} \\
{[4-12]}\end{array}$ & $\begin{array}{l}7^{e} \\
{[2-12]}\end{array}$ & $\begin{array}{l}7^{9} \\
{[4-11]}\end{array}$ & $\begin{array}{l}14^{f} \\
{[6-21]}\end{array}$ \\
\hline
\end{tabular}

P0-59 Tab. 1 Values were expressed as means \pm SD or median (range)

\begin{tabular}{|llllllll}
\hline Ventilator mode & $\mathrm{Pe}\left(\mathrm{cmH}_{2} \mathrm{O}\right)$ & $\begin{array}{l}\mathrm{RR}(\mathrm{breaths} / \\
\mathrm{min})\end{array}$ & $\mathrm{PCO}_{2}(\mathrm{mmHg})$ & $\mathrm{PO}_{2}(\mathrm{mmHg})$ & $\mathrm{StO}_{2}(\%)$ & $\begin{array}{l}\text { Total time of } \\
\text { bradycardia } \\
(\mathrm{sec})\end{array}$ & $\begin{array}{l}\text { Total time of de- } \\
\text { saturation }(\mathrm{sec})\end{array}$ \\
\hline S-NIMV & $5.89 \pm 2.04$ & $69.43 \pm 11.41$ & $57.61 \pm 13.69$ & $49.82 \pm 17.58$ & $68.46 \pm 7.55$ & $16(0-146)$ & $352(0-2252)$ \\
\hline NIMV & $7.13 \pm 2.49$ & $73.13 \pm 10.21$ & $59.55 \pm 15.36$ & $44.47 \pm 9.71$ & $67.85 \pm 7.53$ & $44(0-254)$ & $576(0-878)$ \\
\hline p-value & $0.048^{\mathrm{a}}$ & $0.008^{\mathrm{a}}$ & $0.266^{\mathrm{a}}$ & $0.208^{\mathrm{a}}$ & $0.548^{\mathrm{a}}$ & $0.838^{\mathrm{b}}$ & $0.638^{\mathrm{b}}$ \\
\hline
\end{tabular}


Reduktion der Häufigkeit endotrachealer Intubationen und der Beatmungszeiten bei.

Methoden. In der vorliegenden Studie wurden die im German Neonatal Network erfassten Angaben zum Einsatz von Sedativa und Analgetika von Frühgeborenen $<1500 \mathrm{~g}$ in den Jahren 2003-2010 in Deutschland ausgewertet und in Beziehung zu der verwendeten Art der Atemunterstützung und Form der Surfactantgabe gesetzt.

Ergebnisse. Insgesamt wurden Analgetika und/oder Sedativa in den Jahren 2003-2010 bei 1822 (35,6\%) von 5121 VLBW Frühgeborenen eingesetzt. Frühgeborene, die mit Analgetika/Sedativa behandelt wurden, waren im Mittel signifikant unreifer (27 vs. 30 SSW) und leichter (930 g vs. $1141 \mathrm{~g}$ ). Der Vergleich für den Analgetika/Sedativa Einsatz der Jahre 2003-2009 mit dem Jahr 2010 zeigt, dass sich der Anteil der mit Analgetika und Sedativa behandelten Frühgeborenen nicht signifikant änderte. Es wurden im Zeitraum 1 (2003-2009) 35,1\% und im Zeitraum 2 (2010) 36,8\% der Frühgeborenen mit Analgetika und/oder Sedativa behandelt, $24,8 \%$ vs. $25,3 \%$ nur mit Analgetika und $26,5 \%$ vs. $28,1 \%$ nur mit Sedativa. Betrachtet man die einzelnen Substanzklassen, so fällt bei gleichbleibendem Anteil der mit Opioden behandelten Frühgeborenen (22\%) auf, dass der Einsatz von Morphin und Sufentanil im Jahr 2010 verglichen mit den Vorjahren signifikant anstieg. Fentanyl dagegen wurde etwas seltener eingesetzt (n. s.). Weiter signifikante Änderungen zeigen sich in der vermehrten Anwendung von Acetaminophen (5,6 vs. 8,1\%) und für Propofol, das 2010 bei 3,7\% der Frühgeborenen zum Einsatz kam. Beatmete Frühgeborene wurden häufiger mit Analgetika und oder Sedativa behandelt als Kinder, die nur CPAP oder keine respiratorische Unterstützung erhielten (65 vs. 13 vs. $3 \%$ ). Für alle Gestationsalter zeigt sich, dass die Kinder mit Surfactantgabe ohne Intubation signifikant seltener Analgetika oder Sedativa erhielten im Vergleich zu Kindern mit Surfactantapplikation über Tubus. Extrem unreife Kinder mit einem Gestationsalter von 22 und 23 Wochen erhielten $>50 \%$ Analgetika und/oder Sedativa, erfolgt die Surfactantgabe über Tubus steigt der Anteil auf $>80 \%$. Kinder ab einem Gestationsalter von 24 Wochen erhalten bei Surfactantgabe ohne Intubation in $28-43 \%$ bei Surfactantgabe mit Intubation in $53-69 \%$ Analgetika/Sedativa (• Abb. 1).

Schlussfolgerung. Aktuelle Entwicklungen in der Neontalogie wie Beatmungsvermeidung oder Verzicht auf eine routinemäßige Analgosedierung führten bis zum Jahr 2010 nicht zu einer Reduktion der Anwendung von Analgetika und Sedativa.

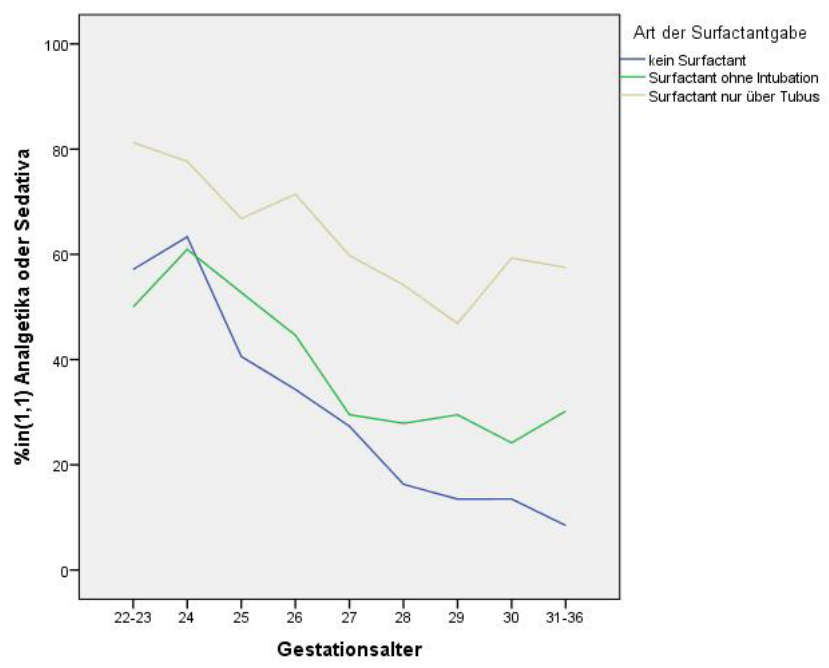

PO-60 Abb. $1 \triangle$ Analgetika/Sedativa in Abhängigkeit von der Art der Surfactantapplikation

\section{PO-61}

Standardisiertes Atemwegsmanagement und frühes Atemversagen bei VLBW-Frühgeborenen

\section{A. Krauth', J. Kühr ${ }^{1}$}

'Städtisches Klinikum gGmbH, Klinik für Kinder- und Jugendmedizin, Karlsruhe, Deutschland

Einleitung. Neben der klassischen Surfactantgabe unter maschineller Beatmung wird sowohl die INSURE-Methode als auch die Applikation über eine dünne, tracheal platzierte Sonde unter CPAP-unterstützter Spontanatmung durchgeführt. Die folgende retrospektive Analyse soll die Frage nach der Machbarkeit im Alltag und nach dem frühen Atemversagen der zuletzt genannten Methode im Rahmen eines standardisierten Erstversorgungskonzeptes für sehr unreife Frühgeborene klären.

Material und Methoden. Weitgehende Vorverlagerung der kompletten Erstversorgung in den Kreißsaalbereich und definiertes Atemwegsmanagement: Atemwegsrekrutierung mittels Masken-Blähmanöver und anschließend assistierte/kontrollierte Maskenbeatmung (Perivent ${ }^{\circledR}$ ); kontinuierliche Masken- bzw. Nasopharyngealtubus-CPAP-Atemhilfe; ohne Analgosedierung Laryngoskopie und nasotracheale Sondenplatzierung, Surfactantapplikation (Curosurf ${ }^{\oplus}, 100 \mathrm{mg} / \mathrm{kg}$ ) unter PEEP 10 mbar; Einschlusskriterien: Gestationsalter (GA) <28 SSW bzw. Geburtsgewicht (GG) <100o g (Ausnahmen im Grenzbereich), klinische RDS-Zeichen, suffizienter Eigenatemantrieb; Ausschlusskriterien: Reanimation, schwerwiegende Fehlbildung, logistische Unzulänglichkeiten; Coffeincitrat und R-NIPPV-Beatmung prophylaktisch bzw. bei frühen Apnoen bis zur Verlegung auf Station; Umstellung auf BinasalCPAP-Atemhilfe (Babylog 8000 Plus/Stephanie); Minimal-HandlingKonzept in den ersten 72 Lebensstunden.

Ergebnisse. Von 346 VLBW-Frühgeborenen im Zeitraum 04/2008 bis 02/2013 wurden 103 (30\%) nach der o. g. Methode behandelt: $56 \%$ männlich, $44 \%$ weiblich; GA-Median: $27+5$ SSW (Range: $24+2 ; 31+2$ ), Anteil <26 SSW: 11\%, Anteil <28 SSW: 57\%, Anteil <30 SSW: 96\%; GG-Median: 940 g (Range: $355 ; 1470$ ), Anteil <750 g: 23\%, Anteil <1000 g: 68\%, Anteil <1250 g: 91\%; „Nonresponder“ (sekundär intubationspflichtiges frühes Atemversagen $<72 \mathrm{~h}$ : RDS oder Apnoe-Bradykardie-Syndrom): Alter bei Atemversagen: Median 24 h (Range: 0,$3 ; 64$ ), Anteil aller Kinder: 37\%, Anteil <26 SSW: 42\%, Anteil <28 SSW: 47\%, Anteil <30 SSW: $36 \%$, Anteil <750 g: $54 \%$, Anteil <100o g: $44 \%$, Anteil <1250 g: $37 \%$.

Diskussion. Inwieweit die von Kribs et al beschriebene Methode der Surfactantgabe unter Umgehung von Intubation und Beatmung die Morbidität bei sehr kleinen Frühgeborenen signifikant beeinflusst, ist noch Gegenstand wissenschaftlicher Diskussion. Die von uns gewählten Einschlusskriterien sind bis auf wenige Ausnahmen im Grenzbereich praktisch umsetzbar. Eine frühe sekundäre Beatmung ist bei etwa einem Drittel der Patienten nötig. Es bestehen Hinweise auf Subgruppenunterschiede. Applikationsfehler oder -abbrüche treten selten auf. Schlussfolgerung. Die Untersuchung zeigt, dass diese Surfactantapplikationsmethode im Alltag machbar und praktikabel ist, aber eine genügend große Patientenzahl zur Etablierung einer Lernkurve erfordert und in ein standardisiertes Erstversorgungskonzept mit definiertem Atemwegsmanagement eingebettet werden sollte.

\section{PO-62}

Neonatal characteristics and risk factors of preterm infants with severe bronchopulmonary dysplasia (BPD): a population-based study

\section{E. Tutdibi', V. Preisegger', B. Misselwitz ${ }^{2}$, L. Gortner ${ }^{1}$}

'Unversitätskinderkliniken des Saarlandes, Klinik für Allg. Pädiatrie und Neonatologie, Homburg, Deutschland, ${ }^{2} \mathrm{GQH}$ - Geschäftsstelle Qualitätssicherung, Landesgeschäftsstelle Hessen, Eschborn, Deutschland

Background. Bronchopulmonary dysplasia (BPD) remains a major threat of very preterm neonates. Affected preterm newborns especially those being discharged on home oxygen therapy are at particularly high 
risk of long-term consequences of BPD including impaired neurodevelopmental outcome and impairment of lung function combined with bronchial hyperresponsiveness. Morphologically lungs were described to show developmental arrest with reduced alveolarization and abnormal vascular growth.

Aim. The present epidemiological study was conducted to evaluate postnatal characteristics of preterm infants with the diagnosis of severe BPD defined as need of supplemental oxygen at time of discharge from hospital to home.

Methods. We reviewed the data reported to the neonatal quality assurance program of the federal state of Hesse (Germany). A total of 3,769 life born preterm infants having a gestational age from $24+0$ to $32+6$ weeks delivered between January 1, 2001 and December 31, 2009 were enrolled. Exclusion criteria were incomplete data and major congenital malformations or chromosomal aberrations ( $\mathrm{n}=563$ in total). Relevant neonatal variables were defined prospectively and analyzed statistically by SPSS version 20 software.

Results. The rate of early BPD defined on the basis of oxygen requirements or mechanical ventilation (MV) for pulmonary reasons at 28 days of life was $18.6 \%(596 / 3,206)$. Newborns with early BPD were more often very low birth weight infants (VLBW: $96 \%$ vs. $51 \%$; $p<0.001$ ) and small for gestational age (SGA: $10.7 \%$ vs. $6.7 \%$; $<<0.001$ ) compared to infants without BPD. In the early BPD group respiratory distress syndrome (RDS), patent ductus arteriosus (PDA), early-onset sepsis, asphyxia, airleaks and MV therapy were more frequently recorded. Out of the 3,206 infants, 2,832 (88.3\%) were discharged to home, 217 (6.8\%) were transferred to another NICU with 66 on supplementary oxygen therapy and 157 (4.9\%) infants died during initial hospitalization. Among the 2,832 infants, who were discharged to home, a total of $32(1.1 \%)$ newborns required home supplementary oxygen therapy because of severe BPD. Multivariate analysis identified VLBW $(\mathrm{OR}=5.4$; 95\%CI $=1.5-19.9$; $\mathrm{p}<0.01)$ and duration of $\mathrm{MV}>7$ days as independent risk factors for developing severe $\mathrm{BPD}(\mathrm{OR}=23.5 ; 95 \% \mathrm{CI}=2.5-222.0$; $\mathrm{p}<0.006)$.

Conclusion. The results of our epidemiological study revealed VLBW and mechanical ventilation as main risk factors predicting severe BPD

\section{P0-63}

Ermittlung der Menge an Budesonid in der bronchialen Absaugflüssigkeit bei Frühgeborenen nach kontinuierlicher, druckunterstützter Atemhilfe (CPAP)

\section{K. Kreutzer', A. Koch', C. Maas', R. Kerb', U. Hofmann², M. Schwab², C. Poets ${ }^{1}$ D. Bassler ${ }^{1}$}

'Eberhard-Karls-Universität Universitätsklinik für Kinderheilkunde und Jugendmedizin, Neonatologie, Tübingen, Deutschland, ${ }^{2}$ Dr. Margarete Fischer-Bosch-Institut für Klinische Pharmakologie, Stuttgart, Deutschland

Hintergrund. Bei Frühgeborenen mit nasaler Atemunterstützung mittels „continuous positive airway pressure“ (CPAP), ist es schwierig abzuschätzen, wieviel Budesonid in der Lunge ankommt, wenn es durch Inhalation verabreicht wird.

Fragestellung. Um die Menge an Budesonid im trachealen Absaugsekret nach Inhalation bei nasalem CPAP zu bestimmen, führten wir eine prospektive Proof-of-principle-Studie durch.

Material und Methoden. Die Studie wurde von der zuständigen Ethikkommission genehmigt. Einschlusskriterien waren: Frühgeburtlichkeit $<28$ Schwangerschaftswochen bei Geburt, nasale Atemunterstützung mittels CPAP und Inhalation mit einem steroidhaltigen Präparat $(\mathrm{Bu}-$ desonid) während der Atemunterstützung am CPAP. Die Inhalation mit Budesonid wurde entsprechend des klinischen Standards mit einem Dosieraerosol, einem Spacer (AeroChamber mini* Aerosol Chamber) und einer Gesichtsmaske durchgeführt. Die Dosierung des Budesonids an Tag 1-14 war $2 \times 400 \mu \mathrm{g} / \mathrm{Tag}$, ab Tag $152 \times 200 \mu \mathrm{g} / \mathrm{Tag}$. Zum Zeitpunkt des Studienbeginns war das Kind, unabhängig von der Studie, bereits aufgrund klinischer Gründe intubiert, hatte aber noch keine endotracheale Inhalation von Budesonid während der maschinellen Beatmung über den Tubus erhalten. Das erste tracheale Absaugsekret nach Intubation, jedoch vor Inhalation über den Tubus, wurde gewonnen und analysiert, nachdem die Eltern ihr schriftliches Einverständnis erteilt hatten. Budesonid wurde in diesem Trachealsekret mittels einer hoch sensitiven Flüssigchromatographie-Massenspektrometrie gemessen.

Ergebnisse. Es wurden bisher insgesamt 3 Frühgeborene mit einem Gestationsalter von $23+4$ (Patient 1), 27+1 (Patient 2) und $27+1$ Wochen (Patient 3) bei Geburt eingeschlossen. Die tägliche Dosis an inhaliertem Budesonid betrug bei Patient 1 und 3 jeweils $800 \mu$ g, bei Patient 2 $400 \mu \mathrm{g} / \mathrm{Tag}$. Die Zeit nach der letzten Inhalation am CPAP und dem Asservieren des Trachealsekrets betrug jeweils 350 min (Patient 1), 90 min (Patient 2) und 20 min (Patient 3). Budesonid konnte in den jeweiligen Proben bei allen 3 Patienten nachgewiesen und quantifiziert werden. Die Budesonidkonzentration im Trachealsekret betrug $220 \mathrm{fmol} / \mathrm{ml}$ (Patient 1), $3227 \mathrm{fmol} / \mathrm{ml}$ (Patient 2) und $1252 \mathrm{fmol} / \mathrm{ml}$ (Patient 3).

Schlussfolgerung. Bei Frühgeborenen mit nasaler CPAP-Atemunterstützung, die Budesonid mittels Dosieraerosol über Spacer und Maske inhalieren, kann Budesonid im endotrachealen Sekret mehr als 5 Stunden nach Medikamentengabe nachgewiesen und quantifiziert werden. Damit erscheint ein lokaler Effekt von Budesonid in der Lunge sehr wahrscheinlich.

\section{PO-64}

Analyse des perioperativen intensivmedizinischen Managements bei Neugeborenen mit intrathorakalen Fehlbildungen (CCAM, Lungensequester)

A. Müller', K. Pommer', F. Kipfmüller', A. Heydweiller' ${ }^{2}$ H. Bachour', S. Bagci' 'Universitätsklinikum Bonn, Zentrum für Kinderheilkunde, Abt. Neonatologie, Bonn, Deutschland, ${ }^{2}$ Klinik und Poliklinik für Allgemein-, Viszeral-, Thorax- und Gefäßchirurgie, Universitätsklinikum Bonn, Kinderchirurgie, Bonn, Deutschland

Einleitung. In der Literatur finden sich nur wenige Daten zum detaillierten perioperativen intensivmedizinischen Management von Neugeborenen mit zystisch-adenomatoider Malformation der Lunge (CCAM) und Lungensequester (LS).

Methode. Wir untersuchten retrospektiv 25 Patienten mit CCAM ( $\mathrm{n}=15)$ und LS ( $\mathrm{n}=10$ ), die über einen Zeitraum von 10 Jahren (1999-2009) in unserer Klinik behandelt wurden. Das perioperative intensivmedizinische Management wurde bzgl. Flüssigkeits- und Katecholaminbedarfs, Beatmung und enteralem Nahrungsaufbau analysiert. Die Ergebnisse wurden als Median (,interquartile range“) dargestellt.

Ergebnisse. Gestationsalter, Geburtsmodus, Geschlechterverteilung, Geburtsgewicht zeigten keine signifikanten Unterschiede zwischen den beiden Gruppen. Das gilt auch für die mütterlichen Daten bezüglich Alter, Gravida und Partum. Postpartal benötigen 1/15 (6,6\%) der CCAM und $1 / 10$ (10\%) Patienten in der LS Gruppe eine Intubation und maschinelle Beatmung ( $>>0,05)$. Zwei weitere Patienten in der CCAM Gruppe benötigten eine Atemunterstützung mittels CPAP. Alle anderen Patienten wurden elektiv zur Operation intubiert. Die perioperativen Beatmungsparameter zeigten zwischen den beiden Gruppen keine Unterschiede bzgl. mittlere Atemwegsdruck (Tag o, 1, 2), postoperativer Beatmungsdauer und CPAP Notwendigkeit nach Extubation ( $\mathrm{p}>0,05)$. Der Volumenbedarf war perioperativ in beiden Gruppen gleich. Perioperativ benötigten 3/15 (20\%) der CCAM- und 8/10 (80\%) der LS-Patienten mindestens 1 Katecholamin $(\mathrm{p}=0,005)$. Auch die Dauer (h) der Katecholamintherapie war signifikant länger in der Gruppe der Patienten mit LS (34,8 h Range: 17-64,5; min. o, max. 265,5 h) im Vergleich zu den Patienten mit CCAM (o h; Range: o-0; min. o, max. 44; $\mathrm{p}=0,003$ ). Die Komplikationsraten bzgl. sekundäre Sepsis (CCAM 4/15 vs. LS o/10), Pleuraerguss (CCAM 7/15 vs. LS 6/10) und Chylothorax (CCAM $1 / 15$ vs. LS $2 / 10$ ) waren nicht signifikant unterschiedlich $(p>0,05)$. Der Nahrungsaufbaus bis zur vollen enteralen Ernährung war bei CCAM (7 Tage, Range: 4-12) und LS Patienten (5 Tage, Range: 4-12) nicht signifikant unterschiedlich $(\mathrm{p}>0,05)$. Die Aufenthaltsdauer war in der CCAM 
Patientengruppe (20 Tage, Range: 18-25) signifikant länger als in der LS Gruppe (15 Tage, Range: $13-25 ; \mathrm{p}=0,043$ ).

Schlussfolgerungen. Außer hinsichtlich der hämodynamischen Unterstützung fand sich kein signifikanter Unterschied bei der perioperativen Betreuung der Patienten mit CCAM und LS.

\section{PO-65}

Brachytelephalangic chondrodysplasia punctata: a rare cause for respiratory failure in a newborn

\section{S. Meyer', C. Wollny'², R. Eymann 3 , L. Gortner', B. Oehl-Jaschkowitz', M. Gencik $^{5}$}

'Universitätsklinikum des Saarlandes, Klinik für Allgemeine Pädiatrie und Neonatologie, Homburg/Saar, Deutschland, ${ }^{2}$ Unversitätsklinik für Kinder und Jugendmedizin Gebäude ${ }^{9}$, Homburg, Deutschland, ${ }^{3}$ Universitätsklinikum des Saarlandes, Klinik für Neurochirurgie, Homburg/Saar, Deutschland, ${ }^{4}$ Biomedizinisches Zentrum, Gemeinschaftspraxis für Humangenetik, Homburg/Saar, Deutschland, ${ }^{5}$ Diagones, Gemeinschaftspraxis für Humangenetik, Osnabrück, Deutschland

Background and study purpose. Chondrodysplasia punctata (CDP) is a heterogenous group of skeletal dysplasias. The hallmark of CDP is aberrant bone mineralization, manifesting radiologically as epiphyseal stippling. Among this group of skeletal dysplasia, brachytelephalangic CDP (BCDP) is probably under-reported. Here, we report on a neonate with BCDP with a new hemizygous missense mutation $[\mathrm{c} .332 \mathrm{G}>\mathrm{A}$ (p.Arg11His) in Exon 5 of the ARSE-gene] and severe spinal compression leading to quadriplegia.

Results. A 3,080-g infant boy was born at 372 weeks' gestation to a gravida III, para II mother by spontaneous vaginal delivery (unrelated parents). The pregnancy was uneventful except for polyhydramnios and absent fetal hiccups, but normal fetal movements. The neonate had a moderately depressed midface with a hypoplastic nose with barely patent nares. The child was initially flaccid (APGAR scores of 2 and 5 at 5 and 10 minutes) with absent spontaneous movements. On chest X-ray pulmonary hypoplasia and bilateral pneumothoraces were seen. Later in the course, slightly improved motor activity of both upper and lower extremities was noted. A skeletal survey demonstrated changes with multiple punctate calcifications of the epiphyses and vertebrae. The infant remained respirator-dependent. On ultrasonography, paresis of the diaphragm was demonstrated. On cerebral MRI and 3-dimensional reconstruction, severe compression of the cervical spine and subluxation of $\mathrm{C}_{1}$ and $\mathrm{C}_{2}$ was noted. On day 27 of life, spinal decompression just below the foramen magnum and stabilization of $\mathrm{C}_{1}$ and $\mathrm{C}_{2}$ was performed. Following surgery, the infant remained ventilator-dependent and a tracheostomy was performed. The infant is alive on a home-respirator without deterioration of neurologic symptoms. In our patient, a new hemizygous missense mutation c.332 G>A (p.Arg11His) could be identified in Exon 5 of the ARSE-gene.

Conclusions. It is important to take into consideration rare causes for respiratory failure. In children with $\mathrm{BCDP}$, respiratory failure may be due to severe cervical spinal compression.

\section{Neugeborenen-Neurologie}

PO-66

Xenon und Fentanyl induzieren keine Neuroapoptose im Gehirn neugeborener Schweine

H. Sabir', S. Bishop', N. Cohen'², E. Maes', X. Liu', J. Dingley³, M. Thoresen ${ }^{1}$ 'University of Bristol, School of Clinical Sciences, Neonatal Neuroscience, St Michael's Hospital, Bristol, United Kingdom, ${ }^{2}$ University of Bristol, School of Clinical Sciences, Neuropathology, Bristol, United Kingdom, ${ }^{3}$ Morriston Hospital, Department of Anaesthetics and Intensive Care, Swansea, United Kingdom

Hintergrund. Der Gebrauch von Inhalationsanästhetika kann für das sich entwickelnde Gehirn verheerende Folgen haben. Xenon, ein Inhalationsanästhetikum, zeigte im neonatalen Hypoxie-Ischämie-Tiermodell eine signifikante Zunahme der Neuroprotektion in der Kombination mit therapeutischer Hypothermie (HT) im Vergleich zu HT allein. Wir untersuchten den möglichen neuroapoptotischen Effekt von $24 \mathrm{~h}$ inhalativem Xenon in Kombination mit i.v. Fentanyl während Normothermie (NT) oder HT in gesunden neugeborenen Schweinen.

Material und Methodik. 26 neugeborene gesunde Schweine (Alter $<24 \mathrm{~h}$ ) wurden in 4 Gruppen randomisiert: (1) $24 \mathrm{~h}$ inhalatives Xenon (50\%) mit i.v. Fentanyl während HT ( $\left.\operatorname{Trec}=33,5^{\circ} \mathrm{C}\right),(2) 24 \mathrm{~h}$ inhalatives Xenon $(50 \%)$ mit i.v. Fentanyl während NT (Trec $\left.=38.5^{\circ} \mathrm{C}\right),(3) 24$ h i.v. Fentanyl während NT und (4) Kontrollgruppe während NT. Zusätzlich wurden 5 weitere Schweine für $24 \mathrm{~h}$ mit $2 \%$ Isofluran beatmet (positive Kontrollgruppe). Nach der jeweiligen Therapie endeten die Experimente. Pathologische Zellen wurden in verschiedenen Gehirnarealen beurteilt und neuroapoptotische Zellen nach immunhistochemischer Färbung quantifiziert (Caspase 3 und TUNEL).

Ergebnisse. Die neuropathologische Untersuchung zeigte keine signifikanten Unterschiede in den untersuchten Hirnarealen zwischen den Gruppen (1)-(4), jedoch eine große Zunahme an neuropathologischen Zellen nach Inhalation von Isofluran. Nach immunhistochemischer Färbung zeigte sich ein signifikanter Unterschied an Caspase-3- und TUNEL-positiven Zellen in der Isofluran-Gruppe für den zerebralen Cortex, den Hippocampus und die weiße Substanz. Für die Gruppen (1)-(4) zeigte sich in der Regressionsanalyse kein signifikanter Anstieg von neuroapoptotischen Zellen in Abhängigkeit von Xenon oder Fentanyl.

Diskussion und Schlussfolgerung. Weder inhalatives Xenon $(50 \%, 24 \mathrm{~h})$ mit i.v. Fentanyl, noch i.v. Fentanyl alleine induzieren Neuroapoptose im Gehirn neugeborener Schweine. Inhalatives Isofluran (2\%) induziert Neuroapoptose im neugeborenen Schweinegehirn.

\section{P0-67}

Einfluss von Hyperoxie und Koffein auf die postnatale, hippokampale Neurogenese und die Neurodegeneration der Ratte

S. Endesfelder', I. Zaak' , U. Weichelt', T. Schmitz', C. Bührer ${ }^{1}$

'Charite Kliniken f. Kinderheilkunde und Kinderchirurgie, Neonatologie, Berlin, Deutschland

Hintergrund. Sehr unreife Frühgeborene haben ein erhöhtes Risiko für neurologische, Verhaltens- und neurobiologische Folgeschäden. Tierexperimentelle Untersuchungen lassen vermuten, dass der postnatale Sauerstoffanstieg dabei eine Rolle spielt. Aus der CAP-Studie hat sich ergeben, dass die Anwendung von Koffein bei Frühgeborenen mit einer signifikant geringeren Inzidenz von kognitiven Defiziten und Zerebralparesen einhergeht.

Fragestellung. In unserer In-vivo-Studie an neugeborenen Wistar-Ratten im Hyperoxie-Schädigungsmodell testeten wir die Hypothese, dass Koffein protektiv auf die postnatale hippokampale Neurogenese und Neurodegeneration wirkt. 
Ergebnisse. Bei 6 Tage alten Wistar-Ratten konnten wir zeigen, dass bereits nach 24 und 48 h Hyperoxie $\left(80 \% \mathrm{O}_{2}\right)$ die Differenzierungs- und Proliferationskapazität reifender und reifer Neurone (immunhistochemische Färbungen für Nestin, DCX, NeuN; Western Blot für DCX, Calretinin) im Gyrus dentatus und die mRNA-Expression für relevante neuronale Transkriptionsfaktoren (Realtime-PCR für Pax6, SOX2, Tbr2, Prox1, Tbr1) signifikant reduziert wurden. Des Weiteren konnte gezeigt werden, dass die Zunahme neurodegenerierter Zellen (TUNEL) mit der Expressionszunahme eines oxidativen Stressmarkers (immunhistochemische Färbung für 8-oxodG) korreliert. Die Applikation von Koffein (10 mg/kg) führte zu einer verbesserten hippokampalen Neurogenese, einer Expressionszunahme der neuronalen Transkriptionsfaktoren und einer Reduktion der apoptotischen Zellen und der 8-oxodG positiven Zellen.

Schlussfolgerung. Diese Ergebnisse weisen auf neuroprotektive Effekte von Koffein, die möglicherweise antioxidativ vermittelt sind.

\section{PO-68 \\ Effekte von Hypothermie und Levetiracetam auf neuroprotektive Mechanismen nach Hypoxie-Ischämie im neonatalen Maushirn}

\section{K. Straßer', B. Reinboth', D. Brait', A. Bertsche ${ }^{2}$, I. Bendix', M. Dzietko ${ }^{1,}$}

J. Fandrey ${ }^{3}$, U. Felderhoff-Müser?

'Universitätsklinikum Essen, Kinderklinik ', Neonatologie, Essen, Deutschland, ${ }^{2}$ Universitätsklinikum Leipzig, Klinik für Kinder- und Jugendheilkunde, Leipzig, Deutschland, ${ }^{3}$ Universitätsklinikum Essen, Physiologie, Essen, Deutschland

Hintergrund. Hypoxisch-ischämische Schädigungen des unreifen ZNS während der Perinatalperiode stellen die häufigsten bekannten Ursachen entwicklungsneurologischer Langzeitdefizite dar. Mit der Ganzkörperkühlung nach einem Insult steht eine Therapie zur Verfügung, aus der sich positive therapeutische Effekte auf die neurologische Langzeitentwicklung ergeben. Inzwischen ist die Hypothermie in nationalen und internationalen Richtlinien empfohlen und zu einer Standardbehandlung bei asphyktischen Neugeborenen geworden. Aktuell ist es jedoch notwendig, 8 Kinder zu behandeln, um das neurologische Outcome eines Kindes zu verbessern. Um die Effektivität der Hypothermie zu steigern, besteht die Notwendigkeit adjuvante Strategien zu entwickeln. Fragestellung. Aus diesen Gründen sollen in einer tierexperimentellen Studie Effekte durch einen kombinierten Therapieansatz von Hypothermie und Levetiracetam auf neuroprotektive Mechanismen nach Hypoxie-Ischämie untersucht werden.

Material und Methoden. 9 Tage alte $\mathrm{C}_{57} \mathrm{BL} / 6$-Mäuse erhielten entweder eine Schein-OP oder eine Hypoxie-Ischämie (HI, modifiziertes Vanucci-Rice-Modell). Nach HI wurden die Jungtiere in 4 Gruppen randomisiert: 1) keine Behandlung, 2) Hypothermie (Ganzkörperkühlung, 4 Stunden, $30^{\circ} \mathrm{C}$ ), 3) hochdosiert Levetiracetam intraperitoneal (70 mg/ $\mathrm{kg} \mathrm{KG),} \mathrm{4)} \mathrm{Kombination} \mathrm{von} \mathrm{Hypothermie} \mathrm{und} \mathrm{hochdosiert} \mathrm{Levetirace-}$ tam intraperitoneal. Die Hirnhemisphären wurden 24 und 96 Stunden nach HI mittels Proteinanalysen und Immunhistochemie analysiert. Zwischen $\mathrm{P}_{28}$ und $\mathrm{P}_{59}$ wurde die sensomotorische Funktion mittels verschiedenen Verhaltenstestungen ermittelt.

Ergebnisse. Nach Hypothermie zeigten sich eine deutliche Verminderung apoptotischer Faktoren sowie ein Anstieg neuroprotektiver Parameter. Unter hochdosierter intraperitonealer Monotherapie mit Levetiracetam sowie in Kombination mit Hypothermie konnte ein Anstieg apoptotischer Faktoren detektiert werden. In den Verhaltenstestungen ergaben sich durch Hypothermie positive Langzeiteffekte.

Schlussfolgerung. Die Ganzkörperkühlung führt zur Neuroprotektion im neonatalen Maushirn durch Apoptosereduktion und Aktivierung neuroprotektiver Faktoren. Eine hochdosierte Levetiracetam-Therapie hat nach hypoxisch-ischämischer Schädigung keine additiven Effekte.

\section{PO-69}

Hypothermiebehandlung bei neonatalen und pädiatrischen Patienten - Daten aus dem Hypothermienetzwerk

\section{H. Schützle', A. Koch', S. Brenner', M. Rüdiger ${ }^{1}$}

'Univ. Klinikum Carl Gustav Carus, Klinik für Kinder- und Jugendmedizin, Neonatologie, Dresden, Deutschland

Hintergrund. Die Hypothermiebehandlung nach schwerer perinataler Asphyxie kann die postasphyktische Schädigung des Gehirns durch Verhinderung eines sekundären Energiemangels reduzieren und somit das neurologische Outcome verbessern. Bei Neugeborenen gilt die Hypothermie daher als Standardtherapie. Für pädiatrische Patienten nach Herz-Kreislauf-Stillstand fehlen bisher eindeutige Daten. Ziel des Hypothermienetzwerkes ist eine Kooperation von Einrichtungen, welche Hypothermiebehandlung anbieten mit dem Ziel des Erfahrungsaustausches, der Qualitätserfassung und der Durchführung wissenschaftlicher Studien. Dargestellt sind die bisherigen Daten seit Bestehen des Netzwerkes (3. Quartal 2010 bis 4. Quartal 2012).

Methode. Quartalsweise erfolgt einer Erfassung der Kinder mit Hypothermiebehandlung. Erfasst werden Kühlmethode (Ganzkörper, selektive Kopfkühlung), Kühlungsindikation, Ort des Ereignisses (in oder außerhalb der Klinik), Entlassmodus (nach Hause, Rehabilitationseinrichtung, Versterben) sowie Art der Nahrungszufuhr bei Entlassung (oral/Sonde). Die Erfassung erfolgt getrennt für Neugeborene (NG), Säuglinge (Sgl), Kleinkinder (KK, <6 Jahre) und Schulkinder (SK, 6-18 Jahre).

Ergebnisse. Insgesamt beteiligen sich 99 Zentren am Hypothermienetzwerk, davon 95 Level 1. Die Rücklaufquote liegt bei 35 bis 52\%. Pro Quartal werden im Median 1-2 Patienten (Range: 1-8) pro Zentrum gekühlt. Bislang wurden 48 o hypothermiebehandelte NG gemeldet, davon $92 \%$ aus klassischer Indikation (perinatale Asphyxie, >36 SSW, >180o g GG). Das Ereignis trat zu nahezu gleichen Anteilen in bzw. außerhalb der Einrichtung auf. $76 \%$ der NG wurden nach Hause entlassen, ca. $5 \%$ in eine Reha-Einrichtung verlegt, $11 \%$ sind verstorben. $87 \%$ waren bei Entlassung/Verlegung voll oral ernährt. Über den gleichen Zeitraum wurden 47 behandelte Sgl. und 26 bzw. 25 Klein- bzw. Schulkinder registriert. Bei Sgl. und KK trat das Ereignis in $70 \%$, bei SK in $96 \%$ außerhalb der Klinik auf. Im Vergleich zu den NG zeigt sich eine deutlich höhere Mortalität (Sgl. 55\%, KK 30\%, SK 48\%). Orale Ernährung bei Entlassung/Verlegung in $47 \%$ (Sgl), 44\% (KK) bzw. 69\% (SK). KK und SK werden viel häufiger ( 38 bzw. 24\%) in Reha-Einrichtungen verlegt als NG und Sgl (4\%).

Schlussfolgerung. Die Daten zeigen, dass die Hypothermie für asphyktische NG bereits in vielen Einrichtungen eine gut etablierte Therapie ist. Bei Säuglingen und Kindern ist ein Herz-Kreislauf-Stillstand ein seltenes Ereignis, umso wichtiger erscheint hier eine multizentrische Datenerfassung, zumal im Median nur 1-2 Patienten pro Zentrum und Quartal einer Hypothermiebehandlung zugeführt werden. Die Daten bestätigen Angaben in der Literatur, wonach die Mortalität bei pädiatrischen Patienten, insbesondere den Säuglingen im Vergleich zu Neugeborenen sehr hoch ist. Neugeborene und Säuglinge werden nur in Einzelfällen in eine Reha-Einrichtung verlegt. Wünschenswert im Hinblick auf aussagekräftigere Daten wäre eine deutlich höhere Rücklaufquote. 


\section{PO-70}

\section{Ambulante Betreuung ehemaliger Neugeborener mit neonatalem} Abstinenzsyndrom

\section{Patschureck', C. de Beer ${ }^{2}$, U. Fleischer ${ }^{3}$, A. Kunze ${ }^{2}$, M. Langhammer ${ }^{2}$ E. Robel-Tillig ${ }^{4}$}

'Universitätskinderklinik, Leipzig, Deutschland, ${ }^{2}$ Klinikum St. Georg gGmbH Kinderklinik, Leipzig, Deutschland, ${ }^{3}$ Klinikum St. Georg gGmbH Kinderklinik, Kinderklinik, Leipzig, Deutschland, ${ }^{4}$ Klinikum St. Georg gGmbH Kinderklinik, FB Neonatologie/IMC, Leipzig, Deutschland

Hintergrund. Die Anzahl Neugeborener die mit einem neonatalem Abstinenzsyndrom (NAS) nach Drogenabusus der Mütter zur Welt kommen, ist den letzten Jahren extrem angestiegen. Diese Kinder bedürfen postnatal einer intensiven medizinischen und pflegerischen Betreuung. Wesentlich ist jedoch die Frage, inwieweit die Kinder auch nach Entlassung neurologische oder psychische Auffälligkeiten bieten.

Material und Methoden. Untersucht werden 32 ehemalige Neugeborene die mit einem NAS 2-17 Wochen stationär betreut wurden und einer mehrwöchigen Substitutionstherapie mit Opiaten bedurften. Die Kinder wurden mindestens 4-mal im ersten Lebensjahr hinsichtlich ihrer motorischen, sozialen und mentalen Entwicklung untersucht.

Ergebnisse. Insgesamt 28 der 32 Kinder wurden zu jedem der vereinbarten Termine vorgestellt. 14 Kinder befanden sich in Betreuung durch die Eltern. In diesen Familien war eine Familienhilfe organisiert. Zehn Mütter waren während mindestens der Hälfte des Untersuchungszeitraums mit ihren Kindern in einem Mutter-Kind-Heim versorgt. Acht Kinder wurden in Pflegefamilien betreut. Die körperliche Entwicklung hinsichtlich Länge und Gewicht lag bei $85 \%$ auf der individuellen Perzentile. Das Kopfumfangswachstum wich jedoch bei fast $40 \%$ nach unten von der Kurve ab. Die motorische Entwicklung der Kinder wurde bei $80 \%$ im Normbereich diagnostiziert oder wich nicht mehr als 2 Monate von der Norm ab. Bei der Einschätzung der mentalen Bereiche fiel eine deutlich verzögerte Sprachentwicklung und Entwicklung der Feinmotorik auf. Bei fast $80 \%$ der Kinder wurden von den pflegenden Personen extreme Unruhe, nächtliche Schreiattacken und Verlustängste beschrieben. Ebenso kommt es häufig (45\%) zu autoaggressivem Verhalten der Kinder.

Schlussfolgerung. Zusammenfassend muss festgestellt werden, dass entwicklungsneurologische Probleme ehemaliger Neugeborener mit NAS weit über die Zeit der Entzugsproblematik hinaus persistieren. Eine spezialisierte Betreuung dieser Patienten ist dringend im Interesse einer positiven Entwicklung zu fordern.

\section{P0-71}

Hämodynamische Reaktion der Sehrinde auf visuelle Stimulation während der augenärztlichen Untersuchung: eine funktionelle NIRS-Untersuchung

\section{B. Hüning', K. Büchsenschütz², B. Jurklies³, U. Felderhoff-Müser', C. Roll'} 'Universitätsklinikum Essen, Universität Duisburg-Essen, Klinik für Kinderheilkunde I, Neonatologie, Essen, Deutschland, ${ }^{2}$ Universitätsklinikum Essen, Universität Duisburg-Essen, Klinik für Hals-Nasen-Ohrenheilkunde, Kopf- und Halschirurgie, Essen, Deutschland, ${ }^{3}$ Helios Klinikum Wuppertal, Klinik für Augenheilkunde, Wuppertal, Deutschland, ${ }^{4}$ Vestische Kinder- und Jugendklinik Datteln, Universität Witten/Herdecke, Neonatologie und Pädiatrische Intensivmedizin, Datteln, Deutschland

Einleitung. Funktionelle Untersuchungen sind wichtig zum Verständnis der Hirnentwicklung Frühgeborener. Das augenärztliche Retinopathie(ROP)-Screening wurde als Reiz verwendet, um die lokale hämodynamische Reaktion der Sehrinde auf einen Lichtreiz mittels funktioneller Nahinfrarotspektroskopie (fNIRS) zu untersuchen.

Patienten und Methodik. 19 Früh- und 1 Reifgeborenes [Gestationsalter (GA): Median 29+4 (23+6-40+0) SSW, Geburtsgewicht: Median 1230 (650-2780) g] wurden im Rahmen des ROP-Screenings augenärztlich untersucht [GA bei Untersuchung: Median 35+2 $(31+1-44+4)$ SSW, Gewicht: Median 2068 (1300-3070) g]. Ausgeschlossen wurden Kinder mit kardiopulmonaler Instabilität, Fehlbildungen des Gehirns oder der Augen. Das Prinzip der NIRS beruht auf dem charakteristischen Absorptionsspektrum des Hämoglobins für nahinfrarotes Licht in Abhängigkeit des Oxygenierungszustandes. Gemessen werden Konzentrationsänderungen des oxygenierten $\left(\mathrm{O}_{2} \mathrm{Hb}\right)$ und des desoxygenierten Hämoglobins ( $\mathrm{HHb}$ ) sowie des Tissue Oxygenation Index (TOI). Parallel werden die Herzfrequenz und arterielle Sättigung aufgezeichnet. Die fNIRS nutzt die Tatsache, dass bei neuronaler Aktivität vermehrt Sauerstoff verbraucht wird. Der Versuchsablauf sah zunächst eine manipulationsfreie Stimulation durch einen Lichtreiz bei geschlossenem Auge, gefolgt von der augenärztlichen ROP-Screening-Untersuchung vor.

Ergebnisse. Die manipulationsfreie Stimulation bei geschlossenem Auge zeigte einen tendenziellen Abfall des $\mathrm{O}_{2} \mathrm{Hb}$ (ns) und Anstieg des $\mathrm{HHb}$ (ns). Während der augenärztliche Untersuchung zeigte sich in den NIRS- und Vitalparametern eine über die Zeit zunehmende Stressreaktion der Kinder: Abfall TOI ( $\mathrm{p}=0,01)$, Anstieg Herzfrequenz $(\mathrm{p}=0,01)$, Abfall art. Sättigung $(\mathrm{p}=0,041)$.

Diskussion. Nur die hämodynamischen Veränderungen auf die manipulationsfreie Stimulation können als reizbedingt interpretiert werden. Der beobachtete tendenzielle Abfall des $\mathrm{O}_{2} \mathrm{Hb}$ entspricht einem $\mathrm{Re}$ aktionsmuster, welches bei älteren Kindern beobachtet wurde. Dies ist möglicherweise der erste Hinweis auf eine akzelerierte Hirnreifung bei Frühgeborener im Vergleich zu Reifgeborenen.

Schlussfolgerung. Hämodynamische Reaktion der Sehrinde auf visuelle Stimulation während des ROP-Screening können nur bedingt mittels funktioneller NIRS erfasst werden. Mit zunehmender Untersuchungsdauer kam es zu einer Stressreaktion bis hin zur Minderung der Sauerstoffsättigung des Gehirns, die die lokale Reizantwort der Sehrinde überlagerte.

\section{PO-72}

Array-basierte genomweite Genotypisierung bei Feten mit syndromalen cerebralen Fehlbildungen

S. Krutzke', M. Draaken², M. Schumann', A. Hilger', S. Moebus', U. Gembruch $^{4}$, M. Ludwig ${ }^{5}$, P. Bartmann', M. Nöthen ${ }^{2}$, H. Reutter', W. Merz ${ }^{4}$ 'Universität Bonn, Zentrum für Kinderheilkunde, Abteilung für Neonatologie, Bonn, Deutschland, ${ }^{2}$ Universitätsklinik Humangenetik, Bonn, Deutschland, ${ }^{3}$ Universitätsklinik Medizinische Informatik, Biometrie und Epidemiologie, Essen, Deutschland, ${ }^{4}$ Universitäts-Frauenklinik Bonn, Klinik für Geburtshilfe und Pränataldiagnostik, Bonn, Deutschland, ${ }^{5}$ Universitätsklinik Klinische Chemie und Pharmakologie, Bonn, Deutschland

Hintergrund. Angeborene Fehlbildungen des Zentralen Nervensystems (ZNS) kommen bei 0,5-2 von 1000 Geburten vor. Damit eingeschlossen sind Neuralrohrdefekte und cerebrale Fehlbildungen, die jeweils isoliert oder kombiniert mit anderen Fehlbildungen auftreten können. Für die meisten cerebralen Fehlbildungen sind die Ursachen ungeklärt. Die genetische Diagnostik beschränkt sich während der Schwangerschaft zumeist auf die Durchführung konventioneller Chromosomenanalysen; die Erfahrungen in der Anwendung neuer Array-basierter Methoden zur Identifizierung genetischer Ursachen sind mangels fehlender systematischer Untersuchungen rudimentär. Allerdings kann der überwiegende Anteil cerebraler Fehlbildungen mittels bildgebender Verfahren (Ultraschall und MRT) pränatal nach anatomischen Gesichtspunkten klassifiziert werden, was dazu geführt hat, dass die meisten der betroffenen Feten aufgrund der assoziierten Entwicklungsstörungen nicht mehr ausgetragen werden.

Fragestellung. Um mehr Erfahrung über den Nutzen von Arrray-basierten Methoden zur Ermittlung von möglichen genetischen Aberrationen bei angeborenen cerebralen Fehlbildungen zu sammeln, wurde ein Kollektiv bestehend aus 33 abortierten Feten mit nicht-isolierten cerebralen Fehlbildungen untersucht. 
Material und Methoden. Da cerebrale Fehlbildungen mit strukturellen Chromosomenanomalien assoziiert sind, macht es das gemeinsame Auftreten von cerebralen und anderen angeborenen Fehlbildungen (nicht-isoliert) wahrscheinlich, ursächliche Kopienzahlveränderungen („copy number variations“, CNVs) zu finden. Alle Feten wurden durch die Abteilung für Geburtshilfe und Pränatalmedizin am Universitätsklinikum Bonn aus über 300 abortierten Feten mit mehrheitlich cerebralen ZNS-Fehlbildungen akquiriert, von denen DNA-Aliquots im Zuge des Fetozids zwischen 2006 und 2012 gewonnen wurden. Für die molekulare Karyotypisierung wurde der Illumina HumanOmniExpress-12V1_H benutzt, der 730.525 Marker enthält. Zur Auswertung der SNP(,single nucleotide polymorphism“)-Floureszenzintensität benutzten wir QuantiSNP mit dem Objective-Bayes Hidden-Markov Modell, um potenziell ursächliche CNVs zu identifizieren.

Ergebnisse. Unsere bisherigen Ergebnisse zeigen möglich-kausative Deletionen in sechs Feten, welche die folgenden chromosomalen Regionen betreffen: 18q21.2-qter (29,6 Mb), 2q35-q37.1 (12,8 Mb), 6q25.3-qter (14,4 Mb), 2q22.2-q23.3 (10,7 Mb), 1q42.3-qter (13,5 Mb) and 5p14.1-pter $(23,2 \mathrm{Mb})$. Der letztgenannte CNV wurde in einem Feten detektiert, der darüber hinaus auch eine $24 \mathrm{Mb}$ große Duplikation auf Chromosom 9932-qter trägt.

Schlussfolgerung. Unsere vorläufigen Ergebnisse deuten darauf hin, dass Array-basierte Methoden in der pränatalen Diagnostik sinnvoll in der ursächlichen Abklärung bei angeborenen syndromalen cerebalen ZNS-Fehlbildungen sind.

\section{PO-73}

\section{Array-basierte genomweite Genotypisierung bei Feten mit isolier- ten cerebralen Fehlbildungen}

\section{Schumann', M. Draaken'2, S. Krutzke', A. Hilger², S. Moebus', U. Gem- bruch $^{4}$, M. Ludwig ${ }^{5}$, P. Bartmann', M. Nöthen' ${ }^{2}$, H. Reutter', W. Merz ${ }^{4}$ 'Universität Bonn, Zentrum für Kinderheilkunde, Abteilung für Neo- natologie, Bonn, Deutschland, ${ }^{2}$ Universitätsklinik Humangenetik, Bonn, Deutschland, ${ }^{3}$ Universitätsklinik Medizinische Informatik, Biometrie und Epidemiologie, Essen, Deutschland, ${ }^{4}$ Universitäts-Frauenklinik Bonn, Klinik für Geburtshilfe und Pränataldiagnostik, Bonn, Deutschland, ${ }^{5}$ Universitäts- klinik Klinische Chemie und Pharmakologie, Bonn, Deutschland}

Hintergrund. Angeborene Fehlbildungen des Zentralen Nervensystems (ZNS) kommen bei 0,5-2 von 1.00o Geburten vor. Damit eingeschlossen sind sowohl Neuralrohrdefekte als auch cerebrale Fehlbildungen. Letztere können isoliert oder kombiniert mit anderen Fehlbildungen auftreten. Für die meisten cerebralen Fehlbildungen wurden bisher keine genetischen oder nicht-genetischen Ursachen gefunden. Die genetischen Untersuchungen während der Schwangerschaft beschränken sich zumeist auf die Durchführung konventioneller Chromosomenanalysen; die Erfahrungen in der Anwendung neuer Array-basierter Methoden zur Identifizierung genetischer Ursachen sind mangels fehlender systematischer Untersuchungen rudimentär. Nichtsdestotrotz kann der überwiegende Anteil angeborener cerebraler Fehlbildungen mittels Ultraschall und/oder MRT pränatal nach anatomischen Gesichtspunkten eindeutig klassifiziert werden, was dazu geführt hat, dass die meisten der betroffenen Feten aufgrund der assoziierten Entwicklungsstörungen nicht mehr ausgetragen werden.

Fragestellung. Um mehr Erfahrung über den Nutzen von Arrray-basierten Methoden zur Ermittlung von möglichen genetischen Aberrationen bei angeborenen zerebralen Fehlbildungen zu sammeln, wurde ein Kollektiv bestehend aus 35 abortierten Feten mit isolierten cerebralen Fehlbildungen untersucht.

Material und Methoden. Alle Feten wurden durch die Abteilung für Geburtshilfe und Pränatalmedizin am Universitätsklinikum Bonn aus über 300 abortierten Feten mit mehrheitlich zerebralen ZNS-Fehlbildungen akquiriert, von denen DNA-Aliquots im Zuge des Fetozids zwischen 2006 und 2012 gewonnen wurden. Für die molekulare Karyotypisierung wurde der Illumina HumanOmniExpress-12v1_H benutzt, der 730.525 Marker enthält. Zur Auswertung der SNP(Single Nucleotide Polymorphism)-Floureszenzintensität benutzten wir QuantiSNP mit dem Objective-Bayes Hidden-Markov Modell, um potentiell ursächliche CNVs zu identifizieren.

Ergebnisse. Unsere bisherigen Ergebnisse zeigen möglich ursächliche Deletionen in zwei Feten, welche folgende chromosomale Regionen betreffen: Die X-chromosomale Region p22.2-p22.33 (9,1 Mb; 32 RefSeq Gene), sowie die Region 6p25.1-6pter (4,38 Mb; 30 RefSeq Gene) in einem anderen Feten. Die Deletion auf dem kurzen Arms des Chromosom 6 betrifft das FOXC1-Gen, von dem man weiß, dass es an der Entstehung der Dandy-Walker-Malformation beteiligt ist.

Schlussfolgerung. Unsere Befunde deuten darauf hin, dass bei isolierten cerebralen Fehlbildungen Array-basierte Methoden sinnvoll in der Abklärung der Ursachen sein können, wobei lediglich bei einem geringen Teil der untersuchten Feten mit auffälligen Befunden zu rechnen ist.

\section{PO-74 \\ Floppy-infant-Syndrom - eine differenzialdiagnostische Heraus- forderung}

\section{U. Brandt', R. Husain ${ }^{2}$, K. Dawczynski ${ }^{3}$, I. Schreyer ${ }^{4}$}

'Universitätsklinikum Jena, Klinik für Kinder- und Jugendmedizin, Neonatologie/Pädiatrische Intensivmedizin, Jena, Deutschland, ${ }^{2}$ Friedrich Schiller Universität/Klinik für Kinder- u. Jugendmed., Neuropädiatrie, Jena, Deutschland, ${ }^{3}$ Friedrich Schiller Universität Klinik f. Kinder- u. Jugendmed., Jena, Deutschland, ${ }^{4}$ Universitätsklinikum Jena, Institut für Humangenetik, Jena, Deutschland

Das Floppy-infant-Syndrom mit seiner breiten Varianz an möglichen Ursachen bietet häufig diagnostische Probleme und benötigt im Einzelfall eine weitreichende molekulargenetische Aufarbeitung.

Ein FG der $30+5$ SSW wurde aufgrund sonographisch und in der MRT nicht darstellbarer Magenblase des Feten sowie Polyhydramnion der Verdacht auf eine Ösophagusatresie gestellt. Eine Amniozentese nach $31+1$ Wochen war unauffällig. Die Kindsbewegungen wurden von der Mutter als normal angegeben, die Familienanamnese bzgl. Muskelerkrankungen war leer.

Die Geburt erfolgte nach 33+o SSW per Sectio bei vorzeitigen Wehen, postpartal zeigte der eutrophe (1780 g) Knabe keine Eigenatmung und einen schlaffen Muskeltonus, APGAR $2 / 3^{*} / 6^{*}\left({ }^{\star} \mathrm{SIPPV}\right), \mathrm{NVpH} 7,32$. Eine Ösophagussondierung war problemlos möglich. Der weitere Verlauf war gekennzeichnet durch muskuläre Hypotonie, Beatmungspflichtigkeit sowie fehlende Spontanmotorik und Muskeleigenreflexe des Kindes.

Laborchemisch ergaben sich keine Auffälligkeiten (infektiologische Serum- und Liquordiagnostik, Creatinkinase, Laktat, überlangkettige Fettsäuren, endokrinologische und metabolische Parameter). Im Schädel-MRT zeigte sich kein Anhalt für einen hypoxischen Hirnschaden oder andere wegweisende Befunde, EEG, Echokardiographie und EKG waren diagnostisch nicht weiterführend.

Molekulargenetisch wurden im Rahmen einer Stufendiagnostik folgende Erkrankungen weitgehend ausgeschlossen: Myotone Dystrophie Typ I, Prader-Willi-Syndrom, Spinale Muskelatrophie (heterozygoter Anlageträger), X-chromosomale sowie autosomal-dominante Myotubuläre Myopathie. Die im Alter von 4 Wochen durchgeführte Muskelbiopsie ergab zu einer konnatalen/primären Myopathie passende Befunde ohne spezifische Strukturelemente. Bezüglich einer nach Referenzbefundung u. a. diskutierten myofibrillären Myopathie ergab eine ACTA1-Mutationsanalyse keinen pathologischen Befund. Die Arbeitsdiagnose einer konnatalen Myopathie konnte somit bisher nicht spezifiziert werden.

In einer postmortal durchgeführten Array-CGH (Microarray-basierten komparativen genomischen Hybridisierung) wurde sehr wahrscheinlich eine De-novo-Mikrodeletion 1q21.1 gefunden. Bei großer klinischer Variabilität dieser Mikrodeletion kann aber hierdurch keine eindeutige Krankheitsverursachung bestätigt werden. 
Der Knabe zeigte im weiteren Verlauf minimale Spontanmotorik sowie Reaktion auf äußere Reize, jedoch weiterhin keine suffiziente Spontanatmung. Nach mehreren Ethikkonsilen wurden nach 2 Monaten die lebenserhaltenden Maßnahmen auf Wunsch der Eltern beendet.

\section{PO-75}

Die Parechovirusenzephalitis - eine wichtige Differenzialdiagnose postnatal erworbener zystischer Marklagerläsionen

\section{Schoberer', M. Häusler', A. Eis-Hübinger', A. Schoberer', K. Heimann", S. Trepels-Kottek', T. Orlikowsky' \\ 'Universitätsklinik für Kinder- und Jugendmedizin, Sektion für Neonato- logie, Aachen, Deutschland, ${ }^{2}$ Universitätsklinik für Kinder- und Jugendme- dizin, Neuropädiatrie, Aachen, Deutschland, ${ }^{3}$ Universität Bonn, Institut für Virologie, Bonn, Deutschland, ${ }^{4}$ Universitätsklinik für Kinder- und Jugend- medizin, SPZ, Aachen, Deutschland}

Einleitung. Im Gegensatz zum sehr unreifen Frühgeborenen führen perinatale Noxen beim späten Frühgeborenen bzw. reif geborenen Kind selten zu zystischen Marklagerdefekten. Ihr Auftreten erfordert daher eine intensive Differentialdiagnostik, einschließlich der Suche nach metabolen und infektiösen Erkrankungen.

Kasuistik. Ein zunächst gut adaptiertes männliches Frühgeborenes (34 SSW; APGAR: 8/9/10; NSa-pH: 7,28; kein Atemnotsyndrom) entwickelt am 5. Lebenstag (LT) Krampfanfälle mit Apnoen, Bradykardien und Irritabilität, welche am 6. LT eine Intubation erforderten. Trotz unauffälliger Werte für CrP und IL-6 im Serum sowie eines normalen Liquorstatus (Zellzahl: $6 \mathrm{M} / \mathrm{l}$; Glucose: $71 \mathrm{mg} / \mathrm{dl}$; Protein: 1,2 g/l; und IL-6: 7 ng/l) wurde eine i.v.-antibiotische Therapie mit Aciclovir, Ceftazidim und Tobramycin begonnen. Auch die Liquorkultur, PCR-Untersuchungen auf HSV-, Coxsackie- und ECHO-Viren, Stuhluntersuchungen auf Rota- und Adenoviren sowie ausführliche Stoffwechseldiagnostik ergaben keinen Befund. Während sequentielle Schädelsonographien ebenfalls unauffällig waren, zeigte die MRT am 7. LT periventrikulär Diffusionsstörungen. Die daraufhin initiierte Liquor-PCR konnte eine Parechovirusinfektion (HPeV, Typ 3) nachweisen. Die MRT-Verlaufsuntersuchung zeigte nach 14 Tagen frontal und occipital zystische Marklagerläsionen. Am 8. LT konnte das Kind extubiert werden. Die Entlassung erfolgte in klinisch unauffälligem Zustand im Alter von 3 Wochen. Retrospektiv hatte die Mutter ca. 10 Tage vor Geburt an einer Gastroenteritis gelitten.

Diskussion. Bei Enzephalopathien des späten Frühgeborenen, die nicht durch perinatale oder metabole Faktoren erklärbar sind, müssen akute infektiöse Ursachen erwogen werden. Wichtige Differenzialdiagnose ist hier die Parechovirus-Infektion. Diese wird durch übliche Enterovirus-PCR-Ansätze nicht erfasst und muss selektiv nachgewiesen werden. Bildmorphologisch richtungsweisend sind in der Frühphase Diffusionsstörungen im Marklager, in der Spätphase frontal und occipital betonte Marklagerzysten, die im Gegensatz zur typischen periventrikulären Leukomalazie des Frühgeborenen nicht mit den Ventrikeln verschmelzen. Der auch aus der Literatur bekannte unauffällige Liquorstatus spricht dafür, dass die eingeschränkte Immunabwehrsituation des Neugeborenen diesen komplizierten Infektionsverlauf begünstigt.

\section{PO-76}

Zerebraler Vasospasmus bei bakterieller Meningitis, Therapieversuch mit lokaler intraarterieller Nimodipin-Dauerinfusion - ein Fallbericht

\section{S. Baranowski', O. Beringer', T. Kapapa ${ }^{2}$, M. Halatsch ${ }^{2}$, B. Schmitz ${ }^{3}$ H. Hummler?}

'Klinik für Kinder- und Jugendmedizin, Universitätsklinikum Ulm, Sektion Neonatologie und Pädiatrische Intensivmedizin, Ulm, Deutschland, ${ }^{2}$ Universitätsklinikum Ulm, Klinik für Neurochirurgie, Ulm, Deutschland, ${ }^{3}$ Klinik für Diagnostische und Interventionelle Radiologie, Sektion Neuroradiologie, Universitätsklinikum Ulm, Günzburg, Deutschland

Hintergrund. Zerebrovaskuläre Manifestationen sind eine gefürchtete Komplikation der bakteriellen Meningitis. $\mathrm{Zu}$ einem ischämischen Schlaganfall kann es durch Vasospasmus, Vaskulitis oder intraarterielle Thrombose kommen. Oft ist damit eine Defektheilung mit neurologischem Defizit verbunden. Die AWMF-Leitlinie zum Thema bakterielle Meningoenzephalitis der Neurologen diskutiert, dass in Analogie zum Vorgehen bei einer Subarachnoidalblutung (SAB) bei einem Vasospasmus großer Hirnarterien eine Therapie mit dem Kalziumantagonisten Nimodipin erwogen werden kann (AWMF 2008). Im British Aneurysm Nimodipine Trial konnte gezeigt werden, dass Nimodipin die zerebrale Infarktrate senkt und das Outcome nach SAB verbessert (Pickard 1989). Fallbericht. Wir berichten den Verlauf einer 10-jährigen Patientin mit Meningoenzephalitis durch Streptococcus intermedius. Seit 5 Tagen Fieber und Kopfschmerzen, im Verlauf Desorientiertheit, ausgeprägter

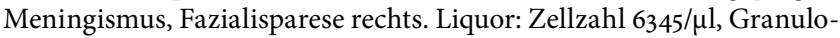
zyten $57 \%$. 48 Stunden nach Therapiebeginn Glasgow Coma Scale von 6 und nahezu lichtstarre Pupillen. Im Aufnahme-CCT kein Hinweis auf erhöhten intrakraniellen Druck, nun dopplersonographisch erniedrigte RI-Werte. Im Re-CT Hirnödem sowie frontale Demarkierungsbereiche. Anlage einer Hirndruckmesssonde nach Ausschluss eines Status epilepticus. Im folgenden Angio-CT V. a. ausgeprägte arterielle Vasospasmen der Aa. cerebri anteriores sowie des Carotis T samt Aa. cerebri mediae bds. Zur permanenten intraarteriellen Spasmolyse wurden je ein Mikrokatheter über Leistenschleusen bds. in die distalen Aa. carotis internae eingebracht. Angio-MRT 5. Tag: Größenkonstanz der bekannten Ischämieareale, unveränderte Darstellung der Vasospasmen, daher Fortführen der lokalen intraarteriellen Nimodipingabe. Keine Demarkierung neuer Infarkte. Verlaufskontrolle 8. Tag: Nachweis eines Spitzenthrombus des linksseitigen Katheters, rechtsseitig Katheterfehllage in der A. carotis externa. Radiologisch zunehmendes Perfusionsdefizit rechts bei rückläufigen Vasospasmen links. Beim Rückzug des linksseitigen Katheters embolischer Verschluss der distalen A. carotis links. Sofortige komplette Rekanalisation mittels Stent-Retriever. Postinterventionell sind die distale A. carotis interna und A. cerebri media links wieder nahezu normalkalibrig. Aufgrund des thrombembolischen Ereignisses keine Katheter-Neuanlage. Im weiteren Verlauf i.v.-DTI mit Nimodipin. Am 9. Tag Nachweis eines Territorialinfarktes im linken Mediastromgebiet sowie von Teilinfarkten im rechten Mediastromgebiet und im Posteriorstromgebiet bds. Entwicklung eines malignen Hirnödems mit ICP-Werten $>55 \mathrm{mmHg}$. Versterben der Patientin am 14. Behandlungstag.

Schlussfolgerung. Obwohl sich Hinweise auf eine Wirksamkeit der intraarteriellen Nimodipingabe auf die Vasospasmen ergaben, ist diese experimentelle Therapie unseres Erachtens aufgrund der fehlenden Datenlage und des erhöhten Risikos thrombembolischer Komplikationen bei Kindern nicht allgemein zu empfehlen. 


\section{PO-77}

\section{Seltene Ursache eines angeborenen Hydrocephalus: kongenitales} Glioblastoma multiforme

\section{Umlauf', S. Trepels-Kottek ${ }^{2}$, M. Hütten ${ }^{3}$, B. Sellhaus', M. Blaum ${ }^{5}$ C. Müller ${ }^{6}$, T. Orlikowsky}

'Universitätsklinikum der RWTH, Klinik für Kinder- und Jugendmedizin - Sektion Neonatologie und Pädiatrische Intensivmedizin, Aachen, Deutschland, ${ }^{2}$ Universitätsklinikum der RWTH, Klinik für Kinder- und Jugendmedizin - Sektion Neonatologie und Pädiatrische Intensivmedizin, Aachen, Deutschland, ${ }^{3}$ Universitätsklinikum der RWTH, Klinik für Kinderund Jugendmedizin - Sektion Neonatologie und Pädiatrische Intensivmedizin, Aachen, Deutschland, ${ }^{4}$ Universitätsklinikum der RWTH, Institut für Neuropathologie, Aachen, Deutschland, ${ }^{5}$ Universitätsklinikum der RWTH, Klinik für Diagnostische und Interventionelle Neuroradiologie, Aachen, Deutschland, ${ }^{6}$ Universitätsklinikum der RWTH, Neurochirurgische Klinik, Aachen, Deutschland, ${ }^{7}$ Universitätsklinikum der RWTH Aachen, Klinik für Kinder- und Jugendmedizin - Sektion Neonatologie und Pädiatrische Intensivmedizin, Aachen, Deutschland

Hintergrund. Kongenitale Hirntumoren sind sehr selten und entfallen auf 0,5-4\% aller Hirntumoren im Kindesalter, wobei Glioblastome gar nur $1 / 6$ der Fälle ausmachen dürften. Davon ausgehend muss mit einer Mortalität von $85 \%$ und einem mittleren Langzeitüberleben von nur wenigen Monaten gerechnet werden. Auf Grund ihrer schlechten Prognose werden sie nach WHO-Klassifikation als Grad IV eingestuft.

Kasuistik. Präpartal erstmaliger sonographischer Verdacht auf Hydrocephalus im Gestationsalter von $37+2$ SSW, so dass im Alter von $37+4$ SSW die primäre Sektio erfolgte. APGAR 7/8/8, NapH 7,40, Geburtsgewicht $3315 \mathrm{~g}$. Initiale Bradykardie, Phasen mit stöhnender Atmung, blasses Hautkolorit, Makrozephalus, opisthotone Körperhaltung, schrilles Schreien.

Diagnostik. In der Schädelsonografie rechtsseitige Erweiterung des Seitenventrikels (SV) sowie Erweiterung des 3. Ventrikels, linksseitig eine Raumforderung (RF) mit unterschiedlichen Echogenitäten des Parenchyms sowie zystischen Arealen. Das kraniale MRT zeigte eine nahezu die gesamte linke Hemisphäre einnehmende RF, die von supratentoriell über infratentoriell bis in den Hirnstamm hineinreichte mit Kompression des angrenzenden Hirnparenchyms mit Mittellinienverlagerung von ca. 1,5 cm nach rechts, schlitzförmiger Kompression des linken SV und starker Erweiterung des linken SV im Sinne eines Liquoraufstaus. Apikal große zystische Anteile in der RF, kaudal hypointense Areale i. S. v. Einblutungen.

Verlauf. In den ersten Lebensstunden traten zunehmend Apnoen auf, so dass die Intubation erfolgte und eine externe Ventrikeldrainage rechts angelegt wurde. Am 2. Lebenstag Ausbildung lichtstarrer, mittelweiter Pupillen. Im cCT zeigte sich nun nach Entlastung des rechten Seitenventrikels eine massive Mittellinienverlagerung zur rechten Seite durch die linksseitige RF sowie eine stammgangliennahe Tumorblutung. Im weiteren Tagesverlauf kam es zu einer zunehmenden Hypotension, einem Abfall des $\mathrm{Hb}$ auf $7 \mathrm{O} \mathrm{mg} / \mathrm{l}$ und einer Laktatazidose ( $\mathrm{pH}$ 7,2, $\mathrm{pCO}_{2}$ $43 \mathrm{mmHg}, \mathrm{BE}-10,2 \mathrm{mmol} / \mathrm{l}$, Laktat 5,1 mmol/l). Auf Grund des ausgeprägten Befundes und der zu erwartenden schlechten Prognose wurde auf weitere operative und therapeutische Maßnahmen verzichtet. Exitus letalis 1 Stunde nach Extubation unter Analgesie. In der neuropathologischen Begutachtung konnte retrospektiv die Diagnose eines kongentialen Glioblastoma multiforme gestellt werden, welche durch die Referenzpathologie bestätigt wurde.

Diskussion. Mit der vorliegenden Kasuistik können wir die bislang in der Literatur beschriebenen weltweit ca. 100 Fälle eines kongenitalen Glioblastoma multiforme um einen weiteren Fall ergänzen. Eine potenzielle Behandlung besteht aus 1. operativer Reduktion, 2. Bestrahlung, und 3. Chemotherapie. Auf Grund des ausgeprägten Befundes unseres Patienten mit rascher neurologischer Verschlechterung sowie sekundärer Tumoreinblutung haben wir jedoch - trotz primärer Unkenntnis der genauen Ätiologie - auf weitere Therapiemaßnahmen verzichtet.
PO-78

Zerebrale arteriovenöse Malformationen bei Früh- und Neugeborenen - eine interdisziplinäre Herausforderung: ein Fallbericht

C. Kannan', M. Lippert ${ }^{2}$, M. von Laer', J. Schneider ', C. Bührer', J. Seemann ${ }^{5}$, H. Fahnenstich ${ }^{7}$

'St. Elisabethenkrankenhaus gGmbH, Zentrum für Kinder- und Jugendmedizin, Lörrach, Deutschland, ${ }^{2}$ St. Elisabethenkrankenhaus gGmbH, Zentrum für Gynäkologie und Geburtshilfe, Lörrach, Deutschland, ${ }^{3}$ Universitäts-Kinderspital beider Basel, Radiologie, Basel, Schweiz, ${ }^{4}$ Charité - Universitätsmedizin Berlin, Klinik für Neonatologie, Berlin, Deutschland, ${ }^{5}$ Werner-ForBmann-Krankenhaus, Kinderklinik, Eberswalde-Finow, Deutschland

Einleitung. Zerebrale arteriovenöse Malformationen bilden eine seltene Entität mit variabler Prävalenz und Inzidenz zwischen 1-10/100.0oo Einwohner/Jahr. Die Inzidenz steigt bis zum Ende der ersten Dekade. Grund hierfür ist die klinisch häufig zunächst blande Präsentation. Symptomatische Neugeborene sind selten. Wir berichten über einen Fall, bei dem pränataldiagnostisch in der 34 . SSW bei fetaler Entwicklungsretardierung, kardialen Belastungszeichen und pathologischem Doppler der A. cerebri media mit V. a. „brain-sparing“ die Geburt eingeleitet wurde.

Fallbericht. Frühgeborenes der $35+3$ SSW, Geburtsgewicht 2390 g, Spontangeburt nach Einleitung, gute kardiorespiratorische Anpassung. Übernahme auf die Intensivstation wegen Hypoglykämie. Postnatal sonographische Diagnose einer cerebralen AV-Malformation mit Darstellung eines pulsierenden Nidus auf Höhe der Sylvischen Furche in der linken Hemisphäre. Eine MRT-Bildgebung am 2. Lebenstag bestätigte die Diagnose mehrerer großer arteriovenöser pialer Fisteln aus der linken A. cerebri media mit Drainage in kortikale Venen der linksseitigen Konvexität, die durch den hohen Fluss zu einer sekundären massiven Erweiterung dieser Gefäße, inkl. Halsgefäße und Sinus geführt haben. Kardial sekundäres "high-output-failure“ bei hohem Shuntvolumen über die linkshemisphärische AV-Malformation, so dass bei klinischen Herzinsuffizienz-Zeichen mit Anasarka, respiratorischer Insuffizienz sowie echokardiographisch pulmonaler Hypertension mit rechtsventrikulärer Dilatation und Rechts-Linksshunt über PDA mit einer diuretischen Therapie begonnen wurde. Nach klinischer Stabilisierung Transfer des Patienten. Interventionelle Selektivembolisation von 4 fistulierenden arteriovenösen High-flow-Shunts der A. cerebri media mittels Coil und Onyx 34 in zweizeitigen neuroangiographischen Eingriffen. Entlassung am 21. Lebenstag unter Enoxaparin-Therapie. Komplizierend trat ein subtotaler Verschluss beider Femoralarterien auf, bei jedoch erhaltener Perfusion der unteren Extremitäten über Kollateralen.

Verlauf. In der Kontrolle deutlich rückläufige Rechtsherzinsuffizienz mit passagerer LV-Funktionsstörung. Partielle Wiedereröffnung der Femoralarterien, so dass die Enoxaparin Therapie nach 8 Wochen beendet wurde. Diskrete neuropathologische Symptomatik mit einer rechtsseitigen Handschwäche.

Schlussfolgerung. Nur die interdisziplinäre und überregionale Zusammenarbeit ermöglicht die erfolgreiche Versorgung großer AV-Malformationen bei Frühgeborenen. Auf Grund der Rarität dieser angeborenen Fehlbildungen erscheint eine bundesweite Zentrumsbildung notwendig. 


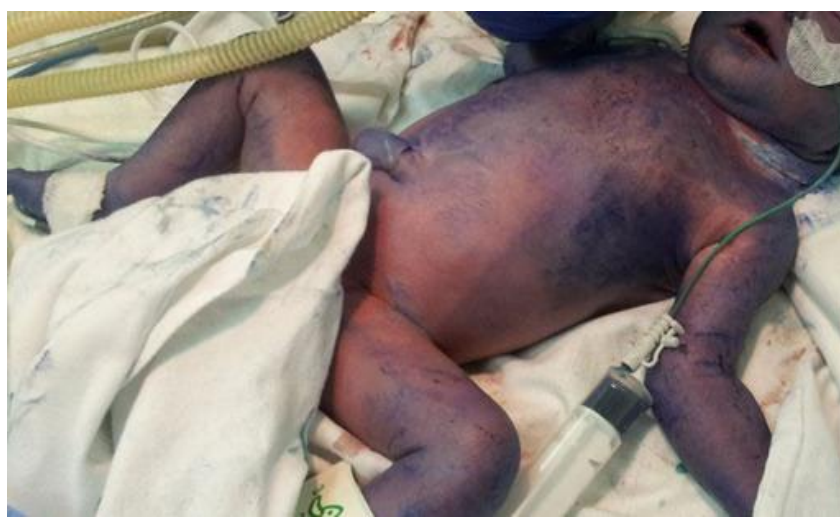

PO-79 Abb. 1 A Frühgeborenes 30 min nach Geburt

\section{Der interessante Fall}

\section{PO-79}

Hämolytische Anämie und Methämoglobinämie bei einem Frühgeborenen nach intraamnialer Toluidinblau-Applikation

\section{K. Mehler', A. Oberthür', G. Weißhaar', A. Vierzig', M. Valter' ', F. Eifinger ${ }^{1}$} 'Univ.-Kinderklinik, Neonatologie und pädiatrische Intensivmedizin, Köln, Deutschland, ${ }^{2}$ Unifrauenklinik Köln, Geburtshilfe, Köln, Deutschland

Methylenblau wurde in den 1960 und 7oer Jahren zur Diagnose eines vorzeitigen Blasensprungs und zur Markierung von Fruchthöhlen bei Zwillingen häufig angewendet. Berichte über schwere Nebenwirkungen „Methylen-blue babies“ und das gehäufte Auftreten von intestinalen Obstruktionen bei Anwendung im zweiten Trimenon führten zu der Empfehlung, den Farbstoff für diese Indikation nicht mehr anzuwenden. Zum Ausschluss eines vorzeitigen Blasensprungs bei unklarem Anhydramnion erfolgte in der 35 . SSW die intraamniale Gabe von $90 \mathrm{mg}$ Toluidinblau. 4,5 Stunden später wurde bei V. a. Amnioninfektionssyndrom das eutrophe Frühgeborene per Sectio entbunden. Das blau gefärbte Frühgeborene (- Abb. 1 ) wurde aufgrund eines Atemnotsyndroms intubiert und beatmet. Es entwickelte eine milde Methämoglobinämie und eine hämolytische Anämie mit austauschpflichtiger Hyperbilirubinämie. Die Hämoglobinanalyse, Sequenzierung des HbA,
HbF Lokus, sowie die Aktivität der Glucose-6-Phosphat Dehydrogenase und der NADH-MetHb-Reduktase waren unauffällig (• Tab. 1). Das Frühgeborene wurde am 15. Lebenstag nach Hause entlassen und zeigte in der neuropädiatrischen Untersuchung mit vier Monaten eine unauffällige Entwicklung. Toluidinblau kann ähnlich wie Methylenblau nach intraamnialer Gabe zu schweren Komplikationen führen. In Situationen, in denen auf eine invasive Diagnostik mittels Farbstoffinjektion nicht verzichtet werden kann, sollte Indigokarmin gewählt werden.

\section{PO-80}

Hyperechogener Kolonrahmen: pränatales Symptom einer Zystinurie - ein Fallbericht

\section{H. Buxmann', D. Lorenz', A. Sewell', H. Böhles ${ }^{3}$, R. Schlößer', E. Merz'} ${ }^{1}$ Klinikum der J.W. Goethe Universität, Klinik für Kinder- und Jugendmedizin, Neonatologie, Frankfurt am Main, Deutschland, ${ }^{2}$ Klinikum der J.W. Goethe Universität, Klinik für Kinder- und Jugendmedizin, Stoffwechsellabor, Frankfurt am Main, Deutschland, ${ }^{3}$ Klinikum der J.W. Goethe Universität, Klinik für Kinder- und Jugendmedizin, Allgemeine Pädiatrie, Frankfurt am Main, Deutschland, ${ }^{4}$ Krankenhaus Nordwest, Gynäkologie und Geburtshilfe, Frankfurt am Main, Deutschland

Einleitung. Die Zystinurie ist eine autosomal-rezessiv vererbte Störung der proximal-tubulären Rückresorption der dibasischen Aminosäuren Zystin, Lysin, Arginin und Ornithin. Führendes Symptom der Erkrankung sind Zystinsteine, die bei der Zystinurie Typ 1 (Defekt im SLC3-A1 Gen) meist vor und bei der Nicht-Typ-1-Zystinurie (Defekt im SLC7A9-Gen) jenseits des 10. Lebensjahres auftreten. Die Diagnosestellung erfolgt klassischerweise nach der Entwicklung von Steinen, meist im Kindesalter. Die Diagnosestellung einer Zystinurie in der Neonatalzeit wurde bisher nicht beschrieben. Sie beinhaltet die Chance, eine Bildung von Zystinsteinen durch Alkalisierung des Urins hinauszuzögern.

Fallbericht. Im pränatalen Ultraschall fällt der Fetus in SSW 30+o durch eine echoreiche Darstellung des gesamten Kolonrahmens auf. Ansonsten finden sich keine sonomorphologischen Auffälligkeiten. Die Geburt des Kindes erfolgt per primärer Re-Sectio in Schwangerschaftswoche 39+6. Gute postnatale Adaptation (APGAR 9/10/10), regelrechter klinischer Status mit normalem Somatogramm: Länge $51 \mathrm{~cm}$ (P50), Gewicht 3200 g (P45), Kopfumfang $35 \mathrm{~cm}$ ( $\left.\mathrm{P}_{55}\right)$. Ein pränatal echoreicher Darm ist beschrieben bei konnataler Cytomegalieinfektion, Trisomie 21, gastrointestinaler Obstruktion, rektourinaler Fistel, zystischer Fibrose und Zystinurie. Bei unserem Patienten waren wiederholte Urinuntersuchungen auf eine konnatale CMV-Infektion in der ersten Lebenswoche

\begin{tabular}{|c|c|c|c|c|c|c|c|c|c|c|c|}
\hline Lebenstag & 1 & 2 & 3 & 4 & 5 & 6 & 6 & 7 & 8 & 10 & 16 \\
\hline $\begin{array}{l}\text { Hämoglo- } \\
\text { bin }\end{array}$ & 18,4 & 16,8 & 14,3 & 11,3 & 8,6 & 10,4 & 10,4 & 13 & 14,5 & nd & nd \\
\hline Bilirubin & 2 & 5,7 & 13 & 17,4 & 21 & 11,6 & 11,6 & 12,1 & 8,7 & 5,1 & nd \\
\hline LDH & nd & 648 & 2085 & 3163 & 2865 & 1108 & 1108 & 1180 & 1139 & 684 & nd \\
\hline GOT & nd & 59 & 188 & nd & 194 & 60 & 60 & 42 & nd & 21 & 38 \\
\hline GPT & nd & 9 & nd & 51 & 14 & 29 & 29 & 23 & nd & 16 & 31 \\
\hline Kreatinin & nd & 0,94 & 0,9 & 0,8 & 0,7 & 0,7 & 0,7 & 0,6 & 0,7 & 0,7 & 0,6 \\
\hline Harnstoff & nd & 57 & 84 & 88 & 86 & 83 & 83 & 56 & 38 & 44 & 31 \\
\hline MetHb & 3 & 0,4 & $10,7^{*}$ & nd & 0,3 & 0,9 & 0,9 & 0,7 & 0,7 & 0,8 & nd \\
\hline
\end{tabular}


negativ. Es fanden sich keine klinischen Stigmata einer Trisomie 21. Der enterale Nahrungsaufbau mit Muttermilch und die Gewichtsentwicklung des Neonaten waren regelrecht. Die Sonographie des Abdomens inklusive Nieren und harnableitenden Wegen waren unauffällig. Immunreaktives Trypsin sowie eine Pilokarpin-Iontophorese erbrachten Normalbefunde. Die Analyse der Aminosäuren im Urin zeigten wiederholt eine erhöhte Ausscheidung von Zystin, Lysin, Arginin und Ornithin. In der Mikroskopie des Urins waren Zystinsteine nachweisbar. Nachfolgend wurde eine orale Therapie mit Calcium-Natrium-Hydrogencitrat $1,5 \mathrm{mmol} / \mathrm{kg} \mathrm{KG/d}$ initiiert. Der mittlerweile 7,5 Monate alte Säugling wird mit Breikost und Muttermilch ernährt. Er gedeiht gut. Bisher haben sich keine Zystinsteine gebildet, die Sonographie der Nieren war im Verlauf wiederholt unauffällig.

Schlussfolgerung. Der Nachweis eines hyperechogenen Kolons im pränatalen Ultraschall kann hinweisend für eine Zystinurie des Feten sein Eine Diagnosestellung in der Neonatalzeit ist durch Bestimmung der Aminosäuren im Urin gut möglich. Sie bietet die Chance, mittels frühzeitiger Alkalisierung des Urins die Bildung von Zystinsteinen zeitlich zu verzögern oder vielleicht auch zu verhindern.

\section{PO-81}

\section{Männliches Frühgeborenes mit partieller Trisomie 10}

\section{K. Deklerk', C. Wild', C. Celanowski', P. Hetzel'}

'Zentrum für Kinder- und Jugendmedizin, St. Elisabethenkrankenhaus, Neonatologie, Lörrach, Deutschland, ${ }^{2}$ Zentrum für Kinder- und Jugendmedizin, St. Elisabethenkrankenhaus, Kinderchirurgie, Lörrach, Deutschland

Hintergrund. Die Trisomie 1op ist eine sehr seltene chromosomale Störung, bei der der komplette kurze Arm des Chromosoms 10 dreifach vorliegt. Daraus entsteht ein komplexes Fehlbildungssyndrom, das verschiedene morphologische Auffälligkeiten und eine meist schwere globale Entwicklungsretardierung beinhaltet und sich in unterschiedlichen Fehlanlagen von Organen äußern kann. Häufiger kommen kleinere Duplikationen des Chromosoms 10 vor. Zahlreiche assoziierte Fehlbildungen sind zwar beschrieben, Aussagen zur Entwicklung sind bei der Seltenheit aber extrem schwierig.

Fallbericht. Sekundäre Sectio eines männlichen Frühgeborenen in der 34+4/7 SSW, Geburtsgewicht 1450 g, nach Einleitung mit seit der 31. SSW bekannter fetaler Retardierung und dringendem Verdacht auf multiple Fehlbildungen; per FISH-Diagnostik Ausschluss einer Trisomie 18 nach eiliger Amniozentese. Postnatal Bestätigung der großen beidseitigen Lippen-Kiefer-Gaumenspalte, der beidseitigen Klumpfüße und der ausgeprägten Dystrophie. Zusätzlich Flexionsstellung der Hände, penile Hypospadie, Hautanhängsel am Hals, beidseitige Nierenzysten, persistierende bilaterale obere Hohlvene ohne weitere kardiale Fehlbildungen, Syrinx des Conus medullaris. Genetisch fand sich eine partielle Trisomie des Chromosoms 10, mit kompletter Duplikation des kurzen Arms sowie eines kurzen Anteils des langen Arms bis zur Bande q11.2 [Karyotyp 47, XY, +del (10) (q11.2)]. Kardiorespiratorisch erfreulich unkomplizierter Verlauf. Seit Geburt allerdings problematischer Nahrungsaufbau, am 8. LT Ileocoecalresektion und Darmdesimpaction aufgrund eines Mekoniumileus bei Atresie des terminalen Ileums. Im Anschluss unkomplizierter Nahrungsaufbau ausschließlich per Magensonde, jedoch zunehmende klinische und laborchemische Zeichen einer schweren exokrinen Pankreasinsuffizienz, begleitend beginnende Cholestasezeichen. Im MRT Nachweis einer Choledochuszyste Typ III sowie einer Pankreaszyste ohne Interventionsbedarf bei rückläufiger Cholestase. Beginn einer Substitution von Pankreasenzymen, fettlöslichen Vitaminen und Gallensäuren. Bei Entlassung weiterhin Sondierung der Mahlzeiten bei ausgeprägten Trinkschwierigkeiten.

Diskussion. Aufgrund der extremen Rarität der partiellen Trisomie 10 ist eine Interpretation bezüglich Entwicklungspotenzial, Lebenserwartung und assoziierter Erkrankungen nicht zuverlässig möglich. Bis zum Alter von inzwischen 6 Monaten ergeben sich weitere Zusatzbefunde, die bisher teilweise schwierig einzuordnen sind. So zeigen sich aktuell weiterhin eine deutliche Gedeihstörung sowie unter anderem eine persistierende Erhöhung der D-Dimere und eine Hyperthyreotropinämie.

\section{PO-82}

\section{Kongenitale Zwerchfellhernie und Cri-du-chat-Syndrom}

P. Barikbin', P. Degenhardt'², L. Graul-Neumann ${ }^{3}$, C. Bührer', C. Czernik ${ }^{5}$

${ }^{1}$ Charité Universitätsmedizin Berlin, Klinik für Neonatologie, Berlin,

Deutschland, ${ }^{2}$ Universitätsmedizin Berlin Charité, Klinik und Poliklinik für Kinderchirurgie, Berlin, Deutschland, ${ }^{3}$ Ambulantes Gesundheitszentrum der Charité Campus Virchow (Humangenetik), Berlin, Deutschland, ${ }^{4}$ Charité Universitätsmedizin Berlin, Klinik für Neonatologie, Berlin, Deutschland, ${ }^{5}$ Charité Universitätsmedizin Berlin, Klinik für Neonatologie, Berlin, Deutschland

Hintergrund. Die kongenitale Zwerchfellhernie ist eine seltene, aber schwerwiegende Fehlbildung, die auch bei optimaler Therapie eine hohe Letalität aufweist. Während die meisten Fälle isoliert auftreten, sind Assoziationen mit verschiedenen syndromalen Erkrankungen beschrieben, insbesondere mit dem Wolf-Hirschhorn-, Fryns- und Brachman-De Lange-Syndrom. Da die Zwerchfellhernie im Rahmen von Syndromen eine schlechtere Prognose zu haben scheint, ist eine umfassende Untersuchung der Patienten auf begleitende Fehlbildungen und wegweisende Dysmorphien unerlässlich.

Fallbericht. Aufnahme eines hypotrophen Neugeborenen (36+6 SSW, Geburtsgewicht $2340 \mathrm{~g}$ ) mit pränatal bekannter linksseitiger Zwerchfellhernie. Nach Spontangeburt primäre endotracheale Intubation und zunächst unkomplizierte Beatmung. Am 3. Lebenstag erfolgte die operative Versorgung der echten Hernia diaphragmatica mit Abtragung des Bruchsacks. Der perioperative Verlauf gestaltete sich unproblematisch, und der Patient konnte am 8. Lebenstag extubiert werden. Aufgrund klinischer Auffälligkeiten (schrilles Schreien, Epikanthus, breiter Nasenrücken, Retrognathie, Ohranhängsel, gotischer Gaumen und Mikrozephalie) wurde der Verdacht auf ein Cri-du-chat-Syndrom gestellt, der durch den Nachweis einer Deletion im kurzen Arm von Chromosom 5 (46 XY, del(5)(:p14.1-qter) bestätigt werden konnte. Am 10. Lebenstag kam es zu einer akuten Verschlechterung mit respiratorischer Insuffizienz und echokardiographischen Zeichen einer schweren pulmonalen Hypertension. Daher erfolgte die Reintubation und erneute maschinelle Beatmung mit inhalativem NO für 5 Tage und überlappend Sildenafil und Dobutamin. Darunter zeigte sich eine langsame Stabilisierung mit erfolgreicher Extubation am 19. Lebenstag. Im Anschluss Atemunterstützung mittels CPAP/Highflowbrille bis zur Verlegung. Echokardiographisch zeigten sich ein persistierender Ductus arteriosus sowie eine persistierende pulmonale Hypertension, die im Verlauf auf knapp subsystemische Werte rückläufig war. Nach einem stationären Aufenthalt von 53 Tagen konnte der Patient mit HighflowBrille (4 1/min, $\mathrm{FiO}_{2}$ o.40) und Sildenafil p.o. in ein heimatnahes Krankenhaus verlegt werden.

Diskussion. Wir berichten den Fall einer Zwerchfellhernie in Verbindung mit einem Cri-du-chat-Syndrom. Eine derartige Assoziation ist bisher nicht beschrieben worden. Bei Kindern mit kongenitaler Zwerchfellhernie und unklaren Dysmorphien kann eine Chromosomenanalyse differenzialdiagnostisch wegweisend sein. 


\section{PO-83}

\section{Pontocerebelläre Hypoplasie mit spinaler Muskelatrophie bei} einem Frühgeborenen

\section{A. Longardt', B. Weschke ${ }^{2}$, B. Spors ${ }^{3}$, D. Horn ${ }^{4}$, W. Stenzel', C. Dame C. Bührer', C. Czernik ${ }^{8}$}

${ }^{1}$ Charite Kliniken f. Kinderheilkunde und Kinderchirurgie, Neonatologie, Berlin, Deutschland, ${ }^{2}$ Charite Kliniken f. Kinderheilkunde und Kinderchirurgie, Berlin, Deutschland, ${ }^{3}$ Charité Universitätsmedizin Berlin, Abteilung für pädiatrische Radiologie, Berlin, Deutschland, ${ }^{4}$ Charité - Universitätsmedizin Berlin, Humangenetik, Berlin, Deutschland, ${ }^{5}$ Charité - Universitätsmedizin Berlin, Institut für Neuropathologie, Berlin, Deutschland, ${ }^{6}$ Charité - Universitätsmedizin Berlin, Campus Virchow-Klinikum, Neonatologie, Berlin, Deutschland, ${ }^{7}$ Charité - Universitätsmedizin Berlin, Klinik für Neonatologie, Berlin, Deutschland, ${ }^{8}$ Charite Campus Virchow-Klinikum, Berlin, Klinik für Neonatologie, Berlin, Deutschland

Hintergrund. Bei der pontocerebellären Hypoplasie Typ 1 (PCH1) tritt neben der Hypoplasie von Cerebellum und Pons ein diffuser Muskelschwund auf. Dieser wird durch den Untergang von motorischen Nervenzellen im Vorderhorn verursacht.

Fallbericht. Wir berichten von einem männlichen Frühgeborenen $(26+6$ Schwangerschaftswochen, $1006 \mathrm{~g}$ Geburtsgewicht), das bei der Geburt mit muskulärer Hypertonie, Beugekontrakturen und Reduktionsfehlbildungen an der rechten Hand und dem rechten Fuß auffiel. Das neurologische klinische Bild war dominiert durch fehlende Reflexe und generalisierte Krampfanfälle. Aufgrund eines unzureichenden Atemantriebs und fehlenden Schutzreflexen musste das Kind von Geburt an beatmet werden. Im Verlauf kam es zu einer deutlichen Zunahme der Krampfanfälle. Der Reflexstatus besserte sich nicht. In der Magnetresonanztomographie zeigte sich eine pontocerebelläre Hypoplasie, in der Muskelbiopsie das typische histologische Bild einer spinalen Muskelatrophie. Daraufhin wurde die Diagnose pontocerebelläre Hypoplasie Typ 1 (PCH1) gestellt und das Therapieziel auf eine Palliativbetreuung hin geändert. Am 33. Lebenstag verstarb das Kind nach Extubation. Schlussfolgerung. Die $\mathrm{PCH}_{1}$ ist eine seltene genetische Erkrankung (OMIM \# 607596). Sie ist definiert durch die Kombination von spinaler Muskelatrophie und pontocerebellärer Hypoplasie. Die Erkrankung ist charakterisiert durch muskuläre Schwäche, ausgeprägte Störung der Motorik sowie Spastiken und eine globale Entwicklungsverzögerung. Das klinische Bild der $\mathrm{PCH} 1$ steht im Gegensatz zur klassischen proximalen spinalen Muskelatrophie (SMA), bei der die muskuläre Hypotonie im Vordergrund steht. Die $\mathrm{PCH} 1$ hat eine infauste Prognose und endet meist früh letal. Die Vererbung ist in der Regel autosomal-rezessiv und konnte in mehreren Fällen auf eine Mutation des Gens EXOSC 3 zurückgeführt werden. EXOSC $_{3}$ kodiert die Exosomen-Komponente 3 (EXOSC 3 ), das an der Reifung („processing“) der ribosomalen RNA beteiligte Protein 40 (Rrp4o). EXOSC 3 ist als zentraler Bestandteil des humanen RNA-Exosomen-Komplex wichtig für den RNA-Metabolismus und evolutionär hoch konserviert. Der Ausfall von $\mathrm{EXOSC}_{3}$ scheint sich in Neuronen von Gehirn und Rückenmark besonders gravierend auszuwirken.

\section{PO-84}

Erhöhtes Risiko für eine intrakranielle Hämorrhagie durch kongenitale Myopathien?

\section{Kuntz', O. Bartsch', C. Hofmann ${ }^{3}$, A. Puhl' ${ }^{4}$, E. Mildenberger ${ }^{5}$}

${ }^{1}$ Universitätsmedizin der Johannes Gutenberg-Universität Mainz, Zentrum für Kinder- und Jugendmedizin, Mainz, Deutschland, ${ }^{2}$ Institut für Humangenetik, Universitätsmedizin Mainz, Mainz, Deutschland, ${ }^{3}$ Klinikum der Johannes-Gutenberg Universität, Zentrum für Kinder- und Jugendmedizin, Mainz, Deutschland, ${ }^{4}$ Klinikum der Johannes-Gutenberg Universität, Klinik und Poliklinik für Geburtshilfe und Frauenkrankheiten, Mainz, Deutschland, ${ }^{5}$ Universitätsmedizin Mainz - Zentrum für Kinder- und Jugendmedizin, Neonatologie, Mainz, Deutschland

Hintergrund. Wir berichten über ein weibliches Neugeborenes mit einem angeborenen Makrozephalus, intrakraniellen Hämorrhagien und Muskelschwäche, bei dem im Verlauf überraschend eine kongenitale myotone Dystrophie (Typ 1, CDM1) nachgewiesen wurde. Kongenitale Myopathien können zum Bild eines „floppy infant“ führen. Eine intrakranielle Hämorrhagie ist eine sehr seltene, noch wenig bekannte Manifestation.

Fallbericht. Während der Schwangerschaft kam es einmalig zu einem geringfügigen Trauma (Stoß an eine Türklinke mit konsekutivem Bauchhauthämatom). Wegen eines ausgeprägten Polyhydramnions wurde zweimalig (SSW 32+o, 35+1) eine Amniondrainage vorgenommen. Der Verlauf des Kopfumfangswachstums war stetig und innerhalb der Norm. Geburt per Sectio bei 37 + o SSW bei BEL. Klinisch bestanden Makrozephalus, respiratorische Insuffizienz (Beatmung) und Trinkschwäche. Das MRT zeigte intrakranielle Hämorrhagien verschiedener Lokalisation und Alters: Hygrome parietal, Subduralblutung occipital, ältere rundliche Blutungsherde intraparenchymal und subdural. Gerinnungsstörungen, Thrombozytopenie, Gefäßmalformationen und Stoffwechselstörungen wurden ausgeschlossen. Aufgrund einer gezielten Exploration der Mutter wurde bei dem Kind eine CDM1 molekulargenetisch gesichert (180o CTG-Repeats im DMPK-Gen). Bei der Mutter lag eine bisher nicht diagnostizierte klassische DM1 (5201000 CTG-Repeats) vor.

Diskussion. Die Genese der intrakraniellen Hämorrhagien ist unklar. Außer dem geschilderten sind keine weiteren Traumata in der Schwangerschaft bekannt, sodass zumindest nicht die Mehrzeitigkeit der Hämorrhagien erklärt werden könnte. Eine Blutungsneigung oder Gefäßmalformationen lagen nicht vor. Amniondrainagen ändern abrupt den intrauterinen Druck. Dadurch können Scherkräfte hervorgerufen werden, die eine vorzeitige Plazentalösung begünstigen. Dieser Mechanismus scheint nicht ursächlich für die intrakraniellen Hämorrhagien unserer Patientin gewesen zu sein, denn pränatal war sonographisch keine sprunghafte Zunahme des Kopfumfanges zu verzeichnen. In der Literatur gibt es lediglich einen Fallbericht eines Neugeborenen mit CDM1 mit subgalealen Hämatomen, Subarachnoidal- und intraventrikulärer Blutung. Bei einer anderen kongenitalen Myopathie, der myotubulären Myopathie, sind bei mehreren Neugeborenen Blutungen verschiedener Lokalisation beschrieben: Hygrome, subdurale Blutung, subkapsuläres Leberhämatom. Die myotubuläre Myopathie kann einen Dolichozephalus begünstigen. Man vermutet, dass die Erweiterung des Subduralraumes die Brückenvenen dehnt und subdurale Blutungen verursacht. Blutungen außerhalb des Subduralraumes können so nicht erklärt werden; weitere, bisher unbekannte Faktoren scheinen ursächlich zu sein.

Schlussfolgerung. Kongenitale Myopathien können mit (intrakraniellen) Hämorrhagien assoziiert sein. Eine Klärung der Ursachen könnte für deren Vermeidung hilfreich sein. 


\section{PO-85}

\section{Spontane Regression einer kongenitalen pulmonalen Hämangio-} matose

\section{Maschke', C. Berg' ${ }^{2}$, A. Müller ${ }^{3}$, S. Schmitt-Grohe', A. Geipel', A. Müller ${ }^{1}$}

'Universitätsklinik Zentrum $\mathrm{f}$. Kinderheilkunde, Neonatologie, Bonn, Deutschland, ${ }^{2}$ Universitätsklinikum Bonn, Geburtshilfe und Pränatale Medizin, Bonn, Deutschland, ${ }^{3}$ Universitätsklinikum Bonn, Institut für Pathologie; Abteilung Kinderpathologie, Bonn, Deutschland, ${ }^{4}$ Universität Bonn, Abteilung für Geburtshilfe und Pränatale Medizin, Bonn, Deutschland

Hintergrund. Hämangiome sind durch exzessive Angiogenese gekennzeichnete benigne Gefäßtumoren, die vor allem in Haut, Bindegewebe und Leber auftreten. Obwohl die Tumoren in jedem Organ lokalisiert sein können, sind sie in der Lunge selten zu finden. In der Literatur sind nur vereinzelte Fälle von pulmonalen Hämangiomen $\mathrm{zu}$ finden, die Verläufe reichen von asymptomatischen radiologischen Zufallsbefunden bis zu fulminant tödlichen Verläufen.

Fallbericht. Wir möchten den Fall eines Frühgeborenen von $34+5$ SSW vorstellen, bei dem pränatal ab der 29. SSW ein generalisierter Hydrops fetalis mit beidseitigen Hydrothoraces bestand. Intrauterin erfolgten fünf Pigtaileinlagen zur Drainage der Pleuraergüsse. Postnatal stabilisierte sich die Kreislauffunktion unter antikongestiver Therapie mit Dobutamin und Noradrenalin zügig. Kompliziert wurde der postoperative Verlauf durch anhaltende Beatmungspflichtigkeit bei persistierenden Pleuraergüssen welche mehrfach drainiert werden mussten, eine zunehmende Septierung der Ergüsse und Verschwartung der Pleura. Im Alter von vier Wochen zeigte ein MRT beidseits zystische Malformationen der Lunge mit interstitiellen Flüssigkeitseinlagerungen sowie einen im rechten Pleuraspalt verbliebenen intrauterin gelegten PigtailKatheter. Daraufhin wurde der Katheterrest operativ entfernt und eine diagnostische Lungenbiopsie durchgeführt, welche mehrherdige kavernöse intrapulmonale Hämangiome (CD 34 und $\mathrm{CD} 31$ positiv) zeigte. Im Alter von 8 Wochen konnte das Kind extubiert werden und war nach anschließend 2 Wochen CPAP-Atemhilfe ohne Sauerstoffbedarf spontanatmend. Eine Behandlung der pulmonalen Hämangiomatose mit alpha-2a-Interferon, die zunächst geplant war, wurde bei spontaner Besserung der klinischen Befunde nicht durchgeführt. Im Alter von 3 Monaten konnte das Kind in stabilem Allgemeinzustand mit noch bestehender leichter Tachydyspnoe nach Hause entlassen werden. Eine Computertomographie im Alter von 6 Monaten zeigte eine Befundregredienz mit noch residuellen kleinen Zysten im linken Unterlappen und rechten Mittel- und Unterlappen. Im Alter von 2 Jahren hatte das Kind sich körperlich und psychomotorisch regelrecht entwickelt, es gab keine pulmonalen Infekte seit Entlassung, der Auskultationsbefund war unauffällig und radiologisch zeigte sich lediglich eine geringe Zeichnungsvermehrung beidseits parahilär ohne neu aufgetretene Veränderungen. Diskussion. Pulmonale Hämangiome stellen eine seltene Differenzialdiagnose angeborener pulmonaler Fehlbildungen dar und führten in diesem Fall sogar zur Ausbildung eines generalisierten Hydrops fetalis. Ohne spezifische Therapie kam es zur Rückbildung der Tumoren in den ersten beiden Lebensjahren.

\section{Versorgungsqualität - „Outcome“}

\section{PO-86}

Der Apgar-Score: Wissensstand der Eltern und dessen Zusammenhang mit sozialen Variablen

\section{Gnigler', C. Staude', U. Kiechl-Kohlendorfer', S. Navarro-Psihas ${ }^{1}$ 'Universitätskinderklinik Innsbruck, Neonatologie, Innsbruck, Österreich}

Hintergrund. Der Apgar-Score dient der postnatalen Beurteilung des klinischen Zustandes des Neugeborenen. Er korreliert mit der Mortalität und Morbidität und wird zur Diagnosestellung der perinatalen Asphyxie verwendet. Da der Apgar-Score im Mutter-Kind-Pass einge- tragen wird und den Eltern frei zugänglich ist, könnte eine mangelnde Aufklärung zu Fehlinterpretationen und falschen Schlussfolgerungen führen.

Fragestellung. Ziel unserer Studie war es herauszufinden, wie der Wissensstand der Eltern über den Apgar-Score ist und ob ein Zusammenhang zwischen diesem und sozialen Variablen vorliegt.

Material und Methoden. In einer prospektiven, Observationsstudie wurden 100 Frauen über die Bedeutung und Wertigkeit des Apgar-Scores befragt, die stationär auf der Mutter-Kind-Station der Universitätsklinik Innsbruck aufgenommen waren und ein gesundes Kind geboren hatten. Die Daten wurden anhand eines standardisierten Fragebogens im Rahmen eines Einzelgesprächs am Tag vor der Entlassung erhoben und mittels $\chi^{2}$ - bzw. T-Test analysiert.

Ergebnisse. Von den 100 befragten Frauen wussten 52 etwas über den Apgar-Score und 10 kannten den aktuellen Apgar-Score ihres Kindes. Es zeigte sich eine positive Korrelation zwischen Ausbildung, Anzahl der Schwangerschaften und Herkunft aus der E.U. mit der Wissen über den Apgar-Score. 95\% der befragten Frauen waren zufrieden mit der Aufklärung über den kindlichen Zustand, 39\% hätten sich mehr Information gewünscht.

Diskussion. Obwohl der Apgar-Score allen Eltern zugänglich ist, ist der Informationsstand der Mütter mangelhaft. Um Missinterpretationen und falsche Schlussfolgerungen zu vermeiden, wäre eine bessere Aufklärung der Eltern über den Apgar-Score von Vorteil.

\section{PO-87}

Qualität von Thoraxaufnahmen auf pädiatrischen Intensivstationen an einer Universitätsklinik

\section{G. Alzen', M. Klinzmann', V. Klingmüller'², M. Läßig', C. Behrens', N. Ham-} scho'

${ }^{1}$ Medizinische Fakultät JLU Gießen, Kinderradiologie, Gießen, Deutschland, 2Phillips Universität Marburg, Zentrum für Radiologie, Gießen, Deutschland, ${ }^{3}$ Asklepiosklinik Lich, Radiologie, Lich, Deutschland

Hintergrund. Röntgenaufnahmen auf pädiatrischen Intensivstationen werden im Tagdienst von MTRAs der Kinderradiologie, nachts von MTRAs der Allgemeinradiologie und von den Ärzten der Intensivstationen angefertigt. Wegen der klinischen Bedeutung solcher Aufnahmen und der zugrunde liegenden gesetzlichen Vorgaben seitens der Röntgenverordnung wurde die Qualität der erstellten Aufnahmen untersucht.

Material und Methode. Aus einem Zeitraum von 6 Jahren wurden 396 repräsentative digitale Thoraxaufnahmen von insgesamt 20 in die Auswertung einbezogenen Mitarbeitern zufällig ausgewählt, sodass von jedem Mitarbeiter und jeder Berufsgruppe annähernd die gleiche Anzahl Aufnahmen ausgewertet werden konnte. Expositionswerte, Einblendung, Verdrehung, Zentrierung, beseitigbare Fremdkörper und die Kollimation wurden vollständig geblindet und nach objektiv messbaren Kriterien ausgewertet. Anschließend wurden die Aufnahmen unabhängig voneinander von 3 Kinderradiologen nach zuvor festgelegten Kriterien bewertet.

Ergebnisse. Die Qualität der Aufnahmen lag deutlich unter dem Qualitätslevel, der von den Mitarbeitern der Kinderradiologie bei Routine Thoraxaufnahmen in der Abteilung Kinderradiologie erzielt wurde (Beiderwellen K. 2010, Ruppert S. 2010). Qualitätsunterschiede zwischen den 3 beteiligten Berufsgruppen konnten nicht festgestellt werden. Lediglich die Anzahl erkennbarer Einblendungsränder nach Kollimation unterschied sich: Bei kinderradiologischen MTRAs waren signifikant mehr Bildränder erkennbar als bei denen der beiden anderen Berufsgruppen.

Schlussfolgerung. Durch die nachträgliche Kollimation digitaler Röntgenaufnahmen gelingt es, zu weit aufgeblendete Aufnahmen bei intensivpflichtigen Kindern und Säuglingen so nachzubearbeiten, dass dosisrelevante Kriterien nicht mehr nachvollzogen werden können. 


\section{PO-88}

\section{Die Veränderung des Outcome von VLBW infants im 5-Jahresver-} gleich des Perinatalzentrums Jena

\section{F. Eichhorn', K. Dawczynski', J. Beck ${ }^{3}$}

${ }^{1}$ Neonatologie, Klinik für Kinder- und Jugendmedizin, Universitätsklinikum Jena, Jena, Deutschland, ${ }^{2}$ Friedrich Schiller Universität Klinik f. Kinder- und Jugendmedizin, Jena, Deutschland, ${ }^{3}$ Klinik für Kinder- und Jugendmedizin, Universitätsklinikum Jena, Jena, Deutschland

Fragestellung. In den letzten Jahren hat es Veränderungen im Behandlungsmanagement von sehr kleinen Frühgeborenen, unter anderem in der Erstversorgung, der Ernährung sowie im Sauerstoffmanagement gegeben. Es stellt sich die Frage, in wie weit sich diese Veränderungen auf die Überlebensrate oder die Morbidität (IVH, ROP und NEC) von Frühgeborenen unter $1500 \mathrm{~g}$ in den letzten 5 Jahren am Perinatalzentrum Jena ausgewirkt haben?

Material und Methoden. Ausgewertet wurden die Fallzahlen der Geburtsjahrgänge 2007 bis 2012 aus dem Perinatalzentrum Jena. Darin eingeschlossen sind alle Frühgeborenen unter 1500 g, die in den ersten 28 Lebenstagen in der Universitätskinderklinik Jena stationär behandelt wurden. Dargestellt wird die Entwicklung der Überlebensrate in Prozent, differenziert nach dem Geburtsgewicht und dem Gestationsalter. Zusätzlich erfolgt eine Gegenüberstellung der Häufigkeiten von NEC, ROP und IVH in der jeweiligen Gewichtsklasse für die entsprechenden Jahrgänge.

Ergebnisse. In den letzten 5 Jahren wurde eine Verbesserung der Überlebensrate der Frühgeborenen unter $1500 \mathrm{~g}$ verzeichnet. Obwohl zunehmende Zahlen von extrem unreifen FG unter 1000 g vorlagen. Besonders bei extrem kleinen Frühgeborenen mit einem Geburtsgewicht unter $500 \mathrm{~g}$ konnte die Überlebensrate auf $67 \%$ gesteigert werden. Damit hat sich die Überlebenschance verdoppelt. 2012 konnte trotz erhöhtem Risikoprofils des Patientenkollektivs (hoher Anteil an ELBW und VLBW) das Auftreten einer behandlungsbedürftigen ROP sowie NECRate deutlich reduziert werden. Die Prozentzahlen der IVH III-IV ${ }^{\circ}$ blieben dagegen nahezu unverändert.

Schlussfolgerung. Das Outcome sehr kleiner Frühgeborener mit einem Geburtsgewicht unter $1500 \mathrm{~g}$ zeigte besonders in den letzten 5 Jahren einen deutlich positiven Trend. Bei steigendem Risikoprofil im Rahmen der deutlich verbesserten Überlebensrate konnte gleichzeitig die Morbiditätsrate gesenkt werden. Hier sind als protektive Einflussfaktoren die kontrollierte Sauerstoffapplikation zusammen mit der signifikant geringeren Notwendigkeit einer maschinellen Beatmung durch Einführung der nichtinvasiven Surfactantapplikation sowie der unmittelbare postnatale enterale Nahrungsaufbau mit Frauen- bzw. Muttermilch zu nennen. Prospektiv werden im Hinblick auf das mittelfristige Outcome sowie das Langzeitoutcome die entwicklungsneurologischen Nachuntersuchungen im korrigierten Alter von 2 Jahren der Geburtsjahrgänge 2007 bis 2012 ausgewertet.

\section{PO-89}

Inzidenz der Retinopathia praematurorum (ROP) über einen Zeitraum von 10 Jahren in der Klinik für Neonatologie am Zentrum für Kinder- und Jugendmedizin des Universitätsklinikums Heidelberg

\section{S. Ronellenfitsch' ${ }^{1}$ F. Schlichtenbrede', J. Poeschl'}

'Universitätsklinikum Heidelberg, Klinik für Kinder- und Jugendmedizin, Heidelberg, Deutschland, ${ }^{2}$ Klinikum Mannheim, Augenklinik, Mannheim, Deutschland

Zielsetzung. Die Zielsetzung dieser retrospektiven Studie ist die Erfassung der Inzidenz der ROP über einen Zeitraum von 10 Jahren an der Klinik für Neonatologie am Zentrum für Kinder- und Jungendmedizin in Heidelberg.

Methoden. Die Patientenakten aller Frühgeborenen mit einem Gestationsalter von weniger als 33 Schwangerschaftswochen (SSW) wurden untersucht, um die Inzidenz einer ROP über einen Zeitraum von 10
Jahren zu bestimmen. Der postpartale Sauerstoffzielbereich der Frühgeborenen lag bis zur ersten augenärztlichen Untersuchung im Alter von 42 Tagen bei $88-94 \%$. Bei Stellung der Diagnose ROP wurde eine stadienabhängige prophylaktische Sauerstofftherapie begonnen. Die Sauerstoffsättigungszielbereiche lagen bei: Stadium I: 92-94\%, Stadium II: $94-96 \%$ und Stadium III: 96-98\%. Lasertherapie wurde bei Stadium III+ durchgeführt.

Ergebnisse. Insgesamt wurden 927 Frühgeborene im Zeitraum von 1999 bis 2008 erfasst. Eine ROP wurde bei 428 Patienten (43,2\%) diagnostiziert. Das ROP Stadium I wurde mit 129 Betroffenen (13,9\% der Studienpopulation) am zweithäufigsten diagnostiziert. Am häufigsten, mit 177 Patienten (entsprechend 19,1\%) lag das Stadium II vor. Gefolgt wird diese Gruppe von 105 Patienten mit Stadium III (11,3\%). Stadium III+ und dementsprechend die Indikation für eine Laserbehandlung wurde bei 14 Patienten (1,5\%) diagnostiziert. Alle 14 Patienten wurden mit einem Gestationsalter von weniger als 28+o SSW entbunden. Bei einer Gesamtzahl von 453 Frühgeborenen kleiner 28+o SSW entspricht dies $3,1 \%$. Bei allen 14 Patienten wurde neben der schweren ROP auch eine bronchopulmonale Dysplasie im Alter von 36 SSW festgestellt. Drei dieser 14 Patienten wurden mit einem Geburtsgewicht unter der zehnten Perzentile geboren, nur bei einem wurde eine nekrotisierende Enterokolitis diagnostiziert.

Schlussfolgerung. Unsere Studie zeigt, dass eine niedrige Schwangerschaftswoche bei Geburt mit einem erhöhten Risiko für die Entstehung einer schweren ROP und der Notwendigkeit einer Lasertherapie korreliert. Wir spekulieren, dass die mit anderen Zentren vergleichbar niedrige Laser-Inzidenz von 1,5\% mit der bilanzierten Sauerstofftherapie zusammenhängt.

\section{PO-90}

Fokal intestinale Perforation vs. nekrotisierende Enterokolitis bei Frühgeborenen mit einem Geburtsgewicht $<1500$ g: zwei Entitäten oder unterschiedliche Ausprägungen eines Krankheitsbildes?

\section{Linge', E. Mayatepek', T. Höhn'}

${ }^{1}$ Med. Einricht. d. Universität Kinderklinik Düsseldorf, Klinik für Allgemeine Pädiatrie, Neonatologie und Kinderkardiologie, Düsseldorf, Deutschland

Hintergrund. Nekrotisierende Enterokolitis (NEC) und fokal intestinale Perforation (FIP) sind derzeit die häufigsten intestinalen Notfälle bei Frühgeborenen (FG) mit einem Geburtsgewicht <1500 g (VLBW). Im klinischen Alltag ist eine Abgrenzung nur schwer möglich. Bezüglich Prophylaxe, Klinik, Therapie und Outcome bestehen keine klaren Unterschiede, sodass auch vor dem Hintergrund noch weitgehend unklarer Pathomechanismen die derzeit etablierte Meinung, es handele sich um zwei unterschiedliche Entitäten, nicht selten in Frage gestellt wird.

Methoden. Im Rahmen einer monozentrisch retrospektiven Studie wurden über einen 11-Jahres-Zeitraum (2000-2011) insgesamt 74 VLBW-FG mit akuter abdominaler Symptomatik erfasst. Nach Überprüfung der Diagnose unter Berücksichtigung definierter Kriterien, konnten $25 \mathrm{~Pa}$ tienten der NEC- und 17 Patienten der FIP-Gruppe zugeordnet werden. Beide Gruppen wurden hinsichtlich epidemiologischer, klinischer, diagnostischer und therapeutischer Daten miteinander verglichen.

Ergebnisse. Bezüglich der erhobenen Daten konnte kein signifikanter Unterschied der Gruppen festgestellt werden. Unreife scheint eher ein prädisponierende Faktor der FIP, Dystrophie tendenziell ein Risikofaktor der NEC zu sein. Alle untersuchten Laborparameter waren letztlich nicht geeignet zwischen den Gruppen zu differenzieren. Hinsichtlich Klinik und Therapie ergaben sich ebenfalls keine relevanten Unterschiede.

Schlussfolgerung. Möglicherweise stellen NEC und FIP unterschiedliche Ausprägungen des gleichen Krankheitsbildes dar. Je nach Konstellation der prädisponierenden Faktoren kann es aufgrund der multifaktoriellen Genese beider Erkrankungen zu einem mehr oder minder schweren, eher langsam oder rasch progredientem Phänotyp kommen. 


\section{PO-91}

\section{Gesundheitskosten von Kindern in den ersten 5 Lebensjahren - eine populationsbasierte Analyse}

\section{J. Reichert' ${ }^{1}$ M. Schemken ${ }^{2}$, R. Manthei' ${ }^{2}$, M. Rüdiger ${ }^{7}$}

'Universitätsklinikum Carl Gustav Carus Kinderklinik, Neonatologie, Dresden, Deutschland, ${ }^{2}$ AOK PLUS - Die Gesundheitskasse für Sachsen und Thüringen, Dresden, Deutschland

Einleitung. Die Entwicklung der Kindermedizin ist in den vergangenen Jahren durch verschiedene Veränderungen gekennzeichnet, was insbesondere ganzheitliche Behandlungsansätze oder Etablierung von Vorsorgemaßnahmen, aber auch die Verlagerung der Behandlung aus dem stationären in den ambulanten Bereich anbetrifft. Zur Beurteilung hierbei entstehender Gesundheitskosten fehlen Referenzwerte. Ziel dieser Untersuchung war es, ein Bezugssystem für die Gesundheitskosten der Behandlung von Neugeborenen, Säuglingen und Kleinkindern zu erhalten.

Methode. Zur Analyse lagen die individuellen Gesundheitskosten der ersten fünf Lebensjahre des Geburtsjahrganges 2005 der bei der AOK PLUS in Sachsen versicherten Kinder vor. Einbezogen wurden die Kosten für die Krankenhausbehandlung, ambulante ärztliche Versorgung, Heilmittel, Hilfsmittel, Arzneimittel sowie Pflegeleistungen.

Ergebnis. Die Kosten pro versichertem Kind und Jahr lagen bei ca. $1277 €(\mathrm{~N}=11.147)$, wobei in den ersten beiden Lebensjahren die höchsten Kosten anfallen. Für einen „durchschnittlichen“ Versicherten sind jährlich $858 €$ aufzuwenden; für ein Kind mit hohen Gesundheitskosten fallen $5691 €$ im Jahr an.

Diskussion. Für etwa 10\% Kinder mit den höchsten Gesundheitskosten werden über fünf Jahre nach der Geburt ungefähr $45 \%$ der gesamten Kosten dieser Geburtskohorte aufgewendet. Dabei sind jährliche Gesundheitsaufwendungen für durchschnittliche pädiatrische Patienten deutlich niedriger als für erwachsene. Die vorliegende Kostenanalyse bildet ein geeignetes Bezugssystem für die orientierende Beurteilung gesundheitsbezogener Aufwendungen.

\section{PO-92}

\section{NIQ - ein europäisches Netzwerk zum Qualitätsvergleich in der Neo-} natologie

\section{H. Küster}

\section{'Universitäts- Kinderklinik, Neonatologie, Göttingen, Deutschland}

Hintergrund. Softwarelösungen im Krankenhaus sind oft wenig benutzerfreundlich: mehrfache Erfassung derselben Daten, umständliche Eingabeformulare, geringe Überprüfung der Datenqualität, kaum Auswertemöglichkeiten und mangelnde Integration eigener Wünsche kennzeichnen viele der vorhandenen Lösungen. Ein Qualitätsvergleich mit anderen Krankenhäusern ist mit keinem der in Deutschland vertriebenen Systeme möglich.

Fragestellung. Lässt sich eine Software mit folgenden Charakteristika entwickeln: 1.) einfache Erfassung der gesetzlich vorgeschrieben Qualitätssicherung, 2.) Integration zusätzlicher Datenfelder, 3.) Generierung von Arztbriefen aus den eingegeben Daten, 4.) Anpassung an lokale Bedürfnisse, 5.) Vernetzung mit dem Krankenhausinformationssystem, 6.) krankenhausübergreifender Qualitätsvergleich, 6) moderne Sicherheitstechnologie.

Material und Methode. Basierend auf Neolink20oo - dem seit 1994 kontinuierlich weiterentwickelten Datenerfassungssystem - wurde ein vollständig neues Softwarepaket entwickelt: NIQ ${ }^{\circ}$. Dieses wurde so ausgelegt, dass sich Module für verschiedene Fachrichtungen, Patientengruppen, Forschungsprojekte oder Qualitätssicherungssysteme frei kombinieren und auswerten lassen.

Ergebnisse. Eine anwenderfreundliche Oberfläche erleichtert Eingabe und Verwertung der Daten aus der Zeit vor, während und nach der stationären Behandlung. Die klare Eingabestruktur, ausführliche Definitionen jedes Datenfeldes und umfangreiche Fehlerprüfungen helfen, eine hohe Datenqualität zu erreichen. NIQ ${ }^{\circledR}$ ist mehrsprachig und unterstützt den deutschen sowie mehrere internationale Standards der Qualitätssicherung (AQUA, GNN, VON, ENN). Darüber hinaus können zusätzliche Datenfelder ausgewählt werden. Im Krankenhaus bereits vorhandene Daten können via Schnittstellen übernommen werden. Eine erhebliche Arbeitserleichterung entsteht durch automatische Übernahme der Daten in adaptierbare Arztbriefe und Formulare. Alle Daten können jederzeit vor Ort krankenhausübergreifend analysiert werden. Damit erlaubt NIQ ${ }^{\bullet}$ erstmals ein Online-Vergleich mit der in anderen Kliniken erreichten Qualität. Die Ergebnisstabellen bzw. -diagramm sind adaptierbar und exportierbar. Abfragen können gespeichert werden und stehen dann jederzeit für eine schnelle erneute Analyse der Daten zur Verfügung. Eine Zertifizierung dokumentiert den hohen Standard des Datenschutzes und der eingesetzten Technologie. Ausblick. NIQ ${ }^{\circ}$ führt bei hoher Datenqualität zur Arbeitsreduktion durch die einmalige Eingabe mit multipler Ausgabe der Daten sowie Schnittstellen und Adaptierbarkeit. Mit NIQ ${ }^{\star}$ soll ein europaweiter Datenverbund geschaffen und wichtige Daten von der Schwangerschaft bis zur Einschulung anonym gesammelt und analysiert werden.

Mehr Informationen sowie eine kostenlose Testversion unter www. NIQ.eu. Förderung durch EU, BMBF und das Land Mecklenburg-Vorpommern.

\section{Neue Studien - systematische Untersuchungen}

\section{PO-93}

Einfluss eines Gehörschutzes gegen Lärm neonataler Intensivstationen (NICU) auf intermittierende Hypoxien und Bradykardien Frühgeborener. Eine randomisierte, kontrollierte Interventionsstudie

\section{T. Bott ${ }^{1}$, M. Urschitz' ${ }^{1}$ A. Poets ${ }^{1}$, C. Poets}

'Universitätskinderklinik Tübingen, Abt. IV Neonatologie, Tübingen, Deutschland

Hintergrund. Atemstörungen bei Frühgeborenen (FG), die mit Hypoxie und Bradykardie einhergehen, sind ein häufiges Problem mit unvollständig verstandener Pathophysiologie. Unruhe des Kindes, z. T. durch Umgebungsreize ausgelöst, scheint ein wichtiger Einflussfaktor zu sein. Fragestellung. Es soll eine klinisch relevante und statistisch signifikante Reduktion der Hypoxien und Bradykardien um 50\% durch Tragen eines spezifischen Gehörschutzes für FG gemessen werden.

Methoden. In einer randomisierten, kontrollierten, verblindeten Interventionsstudie, wurde bei 31 FG Probanden der Effekt von MiniMuffs (NatusMedical Inc., San Carlos, USA), einem Gehörschutz für Säuglinge, Neugeborene und FG getestet. Einschlusskriterien waren das Vorliegen von intermittierenden Hypoxien und Bradykardien, ein Gestationsalter von $24+0 / 7$ bis $36+6 / 7$ Schwangerschaftswochen und das elterliche informierte Einverständnis. Primärer Zielparameter war die Reduktion der Häufigkeit der $\mathrm{SpO}_{2}$-Entsättigungen auf $<80 \%$ pro Stunde (Desaturationsindex 80), sekundärer Zielparameter die Häufigkeit von Bradykardien auf $<80$ Schläge pro Minute (Bradykardieindex 80). Die Zielparameter wurden mit einem handelsüblichen Säuglingsmonitor VitaGuard 3100 (Getemed, Teltow, Deutschland) aufgezeichnet. Innerhalb eines Cross-over-Designs wurden die Probanden in zwei Gruppen randomisiert und zuerst für 2 Stunden mit MiniMuffs und dann 2 Stunden ohne MiniMuffs gemessen, oder, je nach Gruppenzugehörigkeit, in umgekehrter Reihenfolge. Während der 4-stündigen Messung wurden weitere Hypoxie-beeinflussende Parameter, wie z. B. Gabe von Coffein, Lagerung und Beatmungsmodus möglichst konstant gehalten und dokumentiert. Mit dem Lärmpegelmesser Typ 2236 (Bruel \& Kjaer, Naerum, Dänemark) wurde der äquivalente Dauerschallpegel (Leq) in Dezibel $(\mathrm{dB})$ während der Interventionsphase und der Kontrollphase konstant jede Sekunde aufgezeichnet, um nach Abschluss der Datenerhebung die Lärmbelastung beider Studienphasen zu vergleichen. 
Ergebnisse. Die Datenerhebung für diese Studie ist abgeschlossen, die endgültigen Studienergebnisse werden zum GNPI 2013 vorliegen.

\section{PO-94 \\ Analysis of the cardiorespiratory stability of term and preterm newborns}

\section{R. Gerull', N. Préel', M. Nelle \\ IInselspital Bern, Neonatologie, Bern, Schweiz, ${ }^{2}$ Medizinische Kinderklinik/ Inselspital, Abteilungsleiter Neonatologie, Bern, Schweiz}

Objective. A retrospective chart review to assess frequency and changes of cardiorespiratory events over time in term and preterm infants in order to evaluate the existing criteria of cardio respiratory monitoring. Methods. Retrospective chart review of all newborns who were hospitalized in the unit of neonatology in the University Children's Hospita Inselspital Berne in the year 2008 and all newborns with a birth weight of less than $1500 \mathrm{~g}$ or a gestational age of less than 32 weeks in the year 2007. According to Bernese criteria cardiorespiratory stability was defined as absence of desaturation or bradycardia for 72 hours. The number of apneas, bradycardias and desaturations were analyzed for the last 28 days before stop of cardiorespiratory monitoring. Events which needed no intervention, such as stimulation, were classified as type 1 , whereas type 2 events needed interventions such as stimulation or mask ventilation.

Results. A total of 425 patients were analyzed. Bradycardias: 2468 bradycardias type 1 and 250 bradycardias type 2 occurred in the observation period. In the group of infants $>1500 \mathrm{~g}$ and $>32$ weeks of GA 815 bradycardias type 1 and 91 bradycardias type 2 were observed (0.279 bradycardias type 1 per child and per day and 0.031 bradycardias type 2 per child and per day). In the group of infants $\leq 1500 \mathrm{~g}$ and/or $\leq 32$ weeks of GA 1653 bradycardias type 1 and 159 bradycardias type 2 were observed ( 0.542 bradycardias type 1 per child per day and 0.052 bradycardias type 2 per child and per day). Bradycardias type 1 and type 2 declined gradually in infants $>1500 \mathrm{~g}$ and $\leq 1500 \mathrm{~g}$ until monitoring was discontinued. The decrease was not different between the types of bradycardias or between the patients of different age and birth weight. Apneas: 11'352 apneas type 1 and 229 apneas type 2 occurred in the observation period. In the group of infants $\leq 1500 \mathrm{~g}$ and/or $\leq 32$ weeks of GA 8830 apneas type 1 were observed ( 2894 per patient per day). In the group of infants $>1500$ g and $>32$ weeks of GA 2522 apneas type 1 were observed, (o.864 per patient per day). The number of apneas type 1 declined over time to zero. From day -28 to day -9 the number of desaturations per patient per day was almost stable in patients $>1500 \mathrm{~g}$ (decline -0.018 per patient per day). This was considerably different from the other group which showed a gradient of -0.195 . However after day -8 the decline was similar in both groups (gradient of -0.151 and -0.172 , respectively). The analysis of the frequencies and decline of type 2 apneas did not reveal differences between the two groups.

Conclusion. Cardiorespiratory events type 1 were more frequent than type 2. The frequency of apneas type 1 differ between infants with a birth weight of less than $1500 \mathrm{~g}$ or of a GA of less than 32 weeks between day -28 and -9 before reaching cardiorespiratory stability from older or heavier newborns. No differences were detected between day -8 and stop of monitoring. Frequencies of apneas type 2 and bradycardias did not differ between the groups within the last 28 days before reaching cardiorespiratory stability.

\section{PO-95}

Lagerung von Neugeborenen bei der postnatalen Routine-Erstversorgung im Kreißsaal

\section{Konstantelos', R. Bergert ${ }^{1}$, M. Rüdiger ${ }^{1}$ \\ 'Universitätsklinikum Carl Gustav Carus, Neonatologie \& Pädiatrische Intensivmedizin, Dresden, Deutschland}

Hintergrund. Die Erstversorgung von Neugeborenen im Kreißsaal orientiert sich an den jeweils aktuellen Empfehlungen, allerdings ist die Datenlage für viele Empfehlungen eher spärlich. So wir das Neugeborene zur Erstversorgung häufig auf dem Rücken gelagert, allerdings fehlen entsprechende Untersuchungen zur optimalen Lagerung (Seite oder Rücken).

Fragestellung. Beeinflusst die Lagerung des Neugeborenen die postnatale Adaptation?

Material und Methoden. Im Zeitraum von Januar bis Dezember 2012 wurden alle Erstversorgungen von Termingeborenen nach Kaiserschnitt mittels Video aufgezeichnet (keine pränatal bekannte Pathologie, Geburt im Frühdienst). Die Videos wurden anschließend analysiert (Interact ${ }^{\star}$ ) und ausgewertet, wie häufig Kinder im Rahmen der klinischen Routine in Seitenlage versorgt und welche Interventionen (CPAP-Applikation, Stimulieren, Absaugen) in dieser Position verabreicht wurden. Außerdem wurden Vitalparameter (HF, Sättigung) und das Wohlbefinden der Kinder in beiden Positionen verglichen. Das Wohlbefinden der Kinder wurde minütlich mit einem dreistufigen Punktescore ( $1=$ minimale, $3=$ maximale Aufregung) bewertet.

Ergebnisse. In dem Zeitraum wurden 187 Versorgungen aufgezeichnet, bei 89 (48\%) Kinder erfolgte die Versorgung überwiegend (d. h. $>75 \%$ der Zeit) in Rückenlage und bei 57 (30\%) Kinder auf der Seite. In Seitenlage waren die Kinder signifikant $(\mathrm{p}<0,001)$ weniger aufgeregt (mittlerer Score 1,7 ) als in Rückenlage $(2,05)$. CPAP wurde bei 41 Kinder ausschließlich in Rückenlage und lediglich bei einem Kind in Seitenlage appliziert. Eine Stimulation war bei den Kindern in Seitenlage signifikant ( $<<0,001)$ weniger häufig [Median 3,9\% (o-27\%) der Zeit] notwendig als in Rückenlage [12,5\% (o-62\%)]. Es gibt keine signifikanten Unterschiede zwischen beiden Lagen bezüglich der Sättigung oder Herzfrequenz. Die Herzfrequenz war zur 8. Minute 158/min (124-198) im Median in Rückenlage und $162(106-245)$ in Seitenlage $(\mathrm{p}=0,56)$. $\mathrm{SpO}_{2}$ betrug 93\% (70-100) bzw. 95\% (80-100; $\mathrm{p}=0,32$ ).

Diskussion. Eine Versorgung der Neugeborenen ist in Seitenlage gut möglich, auch medizinische Interventionen können appliziert werden. Der niedrigere Aufregungsscore zeigt, dass die Seitenlage für die Kinder deutlich angenehmer ist. Außerdem benötigten die Kinder in Seitenlage weniger Stimulationen, wobei Ursache und Wirkung nicht klar zu unterscheiden sind. Die Vitalparameter zeigen keine deutlichen Unterschiede zwischen beiden Lagen, so dass die Seitenlage mit keinen Nachteilen verbunden zu sein scheint. In einer randomisierten, kontrollierten Studie sollten die Vor- bzw. Nachteile der jeweiligen Lagerung systematisch untersucht werden, bevor entsprechende Empfehlungen in den Reanimationsrichtlinien gegeben werden können.

\section{PO-96}

Die Beziehung von Speichel- und Plasma-Kortisol-Spiegeln bei Frühgeborenen: Ergebnisse einer prospektiven Beobachtungsstudie

\section{Maas', K. Kreutzer', C. Ringwald', K. Weber ${ }^{3}$, C. Poets', G. Binder ${ }^{3,}$ D. Bassler ${ }^{1}$}

'Eberhard-Karls-Universität Universitätsklinik für Kinderheilkunde und Jugendmedizin, Neonatologie, Tübingen, Deutschland, ${ }^{2}$ Universitätskinderklinik Tübingen, Neonatologie, Tübingen, Deutschland, ${ }^{3}$ Eberhard-KarlsUniversität Universitätsklinik für Kinderheilkunde und Jugendmedizin, Sektion Pädiatrische Endokrinologie, Tübingen, Deutschland

Hintergrund. Wir untersuchten die Beziehung von Speichel- und Plasma-Kortisol-Spiegeln bei Frühgeborenen mit dem vorrangigen Ziel, die 
Validität von Speichel-Kortisol-Werten zur Erfassung einer Nebenniereninsuffizienz bei Frühgeborenen zu überprüfen.

Methoden. Wir führten eine prospektive, monozentrische Beobachtungsstudie durch. In diese wurden 58 Frühgeborene mit einem Gestationsalter bei Geburt von $<34$ Schwangerschaftswochen oder einem Geburtsgewicht von $<1500$ g eingeschlossen. Außerdem wurden 19 Kinder und junge Erwachsene im Alter von 5 bis 19 Jahren untersucht. Speichelproben wurden durch Zelluloseschwämmchen jeweils vor Venenpunktion zur Blutentnahme gewonnen. Wir analysierten Speichel- und Plasma-Kortisol-Konzentrationen mittels enzymgekoppeltem Immunadsorptionstest (ELISA). Die Auswertung erfolgte durch eine Korrelationsanalyse. Die Datendarstellung erfolgt unter Angabe von Medianen, Quartilsabständen (IQR) und von Korrelationskoeffizienten.

Ergebnisse. Das mediane (IQR) Gestationsalter bei Geburt lag bei 31,4 $(28,1-32,7)$ Schwangerschaftswochen, das Geburtsgewicht bei 1340 (974-1745) g. Bei 30 Frühgeborenen (51,7\%) konnten wir mindestens $50 \mu 1$ Speichel gewinnen und so Speichel- und Plasma-Kortisol-Spiegel vergleichen. Die Korrelationsanalyse zeigte eine statistisch signifikante Korrelation der Kortisolspiegel in zusammengehörigen Speichel-BlutProben von Frühgeborenen $(\mathrm{r}=0,64 ; \mathrm{p}=0,0001)$. In der Gruppe der größeren Kinder und jungen Erwachsenen war diese Korrelation allerdings deutlicher $(r=0,89 ; p<0,0001)$. In der Frühgeborenenkohorte beobachteten wir einige Probanden, bei welchen Plasma-Kortisol-Spiegel im unteren Bereich mit unerwartet hohen Speichel-Kortisol-Spiegeln einhergingen.

Schlussfolgerungen. Die Validität von Speichel-Kortisol-Spiegeln bei Frühgeborenen scheint insbesondere im niedrigen Wertebereich fragwürdig, welcher von besonderer Bedeutung für die Diagnostik einer (relativen) Nebenniereninsuffizienz ist.

\section{PO-97}

Retrospektiver Vergleich von Urinelektrolyt-Kreatinin-Quotienten in einem Kinderkrankenhaus mit publizierten Referenzbereichen

\section{J. Christoph', E. Kattner', F. Guthmann}

'Auf der Bult, Krankenhaus für Kinder und Jugendliche, Neonatologie, Hannover, Deutschland

Fragestellung. Ist die Verteilung der molaren Urinelektrolyt-KreatininQuotienten von $\mathrm{Na}, \mathrm{K}, \mathrm{Ca}, \mathrm{Cl}$ und Phosphat bei Kindern, die diese Tests im Rahmen ihrer klinischen Untersuchungen erhielten, vergleichbar mit publizierten Referenzbereichen?

Methodik. Von Februar 2004 bis Dezember 2012 wurden mehr als je 2000 Analysen von Natrium, Kalium und Chlorid, mehr als 4000 von Calcium und Phosphat sowie mehr als 8000 von Kreatinin im Urin auf einem Siemens Dimension untersucht. Die 5., 50. und 90. Perzentile wurde für den molaren Elektrolyt/Kreatinin-Quotienten jeden Parameters in verschiedenen Altersgruppen berechnet ( $\mathrm{N}$ für die UrinKreatinin-Quotienten bei Na, K, Cl je 1326; für Ca und P 3915 bzw. 4053).
Ergebnisse. Die goer Perzentile der Natrium- und Chlorid/KreatininQuotienten in der klinischen Routine liegt in allen Altersgruppen außerhalb der Referenzintervalle, die von Mersin/Bianchetti 1995 bzw. Bogaru/Guignard 2002 publiziert wurden. Auch beim Calcium- und Phosphat-Kreatinin-Quotienten im Urin ist unsere 9oer Perzentile höher als die von Guignard angegebenen Referenzwerte, unsere Perzentilen zeigen einen rückläufigem Trend im Untersuchungszeitraum. Die 5er Perzentilen weichen nicht wesentlich von den publizierten Referenzwerten ab (• Tab. 1).

Schlussfolgerung. Gemäß DIN EN ISO 15189 5.5.2 muss das Labor biologische Referenzbereiche oder klinische Entscheidungswerte für die bediente Population festlegen und die Grundlage für diese mitteilen. Analysenergebnisse hospitalisierter Patienten können zur Berechnung von Referenzintervallen genutzt werden [Hoffmann RG: Statistics in the practice of medicine, JAMA 185 (1963), 864-873; zitiert in Soldin SJ: Pediatric Reference Intervals. AACC Press, Washington DC, 7. Auflage 2011, Preface S. XIV] insbesondere wenn Werte einer genügenden Zahl Gesunder nicht zur Verfügung stehen. Eigene Analysenergebnisse können und sollten mit publizierten Daten verglichen werden. Durch klinische Einflussfaktoren können die hausinternen Werte von Referenzwerten abweichen. Um dies zu berücksichtigen und Outlier zu eliminieren, haben wir die 9oer statt der 95er oder 97,5er Perzentile gewählt.

\section{PO-99}

Sildenafil als neue Therapieoption bei großen, zystischen Lymphangiomen

\section{P. Flosdorff', K. Roefke', N. Moschkau', P. Brand', S. Meinig', K. Großer} H. Bittrich'

'Helios Klinikum Erfurt GmbH Klinik für Kinder- und Jugendmedizin, Neonatologie/Kinderintensivmedizin, Erfurt, Deutschland, ${ }^{2}$ Helios Klinikum Erfurt GmbH, Klinik für Anästhesie, Intensivmedizin und Schmerztherapie, Erfurt, Deutschland, ${ }^{3}$ Helios Klinikum Erfurt GmbH, Klinik für Gynäkologie und Geburtshilfe, Erfurt, Deutschland, ${ }^{4}$ Helios Klinikum Erfurt GmbH, Klinik für Kinderchirurgie und Kinderurologie, Erfurt, Deutschland

Einleitung. 2012 veröffentlichte Swetman et al. im NEJM einen Fallbericht mit spontaner Regredienz eines riesigen zystischen Lymphangioms im Bereich der Thoraxwand, welches durch Verdrängung zu einer pulmonalen Hypertension führte. Die langsame Regredienz war zeitlich mit dem Beginn einer Sildenafiltherapie zur Senkung des pulmonalen Widerstandes assoziiert. Eine Pilotstudie konnte bei zwei weiteren Kindern einen positiven Effekt des Sildenafils auf Lymphangiome zeigen. Die pathophysiologischen Zusammenhänge sind nicht bekannt. Fall. Berichtet wird über ein Neugeborenes mit riesigem zystischen Lymphangiom im Halsbereich. Perinatal konnten über eine Exit-Prozedur die Atemwege gesichert werden. Aufgrund der Ausmaße gelang nur eine subtotale initiale Resektion. Bei rascher Progredienz erfolgte

\begin{tabular}{|c|c|c|c|c|c|c|c|c|c|c|}
\hline $\begin{array}{l}\text { Alter | } \\
\text { Analyt }\end{array}$ & $\mathrm{Na}$ & & K & & $\mathrm{Cl}$ & & $\mathrm{Ca}$ & & $\mathbf{P}$ & \\
\hline $\begin{array}{l}\text { Perzen- } \\
\text { tilen }\end{array}$ & 0,05 & 0,9 & 0,05 & 0,9 & 0,05 & 0,9 & 0,05 & 0,9 & 0,05 & 0,9 \\
\hline 1 Woche & 4 & 228 & 3 & 37 & 6 & 204 & 0,1 & 5,0 & 0,1 & 11,3 \\
\hline 1 Monat & 4 & 129 & 7 & 56 & 10 & 137 & 0,5 & 5,7 & 0,4 & 25,5 \\
\hline 3 Monate & 3 & 191 & 10 & 89 & 11 & 193 & 0,6 & 8,3 & 0,5 & 25,8 \\
\hline 6 Monate & 1 & 125 & 7 & 72 & 12 & 149 & 0,2 & 6,6 & 0,8 & 22,3 \\
\hline 1 Jahr & 1 & 106 & 6 & 55 & 10 & 129 & 0,2 & 10,4 & 1,8 & 27,4 \\
\hline 3 Jahre & 7 & 185 & 5 & 57 & 12 & 229 & 0,1 & 4,6 & 0,2 & 15,3 \\
\hline 7 Jahre & 3 & 112 & 4 & 39 & 6 & 121 & 0,1 & 2,6 & 1,2 & 11,2 \\
\hline 14 Jahre & 1 & 95 & 4 & 33 & 4 & 86 & 0,0 & 2,2 & 0,3 & 6,8 \\
\hline 18 Jahre & 1 & 36 & 2 & 12 & 5 & 46 & 0,0 & 1,1 & 0,4 & 5,8 \\
\hline
\end{tabular}


sekundär ein Therapieversuch mit Picibanilinjektionen. Es zeigte sich kein bleibendes Ansprechen und das Tumorausmaß zwang zu einem letzten operativen Resektionsversuch mit erneuter rascher Tumorprogredienz. Als alternativen Heilversuch begannen wir im 6. Lebensmonat die Therapie mit Sildenafil. Darunter zeigte sich eine bis heute eindrucksvolle Regredienz, die wir Ihnen in Bildern und Video gerne vorstellen möchten

Schlussfolgerung. Trotz dieser erst zweiten publizierten Fallbeschreibung könnte Sildenafil - ähnlich wie Propranolol in der Therapie der Hämagiome - zu einer Revolution der ansonsten schwer zu behandelnden großen zystischen Lymphangiome führen.

\section{Frühmorbidität - Langzeit-Outcome}

\section{PO-100}

Die intrauterin entstandene PVL bei IUGR - gibt es einen optimalen Entbindungszeitpunkt?

\section{Kästner', D. Faas', R. Axt-Fliedner' ${ }^{2}$, H. Ehrhardt ${ }^{1}$}

'Zentrum für Kinderheilkunde der Justus-v.-Liebig-Universität, Allgemeine Pädiatrie und Neonatologie, Gießen, Deutschland, ${ }^{2}$ Zentrum für Frauenheilkunde und Geburtshilfe, Pränataldiagnostik, Giessen, Deutschland

Hintergrund. Die periventrikuläre Leukomalazie (PVL) des Frühgeborenen stellt die häufigste Ursache einer spastischen Cerebralparese dar. Die Prognose der Erkrankung ist als schlecht zu bezeichnen. Ursache der intrazerebralen Veränderungen (Zystenbildung) sind hypoxisch-ischämische Ereignisse prä-, peri- oder postnatal, die, getriggert durch freie Radikale und Zytokine, zu Demyelinisierung und Apoptose der periventrikulären Oligodentrozyten führen. Die Diagnose ist sonographisch zu stellen. Während die postnatalen Risikofaktoren für eine PVL gut belegt sind, konnten als pränatale Risikofaktoren in retrospektiven Datenerhebungen lediglich das Amnioninfektionssyndrom (Chorioamnionitis), der vorzeitige Blasensprung und das männliche Geschlecht identifiziert werden. Die Datenlage bezüglich IUGR („,intrauterine growth restriction“) und der fetalen Doppleruntersuchungen als Hinweise für eine uteroplazentare Dysfunktion sowie der Präeklampsie ist sehr widersprüchlich.

Fallberichte. Wir berichten über drei Fälle, in denen die Frühgeborenen nach IUGR bereits intrauterin eine PVL erworben haben: Zunächst über ein Frühgeborenes der 24+5. Schwangerschaftswoche (SSW) mit einer symmetrischen Wachstumsrestriktion, GG $315 \mathrm{~g}\left(135 \mathrm{~g}<\mathrm{P}_{3}\right)$. Die Indikation zur Sectio caesarea (SC) wurde bei pathologischen fetalen Dopplern („revers flow“ in A. uterina) und pathologischen CTG gestellt Das Vollbild einer zystischen PVL war am 14. Lebenstag darstellbar. Als zweites über ein Frühgeborenes mit einer asymmetrischen IUGR, GG $1245 \mathrm{~g}\left(\mathrm{P}_{3}\right)$. Bei pathologischen fetalen Dopplern und pathologischen CTG erfolgt die SC in der 33+4. SSW. Die zystische PVL bestand bereits am 1. Lebenstag. Zuletzt berichten wir von einem Frühgeborenen der 29+3. SSW mit asymmetrischer IUGR, GG $830 \mathrm{~g}(\mathrm{P} 10)$. Die SC wurde bei pathologischen Dopplern („brainsparing“), Wachstumsstillstand und suspekten CTG durchgeführt. Auch bei diesem Patienten war die PVL am 1. Lebenstag sonographisch nachweisbar. Die Gestationsalter und die Begleitmorbiditäten sind bei diesen Fällen unterschiedlich und stehen nicht ursächlich mit der Entwicklung einer PVL in Zusammenhang.

Schlussfolgerung. Ein kausaler Zusammenhang zwischen der intraute rinen Wachstumsrestriktion und der intrauterinen Entwicklung einer PVL ist wahrscheinlich. Obwohl die Konstellation der IUGR in allen drei Fällen unterschiedlich war, entwickelte sich eine PVL. Die Wahl des optimalen Entbindungszeitpunktes in Zusammenschau der fetalen Befunde (somatische Maße im zeitlichen Verlauf, Dopplerbefunde, CTG) stellt eine große interdisziplinäre Herausforderung an Geburtshelfer und Neonatologe dar, da zum einen die Risiken der Frühgeburt- lichkeit aber auch die Entstehung einer irreversiblen Hirnschädigung durch eine pränatal entstandene PVL vermieden werden sollten.

PO-101

Lungenblutung und intraventrikuläre Hämorrhagie als frühe Todesursache bei extrem unreifen Frühgeborenen - Welche prä- und postnatalen Risikofaktoren spielen eine Rolle?

\section{Bendiks', C. Härtel', G. Stichtenoth', M. Rüdiger' ${ }^{2}$, T. Schaible³, U. Felder-} hoff-Müser', F. Heitmann ${ }^{5}$, N. Teig', E. Herting', W. Göpel'

'Universitätsklinikum Schleswig Holstein, Klinik für Kinder- und Jugendmedizin, Lübeck, Deutschland, ${ }^{2}$ Univ. Klinikum Carl Gustav Carus, Klinik für Kinder- und Jugendmedizin, Neonatologie, Dresden, Deutschland, ${ }^{3}$ Klinikum Mannheim, Kinderklinik, Mannheim, Deutschland, ${ }^{4}$ Universitätsklinikum Essen, Zentrum für Kinder- und Jugendmedizin, Essen, Deutschland, ${ }^{5}$ Westfälisches Kinderzentrum, Klinikum Dortmund, Dortmund, Deutschland, ${ }^{6}$ Klinik für Kinder- und Jugendmedizin St. Josefs-Hospital, Abteilung für Neonatologie, Bochum, Deutschland

Hintergrund. Über die Ursachen der frühen Mortalität von extrem unreifen Frühgeborenen existieren in der Literatur nur wenige Daten. In der Kohorte des Geburtsjahrgangs 2010 des Deutschen FrühgeborenenNetzwerks (GNN) waren die Blutungskomplikationen IVH und Lungenblutung relevante Todesursachen in den ersten Lebenstagen [Stichtenoth et al (2012) Klin Paediatr 224:276-81].

Fragestellung. Wie häufig waren IVH und Lungenblutung als Todesursache bei extrem unreifen Frühgeborenen des GNN in den Jahren 2009 bis 2012? Gab es Unterschiede bei den perinatalen Risikofaktoren und Therapiemodalitäten in den ersten Lebenstagen im Vergleich zu Frühgeborenen, die an anderen Komplikationen verstarben?

Methoden. Die hauptsächliche Todesursache der Frühgeborenen während des ersten Klinikaufenthalts wurde von den betreuenden Neonatologen in den einzelnen GNN-Zentren festgelegt. Die Datenqualität wurde durch ein Monitoring vor Ort überprüft.

Ergebnisse. Von insgesamt 660 verstorbenen Frühgeborenen verstarben 46 an einer IVH und 28 an einer Lungenblutung. Beide Komplikationen führten in der Regel während der ersten Lebenswoche zum Tode (Median 4 Tage, Interquartile Range: 3-7 Tage). Das Gestationsalter (Median 24,57 SSW, IQR 23,86-26,29 SSW) und das Geburtsgewicht (Median 640 g, IQR 490-740 g) der an einer IVH oder Lungenblutung verstorbenen Frühgeborenen sowie Geschlechterverteilung und Anzahl der Mehrlinge unterschieden sich nicht signifikant von den an anderen Ursachen verstorbenen Frühgeborenen. Informationen zu den perinatalen Faktoren lagen bis zur Abstracteinreichung von 18 an einer IVH und 8 an einer Lungenblutung verstorbenen Frühgeborenen vor. Die Daten für das Geburtsjahr 2012 werden noch durch ein Monitoring überprüft und in die Endauswertung eingefügt. Keine signifikanten Unterschiede fanden sich bei der Durchführung einer Tokolyse und antenatalen Lungenreife sowie bei den Ursachen der frühzeitigen Geburt. Auch ein Amnioninfektionssyndrom als Geburtsursache trat bei an einer IVH oder Lungenblutung verstorbenen Kindern nicht häufiger auf ( $15,4 \%$ vs. $25,8 \%$ bei Frühgeborenen mit anderen Todesursachen). Bei der Auswertung der postnatalen Faktoren fiel auf, dass bei den an einer IVH oder Lungenblutung verstorbenen Frühgeborenen ein niedrigerer minimaler Blutdruck in den ersten 24 Stunden gemessen wurde. Der Unterschied zu den an anderen Ursachen Verstorbenen war signifikant $(p=0,006)$. Bezüglich der Verabreichung von Surfactant fanden sich keine signifikanten Unterschiede.

Schlussfolgerung. Bei der Auswertung der GNN-Kohorten der Geburtsjahre 2009 bis 2012 fiel als einziger signifikanter Unterschied ein niedrigerer minimaler Blutdruck am ersten Lebenstag bei den an einer IVH oder Lungenblutung verstorbenen Frühgeborenen auf. Hämodynamische Faktoren spielen in der Pathogenese beider Komplikationen eine Rolle und sind potentiell therapeutisch beeinflussbar. Zur Prävention dieser Komplikationen sind weitere Studien notwendig. 


\section{PO-102}

\section{Digitale 3D-Erfassung der Schädelsymmetrie Frühgeborener zur Entlassung}

\section{W. Burkhardt', S. Ifflaender', M. Rüdiger ${ }^{1}$ \\ 'Universitätsklinikum Carl Gustav Carus Kinderklinik, Abteilung für Neo- natologie und Pädiatrische Intensivmedizin, Dresden, Deutschland}

Hintergrund. Frühgeburtlichkeit ist als ein Risikofaktor für einen Plagiocephalus beschrieben. Frühgeborene (FG) mit einem lagebedingten, sog. „deformational plagiocephaly“ (DP) zeigen eine signifikante Entwicklungsverzögerung. Daher ist neben der Erfassung des Kopfwachstumes auch ein Monitoring der Kopfform wichtig. Bisher stehen wenig objektive Routinemethoden zur Messung der Schädelsymmetrie zur Verfügung, die Daten zur Inzidenz des DP bei Frühgeborenen sind daher sehr limitiert.

Ziel. Die objektive Quantifizierung der Schädelsymmetrie bei Frühgeborenen zum Zeitpunkt der Entlassung und die Korrelation des Auftretens von Asymmetrien mit dem Gestationsalter (GA).

Methoden. In einer prospektiven Studie zwischen April 2011 und März 2012 erhielten Frühgeborene ein 3D-Laserscanning des Schädels zum Zeitpunkt der Entlassung. Daten von $n=285$ Frühgeborenen wurden ausgewertet. Die Messungen wurden mittels nichtinvasivem Laserscanner (STARScanner, Vorum research Corp., Vancouver, Canada) durchgeführt. Das Gerät erfasst digital unter Verwendung von 4 Lasern (class-I, eye-safe) die dreidimensionale Schädelform. Die Berechnung der Schädelsymmetrie (Cranial Vault Asymmetry Index, CVAI) wurde mittels STARscanner Laser Data Acquisiton System (Orthomerica, Orlando, FL) durchgeführt. Die Studie wurde von der Ethikkommission genehmigt.

Ergebnisse. Der CVAI zur Entlassung korreliert signifikant mit dem Gestationsalter $(r=-0,2235, p<0,001)$, d. h. je unfeifer die Kinder waren, um so größer war die Asymmetrie. Der mittlere CVAI zur Entlassung war höher bei FG mit einem GA<28 SSW (5,04\%; 95\% CI 3,62-6,45) und bei FG mit einem GA von 28 bis 31+6 SSW (4,43\%; 95\% CI 3,58-5,28) verglichen mit Reifgeborenen (2,97\%; 95\% CI 2,33-3,61; p>0,05). Unter der Annahme, dass ein CVAI $>3,5$ ein Risiko für die Entwicklung eines DP bedeutet, haben $58 \%$ mit einem GA<28 SSW, $61 \%$ mit einem GA 28 to $31+6$ SSW and $31,5 \%$ der Reifgeborenen ein erhöhtes Risiko für die Entwicklung eines DP.

Diskussion. Eine signifikante Anzahl Frühgeborener wird mit einer bereits bestehenden Asymmetrie der Kopfform entlassen. Dies bedeutet ein erhöhtes Risiko für die Entwicklung eines DP und eine verzögerte motorische Entwicklung. Wiederholte Messungen in der neonatologischen Nachsorge sind zur Verlaufskontrolle und gegebenenfalls Einleitung von Therapien notwendig. Die laserunterstützte Erfassung der Kopfform bietet eine sichere und objektive Methode zum Monitoring der Schädelsymmetrie bei Früh- und Reifgeborenen in der klinischen Routine.

\section{PO-103}

Perinatales, neonatales und frühkindliches Outcome nach Blasensprung vor $26+0 / 7 \mathrm{SSW}$

\section{Wieg', O. Stangl', H. Harnack ', A. Teichmann ${ }^{3}$}

'Klinikum Aschaffenburg, Neonatologie und pädiatrische Intensivmedizin, Aschaffenburg, Deutschland, ${ }^{2}$ Klinikum Aschaffenburg, Abteilung Neonatologie u. pädiatrische Intensivmedizin, Aschaffenburg, Deutschland, ${ }^{3}$ Klinikum Aschaffenburg, Frauenklinik, Aschaffenburg, Deutschland

Problemstellung. Das Management des extrem frühen Blasensprungs werdender Mütter stellt sowohl eine peri- als auch neonatologische Herausforderung dar. Ein aktives schwangerschaftserhaltendes Vorgehen vor oder im Bereich der Grenze der Lebensfähigkeit von Frühgeborenen kann für das Kind aber mit einer erheblichen Morbidität und Mortalität verknüpft sein kann. Deshalb ist das Wissen um das zentrumsspezifische Outcome dieser Schwangerschaften bedeutsam.
Methodik. Retrospektive monozentrische Analyse im Perinatalzentrum Aschaffenburg der Jahre 2007 bis 2009 von Schwangerschaften mit Blasensprung 14+o bis-25+6SSW $(n=47)$ hinsichtlich perinatalem, neonatalem und frühkindlichem Outcome ( $24 \mathrm{Mo}$ ) Kontrollgruppe: intrazentrisch Gesamtkohorte adjustiert auf FG $<30$ SSW u. $<1500$ g der Jahrgänge 2007-2009.

Ergebnisse. Pränatale Therapie: Antibiose/Tokolyse (AT, 44\%) Antibiose/Tokolyse/operativer Muttermundsverschluss (OP, 27\%); keine aktive Therapie (NoTh, 29\%). Keine Schwangerschaft ohne Therapie führte zu einer Lebendgeburt. 9 von 33 Schwangerschaften konnten trotz Therapie (6 AT/3 OP) keine Lebendgeburt erreichen. In allen Fällen lag ein Amnioninfektionssyndrom vor. Die durchschnittliche Verlängerung der SS lag bei 15,6 Tagen (o-50 Tage). 26 Kinder aus 47 Schwangerschaften $(61 \% \mathrm{AT} / 39 \% \mathrm{OP})$ wurden geboren (GA $27+2 \mathrm{SSW} / 22+6$ bis $30+0$ SSW ; GG 879 g/330 g bis 1750 g). Mortalität $6 \%$ (vs. 4,7\% Kontrollgruppe), Lungenhypoplasie $19 \%$ (vs. o\% KG) BPD36 8\% (vs. 7\%KG) IVH $>2^{\circ} 8 \%$ (vs. $6 \% \mathrm{KG}$ ) PVL $2 \%$ (vs. $1 \% \mathrm{KG}$ ). Im korrigierten Alter von 24 Monaten PDI u/o MDI $<85$ in $15,8 \%$ vs. $18,4 \%$ in der KG.

Diskussion. Ein aktives pränatales Vorgehen zum Erhalt der Schwangerschaft bei extrem frühem Blasensprung war in $76 \%$ erfolgreich. Das Vorliegen eines floriden AIS zum Zeitpunkt des frühen Blasensprungs führte meist zu einer Terminierung der Schwangerschaft innerhalb weniger Tage. Im Gegensatz zu den Daten von Patkai et al (Eur J Obstet Gynecol Reprod Biol. 2012) war die neonatale Mortalität und Morbidität in unserer Kohorte nicht signifikant höher als in der Kontrollgruppe. Außerdem lag in dieser französischen Arbeit die Quote der Lebendgeburten zudem mit $28 \%$ erheblich niedriger. Auch die frühkindliche Entwicklung zeigte zwischen Frühgeborenen nach extrem frühen BS und der Kontrollgruppe in unserer Kohorte keine Unterschiede.

Schlussfolgerung. Ein individuelles detailliert abgestimmtes Vorgehen zwischen Geburtshilfe und Neonatologie kann beim extrem frühen Blasensprung $\mathrm{zu}$ einem günstigen Outcome führen, was die aktive Therapie zum Erhalt auch sehr früher Schwangerschaften nach Blasensprung rechtfertigt. Zentren sollten ihre Daten kennen, um die betroffenen werdenden Eltern entsprechend differenziert beraten zu können.

\section{PO-104}

Neurologisch-kognitive Langzeitentwicklung von Zwillingen mit FFTS nach intrauteriner Lasertherapie

\section{P. Graeve', C. Prinz', K. Hecher' ${ }^{2}$, P. Bartmann ${ }^{1}$}

'Universität Bonn, Zentrum für Kinderheilkunde, Abteilung für Neonatologie, Bonn, Deutschland, ${ }^{2}$ Universitätsklinikum Hamburg-Eppendorf, Klinik für Geburtshilfe u. Pränatalmedizin, Hamburg, Deutschland

Hintergrund und Fragestellung. Das Zwillingstransfusionssyndrom (ca. 10\% aller monochorialen, diamnioten Schwangerschaften), ist unbehandelt mit einer hohen Mortalität und Morbidität verbunden. Die intrauterine Lasertherapie, die sich seit Mitte der 1990er Jahre etabliert hat, stellt eine effektive, kausale Therapieform dar. In bisherigen Studien zur neurologischen Langzeitentwicklung nach FFTS und intrauteriner Lasertherapie lag das Untersuchungsalter der Patienten meist bei 2 Jahren. Um eine Langzeitprognose bezüglich der neurologischen und kognitiven Entwicklung treffen zu können, führten wir eine Nachuntersuchung im Alter von 9 Jahren und 10 Monaten (Median) durch. Dabei sollte vor allem geprüft werden, inwiefern sich die kognitive Entwicklung von Donatoren und Akzeptoren bei genetischer Identität, aber unterschiedlicher intrauteriner Versorgung, unterscheidet.

Material und Methoden. Im Rahmen der Studie wurden 31 Zwillingspaare im medianen Alter von 9 Jahren und 1o Monaten untersucht. Die Patienten gehören zu einem Studienkollektiv, welches aus 254 überlebenden Einlingen und Zwillingen nach intrauteriner Lasertransfusion bei FFTS besteht. Sie wurden bereits im Alter von 2 und 6 Jahren erstmals untersucht. Die Patienten waren nach verschiedenen Kriterien für die Nachuntersuchung ausgewählt worden, so dass die Studiengruppe nicht das Gesamtkollektiv abbildet. Es erfolgte eine standardisierte kör- 
perliche und neurologische Untersuchung, außerdem führten wir den Hamburg-Wechsler-Intelligenztest-IV (HAWIK-IV) durch. Im Rahmen der Untersuchung wurden die Kinder in drei neurologische Gruppen eingeteilt. Gruppe I beinhaltet neurologisch unauffällige Kinder, Gruppe II Kinder mit leichten Beeinträchtigungen, wie Sprachstörungen oder Defizite in Fein- oder Grobmotorik und normalem Entwicklungstest. Gruppe III beinhaltet Patienten mit schwerer neurologischer Einschränkung und/oder einem pathologischen Entwicklungstest.

Ergebnisse. Unter den 62 Kindern waren 26 Mädchen und 36 Jungen. 52 Kinder wurden der Gruppe I zugeordnet (83,9\%), 4 Kinder der Gruppe II (6,5\%) und 6 Kinder der Gruppe III (9,7\%). Die IQ-Werte der Kinder reichten von 47 bis zu 130 Punkten. Der mediane Intelligenzquotient des Gesamtkollektivs lag bei 92,5. Bei der Einzelauswertung aller Akzeptoren lagen die IQ-Werte zwischen 47 und 130, der Median lag bei 93,5. Bei den Donatoren lagen die erreichten IQ-Werte zwischen 52 und 129, der Median lag bei 92. Es zeigte sich für den Gesamt-IQ der Probanden mit $\mathrm{p}=0,39$ kein statistisch signifikanter Unterschied zwischen Akzeptoren und Donatoren. Auch in den Unterkategorien des HAWIK-IV fanden sich keine signifikanten Unterschiede zwischen Akzeptoren und Donatoren.

Schlussfolgerung. Trotz der unterschiedlichen pränatalen Pathophysiologie der Zwillinge, fanden sich in der vorliegenden Studie keine Unterschiede in der kognitiven Entwicklung zwischen Akzeptoren und Donatoren im Alter von knapp 10 Jahren.

\section{PO-105}

\section{Mentale Rotation bei früh- und reifgeborenen Kindern}

T. Seifen', S. Braun', K. Mehler', A. Kribs'², B. Roth'², M. Kavsek'

'Institut für Psychologie, Entwicklungspsychologie und pädagogische Psychologie, Bonn, Deutschland, ${ }^{2}$ Universitätskinderklinik Köln, Neonatologie und Pädiatrische Intensivmedizin, Köln, Deutschland

Hintergrund. Der Begriff Mentale Rotation (MR) bezeichnet die Fähigkeit, zwei- sowie dreidimensionale Objekte in der Vorstellung, auf der Basis rein kognitiver Prozesse zu drehen. Bisher liegen kaum Befunde zur MR bei Frühgeborenen vor. Bei der Durchführung von MR Aufgaben lassen sich Aktivierungen im superioren parietalen, frontalen und inferotemporalen Cortex nachweisen, die auch an der Verarbeitung von Bewegung beteiligt sind. Mentale Rotationsfähigkeiten korrelieren mit schulischen Leistungen, der neuronalen Verarbeitung von Bewegung und der räumlichen Vorstellung.Fragestellung. Es wurde untersucht, ob sich die mentale Rotationsleistung von ehemaligen VLBW-Frühgeborenen und Reifgeborenen im Alter von zehn und dreizehn Jahren unterscheidet. Neben Gruppenunterschieden wurden Geschlechtsunterschiede und der Entwicklungsverlauf der mentalen Rotationsleistung untersucht.

Methoden. Es wurden zwei Gruppen der Jahrgänge 1999 und 2002 untersucht: 54 ehemals Frühgeborene mit einem Geburtsgewicht unter $1500 \mathrm{~g}$ und 41 Reifgeborene. Allen Teilnehmern wurden die zweidimensionalen Tests MRT-K-Tierversion und MRT-K-Buchstabenversion sowie der dreidimensionale MRT-A vorgelegt. Zur Erhebung von Kontrollvariablen wurde ein Elternfragebogen ausgegeben.

Ergebnisse. Das mittlere Gestationsalter lag in der Gruppe der Frühgeborenen bei 28 SSW, das mittlere Geburtsgewicht bei 906 g, der mediane CRIB-Score bei 5 Punkten. 5\% hatten eine ROP Grad 1,34\% eine ROP Grad 2. Eine höhergradige Hirnblutung lag bei 2 Kindern vor. Alle Frühgeborenen wurden im Alter von 6 Jahren mittels K-ABC nachuntersucht. 88\% waren normal intelligent, bei $7 \%$ lag der IQ $<8$ o, bei $5 \%<70$ Punkten. ANOVAs mit den unabhängigen Variablen Gruppe (Früh- vs. Reifgeborene), Jahrgang und Geschlecht ergaben signifikante Haupteffekte für die Gruppe und den Jahrgang bei allen drei Testverfahren $(p<0,05)$. Ein signifikanter Haupteffekt des Geschlechts zeigte sich bei der MRT-K-Buchstabenversion und dem MRT-A $(\mathrm{p}<0,05) \mathrm{zu}-$ gunsten der Jungen. Ein Entwicklungsfortschritt zeigte sich nur bei den Reifgeborenen bei der MRT-K-Buchstabenversion und im MRT-A. Die besuchte Klassenstufe und die Schulform hatten einen signifikanten
Effekt auf die mentale Rotationsleistung. Eine niedrigere Klassenstufe oder Schulform ging mit einer vergleichsweise niedrigeren Rotationsleistung einher.

Schlussfolgerung. Die Ergebnisse für die MRT-K-Buchstabenversion und den MRT-A zeigen deutlich, dass ehemalige VLBW-Frühgeborene in ihren mentalen Rotationsfähigkeiten schlechter abschneiden als gleichaltrige Reifgeborene und dass nur die Reifgeborenen einen Fortschritt aufweisen. Der Geschlechtsunterschied zugunsten der Jungen zeigt sich auch in vielen anderen Studien zur MR. Die Studie gibt einen ersten Hinweis auf einen Förderbedarf von Frühgeborenen im Bereich der MR. Für die Entwicklung geeigneter Förderkonzepte sind Folgestudien unter Einbezug weiterer Altersgruppen notwendig.

\section{PO-106}

Theory of Mind-Fähigkeiten im Alter von 10 und 13 Jahren bei ehemaligen VLBW-Frühgeborenen im Vergleich zu Reifgeborenen

S. Braun', T. Seifen', K. Mehler', A. Kribs², B. Roth' ${ }^{2}$, M. Kavsek 'Institut für Psychologie, Entwicklungspsychologie und pädagogische Psychologie, Bonn, Deutschland, ${ }^{2}$ Universitätskinderklinik Köln, Neonatologie und Pädiatrische Intensivmedizin, Köln, Deutschland

Hintergrund. Die Fähigkeit, einen nichtzutreffenden mentalen Zustand bei anderen Personen zu erkennen wird als Theory of Mind (ToM) bezeichnet und galt lange als spezifisch für Personen mit einer Autismus Spektrum Störung. Für das Verständnis von ToM-Aufgaben werden exekutive Funktionen und die Arbeitsgedächtniskapazität benötigt. Frühgeborene weisen in diesen beiden Variablen Defizite auf. Bei Frühgeborenen finden sich Auffälligkeiten im präfrontalen Kortex, der eine wichtige Rolle bei der Verarbeitung von ToM-Aufgaben spielt.

Fragestellung. Es wurde untersucht, ob bei der Bewältigung von ToM-Aufgaben Unterschiede zwischen ehemaligen Very-low-birthweight(VLBW)-Frühgeborenen und Reifgeborenen im Alter von 10 und 13 Jahren bestehen. Des Weiteren wurde der Entwicklungsverlauf der ToM von Früh- mit dem von Reifgeborenen verglichen.

Methoden. Es wurden zwei Gruppen der Jahrgänge 1999 und 2002 untersucht: 54 ehemalige Frühgeborene mit einem Geburtsgewicht unter $1500 \mathrm{~g}$ und 41 Reifgeborene. Allen Teilnehmern wurden FauxpasAufgaben und Mental-State-Geschichten in schriftlicher Form vorgelegt. Zusätzlich wurde ein Elternfragebogen zur Erhebung von Kontrollvariablen ausgegeben.

Ergebnisse. Das mittlere Gestationsalter lag in der Gruppe der Frühgeborenen bei 28 SSW, das mittlere Geburtsgewicht bei $906 \mathrm{~g}$, der mediane CRIB-Score bei 5 Punkten. 5\% hatten eine ROP Grad 1, 34\% eine ROP Grad 2. Eine höhergradige Hirnblutung lag bei 2 Kindern vor. Alle Frühgeborenen wurden im Alter von 6 Jahren mittels K-ABC nachuntersucht. $88 \%$ waren normal intelligent, bei $7 \%$ lag der IQ $<80$, bei $5 \%<70$ Punkten. Eine ANOVA mit den unabhängigen Variablen Gruppe (Früh- vs. Reifgeborene), Jahrgang und Geschlecht ergab signifikante Gruppen- $\left[\mathrm{F}(1)=5,59 ; \mathrm{p}<0,05 ; \mathrm{R}_{2}=0,06\right)$ und Jahrgangsunterschiede $[\mathrm{F}(1)=10,03 ; \mathrm{p}<0,01 ; \mathrm{R} 2=0,13)$ in den Antworten auf die Fauxpas-Aufgaben. Eine ANOVA mit der abhängigen Variable der „Mental-State-Geschichten“ ergab einen signifikanten Haupteffekt des Jahrgangs $[\mathrm{F}(1)=21,91 ; \mathrm{p}<0,01 ; \mathrm{R} 2=0,22]$. Deskriptiv zeigte sich auch hier eine Überlegenheit der Reifgeborenen gegenüber den Frühgeborenen. Kontrollvariablen, die einen signifikanten Effekt auf die Performanz in den beiden ToM-Aufgaben hatten, waren die Klassenstufe und die Schulform. Früh- und Reifgeborene des Geburtenjahrgangs 1999, die bereits die siebte Klassenstufe besuchten schnitten besser ab als Kinder in niedrigeren Klassenstufen. Frühgeborene Gymnasiasten schnitten deutlich besser ab als frühgeborene Haupt- und Förderschüler.

Schlussfolgerung. Die vorliegende Studie zeigt, dass Frühgeborene schlechter in ToM-Aufgaben abschneiden als Reifgeborene. Die Daten zeigten allerdings auch, dass einige Frühgeborene, speziell die Gymnasiasten, sehr gute Leistungen in den ToM-Aufgaben erbrachten. Folgestudien unter Berücksichtigung weiterer Altersgruppen, exekutiver 
Funktionen und neurologischer Daten könnten die Entwicklung von ToM-Fähigkeiten ehemaliger Frühgeborener genauer erfassen.

Immunologie - Infektionen - Hygiene

\section{PO-107}

Rolle der TNF-alpha-Internalisierung bei der Apoptoseinduktion in Monozyten

\section{S. Dreschers', F. Seubert', C. Gille², T. Orlikowsky'}

'Universitätsklinik der RWTH Aachen, Kinder- und Jugendmedizin, Neonatologie, Aachen, Deutschland, ${ }^{2}$ Eberhard-Karls-Universität Universitätsklinik für Kinderheilkunde und Jugendmedizin, Neonatologie, Tübingen, Deutschland

Hintergrund. Liganden und Rezeptoren der TNF-Familie (CD95L/R; TNF-alpha/TNFR1) vermitteln Apoptose in Monozyten. Bei Ligation von TNF-alpha und TNFR1 wird eine Internalisierung ausgelöst, die obligatorisch für die Apoptoseinduktion ist. TNF-alpha wird von Monozyten sezerniert, die E. coli phagozytieren und induziert einen Bystander-Zelltod in nichtphagozytierenden Monozyten. Letzterer ist in neonatalen Monozyten (CBMO) reduziert. Hypothese: Die TNFR1 vermittelte TNF-alpha-Internalisierung unterscheidet sich bei adulten Monozyten (PBMO) und CBMO dahingehend, dass TNF-alpha bei $\mathrm{CBMO}$ in geringerem $\mathrm{Maß}$ internalisiert wird und so eine geringere Apoptose bei einer E.-coli-Infektion hervorgerufen wird.

Methodik. Zellen wurden isoliert, phänotypisiert, mit Fluorochrom-exprimierenden E. coli infiziert (GFP-E. coli), Phagozytose- bzw. Apoptoseraten sowie TNF-alpha Internalisierung ermittelt (hypodiploide DNA; FACS-Analyse, ELISA). Cytochalasin D wurde als Inhibitor der Internalisierung verwendet.

Ergebnisse. Bei Zugabe von TNF-alpha unterschied sich die Fähigkeit zur TNF-alpha/TNFR1-Internalisierung und der Apoptose-Induktion zwischen CBMO und PBMO nicht $(42,35 \pm 15,6 \%$ vs. $27,51 \pm 4,1 \%$; $\mathrm{p}<0,05)$. Infektion mit GFP-E. coli führte zur stärkeren TNF-alpha/TNFR1-Internalisierung in nicht phagozytierenden PBMO als in nicht phagozytierenden CBMO (67 $\pm 12,6 \%$ vs. $29,43 \pm 4,6 \%$; $<<0,05)$; nachfolgend zeigte sich der Bystander-Zelltod in CBMO gegenüber PBMO um $73 \%$ reduziert $(\mathrm{p}<0,05)$. Dagegen unterschieden sich die phagozytierenden PBMO und CBMO bei identischer Phagozytoserate nicht in ihrer Sensitivität gegenüber Phagozytose-abhängigem Zelltod.

Schlussfolgerung. Der Mechanismus der TNF-alpha/TNFR1-Internalisierung existiert in PBMO und CBMO. Bei geringerer TNF-alpha-Sekretion wird der Prozess der Internalisierung in CBMO gedrosselt und deren Apoptoseinduktion reduziert.

\section{PO-108}

Apoptose von Monozyten im Infektionsmodell mit Escherichia coli: TNF-alpha-induzierte Aktivierung von Caspasen

\section{S. Dreschers' ${ }^{1}$, F. Seubert', C. Gille ${ }^{2}$, T. Orlikowsky?}

'Universitätsklinik für Kinder- und Jugendmedizin der RWTH Aachen, Neonatologie, Aachen, Deutschland, ${ }^{2}$ Eberhard-Karls-Universität Universitätsklinik für Kinderheilkunde und Jugendmedizin, Neonatologie, Tübingen, Deutschland

Hintergrund. Im Rahmen einer Sepsis wird durch Phagozytose von Bakterien in Monozyten Phagozytose-induzierter Zelltod ausgelöst, der durch Liganden und Rezeptoren der TNF-Familie vermittelt wird. Sezerniertes TNF-alpha aus phagozytierenden adulten Monozyten (PBMO) führt durch Bindung an TNF-Rezeptor 1 (TNFR1) über Internalisierung zur Aktivierung von Caspasen und Apoptose von nichtphagozytierenden PBMO.

Hypothese. Die extrinsische Apoptoseinduktion durch TNF-alpha und CD95L aktiviert die Caspase 3 in neonatalen Monozyten (CBMO) we- niger stark, entweder weil Liganden und/oder Rezeptoren im Vergleich zu PBMO reduziert sezerniert/präsentiert werden. Die Phagozytose ist essenziell für die Induktion der Apoptose.

Methodik. Zellen wurden isoliert, phänotypisiert, mit E. coli infiziert und mit TNF-alpha und LPS stimuliert. Als Inhibitor wurde ein blockierender Antikörper für TNF-alpha verwendet. Apoptoseinduktion wurde im Immunoblot durch Aktivierung der Effektor-Caspase 3 nach Spaltung in die Produkte p17 und pis detektiert und quantifiziert.

Ergebnisse. In PBMO wurde Caspase 3 durch TNF-alpha und E. coli aktiviert, in CBMO nur durch E. coli. Der TNF-alpha-inhibierende Antikörper reduzierte die Aktivität der Caspase 3 in PBMO.

Schlussfolgerung. In CBMO kann die Apoptose nur durch Phagozytose ausgelöst werden. Ein Bystander-Kill durch TNF-alpha aus phagozytierenden CBMO ist nicht möglich.

\section{PO-109}

Newborn plasmacytoid dentritic cells display only limited interferon-alpha expression - a quantitative multiplex real-time PCR analysis

\section{Wisgrill', S. Schüller', A. Spittler ${ }^{2}$, E. Förster-Waldl', K. Sadeghi'}

${ }^{1}$ Medizinische Universität Wien, Universitätsklinik für Kinder- und Jugendheilkunde, Wien, Österreich, ${ }^{2}$ Medizinische Universität Wien, Core Facility Flow Cytometry, Wien, Österreich

Background. Bacterial and viral infections cause high rates of morbidity and mortality in premature newborns. In the setting of viral infection, plasmacytoid dendritic cells (pDCs) play a key role as strong producers of interferon-alpha (IFN- $\alpha$ ) upon TLR9-dependent engagement with molecular viral patterns. The cytokine IFN- $\alpha$ is a crucial factor in host's viral defense.

Methods. To assess IFN- $\alpha$ RNA levels, pDCs from newborns and adults were sorted by means of fluorescence cell sorting and quantitative multiplex RT-PCR was conducted using primer probe sets for IFN- $\alpha_{2}$ and for $18 \mathrm{~S}$ RNA house keeping gene. Furthermore, IFN- $\alpha$ cytokine production was analysed on protein level by flow cytometry and by ELISA. Results. Upon TLR9 challenge with CpG-A DNA motifs we found an up to 6o-fold higher IFN- $\alpha$ RNA expression in adults then in term and preterm newborns. Furthermore, IFN- $\alpha$ cytokine levels were significantly decreased on protein level in both preterm and term neonates.

Conclusion. The reduced functional capacity of newborn pDCs to produce IFN- $\alpha$ on RNA and protein level likely renders such infants more susceptible to viral infections.

PO-110

Myeloide Suppressorzellen könnten zur postpartalen Immunsuppression beim Neugeborenen beitragen

\section{Scheckenbach', N. Köstlin', H. Kugel', B. Spring', C. Poets', C. Gille'}

'Eberhard-Karls-Universität Universitätsklinik für Kinderheilkunde und Jugendmedizin, Neonatologie, Tübingen, Deutschland

Hintergrund. Während der Schwangerschaft ist das fetale Immunsystem zugunsten des Schwangeschaftserhalts unterdrückt. Postpartal muss das neonatale Immunsystem diese Immunsuppression überwinden. Myeloide Suppressorzellen sind Vorläuferzellen, die im Rahmen pathologischer Zustände wie Tumorerkrankungen und Infektionen aber auch im Nabelschnurblut stark vermehrt vorkommen und angeborene und adaptive Immunzellen hemmen können. Es ist unklar wie sich die Anzahl der MDSC postpartal entwickelt und wie sich diese Zellen auf die Adaptation des neonatalen Immunsystems auswirken.

Hypothese. Die Anzahl an MDSC nimmt postpartal rasch ab, kann aber im Rahmen von Inflammationsprozessen hochreguliert werden.

Methoden. Mononukleäre Zellen (PBMC) aus Nabelschnurblut und peripherem Blut Frühgeborener in den ersten zwei Lebenswochen wurden isoliert und der Anteil an granulozytären MDSC (CD66b+/CD $33+1$ 
CD14-/HLA-DR-) wurde durchflusszytometrisch bestimmt. Als Vergleich dienten PBMC aus dem peripheren Blut gesunder Erwachsener. Ergebnisse. Die Anzahl granulozytärer MDSC war im Nabelschnurblut (median 4,5\%; Range: 2,8-11,8; $\mathrm{n}=10$ ) im Vergleich zum peripheren Blut Erwachsener (o,6\%; Range: $0,2-1,4 ; n=8)$ erhöht. In der ersten Lebenswoche blieb die Zahl weiterhin erhöht (median 2,2\%, Range: 0,7-3,4; $\mathrm{n}=7$ ). An Tag 14 war die Anzahl an MDSC im peripheren Blut nicht akut kranker Frühgeborener nicht höher als im Blut Erwachsener (0,99\%; Range: $0,2-1,9 ; n=7$ ). Kinder mit perinatalen Pathologien (Atemnotsyndrom, Sepsis) hatten in diesem Zeitraum deutlich mehr MDSC (median 14,93\%; Range: $9,7-38,8 ; n=5$ ).

Schlussfolgerung. Die hohe Zahl von MDSC im Nabelschnurblut nimmt unter physiologischen Bedingungen in den ersten 14 Lebenstagen stark ab. Unter pathologischen Bedingungen kann die Zahl an MDSC weiter erhöht bleiben. Die Regulation von MDSC könnte für die postpartale Immunadaptation wichtig sein.

\section{PO-111}

\section{Neugeborenensepsis infolge Belastung von Muttermilch mit pathogenen Keimen?}

\section{S. Kirchhoff', M. Kolokythas', A. Kramer', M. Kujawa', M. Dittmann", S. Schubert ${ }^{2}$, A. Flemmer', A. Schulze ${ }^{1}$}

'LMU München, Neonatologie der Kinderklinik am Perinatalzentrum Großhadern, München, Deutschland, ${ }^{2}$ Max von Pettenkofer-Institut, Bakteriologie, München, Deutschland

Einleitung. Muttermilch (MM) wird nicht regelhaft vor der Gabe an Frühgeborene (FG) bakteriologisch untersucht. Häufig wird angenommen, dass Allgemeininfektionen Frühgeborener nicht durch Keime aus der Milch der eigenen Mutter ausgelöst werden können. Folgende Kasuistik dokumentiert die Übertragung einer B-Streptokokkensepsis über Muttermilch.

Kasuistik. Ein FG wird bei Fruchtblasenprolaps in der $23+5$ SSW (GG: $610 \mathrm{~g})$ spontan geboren. Im Vaginalabstrich der Mutter konnten zum Zeitpunkt der Geburt B-Streptokokken nachgewiesen werden. Im postpartal entnommenen Abstrich des Kindes wurden ebenfalls B-Streptokokken nachgewiesen. Am 29. LT entwickelte das Kind eine fulminante Sepsis mit einem max. CRP von 5,o mg/dl. Zu diesem Zeitpunkt erfolgte die Ernährung mit unpasteurisierter MM. Eine antibiotische Therapie mit Meropenem und Vancomycin wurde begonnen. Sowohl in der Blutkultur als auch im Trachealsekret des Kindes konnten BStreptokokken isoliert werden. Parallel zum Auftreten der Infektion konnte die Mutter des Kindes als Spenderin von Frauenmilch gewonnen werden, so dass die MM mikrobiologisch untersucht wurde. Auch hier konnten B-Streptokokken nachgewiesen werden. Mutter und Kind erhielten nach wiederholtem Nachweis von B-Streptokokken zur Sanierung über 7 Tage Amoxicillin bzw. Penicillin. In der Folge erhielt das Kind bis zu einem korrigierten Alter von 32 SSW pasteurisierte MM. In dieser konnten keine B-Streptokokken nachgewiesen werden. Eine Sanierung der rohen MM konnte nicht erreicht werden. Unter kontrollierten stationären Bedingungen wurde die Fütterung mit roher MM erneut begonnen. Klinisch und laborchemisch zeigte sich kein Anhalt für das Auftreten einer Reinfektion. Zwei weitere Fälle legen ebenfalls die Muttermilch als Infektionsquelle nahe. Ein Zwillingsfrühgeborenes der 27+6 SSW (GG: 920 g) wird aufgrund kardialer Dekompensation bei fetofetalem Transfusionssyndrom per Sectio geboren. Am 73. LT kam es zu einer fulminaten Sepsis mit einem CRP von max. 8,o mg/ dl. Staphylococcus aureus und vergrünende Streptokokken konnten sowohl in der MM als auch wiederholt aus dem Augenabstrich des Kindes isoliert werden. Ein weiteres FG wurde bei Zervixinsuffizienz in der 29+1 SSW (GG: 1345 g) per Sectio geboren. Am 10. LT setzte das Kind rezidivierend frischblutige dünne Stühle ab. Im Stuhl des Kindes und in der MM konnte ein hämolysierender E. coli gleichen Resistenzmusters isoliert werden.
Schlussfolgerung. Ursächlich für die schweren Infektionen der vorgestellten Kinder sind vermutlich maternale Bakterien, die über die Muttermilch übertragen wurden. Die Milch wurde in den beschriebenen Fällen ausschließlich unpasteurisiert verabreicht. Für sehr kleine FG sollte abgepumpte Muttermilch mikrobiologisch auf pathogene Keime untersucht werden und diese gegebenenfalls nur pasteurisiert verfüttert werden. Des Weiteren sollte die Anleitung der Mütter in das Vorgehen des Abpumpens optimiert werden, um auch so mögliche Kontaminationen zu vermeiden.

\section{PO-112}

Mikrobiologisches Monitoring von Spendermilch für Frühgeborene nach Gewinnung unter besonderen Standards

A. Kramer', S. Kirchhoff', M. Kujawa', A. Flemmer' ${ }^{2}$ A. Schulze'2, S. Schubert ${ }^{1}$ ${ }^{1} M a x$ von Pettenkofer-Institut für Hygiene und Medizinische Mikrobiologie, LMU, München, Deutschland, ${ }^{2}$ Dr. von Haunersches Kinderspital, Neonatologie der Kinderklinik am Perinatalzentrum Großhadern, München, Deutschland

Hintergrund. Die Frauenmilchbank der Neugeborenen-Intensivstation am Perinatalzentrum Großhadern stellt Spendermilch für sehr kleine Frühgeborene in den ersten Lebenstagen bereit. Die Gewinnung der Spendermilch erfolgt unter folgenden Standards mit dem Ziel, die Spendermilch unpasteurisiert verfüttern zu können: Gewinnung (1) nur vor Ort in separatem Raum, (2) unter ständiger Aufsicht einer Laktationsberaterin, (3) unter Verwendung steriler Hilfsmittel und (4) sofortigem Schockgefrieren der Spendermilch.

Fragestellung. Welches bakteriologische Spektrum weist unter diesen Bedingungen gewonnene Spendermilch auf?

Methode. Mittels kulturellem Verfahren auf unselektivem und selektivem Nährmedium (Blut-Agar und MacConkey-Agar) wurden im Jahr 2012158 Proben frisch abgepumpter Frauenmilch von 1o Spenderinnen auf das Vorkommen und die Menge von Bakterien untersucht. Die Proben wurden qualitativ (Unterscheidung zwischen pathogenen Keimen und Hautflora) und quantitativ (Keimzahlbestimmung) untersucht. Bei jeglichem Nachweis von pathogenen Keimen wurde die Spendermilch verworfen. Bei Nachweis von Hautflora bis 105 koloniebildenden Einheiten (KBE)/ml wurde die Frauenmilch unpasteurisiert verwendet, bei Hautflora in Keimzahlen $>105 \mathrm{KBE} / \mathrm{ml}$ wurde sie pasteurisiert. Diese Kategorisierung basiert auf Kriterien bestehender Frauenmilchbanken und Expertenmeinungen.

Ergebnisse. Bei $131(82,9 \%)$ der insgesamt 158 Proben wurde ein Wachstum von bis zu $105 \mathrm{KBE} / \mathrm{ml}$ Hautflora festgestellt. Diese Spendermilch wurde zur unpasteurisierten Gabe freigegeben. Bei 14 Proben (8,7\%) wurde $>105 \mathrm{KBE} / \mathrm{ml}$ Hautflora festgestellt, so dass die Spendermilch pasteurisiert wurde. In $13(8,2 \%)$ Fällen erfolgte der Nachweis pathogener Keime (Staphylococcus aureus, Enterobacteriaceae, Candida spp.). Diese Frauenmilch stammte von 3 Spenderinnen und wurde verworfen. Bei 2 der 3 Spenderinnen trat die Belastung der Milch mit pathogenen Keimen mit großem zeitlichen Abstand zweimalig bzw. einmalig auf. Bei der dritten Spenderin wurden mehrfach in Folge pathogene Keime nachgewiesen.

Schlussfolgerung. Bei allen untersuchten Proben waren Keime in der Spendermilch nachweisbar. Sowohl Keimarten als auch Keimzahlen in der Milch auch derselben Spenderin unterlagen hohen Schwankungen. Trotz der besonderen Bedingungen bei der Gewinnung der Spendermilch konnten in einem überraschend hohen Anteil der Proben die Kriterien für die unpasteurisierte Freigabe nicht erreicht werden. Um einerseits so wenig wie möglich Frauenmilch zu verwerfen und andererseits die größte mögliche Sicherheit für jede einzelne Probe zu gewährleisten, sollte daher die mikrobiologische Untersuchung jeder Spendermilchprobe durchgeführt werden. 


\section{PO-113}

\section{MRSA - Gehäufte Besiedlungen auf einer gemischten Kinderinten-} sivstation: erfolgreiche Ursachensuche und ihre Konsequenzen

S. Trepels-Kottek', S. Scheithauer' ${ }^{2}$, M. Schoberer', K. Heimann', H. Häfner ${ }^{3}$, T. Ittel' ${ }^{4}$, D. Keller ${ }^{5}$, S. Lemmen ${ }^{3}$, T. Orlikowsky ${ }^{6}$

${ }^{1}$ Universitätsklinik für Kinder- und Jugendmedizin, Sektion Neonatologie und Kinderintensivmedizin, Aachen, Deutschland, ${ }^{2}$ Universitätsklinikum Aachen, Zentralbereich für Krankenhaushygiene und Infektiologie ZfKI, Aachen, Deutschland, ${ }^{3}$ Universitätsklinikum Aachen, Zentralbereich für Krankenhaushygiene und Infektiologie ZfKl, Aachen, Deutschland, ${ }^{4}$ Universitätsklinikum Aachen, Ärztlicher Direktor, Aachen, Deutschland, ${ }^{5}$ Universitätsklinikum Aachen, Hochschulärztin, Aachen, Deutschland, ${ }^{6}$ Universitätsklinik für Kinder- und Jugendmedizin, Sektion für Neonatologie, Aachen, Deutschland

Einleitung. Während in den letzten 3 Jahren in unserem Patientenkollektiv o bis 2 Nachweise von Methicillin resistenten S. aureus (MRSA)/ Jahr auftraten, zeigte sich von Juli bis November 2012 eine zweizeitige Häufung mit insgesamt ${ }_{13}$ für MRSA positiven Abstrichen, jedoch ohne Infektionen. Die Intensivstation umfasst 18 Beatmungsplätze, ist zu 90\% mit Frühgeborenen belegt (Perinatalzentrum Level I) und versorgt pro Jahr etwa 250 Patienten. Im Zuge der Brisanz der Situation führten wir eine erweiterte Ursachensuche durch.

Methodik. Es erfolgten neben standardisierten Schutzmaßnahmen (Schutzkleidung für das Personal, Einzelzimmer, Kohortenpflege) eine Erweiterung um die Schutzkleidung für die Eltern. Im Rahmen des Benchmarking für Händedesinfektion liegt die Station in Deutschland auf der 90. Perzentile mit einem Desinfektionsmittelverbrauch von $158 \mathrm{ml} /$ Patiententag. Alle MRSA-Stämme wurden mittels Pulsfeld-Gelelektrophorese und spa-Typisierung charakterisiert. Ein Screening der Eltern und Umgebungsuntersuchungen brachten keine Erkenntnisse zur Quelle. Ein Personalscreening (Personal der NICU/PICU, Konsiliarien, Geburtshilfe) wurde durch den Hochschularzt initiiert.

Ergebnisse. Die molekulare Typisierung erbrachte in 8 Fällen spa Typ t127, einen in Deutschland seltenen, jedoch in einzelnen osteuropäischen Ländern häufiger Stamm. Die Personaluntersuchungen erbrachte zwei positive Ergebnisse, spa Typ 1265 und spa Typ t127. Letzterer wurde bei einer vor kurzem nach Deutschland gekommenen osteuropäischen Mitarbeiterin gefunden (Zustimmung der Mitarbeiterin zur der Veröffentlichung dieser Daten). Die Mitarbeiterinnen konnten eradiziert werden und arbeiten unter regelmäßiger Kontrolle durch die Hochschulärztin wieder auf der Intensivstation. Keine weiteren Fälle traten seitdem auf.

Schlussfolgerung. Über die Spa-Typisierung können Cluster zugeordnet werden, unter Umständen Übertragungswege aufgezeigt und neue Quellen (Mitarbeiter aus Gegenden mit hoher MRSA Prävalenz) identifiziert werden. Als Konsequenz wurde für alle neuen Mitarbeiter des Hauses, unabhängig ihrer Herkunft, bei der Einstellungsuntersuchung ein MRSA-Screening implementiert.

\section{Hämatologie - Gerinnung - Hämotherapie}

\section{PO-114}

Rezidivierende aplastische Anämie nach persistierender konnataler Parvovirus-B19-Infektion bei frühgeborenen dizygoten Zwillingen Remission nach intravenöser Immunglobulingabe

\section{A. Lejeune', M. Cremer', H. von Bernuth', S. Modrow', C. Bührer'}

${ }^{1}$ Charité Universitätsmedizin Berlin, Klinik für Pädiatrie m. S. Pneumologie und Immunologie, Berlin, Deutschland, ${ }^{2}$ Charité - Universitätsmedizin Berlin, Klinik für Neonatologie, Campus Mitte, Berlin, Deutschland, ${ }^{3}$ Universität Regensburg, Institut für Medizinische Mikrobiologie und Hygiene, Regensburg, Deutschland, ${ }^{4}$ Charité - Universitätsmedizin Berlin, Klinik für Neonatologie, Berlin, Deutschland

Hintergrund. Maternale Primärinfektionen mit humanem Parvovirus B19 (HPV-B19) in der Schwangerschaft sind häufig Ursache eines nichtimmunologisch bedingten Hydrops fetalis. HPV-B19 befällt die erythrogenen Vorläuferzellen, führt so zu einer transienten aplastischen Anämie und kann intrauterin aufgrund des hohen Umsatzes an Erythrozyten zu einem anämiebedingten Hydrops fetalis führen, welcher in 10\% der Fälle letal endet. Etwa 50\% aller schwangeren Frauen zeigen keine ausreichende Immunität gegenüber Parvovirus B19. Die kausale Therapie der Anämie besteht in intrauterinen Bluttransfusionen, welche in der Regel bei erfolgreicher Durchführung und Überleben des Kindes zur terminnahen Geburt eines gesunden Neugeborenen ohne Folgeprobleme führt.

Fallbericht. Wir berichten über eine fetale HPV-B19 Infektion bei einer dichorialen Geminigravidität, die über einen Hydrops fetalis bei Fetus 1 klinisch auffiel. Bei Fetus 1 konnte wegen der ungünstigen Kindslage keine intrauterine Transfusion durchgeführt werden. Hier entwickelte sich ein zunehmender Hydrops, der zur vorzeitigen Entbindung mit $29+4$ SSW führte. Fetus 2 zeigte sich sonographisch stets unauffällig. Postnatal zeigte Zwilling 1 (Geburtsgewicht: 1200 g, Hb 5,2 g/dl) das Vollbild eines Hydrops fetalis mit generalisiertem Ödem, Aszites und hypertropher Kardiomyopathie, der andere Zwilling (Geburtsgewicht: $1495 \mathrm{~g}, \mathrm{Hb}$ fast normal) war klinisch unauffällig. Beide Neugeborenen erhielten während des stationären Aufenthalts mehrere Bluttransfusionen und zeigten eine massive Parvoviruslast im peripheren Blut (>10 Mio. Kopien/ml). Die Kinder wurden stabil nach Hause entlassen, entwickelten jedoch in der Folgezeit einen dramatischen Hb-Abfall mit notfallmäßiger Wiederaufnahme im 3. Lebensmonat mit schwerer aplastischer Anämie beider Kinder (Kind 1: $\mathrm{Hb} 2,8 \mathrm{~g} / \mathrm{dl}$, Kind 2: $\mathrm{Hb} 5,9$ g/ dl) bei persistierender HPV-B19-Infektion mit weiterhin hoher peripherer Viruslast (>10 Mio. Kopien/ml). Nach Rücksprache mit Virologen und Immunologen und Durchsicht der aktuellen Literatur erfolgte ein Therapieversuch mit intravenösem Immunglobulin G (Kiovig ${ }^{\circledR}$ ) in einer Dosierung von $4 \mathrm{~g} / \mathrm{kg}$ KG über 5 Tage. Im Anschluss daran zeigten sich die Kinder klinisch unauffällig mit bis heute (5 Monate nach Therapie) stabilen $\mathrm{Hb}$-Werten und kontinuierlich sinkender Viruslast sowie Serokonversion der spezifischen Antikörper mit Nachweis von IgG und Verschwinden von IgM. Eine Immundefektdiagnostik erbrachte keinen Anhalt für einen zugrundeliegenden Immundefekt als Ursache dieses schweren Verlaufs.

Diskussion. In mehreren Fallberichten wurde bereits zuvor über eine erfolgreiche Reduktion der Parvorirus-B19-Viruslast durch Immunglobulingabe bei immunologisch beeinträchtigten Patienten, z. B. nach Knochenmarktransplantation, berichtet. Das nachhaltige Ansprechen der aplastischen Anämie auf intravenöse Immunglobulingabe unterstreicht den möglichen Stellenwert dieser Therapieoption auch bei Frühgeborenen mit fetaler Parvovirus-B19-Infektion. 


\section{PO-115}

\section{Langzeit-Outcome nach extremer Frühgeburt und Hydrops fetalis bei Parvovirus-B19-Infektion}

\section{S. Welcker', S. Groß', M. Heckmann²}

'Zentrum für Kinderheilkunde der Justus-v.-Liebig-Universität, Neuropädiatrie, Gießen, Deutschland, ${ }^{2}$ Klinik und Poliklinik für Kinder und Jugendmedizin, Abt. Neonatologie u. Päd. Intensivmedizin, Greifswald, Deutschland

Hintergrund. Die Inzidenz einer Parvovirus(PV)-B19-Serokonversion während der Schwangerschaft beträgt 1-1,5\%, in epidemischen Perioden bis zu $13 \%$. Das Risiko einer vertikalen Transmission variiert zwischen $33-51 \%$. In bis zu 12,5\% tritt ein Hydrops fetalis mit einer Mortalität 30 bis $>90 \%$ auf. Es liegen zwar 3 Längsschnittstudien zur neurologischen Entwicklung bei konnataler PV-B19-Infektion vor. Aber nur eine Studie berichtete über ein einziges extrem unreifes Frühgeborenes (FG). Wir berichten hier erstmals das Langzeit-Outcome bis zum 9. Lebensjahr nach extremer Frühgeburt und Hydrops fetalis bei PV-B19-Infektion. Fall 1. 31-jährige Mutter, GII/PI, Sectio in der $25+3$ SSW [GG $730 \mathrm{~g}\left(\mathrm{P}_{25}-\right.$ 50], Länge $31 \mathrm{~cm}\left(\mathrm{P}_{10-25}\right), \mathrm{KU}_{21} \mathrm{~cm}\left(\mathrm{P}_{3}\right), \mathrm{APGAR}_{3} / 6 / 8$, art. NS-pH 7,31) bei HELLP-Syndrom. Pränatal bekannter Hydrops fetalis sowie positive PV-B19-Serologie und Virusnachweis. Intrauterine Transfusion (Hb-Anstieg von $6,8 \mathrm{mg} / \mathrm{dl}$ auf $12 \mathrm{mg} / \mathrm{dl}$ ) und Aszitespunktion in der 24+6SSW. Einschätzung der Prognose als infaust in der Pränatalberatung. Komplizierter postnataler Verlauf durch PPHN mit NO-Therapie, reduzierter linksventrikulärer Funktion, ANS III $^{\circ}$, schwere BPD, PDA, IVH I', keine PVL, ROP Stad 3, Septitiden, schwere Gedeihstörung. Mit korrigiert 20 Monaten Entwicklungsverzögerung von 5 Monaten mit leichter infantiler Cerebralparese. Im Alter von 4 Jahren intellektuelle Fähigkeiten knapp innerhalb der Normtoleranz mit unauffälliger sprachlicher aber deutlich retardierter statomotorischer Entwicklung. Mit 9 Jahren geringe Defizite in Gleichgewicht und Koordination; Besuch der 3. Klasse mit sonderpädagogischer Förderung bei leichter Lernbehinderung.

Fall 2. 33-jährige Mutter, GIII/PIII, Sectio in der $26+3$ SSW [GG $870 \mathrm{~g}$ (P25-50], Länge $33 \mathrm{~cm}\left(\mathrm{P}_{25}-50\right), \mathrm{KU} 23,2 \mathrm{~cm}$ (P10-25), APGAR 6/7/8, art. NS-pH 7,1) bei pathologischem CTG. Pränatal bekannter Hydrops fetalis mit Aszites bei seit 3 Monaten bestehender maternaler PV-B19Infektion. Komplizierter postnataler Verlauf mit Anämie ( $\mathrm{Hb} 4,3 \mathrm{~g} / \mathrm{dl}$ ), PPHN mit NO-Therapie, ANS III-IV ${ }^{\circ}$, Langzeitbeatmung (67 Tage), schwere BPD, PDA, keine IVH, keine PVL, ROP Stad 1/2. Im Verlauf deutlich verzögerte frühkindliche Entwicklung, schwere Gedeihstörung. Sprechen erster Worte und Sitzen mit 2 Jahren, Laufen mit 3 Jahren. Mit 6 Jahren ausgeprägte Lernbehinderung sowie nicht altersentsprechende Motorik. Im Alter von 8 Jahren Besuch der 1. Klasse mit sonderpädagogischer Förderung und Integrationshelfer. Defizite in Gleichgewicht und Koordination.

Schlussfolgerung. Die beiden Fälle zeigen die Verläufe von extrem unreifen FG nach Hydrops fetalis bei konnataler PV-B19-Infektion bis in das Grundschulalter. Trotz postnataler Komplikationen war besonders im ersten Fall eine zufriedenstellende Entwicklung bis zum 9. Lebensjahr zu beobachten. Unsere Daten können bei der schwierigen individuellen pränatalen Beratung bezüglich Frühgeburtlichkeit und Hydrops fetalis bei PV-B19-Infektion helfen.

\section{PO-116}

\section{Transient-myeloproliferatives Syndrom bei Trisomie 21 - Hydrops und Hepatopathie als prognostisch ungünstige Faktoren}

S. Römer' ${ }^{1}$ C. Czernik' , P. Hernaiz-Driever ${ }^{2}$, C. Bührer ${ }^{1}$

${ }^{1}$ Charité - Universitätsmedizin Berlin, Klinik für Neonatologie, Berlin, Deutschland, ${ }^{2}$ Charité Universitätsmedizin Berlin, Klinik für Pädiatrie m. S. Onkologie und Hämatologie, Berlin, Deutschland

Einleitung. Neugeborene mit Trisomie 21 haben ein Risiko von ca. 10\%, ein transient-myeloproliferatives Syndrom (TMD) zu entwickeln, häu- fig in Form einer megakaryozytären AML. Diese kann nach wenigen Monaten spontan ausheilen oder vorübergehend in Remission gehen und nach mehreren Jahren rezidivieren. Die meisten Patienten sind a- bis oligosymptomatisch. Eine Untergruppe von ca. 10\% zeigt jedoch schwere Symptome und potentiell letale peri- und postnatale Komplikationen.

Fallbericht. 44-jährige Mutter, G8, P6, 2-mal Frühabort. Eltern nicht konsanguin. Insulinpflichtiger Gestationsdiabetes. In der Feindiagnostik rechtsseitiger Aortenbogen, keine Amniozentese. Notsectio bei pathologischem CTG nach 38 SSW. Geburtsgewicht 3805 g (90. Perzentile), $\mathrm{NapH}$ 7,02, Apgar 2/4/5. Initial kardiorespiratorisch deprimiert, im Rahmen der Erstversorgung Intubation bei zunehmender respiratorischer Insuffizienz. Auffallend generalisierte Ödeme bei plethorischem Hautkolorit und Hepatomegalie (Leber $5 \mathrm{~cm}$ unter Rippenbogen), Trisomie-21-Aspekt, in der Chromosomenanalyse bestätigt. Im Blutbild Verdacht auf ein TMD (Leukozyten max. 130/nl, Myeloblasten 55\%, Thrombopenie). Nachweis GATA1-Mutation. Bei instabiler klinischer Situation (Ergüsse, pulmonale Hypertonie, Hepatopathie mit Gerinnungsstörung, transfusionswürdige Anämie und Thrombozytopenie) Chemotherapieversuch mit Cytarabin. Ein Tag nach Beginn des zweiten Zyklus progredientes Multiorganversagen, Tod im ausgeprägtem Kapillarlecksyndrom am 50. Lebenstag.

Diskussion. Ein letaler Verlauf ist beim transienten myelodysplastischen Syndrom selten. Bei unserem Patienten erwies sich die ausgeprägte Hepatopathie mit Hydrops und dauerhafter Transfusionspflicht als prognostisch schlechter Faktor. Trotz/nach Cytarabin entwickelte sich eine Leberfibrose und Multiorganversagen, welche zum Tod führten.

\section{PO-117}

Haemophilia in extreme immature preterm infants - increased risk for intracranial haemorrhage?

\section{S. Fink', V. Wiegering', O. Andres', M. Eyrich', S. Kunzmann'}

'Universitäts-Kinderklinik Würzburg, Würzburg, Deutschland

Introduction. While a considerable amount of information on haemophilia in full term neonates has been published over the last decades, data on premature infants with haemophilia remains restricted to a few case reports. The estimation of the bleeding risk as well as the indication and dosage of prophylactic factor replacement therapy therefore are difficult to assess.

Case report. We outline recently published cases on premature haemophiliacs and report about an extreme premature haemophiliac infant $(26+4$ gestational weeks, $635 \mathrm{~g}$ birth weight $)$ without prophylactic factor treatment. Diagnosis of a severe haemophilia A was made by chance at twelve weeks of life before the surgery of bilateral inguinal hernias. No intracranial bleeding and no other bleeding complications before starting substitution therapy before operation were detected.

Conclusion. While prophylaxis substitution of preterm infants with haemophilia A or B can surely minimize the risk of bleeding complications caused by invasive procedures or potential delivery caused brain bleedings, the link of lack of factor VIII or IX as potential risk factor for classical IVH of extreme preterm infants is not so clear.

\section{PO-118}

Diagnostik und Therapie einer Thrombose der Aorta abdominalis bei einem Frühgeborenen

\section{Poralla', M. Born' ${ }^{2}$, P. Bartmann', H. Hertfelder ${ }^{3}$, A. Müller'}

'Universitätsklinik Zentrum f. Kinderheilkunde, Neonatologie, Bonn, Deutschland, ${ }^{2}$ Radiologische Klinik Universitätsklinikum Bonn, Bonn, Deutschland, ${ }^{3}$ Institut für Experimentelle Hämatologie und Transfusionsmedizin/Universitätsklinikum Bonn, Bonn, Deutschland

Hintergrund. Thrombosen der Aorta treten im Neugeborenenalter sehr selten auf, können aber schwere Langzeitschäden verursachen und so- 
gar lebensbedrohlich verlaufen. Diagnostik und Therapie sind aufgrund der Seltenheit des Krankheitsbildes nicht standardisiert.

Fallbericht. Weibliches Frühgeborenes, intrauterine Wachstumsretardierung, Oligohydramnion, Sectio mit $35+6$ SSW bei pathologischer Dopplersonographie. Gewicht: 1680 g $(<3$. Perz.), Länge $42,5 \mathrm{~cm}$ (<3. Perz.), Kopfumfang $29 \mathrm{~cm}$ (<3. Perz.), Hkt 62\%. Infektparameter negativ, unkomplizierte postnatale Adaptation. Am Ende des ersten Lebenstages fiel ein livides Hautkolorit distal des Bauchnabels auf bei nicht tastbaren Pulsen, nicht messbarer Sauerstoffsättigung und nicht messbarem Blutdruck an den unteren Extremitäten. Die Echokardiographie zeigte keinen Hinweis auf eine Aortenisthmusstenose und zunächst unauffällige abdominelle Gefäße. Bei verminderter Flussgeschwindigkeit in den Femoralarterien und erhöhten D-Dimeren bestand der Verdacht auf eine partielle Thrombose der abdominellen Aorta, woraufhin eine Therapie mit Enoxaparin begonnen wurde. Zwei Tage später konnte dopplersonographisch ein Thrombus kranial der Aortenbifurkation mit Verlegung von $70 \%$ des Lumens nachgewiesen werden. Unter einer thrombolytischen Therapie mit rt-PA und fortgesetzter Heparinisierung war der Thrombus schrittweise größenregredient. Nach 4 Wochen wurde die medikamentöse Therapie auf Enoxaparin und ASS umgestellt, bei Verlaufsuntersuchung nach 6 Monaten war kein Thrombus mehr nachweisbar.

Diskussion. Neben einem Nabelarterienkatheter als häufigstem Risikofaktor begünstigen neonatale Asphyxie, Sepsis, Frühgeburtlichkeit, Polyzythämie, pulmonale und kardiale Anomalien, Dehydratation und angeborene Thrombophilien eine Thrombose der Aorta. Unsere Patientin hatte zu keinem Zeitpunkt einen arteriellen Zugang, prothrombotische Gerinnungsstörungen wurden ausgeschlossen.

Schlussfolgerung. Auch bei Frühgeborenen ohne Nabelarterienkatheter muss bei entsprechender Symptomatik an eine Thrombose der Aorta gedacht werden. Bei unserer Patientin erwies sich eine Lysetherapie mit zusätzlicher Heparinisierung als effektive und sichere Therapie.

\section{PO-119}

\section{Symptomatisches, kongenitales Hämangiom der Leber: Verlauf unter Therapie mit Propanolol}

\section{Pocivalnik', F. Reiterer', H. Lackner', M. Riccabona 3 , C. Urban'2, B. Urles- berger ${ }^{1}$ \\ 'Univ.Klinik für Kinder- und Jugendheilkunde Graz, Klinische Abteilung für Neonatologie, Graz, Österreich, ${ }^{2}$ Universitätsklinik für Kinder- und Jugend- heilkunde Graz, Klinische Abteilung für Pädiatrische Hämatologie und Onkologie, Graz, Österreich, ${ }^{3} U n i v$. Klinik für Radiologie, Klinische Abteilung für Kinderradiologie, Graz, Österreich}

Hintergrund. Leberhämangiome (LH) gehören zu den häufigsten benignen Lebertumoren im Kindesalter mit überwiegender Manifestation in den ersten 2 Lebensmonaten. Sie können asymptomatisch bis lebensbedrohlich verlaufen. Die klinische Symptomatik ist abhängig vom Ausmaß der Läsionen (fokal, multifokal, diffus) und dem Auftreten von intrahepatischen Shunts, welche zu einer Herzinsuffizienz führen können. Neben dem Einsatz von Propanolol bei infantilen kutanen Hämangiomen wurde in den letzten Jahren auch über die erfolgreiche Therapie bei LH berichtet.

Fragestellung. Wie verhält sich der klinische Verlauf eines symptomatischen kongenitalen Leberhämangioms unter einer medikamentösen Therapie mit Propanolol.

Material und Methoden. Wir beschreiben die klinischen Charakteristika, Labor- und Songraphiebefunde, sowie den ambulanten Verlauf bis zur letzten Kontrolle im Alter von 1 Jahr bei einem ehemaligen männlichen Frühgeborenen $(36+2 / 7$ SSW) mit symptomatischem, kongenitalem, fokalem LH.

Ergebnisse. Das Neugeborene wurde postpartal auffällig durch Atemnotsymptome, Hepatomegalie, Kardiomegalie, Bradykardien, sowie echokardiographisch verifiziertem, pulmonalem Hypertonus. Die Abdomensonografie zeigte einen über $10 \mathrm{~cm}$ durchmessenden vasku- lären Tumor vorwiegend des vergrößerten rechten Leberlappens mit dilatierter drainierender Lebervene und deutlichen arteriovenösen Shunts. Im kontrastgestützten Ultraschall wies er ein Hämangiom-typisches Enhancement-Muster auf. Laborkontrollen ergaben ein mildes Kasabach-Merritt-Phänomen, erhöhte Herz- und Leberenzymwerte, negative Tumormarker und ein erhöhtes TSH. Unter der Annahme eines symptomatischen LH wurde eine systemische orale Therapie mit Prednisolon (2 mg/kg/Tag) und Propranolol (Inderal $2 \mathrm{mg} / \mathrm{kg} / \mathrm{Tag}$ ) eingeleitet. Dies führte zu einem raschen Rückgang der Akutsymptomatik, so dass das Neugeborene bereits am 17. Lebenstag in ambulante Kontrollen entlassen werden konnte. Ab dem 3. Lebensmonat erfolgte eine Monotherapie mit Propanolol, unter der außer einer initialen Bradykardieneigung keine weiteren Nebenwirkungen auftraten. Im Alter von 12 Monaten zeigte sich sonographisch eine deutliche Tumorregression mit Verkalkungen bei klinisch unauffälligem Kind. Daraufhin wurde mit dem Ausschleichen der Therapie begonnen.

Diskussion. Obwohl fokale LH meist asymptomatisch verlaufen, kam es in unserem Fall durch die Größe des Tumors und die assoziierten Shunt-Phänomene zu klinisch relevanten Begleitsymptomen, insbesondere zu Herzinsuffizienzzeichen, welche eine medikamentöse Therapieeinleitung rechtfertigten. Bei Fehlen aktueller, evidenzbasierter Therapierichtlinien für LH entschieden wir uns auf Grund rezenter positiver Fallberichte in der Literatur für eine längerfristige Therapie mit Propanolol, welche bislang einen günstigen Verlauf zeigte.

Schlussfolgerung. Eine systemische Propanololtherapie ist auch bei symptomatischen, kongenitalen LH eine effektive und relativ nebenwirkungsarme Therapieoption.

\section{PO-120}

Austauschtransfusion in der Neonatologie bei Sepsis mit ausgeprägter Hämolyse

\section{S. Poralla', P. Bartmann', J. Oldenburg', J. Hoch², A. Müller'}

'Zentrum für Kinderheilkunde am Universitätsklinikum Bonn/Neonatologie, Bonn, Deutschland, ${ }^{2}$ Universitätsklinikum Bonn, Institut für Experimentelle Hämatologie und Transfusionsmedizin, Bonn, Deutschland

Hintergrund. Die Austauschtransfusion ist ein etabliertes Therapieverfahren bei Morbus haemolyticus neonatorum. Im Folgenden berichten wir über 4 Frühgeborene, die im Rahmen einer Sepsis eine ausgeprägte Hämolyse entwickelten und aufgrund dessen eine Austauschtransfusion erhielten.

\section{Fallberichte.}

- Kind 1: FG (1. Drilling) mit 30+5 SSW, Geburtsgewicht $1490 \mathrm{~g}$ - Kind 2: FG mit 34+1 SSW, Geburtsgewicht $2470 \mathrm{~g}$

- Kind 3: FG (2. Zwilling) mit 33+2 SSW, Geburtsgewicht $2090 \mathrm{~g}$

- Kind 4: FG mit 35+4 SSW, Geburtsgewicht $2560 \mathrm{~g}$.

Die Kinder 1, 3 und 4 benötigten postnatal kurzzeitig eine Atemunterstützung mittels CPAP, ansonsten verlief die postnatale Adaptation unauffällig. Der Nahrungsaufbau konnte regelrecht begonnen werden, Mekonium wurde zeitgerecht abgesetzt. Zwischen dem 2. und 5. Lebenstag entwickelten alle 4 Kinder eine fulminante Nekrotisierende Enterokolitis. Bei Kind 1 und 4 wurde im Verlauf Clostridium perfringens nachgewiesen, bei Kind 2 und 3 kein Erreger. Alle Kinder wurden zeitnah intubiert, operiert und erhielten eine kreislaufunterstützende Therapie mit Dobutamin und Noradrenalin, Kind 1 und 2 zusätzlich Suprarenin, Kind 3 und 4 zusätzlich Vasopressin. Alle Kinder entwickelten postoperativ eine ausgeprägte Hämolyse. Bei Kind 1 wurde noch am Operationstag eine Austauschtransfusion $(170 \mathrm{ml} / \mathrm{kg})$ durchgeführt, bei Kind 2 und 4 am 1. postoperativen Tag, bei Kind 3 am 2. postoperativen Tag (Austauschmenge jeweils $200 \mathrm{ml} / \mathrm{kg}$ ). Indikation für die Austauschtransfusion war bei Kind 1 und 2 eine rasch progrediente Hämolyse und Lactatazidose mit Kreislaufinsuffizienz, bei Kind 3 eine zunehmende Hämolyse mit rasch ansteigendem Serum-Bilirubin (max. $23 \mathrm{mg} / \mathrm{dl}$ ) und bei Kind 4 eine progrediente Hämolyse mit zügig ansteigender Serum-LDH. Alle Kinder waren während der jeweils über 


\section{Abstracts}

4 Stunden durchgeführten Austauschtransfusion stabil. Bei Kind 2, 3 und 4 konnte die Hämolyse durch die Austauschtransfusion erfolgreich therapiert werden. Kind 2 entwickelte im Verlauf erneut eine schwere Hämolyse und verstarb am 6. Lebenstag infolge einer therapierefraktären Kreislaufinsuffizienz. Kind 1 verstarb am 9. Lebenstag bei ebenfalls nicht beherrschbarer Kreislaufinsuffizienz. Kind 3 und 4 überlebten ohne Folgeschäden.

Ergebnisse. Alle vier Kinder entwickelten in den ersten Lebenstagen im Rahmen einer schweren Nekrotisierenden Enterokolitis eine Sepsis mit ausgeprägter Hämolyse. Die Austauschtransfusion konnte bei allen Kindern komplikationslos durchgeführt werden. Bei drei von vier Kindern konnte die Hämolyse durch die Austauschtransfusion erfolgreich behandelt werden.

Schlussfolgerung. Die Austauschtransfusion sollte als Therapieoption bei Sepsis mit schwerer Hämolyse frühzeitig in Erwägung gezogen werden. 


\section{Autorenindex}

Adams, Mark

Avian, Alexander

$\begin{array}{ll}\text { B } & \\ \text { Bachour, Haitham } & \text { PO-64 } \\ \text { Baden, Winfried } & \text { FV-30 } \\ \text { Bagci, Soyhan } & \text { FV-06, PO 17, } \\ & \text { PO-64 } \\ \text { Bahlmann, Hagen } & \text { PO-33 } \\ \text { Banaschak, Sybille } & \text { FV-59 } \\ \text { Baranowski, Stefanie } & \text { PO-76 } \\ \text { Barikbin, Payman } & \text { PO-82 } \\ \text { Barthlen, Winfried } & \text { PO-33 } \\ \text { Bartmann, Peter } & \text { FV-06, FV-38, } \\ & \text { FV-44, PO-14, } \\ & \text { PO-17, PO-18, } \\ & \text { PO72, PO-73, } \\ \text { Bartram, Claus R. } & \text { PO-104, PO-118, } \\ \text { Bartsch, Oliver } & \text { PO-120 } \\ \text { Bassler, Dirk } & \text { PO-84 } \\ \text { Baumgartner, Sigrid } & \text { PO-63, PO-96 } \\ \text { Beck, James F. } & \text { PO-44 } \\ \text { Becker, S. } & \text { PO-88 } \\ \text { Beckenbach, Elisabeth } & \text { PV-10 } \\ \text { Beckmann, Wiebke } & \text { FV-17 } \\ \text { Behrens, Christopher } & \text { PO-9 } \\ \text { Beker, Friederike } & \text { PO-87 } \\ \text { Bellusci, Saverio } & \text { FV-37 } \\ \text { Bendiks, Meike } & \text { FV-52 } \\ \text { Bendix, Ivo } & \text { PO-101 } \\ \text { Berg, Christoph } & \text { FV-08, FV-10, } \\ \text { Berger, Angelika } & \text { PO-68 } \\ \text { Bergert, Renate } & \text { PO-85 } \\ \text { Beringer, Ortraud } & \text { FV- } 02, \text { FV-07, FV-60, } \\ \text { Berneck, Nicole } & \text { PO-11, PO-20 } \\ & \text { PO-95 } \\ \text { PO-76 } \\ \text { PO-32 } \\ \end{array}$

A

Abel, R.

Abele, Harald

Aicher, Johannes

Akanbi, Sandra

Al Alam, Denise

Albayrak, Bilge

Ali, Anaam

Alshut, Franz-Josef

Alzen, Gerhard

Amon, Oliver

Andres, Oliver

Arenz, Stephan

Arenz, Tina

Arlettaz Mieth, Romaine

Armbrust, Sven

Avenarius, Stefan

Axt-Fliedner, Roland

\section{B}

Bernhard, Wolfgang

Bertling, Frederik

Bertsche, Astrid

Beyersdorff, Anke

Bickle, Myriam

Bielefeld, Thilo

Billing, Heiko

Binder, Christoph

Binder, Corinna

FV-63

FV-33, FV48,

$\mathrm{PO}-87$

PO-28

PO-117

FV-57

FV-57

PO-43

$\mathrm{PO}-1$

FV-33, FV-35

FV-17, PO-47

PO-100

\section{Baden, Winfried}

Baranowski, Stefanie

Barthlen, Winfrie

$\mathrm{PO}-48$

FV-16

$-52$

$-16$

(1)

\section{Burda, Gudrun}

Burgermeister, Carole

Burghard, Rainer

Burgmann, Maximiliane

Burkhardt, Tilo

Burkhardt, Wolfram

Buxmann, Horst

FV-01, FV-22
FV-08, FV10
PO-68
PO-30
FV-58
PO-15
PO-28
FV-02, FV-47,
PO-20
FV-17, FV-18,
PO-46,
PO-47
PO-96
PO-44
FV-54
PO-66
PO-99
PO-77
PO-25
FV-46
FV-36
PO-80
FV33, FV46
PO-18, PO-118
PO-26
FV-58
PO-52
PO-93
V-04
PO-68
PO-99
PO-48
PO-74
PO-24
FV-16
FO-105, PO-106
PO-21, PO-55,
PO-102
FV-33, PO-80
PO-31, PO-69
PO-51, PV-18
FV-43, FV-58
FV-50
PO-71
FV11, FV-13, FV-27,
FV-31, FV-32,
FV-34,
FV-43, PO-26,
PO-41,
PO-42, PO-67,
PO-88,
PO-114,

$\begin{array}{ll}\text { C } & \\ \text { Campbell, Douglas } & \text { FV-26 } \\ \text { Cannizzaro, Vincenzo } & \text { FV-43 } \\ \text { Celanowski, Catrice } & \text { PO-81 } \\ \text { Ceylan, Burcin } & \text { FV-08 } \\ \text { Chao, Cho-Ming } & \text { FV-52 } \\ \text { Cheng, Ji } & \text { FV-26 } \\ \text { Chin, Jennifer } & \text { FV-25 } \\ \text { Christoph, Jürgen } & \text { PO-97 } \\ \text { Cloppenburg, Eva } & \text { FV33 } \\ \text { Cohen, Nicki } & \text { PO-66 } \\ \text { Collins, Jennifer J. } & \text { FV-53 } \\ \text { Cremer, Malte } & \text { PO-114 } \\ \text { Czernik, Christoph } & \text { FV-31, FV-32, } \\ & \text { PO-26, } \\ & \text { PO-82, PO-83, }\end{array}$

D

Dähnert, Ingo

Dame, Christof

Däster, Corinne

Davis, Peter

Dawczynski, Kristin

de Beer, Cornelia

DeCarolis, Boris

Degenhardt, Petra

Deindl, Philipp

Deklerk, Kerstin

Desoye, Gernot

Dieckerhoff, C.

Diehl, Thilo

Dinger, Jürgen

Dingley, John

Dinten-Schmid, Barbara

Dirks, Juliane

Dittmann, Miriam

Dötsch, Jörg

Douwa, Ramadan

Draaken, Markus

Dresbach, Till

Dreschers, Stephan

Dreyhaupt, Jens

Drommelschmidt, Karla

Durina, Renata

Dzietko, Mark

\section{E}

Eaton, Farah

Edle von Oettinger,

Anna-Charlotte

Egender, Friedemann

Ehrhardt, Harald

Eichhorn, Friederike

Eifinger, Frank

Eis-Hübinger, Anna Maria

el Hafid, Zoubida
PO-2, PO-50

$\mathrm{PO}-26, \mathrm{PO}-83$

$\mathrm{PO}-43$

FV-37

PO-74, PO-88

$\mathrm{PO}-70$

$\mathrm{PO}-40$

PO-82

FV-60

PO-81

$\mathrm{V}-17$

PV-12

PO-6

PO-31

PO-66

Pfl.PO-4

PO-41

PO-111

FV-63

PO-38

$\mathrm{PO}-72, \mathrm{PO}-73$

$\mathrm{PO}-14$

PO-107, PO-108

FV-33

FV10

FV47

PO-68

FV-49, PO-53

$\mathrm{PO}-14 \mathrm{a}$

PO-49

FV-50, PO-7,

$\mathrm{PO}-100$

PO-88

FV-63, PO-79

$\mathrm{PO}-75$

PO-5 
el Helou, Salhab

Emmenegger, Ulrike

Endesfelder, Stefanie

Engels, Heike

Engler, Daniela

Entenmann, Andreas

Ergün Süleyman

Eyermann, Richard

Eymann, Regina

Eyrich, Matthias

\section{F}

Faas, Dirk

Fahnenstich, Hubert

Fandrey, Joachim

Fatehpour, Davud

Faust, Kirstin

Fehrholz, Markus

Felberg, K.

Felderhoff-Müser, Ursula

$\begin{array}{ll}\text { Fink, Christoph } & \text { PO-28, PO-32 } \\ \text { Fink, Naomi } & \text { FV23 } \\ \text { Fink, Susanne } & \text { PO-117 } \\ \text { Fischer, Doris } & \text { PO-14a } \\ \text { Fischer, Georg } & \text { PO-44 } \\ \text { Fleischer, Ulrike } & \text { PO-45, PO-70 } \\ \text { Fleming, Ingrid } & \text { FV42 } \\ \text { Flemmer, Andreas W. } & \text { FV-16, FV-39, } \\ & \text { FV-54, } \\ \text { Flosdorff, Patrick } & \text { PO-111, PO-112 } \\ \text { Förster-Waldl, Elisabeth } & \text { PO-99 } \\ \text { Franz, Axel } & \text { FV-07, PO-109 } \\ & \text { FV-01, FV-15, } \\ & \text { FV-22, } \\ \text { Freisinger, Peter } & \text { FV-30 } \\ \text { Frey, Monika } & \text { PO-24 } \\ \text { Frost, Verena } & \text { PO-7 } \\ \text { Frömming-Doll, Nicole } & \text { PO-17 } \\ \text { Fuchs, Hans } & \text { PV-13 } \\ & \text { FV-19, FV-33, } \\ \text { Fusch, Christoph } & \text { FV-61 } \\ & \text { FV-23, FV-24, } \\ & \text { FV-26, } \\ \text { Gerbig, Ines } & \text { FV-41, FV-25, } \\ \text { Fusch, Gerhard } & \text { PO-54 } \\ \text { G } & \text { FV-23, FV-24, } \\ \text { Ganschow, Rainer } & \text { FV-25, } \\ \text { Gebauer, Roman } & \text { FV-41, PO-54 } \\ \text { Geipel, A } & \\ \text { Gembruch, Ullrich } & \text { PO-6 } \\ \text { Gencik, Martin } & \text { PO-2, PO-50 } \\ \text { Genzel-Boroviczény, Orsolya } & \text { PO-10, PO-58, PO-32 } \\ & \text { FV-33, WS-14.1, } \\ & \text { WS-14.4, } \\ & \text { PO-14, PO-73, } \\ & \\ & \\ & \end{array}$

FV-33, PO-7,

PO-100

PO-25, PO-78

$\mathrm{PO}-68$

PO-38

FV-29

FV-55

Pfl.PO-6

FV-08, FV-10,

PO-4, PO-68,

PO-71, PO-101

PO-28, PO-32

$\mathrm{PO}-14 \mathrm{a}$

$\mathrm{PO}-70$

FV-16, FV-39,

FV-54,

$\mathrm{PO}-99$

$\mathrm{FV}-22$,

FV-23, FV-24,

FV-41, FV-25,

FV-25,

\section{Gerstl, Eva}

Gerull, Roland

Gfrörer, Stefan

Gille, Christian

Gnigler, Maria

Goelz, Rangmar

Gohlke, Bettina

Golej, Johann

Göpel, Wolfgang

Görner, Nicole

Gortner, Ludwig

Göttler, Susanne

Graeve, Pauline

Graul-Neumann, Luitgard

Groneck, Peter

Groß, Dominik

Groß, Stephanie

Große Ostendorf, Anna-Lena

Großer, Kay

Gründler, Kerstin

Grundmann, Ulrich

Grunt, Sebastian

Guthmann, Florian

H

Haas, Dorothea

Häfner, Helga

Haiden, Nadja

Halatsch, Marc-Eric

Hambsch, Jörg

Hamelmann, Eckard

Hammel, Markus

Hamscho, Nadia

Hansen, Gesine

Hansmann, Georg

Harnack, Harald

Härtel, Christoph

Hasbargen, Uwe

Hassan, Mohammad

Häusler, Martin

Hecher, Kurt

Heckmann, Matthias

.

Heep, Axel

Heimann, Konrad

Heimberg, Ellen

Heitmann, Friedhelm

Helfer, Sven

Helmer, Hans

Hennermann, Julia

Henrich, Wolfgang

Hentschel, Roland

Herber-Jonat, Susanne
FV-57

FV-57, PO-94

PO-14a

FV-04, FV-05,

PO-107, PO-108,

$\mathrm{PO}-110$

$\mathrm{PO}-86$

PO-48

$\mathrm{PO}-18$

PO-29

FV-29, FV-35,

FV-44,

PO-60, PO-101

FV-48

FV-51, PO-62,

PO-65

FV-26

PO-104

$\mathrm{PO}-82$

FV-34, FV-35

FV-14

PO-115

FV-59

PO-99

$\mathrm{PO}-22$

$\mathrm{V}-21$

FV-58

PO-97

PO-26

PO-113

FV-02, FV-47,

$\mathrm{PO}-20$

$\mathrm{PO}-76$

$\mathrm{PO}-50$

PO-22

FV-54

PO-87

FV-33

PO-41

PO-103

FV-44, FV-29,

FV-35,

PO-60, PO-101

FV-16

PO-56, PO-59

PO-75

PO-104

FV-26, FV-33,

FV-44,

FV-45,

$\mathrm{PO}-30, \mathrm{PO}-33$,

$\mathrm{PO}-115$

FV-44

FV-14, PO-23,

$\mathrm{PO}-75$,

PO-113

$\mathrm{PO}-28, \mathrm{PO}-32$

PO-101

FV-31, FV-32

$\mathrm{FV}-07$

PO-26

FV-13

FV-33

FV-36, FV-39,
Herbst, Johanna
Hermann, Frank
Hermon, Michael
Hernaiz-Driever, Pablo
Herrmann, Ralf
Hertfelder, Hans-Jörg
Herting, Egbert

Hetzel, Patrick

Heubner, Georg

Heydweiller, Andreas

Heyer, Christoph

Hildebrandt, Alexander

Hilgendorff, Anne

Hilger, Alina

Hillebrand, Georg

Hillen, Uwe

Hoch, Jochen

Hinner, Patricia

Hofbeck, Michael

Hoffmann, Wolfgang

Hofmann, Caroline

Hofmann, Nina Kathrin

Hofmann, Ute

Höhn, Thomas

Holzinger, Andreas

Hooper, Stuart

Hopfner, Reinhard

Hoppen, Thomas

Hoppenz, Marc

Horn, Denise

Horsch, Sandra

Howaldt, Hans-Peter

$\mathrm{Hu}$, Jiong

Huang, Li

Hübner, Sarah

Hummler, Helmut

Hüning, Britta

Husain, Ralf

Hüseman, Dieter

Husslein, Peter Wolf

Hütten, Matthias

Hütten, Matthias Christian

|

Ifflaender, Sascha

Irion, Lisa

Ittel, Thomas

J

Jani, Jacques

Jeitler, Valerie

Jellema, Reint K.

Jenke, Andreas

Jobe, Alan $\mathrm{H}$.

Jorch, Gerhard

Jotzo, Martina
FV-54

FV-15

FV-48

PO-29

PO-116

FV-10

PO-118

FV-29, FV-35,

FV-44,

FV-56, PO-60,

$\mathrm{PO}-101$

PO-81

$\mathrm{PO}-13$

$\mathrm{PO}-64$

$\mathrm{PO}-22$

$\mathrm{PO}-8, \mathrm{PO}-38$

$\mathrm{FV}-50$

$\mathrm{PO}-72, \mathrm{PO}-73$

PO-6

PO-4

PO-120

PV-11

PO-32

FV-45

PO-84

FV-50

$\mathrm{PO}-63$

FV-34, FV-35,

PO-90

FV-54

FV-37

FV-19

PO-8, PO-38

FV-34

$\mathrm{PO}-26, \mathrm{PO}-83$

$\mathrm{PO}-16$

$\mathrm{PO}-7$

FV-42

PO-56, PO-59

FV-48

FV-19, FV-33,

FV-36, FV-61,

PO-56, PO-59,

$\mathrm{PO}-76$

PO-71

$\mathrm{PO}-74$

FV-13

FV-07

PO-23

PO-77, FV-09

PO-21, PO-102

PO-15

PO-113

FV-39

PO-57

FV-09

FV-40

FV-09, FV-53

FV-33

PV-10 
Junge, Carl

Jurklies, Bernhard

PO-39

$\mathrm{PO}-22, \mathrm{PO}-71$

\section{K}

Kabus, Maria

Kaiser, Hedwig -J.

Kalaniti, Kaarthigeyan

Kalk, Janina

Kallapur, Suhas

Kannan, Cecil Varna

Kapapa, Thomas

Kasoha, Mariz

Kästner, Lars

Katrin, Manzke

Kattner, Evelyn

Katzer, David

Kavsek, Michael

Keller, D.

Keller, Matthias

Keller, Titus

Kemp, Matthew W

Kerb, Reinhold

Kerzel, Sebastian

Kettenmann, Helmut

Kiechl-Kohlendorfer, Ursula

Kipfmüller, Florian

Kirchhoff, Silke

Kirchner, Lieselotte

Klebermaß-Schrehof, Katrin

Kleinlein, Barbara

Klingmüller, Volker

Klinzmann, Madlen

Klippstein, Tamara

Knauss, Eva

Koch, Alexander

Koch, Arite

Koch, Martina

Koch, Vera

Koehne, Petra

Köhler, Nicole

Kohlhase, Jürgen

Kolokythas, Marie

Konstantelos, Dimitrios

Korall, Herbert

Körber, Andreas

Korinthenberg, Rudolf

Koss, Sarah

Kostelka, Martin

Köstlin, Natascha

Köthe, Lars

Krabbe, Grietje

Kramer, Alexandra

Kramer, Boris W.

Kramer, Hans-Heiner

Krauth, Alexander

Kreissl, Alexandra

Kreutzer, Karen

Kribs, Angela

Krötz, Ariane

Krutzke, Sophia Karoline

Kugel, Hellen
PO-13

PO-25

FV-41

PO-6

FV-09, FV-53

$\mathrm{PO}-78$

PO-76

FV-51

PO-100

$\mathrm{PO}-1$

PO-97

FV-06

PO-105, PO-106

$\mathrm{PO}-113$

FV-08, FV-10

PO-40

FV-09

PO-63

FV-03

FV-11

$\mathrm{PO}-86, \mathrm{~V}-27$

FV-38, PO-64

PO-111, PO-112

PO-57

PO-11

FV-33

$\mathrm{PO}-87$

$\mathrm{PO}-87$

$\mathrm{PO}-42$

FV-36

PO-63

PO-69

PO-6

FV-01, FV-22

PO-41, PO-42

PO-39

$\mathrm{PO}-24, \mathrm{PO}-25$

PO-111

PO-95

PO-24

$\mathrm{PO}-4$

V-37

FV-49

$\mathrm{PO}-2$

FV-04, FV-05,

PO-110

PO-55

FV-11

PO-111, PO-112

FV-09, FV-53,

FV-55,

$\mathrm{V}-22$

PO-49

PO-61

FV-02, PO-20

PO-63, PO-96

FV-34, FV-35,

PO-40, PO-60,

PO-105, PO-106

$\mathrm{PO}-8$

$\mathrm{PO}-72, \mathrm{PO}-73$

PO-110, FV04
Kühr, Joachim

Kujawa, Madelene

Kumpf, Matthias

Kunkel, Philip

Kuntz, Marco

Kunze, Andreas

Kunze, Rebecca

Kunzmann, Steffen

Küster, Helmut

Kuypers, Elke

Lackner, Herwig

L

Lange, Anja $\quad$ FV-45

Lange, Romy FV-56

Langhammer, Marcus

Lasch, Peter

Laschke, Matthias W.

Läßig, Mira

Latal, Bea

Lausch, Ekkehart

Lauterbach, Tina

Laux, Reinhard

Lee, Sau-Young

Lejeune, Alice

Lemmen, Sebastian W.

Lenhof, Susanne

Lettgen, Bernhard

Liebisch, Gerhard

Lindner, Wolfgang

Linge, Mike

Lippert, Miriam

Liu, Xun

Löffelbein, Florian

Longardt, Ann Carolin

Lorenz, Daniel

Lorenz, Laila

Lorenz, Norbert

Loui, Andrea

Ludwig, Michael

M

Maas, Christoph

Mache, Christoph J.

Maes, Elke

Maiberger, Thomas

Maier, Rolf Felix

Manan, Asmaa

Manser, Helen

Manthei, René

Markert, Sabrina

Marmé, Alexander

Marquardt, Thorsten

Marschitz, Ingrid

Maschke, Cornelia

Maurer, Miriam

Mayatepek, Ertan
$\mathrm{PO}-45, \mathrm{PO}-70$

$\mathrm{PO}-37$

PO-61

$\mathrm{PO}-111, \mathrm{PO}-112$

$\mathrm{PO}-28, \mathrm{PO}-32$

PO-39

$\mathrm{PO}-84$

PO-70

FV-01, FV-22

PO-117, FV-53,

FV-55

FV-20, FV-57,

PO-92

FV-09, FV-53

PO-119

FV51

PO-87

FV58

WS- 6

Pfl.PO-2, Pfl.PO-3,

PV-02

FV-34, FV-35

FV-41

PO-114

PO-113

FV-19

PV-10

FV-54

FV-33, FV-61

PO-90

PO-78

FV-12, PO-66

$\mathrm{PO}-2$

$\mathrm{PO}-83$

PO-80

FV-15

PO-13

FV-27

$\mathrm{PO}-72, \mathrm{PO}-73$

FV-01, FV-22,

PO-63,

PO-96

FV-62, PO-27

$\mathrm{PO}-66$

PO-39, PO-52

FV-03

PO-54

FV-57

PO-91

FV-03

FV-04

PO-19

PO-27, PO-34,

$\mathrm{PO}-35$,

PO-36, FV62

PO-85

PO-56

PO-90

McConaghy, Suzanne

Mehler, Katrin

Meier, Carola

Meinig, Silke

Meissner, Thomas

Mendler, Marc Robin

Menken, Udo

Mense, Lars

Merz, Eberhard

Merz, Waltraut Maria

Meyer, Sascha

Meyer Schiffer, Philipp

Michel, Miriam

Mildau, Céline-Fernande

Mildenberger, Eva

Misselwitz, Bernd

Mittal, Rashmi

Möbius, Marius Alexander

Modrow, Susanne

Moebus, Susanne

Mollenhauer, Jan

Möller, Jens

Monz, Dominik

Moschkau, Nicole

Mostafa, Gehan

Muche, Rainer

Mücke, Sonja

Mueller, Andreas

Muenssinger, Jana

Müller, Andreas

Müller, Annette M.

Müller, Christian Andreas

Müller, Hanna

Müller, Wolfgang

Murthy, Prashanth

N

Nagel, Manuela

Natalucci, Giancarlo

Navarro-Psihas, Salvador

Nelle, Mathias

Neuberger, Patrick

Neuhaus, Petra

Neunhoeffer, Felix

Newnham, John P.

Nicolai, Heide

Nikiforou, Maria

Nilles, Louise

Nitsos, llias

Nordwig, Antje

Nöthen, Markus M.

Nußbaum, Claudia

Nüßlein, Thomas 


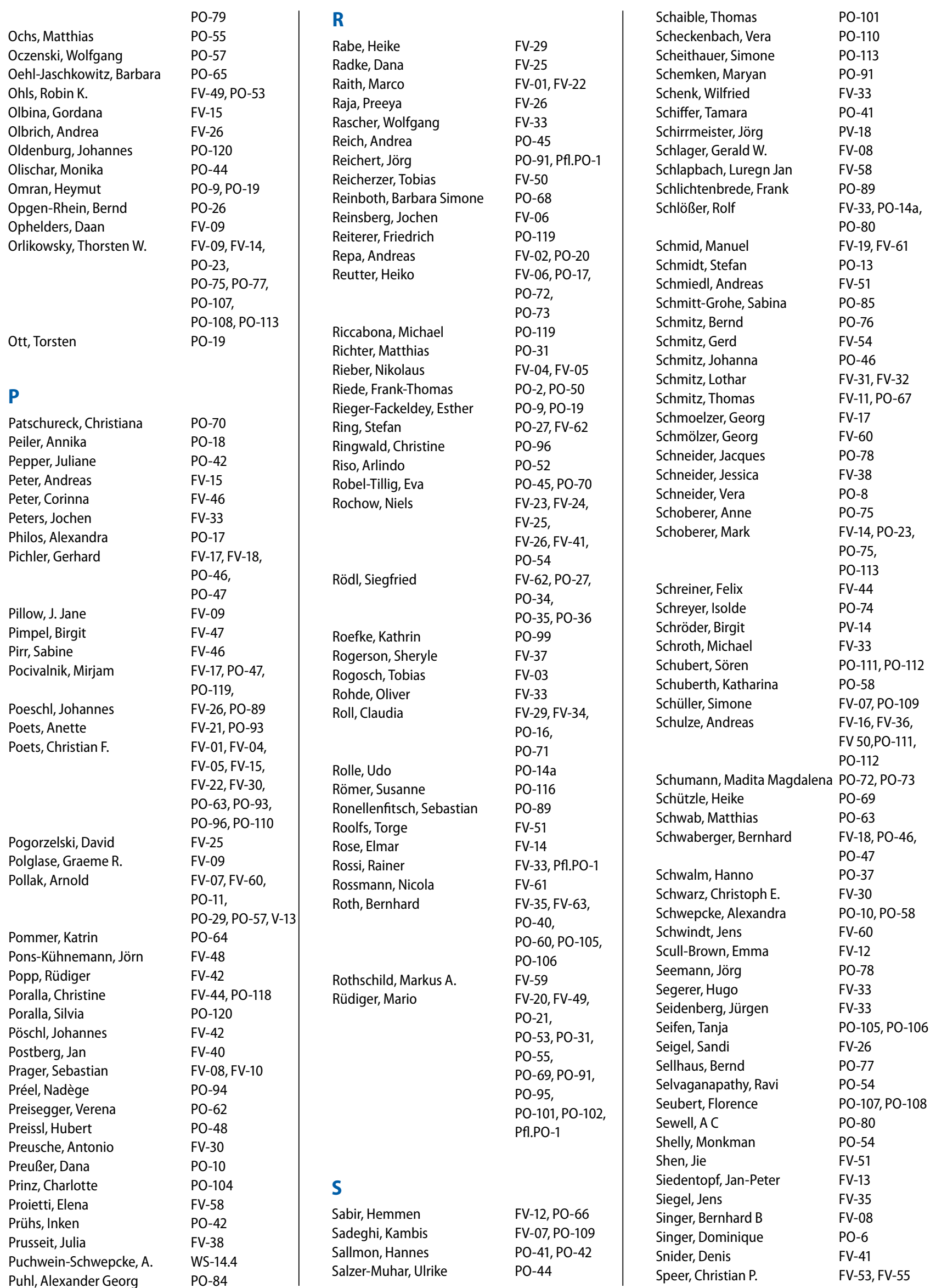


Spieles, Helen

Spittler, Andreas

Sommerfeld, Marion

Spors, Birgit

Spring, Bärbel

Stahl, Anna

Stamm, Daniel

Stangl, Oliver

Staude, Carolin

Stein, Anja

Steiner, Manuel

Steinhardt, Alina

Stenzel, Werner

Stichtenoth, Guido

Sticker, $\mathrm{M}$.

Stoffel, Liliane

Straßer, Katja

Straub, Julia

Streckbein, Philipp

Streiftau, Silke

Stutte, Sonja

Szabo, Miklos

T

Teichmann, Alexander Tobias PO-103

Teig, Norbert

Tekaat, Maria

Thabane, Lehana

Thébaud, Bernard

Thiel, Christian

Thiel, Michael

Thome, Ulrich

Thoresen, Marianne

Thyrian, Rene

Timme, Katharina

Tiozzo, Caterina

Topf, Hans-Georg

Trepels-Kottek, Sonja

Trittenwein, Gerhard

Tröbs, Ralf-Bodo

Trotter, Andreas

Tschernig, Thomas

Tutdibi, Erol

U

Umlauf, Volker

Urban, Christian

Urlesberger, Berndt

Urschitz, Michael S.

\section{V}

v. Wolff, Bodo

Valter, Markus

Vargha, Regina
FV-33, FV-34,

$\mathrm{PO}-22$,

PO-101

FV05

FV07, PO-109

$\mathrm{PV}-11$

FV-04, FV-05,

$\mathrm{PO}-110$

$\mathrm{PO}-22$

PO-37

$\mathrm{PO}-103$

$\mathrm{PO}-86$

$\mathrm{PO}-4$

PO-11, PO-44

Pfl.PO-1

PO-83

FV-56, PO-101

PV-18

Pfl.PO-4

PO-68

PO-11

PO-7

FV36

PO-18

FV-29

PO-9

FV-26

FV-49, PO-53

PO-52

PO-12

FV-33, FV-48,

PO-50

FV-12, PO-66

FV-45

FV-33

FV-52

FV-33

FV-14, PO-23,

$\mathrm{PO}-75$,

PO-77, PO-113

PO-29

PO-16

PO-15

FV-51

FV-51, PO-62

FV-14, PO-77

PO-119

FV-17, FV-18,

PO-46,

PO-47,

PO-119, V-07

PO-93

FV-28

PO-79

PO-29
Vierzig, Anne

Villavicencio Lorini, Pablo

Vlajnic, D

PO-79

WS-6

PV-18

Vochem, Matthias

Voigt, Friedrich

von Bernuth, Horst

von der Wense, Axel

von Laer, Marina

Vuckovic, Aline

W

Wacker-Gußmann, Annette PO-48

Wagner, Johanna-Josophina FV-03

Waitz, Markus

Wald, Martin

Waldhör, Thomas

Walter, Gabi

Weber, Karin

Weber, Sven Christian

Weichelt, Ulrike

Weidner, Anne

Weis, llona

Weiss, Katja

Weiss, Magdalene

Weißhaar, Guido

Weitkämper, Almut

Welcker, Silvia

Weller, Ursula

Wellmann, Sven

Welzing, Lars

Wendelin, Gerald

Weninger, Manfred

Werther, Tobias

Weschke, Bernhard

Westerman, Mark

Wiederkehr, Stephanie

Wieg, Christian

Wiegand, Susanne

Wiegering, Verena

Wien, Sebastian Benedikt

Wilbrand, Jan-Falco

Wilbrand, Martina

Wild, Claudia

Wilke, Mikosch

Wirth, Stefan

Wisgrill, Lukas

Wisniweski, Hans-Georg

Witt, Charlotte

Wolf, Gerhard

Wölfle, Joachim

Wolfs, Tim G.A.M.

Wollny, Caroline

Wong, Connie

Wörnle, Sabine

Wu, Wen

FV-35

FV-36

PO-114

FV-35, PO-39,

PO-52

PO-78

FV39

PO-56, PO-59

PO-44, PO-57

PO-44

FV-56

PO-96

PO-41, PO-42

PO-67

PO-33

$\mathrm{PO}-8$

$\mathrm{PO}-42$

PO-48

$\mathrm{PO}-79$

PO-22

PO-115

FV-35

FV-43

FV-06, FV-34,

PO-17

FV-62,

PO-27,PO-34,

PO-35, PO-36

PO-57

PO-11

PO-83

FV-15

Pfl.PO-5

FV-29, FV-34,

FV-35,

PO-103

PO-22

PO-117

$\mathrm{PO}-8$

$\mathrm{PO}-7$

$\mathrm{PO}-7$

PO-81

FV-29

FV-40

FV-07, PO-109

FV-10

PO-19

PO-24

PO-18

FV-09

PO-65

FV-37

PO-24

PO-54

$\begin{array}{ll}\text { Zaak, Irina } & \text { PO-67 } \\ \text { Zapanta, Bianca } & \text { FV-24 } \\ \text { Zemlin, Michael } & \text { FV-03 } \\ \text { Zenz, Werner } & \text { PO-34, PO-35, PO-36 } \\ \text { Zernickel, Maria } & \text { FV-33 } \\ \text { Zhong, Shumei } & \text { FV-49, PO-53 } \\ \text { Ziehenberger, Evelyn } & \text { FV-18 } \\ \text { Zimmer, Klaus-Peter } & \text { FV-52 } \\ \text { Zimmermann, Andrea } & \text { FV-33 } \\ \text { Zimmermann, Anne } & \text { PO-48 } \\ \text { Zindel, Katharina } & \text { FV-45 } \\ \text { Zobel, Gerfried } & \text { FV-62, PO-27, PO-34, } \\ \text { Zur, Bernd } & \text { PO-35, PO-36 }\end{array}$

\section{Z}

